\title{
ISOTOPE PRODUCTION AND AVAILABILITY FROM POWER REACTORS
}

\author{
D. E. DEONIGI \\ R. W. MCKEE \\ D. R. HAFFNER
}

JULY 1968

\section{AEC RESEARCH \& DEVELOPMENT REPORT}

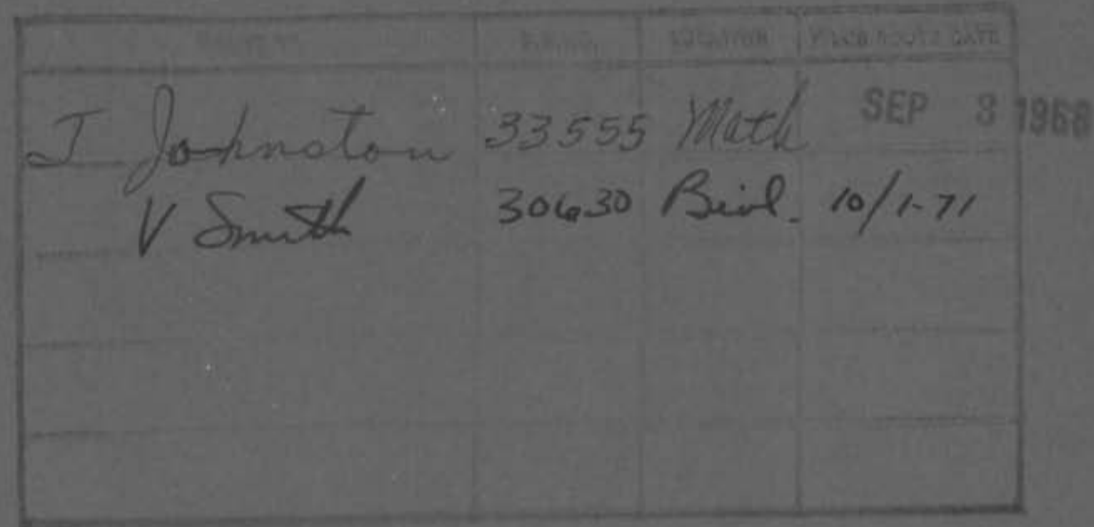

B A T T E L LE BATIELLE MEMORIAL INSTITUTE D PACIFIC NORTHWEST LABORATORY BATTELLE BOULEVARD. P. O. 80X 999 , AICHLAND, WASHINGTON 99352 


\title{
LEGAL NOTICE
}

This report was prepared as an account of Government sponsored work. Neither the United States, nor the Commission, nor any person acting on behalf of the Commission:

A. Makes any warranty or representation, expressed or implied, with respect to the accurocy, completeness, or usefulness of the information contained in this report, or that the use of any informotion, apparatus, method, or process disclosed in this report may not infringe privately owned rights; or

8. Assumes any liabilities with respect to the use of, or for domages resulting from the use of any information, apparatus, method, or process disclosed in this report.

As used in the above, "person acting on behalf of the Commission" includes any employee or contractor of the Commission, or employee of such contractor, to the extent that such employee or contractor of the Commission, or employee of such contractor prepares, disseminates, or provides access to, any information pursuant to his employment or contrat with the Commission, or his employment with such contractor.

\author{
PACIFIC NORTHWEST LABORATORY \\ RICHLAND, WASHINGTON \\ operated by \\ BATTELLE MEMORIAL INSTITUTE \\ for the \\ UNITED STATES ATOMIC ENERGY COMMISSION UNDER CONTRACT AT(45-1)-1830
}




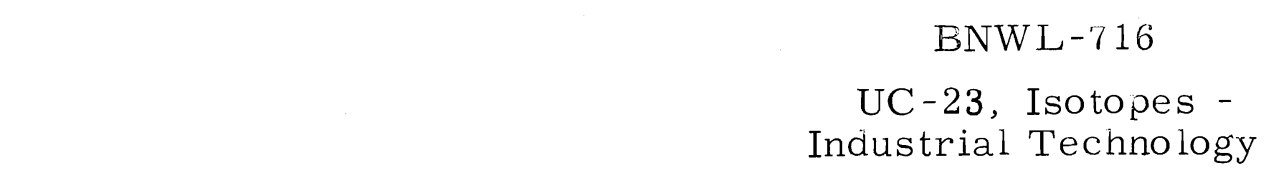

ISOTOPE PRODUCTION AND AVAILABILITY

FROM POWER REACTORS

D. E. Deonigi

R. W. McKee

D. R. Haffner

Engineering Economics Section

Systems Engineering Department

July 1968

PACIFIC NORTHWEST LABORATORY

RICHLAND, WASHINGTON 



\begin{abstract}
This report presents the projected availability of radioactive and stable isotopes from U.S. nuclear power reactors through the year 1990. The availability projections are for total spent fuel, U-235, U-236, total plutonium, Np-237, $\mathrm{Pu}-238, \mathrm{Am}-241, \mathrm{Am}-243, \mathrm{Cm}-242, \mathrm{Cm}-244, \mathrm{Pm}-147$, $\mathrm{Ce}-144, \mathrm{Cs}-137, \mathrm{Sr}-90, \mathrm{Kr}-85, \mathrm{Ru}-106, \mathrm{H}-3, \mathrm{Tc}-99, \mathrm{Rh}-103$, stable palladium, stable xenon, stable krypton, and stable ruthenium. Also included in this report is a complete printout of the ISOPRO computer program for the eight different cases that were evaluated.
\end{abstract}




\section{TABLE OF CONTENTS}

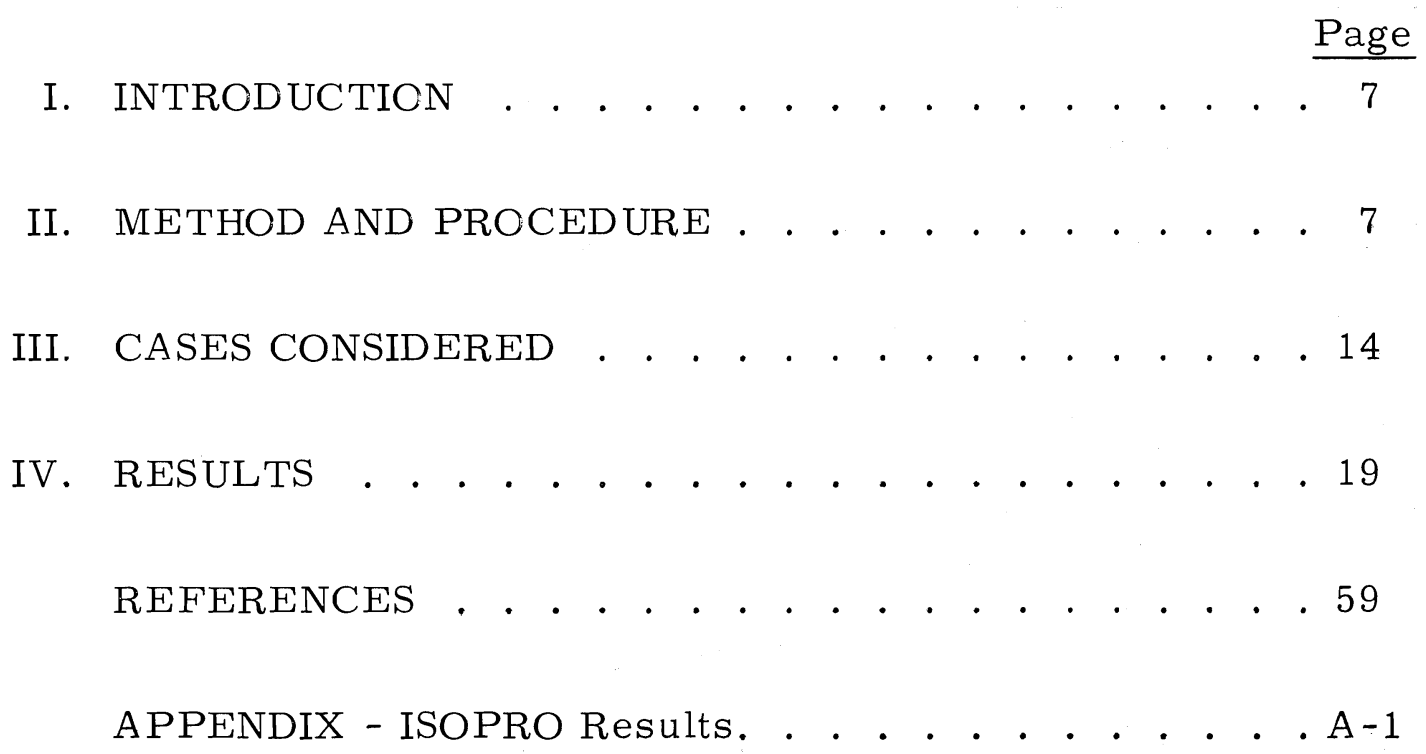




\section{LIST OF TABLES}

Number

Title

Page

I Ground Rules for Isotope Production Projections

II Description of Cases

III Annual Power Reactor Fuel Discharges, MT/yr

IV Comparison of Installed Capacity in Each Case by Reactor Type

$\mathrm{V}$ Comparison of Plutonium Recycle Capacity Installed in Plutonium Recycle Cases

VI Cumulative Plutonium Stockpile, MT

VII Annual Availability of U-236, $\mathrm{MT} / \mathrm{yr}$

VIII Annual Availability of $\mathrm{Np}-237, \mathrm{~kg} / \mathrm{yr}$

IX Annual $\mathrm{Pu}-238$ Availability from Np-237 Target Irradiation, $\mathrm{kg} / \mathrm{yr}$

X Annual Pu-238 Availability from Cm-242 Decay, $\mathrm{kg} / \mathrm{yr}$

XI Annual Pu-238 Availability from Target Irradiation of Mixed Americium Isotopes, $\mathrm{kg} / \mathrm{yr}$

XII Total Annual Pu-238 Availability from All Sources, $\mathrm{kg} / \mathrm{yr}$

XIII Cumulative Availability of $\mathrm{Pu}-238$ from All Sources

XIV Annual Availability of Am-241 in Americium Mixture, $\mathrm{kg} / \mathrm{yr}$

XV Annual Availability of Am-243 in Americium Mixture, $\mathrm{kg} / \mathrm{yr}$

XVI Annual Availability of Am-241 from Processing Plutonium Stockpile, $\mathrm{kg} / \mathrm{yr}$

XVII Annual Availability of Cm-244 Recovered Directly from Fuel, kg/yr

XVIII Annual Availability of Cm-244 from Target Irradiation of Mixed Americium Isotopes, $\mathrm{kg} / \mathrm{yr}$ 


\section{LIST OF TABLES (Continued)}

\begin{tabular}{|c|c|c|}
\hline Number & Title & Page \\
\hline XIX & $\begin{array}{l}\text { Total Annual Availability of } \mathrm{Cm}-244 \text { from Both } \\
\text { Spent Fuel and Americium Target Irradiation, } \mathrm{kg} / \mathrm{yr}\end{array}$ & 40 \\
\hline XX & $\begin{array}{l}\text { Cumulative Availability of } \mathrm{Cm}-244 \text { from Both Spent } \\
\text { Fuel and Americium Target Irradiation }\end{array}$ & 41 \\
\hline XXI & Annual Availability of Pm-147, $\mathrm{kg} / \mathrm{yr}$ & 43 \\
\hline XXII & Annual Availability of $\mathrm{Ce}-144, \mathrm{~kg} / \mathrm{yr}$ & 44 \\
\hline XXIII & Annual Availability of $\mathrm{Cs}-137, \mathrm{~kg} / \mathrm{yr}$ & 45 \\
\hline XXIV & Annual Availability of $\mathrm{Sr}-90, \mathrm{~kg} / \mathrm{yr}$ & 46 \\
\hline XXV & Annual Availability of $\mathrm{Kr}-85, \mathrm{~kg} / \mathrm{yr}$ & 47 \\
\hline XXVI & Annual Availability of $\mathrm{Ru}-106, \mathrm{~kg} / \mathrm{yr}$ & 48 \\
\hline XXVII & Annual Availability of $\mathrm{H}-3$, g/yr & 49 \\
\hline XXVIII & $\begin{array}{l}\text { Cumulative Availability of Principal Radioactive } \\
\text { Fission Products }\end{array}$ & 50 \\
\hline XXIX & Annual Availability of $\mathrm{Tc}-99$, $\mathrm{kg} / \mathrm{yr}$ & 52 \\
\hline $\mathrm{XXX}$ & Annual Availability of Stable Rh-103, kg/yr & 53 \\
\hline XXXI & Annual Availability of Stable Palladium, kg/yr & 54 \\
\hline XXXII & Annual Availability of Stable Xenon, kg/yr & 55 \\
\hline XXXIII & Annual Availability of Stable Krypton, kg/yr & 56 \\
\hline XXXIV & Annual Availability of Stable Ruthenium, kg/yr & 57 \\
\hline XXXV & $\begin{array}{l}\text { Cumulative Availability of Principal Stable Fission } \\
\text { Products }\end{array}$ & 58 \\
\hline
\end{tabular}


LIST OF FIGURES

Number

1 Neptunium-237 Target Irradiation Reaction

2 Neptunium-237 Target Irradiation Cycle in Power Reactors

3 Americium Target Irradiation Reactions

4 Americium Target Irradiation Cycle in Power Reactors
Title

Page

12

13

15

16 


\section{ISOTOPE PRODUCTION AND AVAILABILITY \\ FROM POWER REACTORS}

\section{Introduction}

This report presents projections through the year 1990 of quantities of various isotopes recoverable from spent fuel to be discharged from U.S. nuclear power reactors. Secondary irradiation of recovered neptunium and americium to supplement the production of $\mathrm{Pu}-238$ and $\mathrm{Cm}-244$ is also considered.

The recovery of certain transuranium and fission product isotopes from spent nuclear fuels is of interest because these various radioactive and stable isotopes have uses as heat sources, radiation sources, rare elements, and target isotopes. Some of these isotopes, because they are difficult to contain, are potential contaminants to our environment.

To evaluate the possible useful applications or hazards of these isotopes, as well as the economics of their recovery, estimates are needed of the quantities of these isotopes that are potentially available. The projections presented here were developed to meet this need. The work was sponsored by the U.S. Atomic Energy Commission, Division of Isotopes Development.

An earlier and less detailed version of these projections was published as BNWL-SA-1529. (1) This report represents a complete revision and updating, but the results for similar cases are generally comparable in most respects.

II. Method and Procedure

Estimating the potentially available quantities of these isotopes required us to consider the number and type of reactors that would be operating, the method and schedule of their operation, and the detailed spent-fuel composition data for both startup and equilibrium fuel cycles. The secondary irradiation of neptunium and americium target isotopes to produce $\mathrm{Pu}-238$ and $\mathrm{Cm}-244$ was also considered. 
Spent fuel composition data were obtained by the burnup code ALTHAEA ${ }^{(2)}$ developed at Battelle-Northwest for fuel-cycle analysis studies. This code has been well calibrated with actual light water reactor burnup and critical data, and is capable of providing good estimates of the transuranium and fission-product isotope content in spent fuels.

Separate burnup calculations for each reactor type considered were made for each of the different fuel exposures involved in the startup and equilibrium cycles. With this information a complete schedule of fuel discharges and compositions could be defined for the 30-year operating life of a reactor. Because the ALTHAEA program provides detailed spent-fuel composition data for 11 standard isotopes, plus up to 9 other isotopes, and because some 40 different fission product or transuranium isotopes were involved in these projections, it was necessary to run the program several times for each set of conditions to obtain the complete information required.

This procedure worked very well for the initial projections that were developed, but as the systems under consideration became more complex it proved too cumbersome. For the uranium and transuranium fuel components, the PACTOLUS program, (3) developed for systems analysis studies of the U.S. power economy, was adapted to convert the ALTHAEA output into 30-year fuel discharge and composition histories. To avoid the necessity of repetitive ALTHAEA runs, capability was added to the ISOPRO program (discussed below), to calculate the quantities of the fissionproduct components in the spent fuel. These calculations utilized composite fission yields based on relative number of fissions in each of the fissile isotopes, as calculated by ALTHAEA. The results are not as theoretically precise as they could be had these fission-product compositions been calculated by ALTHAEA, but the results did closely agree with the ALTHAEA results where comparisons could be made.

Fuel discharge histories were developed both for a $19701000 \mathrm{MW}$ boiling water reactor (BWR) based on the TVA Brown's Ferry design and for a $19701000 \mathrm{MW}$ e pressurized water reactor (PWR) based on the Diablo Canyon design. Separate histories for uranium and plutonium recycle 
operation were also prepared. A more advanced design in which the specific power was increased approximately $15 \%$ was assumed to be available in 1980 both for the BWR and the PWR. Because of the limitations on the number of different reactor types that could be considered in ISOPRO and because such a small amount of nuclear capacity is scheduled for operation prior to 1970, the 1970 design-reactor histories were also used for the pre-1970 installations. A fast breeder reactor history was prepared, based on a Westinghouse $1000 \mathrm{MW}_{\mathrm{e}}$ LMFBR design study. (4)

For projecting total isotope availability, the information on individual reactor discharge schedules and fuel compositions was combined with nuclear power growth projections by using a new computer program, ISOPRO, developed specifically for this purpose. This program combines the detailed fuel composition histories from up to 15 different reactor types with specified nuclear growth projections to produce both annual and cumulative projections of discharged fuel quantities for up to 30 fuel components, including target irradiation products, both at the time of reactor discharge and at the time of recovery. In this study, projections were developed for eight uranium and transuranium isotopes, total plutonium and 13 fission product isotopes or combinations of isotopes. A complete printout of the ISOPRO program for eight cases evaluated in this study constitutes the Appendix to this report.

One major advantage of this calculational procedure, compared with simplified methods based on average or equilibrium conditions, is that it accurately reflects the effects of the low-fuel-exposure conditions during startup cycles. In a period of rapid growth, such as is predicted for the next two decades, the startup-cycle effects are quite significant, since new capacity additions for each year range from over $100 \%$ to no less than $10 \%$ of the previously existing capacity through the year 1990. Thus there is always a significant fraction of capacity in the startup phase during this period.

Another advantage of this procedure is the capability to account for time delays and inventory requirements for fuel reprocessing, target irradiations, and fuel recycle operations 
The projection of isotope production and availability from power reactors depends more than anything else on the assumed nuclear power growth projection, there being, of course, a substantial uncertainty in any nuclear power growth projection. The current AEC projection is for 120 to $180 \mathrm{GW}_{\mathrm{e}}$ installed nuclear capacity in 1980 , with the best estimate at $150 \mathrm{GW}_{\mathrm{e}}\left(150,000 \mathrm{MW}_{\mathrm{e}}\right)$. The base projection used in this study predicts that the $150 \mathrm{GW}_{\mathrm{e}}$ installed at the end of 1980 will increase to $500 \mathrm{GW}_{\mathrm{e}}$ installed at the end of 1990. Also included in this report are projections based on upper limits of $180 \mathrm{GW}_{\mathrm{e}}$ in 1980 increasing to $600 \mathrm{GW}_{\mathrm{e}}$ in 1990 and lower limits of $120 \mathrm{GW}_{\mathrm{e}}$ in 1980 increasing to $400 \mathrm{GW}_{\mathrm{e}}$ in 1990 . In effect, an uncertainty of $\pm 20 \%$ is assumed in the nuclear power growth projections, and this is probably the major uncertainty involved in the isotope availability projections presented here.

In addition to the nuclear power growth projection, certain other assumptions and ground rules were required for this study. These are summarized in Table $\mathbb{I}$.

Two secondary target irradiation cycles are involved in these projections: neptunium irradiation to produce $\mathrm{Pu}-238$ and americium irradiation to produce both $\mathrm{Pu}-238$ and $\mathrm{Cm}-244$.

The basic nuclear reactions involved in the $\mathrm{Np}-237$ target irradiation are shown in Figure 1. Other reactions involve formation of plutonium isotopes by neutron capture in $\mathrm{Pu}-239$ and $\mathrm{Pu}-236$ formation by a $\mathrm{Np}-237$ $\mathrm{n}-2 \mathrm{n}$ reaction. A small amount of $\mathrm{Pu}-238$ fission also occurs.

The neptunium target irradiation production cycle used for these projections is illustrated schematically in Figure 2. This cycle is representative of target irradiation in power reactors having a neutron flux of about $2 \times 10^{13} \mathrm{n} / \mathrm{cm}^{2} / \mathrm{sec}$. It was further assumed that before the year 1981 neptunium would be irradiated in AEC reactors having a flux of about $1 \times 10^{14} \mathrm{n} / \mathrm{cm}^{2} / \mathrm{sec}$. For the period prior to 1981 , a one-year neptunium irradiation and recovery cycle was assumed with a $21 \%$ conversion to $\mathrm{Pu}-238$ and a $70 \%$ recovery of neptunium for recycle. After 1980, the powerreactor irradiation cycle that was assumed is that shown in Figure 2. 
TABLE I

\section{GROUND RULES}

\section{FOR ISOTOPE PRODUCTION PROJECTIONS}

1. Installed nuclear capacity and isotope production and availability are always stated as of the end of year shown.

2. Yearly capacity additions startup at mid-year.

3. Nuclear power plant life is 30 years.

4. Nuclear power plant capacity factors are $85 \%$ for 15 years, declining linearly to $50 \%$ at 30 years.

5. Light Water Reactors (LWRs) are 67\% PWRs and 33\% BWRs after 1971. Contract commitments and operating reactors are used for the earlier years.

6. One year is allowed between the reactor discharge and isotope recovery. Isotope recovery is corrected for decay losses.

7. Plutonium and uranium recovery is $98 \%$.

8. All other by-product recovery is $90 \%$.

9. Pm-147 recovery is delayed 3 years after fuel discharge to allow for Pm-148 decay.

10. Cm-244 recovery is delayed 3 years after fuel discharge to allow for $\mathrm{Cm}-242$ decay.

11. Pu-238 formed by Cm-242 decay, except for first year decay prior to separations, is available with the $\mathrm{Cm}-244$. 


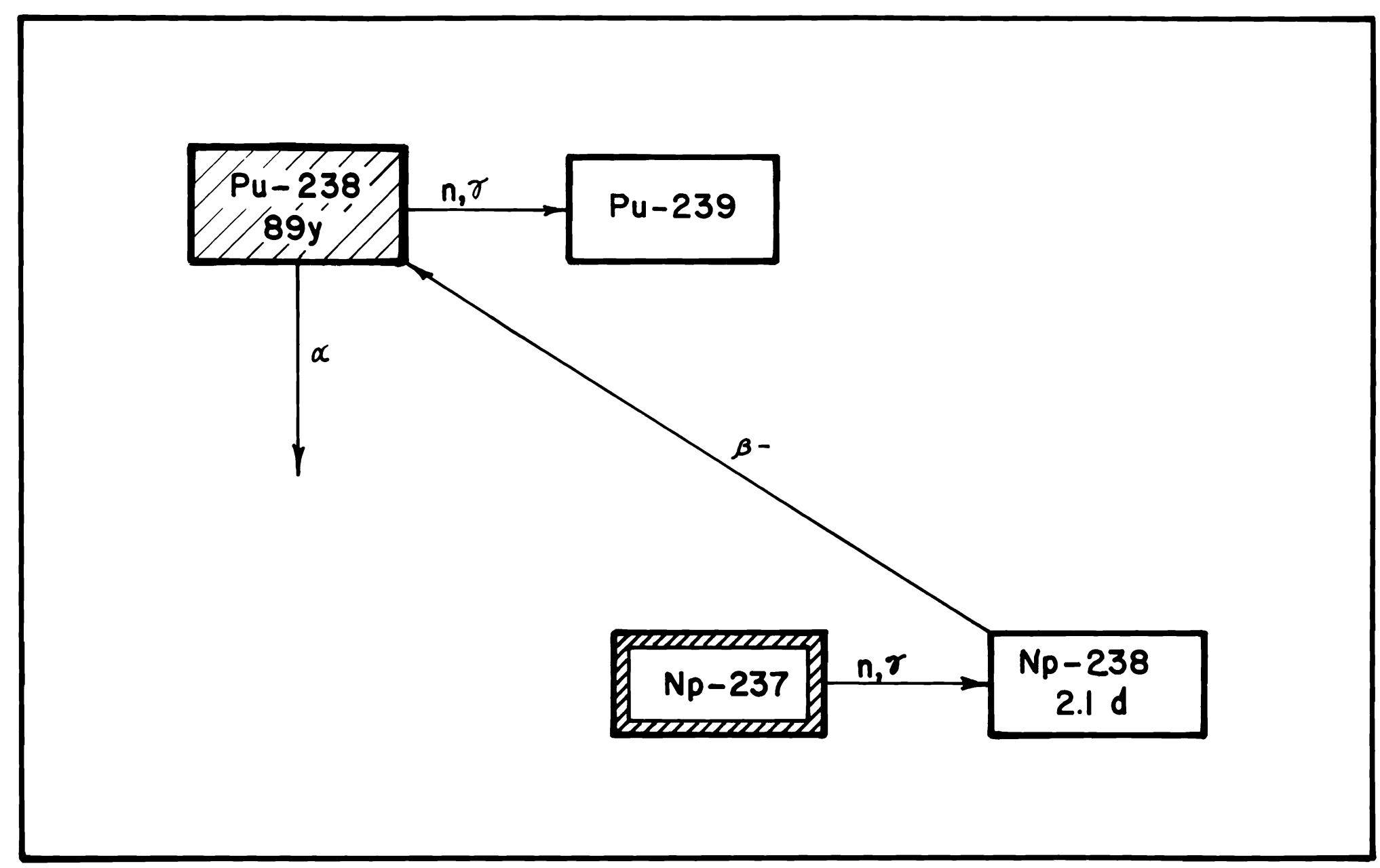

FIGURE 1. Neptunium-237 Target Irradiation Reaction 


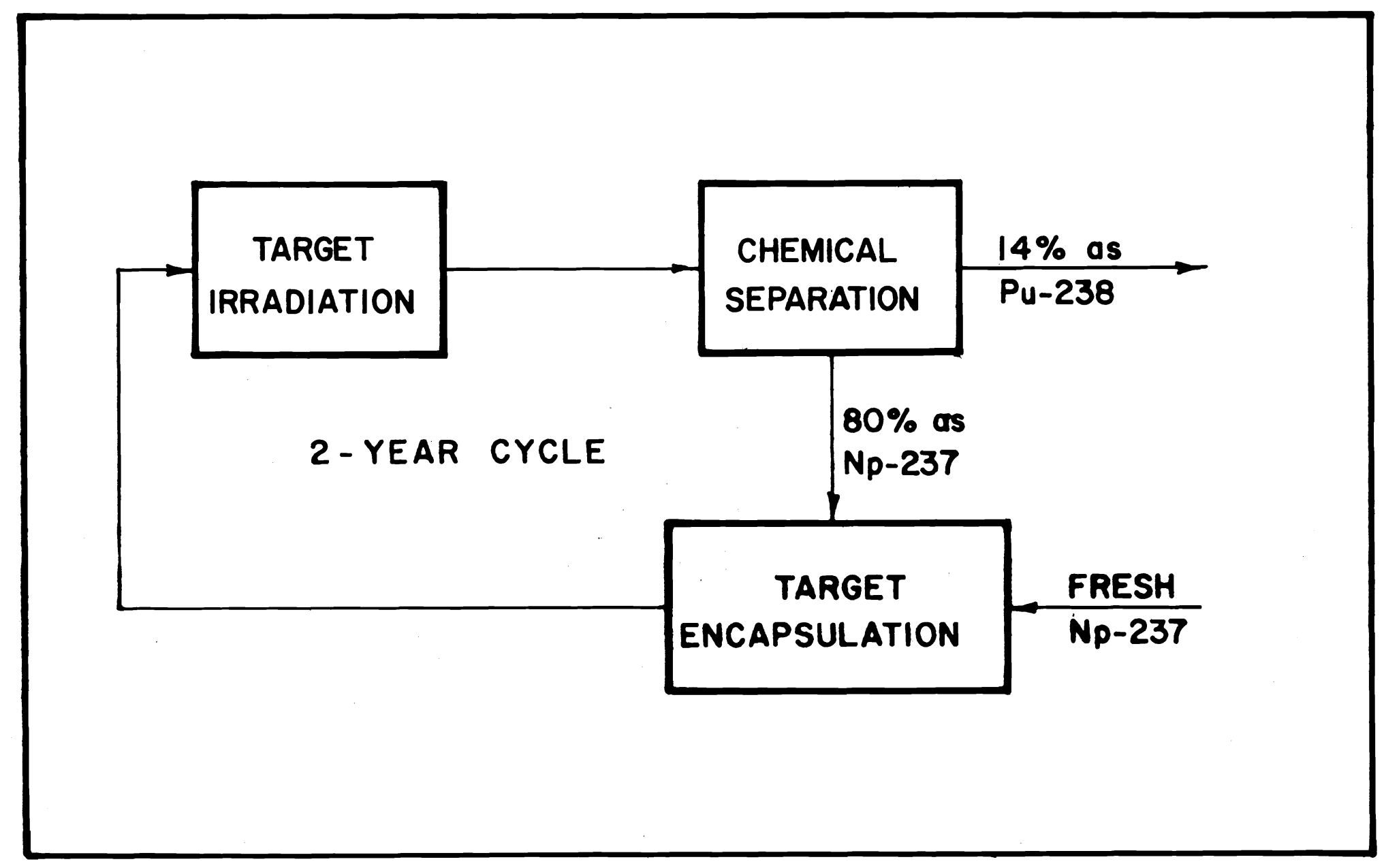

FIGURE 2. Neptunium-237 Target Irradiation Cycle 
The principal nuclear reactions involved in the target irradiation of the mixed americium isotopes (Am-241 and Am-242) are shown in Figure 3 with the ultimate products being $\mathrm{Pu}-238$ and $\mathrm{Cm}-244$. Production of high purity $\mathrm{Pu}-238$ is difficult because of the partial decay of Am-242 to $\mathrm{Pu}-242$, but there is no $\mathrm{Pu}-236$ formation as in $\mathrm{Np}-237$ irradiation. Product recovery is delayed by the 163-day half-life $\mathrm{Cm}-242$. In this study a two-year period was allowed between target irradiation and recovery of the products. This decay period is required to reduce the activity of the curium product enough for processing and to stabilize the heat output of the curium product by allowing the $\mathrm{Cm}-242$ component to decay to the point where the heat output of the $\mathrm{Cm}-242$ is no greater than the heat output of the $\mathrm{Cm}-244$. A two-year decay period also allows for maximum $\mathrm{Pu}-238$ recovery. It has been suggested that during this period the material might be used as a high intensity heat source to take advantage of the decay heat from $\mathrm{Cm}-242$. However, this concept has not attracted great interest.

The americium target irradiation production cycle used for these projections is illustrated schematically in Figure 4, and is based on power reactor target irradiations. No consideration is given here to high flux irradiation of the americium, although it is certainly a possibility.

\section{Cases Considered}

The light-water-reactor concept, both BWR and PWR, is now so well established that it will undoubtedly dominate the power reactor industry for at least the next 5 to 10 years. However, the extent to which plutonium or uranium recycle will be used is uncertain. Also uncertain is the extent to which some other type of reactor, advanced converter or fast breeder, may enter the picture in the late 1970 s or 1980 s.

To encompass these uncertainties as well as the uncertainties previously discussed relating to nuclear power growth projections, the eight cases in Table II were defined for evaluation.

Case 1 assumed that the installed nuclear capacity in 1980 would be $150 \mathrm{GW}_{\mathrm{e}}$, that Light Water Reactors (LWRs) would be the only type of reactor installed, and that there would be no uranium or plutonium recycle. 


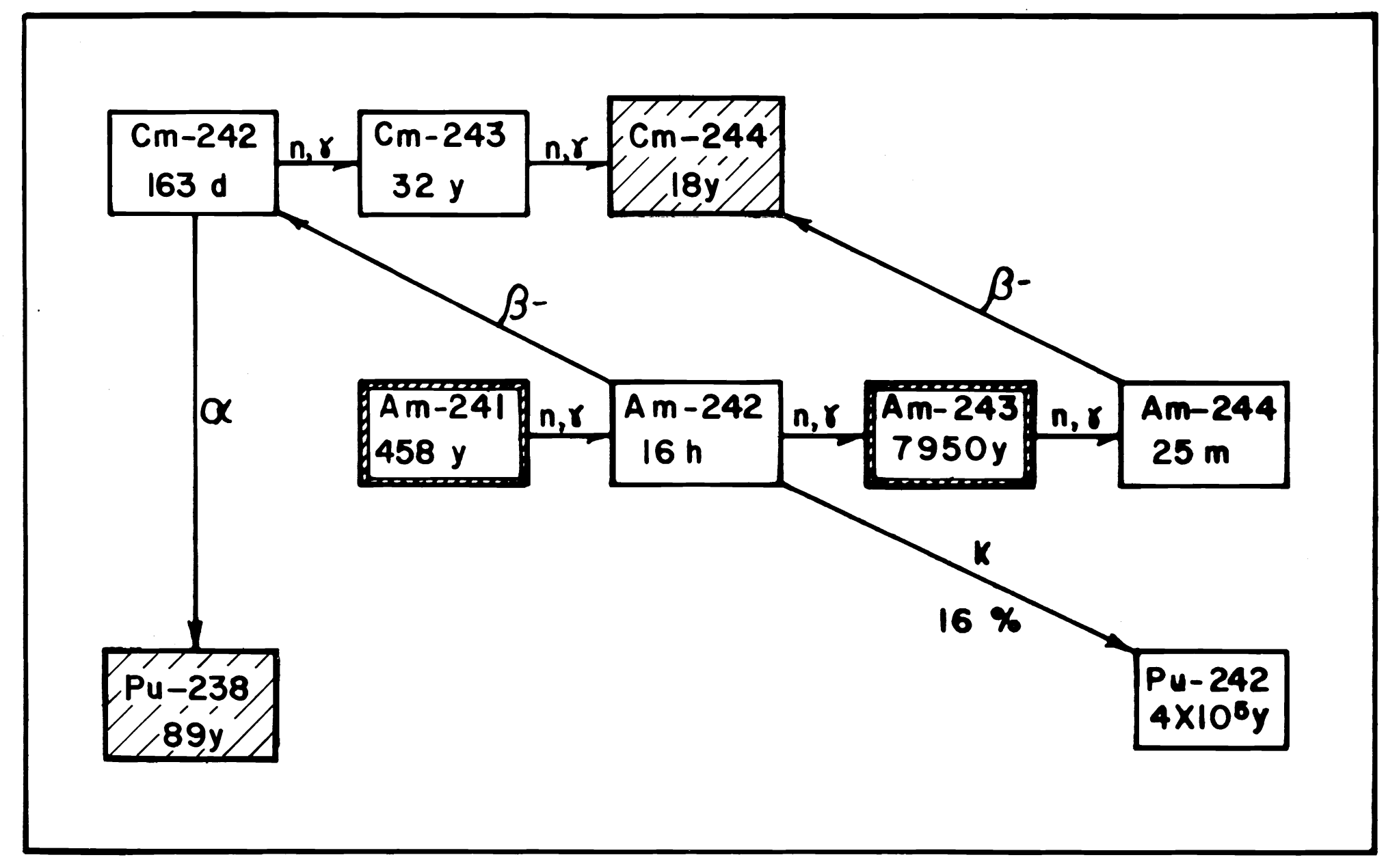




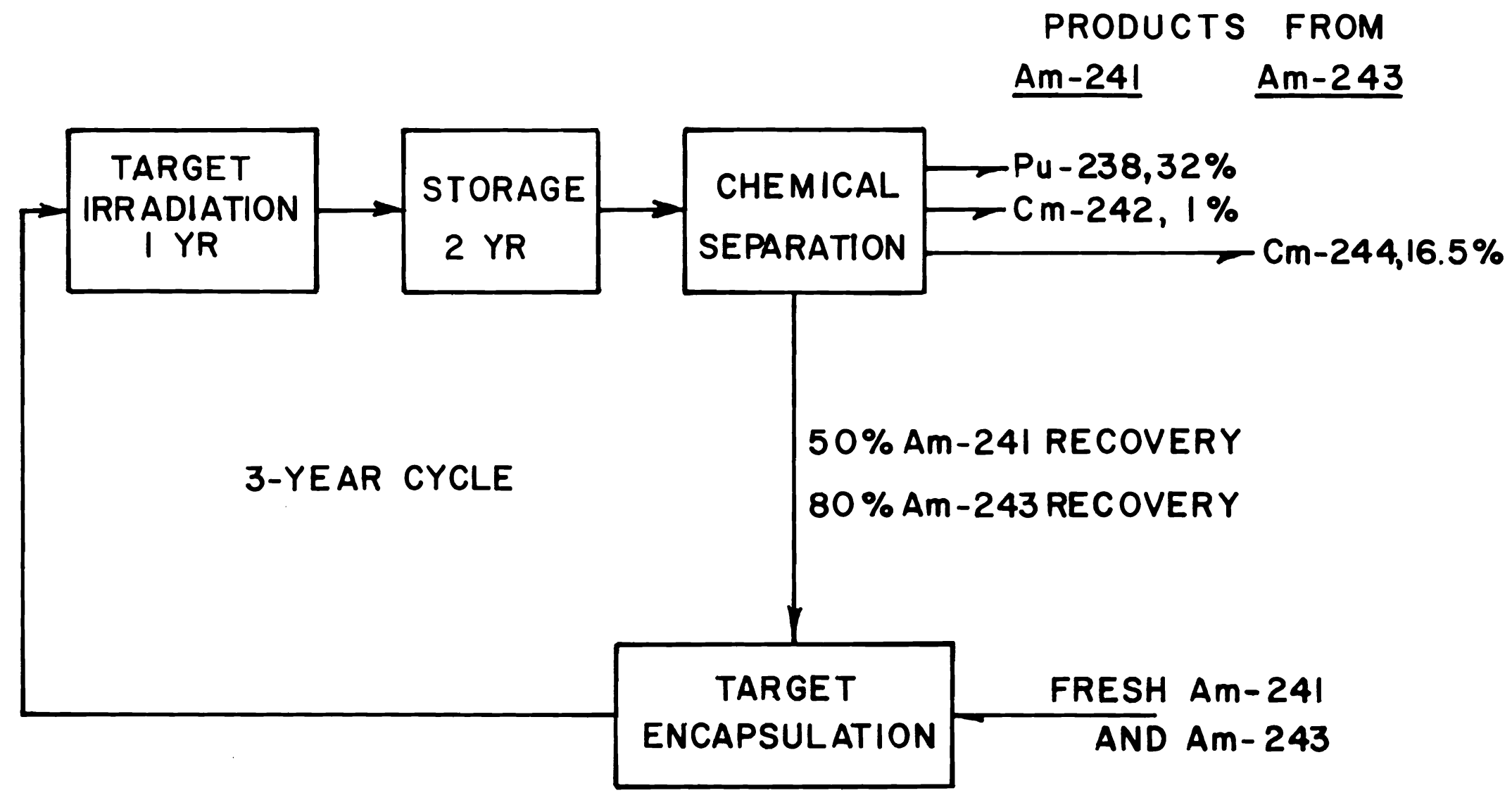


TABLE II

\section{DESCRIPTION OF CASES}

\section{Case}

Description

1 Light Water Reactors (LWRs) fueled with slightly enriched uranium without plutonium or uranium (U-236) recycle. $150 \mathrm{GW}_{\mathrm{e}}$ nuclear capacity in 1980.

2 LWRs as in Case 1, except for $50 \%$ of the U-236 production being recycled.

3 LWRs as in Case 1, except for plutonium being recycled.

4. LWRs as in Case 1, except for both plutonium and $50 \%$ of U-236 being recycled.

5 LWRs with 50\% U-236 recycle and maximum FastBreeder-Reactor (FBR) additions beginning in 1980 .

6 LWRs with $50 \%$ U-236 recycle, limited plutonium recycle, and maximum FBR additions beginning in 1980 .

7 Same as Case 6, except for $180 \mathrm{GW}$ e nuclear capacity in 1980 .

8 Same as Case 6, except for $120 \mathrm{GW}$ e nuclear capacity in 1980 .
Abbreviation

LWRS

LWRS - U

LWRs $-\mathrm{Pu}$

LWRs - U/Pu

LWRs - U and FBRs

LWRs $-\mathrm{U} / \mathrm{Pu}$ and FBRs

Case 6 but $180 \mathrm{GW}_{\mathrm{e}}-1980$

Case 6 but $120 \mathrm{GW}_{\mathrm{e}}-1980$ 
Case 2 is identical to Case 1, except for recycling $50 \%$ of the U-236 present in the discharged spent fuel. This recycle is based on calculations showing that approximately $50 \%$ of the U-236 returned with the spent fuel uranium to the diffusion cascade for re-enrichment will appear in the enriched uranium product. To simplify the calculations, it was assumed that each reactor would recycle its own U-236 after a delay of one charging cycle. Of course it is not likely that each reactor would recycle its own U-236 in such a recycle situation, but the result of the calculation is an accurate reflection of the recycle effects on $\mathrm{Np}-237$ production, and these effects are what we want to identify.

Case 3 is the same as Case 1, except that plutonium is recycled. For plutonium recycle it was assumed that separate plutonium burning reactors would be fueled with natural uranium enriched with plutonium. Separate fueling histories were prepared for LWRs enriched with plutonium for their entire 30-year life. These were substituted for uranium enriched LWRs in the ISOPRO calculation by a trial and error procedure until the out-of-reactor plutonium stockpile was reduced to less than $3 \%$ of the plutonium recovered. It was assumed that one year after fuel discharge, the plutonium would be recovered and available for reactor charging as fabricated plutonium enriched fuel.

Case 4 combines both plutonium recycle and 50\% U-236 recycle. The U-236 produced in the plutonium recycle reactors was not recycled and this may result in some understatement of Np-237 availability. How ever the magnitude of this understatement is less than $5 \%$ because the concentration of U-236 in this portion of the spent fuel is so very low (about one-fifth the concentration of U-236 in uranium enriched fuel) and because less than one-third of the reactors are ever-operating or plutonium recycle. Actually, the final U-235 content is so low (0.35 to 0.40\%) that the justification for returning it to the cascade for re-enrichment is questionable.

Case 5 is like Case 2 with LWRs and 50\% U-236 recycle, except that plutonium-enriched Fast Breeder Reactors (FBRs) are introduced starting in 1980. The introduction rate of FBRs was limited to 4 (at 
$1000 \mathrm{MW}_{\mathrm{e}}$ each) in the first two years and was allowed to double every two years after that until limited by plutonium availability. In terms of annual FBR additions, the limiting sequence was 2, 2, 3, 5, 7, 9, 13, 19, etc. This was assumed to be the maximum rate that the nuclear industry could gear-up for FBR additions, and is essentially the same limit as that adopted in the Systems Analysis Task Force studies for the current AEC Civilian Nuclear Power Study.

Case 6 combines both plutonium and U-236 recycle with FBR additions starting in 1980, and is considered the most plausible case evaluated. In this case, a combination of 4-year and 10 -year plutonium recycle reactors were added to minimize plutonium stockpile accululation and yet allow a high rate of plutonium-enriched FBR additions. The 4-year and 10-year plutonium recycle reactors as opposed to 30 -year recycle in Case 3 , are fueled with plutonium-enriched natural uranium for approximately 4 or 10 years and with enriched uranium for the balance of their lives. The combination of reactors here was obtained by a trial and error process and represents an effort to combine maximum plutonium utilization with maximum FBR additions. Since plutonium is burned in the LWR recycle fuel, the total FBR additions are necessarily less than in Case 5.

Case 7 is like Case 6 , except that the total nuclear capacity is increased to $180 \mathrm{GW}_{\mathrm{e}}$ in 1980 rather than $150 \mathrm{GW}_{\mathrm{e}}$.

Case 8 is like Case 6 , except that the total nuclear capacity is reduced to $120 \mathrm{GW}_{\mathrm{e}}$ in 1980 .

IV. Results

A complete computer printout of the ISOPRO program for all eight cases is included in the Appendix. A summary and discussion of the results is presented in this section.

The annual rate of spent fuel discharges is shown at five-year intervals in Table III. This is the amount of fuel that must be reprocessed to recover the isotopes tabulated in subsequent tables. The main factors in this study that affect the fuel discharge rates are the total installed nuclear power generating capacity and the proportion that is FBR capacity. The fuel 


\section{TABLE III}

ANNUAL POWER REACTOR FUEL DISCHARGES, MT/yr*

\begin{tabular}{|c|c|c|c|c|c|c|c|c|}
\hline & $\begin{array}{l}\text { Case } 1 \\
\text { LWRs } \\
\end{array}$ & $\begin{array}{l}\text { Case } 2 \\
\text { LWRs-U } \\
\end{array}$ & $\begin{array}{l}\text { Case } 3 \\
\text { LWRs-Pu } \\
\end{array}$ & $\begin{array}{c}\text { Case } 4 \\
\text { LWRs - U/Pu }\end{array}$ & $\begin{array}{l}\text { Case } 5 \\
\text { LWRs-U } \\
\& \text { FBRs } \\
\end{array}$ & $\begin{array}{l}\text { Case } 6 \\
\text { LWRs-U/Pu } \\
\text { \& FBRs }\end{array}$ & $\begin{array}{l}\text { Case } 7 \\
\text { Case } 6 \text { but } \\
180 \mathrm{GW}^{-} \\
1980\end{array}$ & $\begin{array}{c}\text { Case } 8 \\
\text { Case } 6 \text { but } \\
120 \mathrm{GW}^{-} \\
1980 \\
\end{array}$ \\
\hline 1970 & 100 & 100 & 100 & 100 & 100 & 100 & 100 & 100 \\
\hline 1975 & 1,830 & 1,830 & 1,860 & 1,850 & 1,830 & 1,850 & 2,010 & 1,640 \\
\hline 1980 & 4,280 & 4,240 & 4,270 & 4,230 & 4,240 & 4,270 & 5,040 & 3,580 \\
\hline 1985 & 10,700 & 10,500 & 10,700 & 10,500 & 10,100 & 10,100 & 12,200 & 8,090 \\
\hline 1990 & 20,700 & 20,200 & 20,400 & 20,100 & 17,400 & 17,700 & 21,600 & 14,200 \\
\hline
\end{tabular}


discharge rate is reduced by introducing FBRs, because of the high fuel exposures in the FBR core fuel and the higher thermal efficiency of FBRs.

A comparison of the amount and types of reactor capacity installed in each of the eight cases is shown in Table IV. Case 6 has less FBR capacity than Case 5 because some of the plutonium was consumed as recycle fuel in the LWRs. Case 7 has more FBR capacity than Case 6 because of more total capacity, but because of recycle, still not as much as Case 5. Case 8 has the least FBR capacity because of the reduced total capacity.

The amount of reactor capacity devoted to plutonium recycle is compared in Table V. In Cases 3 and 4, recycle was limited only by available plutonium. In Cases 6, 7, and 8, the mix of 4-year and 10-year recycle LWRs and FBRs was adjusted to minimize the plutonium stockpile accumulation and to maximize the FBR additions.

Reference to these two tables, as well as the plutonium stockpile and U-236 production tables that follow, will assist in understanding the variations or lack of variations in the isotope availability tabulations.

The accumulation of total plutonium in an unused stockpile is compared for the eight cases in Table VI. This table shows that efficient use of plutonium was achieved in all of the LWR plutonium recycle cases (Cases 3, 4, 6, 7, and 8). The plutonium stockpile reaches $900 \mathrm{MT}$ in 1990 without recycle or FBR additions. With FBR additions, but without LWR plutonium recycle (Case 5), the stockpile accumulation is reduced to less than $200 \mathrm{MT}$ and peaks about 1985. In all of the LWR plutonium recycle cases, the stockpile accumulation is held to $15 \mathrm{MT}$ or less. Plutonium utilization is of interest here primarily because plutonium is the source of the americium and curium isotopes, and efficient utilization means increased availability of these isotopes.

The annual availability rate of U-236 is shown in Table VII. This is not available as a separate isotope, but is a component of the total uranium recovered. The effects of recycling $50 \%$ of this U-236 (Cases 2, 4, 5, 6, 7, and 8) are small prior to 1980 but become significant by 1985 . 
TABLE IV

COMPARISON OF INSTALLED CAPACITY IN EACH CASE BY REACTOR TYPE

\begin{tabular}{|c|c|c|c|c|c|c|c|c|c|c|c|c|c|}
\hline \multirow{2}{*}{\multicolumn{2}{|c|}{ 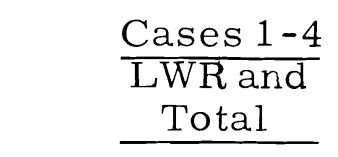 }} & \multicolumn{3}{|c|}{ Case 5} & \multicolumn{3}{|c|}{ Case 6} & \multicolumn{3}{|c|}{ Case 7} & \multicolumn{3}{|c|}{ Case 8} \\
\hline & & $\underline{\mathrm{LWR}}$ & $\underline{\text { FBR }}$ & Total & $\underline{\mathrm{LWR}}$ & $\underline{\text { FBR }}$ & Total & $\underline{\mathrm{LWR}}$ & FBR & Total & $\underline{\mathrm{LWR}}$ & FBR & Total \\
\hline 1970 & 12 & 12 & 0 & 12 & 12 & 0 & 12 & 12 & 0 & 12 & 12 & 0 & 12 \\
\hline 1975 & 70 & 70 & 0 & 70 & 70 & 0 & 70 & 79 & 0 & 79 & 60 & 0 & 60 \\
\hline 1980 & 150 & 148 & 2 & 150 & 148 & 2 & 150 & 178 & 2 & 180 & 119 & 1 & 120 \\
\hline 1985 & 296 & 268 & 28 & 296 & 268 & 28 & 296 & 327 & 28 & 355 & 216 & 21 & 237 \\
\hline 1990 & 500 & 369 & 131 & 500 & 390 & 110 & 500 & 477 & 123 & 600 & 311 & 89 & 400 \\
\hline
\end{tabular}




\section{TABLE V}

COMPARISON OF PLUTONIUM RECYCLE CAPACITY INSTALLED IN PLUTONIUM RECYCLE CASES

(Gigawatts electrical)

\begin{tabular}{|c|c|c|c|c|c|c|c|c|c|c|c|}
\hline \multirow{2}{*}{\multicolumn{2}{|c|}{$\begin{array}{l}\frac{\text { Case } 3}{\text { LWR }} \\
\text { 30 Pu }\end{array}$}} & Case 4 & \multicolumn{3}{|c|}{ Case 6} & \multicolumn{3}{|c|}{ Case 7} & \multicolumn{3}{|c|}{ Case 8} \\
\hline & & $\begin{array}{l}\mathrm{LWR} \\
30 \mathrm{Pu} \\
\end{array}$ & $\begin{array}{l}\text { LWR } \\
4 \mathrm{Pu} \\
\end{array}$ & $\begin{array}{l}\mathrm{LWR} \\
10 \mathrm{Pu} \\
\end{array}$ & FBR & $\begin{array}{l}\mathrm{LWR} \\
4 \mathrm{Pu} \\
\end{array}$ & $\begin{array}{l}\text { LWR } \\
10 \mathrm{Pu} \\
\end{array}$ & FBR & $\begin{array}{l}\overline{\mathrm{LWR}} \\
4 \mathrm{Pu} \\
\end{array}$ & $\begin{array}{l}\text { LWR } \\
10 \mathrm{Pu} \\
\end{array}$ & FBR \\
\hline 1970 & 0 & 0 & 0 & 0 & 0 & 0 & 0 & 0 & 0 & 0 & 0 \\
\hline 1975 & 6 & 5 & 0 & 5 & 0 & 0 & 5 & 0 & 0 & 5 & 0 \\
\hline 1980 & 26 & 26 & 8 & 13 & 2 & 13 & 13 & 2 & 8 & 11 & 1 \\
\hline 1985 & 68 & 67 & 14 & 13 & 28 & 33 & 13 & 28 & 14 & 11 & 21 \\
\hline 1990 & 144 & 142 & 16 & 13 & 110 & 42 & 13 & 123 & 14 & 11 & 89 \\
\hline
\end{tabular}

Notes: $30 \mathrm{Pu}$ denotes reactors operating on Pu recycle for their entire 30-year life.

$4 \mathrm{Pu}$ denotes reactors operating on Pu recycle for 4 years.

$10 \mathrm{Pu}$ denotes reactors operating on Pu recycle for 10 years. 


\section{TABLE VI}

CUMULATIVE PLUTONIUM STOCKPILE, MT

\begin{tabular}{|c|c|c|c|c|c|c|c|c|}
\hline & Case 1 & $\begin{array}{l}\text { Case } 2 \\
\text { LWRs-U }\end{array}$ & LWRs - Pu & LWRs- $\mathrm{L} / \mathrm{Pu}$ & $\begin{array}{l}\text { Case } 5 \\
\text { LWRs-U } \\
\& \text { FBRs } \\
\end{array}$ & $\begin{array}{c}\text { Case } 6 \\
\text { LWRs-U/Pu } \\
\& \text { FBRs } \\
\end{array}$ & $\begin{array}{l}\text { Case } 7 \\
\text { Case } 6 \text { but } \\
180 \mathrm{MW}_{\mathrm{e}} \\
1980 \\
\end{array}$ & $\begin{array}{l}\text { Case } 8 \\
\text { Case } 6 \text { but } \\
120 \mathrm{GW}_{\mathrm{e}} \\
1980 \\
\end{array}$ \\
\hline 1970 & 2 & 2 & 2 & 2 & 2 & 2 & 2 & 2 \\
\hline 1975 & 25 & 25 & 5 & 8 & 25 & 8 & 9 & 7 \\
\hline 1980 & 130 & 127 & 5 & 5 & 115 & 2 & 2 & 7 \\
\hline 1985 & 383 & 375 & 3 & 4 & 191 & 15 & 14 & 14 \\
\hline 1990 & $90^{\prime} 7$ & 892 & 4 & 3 & 5 & 1 & 8 & 4 \\
\hline
\end{tabular}

Note: Plutonium includes $\mathrm{Pu}-239, \mathrm{Pu}-240, \mathrm{Pu}-241$, and $\mathrm{Pu}-242$. 
TABLE VII

ANNUAL AVAILABILITY OF U-236, $\mathrm{MT} / \mathrm{yr}$

\begin{tabular}{|c|c|c|c|c|c|c|c|}
\hline Case 1 & Case 2 & Case 3 & $\underline{L W R S}-\mathrm{L}^{\top} / \mathrm{Pu}$ & $\begin{array}{l}\text { Case } 5 \\
\text { LWRs-U } \\
\& \text { FBRs } \\
\end{array}$ & $\begin{array}{c}\text { Case } 6 \\
\text { LWRs-U/Pu } \\
\& \text { FBRs } \\
\end{array}$ & $\begin{array}{l}\text { Case } 7 \\
\text { Case } 6 \text { but } \\
180 \mathrm{MW}_{\mathrm{e}}^{-} \\
1980\end{array}$ & $\begin{array}{l}\text { Case } 8 \\
\text { Case } 6 \text { but } \\
120 \mathrm{GW}_{\mathrm{e}} \\
1980 \\
\end{array}$ \\
\hline 0.15 & 0.15 & 0.15 & 0.15 & 0.15 & 0.15 & 0.15 & 0.15 \\
\hline 3.6 & 3.7 & 3.6 & 3.7 & 3.7 & 3.7 & 3.9 & 3.5 \\
\hline 11.0 & 12.9 & 9.9 & 11.8 & 12.9 & 11.8 & 13.7 & 10.0 \\
\hline 24.6 & 33.3 & 20.7 & 28.3 & 32.2 & 29.2 & 34.5 & 23.3 \\
\hline 46.4 & 68.9 & 36.8 & 54.4 & 59.4 & 57.2 & 68.3 & 45.4 \\
\hline
\end{tabular}


This is because the recycle effects are overshadowed at first by the growth effects in a rapidly expanding industry. Plutonium recycle reduces U-236 formation since it displaces U-235 which is the precursor of U-236. (Compare Case 3 with Case 1, and Case 4 with Case 2). The U-236 availability is of interest because it is the precursor of $\mathrm{Np}-237$ which is the main source for $\mathrm{Pu}-238$ production.

Annual neptunium availability is shown in Table VIII, and follows, with some lag, the same pattern as U-236 availability.

The principal source of $\mathrm{Pu}_{\mathrm{u}}-238$ is $\mathrm{Np}-237$ target irradiation. The $\mathrm{Pu}-238$ availability from that source, based on the assumptions of this study, is shown in Table IX. There is a marked discontinuity in the rate of change in availability between 1980 and 1985 because of the assumed shift from a high flux $\left(1 \times 10^{14} \mathrm{n} / \mathrm{cm}^{2} / \mathrm{sec}\right)$ target irradiation to a lower power reactor flux irradiation $\left(2 \times 10^{13} \mathrm{n} / \mathrm{cm}^{2} / \mathrm{sec}\right)$ in 1980 , as previously discussed. Such an abrupt shift is unlikely to occur and some smoothing of the data could be justified. It does demonstrate, however, the high sensitivity of $\mathrm{Pu}-238$ availability to the target irradiation flux used.

Plutonium-238 will also be available from Cm-242 decay and from target irradiation of the mixed americium isotopes. Availability from these sources is summarized in Tables X and XI. Total Pu-238 availability from all these sources is shown in Table XII.

Only about 1 to $4 \%$ of the total $\mathrm{Pu}-238$ is available from $\mathrm{Cm}-242$ decay. This quantity could be increased, possibly doubled, since it was rather conservatively assumed that the first year's decay of Cm-242 would not be recoverable (lost to other plutonium isotopes). If it is assumed that the curium isotopes are isolated from the plutonium isotopes within six months of reactor discharge, the $\mathrm{Pu}-238$ from this source is approximately doubled, but it would still be a relatively small source of Pu-238. However, it may be a very important source because of this low radioactivity of the recovered $\mathrm{Pu}-238$, there being no $\mathrm{Pu}-236$ content as in the $\mathrm{Pu}-238$ produced by $\mathrm{Np}-237$ irradiation. 
TABLE VIII

ANNUAL AVAILABILITY OF Np-237, kg/yr*

\begin{tabular}{|c|c|c|c|c|c|c|c|}
\hline Case 1 & $\begin{array}{l}\text { Case } 2 \\
\text { LWRs - U }\end{array}$ & $\begin{array}{l}\text { Case } 3 \\
\text { LWRs-Pu } \\
\end{array}$ & $\underline{L W R s}-\mathrm{L}^{\top} / \mathrm{Pu}$ & $\begin{array}{l}\text { Case } 5 \\
\text { LWRs-U } \\
\& \text { FBRs } \\
\end{array}$ & $\begin{array}{c}\text { Case } 6 \\
\text { LWRs }-U / \mathrm{Pu} \\
\text { \& FBRs } \\
\end{array}$ & $\begin{array}{l}\text { Case } 7 \\
\text { Case } 6 \text { but } \\
180 \mathrm{MW}_{\mathrm{e}^{-}} \\
1980 \\
\end{array}$ & $\begin{array}{l}\text { Case } 8 \\
\text { Case } 6 \text { but } \\
120 \mathrm{GW}_{\mathrm{e}}^{-} \\
1980 \\
\end{array}$ \\
\hline 15 & 15 & 15 & 15 & 15 & 15 & 15 & 15 \\
\hline 376 & 358 & 376 & 358 & 358 & 358 & 370 & 340 \\
\hline 1,220 & 1,260 & 1,130 & 1,190 & 1,260 & 1,180 & 1,370 & 1,010 \\
\hline 2,580 & 3,040 & 2,290 & 2,700 & 2,960 & 2,720 & 3,240 & 2,180 \\
\hline 4,520 & 5,810 & 3,860 & 4,880 & 5,100 & 4,970 & 5,970 & 3,950 \\
\hline
\end{tabular}


$\underline{\text { TABLE IX }}$

ANNUAL Pu-238 AVAILABILITY

FROM Np-237 TARGET IRRADIATION, kg/yr

\begin{tabular}{|c|c|c|c|c|c|c|c|}
\hline Case 1 & Case 2 & Case 3 & Case 4 & Case 5 & Case 6 & $\begin{array}{l}\text { Case } 7 \\
\text { Case } 6 \text { but }\end{array}$ & $\begin{array}{l}\text { Case } 8 \\
\text { Case } 6 \text { but }\end{array}$ \\
\hline WRs & LWRs - U & $\mathrm{LWRs}-\mathrm{Pu}$ & LWRs- $\mathrm{L} / \mathrm{Pu}$ & $\begin{array}{r}\text { LWRs }-U^{\top} \\
\& \text { FBRs } \\
\end{array}$ & $\begin{array}{l}\mathrm{LWRs}-\mathrm{U} / \mathrm{Pu} \\
\& \text { FBRs } \\
\end{array}$ & $\begin{array}{c}180 \mathrm{MW}_{\mathrm{e}}^{-} \\
1980\end{array}$ & $\begin{array}{c}120 \mathrm{GW}_{\mathrm{e}}^{-} \\
1980\end{array}$ \\
\hline
\end{tabular}

1970

6

1975

75

1980

482

472

1985

583

19901,190

1,450
6

75

465

534

1,050
6

72

460

587

1,270
6

72

472

628

1,380
6

72

460

588

1,300
6

73

514

687

1,550

1,040 


\section{TABLE X}

ANNUAL Pu-238 AVAILABILITY FROM Cm-242 DECAY, kg/yr

\begin{tabular}{|c|c|c|c|c|c|c|c|}
\hline ase 1 & Case 2 & Case 3 & Case 4 & Case 5 & Case 6 & $\begin{array}{l}\text { Case } 7 \\
\text { Case } 6 \text { but }\end{array}$ & $\begin{array}{c}\text { Case } 8 \\
\text { Case } 6 \text { b }\end{array}$ \\
\hline & & & & $\begin{array}{l}\text { LWRs } \\
\& \mathrm{FBE}\end{array}$ & LWRs $-\mathrm{U} / \mathrm{Pu}$ & $180 \mathrm{MW}_{\mathrm{e}}^{-}$ & 30 \\
\hline
\end{tabular}

O

1

$1980 \quad 5$

5

1

1

6

25

59
0

$$
0
$$

(1)

1

7

28

39
0

1

6

20 


\section{TABLE XI}

ANNUAL Pu-238 AVAILABILITY FROM TARGET IRRADIATION OF MIXED AMERICIUM ISOTOPES, $\mathrm{kg} / \mathrm{yr}$

\begin{tabular}{|c|c|c|c|c|c|c|c|}
\hline Case 1 & Case 2 & Case 3 & Case 4 & Case 5 & Case 6 & $\begin{array}{l}\text { Case } 7 \\
\text { Case } 6 \text { but }\end{array}$ & $\begin{array}{c}\text { Case } 8 \\
\text { Case } 6 \text { but }\end{array}$ \\
\hline LWRS & LWRs - IT & LWRs - Pu & $\mathrm{LWRs}-\mathrm{L} / \mathrm{Pu}$ & $\begin{array}{r}\text { LWRs -U } \\
\& \text { FBRs } \\
\end{array}$ & $\begin{array}{l}\mathrm{LWRs}-\mathrm{U} / \mathrm{Pu} \\
\& \text { FBRs } \\
\end{array}$ & $\begin{array}{c}180 \mathrm{MW}_{\mathrm{e}}^{-} \\
1980\end{array}$ & $\begin{array}{l}120 \mathrm{GW}_{\mathrm{e}} \\
1980\end{array}$ \\
\hline
\end{tabular}

\begin{tabular}{|c|c|c|c|c|c|c|c|c|}
\hline 1970 & 0 & 0 & 0 & 0 & 0 & 0 & 0 & 0 \\
\hline 1975 & 2 & 2 & 2 & 2 & 2 & 2 & 2 & 2 \\
\hline 1980 & 29 & 29 & 36 & 35 & 29 & 35 & 37 & 32 \\
\hline 1985 & 81 & 81 & 186 & 177 & 82 & 180 & 202 & 155 \\
\hline 1990 & 157 & 157 & 536 & 530 & 192 & 364 & 445 & 310 \\
\hline
\end{tabular}




\section{TABLE XII}

TOTAL ANNUAL Pu-238 AVAILABILITY FROM ALL SOURCES, kg/yr

\begin{tabular}{|c|c|c|c|c|c|c|c|}
\hline Case 1 & Case 2 & Case 3 & $\underline{L W R s}-L^{\top} / \mathrm{Pu}$ & $\begin{array}{l}\text { Case } 5 \\
\text { LWRs-U } \\
\& \text { FBRs } \\
\end{array}$ & $\begin{array}{c}\text { Case } 6 \\
\text { LWRs-U/Pu } \\
\text { \& FBRs } \\
\end{array}$ & $\begin{array}{l}\text { Case } 7 \\
\text { Case } 6 \text { but } \\
180 \mathrm{MW}_{\mathrm{e}^{-}} \\
1980 \\
\end{array}$ & $\begin{array}{l}\text { Case } 8 \\
\text { Case } 6 \text { but } \\
120 \mathrm{GW}_{\mathrm{e}}^{-} \\
1980 \\
\end{array}$ \\
\hline 6 & 6 & 6 & 6 & 6 & 6 & 6 & 6 \\
\hline 78 & 75 & 78 & 75 & 75 & 75 & 75 & 73 \\
\hline 515 & 506 & 507 & 501 & 506 & 500 & 712 & 438 \\
\hline 675 & 722 & 746 & 789 & 721 & 791 & 917 & 666 \\
\hline 1,370 & 1,620 & 1,650 & 1,860 & 1,590 & 1,690 & 2,040 & 1,370 \\
\hline
\end{tabular}


As much as 10 to $30 \%$ of the $\mathrm{Pu}-238$ is available from americium irradiation. The higher proportions are the result of plutonium recycle and are not achieved until the late 1980s. This would also be high-quality $\mathrm{Pu}-238$ from the standpoint of $\mathrm{Pu}-236$ content but would be diluted with $\mathrm{Pu}-242$ resulting from the partial decay of Am-242 to $\mathrm{Pu}-242$.

The cumulative availability of $\mathrm{Pu}-238$ from all three sources is shown in Table XIII. Since $\mathrm{Pu}-238$ availability is of interest primarily as a heat source isotope, the availability in terms of theoretical thermal kilowatts is also shown in Table XIII. The differences between the eight cases in the cululative tabulation turn out to be suprisingly small.

Annual Am-241 and Am-243 availability is shown in Tables XIV and $X V$. The effect of plutonium recycle on americium availability is significant (compare Case 3 with Case 1, for example). For a single batch of fuel, the Am-241 concentration in the spent fuel with plutonium enrichment is 10 to 20 times that for U-235 enrichment, while the Am-243 concentration is as much as 50 times higher with plutonium enrichment. The result is that by 1990, plutonium recycle increases Am-241 availability more than fourfold and Am-243 about eightfold. Use of the plutonium for fueling FBRs reduces americium availability relative to that with plutonium recycle in LWRs, particularly the Am-243. This is because production of the higher transuranium isotopes is reduced in a fast neutron spectrum (where the probability of fission is significantly increased for all transuranium isotopes) and because less fuel is burned as a result of the higher thermal efficiency of the FBRs.

Americium-241 is the daughter of $\mathrm{Pu}-241$ decay. One means of increasing Am-241 availability would be to periodically process the plutonium stockpile for recovery of the accumuiated Am-24.1. The increase in Am-241 annual availability that could be realized by annual processing of the plutonium stockpile is shown in Table. XVI. In the cases where there is no plutonium recycle or no utilization in FBRs (Cases 1, 2, and 5), the potential Am-241 availability from this source is very large. In every case there is potentially more Am-241 available from this source until 1975 than is available directly from the spent fuel. 


\section{TABLE XIII}

CUMULATIVE AVAILABILITY OF Pu-238 FROM ALL SOURCES

\begin{tabular}{|c|c|c|c|c|c|c|c|}
\hline Case 1 & $\underline{L W R S-I T}$ & $\underline{L W R s}-\mathrm{Pu}$ & $\underline{\mathrm{LWRs}}-\mathrm{L} / \mathrm{Pu}$ & $\begin{array}{l}\text { Case } 5 \\
\text { LWRs-U } \\
\& \text { FBRs } \\
\end{array}$ & $\begin{array}{c}\text { Case } 6 \\
\text { LWRs-U/Pu } \\
\text { \& FBRs } \\
\end{array}$ & $\begin{array}{l}\text { Case } 7 \\
\text { Case } 6 \text { but } \\
180 \mathrm{MW}_{\mathrm{e}^{-}} \\
1980 \\
\end{array}$ & $\begin{array}{l}\text { Case } 8 \\
\text { Case } 6 \text { but } \\
120 \mathrm{GW}_{\mathrm{e}}^{-} \\
1980 \\
\end{array}$ \\
\hline \multicolumn{8}{|c|}{ Kilograms } \\
\hline 22 & 22 & 22 & 22 & 22 & 22 & 22 & 22 \\
\hline 176 & 171 & 176 & 171 & 171 & 171 & 172 & 170 \\
\hline 1,720 & 1,670 & 1,710 & 1,670 & 1,670 & 1,670 & 1,790 & 1,520 \\
\hline 4,460 & 4,510 & 4,580 & 4,630 & 4,510 & 4,650 & 5,200 & 4,060 \\
\hline 9,460 & 10,300 & 10,500 & 11,200 & 10,200 & 10,900 & 12,600 & 9,140 \\
\hline \multicolumn{8}{|c|}{ Kilowatts (thermal) } \\
\hline 12 & 12 & 12 & 12 & 12 & 12 & 12 & 12 \\
\hline 99 & 96 & 99 & 96 & 96 & 96 & 96 & 95 \\
\hline 964 & 935 & 958 & 935 & 935 & 935 & 1,000 & 851 \\
\hline 2,500 & 2,530 & 2,560 & 2,540 & 2,530 & 2,600 & 2,910 & 2,270 \\
\hline 5,300 & 5,770 & 5,880 & 6,260 & 5,710 & 6,070 & 7,060 & 5,120 \\
\hline
\end{tabular}


TABLE XIV

ANNUAL AVAILABILITY

OF Am-241 IN AMERICIUM MIXTURE, $\mathrm{kg} / \mathrm{yr}$

\begin{tabular}{|c|c|c|c|c|c|c|c|}
\hline Case 1 & Case 2 & Case 3 & Case 4 & Case 5 & Case 6 & $\begin{array}{l}\text { Case } 7 \\
\text { Case } 6 \text { but }\end{array}$ & $\begin{array}{l}\text { Case } 8 \\
\text { Case } 6 \text { but }\end{array}$ \\
\hline WRs & LWRs - I & LWRs - Pu & LWRs- L / Pu & $\begin{array}{r}\text { LWRs -U } \\
\& \text { FBRs } \\
\end{array}$ & $\begin{array}{l}\mathrm{LWRS}-\mathrm{U} / \mathrm{Pu} \\
\& \text { FBRs }\end{array}$ & $\begin{array}{l}180 \mathrm{MW}_{\mathrm{e}}^{-} \\
1980\end{array}$ & $\begin{array}{l}120 \mathrm{GW}^{-} \\
1980\end{array}$ \\
\hline
\end{tabular}

1970

1

1

1

1

1

1

$\underset{1}{\omega}$

1975

38

39

38

39

39

39

40

38

1980

122

119

231

220

119

225

247

192

1985

237

237

753

745

271

568

695

497

1990

377

372

1,670

1,650

643

695

916

526 
TABLE XV

ANNUAL AVAILABILITY

OF Am-243 IN AMERICIUM MIXTURE, $\mathrm{kg} / \mathrm{yr}$

\begin{tabular}{|c|c|c|c|c|c|c|c|}
\hline Case 1 & Case 2 & Case 3 & Case 4 & Case 5 & Case 6 & $\begin{array}{l}\text { Case } 7 \\
\text { Case } 6 \text { but }\end{array}$ & $\begin{array}{l}\text { Case } 8 \\
\text { Case } 6 \text { but }\end{array}$ \\
\hline LWRS & LWRs - U & LWRs $-\mathrm{Pu}$ & $L W R s-\mathrm{L} / \mathrm{Pu}$ & $\begin{array}{r}\text { LWRs - U } \\
\& \text { FBRs } \\
\end{array}$ & $\begin{array}{l}\text { LWRs - U/Pu } \\
\& \text { FBRs } \\
\end{array}$ & $\begin{array}{l}180 \mathrm{MW}_{\mathrm{e}} \\
1980\end{array}$ & $\begin{array}{l}120 \mathrm{GW}_{\mathrm{e}}^{-} \\
1980\end{array}$ \\
\hline
\end{tabular}

\begin{tabular}{|c|c|c|c|c|c|c|c|c|}
\hline 1970 & 2 & 2 & 2 & 2 & 2 & 2 & 2 & 2 \\
\hline 1975 & 50 & 53 & 50 & 53 & 53 & 53 & 54 & 52 \\
\hline 1980 & 167 & 169 & 387 & 373 & 169 & 384 & 418 & 325 \\
\hline 1990 & 462 & 473 & 3,660 & 3,620 & 477 & 645 & 1,080 & 427 \\
\hline
\end{tabular}




\section{TABLE XVI}

ANNUAL AVAILABILITY

OF Am-241 FROM PROCESSING PLUTONIUM STOCKPILE, kg/yr

\begin{tabular}{|c|c|c|c|c|c|c|c|}
\hline Case 1 & Case 2 & Case 3 & Case 4 & Case 5 & Case 6 & $\begin{array}{l}\text { Case } 7 \\
\text { Case } 6 \text { but }\end{array}$ & $\begin{array}{l}\text { Case } 8 \\
\text { Case } 6 \text { but }\end{array}$ \\
\hline $\mathrm{LWRS}$ & LWRs - U & LWRs - Pu & $\mathrm{LWRs}-\mathrm{L} / \mathrm{Pu}$ & $\begin{array}{r}\text { LWRs-U } \\
\& \text { FBRs }\end{array}$ & $\begin{array}{l}\mathrm{LWRS}-\mathrm{U} / \mathrm{Pu} \\
\& \text { FBRs }\end{array}$ & $\begin{array}{c}180 \mathrm{MW}_{\mathrm{e}}^{-} \\
1980\end{array}$ & $\begin{array}{c}120 \mathrm{GW}^{1980} \\
\mathrm{e}^{-}\end{array}$ \\
\hline
\end{tabular}

$\underline{L W R S}-\underline{L W R S-U} \quad \underline{L W R S-P u} \quad \underline{L W R S-L / P u} \quad$ \&FBRs $\&$ FBRs 1980 1980

\begin{tabular}{|c|c|c|c|c|c|c|c|c|}
\hline 1970 & 10 & 10 & 10 & 10 & 10 & 10 & 10 & 10 \\
\hline 1975 & 118 & 119 & 35 & 64 & 119 & 64 & 65 & 61 \\
\hline 1980 & 823 & 806 & 25 & 47 & 806 & 17 & 46 & 77 \\
\hline 1990 & 6,290 & 6,210 & 34 & 45 & 529 & 29 & 50 & 60 \\
\hline
\end{tabular}


Calculation of Am-241 availability from the plutonium stockpile is based on the assumption that the average $\mathrm{Pu}-241$ content is $17 \%$. The calculation is based on annual processing of the entire plutonium stockpile, but annual processing is not essential to the recovery because the Am-241 will be there, accumulating at the same annual rate, whether the stockpile is processed at one-year or five-year intervals.

The possibility of Am-241 recovery from plutonium stockpile processing was not considered in the $\mathrm{Pu}-238$ availability calculations previously discussed on Page 32.

Annual availability of $\mathrm{Cm}-244$ recovered directly from spent fuel is shown in Table XVII and availability from target irradiation of americium is shown in Table XVIII. The total annual availability from both sources is shown in Table XIX. The two sources are shown to be of about equal importance. Direct recovery is the largest source up through 1980 particularly in the plutonium recycle cases. Beyond that time direct recovery continues to be the largest source in the cases with plutonium recycle, but Am-243 target irradiation is more important otherwise. With FBRs in the system target irradiation becomes the major source of $\mathrm{Cm}-244$.

The cumulative availability of $\mathrm{Cm}-244$ from both sources is shown in Table XX. As in the case of $\mathrm{Pu}-238$, the principal interest in $\mathrm{Cm}-244$ is as a heat-source isotope and availability is shown in terms of both kilograms and thermal kilowatts. The significance of the $\mathrm{Cm}-244$ quantities relative to $\mathrm{Pu}-238$ is more apparent when their projected availabilities are compared on the basis of theoretical heat output rather than kilogram quantities because the theoretical specific heat of $\mathrm{Cm}-244$ is $2.8 \mathrm{~W} / \mathrm{g}$ compared with $0.56 \mathrm{~W} / \mathrm{g}$ for $\mathrm{Pu}-238$. Comparing Table XX with Table XIII, $\mathrm{Pu}-238$ is shown to be the major source of heat energy relative to $\mathrm{Cm}-244$ up through 1980, and on through 1990 for the nonplutonium-recycle cases. With plutonium recycle, however, with or without FBRs, substantially more heat is potentially available from Cm-244 in the 1980 s.

Availability calculations have been made for several of the major radioactive fission products. The ones selected are of interest because they have possible application as heat or radiation sources or because 
TABLE XVII

ANNUAL AVAILABILITY

OF Cm-244 RECOVERED DIRECTLY FROM FUEL, kg/yr**

\begin{tabular}{|c|c|c|c|c|c|c|c|}
\hline Case 1 & $\begin{array}{l}\text { Case } 2 \\
\text { LWRs-U }\end{array}$ & $\begin{array}{l}\text { Case } 3 \\
\text { LWRs-Pu }\end{array}$ & $\underline{\mathrm{LWRS}}-\mathrm{L} / \mathrm{Pu}$ & $\begin{array}{c}\text { Case } 5 \\
\text { LWRs-U } \\
\& \text { FBRs } \\
\end{array}$ & $\begin{array}{c}\text { Case } 6 \\
\text { LWRs - U/Pu } \\
\& \text { FBRs } \\
\end{array}$ & $\begin{array}{l}\text { Case } 7 \\
\text { Case } 6 \text { but } \\
180 \mathrm{MW}_{\mathrm{e}} \\
1980 \\
\end{array}$ & $\begin{array}{l}\text { Case } 8 \\
\text { Case } 6 \text { but } \\
120 \mathrm{GW}_{\mathrm{e}}^{-} \\
1980 \\
\end{array}$ \\
\hline 0 & 0 & 0 & 0 & 0 & 0 & 0 & 0 \\
\hline 3 & 3 & 3 & 3 & 3 & 3 & 3 & 3 \\
\hline 24 & 25 & 52 & 52 & 25 & 48 & 51 & 43 \\
\hline 53 & 56 & 281 & 282 & 55 & 264 & 301 & 223 \\
\hline 66 & 73 & 722 & 722 & 67 & 227 & 316 & 173 \\
\hline
\end{tabular}

*Availability after three-year delay to reduce $\mathrm{Cm}-242$ content. 
TABLE XVIII

ANNUAL AVAILABILITY OF Cm-244

FROM TARGET IRRADIATION OF MIXED AMERICIUM ISOTOPES, $\mathrm{kg} / \mathrm{yr} *$

\begin{tabular}{|c|c|c|c|c|c|}
\hline Case 1 & Case 2 & Case 3 & Case 4 & Case 5 & Case 6 \\
\hline LWRs & LWRS - I? & LWRs - Pu & LWRs - $\mathrm{L}^{\top} / \mathrm{Pu}$ & $\begin{array}{r}\text { LWRS - U } \\
\& \text { FBRs }\end{array}$ & $\begin{array}{c}L W R s-U / P u \\
\& F B R s\end{array}$ \\
\hline
\end{tabular}

1970

1975

1980

1985

1990
0

0

1

20

66

140
0

1

26

166

597
0

1

20

66

141

377

\begin{tabular}{l} 
Case 7 \\
Case 6 but \\
$180 \mathrm{MW}_{\mathrm{e}^{-}}$ \\
1980 \\
\hline
\end{tabular}

0

1

28

184

469
Case 8 Case 6 but $120 \mathrm{GW}_{\mathrm{e}}^{-}$ 1980

0

1

24

145

334

$\because$ Availability aiter two-year delay to reduce $\mathrm{Cm}-242$ content. 
TABLE XIX

TOTAL ANNUAL AVAILABILITY

OF Cm-244 FROM BOTH SPENT FUEL AND AMERICIUM TARGET IRRADIATION, $\mathrm{kg} / \mathrm{yr}$

\begin{tabular}{|c|c|c|c|c|c|c|c|}
\hline Case 1 & Case 2 & $\underline{L W R s}-\mathrm{Pu}$ & LWRs- $\mathrm{L} / \mathrm{Pu}$ & $\begin{array}{l}\text { Case } 5 \\
\text { LWRs-U } \\
\& \text { FBRS } \\
\end{array}$ & $\begin{array}{c}\text { Case } 6 \\
\text { LWRs-U/Pu } \\
\& \text { FBRs } \\
\end{array}$ & $\begin{array}{l}\text { Case } 7 \\
\text { Case } 6 \text { but } \\
180 \mathrm{MW}_{\mathrm{e}}^{-} \\
1980 \\
\end{array}$ & $\begin{array}{l}\text { Case } 8 \\
\text { Case } 6 \text { but } \\
120 \mathrm{GW} \text { e } \\
1980 \\
\end{array}$ \\
\hline 1 & 1 & 1 & 1 & 1 & 1 & 1 & 1 \\
\hline 4 & 4 & 4 & 4 & 4 & 4 & 4 & 4 \\
\hline 44 & 45 & 78 & 73 & 45 & 74 & 78 & 67 \\
\hline 117 & 122 & 455 & 446 & 122 & 428 & 485 & 368 \\
\hline 203 & 213 & 1,323 & 1,314 & 207 & 603 & 785 & 508 \\
\hline
\end{tabular}


TABLE XX

CUMULATIVE AVAILABILITY OF Cm-244

FROM BOTH SPENT FUEL AND AMERICIUM TARGET IRRADIATION

\begin{tabular}{|c|c|c|c|c|c|c|c|}
\hline LWRs & $\underline{L W R S-U}$ & $\underline{L W R s}-\mathrm{Pu}$ & $\underline{L W R s-L / P u}$ & $\begin{array}{l}\text { Case } 5 \\
\text { LWRs-U } \\
\& \text { FBRs } \\
\end{array}$ & $\begin{array}{c}\text { Case } 6 \\
\text { LWRs-U/Pu } \\
\text { \& FBRs } \\
\end{array}$ & $\begin{array}{l}\text { Case } 7 \\
\text { Case } 6 \text { but } \\
180 \mathrm{MW}_{\mathrm{e}} \\
1980 \\
\end{array}$ & $\begin{array}{l}\text { Case } 8 \\
\text { Case } 6 \text { but } \\
120 \mathrm{GW}^{-} \\
1980 \\
\end{array}$ \\
\hline \multicolumn{8}{|c|}{ Kilograms } \\
\hline 2 & 2 & 2 & 2 & 2 & 2 & 2 & 2 \\
\hline 9 & 10 & 9 & 10 & 10 & 10 & 10 & 10 \\
\hline 127 & 134 & 178 & 173 & 134 & 174 & 182 & 162 \\
\hline 503 & 523 & 1,400 & 1,350 & 523 & 1,380 & 1,510 & 1,200 \\
\hline 1,210 & 1,250 & 5,530 & 5,440 & 1,240 & 3,780 & 4,550 & 3,340 \\
\hline
\end{tabular}

\begin{tabular}{|c|c|c|c|c|c|c|c|c|}
\hline 1970 & 6 & 6 & 6 & 6 & 6 & 6 & 6 & 6 \\
\hline 1975 & 24 & 30 & 25 & 30 & 30 & 30 & 30 & 30 \\
\hline 1980 & 356 & 375 & 498 & 484 & 375 & 487 & 510 & 454 \\
\hline 1985 & 1,410 & 1,460 & 3,920 & 3,780 & 1,464 & 3,860 & 4,230 & 3,360 \\
\hline 1990 & 3,390 & 3,500 & 15,500 & 15,200 & 3,470 & 10,600 & 12,700 & 9,350 \\
\hline
\end{tabular}


they are difficult to contain during fuel reprocessing. Annual availability summaries for $\mathrm{Pm}-147, \mathrm{Ce}-144, \mathrm{Cs}-137, \mathrm{Sr}-90, \mathrm{Kr}-85, \mathrm{Ru}-106$, and H-3 are shown in Tables XXI, XXII, XXIII, XXIV, XXV, XXVI, and XXVII respectively.

Even though there are variations in fission yields between uranium and plutonium, the differences in availability with or without plutonium recycle are relatively small compared with the differences in fission yield. For example, there is more than a tenfold difference in fission yield for Ru-106 from U-235 at $0.38 \%$ and $\mathrm{Pu}-239$ at $4.6 \%$, whereas maximum plutonium recycle (Case 3 ) increases $\mathrm{Ru}-106$ availability less than 30\%. Except for the variations resulting from nuclear power growth rates in Cases 7 and 8, fission product availability is closely similar in almost all cases. The principal reasons for this similarity are (1) a significant number of the total fissions are plutonium fissions (on the order of $30 \%$ ) even without plutonium recycle, and (2) because of a steep nuclear growth rate and a low conversion ratio during the period under study, recycle fuel is always outweighed by nonrecycle fuel.

In the case of $\mathrm{H}-3$, a uniform fission yield of one $\mathrm{H}-3$ atom/ $1.25 \times 10^{4}$ fissions ${ }^{(5)}$ was assumed from all fissions. Thus the results in Table XXVII do not represent a measure of fueling effects. The differences in $\mathrm{H}-3$ availability are due to thermal efficiency differences.

The cumulative availability of these radioactive fission products is summarized for Case 6 in Table XXVIII. To give a better measure of their relative importance, their quantities are converted to thermal kilowatts, except that $\mathrm{Kr}-85$ and $\mathrm{H}-3$ (isotopes difficult to contain during reprocessing) are shown in terms of cumulative kilocuries.

Availability calculations were made for another group of fission product isotopes which have attracted interest $(6,7)$ as a possible source of valuable rare materials. These isotopes are all stable or nearly stable and their associated radioactivity is believed to be low. However, two of them, stable krypton and stable ruthenium, can only be recovered with significant amounts of a radioactive component, $\mathrm{Kr}-85$ and $\mathrm{Ru}-106$ respectively, and so are certain to have significant radioactivity. It seems probable that some radioactivity would be associated with all of them, but the levels have not yet been established. 
TABLE XXI

ANNUAL AVAILABILITY OF Pm-147, kg/yr

\begin{tabular}{|c|c|c|c|c|c|c|c|}
\hline Case 1 & Case 2 & Case 3 & $\underline{L W R s}-\mathrm{L} / \mathrm{Pu}$ & $\begin{array}{c}\text { Case } 5 \\
\text { LWRs-U } \\
\& \text { FBRs } \\
\end{array}$ & $\begin{array}{c}\text { Case } 6 \\
\text { LWRs - U / Pu } \\
\text { \& FBRs } \\
\end{array}$ & $\begin{array}{l}\text { Case } 7 \\
\text { Case } 6 \text { but } \\
180 \mathrm{MW}_{\mathrm{e}}^{-} \\
1980 \\
\end{array}$ & $\begin{array}{l}\text { Case } 8 \\
\text { Case } 6 \text { but } \\
120 \mathrm{GW} \mathrm{e}^{-} \\
1980 \\
\end{array}$ \\
\hline ? & 2 & 2 & 2 & 2 & 2 & 2 & 2 \\
\hline 36 & 36 & 36 & 36 & 36 & 36 & 36 & 36 \\
\hline 193 & 180 & 192 & 180 & 180 & 180 & 206 & 153 \\
\hline 492 & 461 & 480 & 456 & 460 & 457 & 542 & 363 \\
\hline 1,020 & 942 & 1,000 & 944 & 907 & 900 & 1,080 & 729 \\
\hline
\end{tabular}




\section{TABLE XXII}

ANNUAL AVAILABILITY OF Ce-144, kg/yr

\begin{tabular}{|c|c|c|c|c|c|c|c|}
\hline Case 1 & Case 2 & Case 3 & Case 4 & Case 5 & Case 6 & $\begin{array}{l}\text { Case } 7 \\
\text { Case } 6 \text { but }\end{array}$ & $\begin{array}{l}\text { Case } 8 \\
\text { Case } 6 \text { but }\end{array}$ \\
\hline LWRs & LWRs - U & LWRs - Pu & LWRs- $\mathrm{U} / \mathrm{Pu}$ & $\begin{array}{l}\text { LWRs-U } \\
\text { \& FBRs }\end{array}$ & $\begin{array}{l}\text { LWRs-U/Pu } \\
\& \text { FBRs }\end{array}$ & $\begin{array}{l}180 \mathrm{MW}_{\mathrm{e}^{-}} \\
1980\end{array}$ & $\begin{array}{c}120 \mathrm{GW}_{\mathrm{e}}^{-} \\
1980\end{array}$ \\
\hline
\end{tabular}

1970

7

7

1975

1980

462

447

447

1,080

2,120

2,180

434

7

172

172

447

1,110

2,070
7

172

432

1,080

2,050

2,460

1,630
161

365

863

可 
TABLE XXIII

ANNUAL AVAILABILITY OF Cs-137, $\mathrm{kg} / \mathrm{yr}$

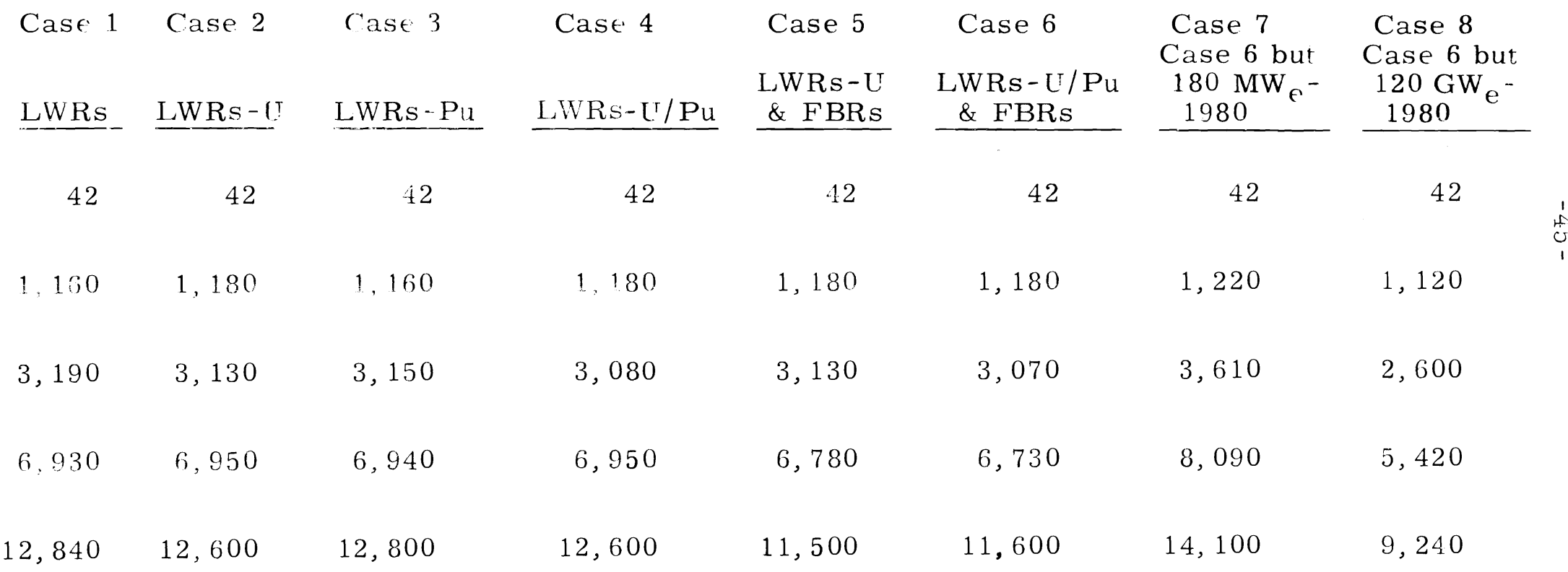




\section{TABLE XXIV}

ANNUAL AVAILABILITY OF Sr-90, $\mathrm{kg} / \mathrm{yr}$

\begin{tabular}{|c|c|c|c|c|c|c|c|c|}
\hline & $\begin{array}{l}\text { Case } 1 \\
\text { LWRs }\end{array}$ & $\begin{array}{l}\text { Case } 2 \\
\text { LWRs-U }\end{array}$ & $\begin{array}{l}\text { Case } 3 \\
\text { LWRs-Pu }\end{array}$ & $\begin{array}{c}\text { Case } 4 \\
\text { LWRs - } \mathrm{L} / \mathrm{Pu} \\
\end{array}$ & $\begin{array}{c}\text { Case } 5 \\
\text { LWRs-U } \\
\& \text { FBRs } \\
\end{array}$ & $\begin{array}{c}\text { Case } 6 \\
\text { LWRs - U/Pu } \\
\& \text { FBRs } \\
\end{array}$ & $\begin{array}{l}\text { Case } 7 \\
\text { Case } 6 \text { but } \\
180 \mathrm{MW}_{\mathrm{e}}^{-} \\
1980 \\
\end{array}$ & $\begin{array}{l}\text { Case } 8 \\
\text { Case } 6 \text { but } \\
120 \mathrm{GW}_{\mathrm{e}}^{-} \\
1980\end{array}$ \\
\hline 1970 & 19 & 19 & 19 & 19 & 19 & 19 & 19 & 19 \\
\hline 1975 & 522 & 532 & 522 & 532 & 532 & 532 & 551 & 505 \\
\hline 1980 & 1,450 & 1,420 & 1,360 & 1,330 & 1,420 & 1,320 & 1,570 & 1,120 \\
\hline 1985 & 3,180 & 3,190 & 2,910 & 2,920 & 3,080 & 2,940 & 3,510 & 2,340 \\
\hline
\end{tabular}


TABLE XXV

ANNUAL AVAILABILITY OF $\mathrm{Kr}-85, \mathrm{~kg} / \mathrm{yr}$

\begin{tabular}{|c|c|c|c|c|c|c|c|}
\hline Case 1 & $\begin{array}{l}\text { Case } 2 \\
\text { LWRs - U } \\
\end{array}$ & $\begin{array}{l}\text { Case } 3 \\
\text { LWRs-Pu } \\
\end{array}$ & $\begin{array}{c}\text { Case } 4 \\
\text { LWRs- } \mathrm{L} / \mathrm{Pu} \\
\end{array}$ & $\begin{array}{l}\text { Case } 5 \\
\text { LWRs-U } \\
\& \text { FBRs } \\
\end{array}$ & $\begin{array}{c}\text { Case } 6 \\
\text { LWRs-U/Pu } \\
\text { \& FBRs } \\
\end{array}$ & $\begin{array}{l}\text { Case } 7 \\
\text { Case } 6 \text { but } \\
180 \mathrm{MW}_{\mathrm{e}}^{-} \\
1980\end{array}$ & $\begin{array}{l}\text { Case } 8 \\
\text { Case } 6 \text { but } \\
120 \mathrm{GW}_{\mathrm{e}} \\
1980 \\
\end{array}$ \\
\hline 1 & 1 & 1 & 1 & 1 & 1 & 1 & 1 \\
\hline 23 & 23 & 23 & 23 & 23 & 23 & 24 & 22 \\
\hline 63 & 62 & 60 & 58 & 62 & 58 & 68 & 58 \\
\hline 140 & 141 & 128 & 128 & 137 & 130 & 155 & 104 \\
\hline 262 & 257 & 230 & 225 & 229 & 223 & 266 & 177 \\
\hline
\end{tabular}




\section{TABLE XXVI}

ANNUAL AVAILABILITY OF Ru-106, kg/yr

\begin{tabular}{|c|c|c|c|c|c|c|c|}
\hline Case 1 & Case 2 & Case 3 & Case 4 & Case 5 & Case 6 & $\begin{array}{l}\text { Case } 7 \\
\text { Case } 6 \text { but }\end{array}$ & $\begin{array}{l}\text { Case } 8 \\
\text { Case } 6 \text { but }\end{array}$ \\
\hline$\underline{\mathrm{LWRS}}$ & LWRs - I? & $\mathrm{LWRs}-\mathrm{Pu}$ & $\mathrm{LWRs}-\mathrm{I} / \mathrm{Pu}$ & $\begin{array}{r}\text { LWRs -U } \\
\& \text { FBRs } \\
\end{array}$ & $\begin{array}{l}\text { LWRs }-[/ P u \\
\& \text { FBRs }\end{array}$ & $\begin{array}{l}180 \mathrm{MW}_{\mathrm{e}}^{-} \\
1980\end{array}$ & $\begin{array}{c}120 \mathrm{GW}_{\mathrm{e}} \\
1980\end{array}$ \\
\hline
\end{tabular}

\begin{tabular}{|c|c|c|c|c|c|c|c|c|}
\hline 1970 & 3 & 3 & 3 & 3 & 3 & 3 & 3 & 3 \\
\hline 1975 & 68 & 69 & 68 & 69 & 69 & 69 & 72 & 65 \\
\hline 1980 & 185 & 180 & 207 & 201 & 180 & 202 & 234 & 169 \\
\hline 1985 & 433 & 430 & 528 & 523 & 456 & 488 & 590 & 404 \\
\hline 1990 & 882 & 857 & 1,130 & 1,100 & 1,090 & 1,150 & 1,390 & 927 \\
\hline
\end{tabular}


TABLE XXVII

ANNUAL AVAILABILITY OF H-3, g/yr

\begin{tabular}{|c|c|c|c|c|c|c|c|}
\hline Case 1 & Case 2 & Case 3 & Case 4 & Case 5 & Case 6 & $\begin{array}{c}\text { Case } 7 \\
\text { Case } 6 \text { but }\end{array}$ & $\begin{array}{l}\text { Case } 8 \\
\text { Case } 6 \text { but }\end{array}$ \\
\hline LWRs & LWRS-U & LWRs - Pu & LWRs - $\mathrm{L} / \mathrm{Pu}$ & $\begin{array}{l}\text { LWRs -U } \\
\& \text { FBRs }\end{array}$ & $\begin{array}{c}\text { LWRs }-U / P u \\
\& \text { FBRs }\end{array}$ & $\begin{array}{c}180 \mathrm{MW}_{\mathrm{e}}^{-} \\
1980\end{array}$ & $\begin{array}{c}120 \mathrm{GW}_{\mathrm{e}} \\
1980\end{array}$ \\
\hline
\end{tabular}

\begin{tabular}{|c|c|c|c|c|c|c|c|c|}
\hline 1970 & 1 & 1 & 1 & 1 & 1 & 1 & 1 & 1 \\
\hline 1975 & 32 & 33 & 32 & 33 & 33 & 33 & 34 & 31 \\
\hline 1980 & 90 & 88 & 88 & 86 & 88 & 86 & 101 & 73 \\
\hline 1990 & 357 & 351 & 354 & 347 & 321 & 324 & 392 & 257 \\
\hline
\end{tabular}


TABLE XXVIII

CUMULATIVE AVAILABILITY

OF PRINCIPAL RADIOACTIVE FISSION PRODUCTS

(Case 6 Basis)

\begin{tabular}{lrrr} 
& \multicolumn{3}{c}{ Kilowatts (thermal) } \\
\cline { 2 - 4 } Kr-85 & $\frac{1970}{2}$ & $\frac{1980}{125}$ & $\frac{1990}{698}$ \\
Pm-147 & 2 & 151 & 906 \\
Cs -137 & 71 & 5,590 & 33,700 \\
Sr-90 & 73 & 5,550 & 32,600 \\
$\mathrm{Ru}-106$ & 132 & 9,660 & 54,200 \\
$\mathrm{Ce}-144$ & 256 & 17,000 & 83,600
\end{tabular}

Kilocuries

$\begin{array}{lrrr}\mathrm{Kr}-85 & 1,200 & 92,000 & 514,000 \\ \mathrm{H}-3 & 39 & 3,400 & 19,000\end{array}$


Annual availability summaries for Tc-99, Rh-103, the stable palladium isotopes, the stable xenon isotopes, the stable krypton isotopes and the stable ruthenium isotopes are shown in Tables XXIX, XXX, XXXI, XXXII, XXXIII, and XXIV. Again, as with the radioactive fission products, there are increases in availability with plutonium recycle, but not nearly as large as one might think just by comparing the uranium and plutonium fission yields.

The cumulative availability for these stable fission products is summarized for Case 6 in Table XXXV. 


\section{TABLE XXIX}

ANNUAL AVAILABILITY OF Tc-99, kg/yr

\begin{tabular}{|c|c|c|c|c|c|c|c|}
\hline Case 1 & Case 2 & Case 3 & Case 4 & Case 5 & Case 6 & $\begin{array}{l}\text { Case } 7 \\
\text { Case } 6 \text { but }\end{array}$ & $\begin{array}{l}\text { Case } 8 \\
\text { Case } 6 \text { but }\end{array}$ \\
\hline
\end{tabular}

LWRs- $\mathrm{L}^{\top} / \mathrm{Pu} \quad \mathrm{LWRs}-\mathrm{C} \quad \mathrm{LWRS}-\mathrm{U} / \mathrm{Pu} \quad 180 \mathrm{MW}_{\mathrm{e}^{-}} 120 \mathrm{GW}_{\mathrm{e}^{-}}$

\begin{tabular}{|c|c|c|c|c|c|c|c|c|}
\hline 1970 & 29 & 29 & 29 & 29 & 29 & 29 & 29 & 29 \\
\hline 1975 & 798 & 809 & 798 & 809 & 809 & 809 & 836 & 768 \\
\hline 1980 & 2,200 & 2,140 & 2,170 & 2,100 & 2,140 & 2,090 & 2,460 & 1,770 \\
\hline 1990 & 8,940 & 8,670 & 8,820 & 8,590 & 7,920 & 7,980 & 9,670 & 6,340 \\
\hline
\end{tabular}


TABLE XXX

ANNUAL AVAILABILITY OF STABLE Rh-103, kg/yr

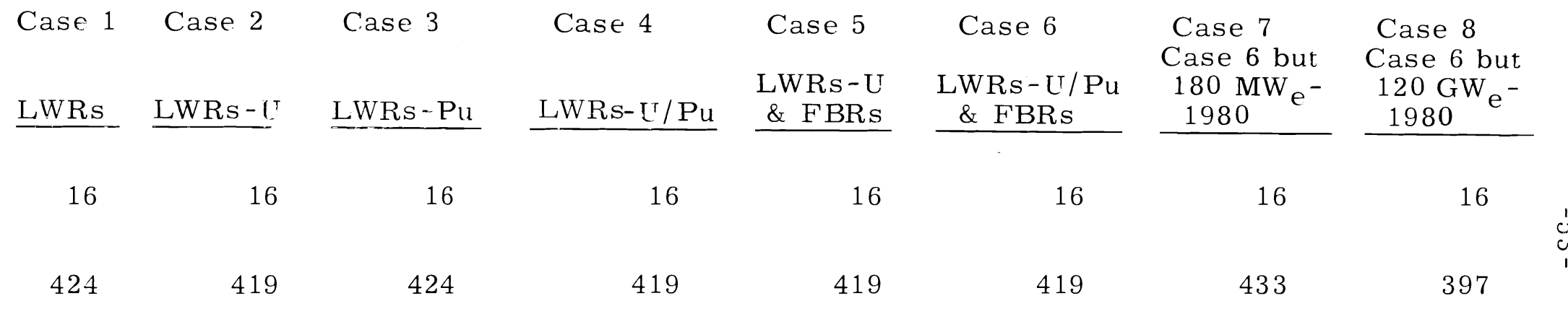

\begin{tabular}{|c|c|c|c|c|c|c|c|c|}
\hline 1975 & 424 & 419 & 424 & 419 & 419 & 419 & 433 & 397 \\
\hline 1980 & 1,150 & 1,080 & 1,180 & 1,120 & 1,080 & 1,110 & 1,300 & 936 \\
\hline 1985 & 2,600 & 2,460 & 2,780 & 2,670 & 2,390 & 2,440 & 2,950 & 1,990 \\
\hline 1990 & 5,040 & 4,640 & 5,460 & 5,150 & 4,110 & 4,280 & 5,300 & 3,410 \\
\hline
\end{tabular}


TABLE XXXI

ANNUAL AVAILABILITY OF STABLE PALLADIUM, $\mathrm{kg} / \mathrm{yr}$

\begin{tabular}{|c|c|c|c|c|c|c|c|}
\hline Case 1 & Case 2 & Case 3 & Case 4 & Case 5 & Case 6 & $\begin{array}{l}\text { Case } 7 \\
\text { Case } 6 \text { but }\end{array}$ & $\begin{array}{l}\text { Case } 8 \\
\text { Case } 6 \text { but }\end{array}$ \\
\hline$\underline{\mathrm{LWRS}}$ & $\underline{L W R S-U}$ & $\underline{L W R s}-\mathrm{Pu}$ & $\underline{L W R s-\mathrm{L}^{\top} / \mathrm{Pu}}$ & $\begin{array}{l}\text { LWRs-U } \\
\& \text { FBRS } \\
\end{array}$ & $\begin{array}{l}\mathrm{LWRs}-\mathrm{U} / \mathrm{Pu} \\
\& \text { FBRs } \\
\end{array}$ & $\begin{array}{l}180 \mathrm{MW}_{\mathrm{e}} \\
1980\end{array}$ & $\begin{array}{l}120 \mathrm{GW}^{-} \\
1980\end{array}$ \\
\hline 24 & 24 & 24 & 24 & 24 & 24 & 24 & 24 \\
\hline 695 & 706 & 695 & 706 & 706 & 706 & 727 & 674 \\
\hline
\end{tabular}

$\begin{array}{rrrrrrrrr}1980 & 1,910 & 1,860 & 2,060 & 2,010 & 1,860 & 2,010 & 2,340 \\ 1985 & 4,030 & 4,040 & 4,770 & 4,760 & 4,070 & 4,340 & 5,280 & 3,580 \\ 1990 & 7,450 & 7,300 & 9,210 & 9,050 & 7,780 & 8,300 & 10,200 & 6,660\end{array}$


TABLE XXXII

ANNUAL AVAILABILITY OF STABLE XENON, $\mathrm{kg} / \mathrm{yr}$

\begin{tabular}{|c|c|c|c|c|c|c|c|}
\hline Case 1 & Case 2 & Case 3 & Case 4 & Case 5 & Case 6 & $\begin{array}{l}\text { Case } 7 \\
\text { Case } 6 \text { but }\end{array}$ & $\begin{array}{l}\text { Case } 8 \\
\text { Case } 6 \text { but }\end{array}$ \\
\hline$\underline{L} \underline{W} \mathrm{~s}$ & LWRs - I & $\underline{\mathrm{LWR}} \mathrm{s}-\mathrm{Fu}$ & $\underline{L W R s-\mathrm{U} / \mathrm{Pu}}$ & $\begin{array}{l}\text { LWRs -U } \\
\& \text { FBRs } \\
\end{array}$ & $\begin{array}{l}\text { LWRs }-U / \mathrm{Pu} \\
\& \text { FBRs } \\
\end{array}$ & $\begin{array}{l}180 \mathrm{MW}_{\mathrm{e}} \\
1980 \\
\end{array}$ & $\begin{array}{l}120 \mathrm{GW}_{\mathrm{e}}- \\
1980\end{array}$ \\
\hline 167 & 167 & 167 & 167 & 167 & 167 & 167 & 167 \\
\hline 4,560 & 4,640 & 4,560 & 4,640 & 4,640 & 4,640 & 4,800 & 4,400 \\
\hline 12,600 & 12,300 & 21,400 & 12,100 & 12,300 & 12,100 & 14,200 & 10,200 \\
\hline 27,600 & 27,700 & 27,600 & 27,700 & 27,000 & 26,800 & 32,200 & 21,600 \\
\hline 51,700 & 50,800 & 51,500 & 50,700 & 46,700 & 47,200 & 57,200 & 37,500 \\
\hline
\end{tabular}


TABLE XXXIII

ANNUAL AVAILABILITY OF STABLE KRYPTON, kg/yr

\begin{tabular}{|c|c|c|c|c|c|c|c|}
\hline Case 1 & Case 2 & Case 3 & Case 4 & Case 5 & Case 6 & $\begin{array}{l}\text { Case } 7 \\
\text { Case } 6 \text { but }\end{array}$ & $\begin{array}{l}\text { Case } 8 \\
\text { Case } 6 \text { but }\end{array}$ \\
\hline LWRs & LWRs - I & LWRs - Pu & LWRs- $\mathrm{L}^{\top} / \mathrm{Pu}$ & $\begin{array}{r}\text { LWRs - U } \\
\& \text { FBRs }\end{array}$ & $\begin{array}{c}\text { LWRs - U / Pu } \\
\& \text { FBRs }\end{array}$ & $\begin{array}{l}180 \mathrm{MW}_{\mathrm{e}}^{-} \\
1980\end{array}$ & $\begin{array}{c}120 \mathrm{GW}^{1980} \\
\mathrm{e}^{-}\end{array}$ \\
\hline
\end{tabular}

\begin{tabular}{|c|c|c|c|c|c|c|c|c|}
\hline 1970 & 12 & 12 & 12 & 12 & 12 & 12 & 12 & 12 \\
\hline 1975 & 334 & 339 & 334 & 339 & 339 & 339 & 351 & 322 \\
\hline 1980 & 923 & 902 & 867 & 846 & 902 & 841 & 995 & 712 \\
\hline 1990 & 3,720 & 3,650 & 3,270 & 3,200 & 3,330 & 3,240 & 3,850 & 2,570 \\
\hline
\end{tabular}


TABLE XXXIV

ANNUAL AVAILABILITY OF STABLE RUTHENIUM, kg/yr

\begin{tabular}{|c|c|c|c|c|c|c|c|}
\hline Case 1 & Case 2 & LWRs-Pu & LWRS- $\mathrm{T} / \mathrm{Pu}$ & $\begin{array}{l}\text { Case } 5 \\
\text { LWRs-U } \\
\& \text { FBRS } \\
\end{array}$ & $\begin{array}{c}\text { Case } 6 \\
\text { LWRs }-U / P u \\
\& \text { FBRs } \\
\end{array}$ & $\begin{array}{l}\text { Case } 7 \\
\text { Case } 6 \text { but } \\
180 \mathrm{MW}_{\mathrm{e}}- \\
1980 \\
\end{array}$ & $\begin{array}{l}\text { Case } 8 \\
\text { Case } 6 \text { but } \\
120 \mathrm{GW}_{\mathrm{e}}^{-} \\
1980 \\
\end{array}$ \\
\hline 70 & 70 & 70 & 70 & 70 & 70 & 70 & 70 \\
\hline 1,970 & 2,000 & 1,970 & 2,000 & 2,000 & 2,000 & 2,070 & 1,910 \\
\hline 5,430 & 5,310 & 5,480 & 5,340 & 5,310 & 5,330 & 6,250 & 4,510 \\
\hline 1,700 & 11,700 & 12,200 & 12,200 & 11,500 & 11,600 & 14,000 & 9,400 \\
\hline 21,600 & 21,100 & 22,700 & 22,300 & 19,900 & 20,400 & 24,900 & 16,300 \\
\hline
\end{tabular}




\section{TABLE XXXV}

CUMULATIVE AVAILABILITY OF

PRINCIPAL STABLE FISSION PRODUCTS

(Case 6 Basis)

\begin{tabular}{llcc} 
& \multicolumn{3}{c}{ Metric Tons } \\
\cline { 2 - 3 } $\begin{array}{l}\text { Technetium-99 } \\
\text { Rhodium-103 }\end{array}$ & 0.1 & $\frac{1980}{9.6}$ & $\frac{1990}{61}$ \\
$\begin{array}{l}\text { Stable Palladium } \\
(104,105,106,107,108, \\
\text { and 110) }\end{array}$ & 0.06 & 5.1 & 60 \\
$\begin{array}{l}\text { Stable Xenon } \\
(131,132,134,136)\end{array}$ & 0.1 & 8.8 & 358 \\
$\begin{array}{l}\text { Stable Krypton* } \\
(83,84, \text { and 86) }\end{array}$ & 0.7 & 56 & 25 \\
$\begin{array}{l}\text { Stable Ruthenium*** } \\
(99,100,101,102, \text { and 104) }\end{array}$ & 0.3 & 4.0 & 156
\end{tabular}

*Contaminated with radioactive $\mathrm{Kr}-85$.

$* *$ Contaminated with radioactive $\mathrm{Ru}-106$. 
$\underline{\text { REFERENCES }}$

1. R. W. McKee, D. E. Deonigi, and D. R. Haffner, Isotope Production Projections for U.S. Nuclear Power Reactors, BNWL-SA-1529, paper presented at American Chemical Society Meeting, San Francisco, California, March 31 - April 5, 1968.

2. W. W. Porath, D. E. Deonigi, and E. T. Merrill, The Calibration of a High-Speed Burnup Code for Nuclear Fuel Surveys, BNWL-183, November 1965.

3. J. H. Nail, CLOTHO: A Code for Communicating Reactor Mass Balance Data to Reactor Economics Codes, BNWL-770, July 1968.

4. Westinghouse Electric Corp., Liquid Metal Fast Breeder Reactor Design Study, Report WCAP-3251-1, January 1964.

5. E. N. Sloth, D. L. Horrocks, E. J. Boyce and M. H. Studier, 'Tritium in Thermal Neutron Fission of Uranium-235?" J. Inorg. Nucl. Chem., vol. 24, pp. 337-341, 1962.

6. C. A. Rohrmann, Values in Spent Fuel from Power Reactors, BNWL-25, March 1965.

7. L. W. Lang, D. E. Deonigi, and C. A. Rohrmann, "Power Cost Reduction from Isotope Revenues," Nuclear Applications, vol. 3, p. 665, November 1967. 



\section{APPENDIX}

\section{ISOPRO RESULTS}

A complete printout of the ISOPRO program for the eight cases evaluated in this study is presented in this Appendix. 


\section{SOTOPE PRODUCTION AND}

A V A I L A B I L I T Y REPORT

JULY 1,1968 
IT IS THE OBJECTIVE OF THIS REPORT TO PROVIUE ANIJUAL ANO CUMULATIVE LISTINGS OF ISOTOPES OF INTEREST FOR UIFFERENT REACTOR COMBINATIONS AHO REACTOR FUELING STRATEGIES FROM 1960 THROUGH 1990. THPOUGHOUT THIS INTERVAL, VARIOUS ENERGY DEMAIND CURVES CAN BE SATISFIED. THE REACTOR COMBINATIONS ANO ENERGY DEMANDS MET ARE REPORTED AT THE BEGININING OF EACH CASE.

THIS REPORT IS ARRANGED IN THE FOLLOWING ORLER--

PAGE

DESCRIPTION OF CASES....................... 2

GROUNU RULES.............................. 3

ISOTOPE DEFINITIONS AND HALF LIFE TABLE........... 6

REACTUR TYPE DESCRIPTION.................... 7

DISCHARGE SCHEDULE FOK EACH REACTOR

AND ITS FUELING MODE..................... 
ISOTOPE PRODUCTION TABLES (ANNUAL AND CUMULATIVE)

- INDICATES ISOTOPE PRONUCTION AT DISCHARGE WITH NO REPROCESSING TIME OR LOSSES. EXCEPT CUMULATIVE TABLES INCLUDE DECAY LOSSES.

ISOTOPE AVAILABILITY TABLES (ANNUAL AND CUMULATIVE)

- INDICATE ISOTOPE AVAILABILITY ALLOWING FOR REPROCESSING TIME AND LOSSES DUE TO DECAY AND REPROCESSING.

DESCRIPTION OF CASES

CASE
PAGE

IIGHT WATER REACTORS (LWR'S) FUELED WITH SLIGHTLY ....... ENRICHED URANIUM WITHOUT PLUTONIUM OR URANIUM RECYCLE.

150 GWE NUCLEAR CAPACITY IN 1980.

LWR'S AS IN CASE 1 EXCEPT 50\% OF U-236 PRODUCTION........ IS RECYCLED.

LWR'S AS IN CASE 1 EXCEPT PLUTONIUM IS RECYCLED......... 64

LWR'S AS IN CASE 1 EXCEPT PLUTONIUM ANU $50 \%$ OF $U-236 \ldots \ldots . . .78$ IS RECYCLED.

5 LWR'S WITH $50 \%$ U-236 RECYCLE AND MAXIMUM FAST BREEDER.... REACTOR (FBR) ADDITIONS BEGINNING IN 1980.

LWR'S WITH 50\% U-236 RECYCLE, LIMITED PLUTONIUM RECYCLE... 106 AND MAXIMUM FBR ADDITIONS BEGINNING IN 1980.

7 CASE 6 EXCEPT 180 GWE NUCLEAR CAPACITY IN $1980 \ldots \ldots \ldots \ldots \ldots \ldots 120$

8 CASE 6 EXCEPT 120 GWE NUCLEAR CAPACITY IN $1980 \ldots \ldots \ldots \ldots \ldots \ldots 134$ 


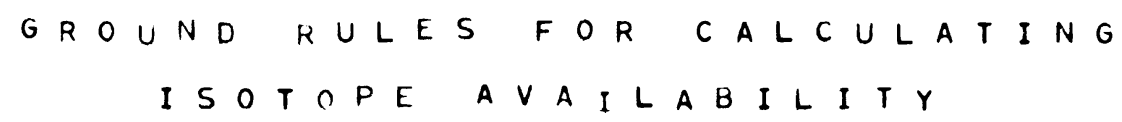

1. INSTALLEO NUCLEAR CAPACITY AND ISOTOPE PRODUCTION AND AVAILABILITY ARE ALWAYS STATED AS OF END OF YLAR SHOWN.

2. YEARLY CAPACITY ADDITIONS STARTUP AT MID-YEAR.

3. NUCLEAR POWER PLANT LIFE IS 30 YEARS.

4. NUCLEAR POWER PLANT CAPACITY FACTORS ARE 85\% FOR 15 YEARS, DECLINING LINEARLY TO $50 \%$ AT 30 YEARS.

5. LIGHT WATER REACTORS (LWR'S) ARE 67\% PWR'S ANU 33\% BWR'S AFTER 1971. CONTRACT COMMITMENTS AND OPERATING REACTORS ARE USED FOR PRIOR YEARS.

6. ONE YEAR BETWEEN REACTOR DISCHARGE AND RECOVERY OF ANY ISOTOPE. RECOVERY CORRECTED FOR DECAY LOSSES.

7. $98 \%$ RECOVERY OF PLUTONIUM AND URANIUM.

8. 90\% RECOVERY OF ALL OTHER BY-PROUUCTS.

9. PM-147 RECOVERY DELAYED 3 YEARS AFTER FUEL DISCHARGE TO ALLOW FOR PM-148 DECAY.

10. CM-244 RECOVERY DELAYED 3 YEARS AFTER FUEL DISCHARGE TO ALLOW FOR CM-242 DECAY.

11. PU-238 FORMED BY CM-242 DECAY. EXCEPT FOR FIRST YEAR PRIOR TO SEPARATIONS, IS AVAILABLE WITH THE CM-244. 
THE FOLLOWING DEFINED ISOTOPES ARE REPORTEU.

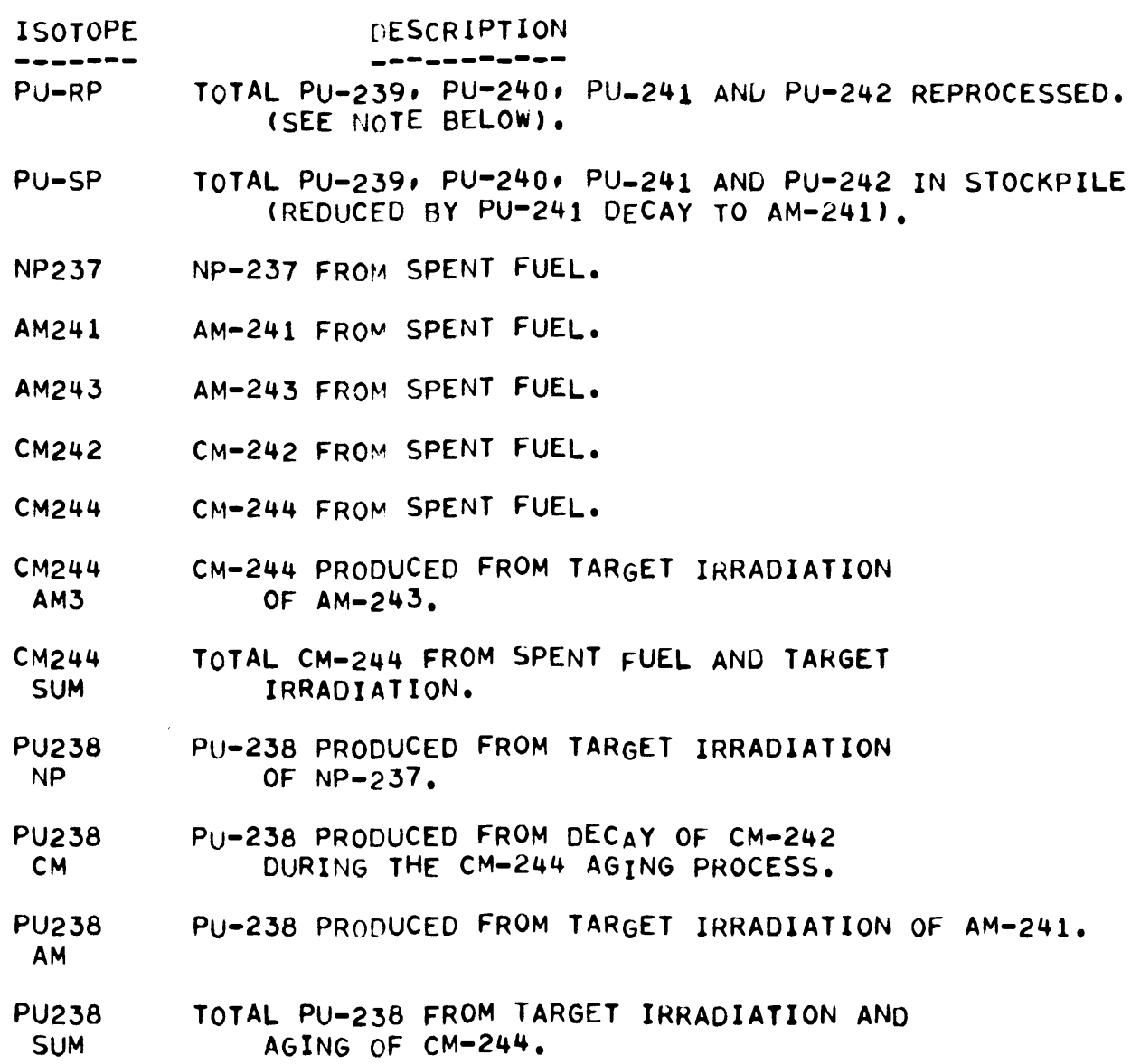




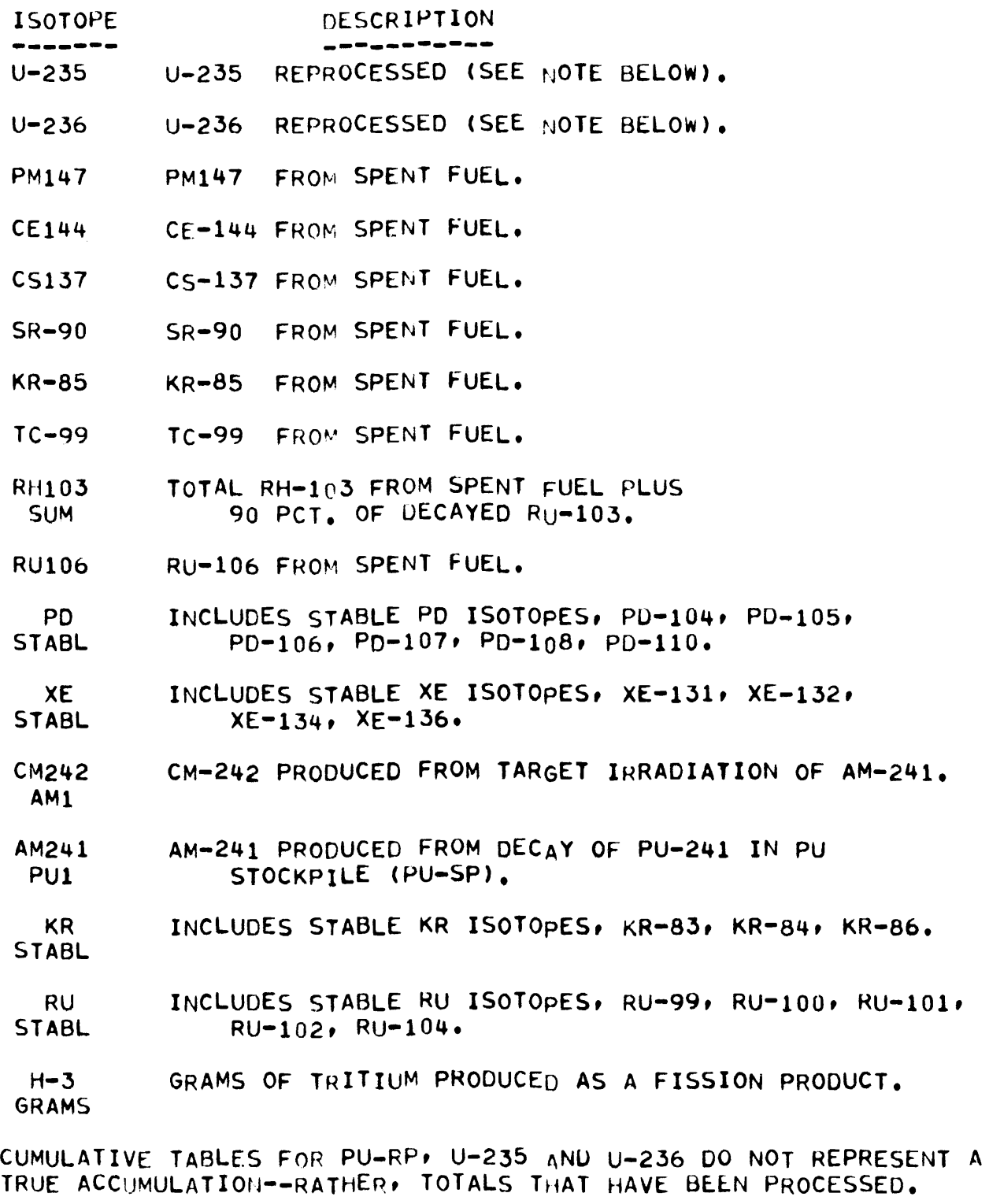




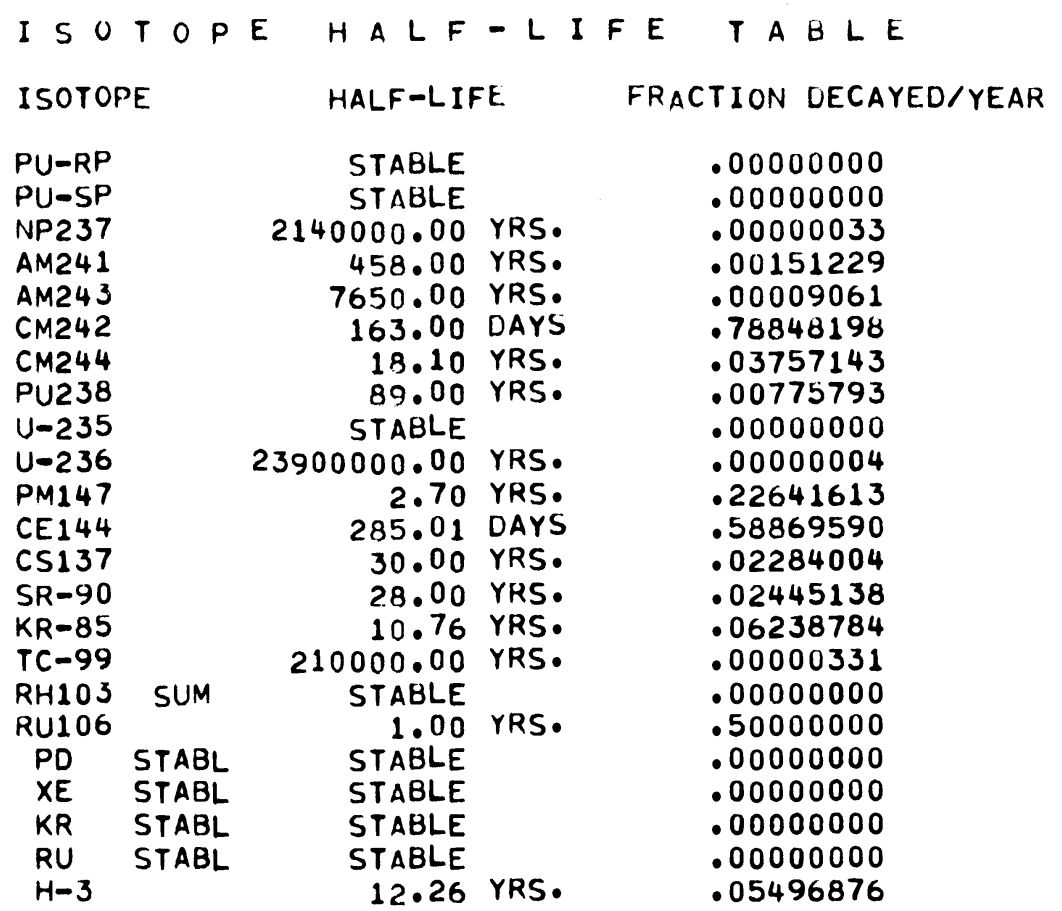




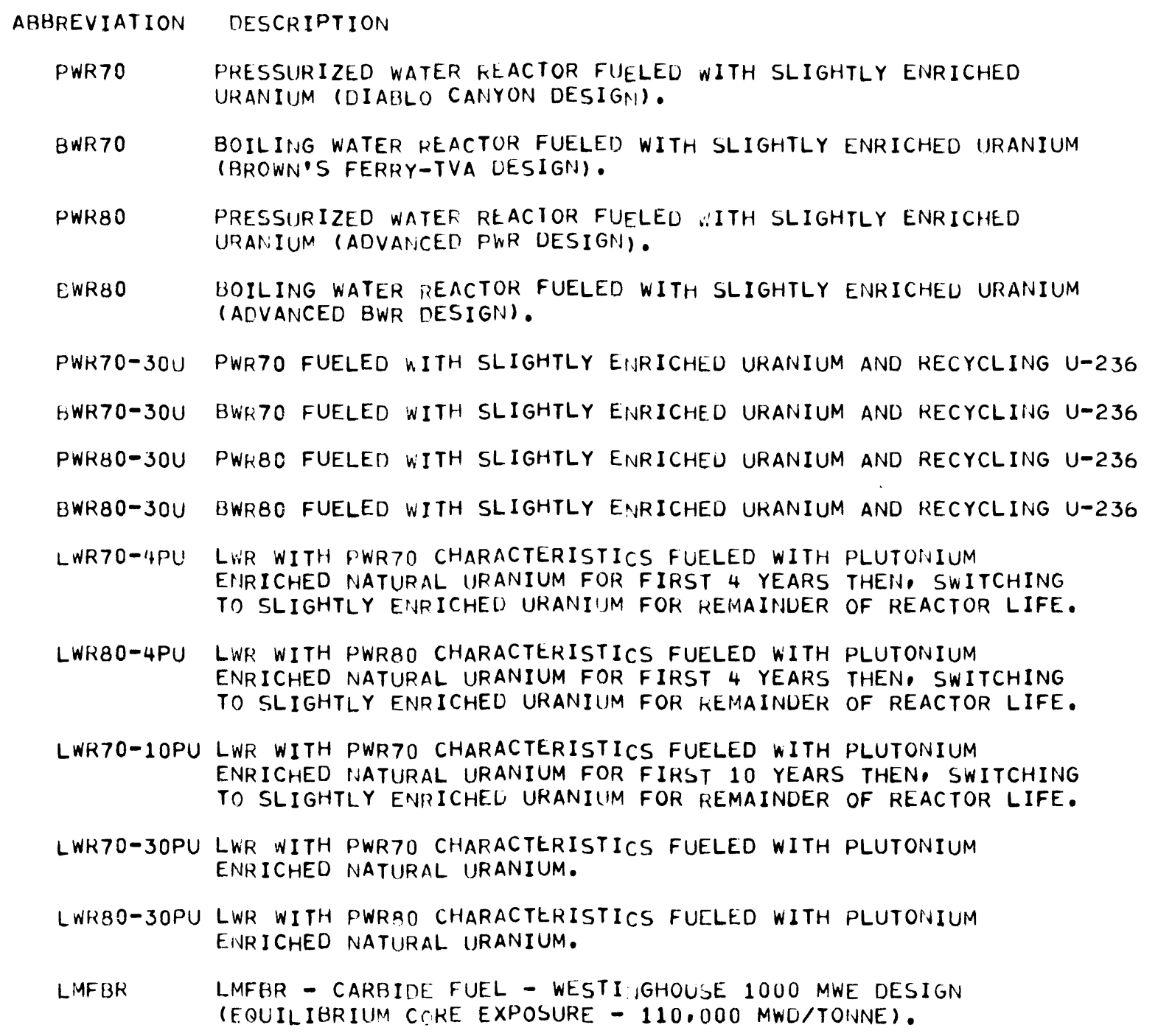


DISCHARGE

CYCLE
NUMBE
1
5
3
4
5
0
7
8
9
10
11
12
13
14
15
16
17
18
19
20
21
22
23
24
25
20
27
26
29
30
31
32
33
34
35
30
37
30
34
40

ISOTOPE PRODUCTION. GRAMS/TONNE

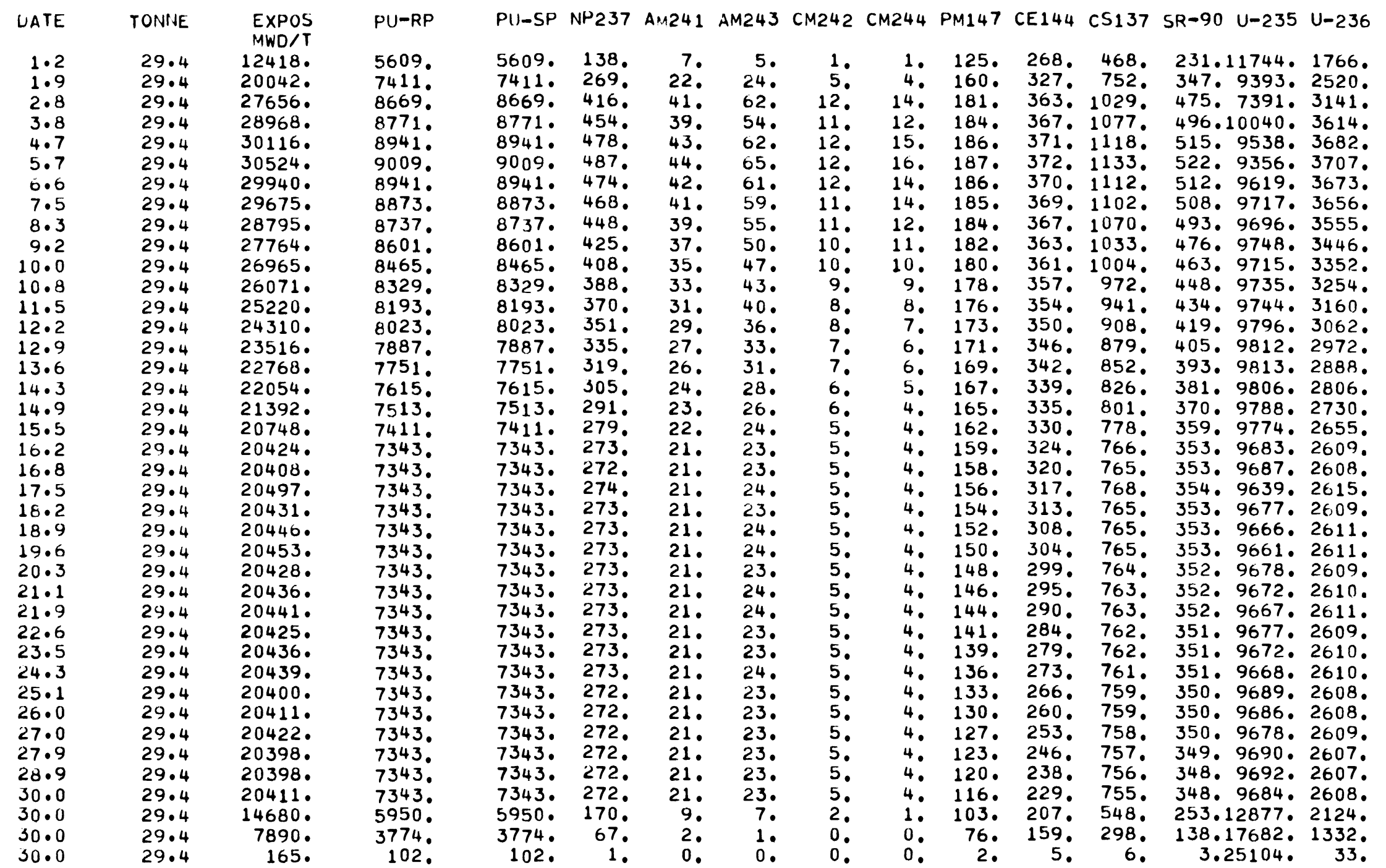


DISCHARGE

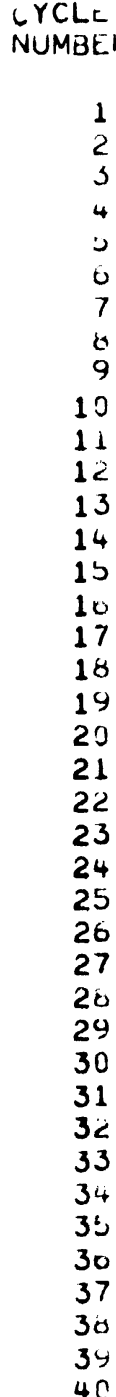

ISOTOPE PRODUCTION, GRAMS/TUNINE

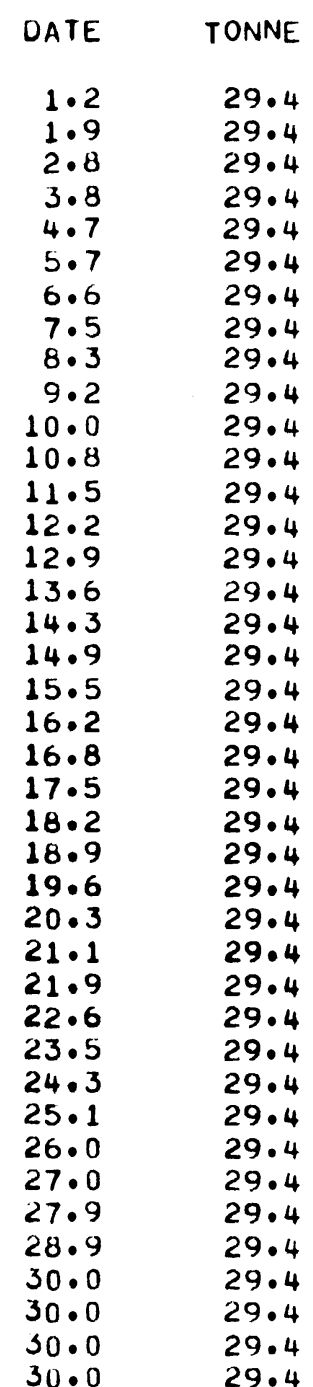

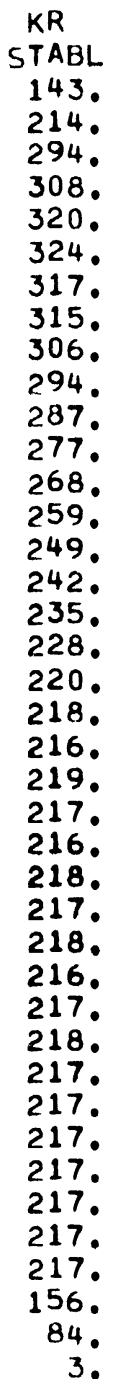

TC-99 RH103 RU106 RU

$$
\begin{array}{lllll}
\text { SUM } & \text { RU106 RU } & \text { PD } & \text { XE } \\
\text { 323. } & \text { STABL STABL STABL } & \text { ST. } \\
\text { 185. } & 63 . & 742 . & 224 . & 1969 .
\end{array}
$$$$
\text { 323. 185. 63. 742. 224. 1969. }
$$$$
510 \text {. 283. 101. 1233. 423. } 2967 .
$$$$
\text { 692. 353. 116. 1693. 581. 3956. }
$$$$
\text { 722. 364. 118. 1775. 609, } 4130 \text {. }
$$$$
749.373 .6119 .1842 .632 .4276 .
$$$$
758 \text {. 376. } 120.1869 .641 .4334 .
$$$$
\text { 745. 372. 119. 1829. 627. } 4247^{\circ} \text {. }
$$$$
\text { 739. 369. 119. 1815. 623. } 4217 .
$$$$
\text { 718. 363. 118. 1761. 604. 4101. }
$$$$
\text { 694. 354. 116. 1693. 581. 3956. }
$$$$
\text { 676. 348. 115. 1653. 567. 3868. }
$$$$
\text { 655. 340. 113. 1598. 548. 3752. }
$$$$
\text { 634. 333. 112. 1544. 530. 3636。 }
$$$$
\text { 613. 325. 110. 1490. 511. 3519. }
$$$$
\text { 594. 317. 109. 1436. 493. } 3403 .
$$$$
\text { 576. 310. 107.1395. 479. 3316. }
$$$$
\text { 559. 304. 106. 1355. 465. 3228. }
$$$$
\text { 543. 297. 104. 1314. 451. 3141. }
$$$$
\text { 527. 290. 102. 1266. 434. 3036. }
$$$$
\text { 519. 285. 101. 1257. 431.3011. }
$$$$
\text { 518. 284. 100.1247. 428. 2983. }
$$$$
\text { 520. 283. 99. 1261. 433. 3008. }
$$$$
\text { 518. 281. 98. 1249. 428. 2976. }
$$$$
\text { 517. 279. 97. } 1247^{\circ}, 428.2967^{\circ}
$$

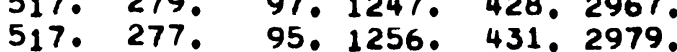$$
\text { 516. 275. 94. 1251. 429. 2963. }
$$$$
\text { 515. 273. 93. 1256. 431.2967. }
$$$$
\text { 515. 271. 92. 1247. 428.2941。 }
$$$$
\text { 513. 268. 90.1247. 428.2934. }
$$$$
\text { 513. 266. 89. 1256. 431. 2945. }
$$

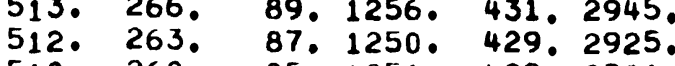$$
510.260 .85 .1251 .429 .2921 .
$$$$
\text { 509. 257. 83. 1249. 428.2908. }
$$$$
\text { 508. 254. 81. 1251. 429.2904. }
$$$$
\text { 506. 250. 79. 1248. 428.2891. }
$$$$
\text { 505. 246. 77. } 1249.429 .2884^{\circ} \text {. }
$$

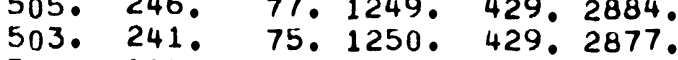$$
370.194 .66 .901 .9309 .2123 .
$$$$
\text { 204. 122. 47. 486. 167. } 1232 .
$$ 
DISCHARGE

CYCL

DATE TONNE

$\begin{array}{ll}2.6 & 67.8 \\ 3.5 & 33.9\end{array}$

$4.4 \quad 33.9$

$\begin{array}{lll}5.5 & 33.9 & 25692 .\end{array}$

$\begin{array}{lll}7.3 & 33.9 & 27603 .\end{array}$

$8.2 \quad 33.9 \quad 27222$.

$9.1 \quad 33.9 \quad 25795$.

$9.9 \quad 33.9 \quad 25127$.

$10.8 \quad 33.9 \quad 24259$.

$11.5 \quad 33.9 \quad 23379$.

$12.3 \quad 33.922394$.

$\begin{array}{lll}12.3 & 33.9 & 22394 . \\ 13.0 & 33.9 & 21598 .\end{array}$

$\begin{array}{lll}13.7 & 33.9 & 20804 . \\ 14.4 & 33.9 & 19973 .\end{array}$

$\begin{array}{lll}14.4 & 33.9 & 19973 . \\ 15.0 & 33.9 & 19203 .\end{array}$

$15.6 \quad 33.9 \quad 18593$.

$16.3 \quad 33.9 \quad 18127$.

$17.0 \quad 33.9 \quad 17843$.

$17.6 \quad 33.9 \quad 17832$.

$18.3 \quad 33.9 \quad 17814$.

$19.7 \quad 33.9 \quad 17821$.

$\begin{array}{lll}19.7 & 33.9 & 17821 . \\ 20.5 & 33.9 & 17820 .\end{array}$

$\begin{array}{lll}20.5 & 33.9 & 17820 . \\ 21.2 & 33.9 & 17779 .\end{array}$

$22.0 \quad 33.9 \quad 17819$.

$22.8 \quad 33.9 \quad 17818$.

$23.6 \quad 33.9 \quad 17775$.

$24.4 \quad 33.9 \quad 17817$.

$25.3 \quad 33.9 \quad 17817$.

$\begin{array}{lll}26.2 & 33.9 & 17811 . \\ 27.1 & 33.9 & 17822 .\end{array}$

$28.1 \quad 33.9 \quad 17825$

$29.1 \quad 33.9 \quad 17819$ 。

$30.0 \quad 33.9 \quad 17225$.

30.0

$30 \cdot 0$

30.0

33.9

33.9

$17225 \circ$
13018.

13018.
8527.

3943.

ISOTOPE PRODUCTION. GRAMS/TONNE

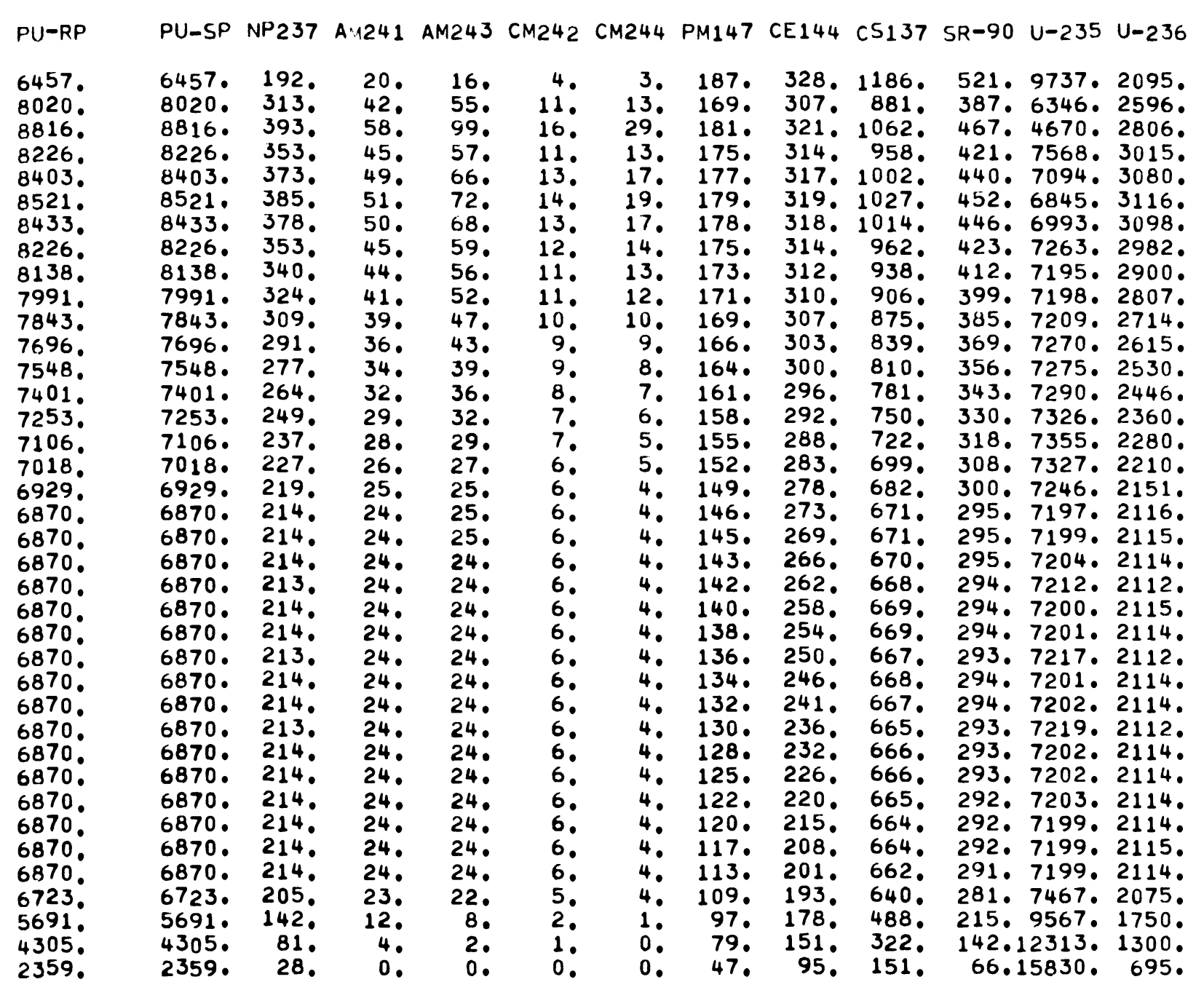


DISCHARGE

\begin{tabular}{|c|c|c|}
\hline $\begin{array}{l}\text { CYCLE } \\
\text { NUMBLKK }\end{array}$ & UATE & TONNE \\
\hline 1 & $2 \cdot 6$ & 67.8 \\
\hline$<$ & $3 \cdot 5$ & 33.9 \\
\hline 3 & 4.4 & 33.9 \\
\hline 4 & $5 \cdot 5$ & 33.9 \\
\hline 5 & 6.4 & 33.9 \\
\hline bo & $7 \cdot 3$ & 33.9 \\
\hline 7 & 8.2 & 33.9 \\
\hline 8 & 9.1 & 33.9 \\
\hline 9 & 9.9 & 33.9 \\
\hline 10 & 10.8 & 33.9 \\
\hline $1 i$ & $11 \cdot 5$ & 33.9 \\
\hline 12 & $12 \cdot 3$ & 33.9 \\
\hline 13 & 13.0 & 33.9 \\
\hline 14 & 13.7 & 33.9 \\
\hline 15 & $14 \cdot 4$ & 33.9 \\
\hline 10 & $15 \cdot 0$ & 33.9 \\
\hline 17 & 15.6 & 33.9 \\
\hline 10 & $16 \cdot 3$ & 33.9 \\
\hline 19 & 17.0 & 33.9 \\
\hline 20 & 17.6 & 33.9 \\
\hline 21 & 18.3 & 33.9 \\
\hline $2 a$ & 19.0 & 33.9 \\
\hline 25 & 19.7 & 33.9 \\
\hline 24 & $20 \cdot 5$ & 33.9 \\
\hline 25 & $21 \cdot 2$ & 33.9 \\
\hline 20 & $22 \cdot 0$ & 33.9 \\
\hline 27 & $22 \cdot 8$ & 33.9 \\
\hline 20 & 23.6 & 33.9 \\
\hline 29 & $24 \cdot 4$ & 33.9 \\
\hline 30 & 25.3 & 33.9 \\
\hline 31 & $26 \cdot 2$ & 33.9 \\
\hline 32 & $27 \cdot 1$ & 33.9 \\
\hline $3 j$ & $28 \cdot 1$ & 33.9 \\
\hline 34 & 29.1 & 33.9 \\
\hline 35 & 30.0 & 33.9 \\
\hline 36 & $30 \cdot 0$ & 33.9 \\
\hline 37 & $30 \cdot 0$ & 33.9 \\
\hline 30 & 30.0 & 33 \\
\hline
\end{tabular}

ISOTOPE PROUUCTION. GRAMS/TONNE

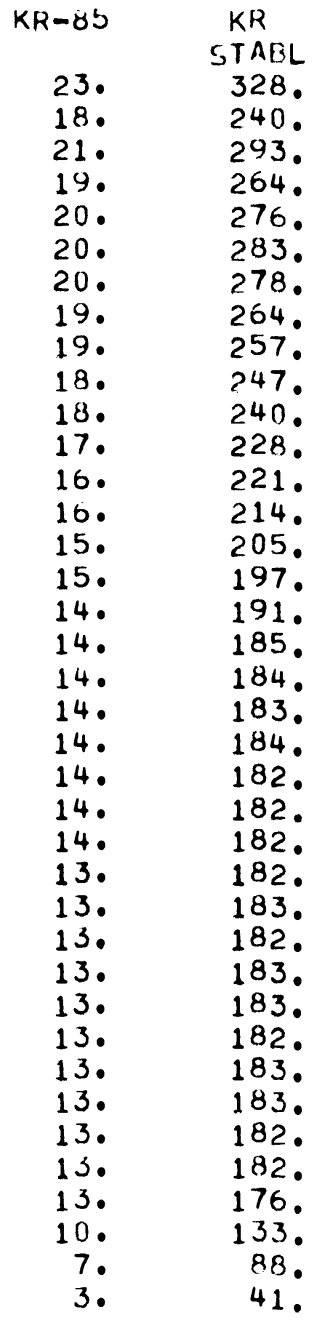

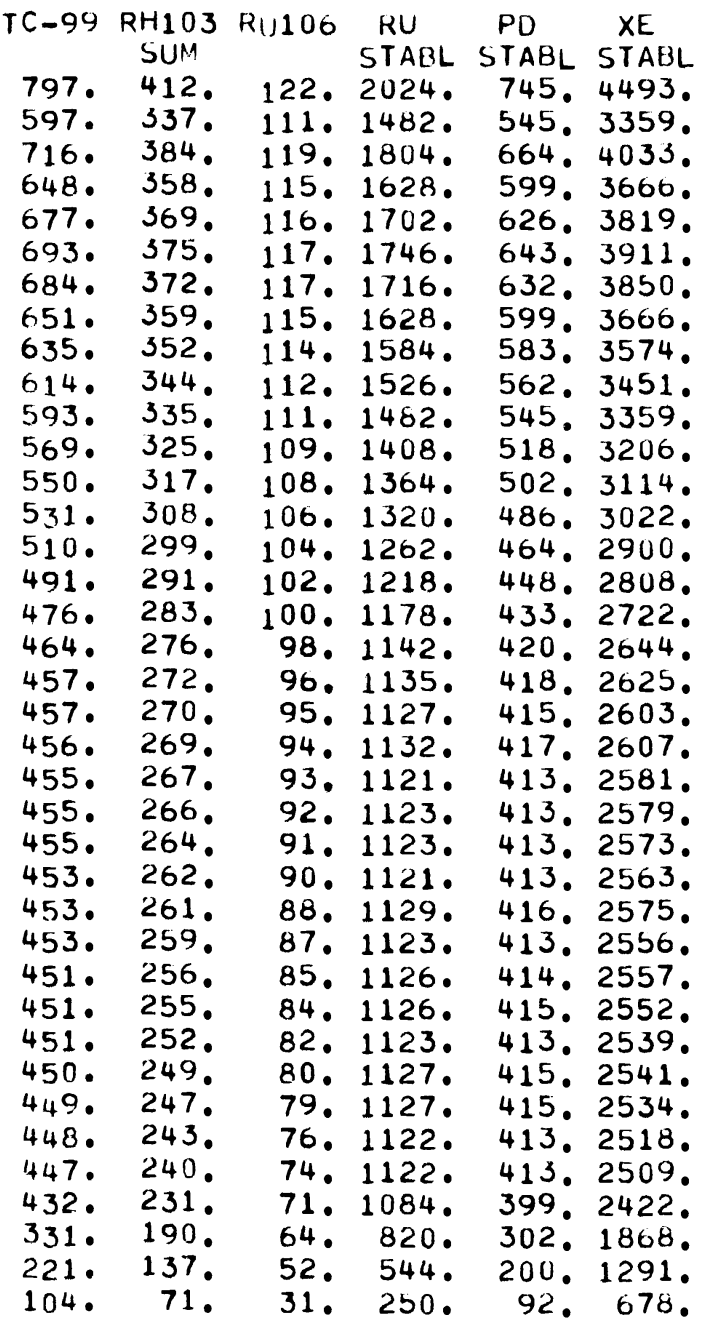


DISCHARGE SCHEUULE FOR 1000 MW (E) PWRO-

PAGE 12

DISCHARGE

CYCL

\begin{tabular}{|c|c|c|c|}
\hline UATE & TONNE & $\begin{array}{l}\text { EXPOS } \\
\text { MWD/T }\end{array}$ & $P U-R P$ \\
\hline .8 & $25 \cdot 7$ & 9977. & 4857. \\
\hline $1 \cdot 4$ & $25 \cdot 7$ & 16070 & 6527 \\
\hline $2 \cdot 0$ & $25 \cdot 7$ & 22631 . & 7851 \\
\hline 2.7 & 25.7 & $23914^{\circ}$ & 7929 \\
\hline $3 \cdot 3$ & $25 \cdot 7$ & 24368 . & 8046 \\
\hline 3.9 & $25 \cdot 7$ & 23700 & 7929 \\
\hline $4 \cdot 5$ & $25 \cdot 7$ & 22516 & 7696 \\
\hline $5 \cdot 1$ & $25 \cdot 7$ & 21773 & 7579 \\
\hline $5 \cdot 6$ & 25.7 & 21082 & 7463. \\
\hline $6 \cdot 2$ & 25.7 & 20513 & 7346 \\
\hline 6.7 & 25.7 & 20340 . & 7307 \\
\hline $7 \cdot 3$ & 25.7 & 20311 . & 7307. \\
\hline $7 \cdot 8$ & $25 \cdot 7$ & 20323 & 7307 \\
\hline $8 \cdot 4$ & 25.7 & 20332 . & 7307 \\
\hline $8 \cdot 9$ & 25.7 & 20318 & 7307. \\
\hline $9 \cdot 5$ & 25.7 & 20324 & 7307 . \\
\hline $10 \cdot 0$ & 25.7 & 20333 & 7307. \\
\hline $10 \cdot 6$ & 25.7 & 20312 & 7307. \\
\hline $11 \cdot 1$ & 25.7 & 20321. & 7307. \\
\hline 11.7 & 25.7 & 20329 . & 7307 \\
\hline $12 \cdot 2$ & 25.7 & 20308 & 7307 \\
\hline $12 \cdot 8$ & 25.7 & 20318 . & 7307. \\
\hline $13 \cdot 3$ & $25 \cdot 7$ & 20340 . & 7307. \\
\hline 13.9 & 25.7 & 20407. & 7346. \\
\hline $14 \cdot 4$ & $25 \cdot 7$ & 20350 . & 7307. \\
\hline $15 \cdot 0$ & $25 \cdot 7$ & 20349. & 7307. \\
\hline $15 \cdot 5$ & $25 \cdot 7$ & 20372 . & 7346 . \\
\hline $16 \cdot 1$ & $25 \cdot 7$ & 20350 . & 7307. \\
\hline $16 \cdot 7$ & $25 \cdot 7$ & 20350 . & 7307. \\
\hline $17 \cdot 3$ & $25 \cdot 7$ & 20361 . & 7307 \\
\hline $17 \cdot 9$ & $25 \cdot 7$ & 20348 . & 7307 \\
\hline $18 \cdot 5$ & 25.7 & 20362 . & 7307 \\
\hline 19.1 & $25 \cdot 7$ & 20361 . & 7307 \\
\hline 19.7 & 25.7 & 20353. & 7307 \\
\hline $20 \cdot 3$ & 25.7 & 20350 . & 7307 \\
\hline $21 \cdot 0$ & $25 \cdot 7$ & 20358 . & 7307 \\
\hline 21.7 & $25 \cdot 7$ & 20354 & 7307 \\
\hline $22 \cdot 3$ & $25 \cdot 7$ & 203 & $730^{\circ}$ \\
\hline $23 \cdot 0$ & 25.7 & 20349. & 7307 \\
\hline 23 & $25 \cdot 7$ & 20 & 730 \\
\hline
\end{tabular}

ISOTOPE PRODUCTION, GRAMG/TONNE

PU-SP NP237 A:1241 AM243 CM242 CM244 PM147 CE144 CS137 SK-90 U-235 U-236

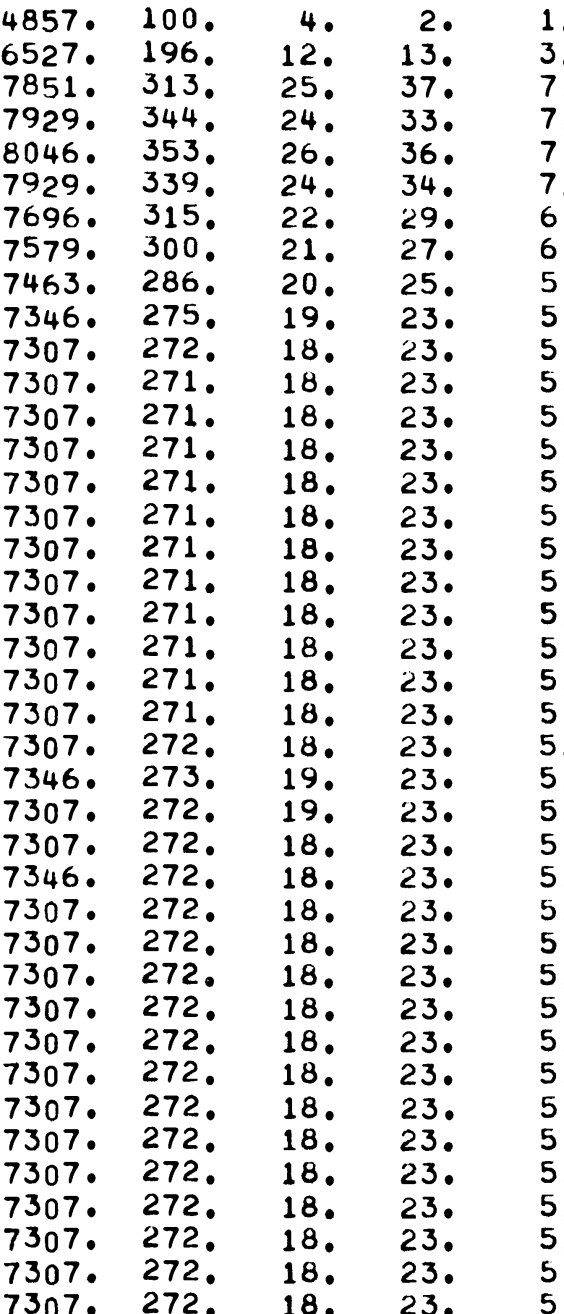

0. 113. 245. 377, 186.11980. 1469 2. 155. 328. 604. 298. 9621. 2090 7. 182. 372. 849. 392.7789. 2666. 6. 186. 380. 896. 414.10451.3080. 7. 188. 382. 913. 421.9924. 3080. 6. 186. 378. 889. 410.9870. 2999. 5. 181. 371. 845. 390.10019. 2877. b. 179. 366. 818. 377.10005. 2791. 4. 176. 361. 792. 366.9978. 2709. 4. 174. 357. 771, 356.9893.2636. 4. 173. 355. 765. 353.9866. 2614. 4. 173. 355. 764. 353.9874. 2612. 4. 173. 355. 764. 353.9873.2613. 4. 173. 355. 765. 353.9869.2613. 4. 173. 355. 764. 353.9871. 2613. 4. 173. 355. 764. 353.9873.2613. 4. 173. 355. 765. 353.9869.2613. 4. 173. 355. 764. 353.9874. 261?. 4. 173. 355. 764. 353.9874. 2612 4. 173. 355. 765. 353.9871.2613. 4. 173. 355. 764. 353.9875. 2612. 4. 173. 355. 764. 353.9876.2612. 4. 173. 355. 765. 353. 9865. 2614. 4. 173. 356. 767. 354.9825.2619. 4. 173. 355. 765. 353.9859. 2614. 4. 173. 355. 765. 353.9859. 2615. 4. 172. 354. 766. 354.9842. 2617. 4. 171. 351. 765. 353.9860. 2614. 4. 169. 348. 765. 353.9858. 2615. 4. 168. 344. 765. 353.9848.2616. 4. 166. 341. 764. 353.9861.2614. 4. 165. 337. 764. 353.9851.2616. 4. 163. 334. 764. 353.9849. 2616. 4. 162. 330. 764. 352. 9859. 2615. 4. 160. 326. 763. 352.9857. 2615. 4. 158. 322. 763. 352.9850. 2616. 4. 156. 317, 762, 352.9858, 2615. 4. 154. 313. 762. 351.9858.2615. 4. 152. 308. 761. 351.9855. 2615. 4. 150. 303. 761. 351.9862.2614. 
DISCHARGE

$\begin{array}{crr}\text { CYCLE } & & \\ \text { NUMBEK } & \text { UATE } & \text { TONNE } \\ & & \\ 1 & .8 & 25.7 \\ 2 & 1.4 & 25.7 \\ 3 & 2.0 & 25.7 \\ 4 & 2.7 & 25.7 \\ 5 & 3.3 & 25.7 \\ 0 & 3.9 & 25.7 \\ 7 & 4.5 & 25.7 \\ 0 & 5.1 & 25.7 \\ 9 & 5.6 & 25.7 \\ 10 & 6.2 & 25.7 \\ 11 & 6.7 & 25.7 \\ 12 & 7.3 & 25.7 \\ 13 & 7.8 & 25.7 \\ 14 & 8.4 & 25.7 \\ 15 & 8.9 & 25.7 \\ 10 & 9.5 & 25.7 \\ 17 & 10.0 & 25.7 \\ 10 & 10.6 & 25.7 \\ 13 & 11.1 & 25.7 \\ 20 & 11.7 & 25.7 \\ 21 & 12.2 & 25.7 \\ 22 & 12.8 & 25.7 \\ 23 & 13.3 & 25.7 \\ 24 & 13.9 & 25.7 \\ 25 & 14.4 & 25.7 \\ 20 & 15.0 & 25.7 \\ 27 & 15.5 & 25.7 \\ 20 & 16.1 & 25.7 \\ 29 & 16.7 & 25.7 \\ 30 & 17.3 & 25.7 \\ 31 & 17.9 & 25.7 \\ 32 & 18.5 & 25.7 \\ 33 & 19.1 & 25.7 \\ 34 & 19.7 & 25.7 \\ 35 & 20.3 & 25.7 \\ 30 & 21.0 & 25.7 \\ 37 & 21.7 & 25.7 \\ 30 & 22.3 & 25.7 \\ 39 & 23.0 & 25.7 \\ 40 & 23.8 & 25.7\end{array}$

ISOTOPE PRODUCTION, GRAMS/TONNE

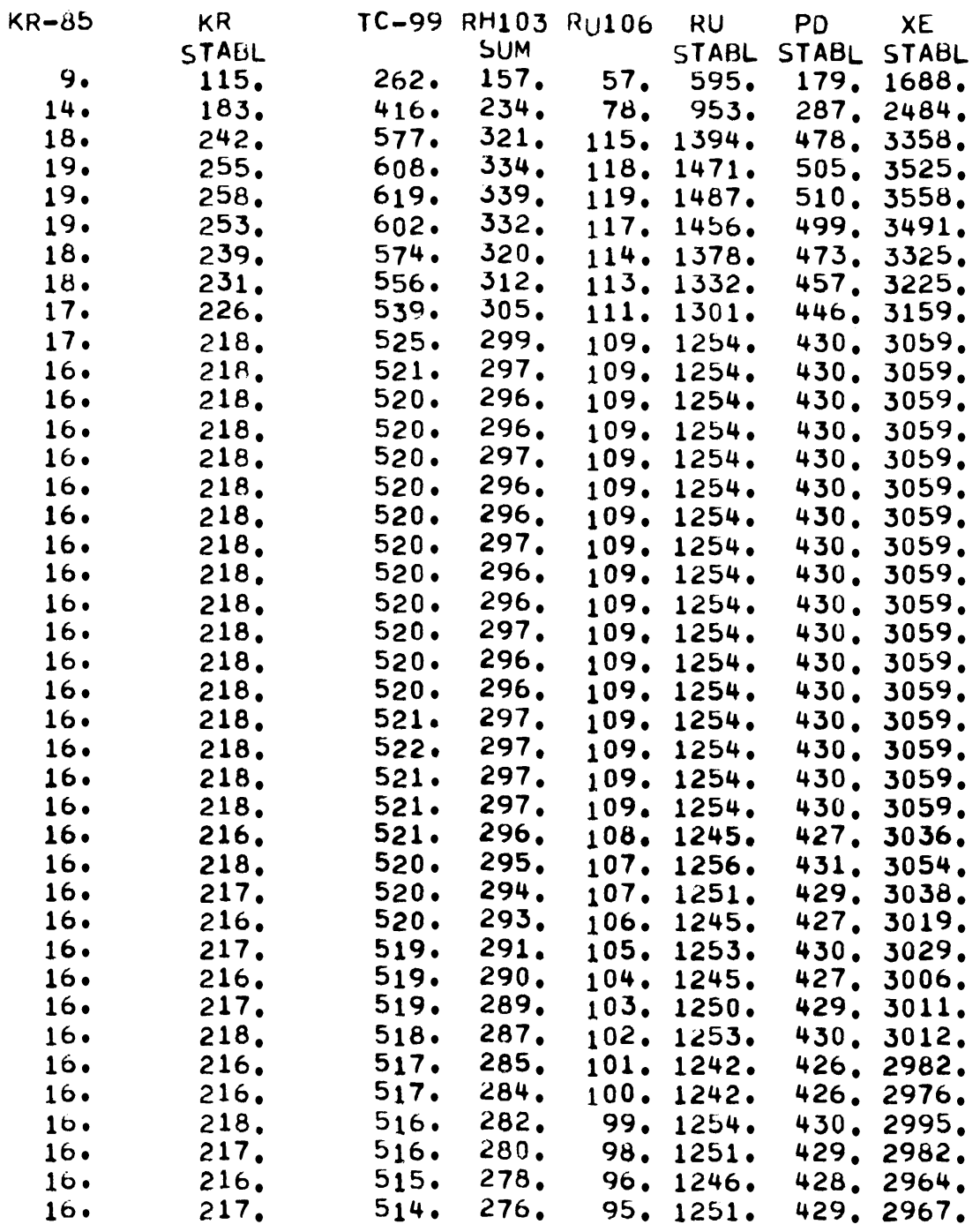


DISCHARGE SCHEDULE FOR 1000 MW ( E) BWR80-

PAGE 14

DISCHARGE

CYCL NUMBER DATE TONNE

\begin{tabular}{|c|c|c|}
\hline $\begin{array}{l}1 \cdot 8 \\
2 \cdot 3 \\
2 \cdot 9 \\
3 \cdot 6 \\
4 \cdot 1 \\
4 \cdot 7 \\
5 \cdot 2 \\
5 \cdot 8 \\
6 \cdot 3 \\
6 \cdot 8 \\
7 \cdot 4 \\
7 \cdot 9 \\
8 \cdot 4 \\
9 \cdot 0 \\
9 \cdot 5 \\
10 \cdot 0 \\
10 \cdot 5 \\
11 \cdot 1 \\
11 \cdot 6 \\
12 \cdot 1 \\
12 \cdot 7 \\
13 \cdot 2 \\
13 \cdot 7 \\
14 \cdot 2 \\
14 \cdot 8 \\
15 \cdot 3 \\
15 \cdot 8 \\
16 \cdot 4 \\
16 \cdot 9 \\
17 \cdot 5 \\
18 \cdot 1 \\
18 \cdot 7 \\
19 \cdot 3 \\
19 \cdot 9 \\
20 \cdot 5 \\
21 \cdot 1 \\
21 \cdot 8 \\
22 \cdot 4 \\
23 \cdot 1 \\
23 \cdot 8\end{array}$ & $\begin{array}{l}58 \cdot 5 \\
29 \cdot 2 \\
29 \cdot 3 \\
29 \cdot 3 \\
29 \cdot 3 \\
29 \cdot 3 \\
29 \cdot 3 \\
29 \cdot 3 \\
29 \cdot 3 \\
29 \cdot 3 \\
29 \cdot 3 \\
29 \cdot 3 \\
29 \cdot 3 \\
29 \cdot 3 \\
29 \cdot 3 \\
29 \cdot 3 \\
29 \cdot 3 \\
29 \cdot 3 \\
29 \cdot 3 \\
29 \cdot 3 \\
29 \cdot 3 \\
29 \cdot 3 \\
29 \cdot 3 \\
29 \cdot 3 \\
29 \cdot 3 \\
29 \cdot 3 \\
29 \cdot 3 \\
29 \cdot 3 \\
29 \cdot 3 \\
29 \cdot 3 \\
29 \cdot 3 \\
29 \cdot 3 \\
29 \cdot 3 \\
29 \cdot 3 \\
29 \cdot 3 \\
29 \cdot 3 \\
29 \cdot 3 \\
29 \cdot 3 \\
29 \cdot 3 \\
29 \cdot 3\end{array}$ & 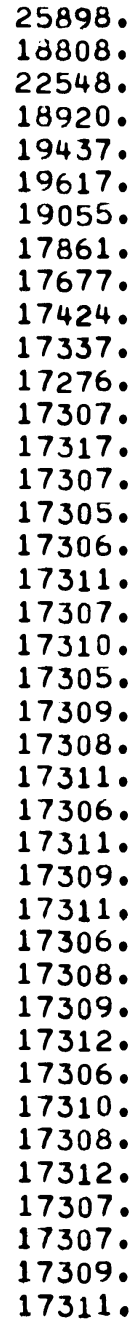 \\
\hline
\end{tabular}

ISOTOPE PRAUUCTION. GRAMS/TONNE

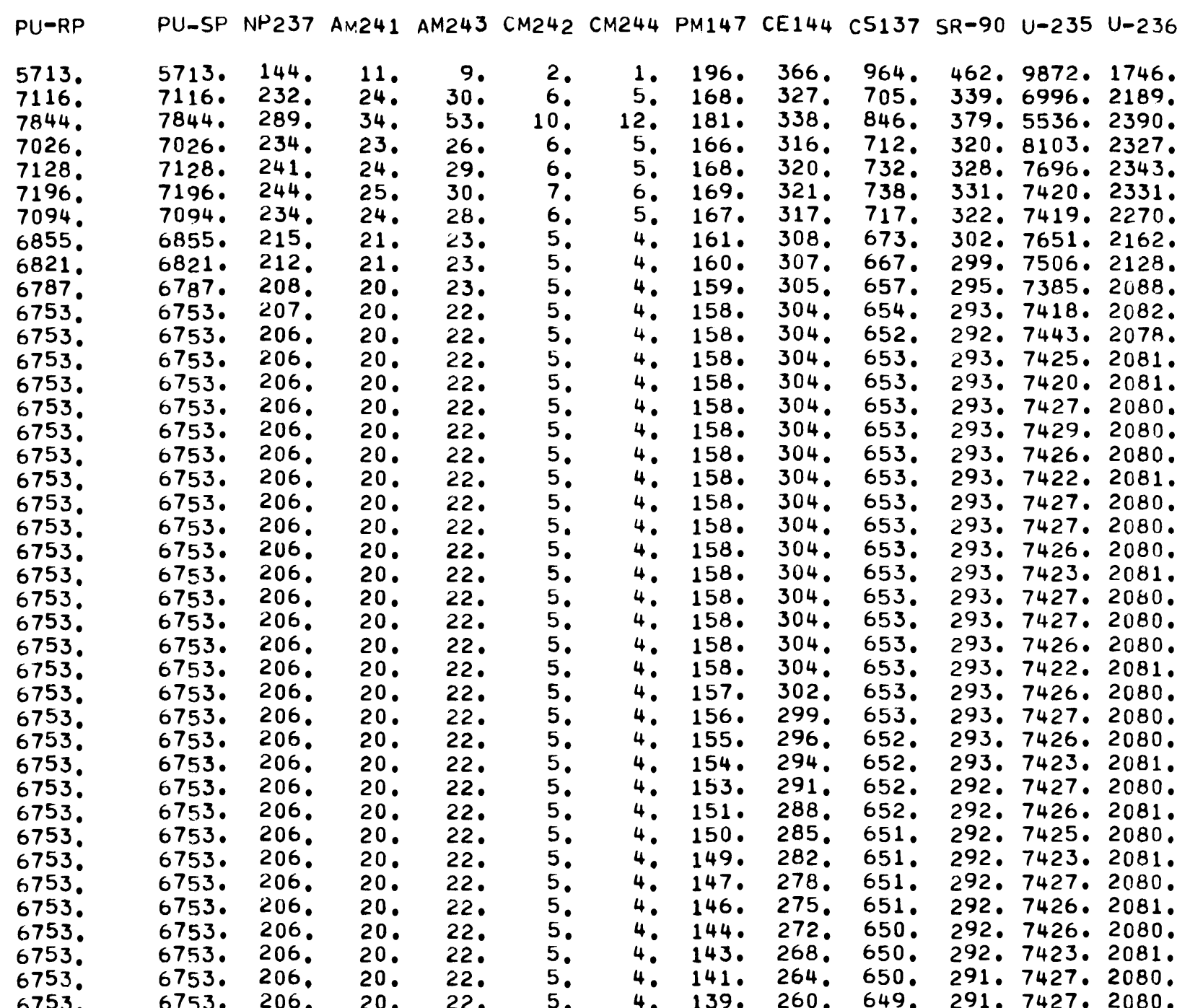


DISCHARGE

\begin{tabular}{|c|c|c|}
\hline $\begin{array}{l}\text { CYCLE } \\
\text { INUMBER }\end{array}$ & DATE & TONNE \\
\hline 1 & $1 \cdot 8$ & 58.5 \\
\hline 2 & 2.3 & 29.2 \\
\hline 3 & 2.9 & $29 \cdot 3$ \\
\hline 4 & 3.6 & 29.3 \\
\hline$b$ & 4.1 & $29 \cdot 3$ \\
\hline 6 & $4 \cdot 7$ & 29.3 \\
\hline 7 & $5 \cdot 2$ & 29.3 \\
\hline ¿ & 5.8 & 29.3 \\
\hline 9 & $6 \cdot 3$ & 29.3 \\
\hline 10 & 6.8 & 29.3 \\
\hline 11 & 7.4 & $29 \cdot 3$ \\
\hline 12 & $7 \cdot 9$ & $29 \cdot 3$ \\
\hline 13 & 8.4 & 29.3 \\
\hline 14 & 9.0 & 29.3 \\
\hline 15 & 9.5 & 29.3 \\
\hline 16 & $10 \cdot 0$ & 29.3 \\
\hline 17 & $10 \cdot 5$ & 29.3 \\
\hline 18 & $11 \cdot 1$ & 29.3 \\
\hline 19 & 11.6 & 29.3 \\
\hline 23 & $12 \cdot 1$ & $29 \cdot 3$ \\
\hline 21 & 12.7 & $29 \cdot 3$ \\
\hline $\begin{array}{l}22 \\
23\end{array}$ & $\begin{array}{l}13.2 \\
13.7\end{array}$ & $\begin{array}{l}29.3 \\
29.3\end{array}$ \\
\hline 24 & 14.2 & 29.3 \\
\hline 25 & $14 \cdot 8$ & $29 \cdot 3$ \\
\hline 26 & $15 \cdot 3$ & 29.3 \\
\hline 27 & $\begin{array}{l}15.8 \\
16.4\end{array}$ & 29.3 \\
\hline $\begin{array}{l}28 \\
24\end{array}$ & 16.9 & $\begin{array}{l}29.3 \\
29.3\end{array}$ \\
\hline 30 & 17.5 & 29.3 \\
\hline 31 & $18 \cdot 1$ & $29 \cdot 3$ \\
\hline 32 & 18.7 & 29.3 \\
\hline 33 & $19 \cdot 3$ & $29 \cdot 3$ \\
\hline 34 & 19.9 & $29 \cdot 3$ \\
\hline 35 & $20 \cdot 5$ & 29.3 \\
\hline 36 & $21 \cdot 1$ & $29 \cdot 3$ \\
\hline 37 & $21 \cdot 8$ & $29 \cdot 3$ \\
\hline 38 & $22 \cdot 4$ & 29.3 \\
\hline 39 & $23 \cdot 1$ & 29.3 \\
\hline 40 & 23.8 & 29. \\
\hline
\end{tabular}

ISOTOPE PRCDUCTION. GRAMS/TONNE

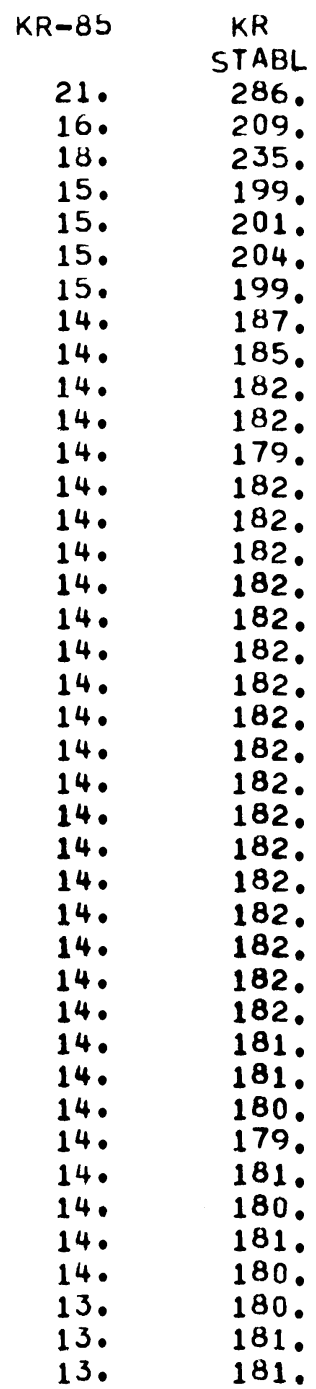

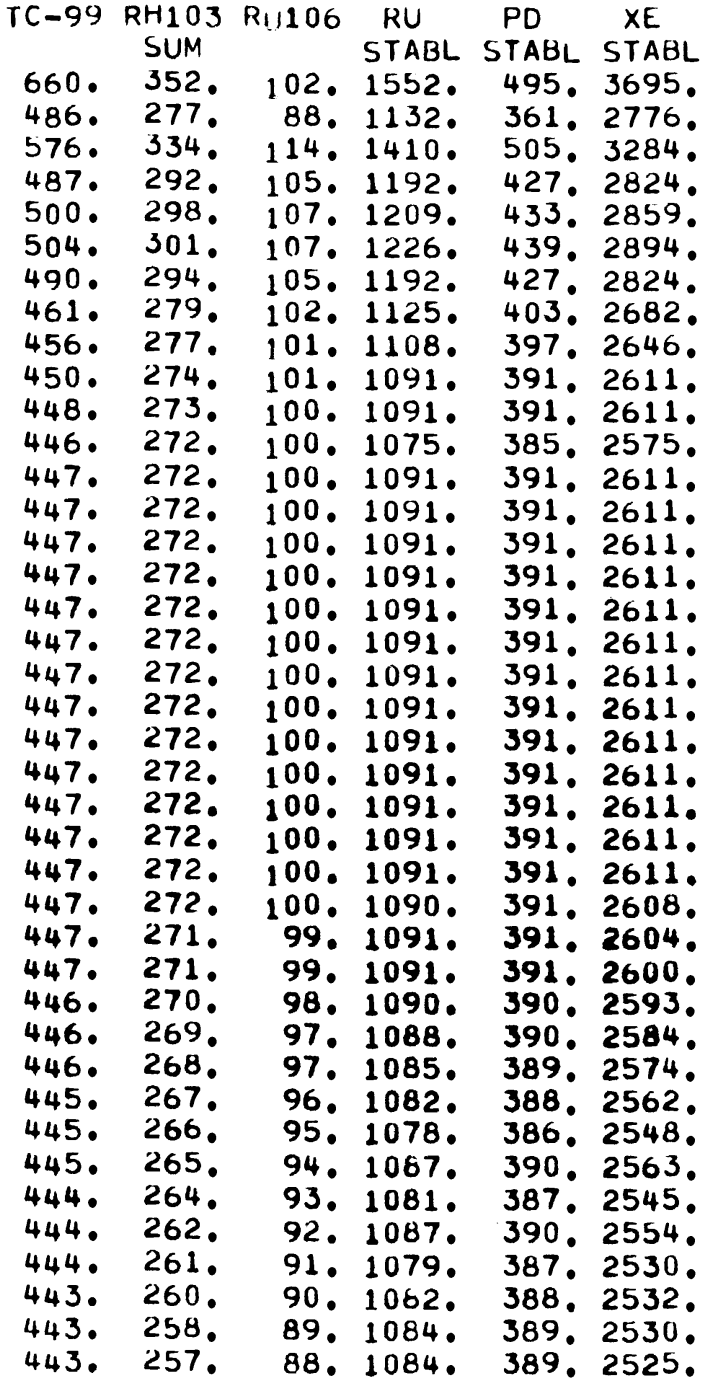


DISCHARGE SCHEDULE FOR 1000 MW ( E) PWR70- 30U PAGE 16

DISCHARGE

INUMBE
1
2
3
4
5
6
7
8
9
10
11
12
13
14
15
16
17
18
19
20
21
22
23
24
25
20
27
28
29
30
31
32
33
34
35
36
37
38

ISOTOPE PRODUCTION. GRAMS/TONNE

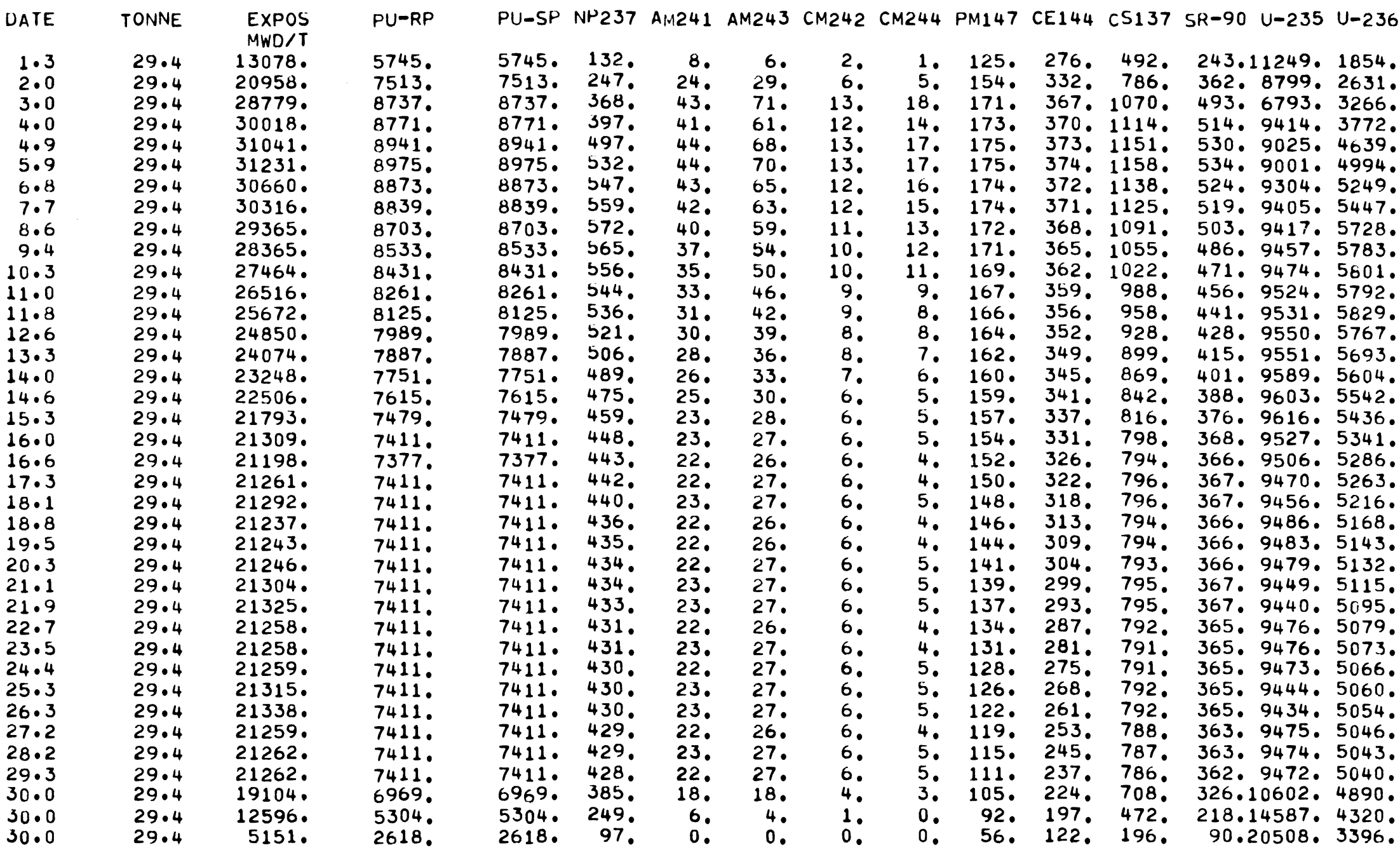


DISCHARGE

\begin{tabular}{|c|c|c|}
\hline $\begin{array}{l}\text { CYCLE } \\
\text { NUMBER }\end{array}$ & UATE & TONNE \\
\hline 1 & $1 \cdot 3$ & 29.4 \\
\hline$<$ & $2 \cdot 0$ & 29.4 \\
\hline 3 & 3.0 & 29.4 \\
\hline 4 & $4 \cdot 0$ & 29.4 \\
\hline 5 & $4 \cdot 9$ & 29.4 \\
\hline 6 & 5.9 & 29.4 \\
\hline 7 & 6.8 & 29.4 \\
\hline 8 & $7 \cdot 7$ & 29.4 \\
\hline 9 & $8 \cdot 6$ & 29.4 \\
\hline 10 & 9.4 & 29.4 \\
\hline 11 & 10.3 & 29.4 \\
\hline 12 & 11.0 & 29.4 \\
\hline 13 & $11 \cdot 8$ & 29.4 \\
\hline 14 & $12 \cdot 6$ & $29 \cdot 4$ \\
\hline 15 & $13 \cdot 3$ & 29.4 \\
\hline 16 & $14 \cdot 0$ & 29.4 \\
\hline 17 & $14 \cdot 6$ & $29 \cdot 4$ \\
\hline 18 & $15 \cdot 3$ & 29.4 \\
\hline 19 & $16 \cdot 0$ & $29 \cdot 4$ \\
\hline 20 & $16 \cdot 6$ & 29.4 \\
\hline 21 & $17 \cdot 3$ & 29.4 \\
\hline 22 & $18 \cdot 1$ & $29 \cdot 4$ \\
\hline 23 & 18.8 & $29 \cdot 4$ \\
\hline 24 & 19.5 & 29.4 \\
\hline 25 & $20 \cdot 3$ & 29.4 \\
\hline 25 & $21 \cdot 1$ & $29 \cdot 4$ \\
\hline 27 & $21 \cdot 9$ & 29.4 \\
\hline 20 & 22.7 & 29.4 \\
\hline 29 & 23.5 & 29.4 \\
\hline 30 & $24 \cdot 4$ & $29 \cdot 4$ \\
\hline 31 & $25 \cdot 3$ & 29.4 \\
\hline 32 & $26 \cdot 3$ & 29.4 \\
\hline 33 & $27 \cdot 2$ & 29.4 \\
\hline 34 & 28.2 & $29 \cdot 4$ \\
\hline ذ3 & $29 \cdot 3$ & 29.4 \\
\hline 36 & 30.0 & $29 \cdot 4$ \\
\hline 37 & $30 \cdot 0$ & 29.4 \\
\hline 38 & 30.0 & 29. \\
\hline
\end{tabular}

ISOTOPE PROUUCTION. GRAMS/TONNE

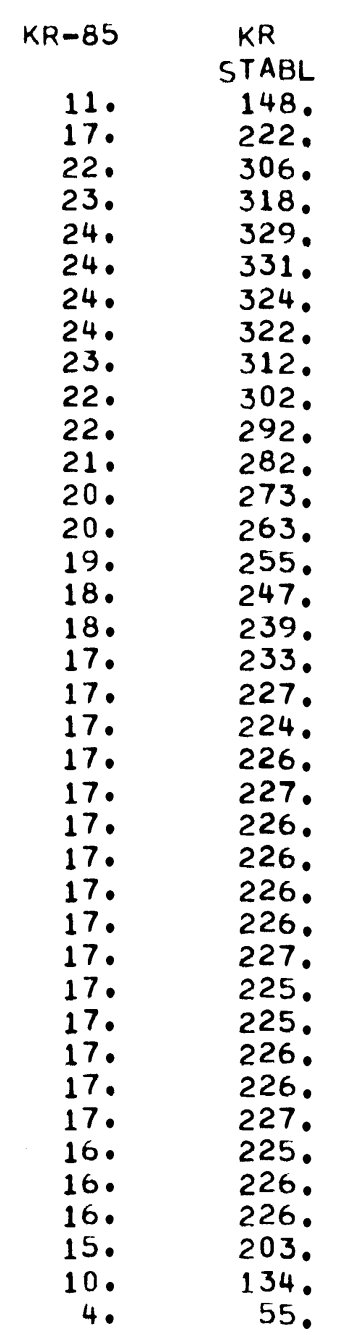

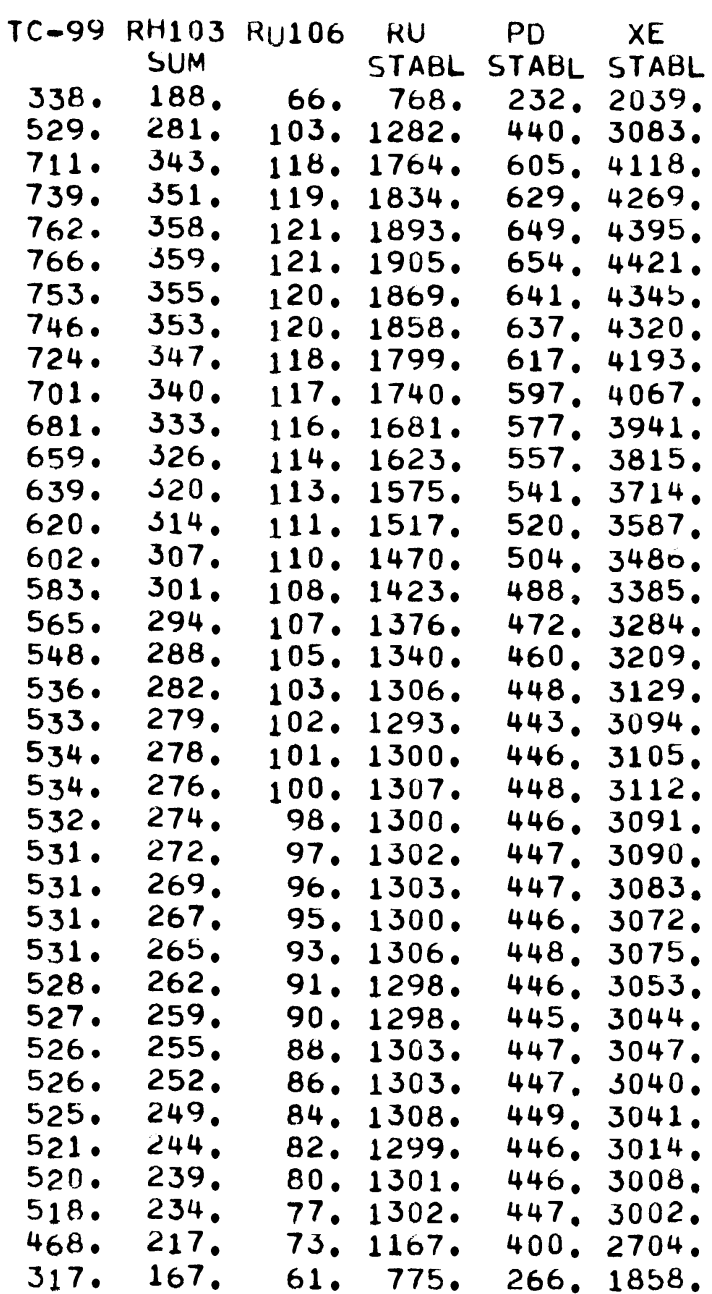


DISCHARGE

CYCLE UATE TONNE

\begin{tabular}{|c|c|c|}
\hline & & $M W D / T$ \\
\hline $2 \cdot 6$ & $67 \cdot 8$ & 32056 \\
\hline $3 \cdot 5$ & 33.9 & 23534 \\
\hline $4 \cdot 4$ & 33.9 & 28503 \\
\hline $5 \cdot 5$ & 33.9 & 5597 . \\
\hline $6 \cdot 4$ & 33.9 & 26681 . \\
\hline $7 \cdot 3$ & 33.9 & 27303 \\
\hline $8 \cdot 2$ & 33.9 & 26947 . \\
\hline $9 \cdot 1$ & 33.9 & 25504 \\
\hline 9.9 & 33.9 & 24813 \\
\hline 10.7 & 33.9 & 24035 . \\
\hline $11 \cdot 4$ & 33.9 & 23079 . \\
\hline $12 \cdot 2$ & 33.9 & 22115 \\
\hline $12 \cdot 9$ & 33.9 & 21370 \\
\hline $13 \cdot 6$ & 33.9 & 20552 . \\
\hline $14 \cdot 2$ & 33.9 & $19784^{\circ}$ \\
\hline $14 \cdot 9$ & 33.9 & 19074. \\
\hline $15 \cdot 5$ & 33.9 & 18456 \\
\hline $16 \cdot 2$ & $\begin{array}{l}33.9 \\
33.0\end{array}$ & 18000 \\
\hline $\begin{array}{l}16 \cdot 8 \\
17.5\end{array}$ & $\begin{array}{l}33.9 \\
33.9\end{array}$ & 17644 . \\
\hline $18 \cdot 2$ & 33.9 & 17680 \\
\hline $18 \cdot 9$ & 33.9 & 17690. \\
\hline $19 \cdot 6$ & 33.9 & 17665 \\
\hline $20 \cdot 3$ & 33.9 & 17709. \\
\hline $21 \cdot 0$ & 33.9 & $17709^{\circ}$ \\
\hline $21 \cdot 8$ & 33.9 & 17675 . \\
\hline $22 \cdot 6$ & 33.9 & 17722 . \\
\hline $23 \cdot 4$ & 33.9 & 17723. \\
\hline $24 \cdot 2$ & 33.9 & 17686 \\
\hline $25 \cdot 1$ & 33.9 & 17729. \\
\hline $25 \cdot 9$ & 33.9 & 17728 \\
\hline $26 \cdot 9$ & 33.9 & 17691 \\
\hline $27 \cdot 8$ & 33.9 & 17734 \\
\hline $28 \cdot 8$ & 33.9 & 17732 \\
\hline 29.8 & 33.9 & 17694 \\
\hline $30 \cdot 0$ & $33 \cdot 9$ & 14250 \\
\hline $30 \cdot 0$ & 33.9 & 9851. \\
\hline 30.0 & 33.9 & 5310 \\
\hline $30 \cdot 0$ & 33.9 & 758 \\
\hline
\end{tabular}

ISOTOPE PRODUCTION• GRAMS,TONNE

$P U-R P$

6457

8020

8816 .

8197

8374

8462

8403 .

8167

8079

7961

7784

7637 .

7489.

7342 .

7224.

7077 .

6959

6870 .

6841.

6811.

6811

6811 .

6811 .

6841 .

6841

6811.

6841 .

6841 .

6811

6841 .

6841

6811 .

6841 .

6841

6811

6015

4718 .

2978
PU-SP NP237 AM241 AM243 CM242 CM244 PM147 CE144 CS137 SR-90 U-235 U-236

6457. 193.

8020.313.

8816.392.

8197. 351.

8374.460 .

8462 . 488 .

8403.494.

8167.474.

8079.469.

7961.486.

$7784.474^{\circ}$

7637.458 .

7489.442 .

7342 . 426 .

7224.420.

7077.405.

$69590^{\circ} 391$.

6841.372.

6811.372.

6811.371.

6811.368.

6811.365.

6841.364.

6841.365.

6811.363.

6841.363.

6841.362 .

6811.360 .

6841.361.

6841.361.

6811.359.

6841.360.

$\begin{aligned} & 6841 . \\ & 6811 .\end{aligned} 359$.

6015.288.

4718.196.

2978.102.

501.14.

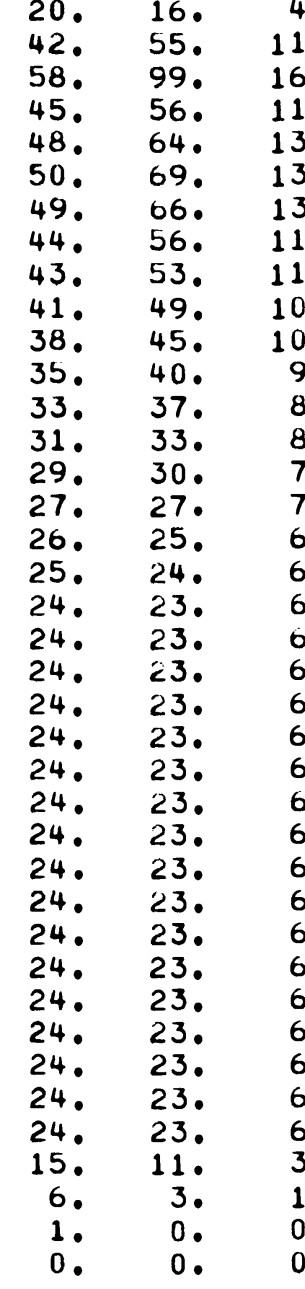

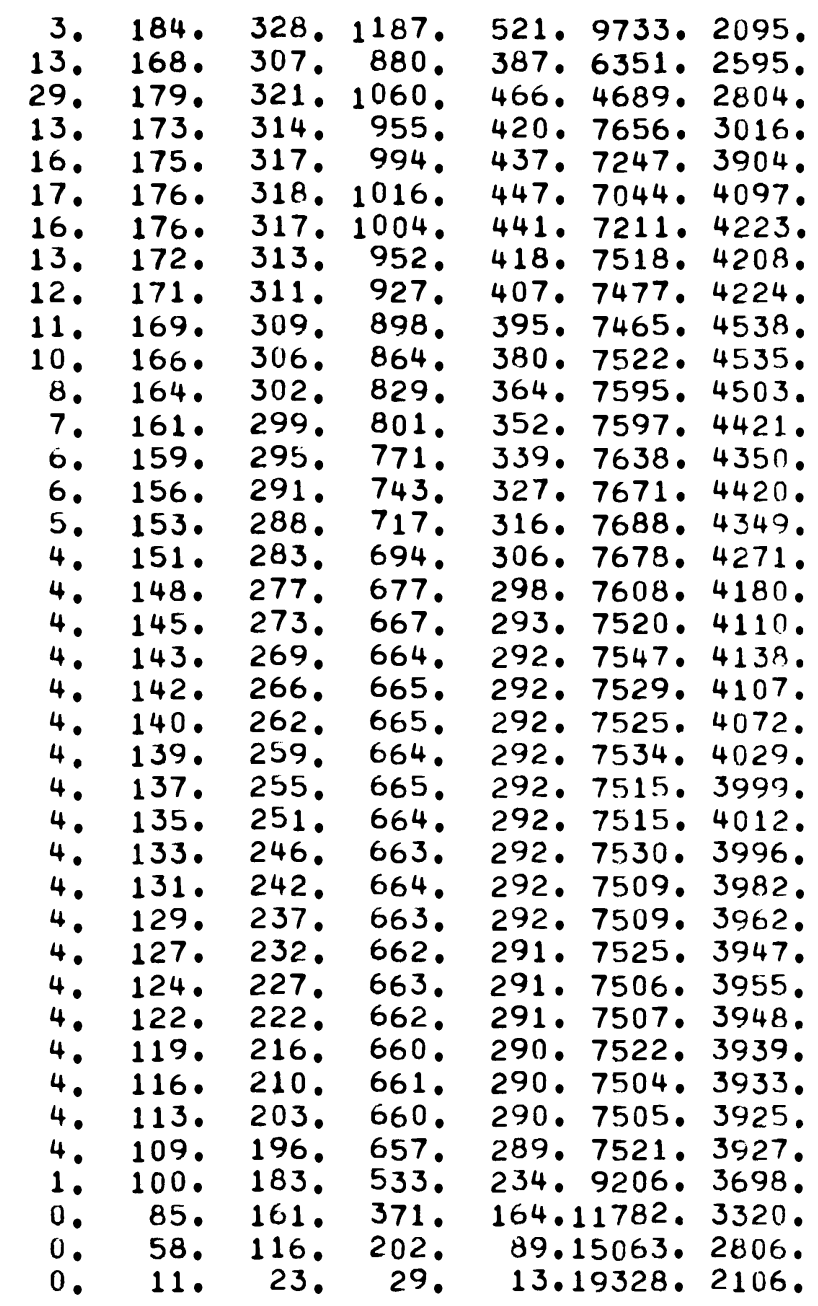


DISCHARGE

CYCLE

NUMBLR

VATE TONIVE

$2.6 \quad 67 \cdot 8$

$3.5 \quad 33.9$

4.4
5.5

5.5

7.3

$8 \cdot 2$

9.1
9.9

10.7

11.4

12.2

12.9
13.6

13.6
14.2

14.9

$15 \cdot 5$

16.2

16.9

17.5

18.2

18.9

19.6

20.3

$21 \cdot 0$

21.8

22.6

$25 \cdot 1$
25.9

$25 \cdot 9$
26.9

27.8

28. is

29.8

30.0

30.0

30.0

$$
\begin{aligned}
& 33.9 \\
& 33.9
\end{aligned}
$$

33.9

33.9

33.9

33.9

33.9

33.9

33.9

33.9

33.9

33.9

33.9

33.9

33.9

33.9

33.9

33.9

33.9

33.9

33.9

33.9

33.9

33.9

33.9

33.9

33.9

33.9

33.9

33.9

33.9

33.9

33.9

33.9
ISOTOPE PRODUCTION, GR,AMS/TONINE

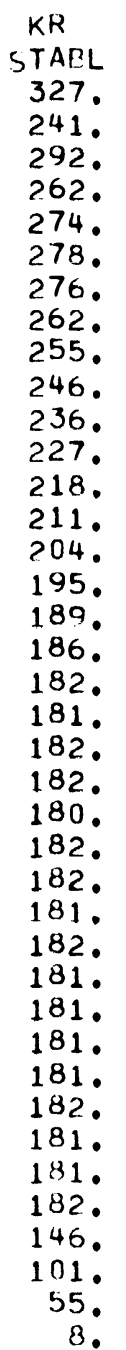

TC-99 RH103 RU106 RL
SUM

796. 408. 122. 2015. 742. 4476.

596. 334. 111. 1487. 547. 3372.

713. 380. 119. 1801. 663. 4028.

645. 534. 115. 1615. 594. 3640.

671. 364. 116.1687. 621.3790.

685. 369. 117. 1715. 631. 3849.

677. 366. 116. 1701. 626. 3819.

643. 353. 114. 1615. 594. 3640.

626. 346. 113. 1572. 579. 3551.

608.339 .112 .1515 .558 .3431$.

585.330 .110 .1458 .537 .3312$.

562. 320. 109.1401. 516.3192.

544. 312. 107. 1344. 495. 3073 .

524. 303. 105. 1301. 479. 2984.

505. 295. 104. 1258. 463. 2894.

287. 102.1201. 442. 2775 .

472.280 .200 .1165 .429 .2700$.

461. 273. 98.1145. 421.2652.

454. 269. 96. 1124. 414.2604.

452. 267. 95. 1117. 411.2583.

452. 266. 94. 1121. 413.2587.

452. 265. 93. 1124. 414.2589.

451 . 263. 92. 1113. 410.2560.

451. 262. 91. 1125. 414.2580.

451 . 260. 90. 1123. 413.2570.

450. 258. 88. 1119. 412. 2557.

$450^{\circ} 256.87 .1125 .414 .2563$.

$448,252.84 .1117 .411 .2535$.

448. 250. 83.1115. 410.2524.

$447.247 . \quad 81.1119 .412 .2527$.

446.244 .49 .1120 .412 .2522$.

446. 241. 77. 1116. 411. 2507.

444.238 .2501116 .411 .2500$.

442. 234. 72. 1119. 412.2500.

360 . 201. 66. 903. 332. 2044.

253. 153. 56. 022. 229. 1450.

$139^{\circ} \quad 92 . \quad 39^{\circ}$ 336. $124^{\circ} .859^{\circ}$ 
UISCHARGE SCHEDULE FOR 1000 iN W 1 E I PWR8O- 3OU PAGE 20

DISCHARGE

$\begin{array}{crr}\text { CYCLE } & & \\ \text { NUMBEK } & \text { UATE } & \text { TONNE } \\ & & \\ 1 & .9 & 25.7 \\ 2 & 1.4 & 25.7 \\ 3 & 2.1 & 25.7 \\ 4 & 2.8 & 25.7 \\ 5 & 3.5 & 25.7 \\ 6 & 4.1 & 25.7 \\ 7 & 4.7 & 25.7 \\ 8 & 5.3 & 25.7 \\ 9 & 5.8 & 25.7 \\ 10 & 6.4 & 25.7 \\ 11 & 7.0 & 25.7 \\ 12 & 7.6 & 25.7 \\ 13 & 8.1 & 25.7 \\ 14 & 8.7 & 25.7 \\ 13 & 9.3 & 25.7 \\ 16 & 9.9 & 25.7 \\ 17 & 10.4 & 25.7 \\ 18 & 11.0 & 25.7 \\ 19 & 11.6 & 25.7 \\ 20 & 12.2 & 25.7 \\ 21 & 12.8 & 25.7 \\ 21 & 13.3 & 25.7 \\ 23 & 13.9 & 25.7 \\ 24 & 14.5 & 25.7 \\ 25 & 15.0 & 25.7 \\ 26 & 15.6 & 25.7 \\ 27 & 16.2 & 25.7 \\ 28 & 16.8 & 25.7 \\ 29 & 17.4 & 25.7 \\ 30 & 18.1 & 25.7 \\ 31 & 18.7 & 25.7 \\ 32 & 19.3 & 25.7 \\ 33 & 20.0 & 25.7 \\ 34 & 20.7 & 25.7 \\ 35 & 21.4 & 25.7 \\ 30 & 22.1 & 25.7 \\ 37 & 22.8 & 25.7 \\ 30 & 23.5 & 25.7 \\ 39 & 24.3 & 25.7 \\ 40 & 25.1 & 25.7 \\ & & \end{array}$

ISOTOPE PRODUCTION. GRAMS/TONNE

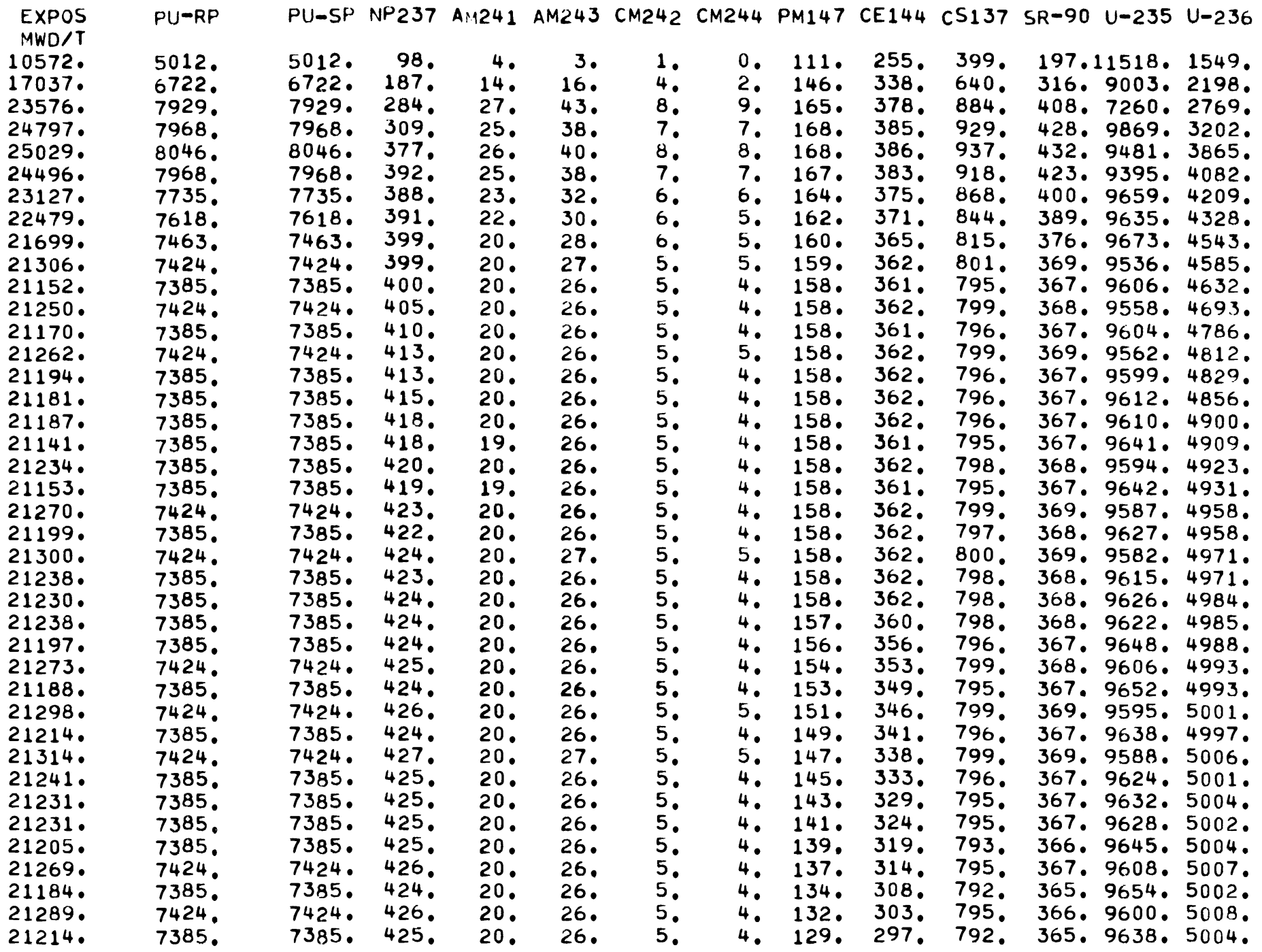


DISCHARGE

WCLE
NUMBE
1
1
2
3
4
5
6
7
8
9
10
11
12
13
14
15
10
17
16
19
20
21
22
23
24
25
20
27
20
29
30
31
32
33
34
35
30
37
30
34
40

ISOTOPE PRODUCTION. GRAMS/TONNE

\begin{tabular}{|c|c|c|c|}
\hline LAIE & TONNE & $K R-8 b$ & $\begin{array}{c}\text { KR } \\
\text { STABL }\end{array}$ \\
\hline .9 & 25.7 & 9. & 121. \\
\hline $1 \cdot 4$ & 25.7 & 15. & 192. \\
\hline $2 \cdot 1$ & $25 \cdot 7$ & 19. & 251. \\
\hline 2.8 & 25.7 & 20. & 264. \\
\hline 3.5 & $25 \cdot 7$ & 20. & 266. \\
\hline $4 \cdot 1$ & 25.7 & 20. & 259. \\
\hline $4 \cdot 7$ & $25 \cdot 7$ & 19. & 245. \\
\hline $5 \cdot 3$ & 25.7 & 18. & 239. \\
\hline 5.8 & $25 \cdot 7$ & 17. & 231. \\
\hline 6.4 & 25.7 & 17. & 227. \\
\hline 7.0 & 25.7 & 17. & 225. \\
\hline $7 \cdot 6$ & $25 \cdot 7$ & 17. & 225. \\
\hline $8 \cdot 1$ & 25.7 & 17. & 225 . \\
\hline 8.7 & 25.7 & 17. & 227. \\
\hline 9.3 & $25 \cdot 7$ & 17. & 225. \\
\hline 9.9 & 25.7 & 17. & 225. \\
\hline 10.4 & 25.7 & 17. & 225. \\
\hline $11 \cdot 0$ & $25 \cdot 7$ & 17. & 225. \\
\hline $11 \cdot 0$ & 25.7 & 17. & 225. \\
\hline $12 \cdot 2$ & $25 \cdot 7$ & 17. & 225. \\
\hline $12 \cdot 8$ & $25 \cdot 7$ & 17. & 227. \\
\hline 13.3 & 25.7 & 17. & 225. \\
\hline 13.9 & 25.7 & 17. & 227. \\
\hline $14 \cdot 5$ & 25.7 & 17. & 225. \\
\hline 15.0 & 25.7 & 17. & 225. \\
\hline $15 \cdot 6$ & 25.7 & 17. & 225. \\
\hline $16 \cdot 2$ & 25.7 & 17. & 225. \\
\hline $16 \cdot 8$ & $25 \cdot 7$ & 17. & 225. \\
\hline 17.4 & 25.7 & 17. & 225. \\
\hline $18 \cdot 1$ & 25.7 & 17. & 227. \\
\hline $18 \cdot 7$ & $25 \cdot 7$ & 17. & 226. \\
\hline 19.3 & 25.7 & 17. & 227. \\
\hline $20 \cdot 0$ & 25.7 & 17. & 226. \\
\hline 20.7 & 25.7 & 17. & 225 . \\
\hline 21.4 & 25.7 & 17. & 225 . \\
\hline $22 \cdot 1$ & 25.7 & 17. & 225. \\
\hline $22 \cdot 8$ & 25.7 & 17. & 226. \\
\hline $23 \cdot 5$ & 25.7 & 17. & 225 . \\
\hline $24 \cdot 3$ & $25 \cdot 7$ & 17. & 227. \\
\hline $25 \cdot 1$ & $25 \cdot 7$ & 17. & 225. \\
\hline
\end{tabular}

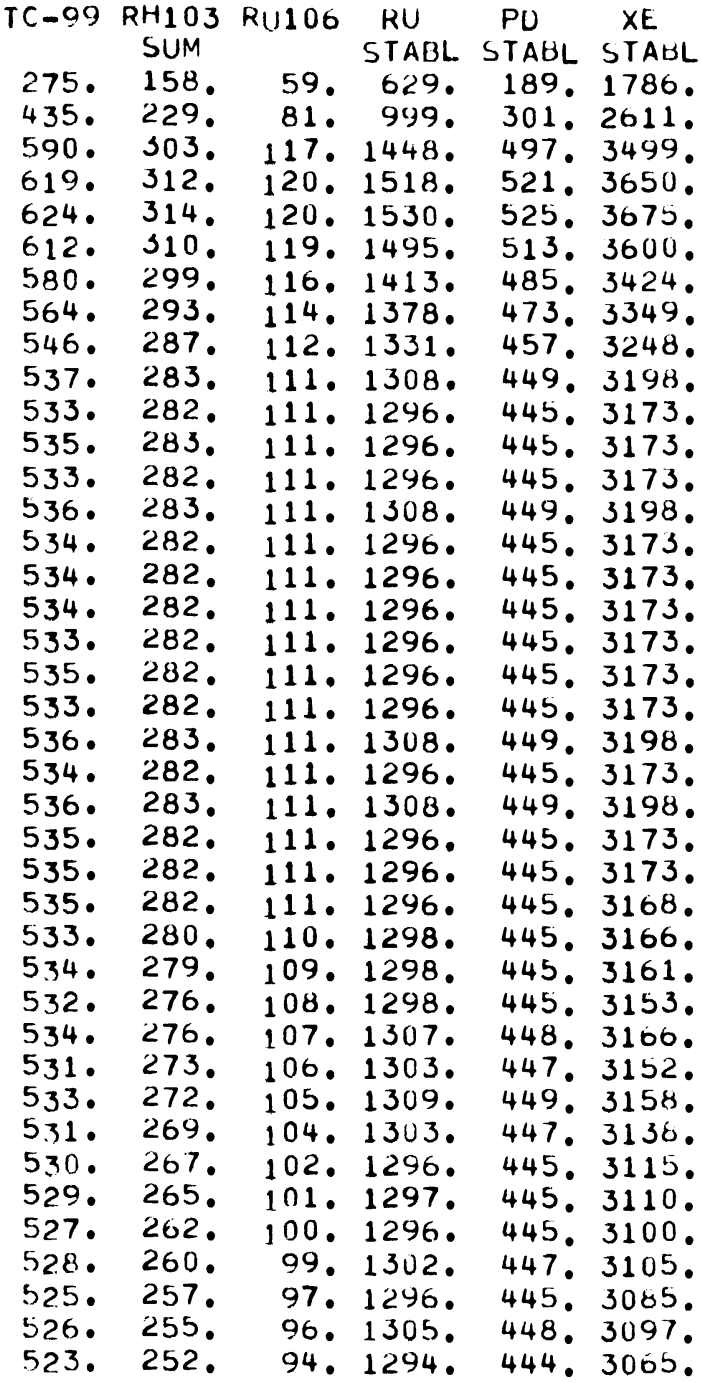


DISCHAKGE SCHEDULE FOR 1000 M W ( E ) BWR80- 30U PAGE 22

DISCHARGE

\begin{tabular}{|c|c|c|c|c|}
\hline NUMBEK & UATE & TOINNE & $\begin{array}{l}\text { EXPOS } \\
M W D / T\end{array}$ & FU-RP \\
\hline 1 & 1.8 & 58.5 & 25923 . & 5713. \\
\hline 2 & 2.3 & 29.2 & 18818 . & $7116^{\circ}$ \\
\hline 4 & 3.6 & 29.3 & 18911. & 7026 \\
\hline ؛ & $4 \cdot 1$ & $29 \cdot 3$ & 19346. & 7128. \\
\hline 6 & 4.7 & $29 \cdot 3$ & 19524 & 7162. \\
\hline 7 & $5 \cdot 2$ & $29 \cdot 3$ & 13995. & 7060 . \\
\hline c) & 5.8 & $29 \cdot 3$ & $17819^{\circ}$ & 6821. \\
\hline$\Rightarrow$ & $6 \cdot 3$ & $29 \cdot 3$ & 17672 . & 6821. \\
\hline 10 & $6 \cdot 8$ & $29 \cdot 3$ & 17418. & 6787. \\
\hline 11 & $7 \cdot 4$ & 29.3 & 17318 & 6753. \\
\hline $1<$ & $7 \cdot 9$ & $29 \cdot 3$ & 17245. & 6719. \\
\hline 13 & $8 \cdot 4$ & $29 \cdot 3$ & 17272 & 6719. \\
\hline 14 & $8 \cdot 9$ & $29 \cdot 3$ & $17289^{\circ}$ & 6719. \\
\hline 15 & $9 \cdot 5$ & $29 \cdot 3$ & $17264^{\circ}$ & 6719. \\
\hline 10 & $10 \cdot 0$ & $29 \cdot 3$ & 17263. & $6719^{\circ}$ \\
\hline 17 & $10 \cdot 5$ & $29 \cdot 3$ & 17262 & 6719. \\
\hline 10 & $11 \cdot 1$ & $29 \cdot 3$ & 17278 . & 6719. \\
\hline 13 & 11.0 & $29 \cdot 3$ & 17278 & 6719. \\
\hline 20 & $12 \cdot 1$ & 29.3 & 17275 & 6719. \\
\hline 21 & 12.6 & $29 \cdot 3$ & 17270 & 6719. \\
\hline 22 & $13 \cdot 2$ & $29 \cdot 3$ & $17284^{\circ}$ & $6719^{\circ}$ \\
\hline 2.3 & 13.7 & $29 \cdot 3$ & 17286. & 6719. \\
\hline 24 & $14 \cdot 2$ & $29 \cdot 3$ & 17301. & $6719^{\circ}$ \\
\hline $2 b$ & 14.7 & 29.3 & 17300 . & 6753. \\
\hline 20 & $15 \cdot 3$ & $29 \cdot 3$ & 17303. & 6753. \\
\hline 27 & 15.8 & $29 \cdot 3$ & 17309. & 6753. \\
\hline 23 & $16 \cdot 4$ & $29 \cdot 3$ & $17310^{\circ}$ & 6753. \\
\hline 29 & 16.9 & 29.3 & 17315 & 6753. \\
\hline 30 & $17 \cdot 5$ & $29 \cdot 3$ & 17312. & 6753. \\
\hline 31 & $18 \cdot 1$ & $29 \cdot 3$ & 17308 & 6753. \\
\hline 32 & $18 \cdot 6$ & $29 \cdot 3$ & 17313. & 6753 . \\
\hline 33 & 19.2 & $29 \cdot 3$ & 17316 . & 6753. \\
\hline 34 & 19.8 & $29 \cdot 3$ & 17311. & 6753. \\
\hline 3ذ & $20 \cdot 5$ & $29 \cdot 3$ & 17310 & 6753. \\
\hline 30 & $21 \cdot 1$ & $29 \cdot 3$ & 17305. & 6753. \\
\hline 37 & $21 \cdot 7$ & $29 \cdot 3$ & 17313. & 6753. \\
\hline 38 & $22 \cdot 4$ & $29 \cdot 3$ & 17308 . & 6753. \\
\hline 39 & $23 \cdot 1$ & $29 \cdot 3$ & 17313 & 6753. \\
\hline 42 & 23.7 & $29 \cdot 3$ & 17309. & 6753 \\
\hline
\end{tabular}

ISOTOPE PRODUCTION, GRAMS/TONINE

\begin{tabular}{|c|c|c|c|c|c|c|c|c|c|c|c|}
\hline$p$ & 7 & 41 & +3 & 42 & & 1147 & & & & & 0 \\
\hline $\begin{array}{l}713 . \\
116 . \\
844^{\circ} \\
026 . \\
128 . \\
162 . \\
060^{\circ} \\
821 .\end{array}$ & $\begin{array}{l}144^{\circ} \\
232^{\circ} \\
289^{\circ} \\
234^{\circ} \\
307^{\circ} \\
323^{\circ} \\
324^{\circ} \\
309^{\circ} \\
304^{\circ} \\
324^{\circ} \\
327^{\circ} \\
327^{\circ} \\
328^{\circ} \\
326^{\circ} \\
335^{\circ} \\
337^{\circ} \\
338^{\circ} \\
338^{\circ} \\
337^{\circ} \\
341^{\circ} \\
342^{\circ} \\
343^{\circ} \\
343^{\circ} \\
343^{\circ} \\
345^{\circ} \\
345^{\circ} \\
345^{\circ} \\
3460^{\circ} \\
3460^{\circ} \\
3460^{\circ} \\
345^{\circ} \\
3460^{\circ} \\
3460^{\circ} \\
346^{\circ} \\
346^{\circ} \\
345^{\circ} \\
346^{\circ} \\
345^{\circ} \\
346^{\circ} \\
3460^{\circ}\end{array}$ & $\begin{array}{l}20 \\
20 \\
20 \\
20 \\
20 \\
20 \\
20 \\
20 \\
20 \\
20 \\
20 \\
20 \\
20 \\
20 \\
20 \\
20 \\
20 \\
20 \\
20 \\
20 \\
20 \\
20 \\
20\end{array}$ & $\begin{array}{l}9 . \\
30 . \\
53 . \\
26 .\end{array}$ & 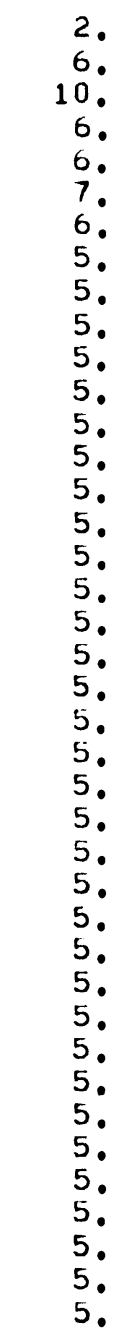 & $\begin{array}{r}1 . \\
5 . \\
12 . \\
4 . \\
5 . \\
5 . \\
5 . \\
4 . \\
4 . \\
4 . \\
4 . \\
3 . \\
4 . \\
4 . \\
3 . \\
3 . \\
4 . \\
3 . \\
3 . \\
4 .\end{array}$ & $\begin{array}{l}148 \\
148 \\
148 \\
148 \\
147 \\
146 \\
145 \\
144 \\
143 \\
141 \\
140 \\
139 \\
137 \\
135 \\
134 \\
132 \\
130 \\
125\end{array}$ & $\begin{array}{l}0 \\
0 \\
0 \\
0 \\
0 \\
0 \\
0 \\
0 \\
0 \\
0 \\
0 \\
0 \\
0 \\
0 \\
0 \\
0 \\
0 \\
0 \\
0 \\
0 \\
0 \\
0\end{array}$ & $\begin{array}{l}651 . \\
652 . \\
652 . \\
652 . \\
651 . \\
652 . \\
652 . \\
653 . \\
653 . \\
653 . \\
653 . \\
653 . \\
653 . \\
652 . \\
652 . \\
652 . \\
652 . \\
651 . \\
651 . \\
651 . \\
651 . \\
650 . \\
650 . \\
649 .\end{array}$ & $\begin{array}{l}463 . \\
339 . \\
379 . \\
319 . \\
327 . \\
330 . \\
321 . \\
301 . \\
299 . \\
295 . \\
293 . \\
292 . \\
292 . \\
293 . \\
292 . \\
292 . \\
292 . \\
292 . \\
292 . \\
292 . \\
292 . \\
292 . \\
293 . \\
293 . \\
293 . \\
293 . \\
293 . \\
293 . \\
293 . \\
293 . \\
292 . \\
292 . \\
292 . \\
292 . \\
292 . \\
292 . \\
292 . \\
291 . \\
291 . \\
291 . \\
.\end{array}$ & 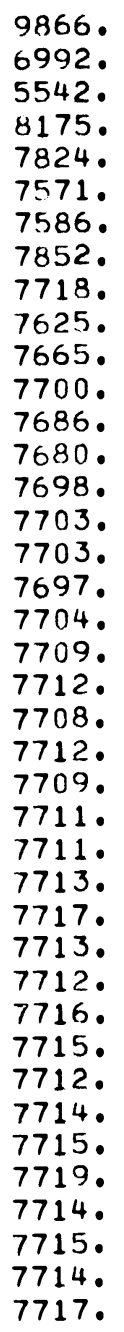 & 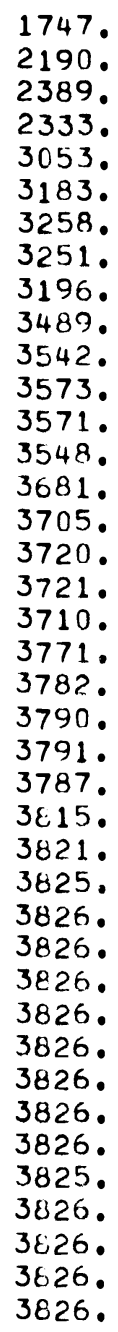 \\
\hline
\end{tabular}


DISCHARGE

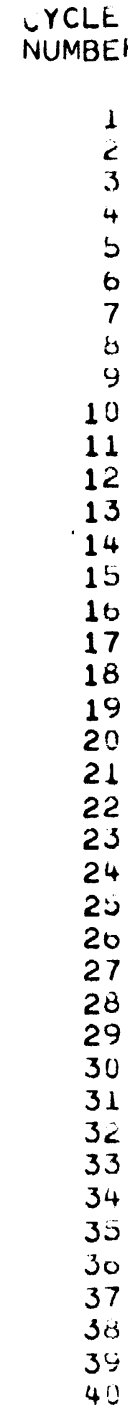

ISOTOPE PRODUCTION. GRAMS/TONNE

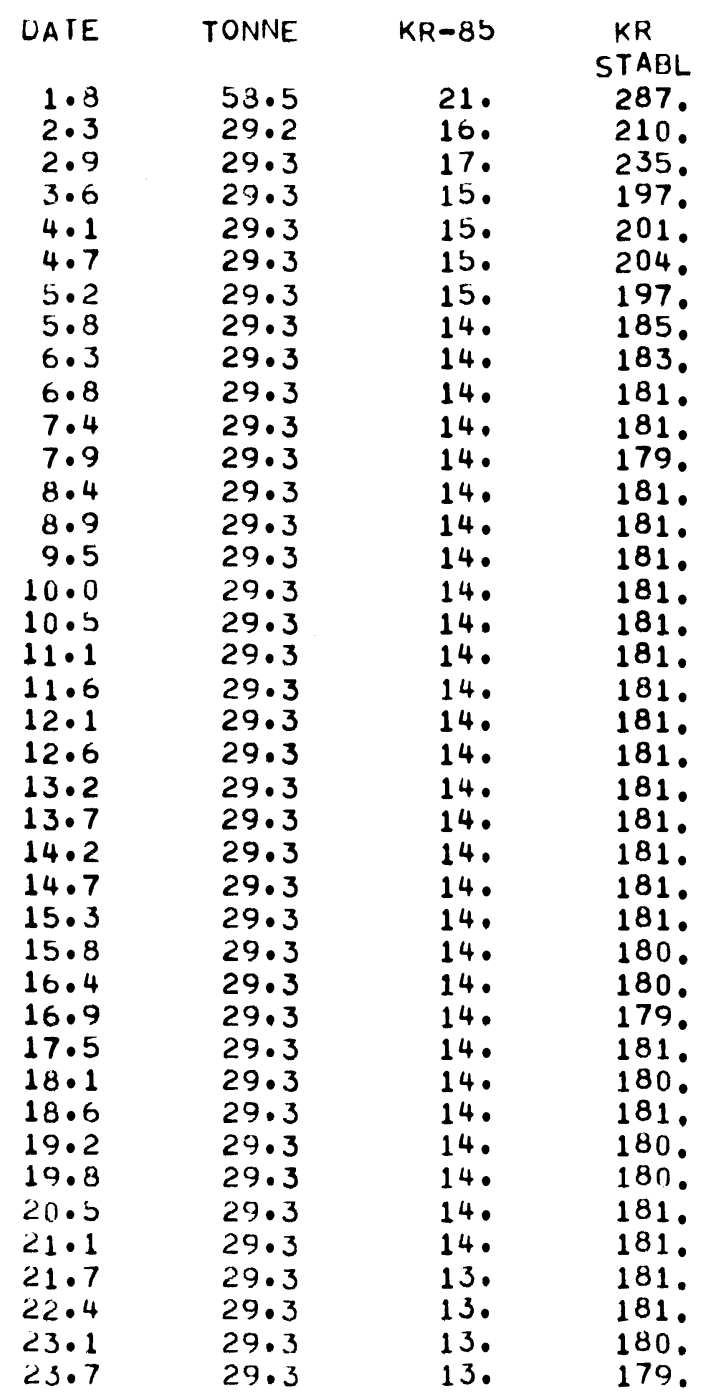

TC-99 RH103 RU106 RU

SUM RU106 RTABL STABL STABL

652. 330. 102. 1556. 496. 3720.

$482.264 .88,1138,362.2806$.

570, 315, 114. 1412, 506. 3305.

483. 278, 105. 1181, 423, 2818.

493. 282. 106. 1208. 433. 2875 .

498. 284. 107, 1222. 438, 2904 .

485. 279, 105. 1181, 423, 2818.

456. 266. 102. 1113. 399. 2675

452. 264. 101. 1100. 394. 2646.

446. 261, 101. 1086. 389. 2617.

444. 260, 100. 1086. 389. 2617 .

442. 259. 100. 1072. 384. 2589.

443. 259. 100. 1086. 389. 2617.

443. 260. 100, 1086, 389. 2617.

442. 259. 100. 1086. 389. 2617.

442. 259. 100. 1086, 389. 2617.

442. 259. 100. 1086. 389. 2617.

443. 259. 100. 1086. 389. 2617.

443. 259. 100. 1086. 389. 2617.

443. 259. 100. 1086. 389. 2617 .

442. 259. 100. 1086. 389. 2617.

443. 260. 100. 1086. 389. 2617.

443. 260. 100. 1086. 389. 2617 .

443. 260. 100. 1086. 389. 2617 .

443. 260. 100. 1086. 389. 2617 .

443. 260. 100. 1085. 389. 2616.

443. 259. 100, 1083. 388. 2606.

443. 258. 99. 1080. 387, 2594.

443. 257. 98. 1076. 386. 2581.

442. 256. 97. 1084. 389. 2594.

442. 255. 97. 1079. 387. 2577.

442. 254. 96. 1085. 389. 2585.

441. 252. 95. 1078. 386, 2565.

441. 251. 94, 1082, 388, 2568.

$440.250 .93 .1084,388,2567$.

440.248 .92 .1085 .389 .2565$.

440 . 247. 91. 1085. 389. 2559.

439. 245. 90. 1084. 388. 2550.

$4390^{\circ} 244.289 .1080^{\circ}, 387.2537$. 
DISCHARGE SCHE DULE FOR 1000 M W I I I LWR70- 4PU PAGE 24

DISCHARGE

\begin{tabular}{|c|c|c|c|}
\hline INUMBLK & LATE & TONNE & $\begin{array}{l}\text { EXPOS } \\
\text { MWD/T }\end{array}$ \\
\hline 1 & $-1 \cdot 0$ & 117.7 & 0. \\
\hline 2 & $1 \cdot 1$ & $29 \cdot 4$ & 11563. \\
\hline 3 & 1.8 & 29.4 & 18515. \\
\hline 4 & $2 \cdot 7$ & $29 \cdot 4$ & 26981. \\
\hline ن & $3 \cdot 8$ & $29 \cdot 4$ & 29275 . \\
\hline u & $4 \cdot 6$ & 29.4 & 29993. \\
\hline 7 & $5 \cdot 0$ & $29 \cdot 4$ & 31657 . \\
\hline os & $6 \cdot 6$ & $29 \cdot 4$ & 31075. \\
\hline 9 & $7 \cdot 5$ & $29 \cdot 4$ & 29640 . \\
\hline 10 & $8 \cdot 3$ & $29 \cdot 4$ & $28607^{\circ}$ \\
\hline 11 & $9 \cdot 1$ & 29.4 & 27431. \\
\hline 1. & 9.9 & $29 \cdot 4$ & 26598 . \\
\hline 13 & $10 \cdot 7$ & $29 \cdot 4$ & 25742 . \\
\hline 14 & $11 \cdot 4$ & $29 \cdot 4$ & 24904 . \\
\hline 15 & $12 \cdot 2$ & $29 \cdot 4$ & 24029 . \\
\hline 10 & $12 \cdot 8$ & $29 \cdot 4$ & 23250 . \\
\hline 17 & 13.5 & $29 \cdot 4$ & 22516 . \\
\hline $1 i$ & $14 \cdot 2$ & $29 \cdot 4$ & $21799^{\circ}$ \\
\hline 19 & $14 \cdot 8$ & $29 \cdot 4$ & 21007 . \\
\hline 20 & $15 \cdot 4$ & $29 \cdot 4$ & 20460 . \\
\hline 21 & $16 \cdot 0$ & $29 \cdot 4$ & 20079 . \\
\hline $2 c$ & 16.7 & $29 \cdot 4$ & $20047^{\circ}$ \\
\hline 23 & $17 \cdot 4$ & $29 \cdot 4$ & 20059 . \\
\hline 24 & $18 \cdot 0$ & $29 \cdot 4$ & 20062 . \\
\hline 20 & 18.7 & $29 \cdot 4$ & 20053 . \\
\hline 26 & $19 \cdot 4$ & 29.4 & 20069 . \\
\hline 27 & $20 \cdot 1$ & $29 \cdot 4$ & 19969. \\
\hline 20 & $20 \cdot 8$ & $29 \cdot 4$ & 20028 . \\
\hline 29 & $21 \cdot 6$ & $29 \cdot 4$ & 20037 . \\
\hline 30 & $22 \cdot 3$ & $29 \cdot 4$ & 20027 \\
\hline 31 & 23.1 & 29.4 & 20051 . \\
\hline 32 & $24 \cdot 0$ & $29 \cdot 4$ & 20053. \\
\hline 33 & $24 \cdot 9$ & $29 \cdot 4$ & 20053 . \\
\hline 34 & $25 \cdot 6$ & $29 \cdot 4$ & 20066 . \\
\hline 35 & $26 \cdot 6$ & $29 \cdot 4$ & 20065 . \\
\hline 36 & $27 \cdot 5$ & $29 \cdot 4$ & 20059 . \\
\hline 37 & $28 \cdot 4$ & 29.4 & 20073 . \\
\hline 30 & $29 \cdot 4$ & $29 \cdot 4$ & 19966. \\
\hline 34 & $30 \cdot 0$ & $29 \cdot 4$ & 17416. \\
\hline 40 & $30 \cdot 0$ & $29 \cdot 4$ & 11185. \\
\hline
\end{tabular}

ISOTOPE PRODUCTION. GRAMS/TUINAE

PU-RP PU-SP NP237 A :241 AM243 CM242 CM244 PM147 CE144 CS137 SR-90 U-235 U-236

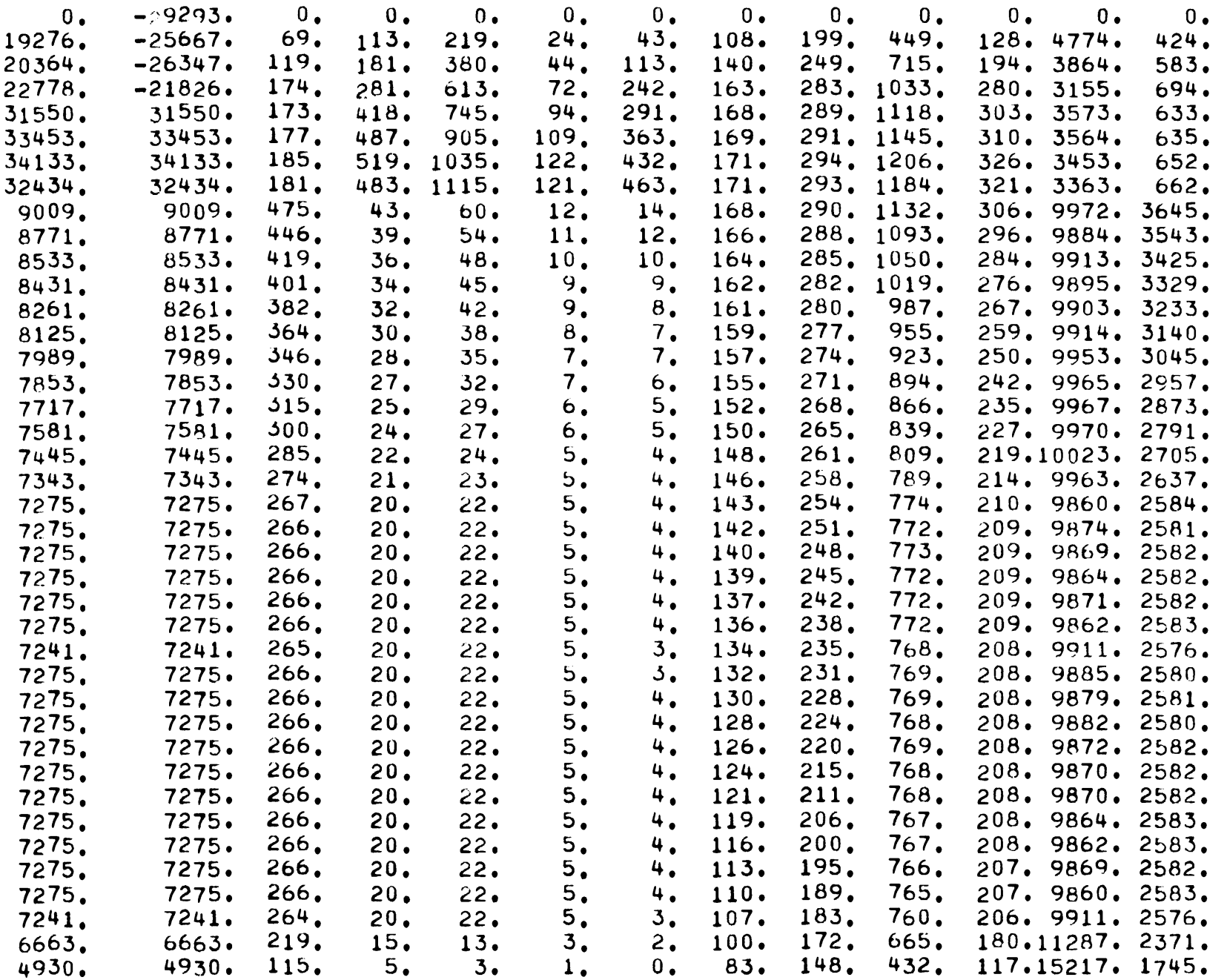


DISCHARGE

$\begin{array}{crr}\text { CYCLE } & & \\ \text { NUMBER } & \text { UATE } & \text { TONNE } \\ & & \\ 1 & -1.0 & 117.7 \\ 2 & 1 \cdot 1 & 29.4 \\ 3 & 1.8 & 29.4 \\ 4 & 2.7 & 29.4 \\ 5 & 3.8 & 29.4 \\ 0 & 4.6 & 29.4 \\ 7 & 5.6 & 29.4 \\ 8 & 6.6 & 29.4 \\ 9 & 7.5 & 29.4 \\ 10 & 8.3 & 29.4 \\ 11 & 9.1 & 29.4 \\ 12 & 9.9 & 29.4 \\ 13 & 10.7 & 29.4 \\ 14 & 11.4 & 29.4 \\ 15 & 12.2 & 29.4 \\ 16 & 12.8 & 29.4 \\ 17 & 13.5 & 29.4 \\ 18 & 14.2 & 29.4 \\ 19 & 14.8 & 29.4 \\ 21 & 15.4 & 29.4 \\ 21 & 16.0 & 29.4 \\ 22 & 16.7 & 29.4 \\ 23 & 17.4 & 29.4 \\ 24 & 18.0 & 29.4 \\ 25 & 18.7 & 29.4 \\ 26 & 19.4 & 29.4 \\ 27 & 20.1 & 29.4 \\ 20 & 20.8 & 29.4 \\ 29 & 21.6 & 29.4 \\ 30 & 22.3 & 29.4 \\ 31 & 23.1 & 29.4 \\ 32 & 24.0 & 29.4 \\ 33 & 24.8 & 29.4 \\ 34 & 25.6 & 29.4 \\ 35 & 26.6 & 29.4 \\ 30 & 27.5 & 29.4 \\ 37 & 28.4 & 29.4 \\ 30 & 29.4 & 29.4 \\ 39 & 30.0 & 29.4 \\ 40 & 30.0 & 29.4 \\ & & \end{array}$

ISOTOPE PRODUCTION, GRAMS/TONNE

$$
\begin{aligned}
& \text { KR } \\
& \text { STABL } \\
& 77 . \\
& 116 \text {. } \\
& 168 . \\
& 182 . \\
& 187 . \\
& 197 . \\
& 194 . \\
& 178 . \\
& 171 . \\
& 165 \text {. } \\
& 160 \text {. } \\
& 156 . \\
& 150 \text {. } \\
& 144 . \\
& 140 \text {. } \\
& 136 . \\
& 132 . \\
& 127 . \\
& 125^{\circ} \\
& 125 . \\
& 124 . \\
& 124 . \\
& 125 . \\
& 126 . \\
& 125^{\circ} \\
& 124 . \\
& 125 . \\
& 125 . \\
& 125 . \\
& 124 . \\
& 125 \text {. } \\
& 125 \text {. } \\
& 125 \text {. } \\
& 125{ }^{\circ} . \\
& 109 .
\end{aligned}
$$

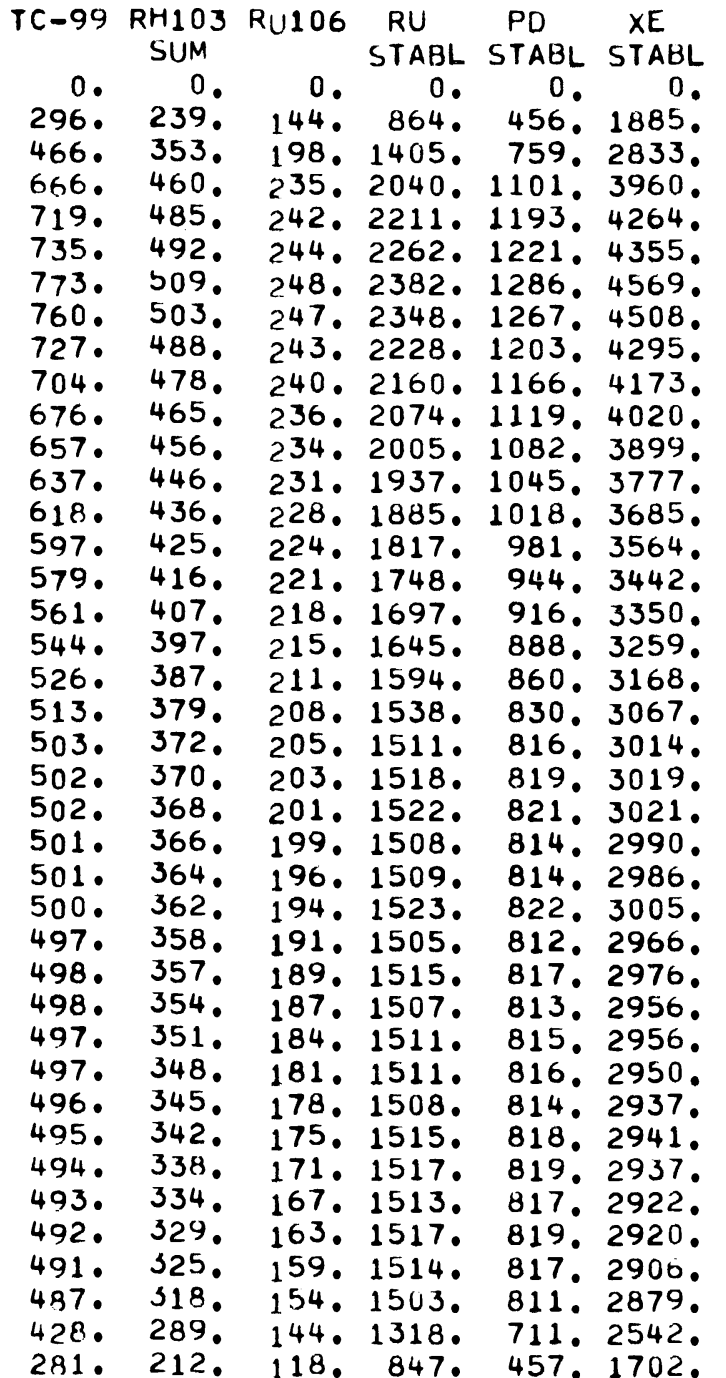


1) ISCHARGE SCH: C IILE FOR 10000 IN W I I I LWR80- 4PU

DISCHARGE

CYCLE

NUMBER UATE TONNE

$\begin{array}{ll}-1.0 & 102.9\end{array}$

$1 \cdot 3$

$1 \cdot 3$
$2 \cdot 0$

$2 \cdot 7$

$3 \cdot 3$

3.9

$4 \cdot 5$

$5 \cdot 1$

$5 \cdot 6$

6.7

$7 \cdot 3$

$7 \cdot 8$

$8 \cdot 3$

8.9

10.0

10.5

$11 \cdot 0$

$11 \cdot 6$

$12 \cdot 1$

$12 \cdot 7$

13.2

$13 \cdot 8$

$14 \cdot 3$
14.8

$15 \cdot 4$

15.9

$16 \cdot 5$

$17 \cdot 1$

17.6

18.2

$18 \cdot 8$

19.4

$20 \cdot 0$

$20 \cdot 7$

$21 \cdot 3$

$22 \cdot 0$

$22 \cdot 7$

\section{ISOTOPE PROUUCTION, GRAMS/TONNE}

EXPOS

0.

$25 \cdot 7$

$25 \cdot 7$

$25 \cdot 7$
$25 \cdot 7$

$25 \cdot 7$

$25 \cdot 7$

$25 \cdot 7$

$25 \cdot 7$

25.7
25.7

$25 \cdot 7$

$25 \cdot 7$

$25 \cdot 7$

$25 \cdot 7$

$25 \cdot 7$

25.7

$25 \cdot 7$

$25 \cdot 7$

$25 \cdot 7$

$25 \cdot 7$

$25 \cdot 7$

$25 \cdot 7$

$25 \cdot 7$

$25 \cdot 7$

$25 \cdot 7$

$25 \cdot 7$

$25 \cdot 7$

$25 \cdot 7$

$25 \cdot 7$

25.7

$25 \cdot 7$

$25 \cdot 7$

25.7
10140 .

15836.

22467.

24287 .

23212 .

22708.

21844 .

20777.

20071 .

19939.

19995.

20017.

19987.

$20004^{\circ}$

$20009^{\circ}$

19998.

19977.

19993.

19976.

19992.

20013.

19978.

19995.

20006.

20007 .

19919.

19980 .

20001 .

19985.

19997.

20006.

19982.

19975.

19984.

19952.
PU-RP

0 .

$18300^{\circ}$

$19155^{\circ}$.

$20639^{\circ}$

27674 .

8840.

$29618^{\circ}$

0239.

27208.
7502.

7346 .

7268 .

7229.

7268.

$7268^{\circ}$.

7268 .

7268 .

7268 .

7268 .

7229 .

7268 .

7268 .

7268 .

7268 .

7268 .

7268 .

7268 .

7268 .

7268 .

7229.

7268 .

7268 .

7268 .

7268 .

7268 .

7268 .

7229.

7268 .

7268.
PUI-SP NP237 AM241 AM243 CM242 CM244 PM147 CE144 CS137 SR-90 U-235 U-236

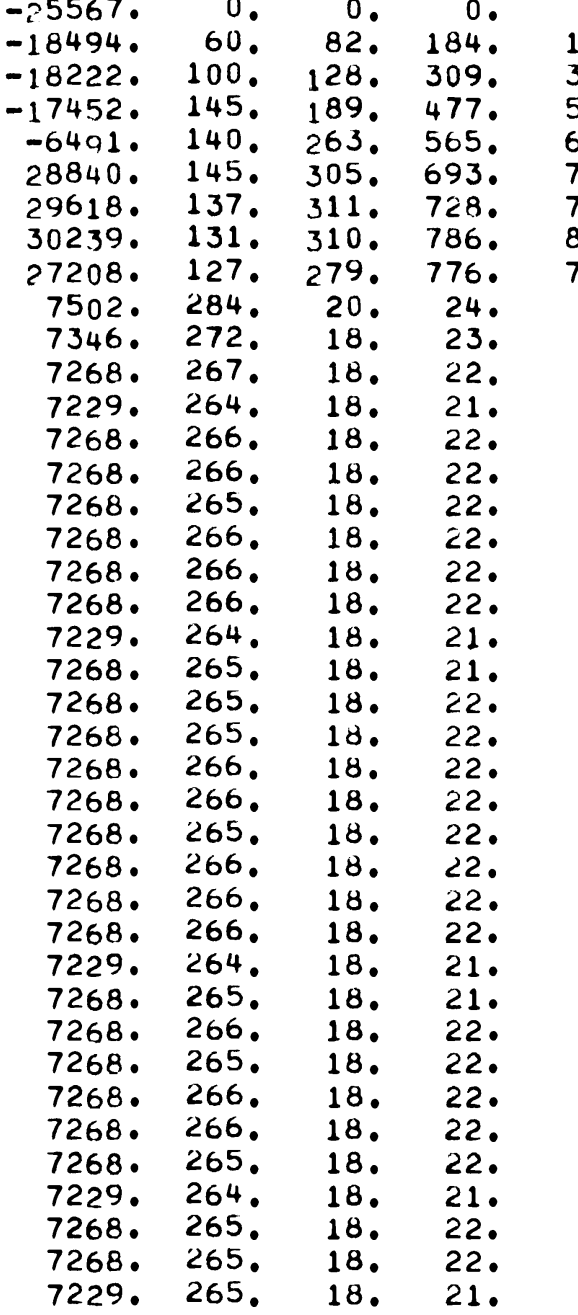

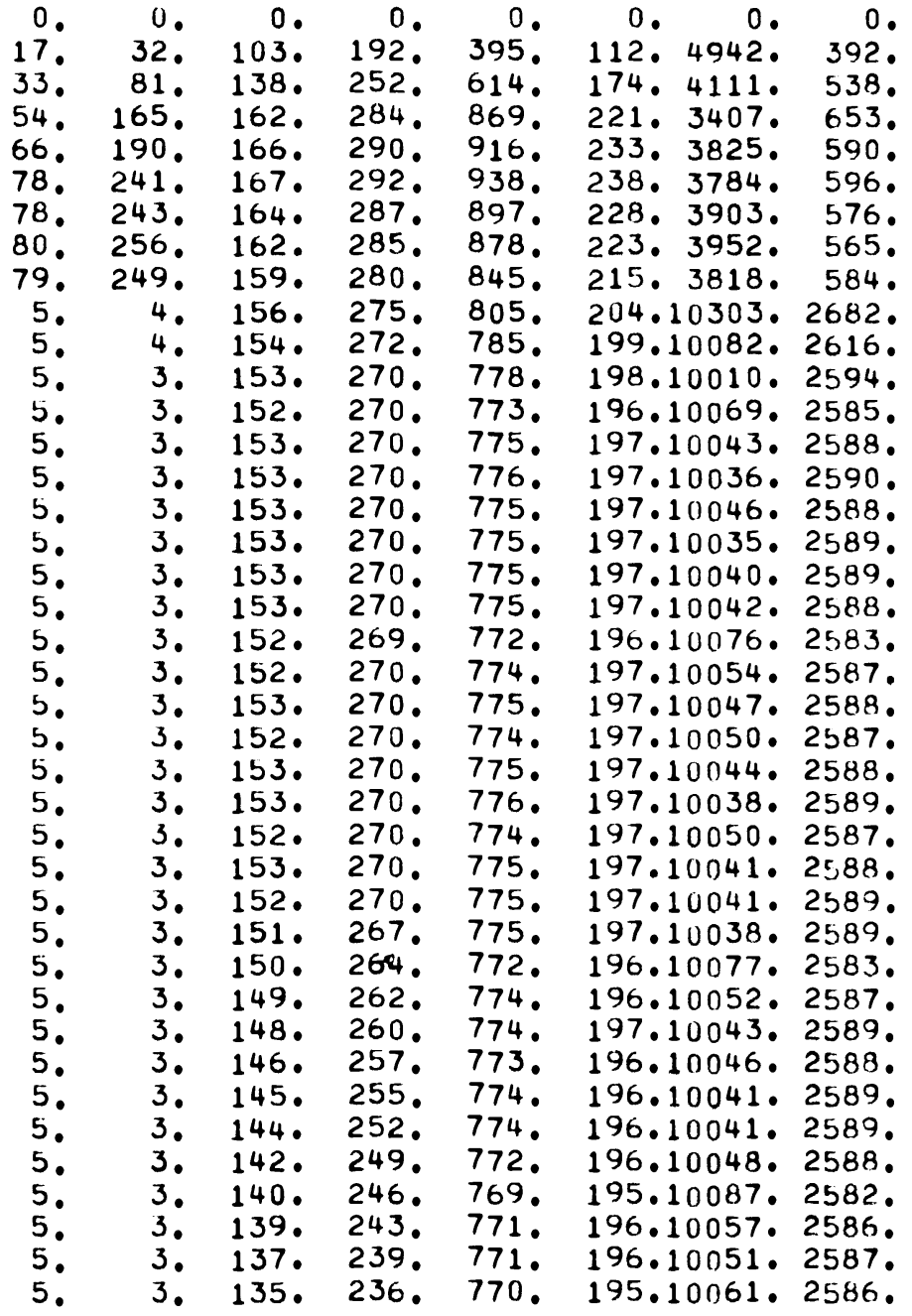


DISCHARGE

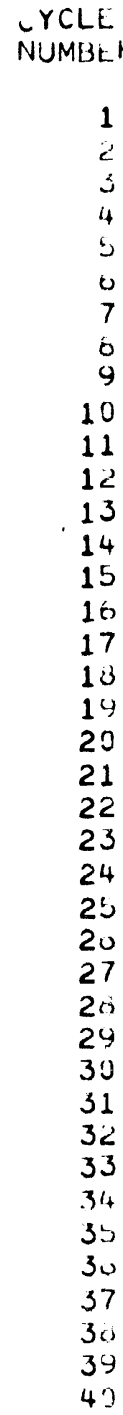

ISOTOPE PROUUCTION. GRAMS/TOINIE

$$
\begin{aligned}
& \begin{array}{c}
\text { KR } \\
\text { STABL }
\end{array} \\
& 0 . \\
& \begin{array}{r}
69 . \\
105 .
\end{array} \\
& 105 . \\
& 135^{\circ} \\
& 138 . \\
& 129^{\circ} . \\
& 123^{\circ} . \\
& 118 . \\
& 115^{\circ} \\
& 115 \text {. } \\
& 113 \text {. } \\
& 113 . \\
& 113 . \\
& 113 \text {. } \\
& 113 \text {. } \\
& 113 . \\
& 113 . \\
& 113 . \\
& 113 . \\
& 113 \text {. } \\
& 113 \text {. } \\
& 113^{\circ} \\
& 113 . \\
& 113 \text {. } \\
& 114 . \\
& 114 . \\
& 113 \text {. } \\
& 114 . \\
& 114 . \\
& 113 . \\
& 113 . \\
& 114 . \\
& 113 . \\
& 113 . \\
& 113 .
\end{aligned}
$$

TC-99 RH103 RU106 RU

$$
\text { SUM }
$$

STABL

PD $\quad X E$
STABL STA

26. $\quad 0^{\circ} \quad 0^{\circ} \quad 0^{\circ} \quad 0^{\circ} \quad 0^{\circ}$ $403.317^{\circ} 1370^{\circ} 775 \circ 409^{\circ} 1772$. $403.317 \cdot 185.1182 \cdot 624.2502$. 563. 429. 245. 1729. 959. 3421.

593. 447. 251.1829. 1014. 3595.

606.454 .254 .1868 .1036 .3665$.

580.440 .249 .1789 .992 .3525$.

568. 433. 246. 1749.970 .3455$.

$548^{\circ} 420.9241 .1670^{\circ}$ 926. 3316.

522. 405. 235. 1590. 882. 3176.

510 . 397. 232. 1550. 860. 3106.

505. 394. 231. 1550. 860.3106.

502. 392. 230. 1531. 849. 3072 .

503. 393. 230. 1531. 849.3072.

504. 394. 230. 1531. 849. 3072.

503. 393. 230. 1531. 849. 3072.

503. 393. 230.1531. 849. 3072.

504. 394. 230. 1531. 849.3072.

503. 393. 230. 1531. 849.3072.

501. 392. 230.1531. 849.3072。

503. 393. 230. 1531. 849. 3072.

503. 393. 230. 1531. 849. 3072 .

503. 393. 230.1531. 849.3072.

503. 393. 230.1531. 849.3072.

504. 394. 230. 1531. 849.3072.

503. 393. 230. 1531. 849.3072.

503. 393. 230. 1531. 849. 3072.

503. 393. 230. 1526. 846. 3063.

503. 392. 228. 1543. 855. 3086.

$501.389 .226 .1538,853,3072$.

$502,388.225 .1532 .849 .3056$.

$500^{\circ} 387.227^{\circ} 1544^{\circ} 89^{\circ} 3050^{\circ}$

$501.385^{\circ} 223.1544 .0850^{\circ} 3070$.

501.385 .221 .1536 .851 .3050 .

501.384 .219 .1527 . 846. 3027.

$501.382 .217 .1534 .850,3034$.

500 . 380. 215. 1539. 853. 3037.

498. 377. 213.1526. 840, 3007 .

499. 375. 211. 1528. 847. 3003.

498. 373. 208. 1527. 847. 2995.

497. 370. 206. 1525. 845. 2984. 
1) IS C I AKGE SCHELULE FOR 1000 MWIE ) LWR70- 10PU PAGE 28

8

DISCHARGE

LYCLF

IVMBEK

UATE TONIVE

$-1 \cdot 0 \quad 117 \cdot 7$

$1.1 \quad 27.4$

$1.8 \quad 29.4$

$2.7 \quad 29.4$

$\begin{array}{ll}3.7 & 29.4 \\ 4.6 & 29.4\end{array}$

$5.6 \quad 29.4$

$6.5 \quad 29.4$

$7.4 \quad 29.4$

$8.2 \quad 29.4$

$\begin{array}{ll}9.0 & 29.4 \\ 9.7 & 29.4\end{array}$

10.6

$11 \cdot 4$

$12 \cdot 1$
$12 \cdot 8$

13.5

$14 \cdot 1$

14.8
15.4

16.0

16.7

17.3

$18 \cdot 0$

18.7

19.4

20.1

$20 \cdot 8$

$21 \cdot 5$

$22 \cdot 3$

$23 \cdot 1$

23.9

$24 \cdot 7$

$25 \cdot 6$

$26 \cdot 5$

$27 \cdot 4$

28.4

29.4

30.0

$30 \cdot 0$

ISOTOPE PRODUCTION. GRAMS/TONIIE

EXPOS
IWD/T

0 .

$18369^{\circ}$

26854 .

29221.

30455 .

29481 .

29342 .

28210 .

27303 .

$25734^{\circ}$

26706.

25922 .

24425 .

23522 .

22692.

$218060^{\circ}$

20522 .

19986.

20030 .

20030 .

20014 .

$20043^{\circ}$

$20047^{\circ}$.

$20064^{\circ}$

20055 .

20063.

20070 .

19968 .

20038 .

20038 .

20018 .

20043.

20046 .

20060 .

17751 .

11560 .
PU-RP

0.

20364 .

22948.

31754 .

34949 .

37057 .

38621 .

39539.

40627 .

40117.

35391 .

8193 .

7955.

7751.

7445 .

7343

7343

7275 .

72.75 .

7275 .

7275 .

7275 .

7275 .

7275 。

7275 .

7275 .

7275 .

7275 .

7241.

7275 .

7275 .

$7275^{\circ}$

7275 .

7275 .

7275 .

6765 .

5066 .
PU-SP NP237 A.1241 AM243 CM242 CM244 PM147 CE144 CS137 SR-90 U-235 U-236

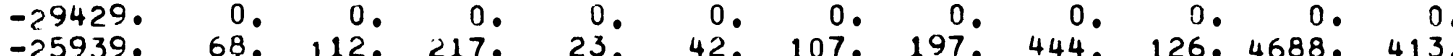
$-26687.117 .181 .378,44.112 .138 .248,710.192 .3782 .567$. $-26280.171 .284 .614 .22 .241 .262 .283 .1028 .278 .3086 .672$.

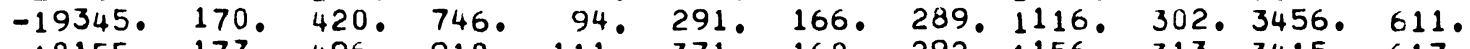
-18155 . 177. 496. 918. 111. 371. 168, 292. 1156, 313.3415.617.

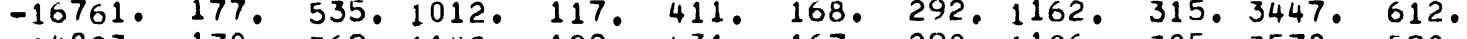
-14823 . 170. 569. 1100. 122. 431. 167. 290, 1126. 305.3578. 590. $-13327,168,618,1147,131,442,166,290,1120,303,3632.581$. $-6562 \cdot 160 \cdot 628 \cdot 1212 \cdot 131 \cdot 451 \cdot 164 \cdot 287 \cdot 1079 \cdot 292 \cdot 3721 \cdot 565$. 39879. 155. 625. 1235. 129. 448. 163. 284. 1045. 283.3785. 554. 40627. 144. 611. 1238. 125. 425. 159. 280. 986. 267.3910. 531. 40117. 148. 609. 1305. 134. 459. 161. 283. 1023. 277. 3806. 547. 35391. 146. 513. 1328. 125. 469. 160. 280. 993. 269.3632. 573. 8193. 359. 30. 37. 8. 7. 156. 275. 938. 254.10056.3070. 7955. 336. 28. $33 . \quad 7 \cdot \quad 6.254 .272 .904 .245 .9948 .2977$.

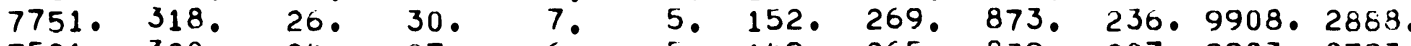

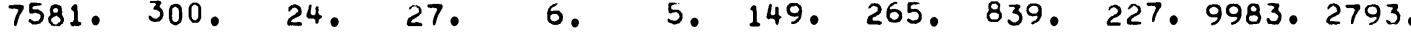
$7445.286 .22 .25 . \quad 6.24 .447 .262 .812 .220 .10005 \cdot 2711$. $7343.275 .21 .23 . \quad 5 . \quad 4.245 .259 .791 .214 .9948 .2642$. $7275.265 .20 .22 .25 . \quad 3.242 .253 .770 .209 .9906 .2577$. 7275. 266. 20. 22. 5. 4. 141. 251. 772. 209.9886. 2580. 7275. 266. 20. 22. 5. 4. 140. 248. 772. 209.9883. 2580. 7275. 265. 20. 22. 5. 3. 138. 245. 771. 209.9888. 2579. 7275. 266. 20. 22. 5. 4. 136. 242. 771. 209.9881. 2581. 7275. 266. 20. 22. 5. 4. 135. 238. 771. 209.9876.2581. 7275. 266. 20. 22. 5. 4. 133. 235. 771. 209.9873. 2582. 7275. 266. 20. 22. $\quad$ 5. 4. 131. 232. 771. 209.9867. 2583. 7275. 266. 20. 22. 5. 4. 129. 228. 770. 209.9869.2582. $7275.266 .20 .22 . \quad 5 . \quad 4.427 .224 .770 .209 .9867 .2583$. 7275. 266. 20. 22. 5. 4. 125. 220. 770. 208.9862. 2583. $7241.264 .20,22, \quad 5, \quad 3,123,215,765,207.9911 .2576$.

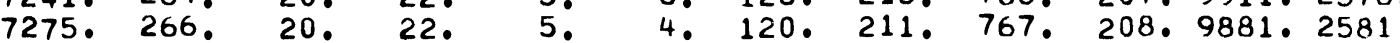
7275. 266. 20. 22. 5. 4. 118. 206. 766. 208.9879.2581. 7275. 265. 20. 22. 5. 3. 115. 201. 765. 207.9887. 2579. 7275. 266. 20. 22. 5. 4. 112. 195. 765. 207.9878. 2581. 7275. 266. 20. 22. 5. 4. 109. 189. 764. 207.9874.2581. 7275. 266. 20. 22. 5. 4. 106. 183. 764. 207.9867.2582.

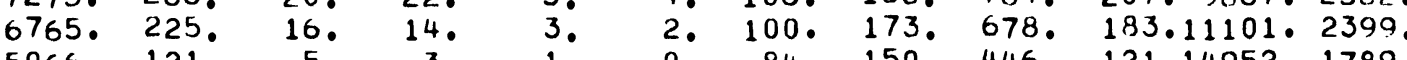

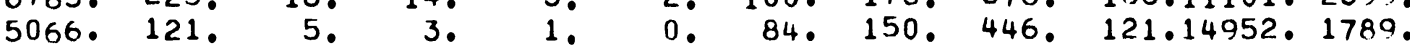




\begin{tabular}{|c|c|c|}
\hline \multirow{2}{*}{$\begin{array}{l}\text { CYCLL } \\
\text { NUMBER }\end{array}$} & \multicolumn{2}{|c|}{ DISCHARGE } \\
\hline & DATE & TONIVE \\
\hline 1 & $-1 \cdot 0$ & $117 \cdot 7$ \\
\hline 2 & $1 \cdot 1$ & 29.4 \\
\hline 3 & $1 \cdot 8$ & 29.4 \\
\hline 4 & $2 \cdot 7$ & $29 \cdot 4$ \\
\hline 5 & 3.7 & 29.4 \\
\hline 0 & $4 \cdot 6$ & 29.4 \\
\hline 7 & 5.6 & 29.4 \\
\hline 8 & 6.5 & 29.4 \\
\hline 9 & $7 \cdot 4$ & $29 \cdot 4$ \\
\hline 10 & $8 \cdot 2$ & 29.4 \\
\hline 11 & 9.0 & 29.4 \\
\hline 12 & 9.7 & 29.4 \\
\hline 13 & $10 \cdot 6$ & 29.4 \\
\hline 14 & 11.4 & 29.4 \\
\hline 15 & $12 \cdot 1$ & 29.4 \\
\hline 10 & $12 \cdot 8$ & 29.4 \\
\hline 17 & 13.5 & 29.4 \\
\hline 16 & $14 \cdot 1$ & 29.4 \\
\hline 19 & $14 \cdot 8$ & 29.4 \\
\hline 20 & 15.4 & 29.4 \\
\hline 21 & $16 \cdot 0$ & $29 \cdot 4$ \\
\hline 22 & 16.7 & $29 \cdot 4$ \\
\hline 23 & $17 \cdot 3$ & 29.4 \\
\hline 24 & 18.0 & 29.4 \\
\hline 25 & 18.7 & 29.4 \\
\hline 26 & 19.4 & 29.4 \\
\hline 27 & $20 \cdot 1$ & 29.4 \\
\hline 20 & $20 \cdot 8$ & $29 \cdot 4$ \\
\hline 29 & $21 \cdot 5$ & $29 \cdot 4$ \\
\hline 30 & $22 \cdot 3$ & 29.4 \\
\hline 31 & $23 \cdot 1$ & 29.4 \\
\hline 32 & 23.9 & $29 \cdot 4$ \\
\hline 33 & 24.7 & 29.4 \\
\hline 34 & $25 \cdot 6$ & 29.4 \\
\hline 35 & $26 \cdot 5$ & $29 \cdot 4$ \\
\hline 30 & 27.4 & 29.4 \\
\hline 37 & 28.4 & 29.4 \\
\hline $3 k$ & 29.4 & 29.4 \\
\hline 39 & $30 \cdot 0$ & 29.4 \\
\hline 40 & 30.0 & 29.4 \\
\hline
\end{tabular}

ISOTOPE PRODUCTION. GRAMS/TONNE

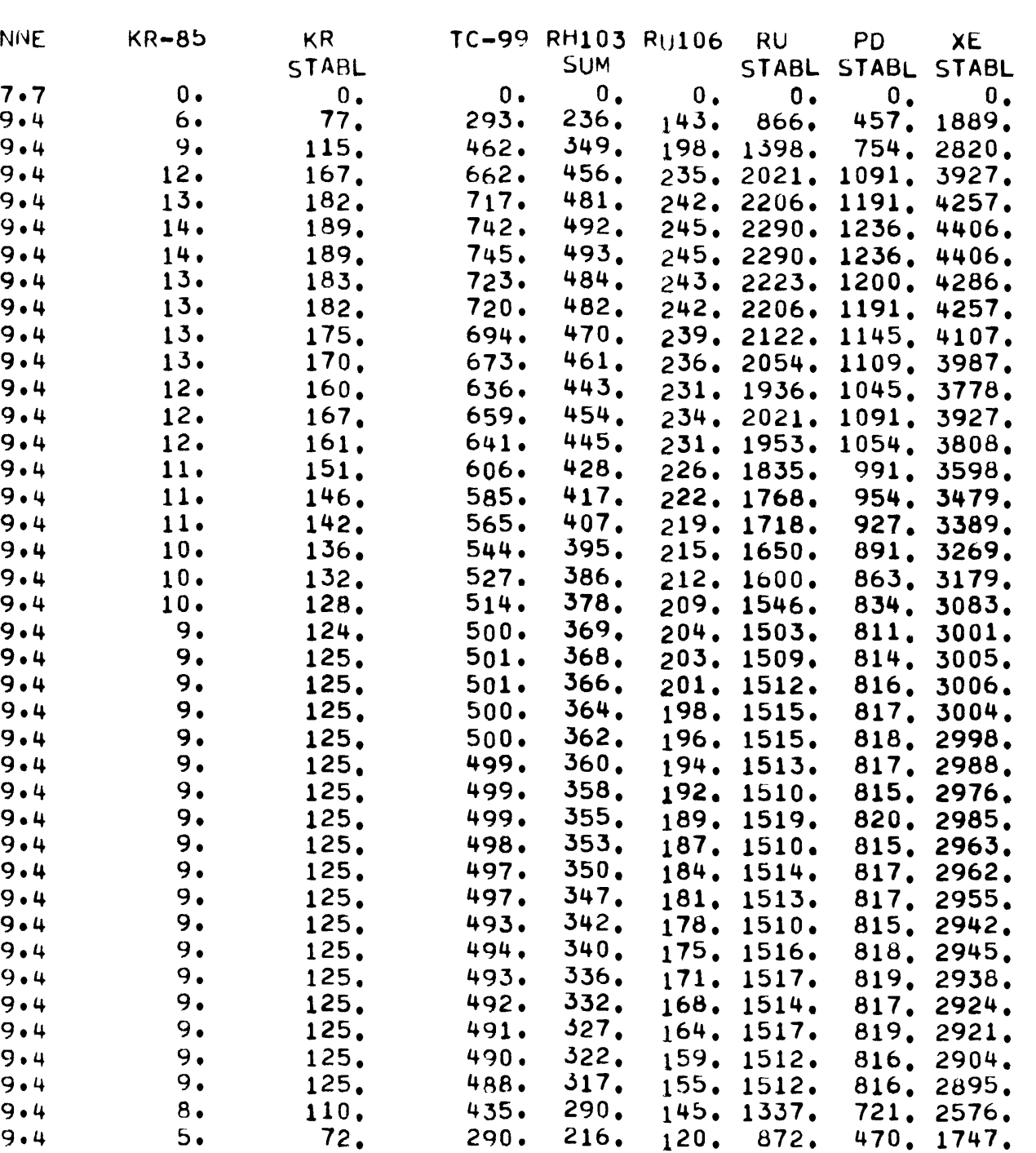


DISCHARGE SCHEDULE FOR 1000 MW ( E ) LWR70- 3OPU PAGE 30

DISCHARGE

CYCL
NUMBE









1
1
1
1
1
1
1
1
1
1
2
2
2
2
2
2
2
2
2
2
3
3
3
3
3
3
3
3
3
3
4

ISOTOPE PRODUCTION. GRAMS/TONNE

\begin{tabular}{|c|c|c|}
\hline UATE & TONNE & $\begin{array}{l}\text { EXPOS } \\
\text { MWD/T }\end{array}$ \\
\hline$-1 \cdot 0$ & $117 \cdot 7$ & 0. \\
\hline $1 \cdot 1$ & 29.4 & $11524^{\circ}$ \\
\hline 1.9 & 29.4 & 13469. \\
\hline 2.7 & 29.4 & 26943 . \\
\hline 3.8 & 29.4 & 29403. \\
\hline 4.7 & 29.4 & 30426 . \\
\hline $5 \cdot 6$ & 29.4 & 30563 . \\
\hline $6 \cdot 5$ & 29.4 & 29511 . \\
\hline 7.4 & 29.4 & 29361 . \\
\hline 8.2 & 29.4 & 28128 \\
\hline 9.0 & 29.4 & 27124 \\
\hline 9.8 & 29.4 & $20077^{\circ}$ \\
\hline 10.6 & 29.4 & $25224^{\circ}$ \\
\hline $11 \cdot 3$ & $29 \cdot 4$ & 24463. \\
\hline 12.0 & 29.4 & 23822 \\
\hline 12.8 & 29.4 & 23237 . \\
\hline 13.4 & 29.4 & 22561 \\
\hline 14.1 & 29.4 & 21818 \\
\hline 14.7 & 29.4 & 21301. \\
\hline 15.4 & 29.4 & 20834 \\
\hline 16.0 & 29.4 & 20413 . \\
\hline $16 \cdot 7$ & 29.4 & 20313. \\
\hline $17 \cdot 3$ & 29.4 & 20286 \\
\hline 18.0 & 29.4 & 20189. \\
\hline 18.7 & 29.4 & 20147 . \\
\hline 19.4 & 29.4 & 20137 . \\
\hline $20 \cdot 1$ & 29.4 & 20085 \\
\hline 20.9 & 29.4 & 20071 . \\
\hline 21.6 & 29.4 & 20079 \\
\hline $22 \cdot 4$ & 29.4 & 20028 \\
\hline $23 \cdot 2$ & 29.4 & 20040 . \\
\hline $24 \cdot 0$ & 29.4 & 20042 . \\
\hline $24 \cdot 8$ & 29.4 & 20029 \\
\hline $25 \cdot 7$ & 29.4 & 20038 \\
\hline $26 \cdot 6$ & 29.4 & 20069. \\
\hline 27.5 & 29.4 & 20047 \\
\hline $28 \cdot 5$ & 29.4 & 20069. \\
\hline $29 \cdot 5$ & $29 \cdot 4$ & 20092 \\
\hline $30 \cdot 0$ & 29.4 & $16767^{\circ}$ \\
\hline & & 10386 \\
\hline
\end{tabular}

PU-RP

9276

20364 .

22778 .

31516 .

33317 .

34711 .

36853 .

38383 .

39165.

39811 .

40117 .

40321 .

40321 .

40355 .

40389 .

40491 .

40355 .

40287 .

40151 .

40423 .

40593 .

40831 .

41001 .

41239 .

41409 .

41579 .

41749.

41953.

42089 .

42259.

42429 .

$42599^{\circ}$

42701 .

42871 .

43007 .

$43177^{\circ}$

44707 .

47698 .
PU-SP NP237 AM241 AM243 CM242 CM244 PM147 CE144 CS137 SR-90 U-235 U-236

$\begin{array}{rrrr}-29293^{\circ} & 0 . & 0 . & 0 \\ -25667 . & 69 . & 114 . & 219\end{array}$

$-26381.119 .182 .380$.

$-26144.174,281.612$.

$-19277.174 .418 .747$.

-17883.181 .

493. 918 .

$-14177.172$.

532.1013.

564. 1097, 121. 431.

12613.170.

-11729.162 .

$\begin{array}{lll}-11151 . & 156 . \\ -10573 . & 149 .\end{array}$

$620.1206 .130 .449^{\circ}$.

613. 1225. 128. 443 .

$-9995^{\circ} 144^{\circ} 608.12488^{\circ} 125.436$.

-9553. 139. 600.1283. 122. 424.

-9179. 135. 588. 1287. 119. 416.

-8839.132 .

$567.1295 .114 .399^{\circ}$.

$-8941^{\circ} 1200^{\circ} 540^{\circ} 1290.127^{\circ} 379^{\circ}$

$-9247.117$.

528. 1291. 105. 372.

$-9553.115 .520 .1288^{\circ}$. 102. 365.

$-9553.114$.

$-9587.114$.

$\begin{array}{llll}518.1306 . & 102, & 368 . \\ 518 . & 1316 . & 101 . & 371 .\end{array}$

$-9553.113$.

$517.1326,101.371$.

-9519. 112. 518.1352. 100. 377.

-9519. 112. 518. 1357. 100. 377.

$\begin{array}{llllll}-9553^{\circ} & 112 . & 519.1368 . & 100 . & 380 . \\ -9587 . & 112 . & 520.1377 . & 100 . & 382 .\end{array}$

-9587.112 .

111.

523.1394 .100 .385

$-9587.111 .524 .1404 .100 .388$.

$-9587.111 .526 .1410 .100 .389$.

$-9621.111$.

527.1421 .100 .391$.

$-9655^{\circ} 111.531^{\circ} 14284^{\circ} 101.394^{\circ}$

-9655. 111. 533. 1440. 101. 396.

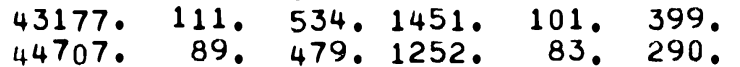

$\begin{array}{llll}44707 . & 89 . & 479, & 1252 . \\ 47698 . & 50 . & 333 . & 823 .\end{array}$
111. 198. 448. 127.4780.423. 147. 248. 713. 193. 3871. 582 . 175. 283. 1031. 279. 3159. 694. 180.290 .1123 .604 .3564 .636$. 182. 292. 1161. 314. 3529. 641. 183. 292. 1166. 316. 3562. 636. 180. 290. 1127. 305. 3706. 612. 180.290 .1121 .304 .3762 .602$. 177. 287. 1076. 291. 3856. 586. 175. 284. 1038. 281. 3927. 573. 172. 281. 999. 271.4005. 559. 170. 278. 967. 262.4067. 547. 168. 275. 939. 254. 4120. 537. 166. 273. 915. 248.4163. 530. 164. 271. 893. 242. 4201. 522. 162. 268. 868. 235. 4250. 513. 160. 265. 840. 228. 4306. 503. 158. 263. 820. 222. 4340.496. 156. 260. 803. 218.4370. 490. 153. 256. 787. 213.4397. $4850^{\circ}$ $\begin{array}{llllll}151 . & 252 & 782 . & 212 . & 4409 \cdot & 483 . \\ 150 . & 249 & 781 . & 212 . & 4414 . & 482 .\end{array}$ 148. 246. 777. 211.4426. 479. 146. 242. 775. 210.4433. 478. 144. 239, 774. 210,4437, 477 . 142. 235. 772. 209. 4444. 476. 140. 231. 771. 209. 4448. 475. 139. 228. 771. 209.4450. 474. 134. 219. 768. 208. 4459. 472 . 132. 215. 768. 208. 4462. 472 . 130. 210. 767. 208. 4465. 471 . 127. 205. 766. 208.4467. 471 . 115. 182. 765. 207. 4473. 469. \begin{tabular}{llllll}
$1060^{\circ}$ & $170^{\circ}$ & 641. & 174.4822. & 403. \\
\hline
\end{tabular} 136. 223. 768. 208. 4457. 473 . 124. 200. 767. 208. 4467. $471^{\circ}$. 118. 189. 765. 207.4472. 469. 
DISCHARGE

\begin{tabular}{|c|c|c|}
\hline $\begin{array}{l}\text { ¿YCLE } \\
\text { NUMBER }\end{array}$ & UATE & TONNE \\
\hline 1 & $-1 \cdot 0$ & 117.7 \\
\hline 2 & $1 \cdot 1$ & 29.4 \\
\hline 3 & 1.9 & 29.4 \\
\hline 4 & 2.7 & 29.4 \\
\hline 5 & 3.8 & 29.4 \\
\hline 6 & 4.7 & 29.4 \\
\hline 7 & $5 \cdot 6$ & 29.4 \\
\hline 8 & 6.5 & 29.4 \\
\hline 9 & 7.4 & 29.4 \\
\hline 10 & $8 \cdot 2$ & 29.4 \\
\hline 11 & 9.0 & 29.4 \\
\hline 12 & 9.8 & 29.4 \\
\hline 13 & 10.6 & 29.4 \\
\hline 14 & 11.3 & 29.4 \\
\hline 15 & 12.0 & 29.4 \\
\hline 10 & 12.8 & 29.4 \\
\hline 17 & 13.4 & 29.4 \\
\hline 18 & $14 \cdot 1$ & 29.4 \\
\hline 19 & $1: 0.7$ & 29.4 \\
\hline 20 & 15.4 & 29.4 \\
\hline 21 & 16.0 & 29.4 \\
\hline $2 z$ & 16.7 & 29.4 \\
\hline 23 & 17.3 & 29.4 \\
\hline 24 & 18.0 & 29.4 \\
\hline 25 & 18.7 & 29.4 \\
\hline 26 & 19.4 & 29.4 \\
\hline 27 & $20 \cdot 1$ & 29.4 \\
\hline 28 & 20.9 & 29.4 \\
\hline 29 & $21 \cdot 6$ & 29.4 \\
\hline 35 & 22.4 & 29.4 \\
\hline 31 & 23.2 & 29.4 \\
\hline 32 & 24.0 & 29.4 \\
\hline 33 & 24.8 & 29.4 \\
\hline 34 & 25.7 & 29.4 \\
\hline 35 & $26 \cdot 6$ & 29.14 \\
\hline 30 & $27 \cdot 5$ & 29.4 \\
\hline 37 & 28.5 & 29.4 \\
\hline 33 & 29.5 & 29.4 \\
\hline 39 & $30 \cdot 0$ & 29.4 \\
\hline 40 & 30.0 & 29.4 \\
\hline
\end{tabular}

ISOTOPE PRCUUCTION. GRAMS/TONNE

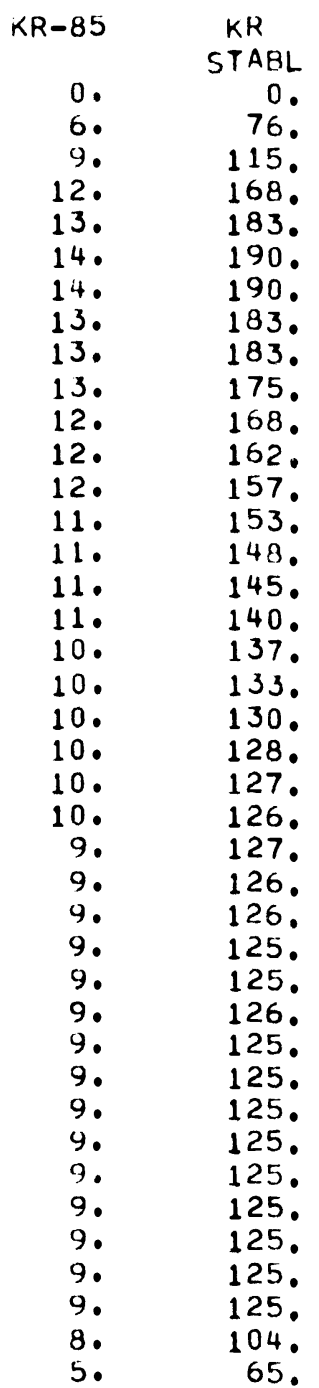

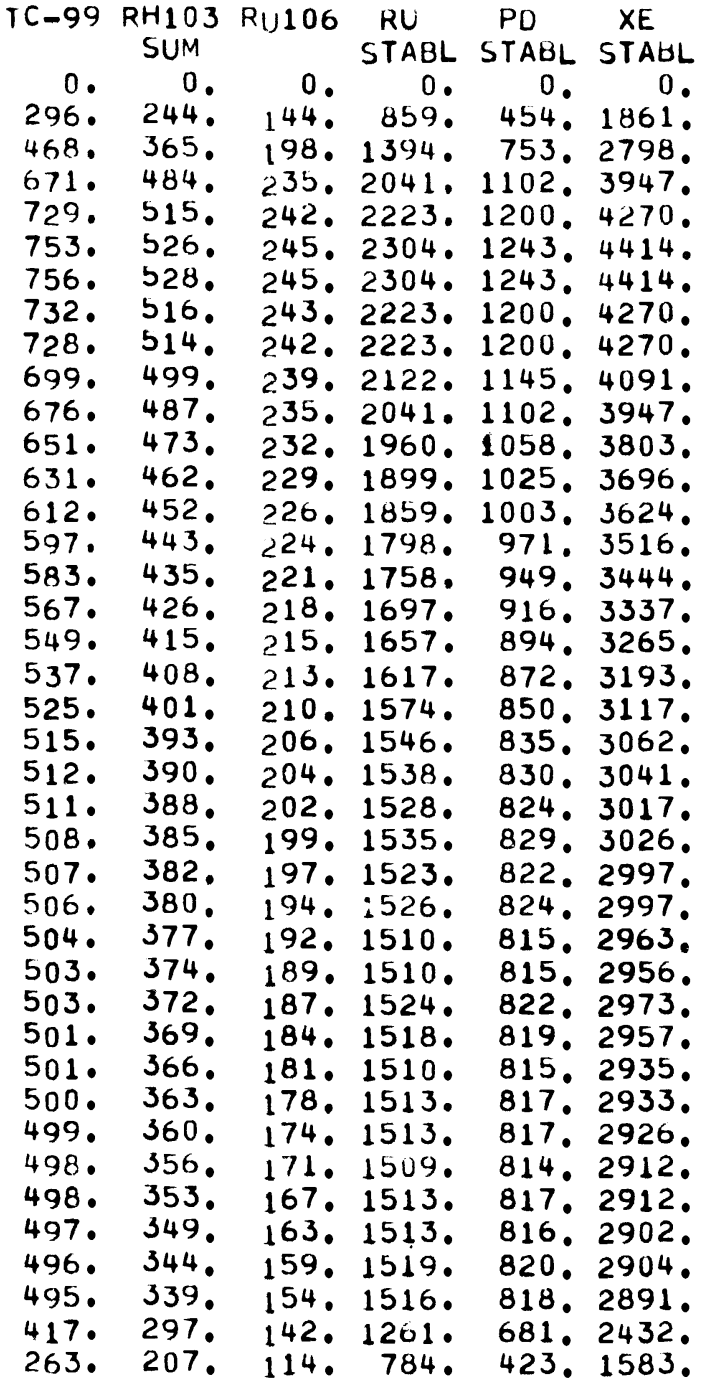


DISCHARGE

CYCLE

\begin{tabular}{|c|c|c|c|}
\hline INUMBEK & DATE & TONNE & $\begin{array}{l}\text { EXPOS } \\
\text { MWD/T }\end{array}$ \\
\hline 1 & $-1 \cdot 0$ & 102.9 & 0 . \\
\hline i & .9 & $25 \cdot 7$ & 10134 \\
\hline 3 & $1 \cdot 4$ & $25 \cdot 7$ & 15833 \\
\hline 4 & $2 \cdot 0$ & $25 \cdot 7$ & 22543 \\
\hline 5 & $2 \cdot 7$ & $25 \cdot 7$ & 23802 \\
\hline 0 & $3 \cdot 4$ & $25 \cdot 7$ & 24395 . \\
\hline 7 & $4 \cdot 0$ & $25 \cdot 7$ & 23819. \\
\hline b & $4 \cdot 6$ & $25 \cdot 7$ & $22517^{\circ}$ \\
\hline$y$ & $5 \cdot 1$ & $25 \cdot 7$ & 21997 . \\
\hline 10 & $5 \cdot 7$ & $25 \cdot 7$ & 21478 \\
\hline 11 & $6 \cdot 3$ & $25 \cdot 7$ & 20925 . \\
\hline 12 & $6 \cdot 8$ & $25 \cdot 7$ & 20817 . \\
\hline 13 & $7 \cdot 4$ & $25 \cdot 7$ & 20624 . \\
\hline 14 & $8 \cdot 0$ & $25 \cdot 7$ & 20355 . \\
\hline $1 \mathrm{~s}$ & $8 \cdot 5$ & $25 \cdot 7$ & 20373 . \\
\hline 10 & $9 \cdot 1$ & $25 \cdot 7$ & 20239 . \\
\hline 17 & $9 \cdot 6$ & $25 \cdot 7$ & 20167 \\
\hline 18 & $10 \cdot 2$ & $25 \cdot 7$ & 20120 . \\
\hline 19 & $10 \cdot 7$ & $25 \cdot 7$ & 20097 . \\
\hline 20 & $11 \cdot 3$ & $25 \cdot 7$ & 20045 . \\
\hline 21 & $11 \cdot 8$ & $25 \cdot 7$ & 20076 . \\
\hline 22 & $12 \cdot 3$ & $25 \cdot 7$ & 20038 \\
\hline 20 & $12 \cdot 9$ & $25 \cdot 7$ & 20042 . \\
\hline 24 & $13 \cdot 4$ & $25 \cdot 7$ & 20081 • \\
\hline 25 & $14 \cdot 0$ & $25 \cdot 7$ & 20101 . \\
\hline 26 & $14 \cdot 5$ & $25 \cdot 7$ & 20087 . \\
\hline 27 & $15 \cdot 1$ & $25 \cdot 7$ & 20155 . \\
\hline 20 & $15 \cdot 6$ & $25 \cdot 7$ & 20155. \\
\hline 29 & $16 \cdot 2$ & $25 \cdot 7$ & 20131 . \\
\hline 30 & $16 \cdot 8$ & $25 \cdot 7$ & 20139 . \\
\hline 31 & $17 \cdot 4$ & $25 \cdot 7$ & $20124^{\circ}$ \\
\hline 32 & $17 \cdot 9$ & $25 \cdot 7$ & 20097 . \\
\hline 33 & $18 \cdot 6$ & $25 \cdot 7$ & 20134 . \\
\hline 34 & $19 \cdot 2$ & $25 \cdot 7$ & 20113. \\
\hline 35 & 19.8 & $25 \cdot 7$ & 20109 . \\
\hline 36 & $20 \cdot 4$ & $25 \cdot 7$ & 20146 . \\
\hline 37 & $21 \cdot 1$ & $25 \cdot 7$ & 20150 . \\
\hline 38 & $21 \cdot 8$ & $25 \cdot 7$ & 20140 . \\
\hline 39 & $22 \cdot 5$ & $25 \cdot 7$ & 20191. \\
\hline 40 & $23 \cdot 1$ & 25.7 & 20200 . \\
\hline
\end{tabular}

\section{ISOTOPE PRODUCTION. GRAMS/TONINE}

PU $-R P$

0 .

$18300^{\circ}$

19155.

20678 .

27713.

$29462^{\circ}$

30473 .

31095 .

31833 .

32144.

$32610^{\circ}$

$33854^{\circ}$.

$34165^{\circ}$.

34670 .

35020 .

35525 .

35836 .

36186 .

$36497^{\circ}$

36925.

37158.

$37469^{\circ}$

$38091^{\circ}$.

38363 .

38635 .

38829.

39063.

39257 .

39451 .

39607.

39840.

40112 .

$40267^{\circ}$.

40462 .

40578 .

40734 .
PU-SP NP237 AM241 AM243 CM242 CM244 PM147 CE144 CS137 SR-90 U-235 U-236

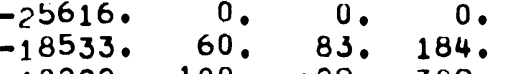
$-18222.100 \cdot 129.309$ $-17452,144.191,482$. $-10572 . \quad 145.306 .695$ $-10106.741 .317 .745$. $-9600 . \quad 132.322 .788$. -9562 . 128. 338.804

$-9562.125 .347 .867$

$-96399^{\circ} 121.346 .884$.

-9562. 119. 360. 942.

$-9406.9117 .366 .984$.

$-9445.117 .369 .1006$.

$-9367.116 .373 .1032$.

$-9367.115 .378 .1047$.

$-9367.114 .383 .1082$.

-9328. 114. 386. 1098.

$-9367.114 .390 .1117$

-9406. 114. 394. 1133.

$-93677^{\circ} 113.397 .1159$.

$-9484^{\circ} 113.404 .1190$

$-9367.113 .408 .1202$.

-9328 . 113. 412. 1222.

$-9289^{\circ} 113.415 .1235$.

$-92511^{\circ} 113.418 .1248$.

$-9251.113 .423 .1273$.

$-9212.113 .425 .1280$

$-9212.112 .427 .1289$

$-9212.113 .429 .1298$.

$-92120^{\circ} 112.430 .1310$.

112. 432. 1316.

112. 435. 1326.

$-9328.112 .436 .1332$

$-9328.112 .438 .1341$

$-9328.113 .440 .1349$.

-9367 . 113. 442. 1356 .

\begin{tabular}{|c|c|c|c|c|c|c|c|}
\hline $\begin{array}{l}0 . \\
8 . \\
3 . \\
4\end{array}$ & 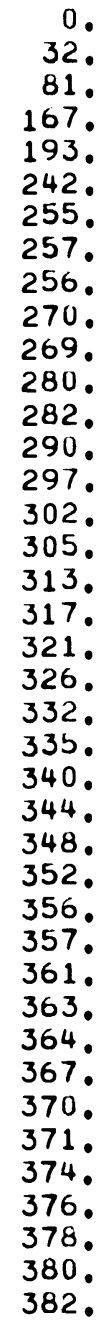 & $\begin{array}{l}00^{\circ} \\
106^{\circ} \\
144^{\circ} \\
171^{\circ} \\
175^{\circ} \\
178^{\circ} \\
176^{\circ} \\
171^{\circ} \\
169^{\circ} \\
167^{\circ} \\
164^{\circ} \\
164^{\circ} \\
163^{\circ} \\
162^{\circ} \\
162^{\circ} \\
161^{\circ} \\
161^{\circ} \\
161^{\circ} \\
1611^{\circ} \\
160^{\circ} \\
161^{\circ} \\
160^{\circ} \\
160^{\circ} \\
161^{\circ} \\
161^{\circ} \\
1611^{\circ} \\
161^{\circ} \\
160^{\circ} \\
159^{\circ} \\
1580^{\circ} \\
157^{\circ} \\
155^{\circ} \\
154^{\circ} \\
152^{\circ} \\
151^{\circ} \\
150^{\circ} \\
1480^{\circ} \\
146^{\circ} \\
145^{\circ} \\
143^{\circ}\end{array}$ & 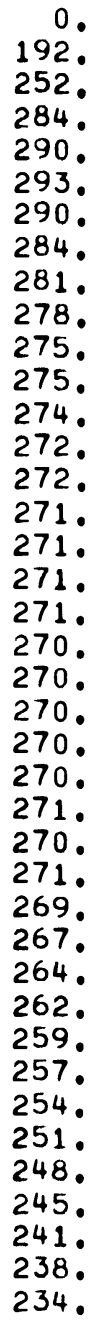 & $\begin{array}{l}0 . \\
395 . \\
613 . \\
872 . \\
919 . \\
942 . \\
920 . \\
871^{\circ} \\
851 . \\
831 . \\
810 . \\
806 . \\
799 . \\
789 . \\
789 . \\
784 . \\
782 . \\
780 . \\
779 . \\
777 . \\
778 . \\
777 . \\
777 . \\
778 . \\
779 . \\
778 . \\
781 . \\
781 . \\
780 . \\
780 . \\
779 . \\
778 . \\
779 . \\
778 . \\
777 . \\
778 . \\
778 . \\
777 . \\
779 . \\
779 .\end{array}$ & 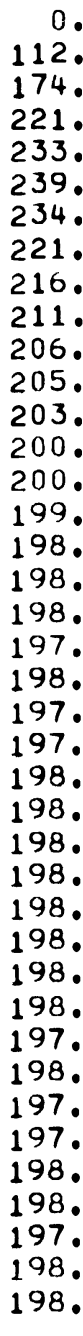 & 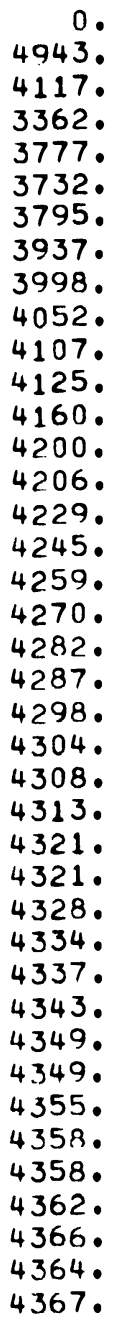 & \\
\hline
\end{tabular}


DISCHARGE

YCLE
NUMBLK
1
2
3
4
5
0
7
0
9
10
11
12
13
14
15
16
17
10
19
20
21
22
23
24
25
20
27
20
29
30
31
32
33
34
35
30
37
30
39
40

ISOTOPE PRODUCTION. GRAMS/TONINE

$\begin{array}{rr}\text { UATE } & \text { TONNE } \\ -1.0 & 102.9 \\ .9 & 25.7 \\ 1.4 & 25.7 \\ 2.0 & 25.7 \\ 2.7 & 25.7 \\ 3.4 & 25.7 \\ 4.0 & 25.7 \\ 4.6 & 25.7 \\ 5.1 & 25.7 \\ 5.7 & 25.7 \\ 6.3 & 25.7 \\ 6.8 & 25.7 \\ 7.4 & 25.7 \\ 8.0 & 25.7 \\ 8.5 & 25.7 \\ 9.1 & 25.7 \\ 9.6 & 25.7 \\ 10.2 & 25.7 \\ 10.7 & 25.7 \\ 11.3 & 25.7 \\ 11.8 & 25.7 \\ 12.3 & 25.7 \\ 12.9 & 25.7 \\ 13.4 & 25.7 \\ 14.0 & 25.7 \\ 14.5 & 25.7 \\ 15.1 & 25.7 \\ 15.6 & 25.7 \\ 16.2 & 25.7 \\ 16.8 & 25.7 \\ 17.4 & 25.7 \\ 17.9 & 25.7 \\ 18.6 & 25.7 \\ 19.2 & 25.7 \\ 19.8 & 25.7 \\ 20.4 & 25.7 \\ 21.1 & 25.7 \\ 21.8 & 25.7 \\ 22.5 & 25.7 \\ 23.1 & 25.7\end{array}$

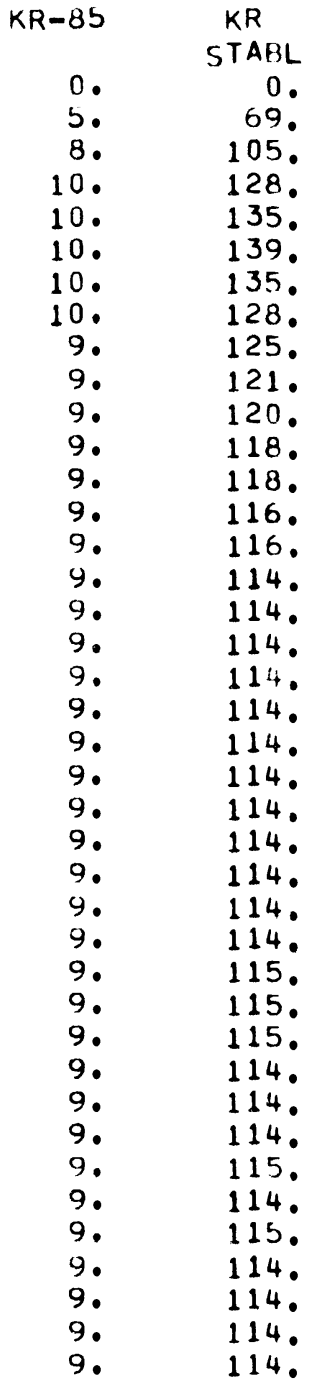

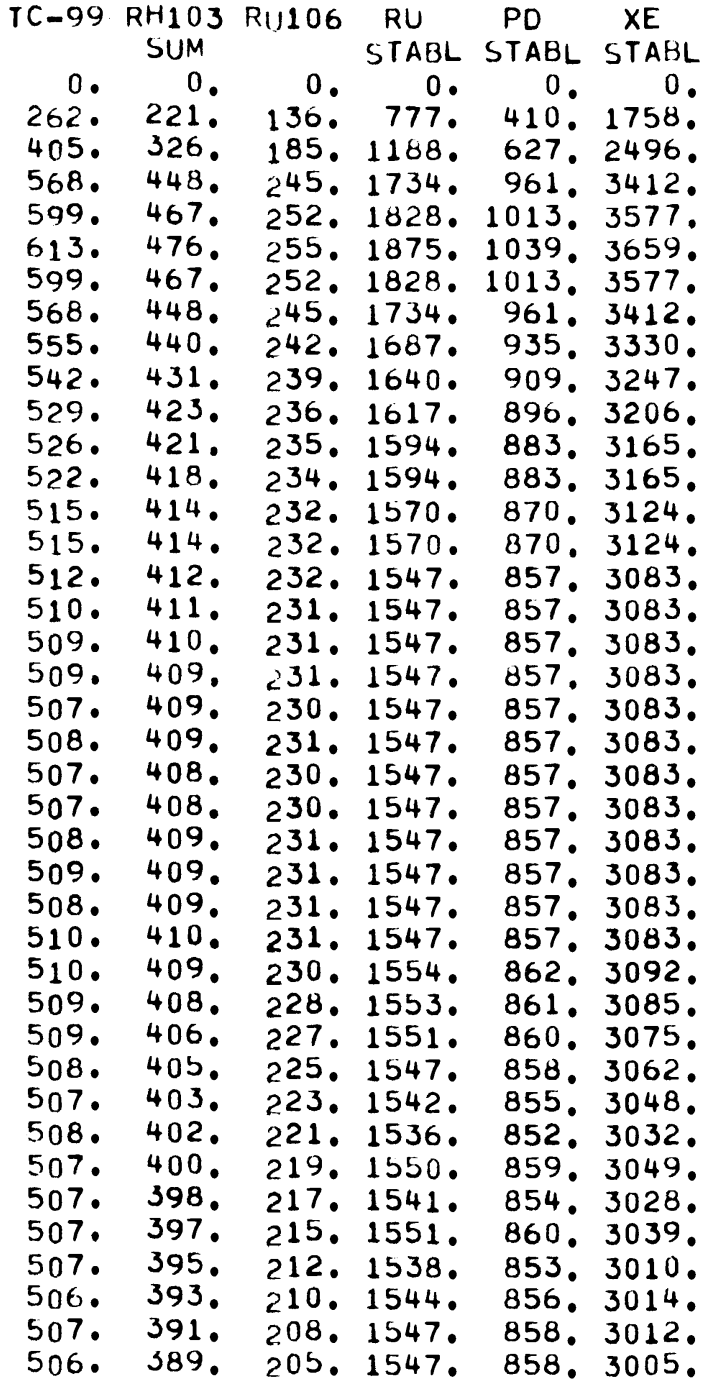


DISCHARGE

$\begin{array}{crr}\begin{array}{rr}\text { YCLK } \\ \text { NUMBCK }\end{array} & \text { UATE } & \text { TONNE } \\ 1 & -1.0 & 32.8 \\ 1 & .9 & 12.1 \\ 3 & .9 & 8.2 \\ 3 & 2.1 & 12.1 \\ 4 & 2.1 & 8.2 \\ 5 & 3.4 & 12.1 \\ 0 & 3.4 & 8.2 \\ 7 & 5.0 & 12.1 \\ 0 & 5.0 & 8.2 \\ 9 & 6.7 & 12.1 \\ 10 & 6.7 & 8.2 \\ 11 & 8.3 & 12.1 \\ 12 & 8.3 & 8.2 \\ 13 & 10.0 & 12.1 \\ 14 & 10.0 & 8.2 \\ 13 & & \end{array}$

ISOTOPE PRODUCTION. GRAMS/TONNE
EXPOS MWD/T

604. 27563.

2427.

60643.

5981.

89453.

12919.

102326.

17254

109880 。

17254 .

109880 .

17254 .

109880 .
$P U-R P$

PU-SP NP237 AM241 AM243 CM242 CM244 PM147 CE144 CS137 SR-90 U-235 U-236

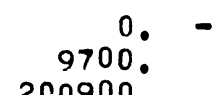

$199000 . \quad-0, \quad-0 . \quad-0$

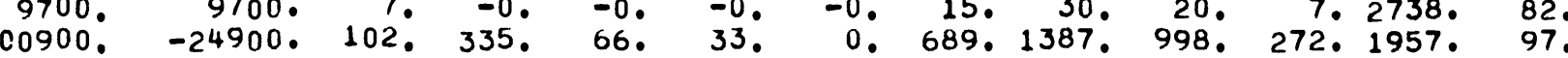
22600. 199700 .

36100 .

198900 . 22600.25 .200$.

36100 .

-26900 . $209.697^{\circ}$

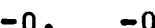

$-0 .-0$.

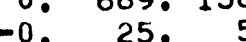

88. 30.2410 .174

1. 624. 1256.2224. 656.1550. 184.

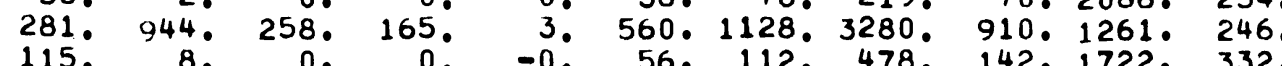

52000

115 .

8.

0.

-0 .

59300.

14. 1105. 307

84.

193200

146. 13. 0 .

1.

441.888 .3724 .1014 .1142 .271$.

59200

59300 .

1433. 1165. 337.194.

193200 .

$-32300$

1433. $1165^{\circ}$. $337^{\circ}, 194^{\circ}$.

93200.

-32300 .

5. 355.714.3980,1080,1078, 285

$-0.56 .212 .635 .63 .1560 .363$.

5. 355. 714.3980. 1080. 1078. 285.

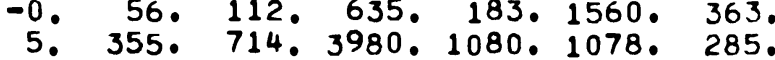


DISCHARGE

$\begin{array}{rrr}\text { UATE } & \text { TUNNE } & K R-85 \\ -1.0 & 32.8 & -0 . \\ .9 & 12.1 & 0 . \\ .9 & 8.2 & 17 . \\ 2.1 & 12.1 & 1 . \\ 2.1 & 8.2 & 42 . \\ 3.4 & 12.1 & 4 . \\ 3.4 & 8.2 & 56 . \\ 5.0 & 12.1 & 8 . \\ 5.0 & 8.2 & 63 . \\ 6.7 & 12.1 & 11 . \\ 6.7 & 8.2 & 68 . \\ 8.3 & 12.1 & 11 . \\ 8.3 & 8.2 & 68 . \\ 10.0 & 12.1 & 11 . \\ 10.0 & 8.2 & 68 .\end{array}$

ISOTOPE PRODUCTION. GRAMS/TONNE

$$
\begin{gathered}
K R \\
\text { STABL } \\
-0 . \\
10^{\circ} \\
276^{\circ} \\
33^{\circ} \\
676^{\circ} \\
73^{\circ} \\
936^{\circ} \\
147 . \\
1040^{\circ} \\
189^{\circ} \\
1104^{\circ} \\
189^{\circ} \\
1104^{\circ} \\
189^{\circ} \\
1104^{\circ}
\end{gathered}
$$

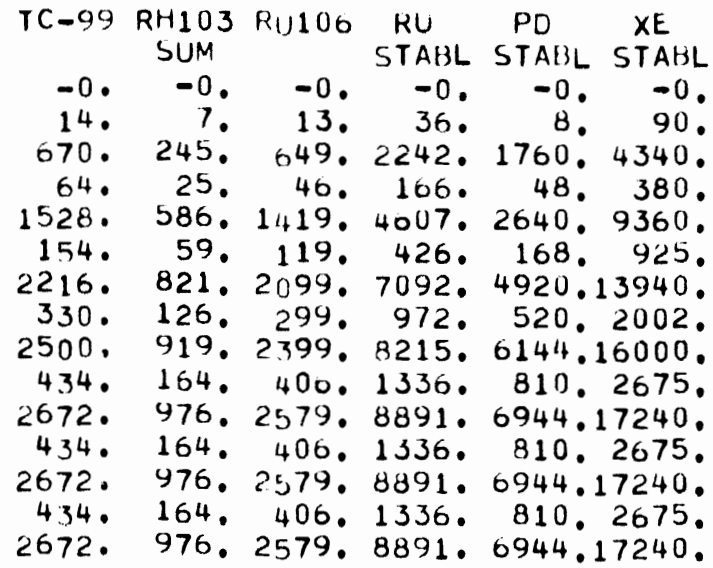


I SOTOPE PRODUCTION

LWR' S - NO RECYCLE 


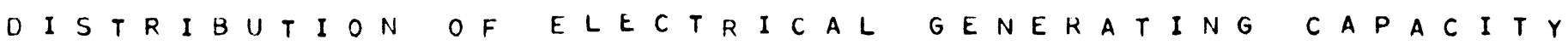

PAGE 36

LWR ' S - NO RECYCLE

CASE 1

TUTAL NEWLY

YEAR INSTALLED INSTALLED

ENDIIVG CAPACITY CAPACITY

GW(E) GW(E)

NEWLY INSTALLED CAPACITY BY REACTOR TYPE, GIGAWATTS ELECTRICAL

$\begin{array}{lrr}1960 . & .3 & .3 \\ 1961 . & .5 & .2 \\ 1962 . & .5 & .0 \\ 1963 . & .6 & .1 \\ 1964 . & .6 & .0 \\ 1965 . & .6 & .0 \\ 1966 . & 1.0 & .4 \\ 1967 . & 1.0 & .0 \\ 1968 . & 3.0 & 2.0 \\ 1969 . & 5.7 & 2.7 \\ 1970 . & 12.0 & 6.3 \\ 1971 . & 22.7 & 10.7 \\ 1972 . & 33.7 & 11.0 \\ 1973 . & 45.2 & 11.5 \\ 1974 . & 57.2 & 12.0 \\ 1975 . & 69.7 & 12.5 \\ 1976 . & 82.7 & 13.0 \\ 1977 . & 96.7 & 14.0 \\ 1978 . & 112.2 & 15.5 \\ 1979 . & 129.7 & 17.5 \\ 1980 . & 150.0 & 20.3 \\ 1981 . & 173.7 & 23.7 \\ 1982 . & 200.2 & 26.5 \\ 1983 . & 229.5 & 29.3 \\ 1984 . & 261.4 & 31.9 \\ 1985 . & 295.7 & 34.3 \\ 1986 . & 332.2 & 36.5 \\ 1987 . & 370.7 & 38.5 \\ 1988 . & 411.2 & 40.5 \\ 1989 . & 454.1 & 42.9 \\ 1990 . & 500.0 & 45.9 \\ 190.0 & & \end{array}$

$\begin{array}{rrrr}\text { PWR70 } & \text { BWR70 } & \text { FWR8O } & \text { BWR80 } \\ .1 & .2 & .0 & .0 \\ .2 & .0 & .0 & .0 \\ .0 & .0 & .0 & .0 \\ .0 & .1 & .0 & .0 \\ .0 & .0 & .0 & .0 \\ .0 & .0 & .0 & .0 \\ .3 & .1 & .0 & .0 \\ .0 & .0 & .0 & .0 \\ .9 & 1.1 & .0 & .0 \\ 1.4 & 1.3 & .0 & .0 \\ 3.1 & 3.2 & .0 & .0 \\ 6.3 & 4.4 & .0 & .0 \\ 7.3 & 3.7 & .0 & .0 \\ 7.7 & 3.8 & .0 & .0 \\ 8.0 & 4.0 & .0 & .0 \\ 8.3 & 4.2 & .0 & .0 \\ 8.7 & 4.3 & .0 & .0 \\ 9.3 & 4.7 & .0 & .0 \\ 10.3 & 5.2 & .0 & .0 \\ 11.7 & 5.8 & .0 & .0 \\ .0 & .0 & 13.5 & 6.8 \\ .0 & .0 & 15.8 & 7.9 \\ .0 & .0 & 17.7 & 8.8 \\ .0 & .0 & 19.5 & 9.8 \\ .0 & .0 & 21.3 & 10.6 \\ .0 & .0 & 22.9 & 11.4 \\ .0 & .0 & 24.3 & 12.2 \\ .0 & .0 & 25.7 & 12.8 \\ .0 & .0 & 27.0 & 13.5 \\ .0 & .0 & 28.6 & 14.3 \\ .0 & .0 & 30.6 & 15.3\end{array}$

$\begin{array}{ll}\text { PWR70 } & \text { BWR } \\ 30 U & 30 U \\ .0 & .0 \\ .0 & .0 \\ .0 & .0 \\ .0 & .0 \\ .0 & .0 \\ .0 & .0 \\ .0 & .0 \\ .0 & .0 \\ .0 & .0 \\ .0 & .0 \\ .0 & .0 \\ .0 & .0 \\ .0 & .0 \\ .0 & .0 \\ .0 & .0 \\ .0 & .0 \\ .0 & .0 \\ .0 & .0 \\ .0 & .0 \\ .0 & .0 \\ .0 & .0 \\ .0 & .0 \\ .0 & .0 \\ .0 & .0 \\ .0 & .0 \\ .0 & .0 \\ .0 & .0 \\ .0 & .0 \\ .0 & .0 \\ .0 & .0 \\ .0 & .0 \\ 0\end{array}$

\begin{tabular}{lc}
$P W R 80$ & BWR80 \\
$30 U$ & $30 U$ \\
.0 & .0 \\
.0 & .0 \\
.0 & .0 \\
.0 & .0 \\
.0 & .0 \\
.0 & .0 \\
.0 & .0 \\
.0 & .0 \\
.0 & .0 \\
.0 & .0 \\
.0 & .0 \\
.0 & .0 \\
.0 & .0 \\
.0 & .0 \\
.0 & .0 \\
.0 & .0 \\
.0 & .0 \\
.0 & .0 \\
.0 & .0 \\
.0 & .0 \\
.0 & .0 \\
.0 & .0 \\
.0 & .0 \\
.0 & .0 \\
.0 & .0 \\
.0 & .0 \\
.0 & .0 \\
.0 & .0 \\
.0 & .0 \\
.0 & .0 \\
.0 & .0 \\
\hline 0 &
\end{tabular}

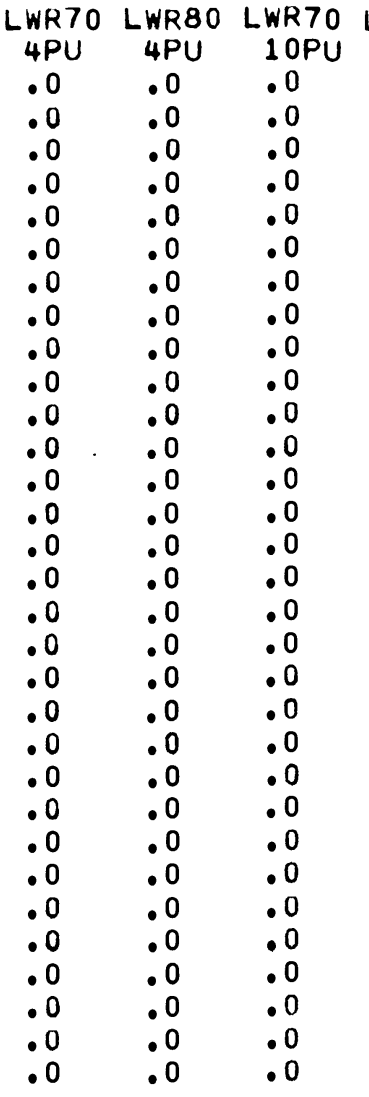

$\begin{array}{ccc}\text { LWR70 } & \text { LWR80 } & \text { LMFBR } \\ 30 P U & 30 P U & \\ .0 & .0 & .0 \\ .0 & .0 & .0 \\ .0 & .0 & .0 \\ .0 & .0 & .0 \\ .0 & .0 & .0 \\ .0 & .0 & .0 \\ .0 & .0 & .0 \\ .0 & .0 & .0 \\ .0 & .0 & .0 \\ .0 & .0 & .0 \\ .0 & .0 & .0 \\ .0 & .0 & .0 \\ .0 & .0 & .0 \\ .0 & .0 & .0 \\ .0 & .0 & .0 \\ .0 & .0 & .0 \\ .0 & .0 & .0 \\ .0 & .0 & .0 \\ .0 & .0 & .0 \\ .0 & .0 & .0 \\ .0 & .0 & .0 \\ .0 & .0 & .0 \\ .0 & .0 & .0 \\ .0 & .0 & .0 \\ .0 & .0 & .0 \\ .0 & .0 & .0 \\ .0 & .0 & .0 \\ .0 & .0 & .0 \\ .0 & .0 & .0 \\ .0 & .0 & .0 \\ .0 & .0 & .0\end{array}$


DISTRIBUTION OF ELECTRICAL GENERATINGACA PACITY

PAGE 37

$L W R$ ' S - NO RECYCLE

CASE 1

TUTAL NEWLY

YEAR INSTALLED INSTALLED

ENDIIIG CAFACITY CAPACITY GW(E) GW(E)

CUMULATIVE INSTALLED CAPACITY BY REACTOR TYPE, GIGAWATTS ELECTRICAL PWR70 BWR70 PWR80 BWR80 PWR70

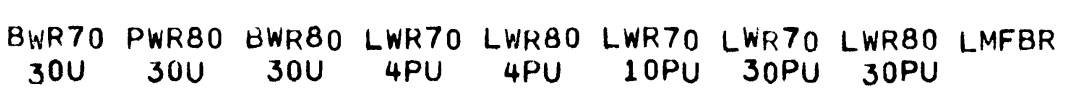

$\begin{array}{lrr}1960 . & .3 & .3 \\ 1961 . & .5 & .2 \\ 1962 . & .5 & .0 \\ 1963 . & .6 & .1 \\ 1964 . & .6 & .0 \\ 1965 . & .6 & .0 \\ 1966 . & 1.0 & .4 \\ 1967 . & 1.0 & .0 \\ 1968 . & 3.0 & 2.0 \\ 1969 . & 3.7 & 2.7 \\ 1970 . & 12.0 & 6.3 \\ 1971 . & 22.7 & 10.7 \\ 1972 . & 33.7 & 11.0 \\ 1973 . & 45.2 & 11.5 \\ 1974 . & 57.2 & 12.0 \\ 1975 . & 69.7 & 12.5 \\ 1976 . & 82.7 & 13.0 \\ 1977 . & 96.7 & 14.0 \\ 1978 . & 112.2 & 15.5 \\ 1979 . & 129.7 & 17.5 \\ 1980 . & 150.0 & 20.3 \\ 1981 . & 173.7 & 23.7 \\ 1982 . & 200.2 & 26.5 \\ 1983 . & 229.5 & 29.3 \\ 1984 . & 261.4 & 31.9 \\ 1985 . & 295.7 & 34.3 \\ 1986 . & 332.2 & 36.5 \\ 1987 . & 370.7 & 38.5 \\ 1988 . & 411.2 & 40.5 \\ 1989 . & 454.1 & 42.9 \\ 1990 . & 300.0 & 45.9\end{array}$

$\begin{array}{rrrr}.1 & .2 & .0 & .0 \\ .2 & .2 & .0 & .0 \\ .2 & .2 & .0 & .0 \\ .2 & .4 & .0 & \cdot 0 \\ .3 & .4 & .0 & .0 \\ .3 & .4 & .0 & .0 \\ .6 & .4 & .0 & .0 \\ .6 & .4 & .0 & .0 \\ 1.5 & 1.5 & .0 & .0 \\ 2.9 & 2.8 & .0 & .0 \\ 6.0 & 6.0 & .0 & .0 \\ 12.3 & 10.4 & .0 & .0 \\ 19.6 & 14.1 & .0 & .0 \\ 27.3 & 17.9 & .0 & .0 \\ 35.3 & 21.9 & .0 & .0 \\ 43.6 & 26.1 & .0 & .0 \\ 52.3 & 30.4 & .0 & .0 \\ 61.6 & 35.1 & .0 & .0 \\ 71.9 & 40.2 & .0 & .0 \\ 83.6 & 46.1 & .0 & .0 \\ 83.6 & 46.1 & 13.5 & 6.8 \\ 83.6 & 46.1 & 29.3 & 14.7 \\ 83.6 & 46.1 & 47.0 & 23.5 \\ 83.6 & 46.1 & 66.5 & 33.3 \\ 83.6 & 46.1 & 87.8 & 43.9 \\ 83.6 & 46.1 & 110.7 & 55.3 \\ 83.6 & 46.1 & 135.0 & 67.5 \\ 83.6 & 46.1 & 160.7 & 80.3 \\ 83.6 & 46.1 & 187.7 & 93.8 \\ 83.6 & 46.1 & 216.3 & 108.1 \\ 83.6 & 46.1 & 246.9 & 123.4\end{array}$

$\begin{array}{ll}.0 & .0 \\ .0 & .0 \\ .0 & .0 \\ .0 & .0 \\ .0 & .0 \\ .0 & .0 \\ .0 & .0 \\ .0 & .0 \\ .0 & .0 \\ .0 & .0 \\ .0 & .0 \\ .0 & .0 \\ .0 & .0 \\ .0 & .0 \\ .0 & .0 \\ .0 & .0 \\ .0 & .0 \\ .0 & .0 \\ .0 & .0 \\ .0 & .0 \\ .0 & .0 \\ .0 & .0 \\ .0 & .0 \\ .0 & .0 \\ .0 & .0 \\ .0 & .0 \\ .0 & .0 \\ .0 & .0 \\ .0 & .0 \\ .0 & .0 \\ .0 & .0\end{array}$

.0
.0
.0
.0
.0
.0
.0
.0
.0
.0
.0
.0
.0
.0
.0
.0
.0
.0
.0
.0
.0
.0
.0
.0
.0
.0
.0
.0
.0
.0
.0

$\begin{array}{ll}.0 & .0 \\ .0 & .0 \\ .0 & .0 \\ .0 & .0 \\ .0 & .0 \\ .0 & .0 \\ .0 & .0 \\ .0 & .0 \\ .0 & .0 \\ .0 & .0 \\ .0 & .0 \\ .0 & .0 \\ .0 & .0 \\ .0 & .0 \\ .0 & .0 \\ .0 & .0 \\ .0 & .0 \\ .0 & .0 \\ .0 & .0 \\ .0 & .0 \\ .0 & .0 \\ .0 & .0 \\ .0 & .0 \\ .0 & .0 \\ .0 & .0 \\ .0 & .0 \\ .0 & .0 \\ .0 & .0 \\ .0 & .0 \\ .0 & .0 \\ .0 & .0 \\ & 0\end{array}$

.0
.0
.0
.0
.0
.0
.0
.0
.0
.0
.0
.0
.0
.0
.0
.0
.0
.0
.0
.0
.0
.0
.0
.0
.0
.0
.0
.0
.0
.0
.0

.0
.0
.0
.0
.0
.0
.0
.0
.0
.0
.0
.0
.0
.0
.0
.0
.0
.0
.0
.0
.0
.0
.0
.0
.0
.0
.0
.0
.0
.0
.0

$\begin{array}{lll}.0 & .0 & .0 \\ .0 & .0 & .0 \\ .0 & .0 & .0 \\ .0 & .0 & .0 \\ .0 & .0 & .0 \\ .0 & .0 & .0 \\ .0 & .0 & .0 \\ .0 & .0 & .0 \\ .0 & .0 & .0 \\ .0 & .0 & .0 \\ .0 & .0 & .0 \\ .0 & .0 & .0 \\ .0 & .0 & .0 \\ .0 & .0 & .0 \\ .0 & .0 & .0 \\ .0 & .0 & .0 \\ .0 & .0 & .0 \\ .0 & .0 & .0 \\ .0 & .0 & .0 \\ .0 & .0 & .0 \\ .0 & .0 & .0 \\ .0 & .0 & .0 \\ .0 & .0 & .0 \\ .0 & .0 & .0 \\ .0 & .0 & .0 \\ .0 & .0 & .0 \\ .0 & .0 & .0 \\ .0 & .0 & .0 \\ .0 & .0 & .0 \\ .0 & .0 & .0 \\ .0 & .0 & .0\end{array}$



ANNUAL ISOTOPE PRODUCTION AT D I S CHARGE (NO LOSS BASIS)

LWR $S$-- NO RECYCLE

CASE 1

TUTAL

DURIIG INSTALLEU

PERIUU CARACITY

ISOTOPE PRODUCTION, KILOGRAMS/YEAR

\begin{tabular}{|c|c|c|c|c|c|c|c|c|}
\hline 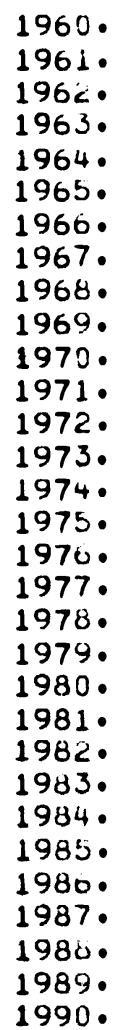 & $\begin{array}{r}.3 \\
.5 \\
.5 \\
.6 \\
.6 \\
.0 \\
1.0 \\
1.0 \\
3.0 \\
5.7 \\
12.0 \\
22.7 \\
33.7 \\
45.2 \\
37.2 \\
09.7 \\
82.7 \\
96.7 \\
112.2 \\
129.7 \\
100.0 \\
173.7 \\
200.2 \\
229.5 \\
201.4 \\
245.7 \\
332.2 \\
370.7 \\
411.2 \\
404.1 \\
500.0\end{array}$ & 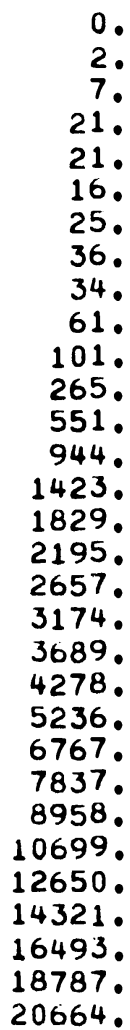 & 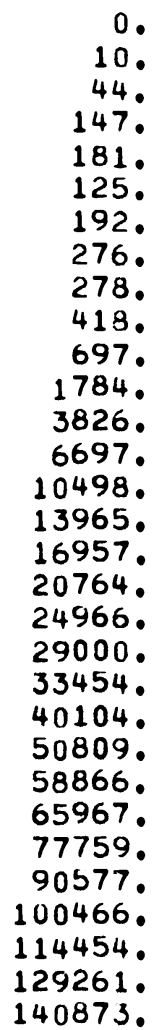 & 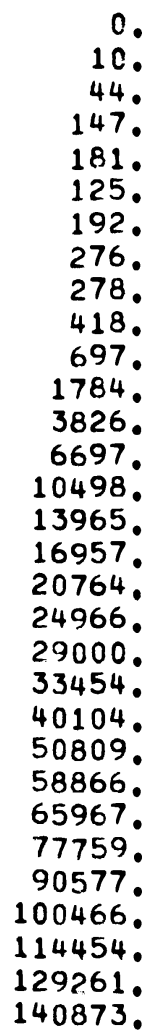 & 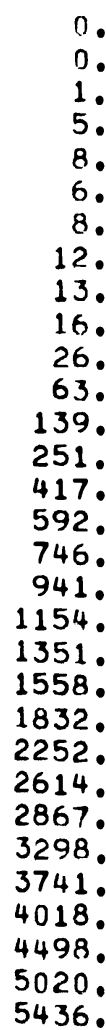 & 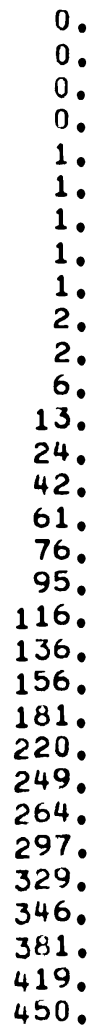 & 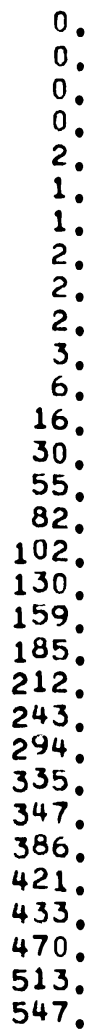 & $\begin{array}{l}0 . \\
0 . \\
0 . \\
0 . \\
0 . \\
0 . \\
0 . \\
0 \\
0 . \\
0 . \\
1 . \\
1 . \\
3 . \\
6 . \\
11 . \\
16 . \\
20 . \\
25 . \\
31 . \\
36 . \\
42 . \\
48 . \\
58 . \\
67 . \\
70 \\
79 . \\
87 . \\
91 . \\
100 . \\
109 . \\
117 .\end{array}$ \\
\hline
\end{tabular}

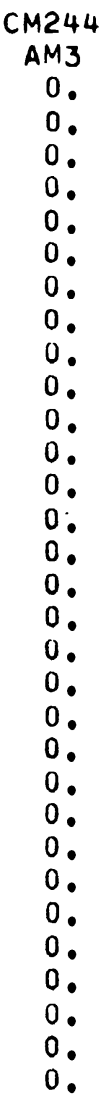

CM244

$\begin{array}{ccccc}\text { CM2 } 44 & \text { PU238 } & \text { PU238 } & \text { PU238 } & \text { PU238 } \\ \text { SUM } & \text { NP } & \text { CM } & \text { AM } & \text { SUM }\end{array}$

$\begin{array}{ccccc}0 . & 0 . & 0 . & A M & S \\ 0.0 . & 0.0 & 0.0\end{array}$

$\begin{array}{lllll}0 . & 0 . & 0 . & 0 . & 0 \\ 0 . & 0 . & 0 . & 0 . & 0 .\end{array}$

0.00 .000 .000

0.0 .0 .0 .000$.

0.0 .0 .000 .000

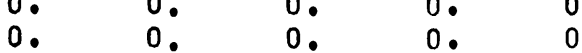

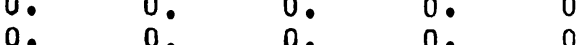

0.0 .0 .00000

1. $\quad 0.00 .000$

1.

6.

13.

19.

24.

31 .

37.

43.

56.

66.

66.

75.

80.

84.

83.

87.

93.

98.

0
0
0
0

0.

0 .

0.

0. 
ANNUAL ISOTOPE PRODUCTION AT DISCHARGE

PAGE 39

(NO LOSS BASIS)

$L W R \cdot S$ - NO RECYCLE

CASE 1

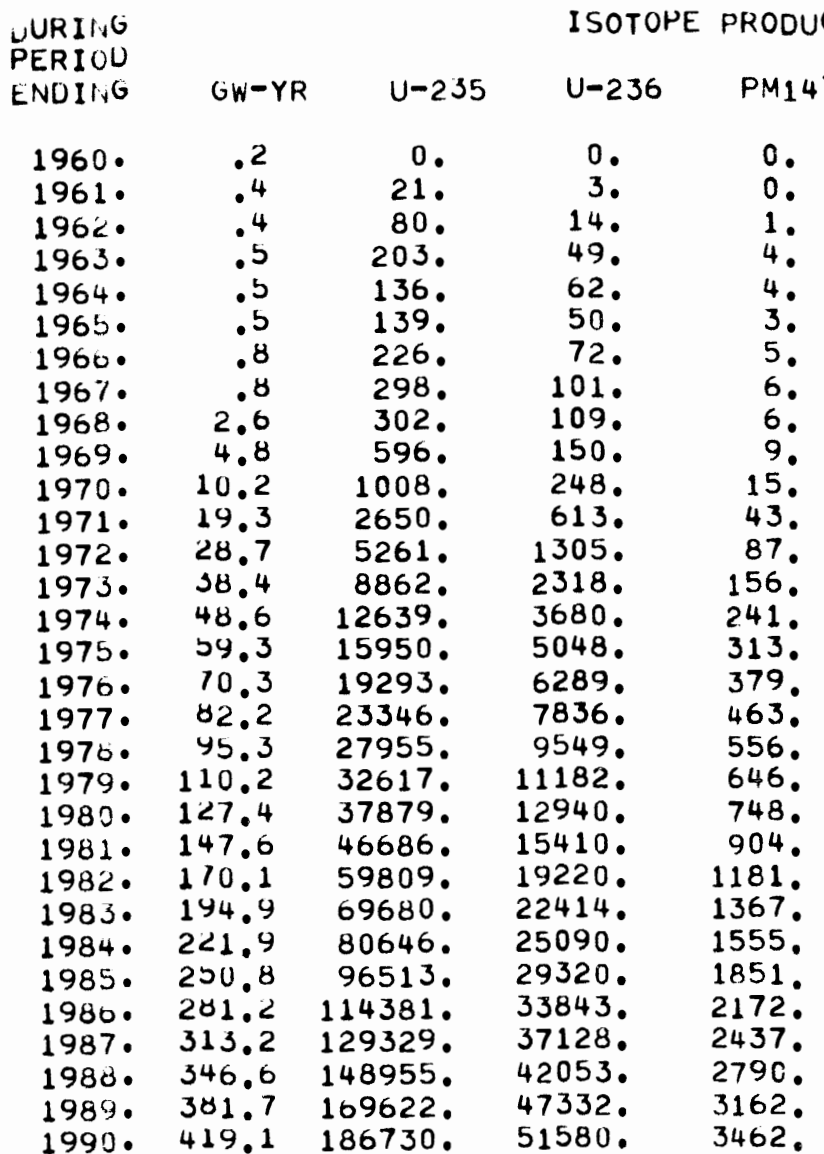

\begin{tabular}{|c|c|c|}
\hline CE 144 & $\operatorname{CS} 137$ & $S R-90$ \\
\hline $\begin{array}{r}0 . \\
0 . \\
2 . \\
7 . \\
7 \\
5 . \\
9 . \\
11 . \\
12 . \\
19 . \\
31 . \\
83 . \\
171 . \\
301 . \\
462 . \\
602 . \\
729 \\
891 . \\
1071 . \\
1247 . \\
1444 . \\
1758 . \\
2293 . \\
2675 . \\
3055 . \\
3649 . \\
4295 . \\
4831 . \\
5540 . \\
6290 \\
6892 \\
\end{array}$ & 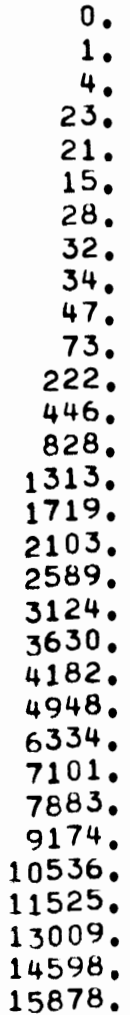 & 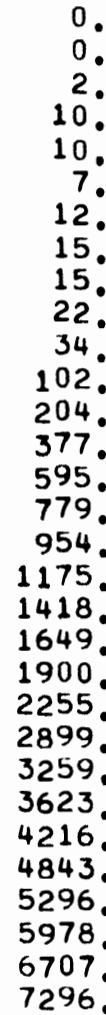 \\
\hline
\end{tabular}

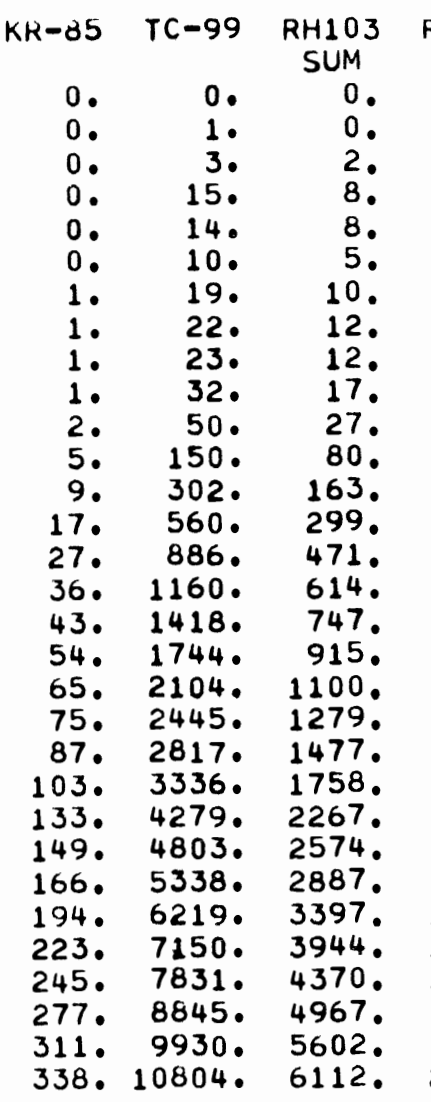

RU106

PD

XE

CM242 AM241

STABL STABL AM

PU1

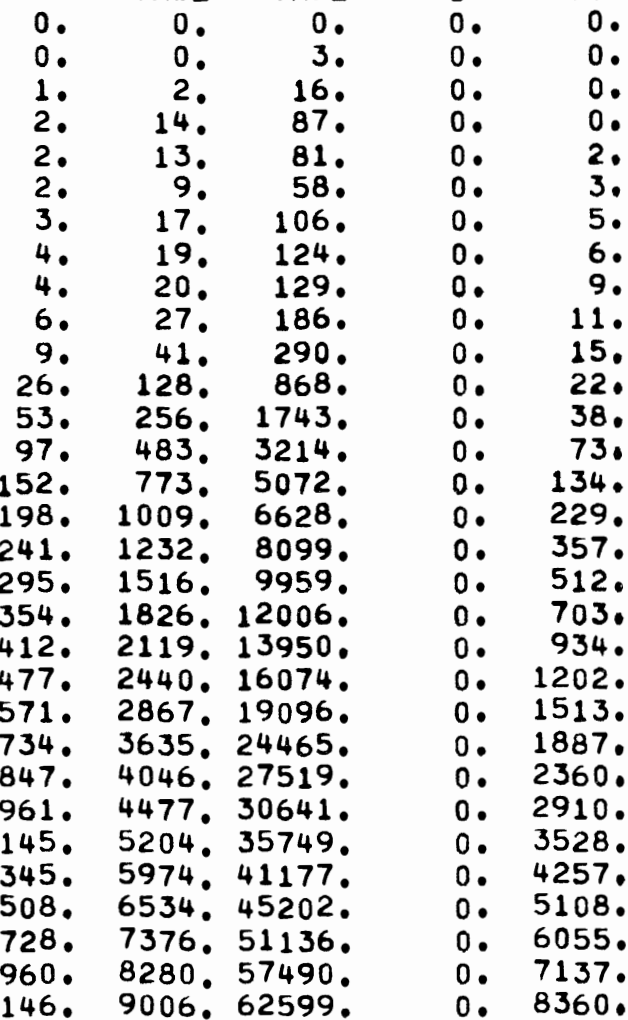


(NO LOSS BASIS)

$L W R$, S - NO RECYCLE

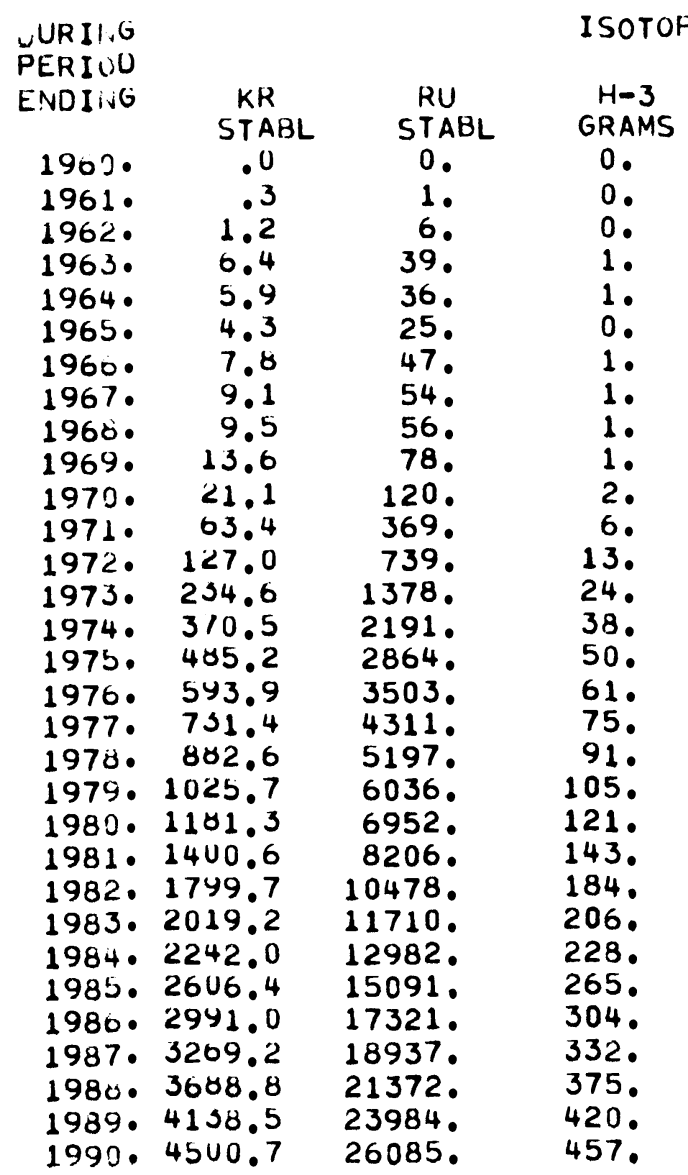


(NO LOSSES EXCEPT FOR RADIOACTIVE LECAY)

TUTAL

INS I ALLED

YEAR CAPACITY

YEAR CAPACITY

ISOTOPE PRODUCTION, KGS

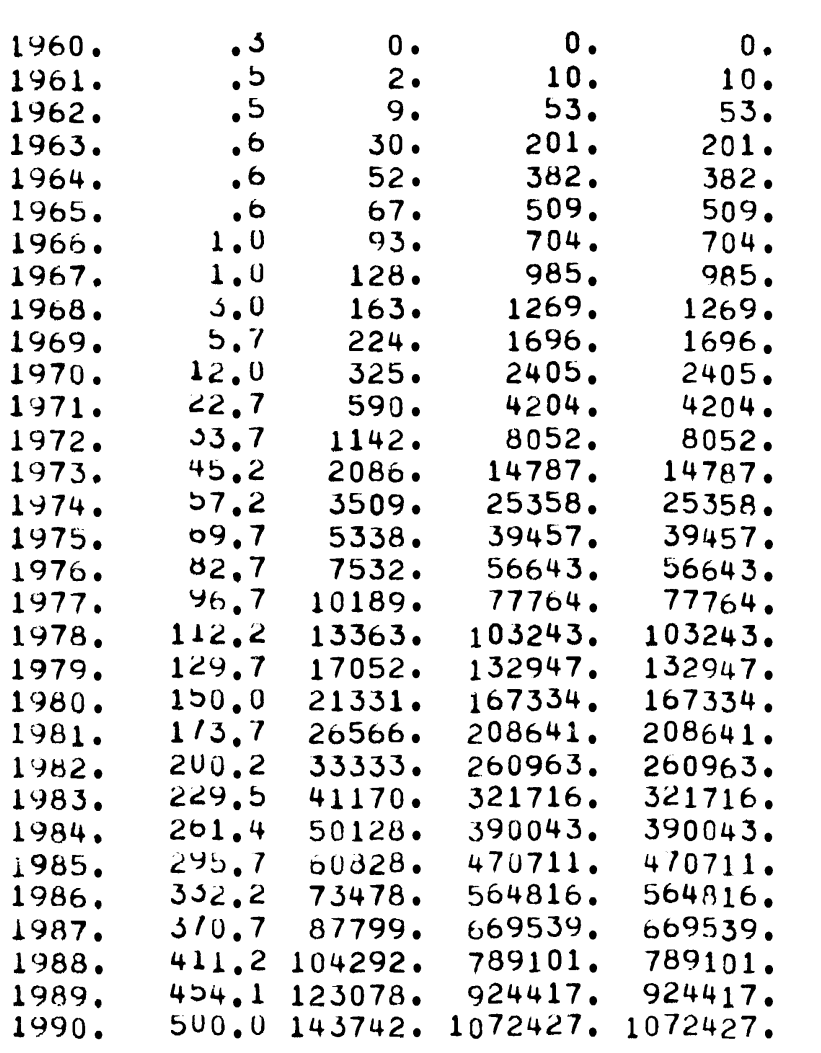

\begin{tabular}{|c|c|c|c|c|c|}
\hline NP2 37 & AM241 & AM243 & CM242 & CM244 & $\begin{array}{c}\text { CM24L } \\
\text { AM3 }\end{array}$ \\
\hline 0. & 0 . & 0 . & 0. & 0. & 0 \\
\hline 0 . & 0 . & 0. & 0. & 0 . & -0 \\
\hline 1. & 0 . & 0 . & 0. & 0. & . \\
\hline 6. & 1. & 1. & 0. & 0 . & -0 \\
\hline 14. & 2. & 2. & 0. & 0 . & - \\
\hline 20. & 2. & 3. & 0. & 1 . & \\
\hline 29. & 3. & 4. & 0. & 1. & \\
\hline 41. & 4. & 6. & 0. & 1. & -0 \\
\hline 54. & 6. & 8. & 0. & 2. & $-c$ \\
\hline 70. & 7. & 10. & 0 . & 2. & \\
\hline 97. & 10 & 13. & 1. & 3. & -0 \\
\hline 159. & 15. & 19. & 2. & 4. & \\
\hline 298 . & 28. & 35. & 4. & 7. & \\
\hline 549. & 52 . & 65 . & 7. & 13. & -0 \\
\hline 967. & 95. & 120. & 12. & 26. & $-c$ \\
\hline $1559^{\circ}$ & 156. & 202 . & 19. & 44. & \\
\hline 2305 . & 231. & 304. & 24. & 66. & -8 \\
\hline 3246 . & 326. & 434. & 30. & 94. & $\begin{array}{l}-0 \\
-0\end{array}$ \\
\hline 4400 . & 442. & 593. & 38. & 128. & $\begin{array}{l}-0 \\
-0\end{array}$ \\
\hline 5751. & 577 . & 778. & 44. & 167. & -0 \\
\hline 7309 . & 733. & 990. & 51. & 209. & \\
\hline 9142 . & 912. & $1233^{\circ}$ & 59. & 257 . & $\begin{array}{l}-0 \\
-0\end{array}$ \\
\hline 11394 . & 1131. & 1527. & 71. & 314. & \\
\hline 14008. & 1378 . & 1862 . & 82. & 377. & \\
\hline 16875. & $1640^{\circ}$ & 2209 . & 88. & 437. & \\
\hline 20173 & $1934^{\circ}$ & 2595. & 97. & 500 . & -0 \\
\hline $\begin{array}{l}23915 . \\
27933\end{array}$ & $\begin{array}{l}2260 . \\
2602 .\end{array}$ & $\begin{array}{l}3016 . \\
3449^{\circ}\end{array}$ & 108. & 565. & - \\
\hline $\begin{array}{l}279 \\
324\end{array}$ & 2979 & $3919^{\circ}$. & $\begin{array}{l}114^{\circ} \\
124^{\circ}\end{array}$ & $\begin{array}{l}621 . \\
691 .\end{array}$ & $\begin{array}{l}-0 \\
-0\end{array}$ \\
\hline 37 & 3394 . & 4432 . & 136. & 758. & -0 \\
\hline & $3839^{\circ}$ & 4978 . & 146. & 827. & \\
\hline
\end{tabular}

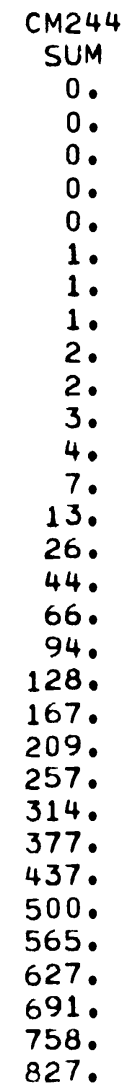

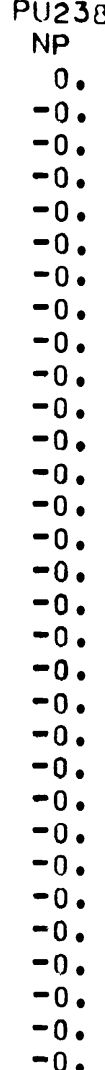

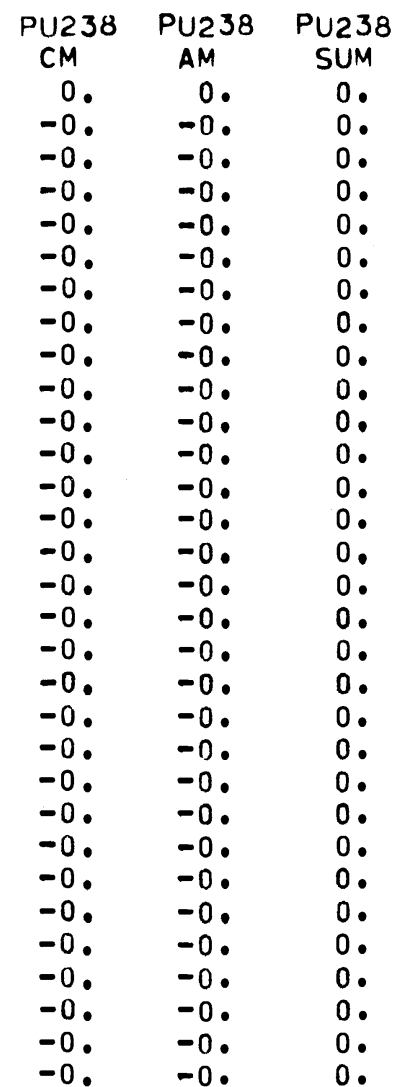


CUMULATIVE ISOTOPE PROOUCTI ON A T D I S C HAR GE (NO LOSSES EXCEPT FOR RADIOACTIVE DECAY)

$L W R \cdot S$ - NO RECYCLE

CASE 1

ISOTOPE PRODUCTION, KGS

\begin{tabular}{|c|c|c|c|c|}
\hline $\begin{array}{l}\text { YEAR } \\
\text { ENDING }\end{array}$ & $G W-Y R$ & $U-235$ & $U-236$ & PM147 \\
\hline 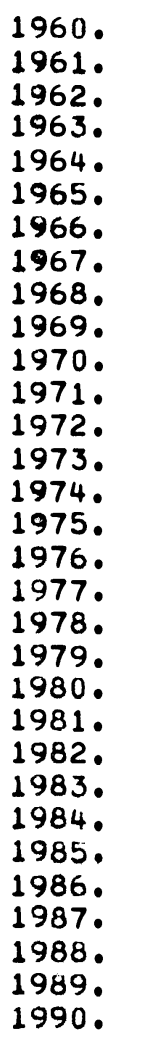 & $\begin{array}{r}.2 \\
.6 \\
1.0 \\
1.5 \\
2.1 \\
2.6 \\
3.4 \\
4.3 \\
6.8 \\
11.7 \\
21.9 \\
41.2 \\
69.9 \\
108.3 \\
136.9 \\
216.2 \\
286.5 \\
368.7 \\
464.0 \\
574.2 \\
701.6 \\
849.2 \\
1019.3 \\
1214.2 \\
1436.0 \\
1606.8 \\
1968.1 \\
2281.2 \\
2627.8 \\
3009.51 \\
3428.61\end{array}$ & 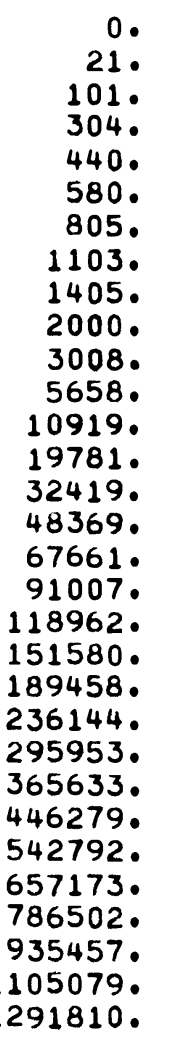 & 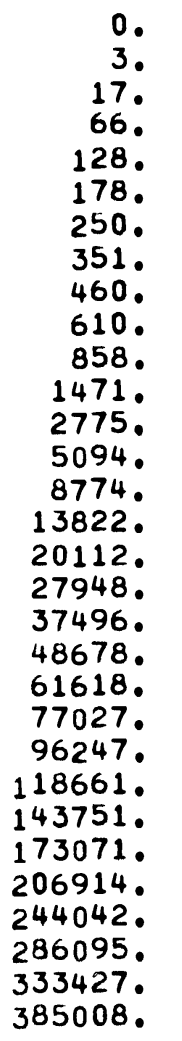 & 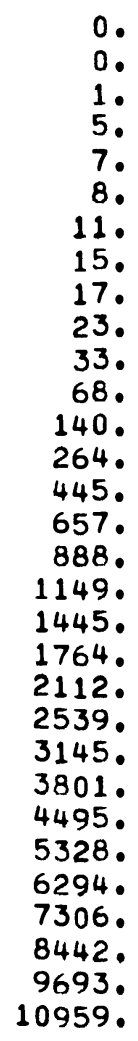 \\
\hline
\end{tabular}

CE 144

CS137 SR 90

KR-85 TC-99 RH103 RU106

$\begin{array}{ccc}\text { RU106 } & \text { PD } & \text { XE } \\ & \text { STABL } & \text { STABL } \\ 0 . & 0 . & 0 .\end{array}$

CM242 AM241

0.10

$\begin{array}{rr}0 . & 10^{\circ} \\ 2 . & 5 \circ \\ 8 . & 27 . \\ 10 . & 48 . \\ 9 . & 62 . \\ 12 . & 89 .\end{array}$

12.89.

19. 150.

26. 193.

100.479 .119$.

$\begin{array}{lll}100 . & 4790^{\circ} & 218 . \\ 213^{\circ} & 914 . & 417 .\end{array}$

389.1721 .784 .

622. 2995. 1359.

857. 4645. 2105 .

1082. 6642. 3008.

$\begin{array}{lll}1336^{\circ} & 9080^{\circ} \quad 4109^{\circ} \\ 1620^{\circ} & 11996 . & 5427 .\end{array}$

$\begin{array}{lll}1620 & 11996 . & 5427^{\circ} \\ 1914^{\circ} & 15352 . & 6944^{\circ}\end{array}$

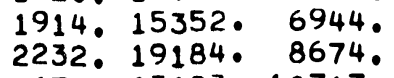

2676. 23693. 10717.

3393. 29486. 13354 .

4071 . 35914. 16287.

4730.42976 .19511$.

5594.51169 .23250 .

7544.70678 . 32148.

8643 . 82073. 37340 .

9845 . 94796. 43135 .

SUM

10941.108509 . 49375 .

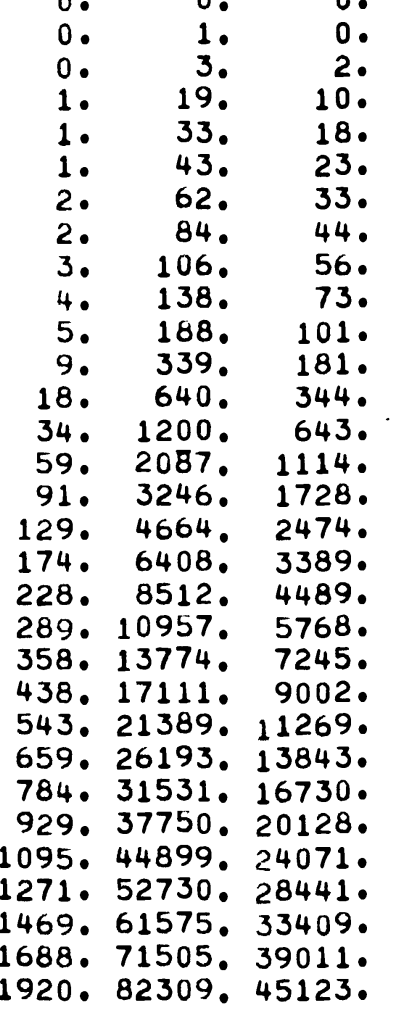

3.

4.29

4.38

6. 74.

9. 120.

14. 161 .

$33 . \quad 289$.

70.545.
$132 . \quad 1028$.

217. 1800. 11979.

307. 2809. 18607.

394. 4041. 26706.

492. 5557. 36665 .

600.7383 .48670 .

712 . 9502. 62620 .

833. 11942. 78694 .

987.14810 .97790 .

1227.18445 .122255 .

1460 . 22491. 149774 .

1692. 26968. 180415.

1991. 32172. 216164.

2340. 38146. 257341.

2678. 44680. 302543.

3067 . 52056. 353680 。

3494 . 60336. 411169 .

3893 . 69342. 473769 .

$\begin{array}{rr}0 . & 0 . \\ -0 . & -0 . \\ -0 . & 0 . \\ -0 . & 1 . \\ -0 . & 2 . \\ -0 . & 6 . \\ -0 . & 10 . \\ -0 . & 17 . \\ -0 . & 26 . \\ -0 . & 37 . \\ -0 . & 52 . \\ -0 . & 74 . \\ -0 . & 112 . \\ -0 . & 185 . \\ -0 . & 318 . \\ -0 . & 547 . \\ -0 . & 903 . \\ -0 . & 1414 . \\ -0 . & 2115 . \\ -0 . & 3046 . \\ -0 . & 4243 . \\ -0 . & 5750 . \\ -0 . & 7629 . \\ -0 . & 9977 . \\ -0 . & 12872 . \\ -0 . & 16380 . \\ -0 . & 20612 . \\ -0 . & 25689 . \\ -0 . & 31706 . \\ -0 . & 38794 . \\ -0 . & 47096 .\end{array}$



CUMULATIVE ISOTOPE PRODUCTION AT T D I SCHARGE
(NO LOSSES EXCEPT FOR RADIOACTIVE UECAY)

$$
L W R \text { ' } S \text {-- NO RECYCLE }
$$

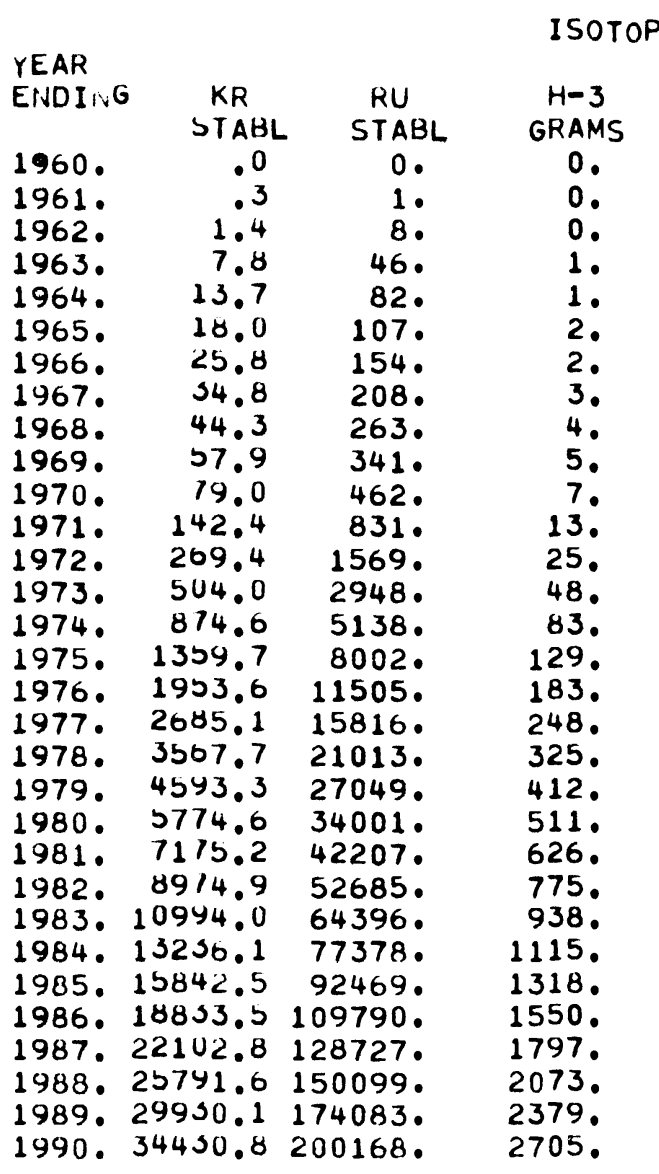


NET ANNUAL I S O T O P E AVA I LAB I L I T Y

(WITH TARGET IRRADIATION, PROCESS LOSSES AND DECAY LOSSES)

$$
\text { LWR ' S -- NO RECYCLE }
$$

CASE 1

TUTAL

DURING INSTALLED
PERIOU CATACITY

\begin{tabular}{|c|c|c|c|c|}
\hline $\begin{array}{l}1960^{\circ} \\
1961^{\circ} \\
1962^{\circ} \\
1963^{\circ} \\
1964^{\circ} \\
1965^{\circ} \\
1960^{\circ} \\
1967^{\circ} \\
1968^{\circ} \\
1969^{\circ} \\
1970^{\circ} \\
1971^{\circ} \\
1970^{\circ} \\
1973^{\circ} \\
1974^{\circ} \\
1975^{\circ} \\
1970^{\circ} \\
1977^{\circ} \\
1978^{\circ} \\
1979^{\circ} \\
1980^{\circ} \\
1981^{\circ} \\
1982^{\circ} \\
19830^{\circ} \\
1984^{\circ} \\
1985^{\circ} \\
1980^{\circ} \\
1980^{\circ} \\
1980^{\circ} \\
19890^{\circ} \\
1990^{\circ}\end{array}$ & $\begin{array}{r}.3 \\
.5 \\
.5 \\
.6 \\
.6 \\
.6 \\
1.0 \\
1.0 \\
3.0 \\
5.7 \\
12.0 \\
22.7 \\
33.7 \\
45.2 \\
57.2 \\
09.7 \\
82.7 \\
96.7 \\
112.2 \\
129.7 \\
100.0 \\
113.7 \\
200.2 \\
229.5 \\
201.4 \\
295.7 \\
352.2 \\
310.7 \\
411.2 \\
404.1 \\
500.0\end{array}$ & 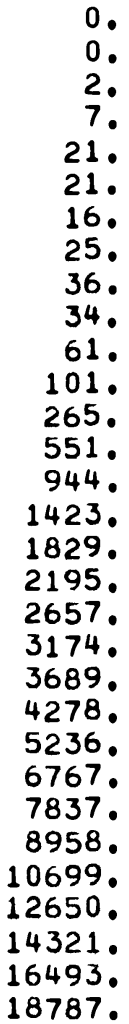 & $\begin{array}{r}0 \\
0 \\
10 \\
43 \\
144^{\circ} \\
177^{\circ} \\
123^{\circ} \\
189 \\
271 \\
273 \\
410 \\
685 \\
1751 \\
3753 \\
6571 \\
10301 \\
13709 \\
16653 \\
20400 \\
24537 \\
28512 \\
32904 \\
39452 \\
49979 \\
57920^{\circ} \\
64934 \\
76552 \\
89186 \\
98960 \\
112763 \\
127380\end{array}$ & $\begin{array}{r}0 . \\
0 . \\
10^{\circ} \\
43^{\circ} \\
144 . \\
177 . \\
123 . \\
189 . \\
271 . \\
273 . \\
410^{\circ} \\
685 . \\
1751 . \\
3753 . \\
6571 . \\
10301 . \\
13709 . \\
16653 . \\
20400 . \\
24537 . \\
28512 . \\
32904^{\circ} \\
39452 . \\
49979 . \\
57922 . \\
64934 . \\
76552 . \\
89186 . \\
98960 . \\
112763 . \\
127380 .\end{array}$ \\
\hline
\end{tabular}

ISOTOPE PRODUCTION, KILOGRAMS/YEAR

PU-RP PU-SP NP237 AM241 AM243 CM242

$\mathrm{CM} 244$

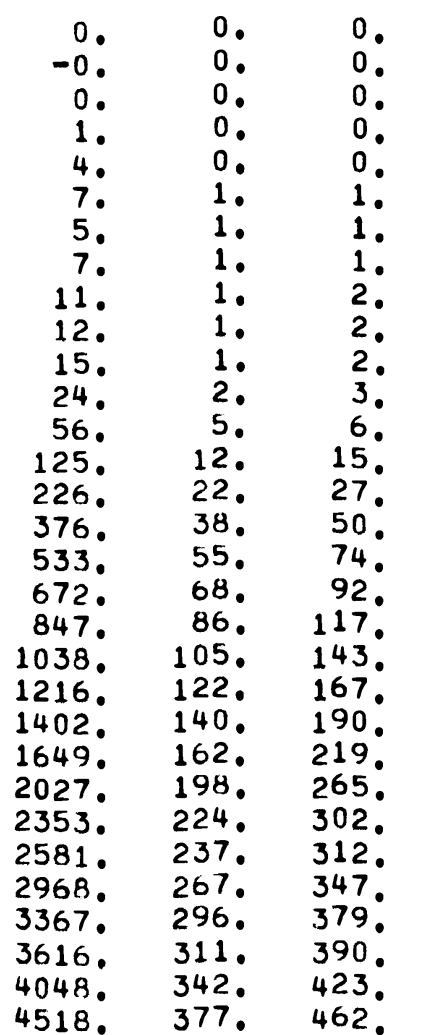

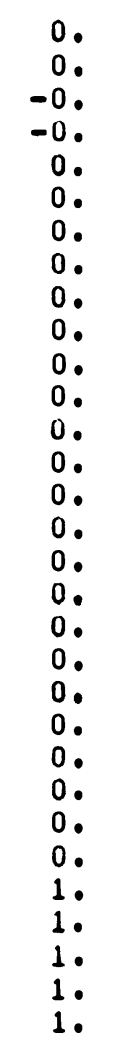
0
-0
-0

-0
-0

0.

0

0.

0.

0.

0.

0.

0.

1.

3.

10.

15.

19.

24.

35.

35.

39.

53.

60.

59.

64.

$670^{\circ}$

AM
0
0
0
0
0
0
0
0
0
0
0
0
0
0
1
1
3
5
9
14
19
27
35
43
53
64
78
92
103
120
136

CM244 $\mathrm{CM} 244 \mathrm{CM} 244$
$\mathrm{AM} 3$

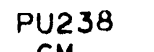

$\begin{array}{lll}\text { CM } & \text { PU238 } & \text { PU23 } \\ \text { AM } & \text { SUM }\end{array}$

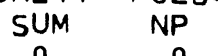

$\begin{array}{ll}0 . & 0 . \\ 0 . & 0 .\end{array}$

0.

0.

0 .

0 .

0.

0.

1.

1.

2.

4
15

15.

25.

44

57.

70.

82.

82.
97.

117.

583

151.784.

$167^{\circ} 784^{\circ}$

$\begin{array}{rr}167 . & 876 . \\ 187 . & 1077^{\circ}\end{array}$

$\begin{array}{ll}187 . & 1077 . \\ 203 . & 1192 .\end{array}$

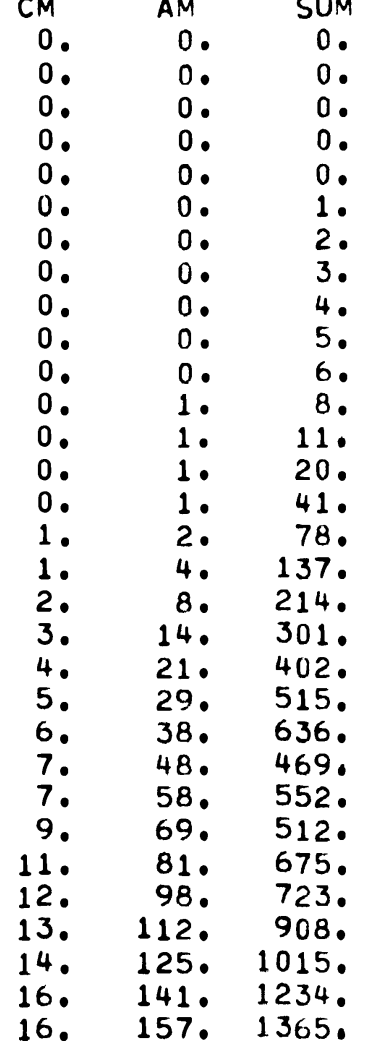


NET ANNUAL I SOOTOPF AVAILABILI T Y

PAGE 45

(WITH TARGET IRRADIATION, PROCESS LOSSES ANU DECAY LOSSES)

$$
L W R \cdot S \text { - NO RECYCLE }
$$

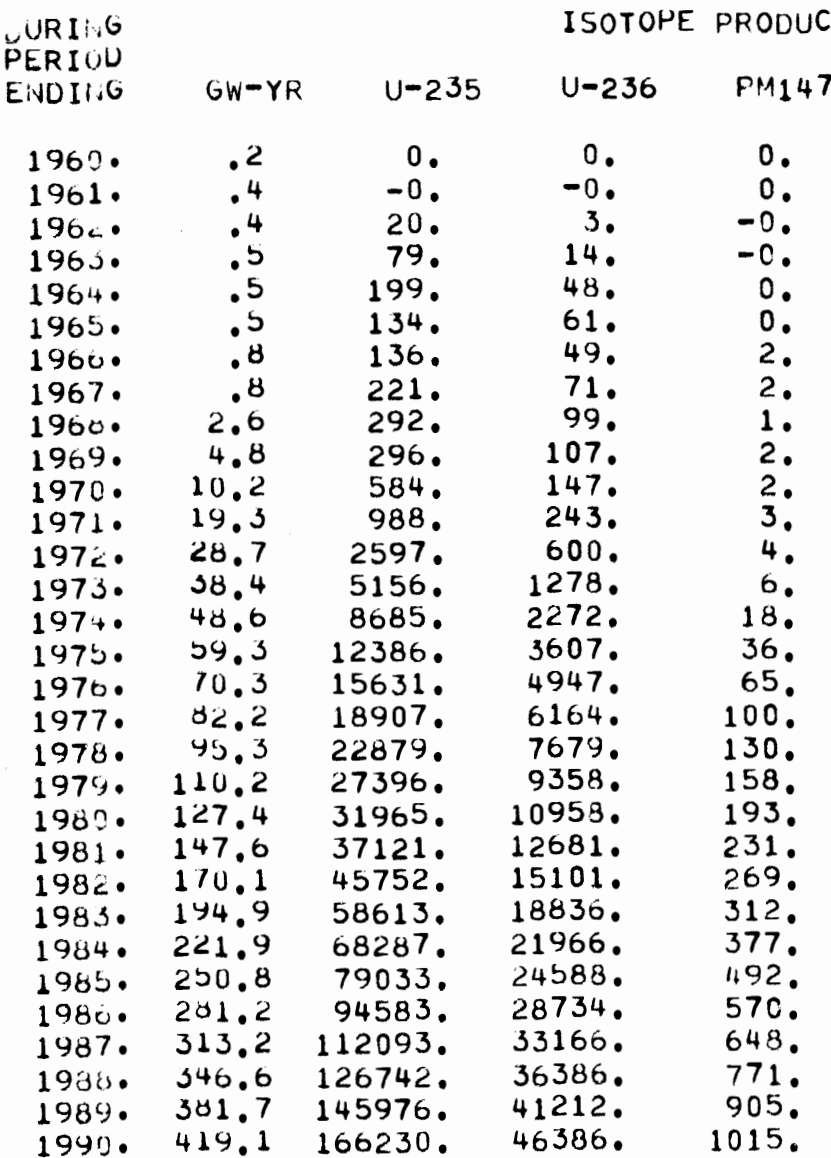

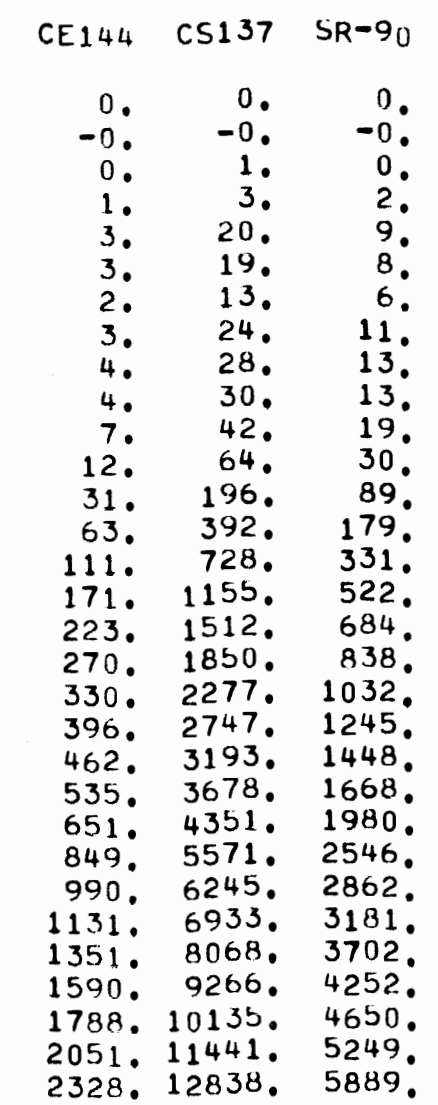

\begin{tabular}{|c|c|c|}
\hline$K R-85$ & $T C-99$ & $\begin{array}{c}\text { RH103 } \\
\text { SUM }\end{array}$ \\
\hline 0. & 0. & 0. \\
\hline-0 & -0. & -0 . \\
\hline 0 . & 1. & 0. \\
\hline 0 . & 2. & 1. \\
\hline 0 . & 14. & 7. \\
\hline 0. & 13. & 7. \\
\hline 0 & 9. & • \\
\hline 0 & 17. & 9. \\
\hline 1. & 20. & 10. \\
\hline 1. & 20 . & 11. \\
\hline 1. & 29. & 16. \\
\hline 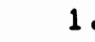 & 45. & 25. \\
\hline $\begin{array}{l}4 \\
8\end{array}$ & $\begin{array}{l}135^{\circ} \\
272 .\end{array}$ & $\begin{array}{r}72 \\
147 .\end{array}$ \\
\hline 15 & 504. & 269. \\
\hline 23 & 798. & 424. \\
\hline 30 & $1044^{\circ}$ & $\begin{array}{l}553 . \\
672\end{array}$ \\
\hline $\begin{array}{l}37 \\
45\end{array}$ & $\begin{array}{l}1276 \\
1570\end{array}$ & $\begin{array}{l}672 . \\
823 .\end{array}$ \\
\hline 55 & 1893. & 990 . \\
\hline 63 & 2200 & 1151. \\
\hline & $\begin{array}{l}2536 \\
3003\end{array}$ & $\begin{array}{l}1329 \\
1582\end{array}$ \\
\hline 112 & 3851 & 2040 . \\
\hline 126 & 4323 & 2316 . \\
\hline 140 & 4804 & 2599. \\
\hline 164 & $\begin{array}{l}5597 \\
0435\end{array}$ & $\begin{array}{l}3058^{\circ} \\
3549^{\circ}\end{array}$ \\
\hline $\begin{array}{l}188 \\
207\end{array}$ & 7048 & 3933. \\
\hline 233 & 7961 & $4470^{\circ}$ \\
\hline & & 5042 \\
\hline
\end{tabular}

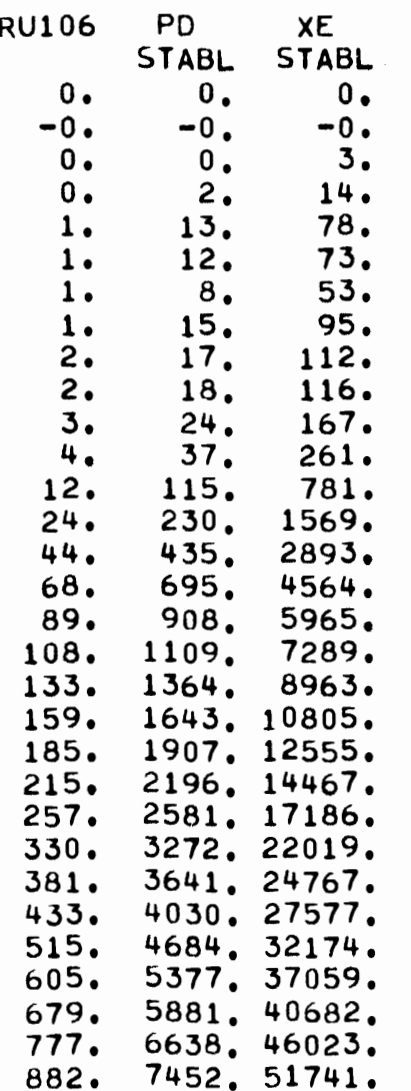

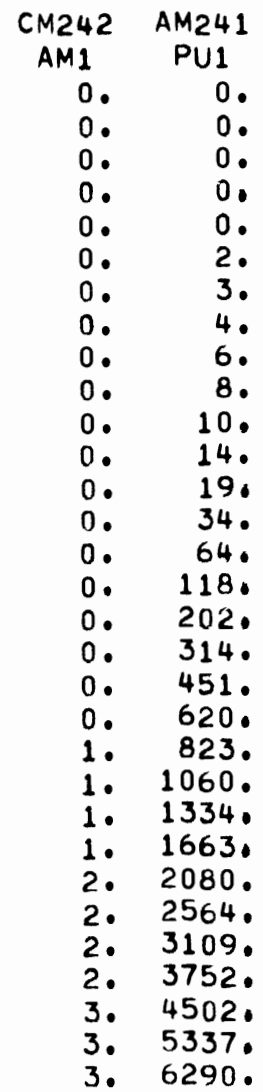


NET ANNUAL I SOTOPE A $T$ T A I L A B I L I T Y

(WITH TARGET IRRADIATION. PROCESS LOSSES AND DECAY LOSSES)

$$
L W R \text { ' } S \text { - NO RECYCLE }
$$

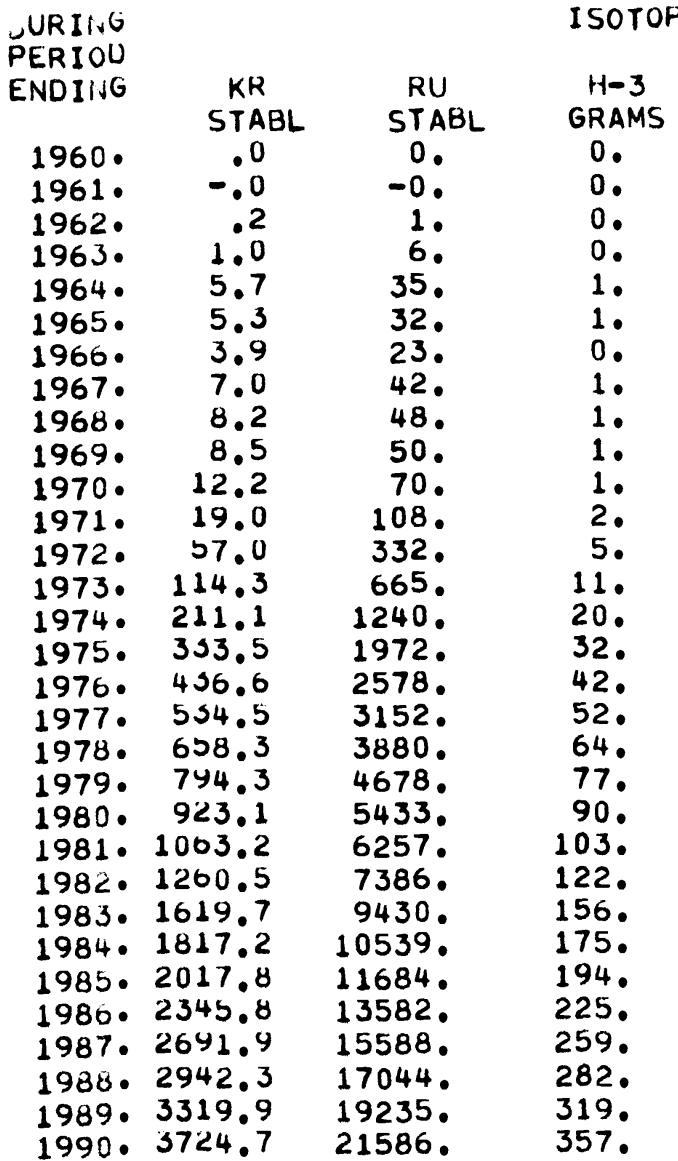


(WITH TARGET IRRAIIATION, PROCESS LOSSES AND DECAY LOSSES)

$$
\text { LWR } \text { ' } S \text {-- NO RECYCLE }
$$

TUTAL

INS I ALLED

YEAR CAPACITY

ENDIIG GWIE) TONNE

ISOTOPE PRODUCTION, KGS

\begin{tabular}{|c|c|c|c|c|}
\hline $\begin{array}{l}1960^{\circ} \\
1961^{\circ} \\
1962^{\circ} \\
1963^{\circ} \\
1964^{\circ} \\
1965^{\circ} \\
1960^{\circ} \\
1967^{\circ} \\
1968^{\circ} \\
1969^{\circ} \\
1970^{\circ} \\
1971^{\circ} \\
1972^{\circ} \\
1973^{\circ} \\
1974 . \\
1975^{\circ} \\
1970^{\circ} \\
1977^{\circ} \\
1978^{\circ} \\
1979^{\circ} \\
1980^{\circ} \\
1981^{\circ} \\
1982^{\circ} \\
1983^{\circ} \\
1984^{\circ} \\
1985^{\circ} \\
19860^{\circ} \\
1987^{\circ} \\
1980^{\circ} \\
1989^{\circ} \\
1991 .\end{array}$ & $\begin{array}{r}.3 \\
.5 \\
.5 \\
.6 \\
.6 \\
.6 \\
1.0 \\
1.0 \\
3.0 \\
5.7 \\
12.0 \\
22.7 \\
33.7 \\
45.2 \\
37.2 \\
69.7 \\
32.7 \\
90.7 \\
112.2 \\
129.7 \\
130.0 \\
113.7 \\
200.2 \\
229.5 \\
201.4 \\
295.7 \\
352.2 \\
310.7 \\
411.2 \\
454.1 \\
500.0\end{array}$ & 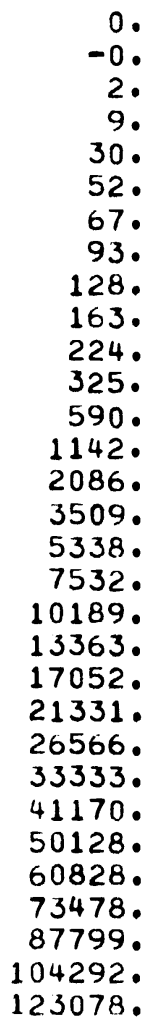 & 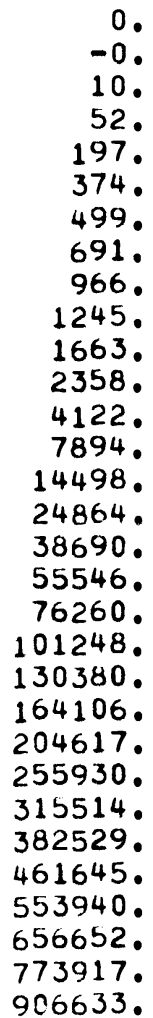 & 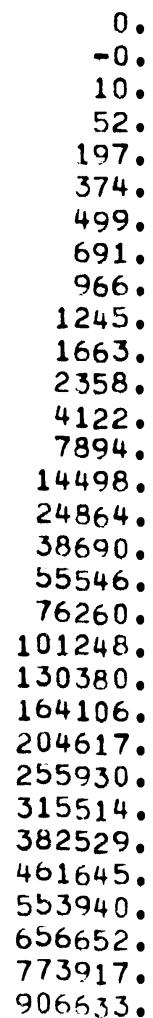 \\
\hline
\end{tabular}

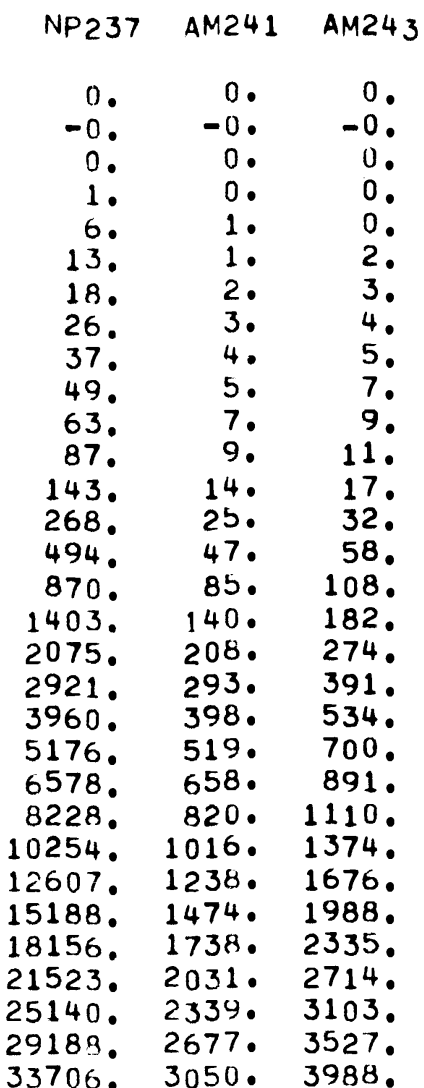

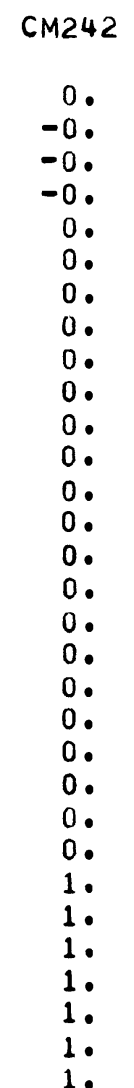

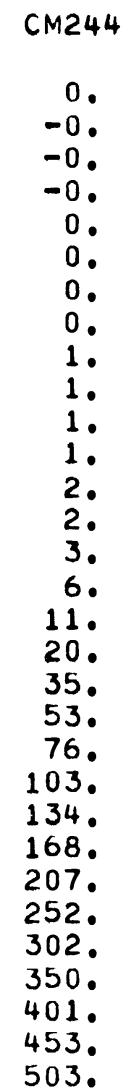

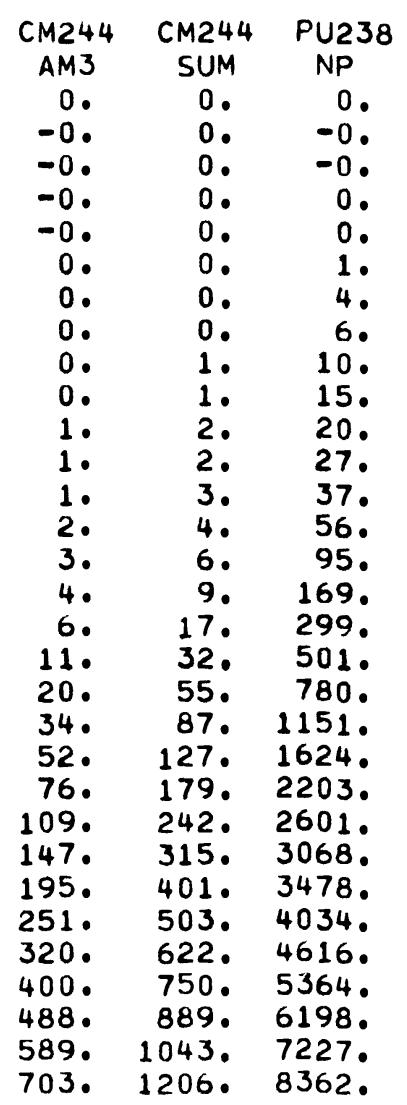

$\begin{array}{ccc}\text { PU238 } & \text { PU238 } & \text { PU238 } \\ \text { CM } & \text { AM } & \text { SUM } \\ 0 . & 0 . & 0 . \\ -0 . & -0 . & 0 . \\ -0 . & -0 . & 0 . \\ -0 . & -0 . & 0 . \\ 0 . & -0 . & 0 . \\ 0 . & 0 . & 1 . \\ 0 . & 0 . & 4 . \\ 0 . & 0 . & 7 . \\ 0 . & 0 . & 10 . \\ 0 . & 1 . & 15 . \\ 0 . & 1 . & 22 . \\ 0 . & 2 . & 29 . \\ 0 . & 2 . & 40 . \\ 0 . & 3 . & 59 . \\ 1 . & 4 . & 99 . \\ 1 . & 6 . & 176 . \\ 2 . & 10 . & 311 . \\ 4 . & 18 . & 523 . \\ 7 . & 32 . & 820 \\ 11^{\circ} & 53 . & 1215 . \\ 15 . & 82 . & 1721 . \\ 21 . & 119 . & 2344 . \\ 27 . & 166 . & 2795 . \\ 34 . & 223 . & 3325 . \\ 43 . & 290 . & 3811 . \\ 53 . & 369 . & 4456 . \\ 65 . & 464 . & 5145 . \\ 77 . & 572 . & 6013 . \\ 90 . & 693 . & 6981 . \\ 105 . & 829 . & 8161 . \\ 121 . & 979 . & 9463 . \\ & & \end{array}$


(WITH TARGET IRRAOIATION, PROCESS LOSSES AND DECAY LOSSES)

YEAR

1960.

1960.

1961.

1963.

1964.

1965.

1966.

1967.

1968.

1969.

1970.

1971.

1972 .

1973.

1974.

1975.

1976.

1977.

1978.

1979.

$1980^{\circ}$

1982.

1983.

1984.

$1985^{\circ}$

1986.

$1987^{\circ}$

1988.

1990 .
ISOTOPE PRODUCTION, KGS

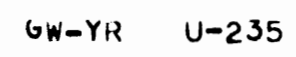

CE144

CS137

0

-0
-0

-0 .

0 .

65.

126.

174 .

245.

344 .

450 .

598 .

841.

1441 .

2720 .

4992 .

8599.

13546 .

19710 .

27389.

36747 .

47705 .

60385.

94322 .

116288 .

169610 .

202776.

239161.

280373 .

326759 .

0
3
3

3.

4.

24.

55.

$$
78 .
$$

5. 18.

7.132.

10.2170

1583.

2623. 3044 .
140876 . $\begin{array}{rrr}0 . & 0 . & 0 \\ -0 . & -0 . & -0\end{array}$

16. 231. 105 .

37. 421. 192 .

$144^{\circ} \quad 1513.688$.

317 . 4085. 1848

401. 5841. 2641 .

495. 7985. 3608 .

$600.10550,4765$.

708 . 13501. 6097.

826. 16871. 7616.

$991.20837 \circ 11725$

1507.31584 .14300 .

1751 . 37795. 17131 .

1751. 37795. 17131 .

2442. 53238. 24166 . 2792.62158 .28226 . 1073.47457 .25597$.

$\begin{array}{lll}0 . & 1 . & 0 .\end{array}$

0.17 .29$.

1. 30.16.

1. 39. 21.

1.56 .29$.

2. $96.950^{\circ}$.

3. 124.66.

8. 305.2163.

15. 576. 309.

29. 1080 . 578.

50. 1878. 1002 .

77. 2922. 1555.

$1090^{\circ} 4197.22270^{\circ}$

192. 7661. 4040 .

244. 9861. 5191.

302. 12397. 6520.

370. 15400 . 8102.

556. 23573. 12459 .

662 . 28378. 15057 .

784. 33975. 18115.

$924 \cdot 40409 \cdot 21664$.

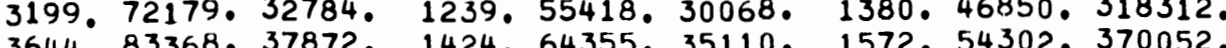

$\begin{array}{ccc}K R-85 & T C-99 \quad \text { RH103 } \\ \text { SUM } & \text { TC }\end{array}$

RU106

STAB

$\begin{array}{ccc}\text { XE } & \text { CM242 } & \text { AM241 } \\ \text { STABL } & \text { AM1 } & \text { PU1 }\end{array}$

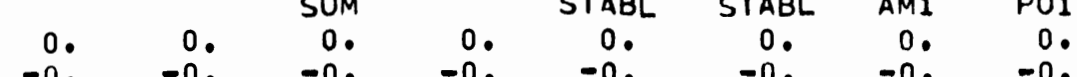

$-0.00$.

0.00

1. 2.

2. $26.269^{\circ}$.

2. 34.222.

2. 49. 317 .

3. 66. 429.

3. $84^{\circ}$ 545.

4. 108. 712.

6. 145. 973.

31. 490. 3324.

59. 925. 6216.

98. 1620. 10781.

138. 2528. 16746 .

177. 3637. 24035 .

221. 5001. 32998.

270. 6645. 43803.

320. 8552. 56358.

375. 10748. 70825.

444. 13329. 88011.

552. 16600. 110029 .

$657,20242.134796$ 。

761 . 24271 . 162374.

896. 28955. 194548.

1053. 34332. 231607 .

1205.40212 . 272289 .

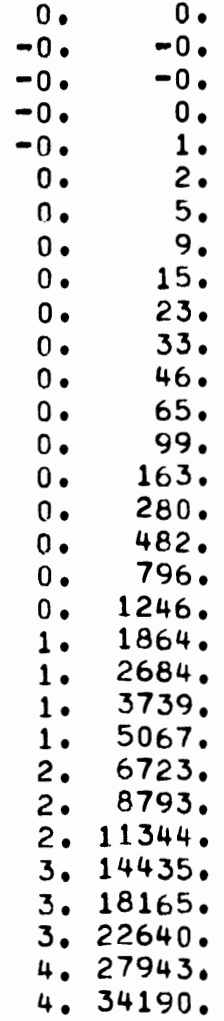


CUMULATIVE I SOT OP E AVA I LA B I L I T Y

(WITH TARGET IRRACIATION, PROCESS LOSSES AND DECAY LOSSES)
CASE
ISOTOPE PRODUCTION, KGS

\section{YEAR}

EINDII.G

1960.

1961.

1962.

1963.

1964.

1965

1965

1966

1967.

1968.

1969.

1970.

1971.

1972

1973.

$1974^{\circ}$

1974.

1977.1758 .310355$.

1978. $2416.6 \quad 14234$.

1979. 3210.918912 .

1980.4134 .024344$.

1981.5197 .230601

1982. 6457.737986

1983. 8017.447417 .

$1984.9894 .6 \quad 57956$.

1985. 11912.569640 .

1986.14258 .283222$.

1987.16950 .298811$.

1988. 19842.5115854 .

1989.23212 .4135089$.

1990 . 26937.1 156675.
$\mathrm{H}-3$

GRAMS

0 .

-0 .

0.

1 .

1.

1.

2.

3.

3.

6.

11.

11.

21.

41.

71 .

109.

276.

351.

434.

533 .

659.

798.

948.

1121.

1318 .

1528.

1763.

2023. 
I SOTUPE PRODUCTION

LWR' S - U236 RECYCLE 
DISTRIBUTION OF ELECTRICAL GENERATANG CAPACITY

PAGE 50

LWR S - U U 36 RECYCLE

CASE 2

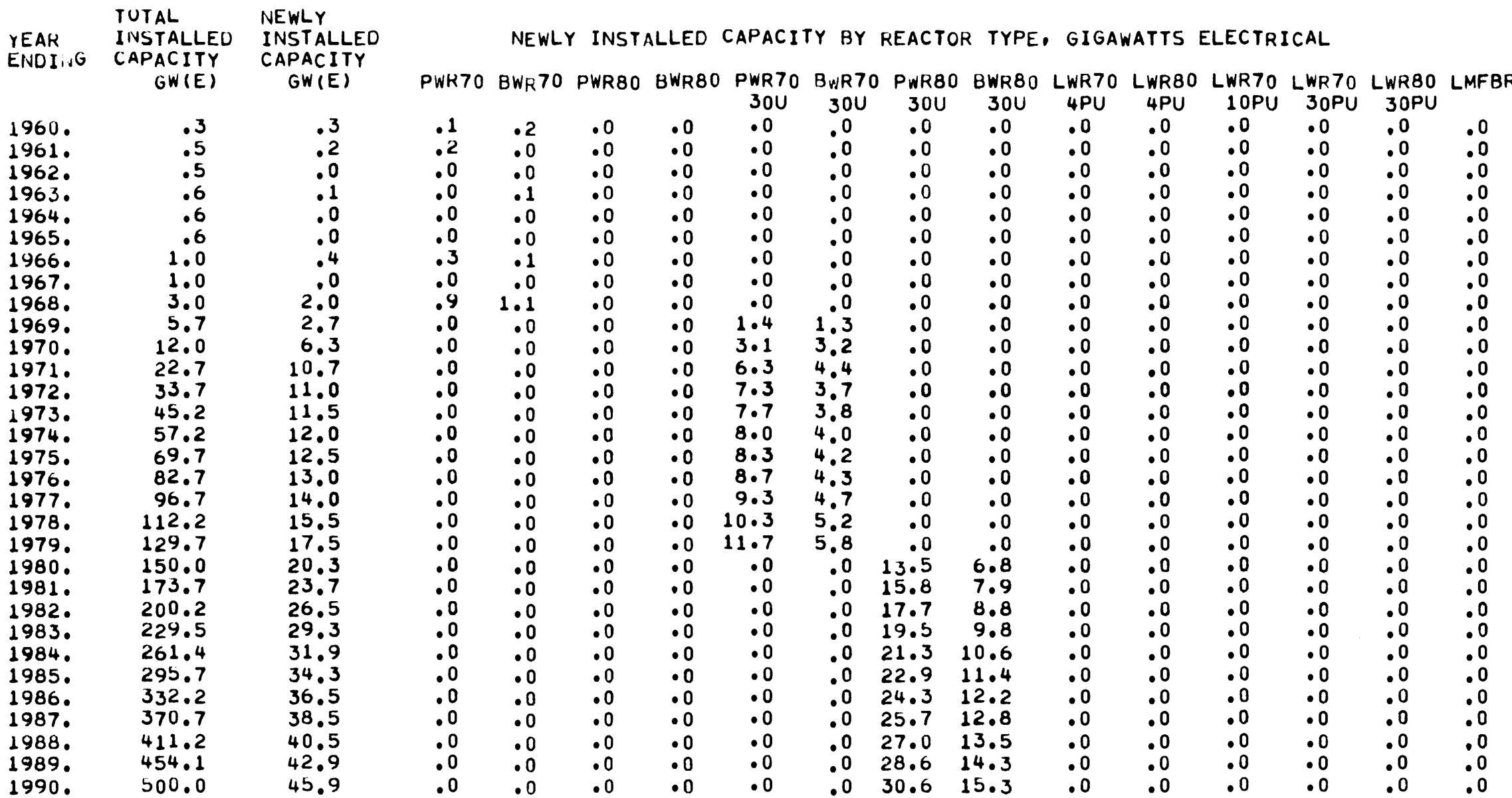




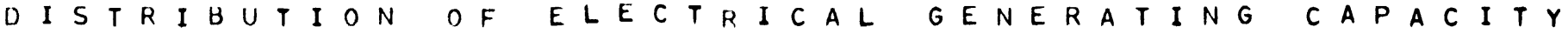
PAGE 51
LWR ' S -- U236 RECYCLE

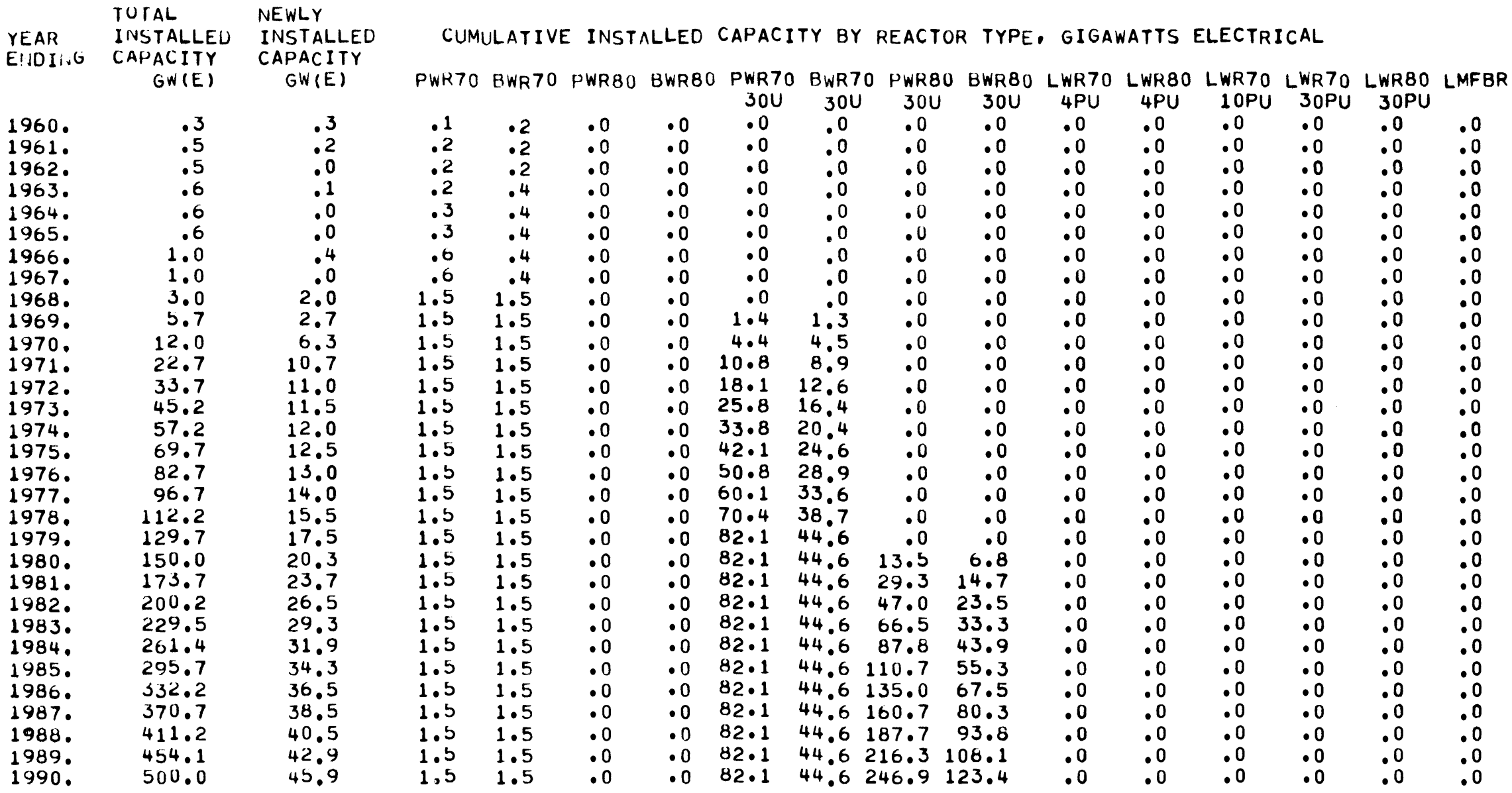


TUTAL

DURIING INSTALLED

PERIOU CAPACITY

ENDING GW (E)

ISOTOPE PRODUCTION, KILOGRAMS/YEAR

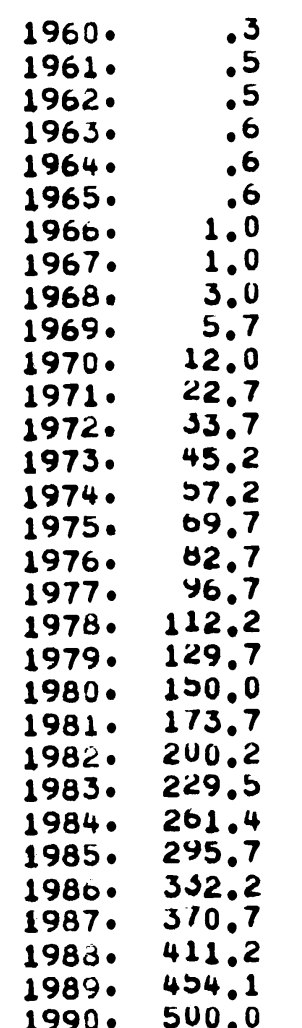

PU-RP PU-SP

NP237

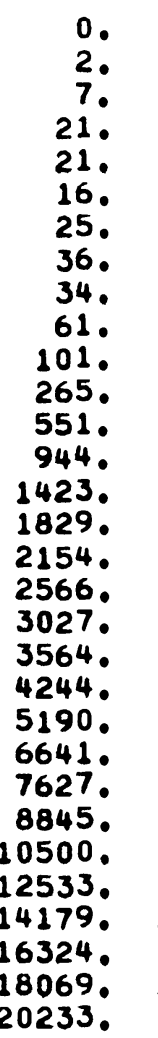

\section{0.} $44^{\circ}$

147.

181.

125.

192.

276.

278 .

418.

703 .

1801 .

3863 .

6751 .

10561 .

14027 .

$16654^{\circ}$

20015 .

23711.

27939.

33165.

39791.

$49844^{\circ}$

65166 .

76330 .

89764 .

100130.100130 .

114040 . 114040 .

$\begin{array}{ll}124499^{\circ} & 124499^{\circ} \\ 138340^{\circ} & 138340^{\circ}\end{array}$
0
10
44
147
181
125
192
276
278
418
703

703.
1801.

3863 .

6751.

10561 .

$14027^{\circ}$

16654 .

20015 .

23711.

$27939^{\circ}$

33165

$49891^{\circ}$

57266 .

65166.

76330

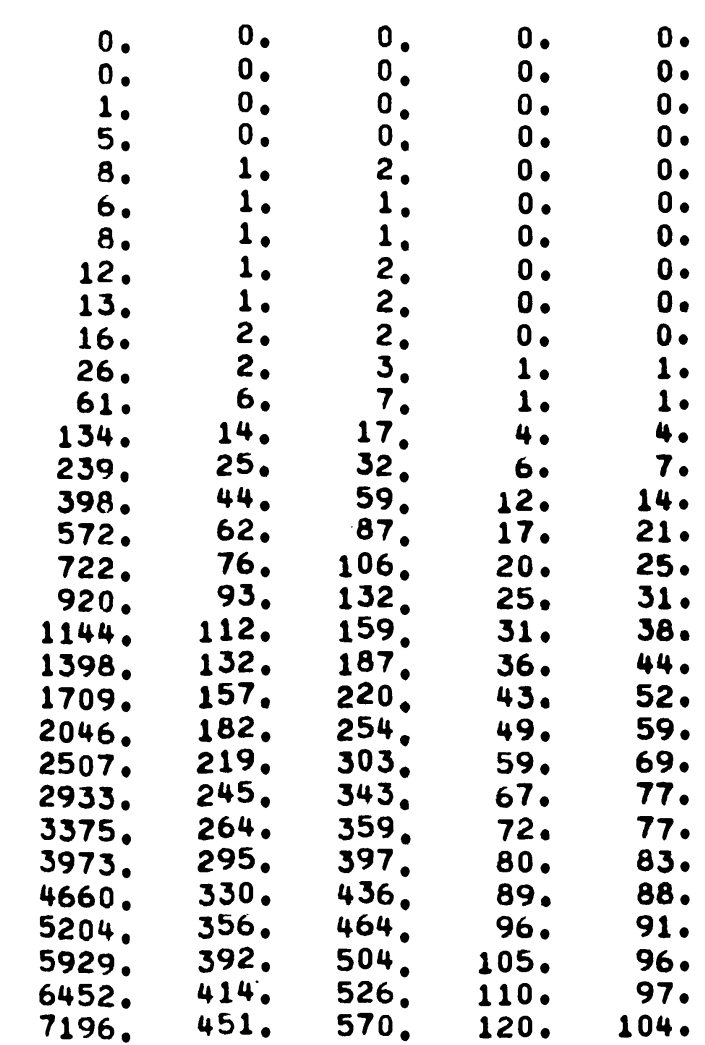

$\mathrm{CM}_{\text {AM3 }} 44$

CM244

PU238

PU238

PU238 PU238

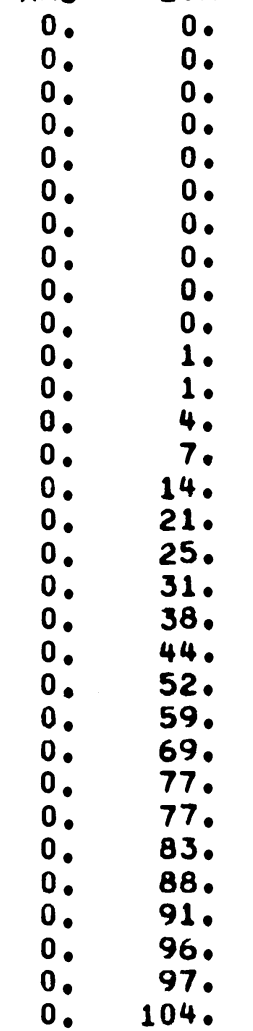

0.
0.
0.
0.
0.
0.
0.
0.
0.
0.
0.
0.
0.
0.
0.
0.
0.
0.
0.
0.
0.
0.
0.
0.
0.
0.
0.
0.
0.
0

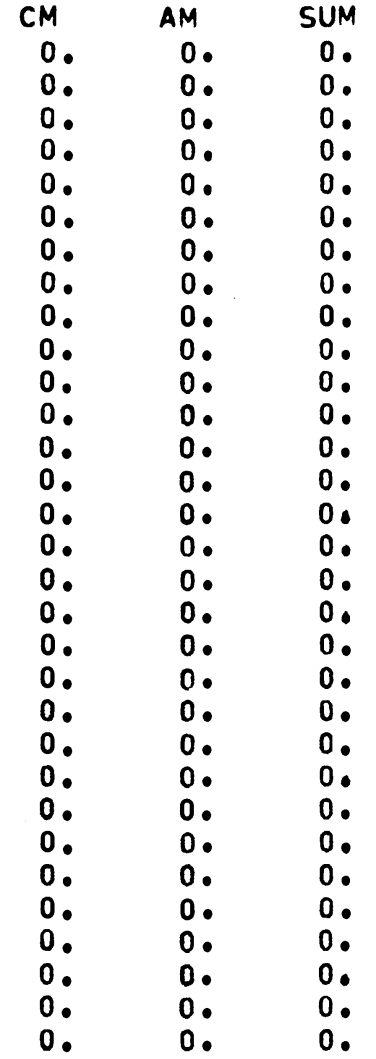


(NO LOSS BASIS)

UURING

PERIOU

PEIIV

1960 .

1961.

1962.

1963.

$1964^{\circ}$

1965.

1966.

1967.

$1960^{\circ}$

1969.

1970 .

1971.

1972.

1973.

1974.

1975 .

1976.

1977.

$1974 \circ$

1980

1981 .

1983.144 .9

1984.2219

1980.200 .8

$1980^{\circ} 281.2$

$1987 \cdot 313.2$

$1980^{\circ}$

1989.

301.7

$G W-Y R \quad U-235$

.2000

.421.

.5203.

.8

4.8

19.3

28.7

48.6
59.3

10.3
82.2

प5. 3

10.2

226.

298.

302 .

596.

988.

2581

5090 .

8565 .

12216 .

15412 .

$18270^{\circ}$

21765.

25747 .

30527 .

36457 .

44827 .

57117 .

66034

92605 .

110910 .

125413.

144569 .

$159928^{\circ}$.

\section{ISOTOPE PRODUCTION, KILOGRAMS/YEAR}

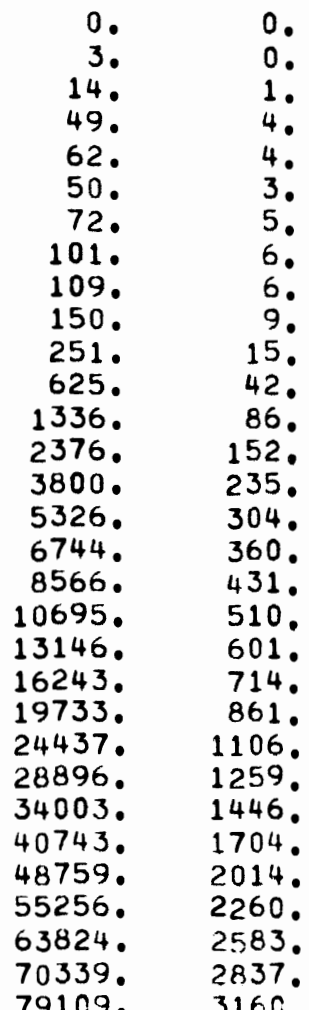

79109.

\begin{tabular}{|c|c|c|}
\hline CE 144 & $\operatorname{cs} 137$ & $S R-90$ \\
\hline 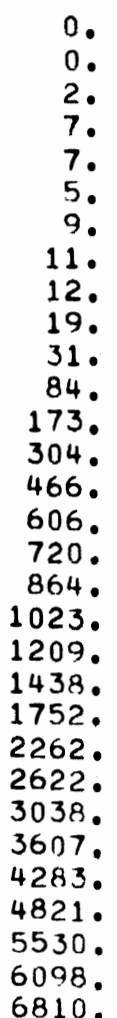 & 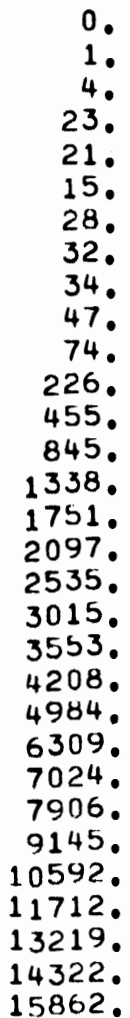 & 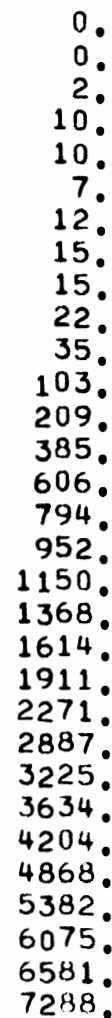 \\
\hline
\end{tabular}

6810.15862 .7288$.
$K F-85$

$\begin{array}{rr}0 . & \\ 0 . & 1 \\ 0 . & 3 \\ 0 . & 15\end{array}$

0.14.

0.10.

1. 19.

1.22.

1.23

1. $\quad 32$.

5. 152 .

10. 307 .

18. 568 .

36. $1174^{\circ}$

43. 1405.

52. 1697 .

62. 2017. 1023。

73. 2377. 1204 .

87. 2815. 1428 .

104. 3336. 1698.

132. 4227. 2158 .

148. 4708. 2414.

133. 6137.

248.7871 .

280. 8889 .

304.9637.

RH103
SUM

2.

8.

10.

12.

$177^{\circ}$

80.

296.

465 .

604.
PTAB

XE

CM242 AM241

0. AMI PU1

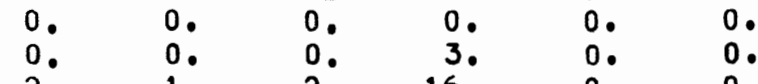

1. 2. 16.

2. 14.87.

13. 81.

9. 58.

3. 17.106.

4. $20^{\circ} \quad 129^{\circ}$

4. $27^{\circ} \quad 129$.

9. 41.2293.

26. 129.879.

54. 260 . 1772 .

98. 490. 3268.

153. 784. 5154 .

200. 1024. 6738 .

238. 1225.8061.

286. 1481. 9734 .

339. 1760. 11572 .

400. 2071. 13643.

476. 2453. 16164.

570. 2886. 19235.

725. 3618. 24390 .

830 . 3996. 27269.

957. 4483. 30782.

483.30782.

5990.41499.

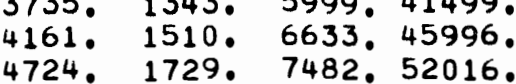

4724. 1729. 7482, 52016.

5721. 2125. 8975. 62645.

$\begin{array}{ll}0 . & 0 . \\ 0 . & 0 . \\ 0 . & 0 . \\ 0 . & 2 . \\ 0 . & 3 \circ \\ 0 . & 5 . \\ 0 . & 6 . \\ 0 . & 9 . \\ 0 . & 11 . \\ 0 . & 15 . \\ 0 . & 22 . \\ 0 . & 38 . \\ 0 . & 73 . \\ 0 . & 135 . \\ 0 . & 231 . \\ 0 . & 359 . \\ 0 . & 512 . \\ 0 . & 696 . \\ 0 . & 915 . \\ 0 . & 1174 . \\ 0 . & 1482 . \\ 0 . & 1853 . \\ 0 . & 2317 . \\ 0 . & 2852 . \\ 0 . & 3462 . \\ 0 . & 4178 . \\ 0 . & 5021 . \\ 0 . & 5965 . \\ 0 . & 7041 . \\ 0 . & 8221 . \\ & \end{array}$



ANNUAL ISOTOPE PRODUCTION AT DISCHARGE (NO LOSS BASIS)

LWR S $S$ - U236 RECYCLE

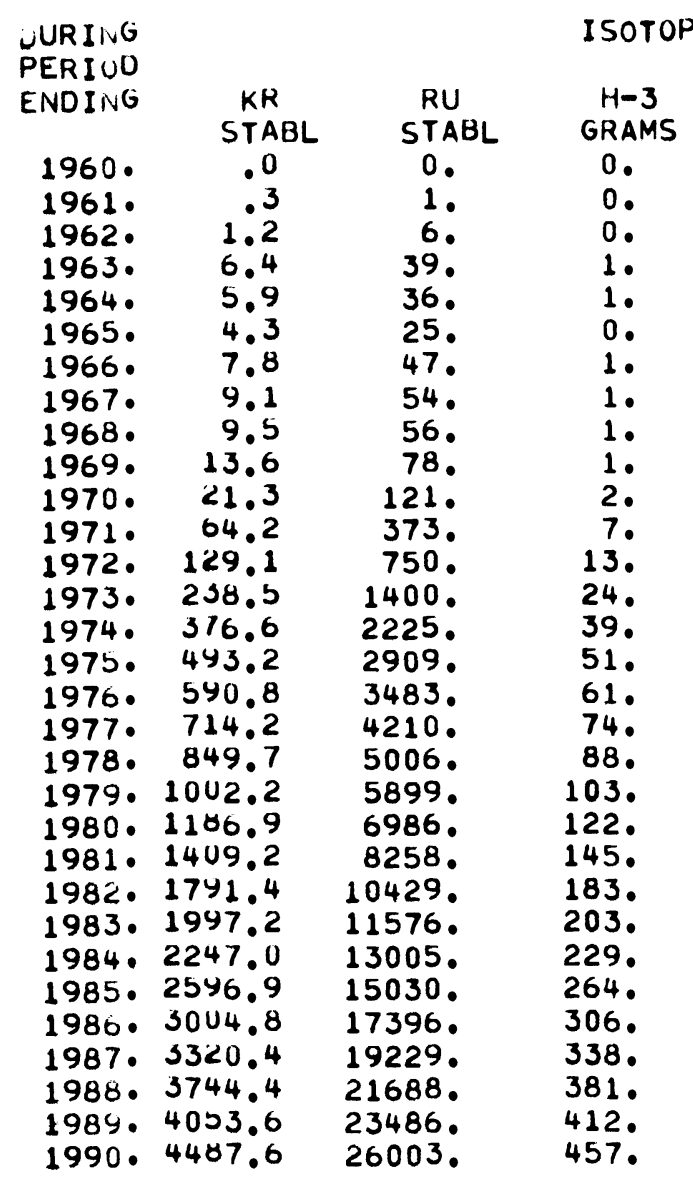


(NO LOSSES EXCEPT FOR RADIOACTIVE LECAY)

TUTAL

INS I ALLEU

IEAR CAPACITY

EIJDIIG GW(E)

1960.

1961.

1963.

$1964^{\circ}$

1965.

1966.

1967.

1968.

1969.

1970.

1971 .

1972 .

1973.

1974 .

1975.

1976.

1977.

1978.

1979 .

1980.

1981.

1982

1983.

1984.

1985.

1986.

1989.

1990 .

.6

.6

1.0

1.0
ISOTOPE PRODICTION, KGS

TONNE PU-RP PU-SP

NP237

$A M 241$

AM243

CM242

CM244

CM244

AM3 3244 PU238

SUM NP

0. -0 .

$0 . \quad-0$.

$3.0 \quad 128$

5.7224 .

12.0325 .

22.7590 .

$33.7 \quad 1142$.

37.23509.

69.75338 .

02.77492 .

46.710058 .

112.213086 .

129.7 16650. $129814^{\circ} 129814^{\circ}$

150.020894 .163894 .163894$.

173.7 26084. 204859. 204859.

200.2 32725. 256185. 256185.

229.540352 . 315304. 315304.

201.4 49197. 382786. 382786.

295.759698.

461968.461968.

72231. 555194. 555194.

410.7 86409. 659503. 659503.

454.1 120802. 909027. 909027.

500.0141036 .1054408 . 1054408 . $\begin{array}{rr}0 . & 0 \\ 0 . & \\ 1 . & 0 \\ 6 . & 1 \\ 14 . & 2 \\ 20 . & 20\end{array}$

20.02.

41.

54.6 .

97.

97.
158.

158.

531.

928.

1501.

$2223^{\circ}$

3142. 328 .

4286.

5684.

571.799.

94390908.1019.

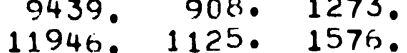

$14879^{\circ} 1369.1918$.

18254.1630 .2278 .

22227. 1922. 2675 .

$268870^{\circ} 22490^{\circ} 3111$.

$38010^{\circ} 26020^{\circ} 3574$.

$\begin{array}{lll}38019 . & 2989 . & 4078 . \\ 44471 . & 3399 . & 4604 .\end{array}$

$\begin{array}{lll}44471^{\circ} & 3399^{\circ} & 4604^{\circ} \\ 51666^{\circ} & 38455^{\circ} & 5174^{\circ}\end{array}$
0.

0. $\quad 0 . \quad-0$.

$0 . \quad 0 . \quad-0$.

0.

0.

0.

0.

0.

1.

1.

4.

13.

20.

25.

31.

37.

173.

52. 218.

72. 328.

82.393.

89. 455.

99.521.

110.590.

119.659.

130.730 .

138.800 .

149. 374 .
0 .

$-0$

-0
-0

-0
-0

-0 .

-0 .

-0.
-0.

-0.
-0.

-0.
-0

$-0$.

-0 .

$-0$.

-0 .

-0.
-0.

-0 .

-0 .

$-0$.

-0 .

-0.
-0.

521 .

$-0.659$.

-0. 800 .

-0.874 .
-0.730 .
-0.
-0.

-0
-0

-0 .

-0 .

-0 .

-0 .

-0 .

-0.
-0.

-0 .

-0 .

-0 .

-0 .

-0 .

-0.
-0.

-0 .

-0 .

-0 .

-0 .

-0 .

-0
-0

-0 .

-0 .

$-0$.

$-0$.

-0.
-0.

-0 .
PU238 PU238 PU238

$\begin{array}{ccc}C M & A M & \text { SUM } \\ 0 . & 0 . & 0\end{array}$

$-0 . \quad-0.00$

$\begin{array}{lll}-0 . & -0 . & 0\end{array}$

$-0.0 .000$

$\begin{array}{lll}-0 . & -0 . & 0 \\ -0 . & -0 . & 0\end{array}$

$-0 . \quad-0$.

$\begin{array}{lll}-0 & -0 . & 0\end{array}$

$-0 . \quad-0 . \quad 0$.

$\begin{array}{lll}-0 . & -0 . & 0 . \\ -0 . & -0 . & 0 .\end{array}$

$-0 . \quad-0.0$.

-0 .

-0 .

$-0$

-0 .

-0
-0.

-0 .

-0 .

$-0$.

-0 .

-0
-0

-0 .

-0 .

-0 .

$-0$.

-0 .

-0.
-0.

$-0$

-0 .

-0 .

-0.
-0 .

-0 .

-0 .

$-0$.

-0
-0

-0 .

-0 .

$-0$.

$-0$.

-0 .

-0.
-0. 


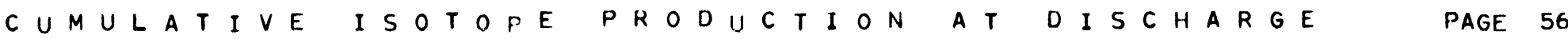
(NO LOSSES EXCEPT FOR RADIOACTIVE LECAY)

L W R S $\mathrm{S}$ - U236 RECYCLE

CASE 2

YEAR

ISOTOPE PRODUCTION, KGS

ENDING

GW-YR U-235

ISOTOP

1960.

1961.

1963.

$1964^{\circ}$

1965.

1966.

1967.

1968 .

$1969^{\circ}$

1970.

1971.

1972 .

1973.

1974.

1976 .

1976.

1978.

1979.

1980 .

1981.

$1983^{\circ}$

1984 .

1985.

1986.

1987

1988.

1989.

$\begin{array}{rr}.2 & 0 \\ .6 & 21 \\ 1.0 & 101 \\ 1.5 & 304 \\ 2.1 & 440 \\ 2.6 & 580 \\ 3.4 & 805 \\ 4.3 & 1103 . \\ 6.8 & 1405 . \\ 11.7 & 2000 \\ 21.9 & 2988 \\ 41.2 & 5569 \\ 69.9 & 10659\end{array}$
09.910659 . 108.319225 . 106.931440 . 216.246852 . 286.565123 . 368.786887 . 464.0112634 . 574.2143161. 701.6179618 . 849.2224445 . 1019.3281562 . 1214.2347596 . 1436.0425363. 1968.1627968. 2281.2754290 . 2627.8898859 . 3009.51058787 . $u-236$ PM147

CE1 144

$\begin{aligned} 0 . & \\ 3 . & \\ 17 . & \\ 66 . & \\ 128 . & \end{aligned}$

\begin{tabular}{|c|c|}
\hline $\begin{array}{c}0 . \\
0 . \\
10^{\circ} \\
50^{\circ} \\
8 . \\
110^{\circ} \\
150^{\circ} \\
170^{\circ} \\
330^{\circ}\end{array}$ & $\begin{array}{r}0 \\
0 \\
20 \\
10 \\
12 \\
17 \\
19 \\
26 \\
42 \\
101\end{array}$ \\
\hline
\end{tabular}

$S R=90$

$K R-85 \quad T C-99$

RH103
SUM

RU106

PD

XE

CM242 AM241

250 .

351.

460.

610.

862.

1486.

5198 .

8999 .

14324.

21068 .

29634.

40329.

53475 .

89452.

$113889^{\circ}$

$113889^{\circ}$

176788 .

217532 .

266291.

321547 .

385372 .

139.

260.

436.

641.

856.

1094.

1650 .

1991 .

2401.

2963.

3551 .

4193.

4948 .

5842 .

6779.

7827.

$8892^{\circ}$

215. $927^{\circ} \quad 424^{\circ}$.

393. 1751. 798.

627. 3049. 1385.

864. 4731. 2145 .

1075. 6720. 3044.

1306. 9102. 4120 .

1560 • 11909 . 5388.

2199. 19051. 8613.

2656. 23599. 10673.

3354 . 29369. 13299 .

4002 . 35722. 16199 .

4683 . 42812. 19437 .

5533 . 50979. 23165 .

7519.70738 . 32177.

0.
0.
0.
1.

$\begin{array}{ll}0 . & 0 . \\ 1 . & 0 . \\ 3 . & 2 .\end{array}$

9. 10.

33. 18.

1. 43. 23.

$\begin{array}{lll}1 . & 42^{\circ} & 23 . \\ 2 . & 84 . & 4{ }^{\circ}\end{array}$

3. 106.56.

4. 138.73.

$\begin{array}{lll}5 . & 189 . & 101 . \\ 9 . & 341 . & 181 .\end{array}$

18. 648. 344.

35. 1217. 640.

93. 3289. 1709 .

130 . 4694. 2428 .

174. 6391. 3291.

226.8408 . 4314.

285. 10785.5519.

436. 16936. 8645.

540.21163 .10803$.

654.25871 .13217 .

780.31172 .15954$.

925. 37309, 19148.

1473 . 61182. 31768.

9644.94783 .43130 .

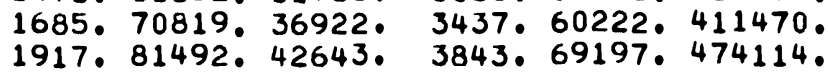

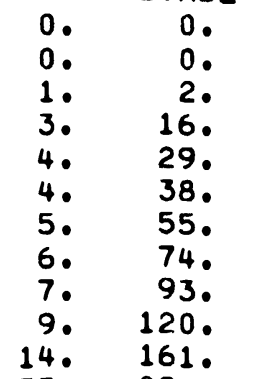

$\begin{array}{ccc}\text { STABL } & \text { AM1 } & \text { PU1 } \\ 0 . & 0 . & 0 .\end{array}$

3428.61238294 . 534819 .

10039 .

10776.108481 . 49364 .

$\begin{array}{rrr}0 . & 0 . & -0 \\ 19 . & -0 . & 0 \\ 107 . & -0 . & 1\end{array}$

$188 .-0.00$

246. -0.06

352. -0.10

$477 . \quad-0 . \quad 17$.

$-0.260$

14. 161. 1084. $\quad-0.052$.

70. 550. 3735. -0.112.

133. 1040. 7003. -0. 185 .

1824. 12157. -0. $320^{\circ}$

$393^{\circ} \quad 2848^{\circ} 18896^{\circ}$

482. 5554. 36691. -0. 1419 。

$580.7314 .48262 . \quad-0.2113$.

690. 9385. 61905. -0. 3025.

. 11838. 78069.

$1215.18342^{\circ} 97305^{\circ} \quad-0.5670$

1438.22338 . 148964. $-0.9820^{\circ}$

1675.26821. 179746. -0. 12656 .

1971.32002. 215464. -0. 16099 .

$2328.38000 .256963^{\circ}-0.20253$.

2674. 44634. 302958. -0.25243.

-0. 31170

-0.38164.
-0.46328. 
CUMULATIVE ISOTOPE PROOUCTION AT DISCHARGE

(NO LOSSES EXCEPT FOR RADIOACTIVE DECAY)

\section{ISOTOPE PRODUCTION, KGS}

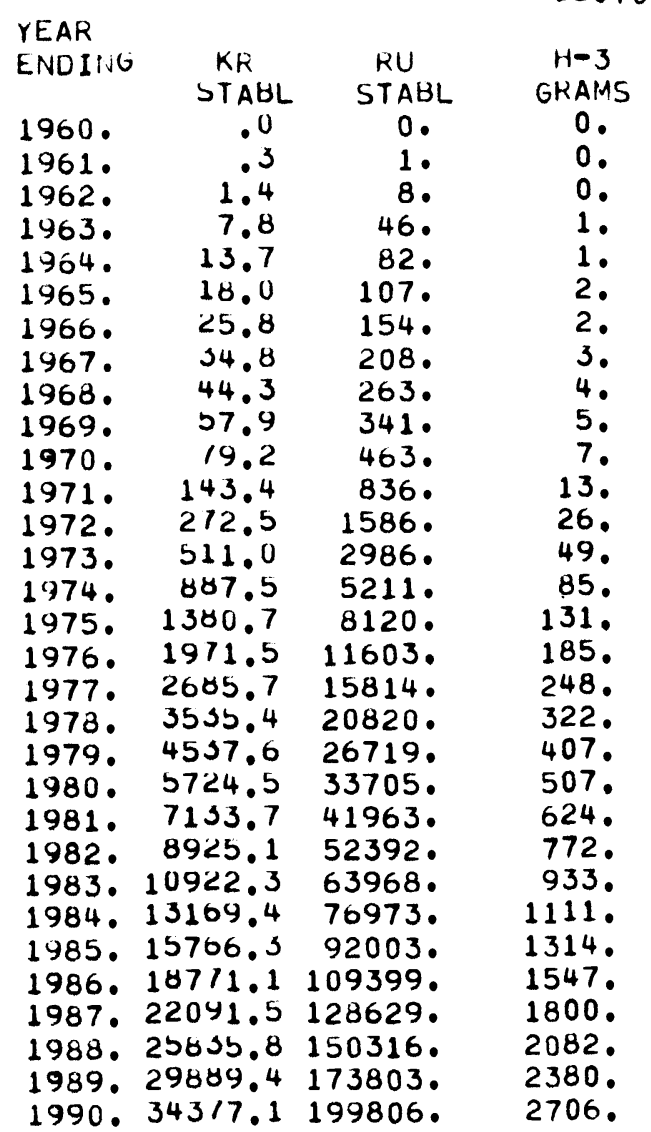


NET ANNUAL I SOTOPE AVAILABILI T Y

PAGE 58

(WITH TARGET IRRAUIATION. PROCESS LOSSES AND DECAY LOSSES)

$$
\text { LWR ' S -- U236 RECYCLE }
$$

CASE 2

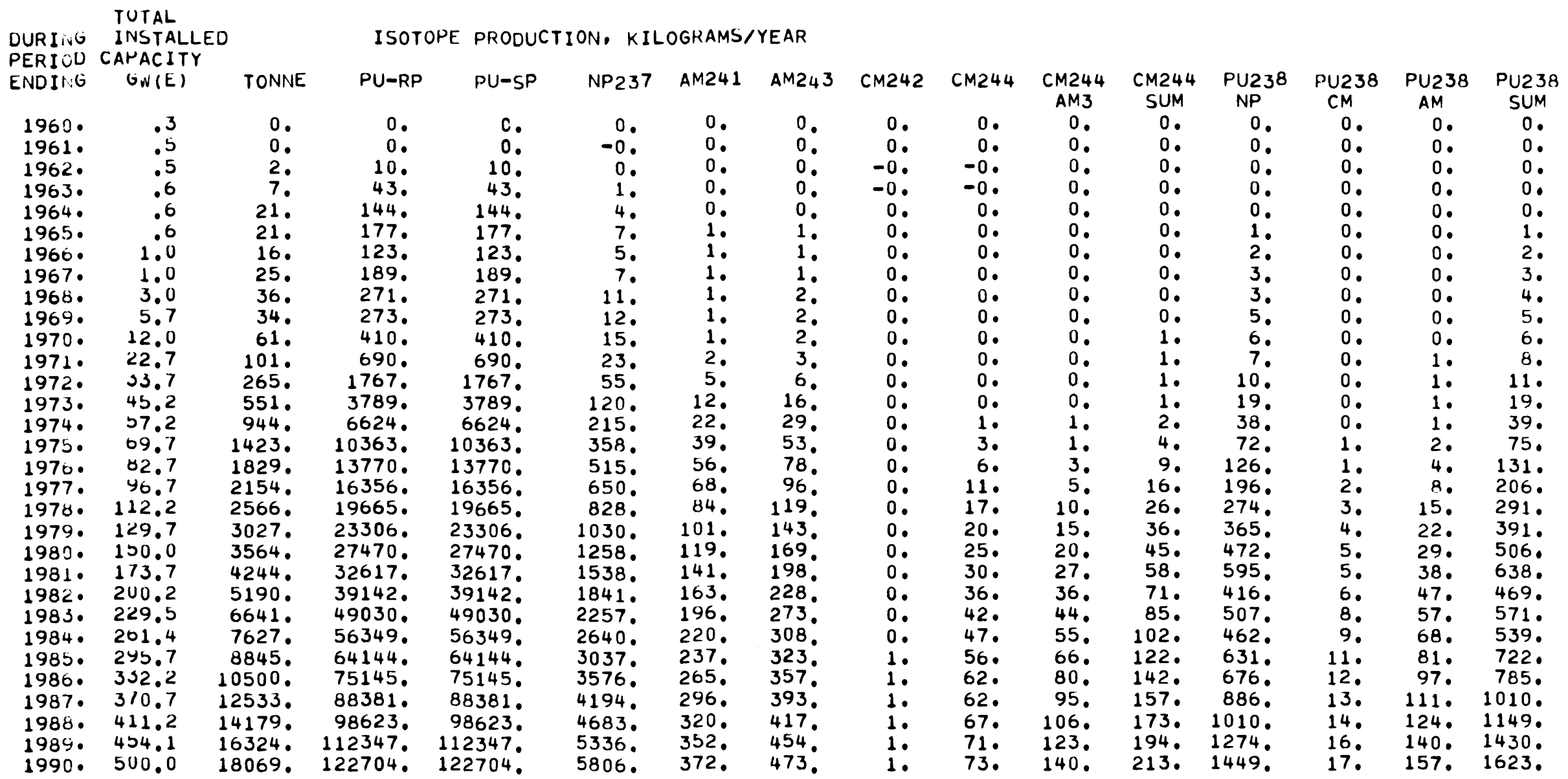


NET ANNUAL I SOTOPE A V A I LAB I L I T Y

(WITH TARGET IRRADIATION. PROCESS LOSSES AIND DECAY LOSSES)

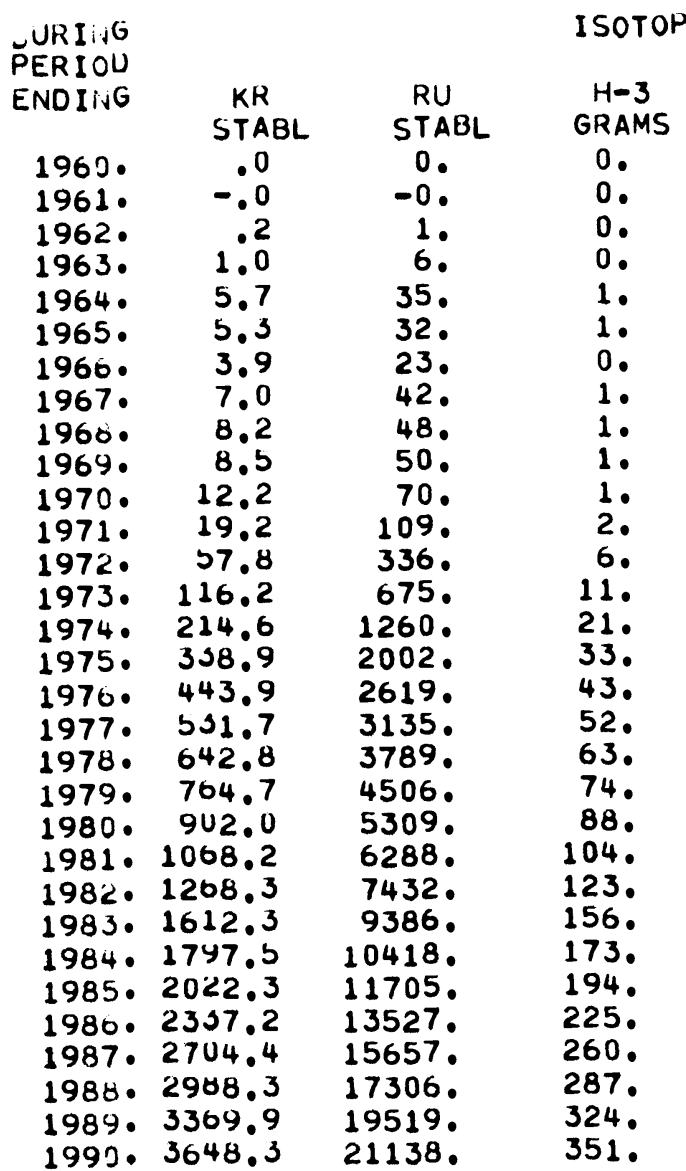


(WITH TANGET IRRADIATION, PROCESS LOSSES AND DECAY LOSSES)

TUTAL

INS I ALLED

EAR CAPACITY

ISOTOPE PRODUCTION, KGS

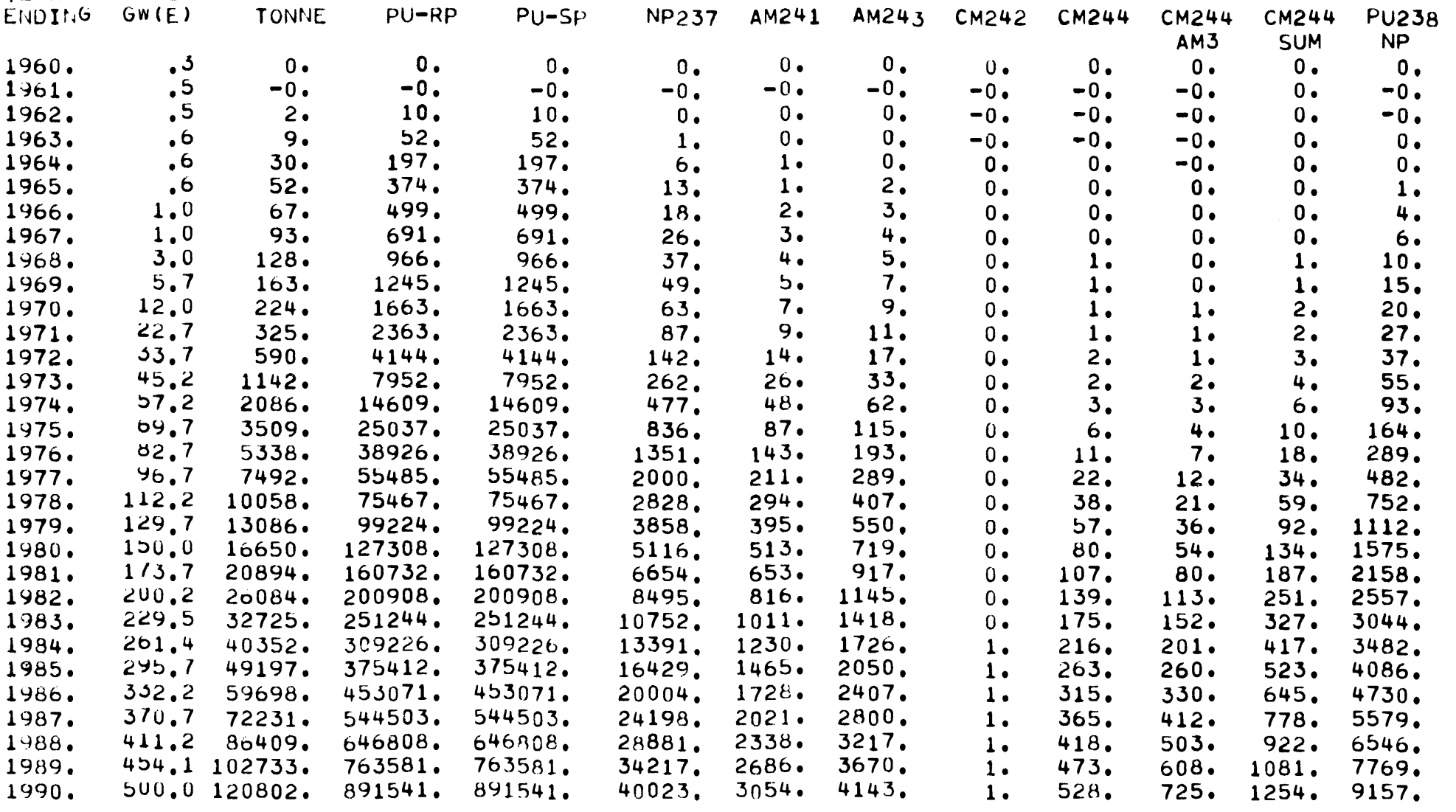

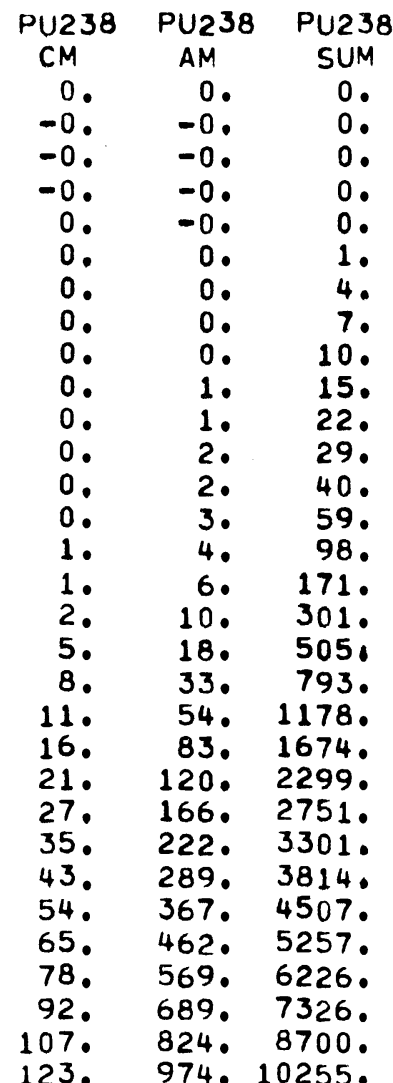


(WITH TARGET IRRAMIATION, PROCESS LOSSES AND DECAY LOSSES)

YEAR

ENDIING

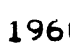

1960

1961.

1963.

1964.

1965.

1966.

1967.

1968.

1969.

1970 .

1971.

1972.

1973.

1974.

1976.

1976

1978 .

1979.

1980 .

1981

1982

1983

1984.

1986.

1987.

1988.

$1990^{\circ}$
ISOTOPE PRODUCTION, KGS

\section{GW-YR U-235}

$.2 \quad 0$.

$$
1 \div 0
$$

1.5

2.1

2.6

3.4

4.3
6.8
11.7

11.7

41.21960.

69.95458 .

108.310446.

156.918840 .

216.230812.

286.545915 .

368.763820 .

404.085150 .

574.2110382 .

701.6140298 .

849.2176026.

1019.3219956.

1214.2275931.

1436.0340644 .

1606.8416856.

2281.2616300 .

2627.8739204.

3009.5880882 .

3428.61037611 .
U-236 PM147

$\begin{array}{rr}0 . & 0 \\ -0 . & -0\end{array}$

0.
-0.

3.

17.
65.
126.

174.

245 .

344 .

450 .

598.

844.

1457 .

2766 .

5094

8819

14038 .

20647.

29041 .

39523 .

52406 .

68324.

87663.

111611 .

$139929^{\circ}$

173253 .

213181 .

260965 .

315116 .

377664 .

446596 .

$\begin{array}{r}0 . \\ -0 . \\ -0 . \\ -0 . \\ 0 \\ 0 \\ 2 . \\ 3 . \\ 4 . \\ 5 . \\ 6 . \\ 7 \\ 10 \\ 14 \\ 28 \\ 58 \\ 108 \\ 182 . \\ 267 \\ 357 \\ 456 . \\ 565 \\ 688 \\ 829 \\ 1000 \\ 1235 \\ 1480 \\ 1747 \\ 2062 \\ 2434 \\ 2824^{\circ} \\ \hline\end{array}$

1

1. 24.

4. 24.

4.55.

5. 78.

$\begin{array}{lll}7 . & 132 . & 47 .\end{array}$

$10.70^{\circ} \quad 170$.

38.425 .194$.

$\begin{array}{rrr}80 . & 815^{\circ} & 372 . \\ 145 . & 1540 . & 700 .\end{array}$

232. 2681.1216 .

320 . 4160. 1883 .

398 . 5910. 2673.

483. 8004. 3617.

578. 10473 . 4730 .

685.13359 .6032$.

814.16754 .7562 .

1242.25828. $11677^{\circ}$

$1481^{\circ} 31416.14223^{\circ}$

1734 . 37651. 17065 .

2048 . 44833. 20339.

2428 . 53124. 24116.

2783.62210 . 28252 .

3570 . 83357 • 37868 .
CE144 CS137 SR-90

KR-85 TC-99 RH103

RU106

PD
STABL

$X E$

CM242 AM241

$\begin{array}{rrr}0 . & 0 . & 0 \\ -0 . & -0 . & -0\end{array}$

16. 232. 105 .
160

0.12

$\begin{array}{rrr}0 . & 3 . & 2 . \\ 0 . & 17 . & 9 . \\ 1 . & 30 . & 16 .\end{array}$

1.39.

1. 76.

2. 96.

3. 124.

8. 307.

16. 583.

51. 1904.

110.4225 .1538

147.5752 .2962$.

191. 7568. 3883.

241.9706 .4967$.

299. 12240. 6252.

368. 15242. 7781 .

456. 19047.9723.

552. 23283. 11895 .

780 . 33578. 17233.

921 . 39980 . 20595.

1073. 47064. 24340.

1243. 55064. 28591 .

1422. 63737. 33229 .
658 . 28055. 14359 . $\begin{array}{rrr}0 . & 0 . & 0 . \\ -0 . & -0 . & -0 . \\ 0 . & 0 . & 3 . \\ 0 . & 2 . & 17 . \\ 1 . & 15 . & 96 . \\ 2 . & 26 . & 169 .\end{array}$

169.

222.

317.

429 .

545 .

712 .

6. 145. 976 .

15. 261. 1767 .

32. 495. 3361.

60. 936.6303

139. 2563. 17006 。

$177.3666^{\circ} 24261$.

217. 4999. 33022

261. 6582. 43436 .

370. 10654. 70262。

441.13251 .87574$.

$547,16508,109525$

647.20104 .134067 .

754. 24139. 161771.

887 . 28802. 193917.

1048 . 34200. 231266.

1203. 40170 . 272663 .

1380 . 46904. 319477.

1547 . 54200. $370323^{\circ}$
311 . 8446. 55714.
AM1 PU1

$\begin{array}{rr}0 . & 0 . \\ -0 . & -0 .\end{array}$

$\begin{array}{ll}-0 . & -0 . \\ -0 . & 0 .\end{array}$

$-0 . \quad 1$.

2.2

0.9

0.15.

0.33

0.46

0.65.

0.163.

0.282.

0.485

0.1250 .

1. 1862 .

1. 3696

1. 4997 .

2. 6622 .

2. 8654

2. 11154 .

3. 14188 .

3. 17849

4. 27470 .

4. 33635 . 
(WITH TARGET IRRADIATION, PROCESS LOSSES AND DECAY LOSSES)

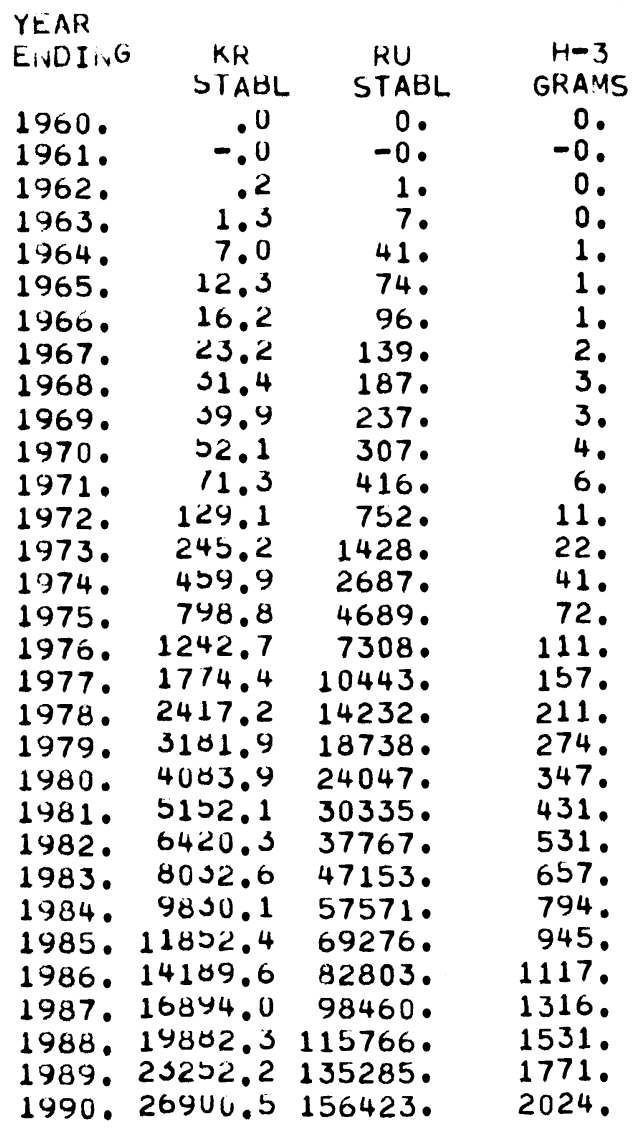


I SOTOPE PRODUCTION

LWR ' S - PU RECYCLE 
D I S T R I BUTION OF ELECTRICAL GENERATA

PAGE $\quad 64$

L W ' S -- PU RECYCLE

CASE 3

\begin{tabular}{|c|c|c|c|c|c|c|c|c|c|c|c|c|c|c|c|c|}
\hline $\begin{array}{l}\text { YEAR } \\
\text { ENDI/,G }\end{array}$ & $\begin{array}{l}\text { TUTAL } \\
\text { INSTALLED } \\
\text { CAPACITY }\end{array}$ & $\begin{array}{l}\text { NEWLY } \\
\text { INSTALLEU } \\
\text { CAPACITY }\end{array}$ & & NEWL & $Y$ INST & ALLED & APACI & TY BY F & REACTOF & Q TYPE, & - GIGAL & NATTS & ELECTR & ICAL & & \\
\hline & GW $(E)$ & $G W(E)$ & PWR 70 & BWR 70 & PWR80 & BWR 80 & $\begin{array}{c}\text { PWR 70 } \\
30 U\end{array}$ & $\begin{array}{c}B, 1 R 70 \\
30 U\end{array}$ & $\begin{array}{c}\text { PWK80 } \\
30 U\end{array}$ & $\begin{array}{c}\text { BWR80 } \\
30 U\end{array}$ & $\begin{array}{c}\text { LWR70 } \\
4 P U\end{array}$ & $\begin{array}{c}\text { LWR80 } \\
\text { 4PU }\end{array}$ & $\begin{array}{r}\text { LWR70 } \\
10 P U\end{array}$ & $\begin{array}{l}\text { LWR } 70 \\
30 P U\end{array}$ & $\begin{array}{r}\text { LWR80 } \\
30 P U\end{array}$ & LMFBF \\
\hline 1960. & .3 & .3 & .1 & .2 & .0 & .0 & $\bullet 0$ & .0 & .0 & .0 & .0 & .0 & .0 & $\cdot 0$ & .0 & .0 \\
\hline 1961. & .5 & .2 & .2 & .0 & .0 & $\cdot 0$ & $\cdot 0$ & .0 & .0 & .0 & .0 & .0 & .0 & $\cdot 0$ & .0 & .0 \\
\hline 1962. & .5 & .0 & .0 & .0 & .0 & .0 & $\cdot 0$ & .0 & .0 & .0 & .0 & .0 & .0 & .0 & .0 & .0 \\
\hline 1963. & .6 & .1 & .0 & .1 & .0 & .0 & $\bullet 0$ & .0 & .0 & .0 & .0 & .0 & .0 & .0 & .0 & .0 \\
\hline 1964. & .6 & .0 & .0 & .0 & .0 & .0 & .0 & .0 & .0 & .0 & .0 & .0 & .0 & .0 & .0 & .0 \\
\hline 1965. & .6 & .0 & .0 & .0 & .0 & .0 & .0 & .0 & .0 & .0 & .0 & .0 & .0 & .0 & .0 & .0 \\
\hline 1966. & 1.0 & .4 & .3 & .1 & .0 & .0 & $\cdot 0$ & .0 & .0 & .0 & .0 & .0 & .0 & .0 & .0 & .0 \\
\hline 1967. & 1.0 & .0 & .0 & .0 & .0 & .0 & .0 & .0 & .0 & .0 & .0 & .0 & .0 & .0 & .0 & .0 \\
\hline 1968. & 3.0 & 2.0 & .9 & 1.1 & .0 & .0 & .0 & .0 & .0 & .0 & .0 & .0 & .0 & .0 & .0 & .0 \\
\hline 1969. & 3.7 & 2.7 & 1.4 & 1.3 & .0 & .0 & .0 & .0 & .0 & .0 & .0 & .0 & .0 & .0 & .0 & .0 \\
\hline 1970 . & 12.0 & 6.3 & 3.1 & 3.2 & .0 & .0 & .0 & .0 & .0 & .0 & .0 & .0 & .0 & .0 & .0 & .0 \\
\hline 1971. & 22.7 & 10.7 & 6.3 & 4.4 & .0 & .0 & .0 & .0 & .0 & .0 & .0 & .0 & .0 & .0 & .0 & .0 \\
\hline 1972. & 33.7 & 11.0 & 7.3 & 3.7 & .0 & .0 & $\bullet 0$ & .0 & .0 & .0 & .0 & .0 & .0 & .0 & .0 & .0 \\
\hline 1973. & 45.2 & 11.5 & 7.7 & 3.8 & .0 & .0 & .0 & .0 & .0 & .0 & .0 & .0 & .0 & .0 & .0 & .0 \\
\hline 1974. & 57.2 & 12.0 & 6.0 & $3 \cdot 0$ & .0 & $\cdot 0$ & $\cdot 0$ & .0 & .0 & .0 & .0 & .0 & $\cdot 0$ & $3 \cdot 0$ & $\cdot 0$ & .0 \\
\hline 1975. & 69.7 & 12.5 & 6.5 & 3.0 & .0 & .0 & $\cdot 0$ & .0 & .0 & .0 & .0 & .0 & .0 & $3 \cdot 0$ & .0 & .0 \\
\hline 1976. & 82.7 & 13.0 & 6.7 & $3 \cdot 3$ & .0 & $\cdot 0$ & $\bullet 0$ & .0 & .0 & .0 & .0 & .0 & .0 & $3 \cdot 0$ & .0 & .0 \\
\hline 1977. & 96.7 & 14.0 & 7.0 & 3.5 & .0 & $\cdot 0$ & $\cdot 0$ & .0 & .0 & .0 & .0 & .0 & .0 & $3 \cdot 5$ & .0 & .0 \\
\hline 1978. & 112.2 & 15.5 & 7.7 & 3.8 & .0 & .0 & $\cdot 0$ & .0 & .0 & .0 & .0 & .0 & .0 & $4 \cdot 0$ & .0 & .0 \\
\hline 1979. & 129.7 & 17.5 & 8.7 & $4 \cdot 3$ & .0 & $\cdot 0$ & $\cdot 0$ & .0 & .0 & .0 & .0 & .0 & . 0 & $4 \cdot 5$ & .0 & .0 \\
\hline 1980. & 150.0 & 20.3 & .0 & .0 & 10.2 & $5 \cdot 1$ & $\bullet 0$ & .0 & .0 & .0 & .0 & .0 & .0 & .0 & 5.0 & .0 \\
\hline 1981. & 173.7 & 23.7 & .0 & .0 & 11.8 & 5.9 & $\bullet 0$ & .0 & .0 & .0 & .0 & .0 & .0 & .0 & 6.0 & .0 \\
\hline 1982. & 200.2 & 26.5 & .0 & .0 & 12.7 & 6.3 & .0 & .0 & .0 & .0 & .0 & .0 & .0 & .0 & 7.5 & .0 \\
\hline 1983. & 229.5 & 29.3 & .0 & .0 & 13.7 & 6.8 & .0 & .0 & .0 & .0 & .0 & .0 & .0 & .0 & 8.8 & .0 \\
\hline 1984. & 261.4 & 31.9 & .0 & .0 & $15 \cdot 0$ & 7.4 & $\cdot 0$ & .0 & .0 & .0 & .0 & .0 & .0 & .0 & 9.5 & .0 \\
\hline 1985. & 295.7 & 34.3 & .0 & .0 & 16.0 & 8.0 & .0 & 0 & .0 & .0 & .0 & .0 & .0 & .0 & 10.3 & .0 \\
\hline 1986. & 332.2 & 36.5 & .0 & .0 & 16.4 & 8.2 & .0 & .0 & .0 & .0 & .0 & .0 & .0 & .0 & 11.9 & .0 \\
\hline 1987. & 370.7 & 38.5 & .0 & .0 & 16.3 & 8.2 & .0 & .0 & .0 & .0 & .0 & .0 & .0 & .0 & 14.0 & .0 \\
\hline 1988. & 411.2 & 40.5 & .0 & .0 & 16.7 & $8 \cdot 3$ & .0 & .0 & .0 & .0 & .0 & .0 & .0 & .0 & 15.5 & .0 \\
\hline 1989. & 454.1 & 42.9 & .0 & .0 & 17.6 & 8.8 & $\cdot 0$ & .0 & .0 & .0 & .0 & .0 & .0 & .0 & 16.5 & .0 \\
\hline 1990 & 500.0 & 45.9 & .0 & .0 & 18.9 & 9.5 & $\cdot 0$ & .0 & .0 & .0 & .0 & .0 & .0 & $\cdot 0$ & 17.5 & 0 \\
\hline
\end{tabular}


DISTRIBUTION OF ELECTRICAL GENERATINGACAPACITY

PAGE 65

$L W R \cdot S$ - PU RECYCLE

CASE 3

TUTAL NEWLY

YEAK IINSTALLED INSTALLEU CUMULATIVE INSTALLED CAPACITY BY REACTUR TYPE. GIGAWATTS ELECTRICAL

PWR70 BWR70 PWR80 BWR80 PWR70 BWR70 PWR80 BWR80 LWR70 LWR80 LWR70 LWR70 LWR80 LMFBR

1960 .

1961.

1963.

1964.

1965.

1966.

1968.

1969.

1970 .

1971 .

GW(E)
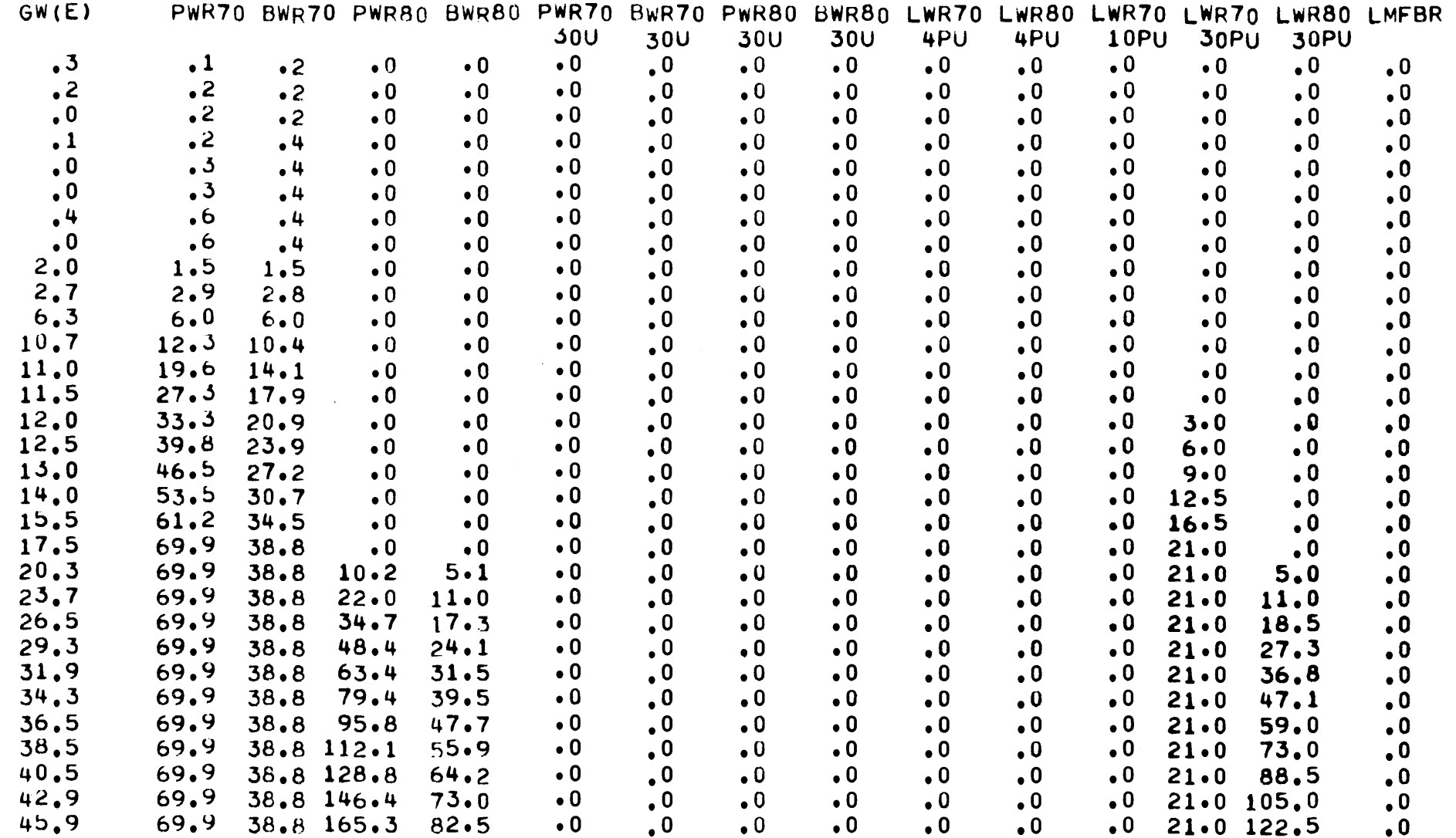

$\begin{array}{rrrr}.1 & .2 & .0 & .0 \\ .2 & .2 & .0 & .0 \\ .2 & .2 & .0 & .0 \\ .2 & .4 & .0 & .0 \\ .3 & .4 & .0 & .0 \\ .3 & .4 & .0 & .0 \\ .6 & .4 & .0 & .0 \\ .6 & .4 & .0 & .0 \\ 1.5 & 1.5 & .0 & .0 \\ 2.9 & 2.8 & .0 & .0 \\ 6.0 & 6.0 & .0 & .0 \\ 12.3 & 10.4 & .0 & .0 \\ 19.6 & 14.1 & .0 & .0 \\ 27.3 & 17.9 & .0 & .0 \\ 33.3 & 20.9 & .0 & .0 \\ 39.8 & 23.9 & .0 & .0 \\ 46.5 & 27.2 & .0 & .0 \\ 53.5 & 30.7 & .0 & .0 \\ 61.2 & 34.5 & .0 & .0 \\ 69.9 & 38.8 & .0 & .0 \\ 69.9 & 38.8 & 10.2 & 5.1 \\ 69.9 & 38.8 & 22.0 & 11.0 \\ 69.9 & 38.8 & 34.7 & 17.3 \\ 69.9 & 38.8 & 48.4 & 24.1 \\ 69.9 & 38.8 & 63.4 & 31.5 \\ 69.9 & 38.8 & 79.4 & 39.5 \\ 69.9 & 38.8 & 95.8 & 47.7 \\ 69.9 & 38.8 & 112.1 & 55.9 \\ 69.9 & 38.8 & 128.8 & 64.2 \\ 69.9 & 38.8 & 146.4 & 73.0 \\ 69.9 & 38.8 & 165.3 & 82.5\end{array}$

.0
.0
.0
.0
.0
.0
.0
.0
.0
.0
.0
.0
.0
.0
.0
.0
.0
.0
.0
.0
.0
.0
.0
.0
.0
.0
.0
.0
.0
.0
.0

.0
.0
.0
.0
.0
.0
.0
.0
.0
.0
.0
.0
.0
.0
.0
.0
.0
.0
.0
.0
.0
.0
.0
.0
.0
.0
.0
.0
.0
.0
.0

.0
.0
.0
.0
.0
.0
.0
.0
.0
.0
.0
.0
.0
.0
.0
.0
.0
.0
.0
.0
.0
.0
.0
.0
.0
.0
.0
.0
.0
.0

.0
.0
.0
.0
.0
.0
.0
.0
.0
.0
.0
.0
.0
.0
.0
.0
.0
.0
.0
.0
.0
.0
.0
.0
.0
.0
.0
.0
.0
.0
.0

- 0

.0
.0
.0

10
.0

1973. 45.2

$1974 . \quad 57.2$

1977.

82.7
96.7

112.2

129.7

1980.150 .0

$1981 . \quad 173.7$

1982.

200.2

261.4

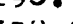

454.1

45.9

$69.9 \quad 38.8 \quad 165.3 \quad 82.5$ 

ANNUAL ISOTOPE PRODUCTION AT DISCHARGE (NO LOSS BASIS)

LWR P $\mathrm{S}$ - PU RECYCLE

CASE 3

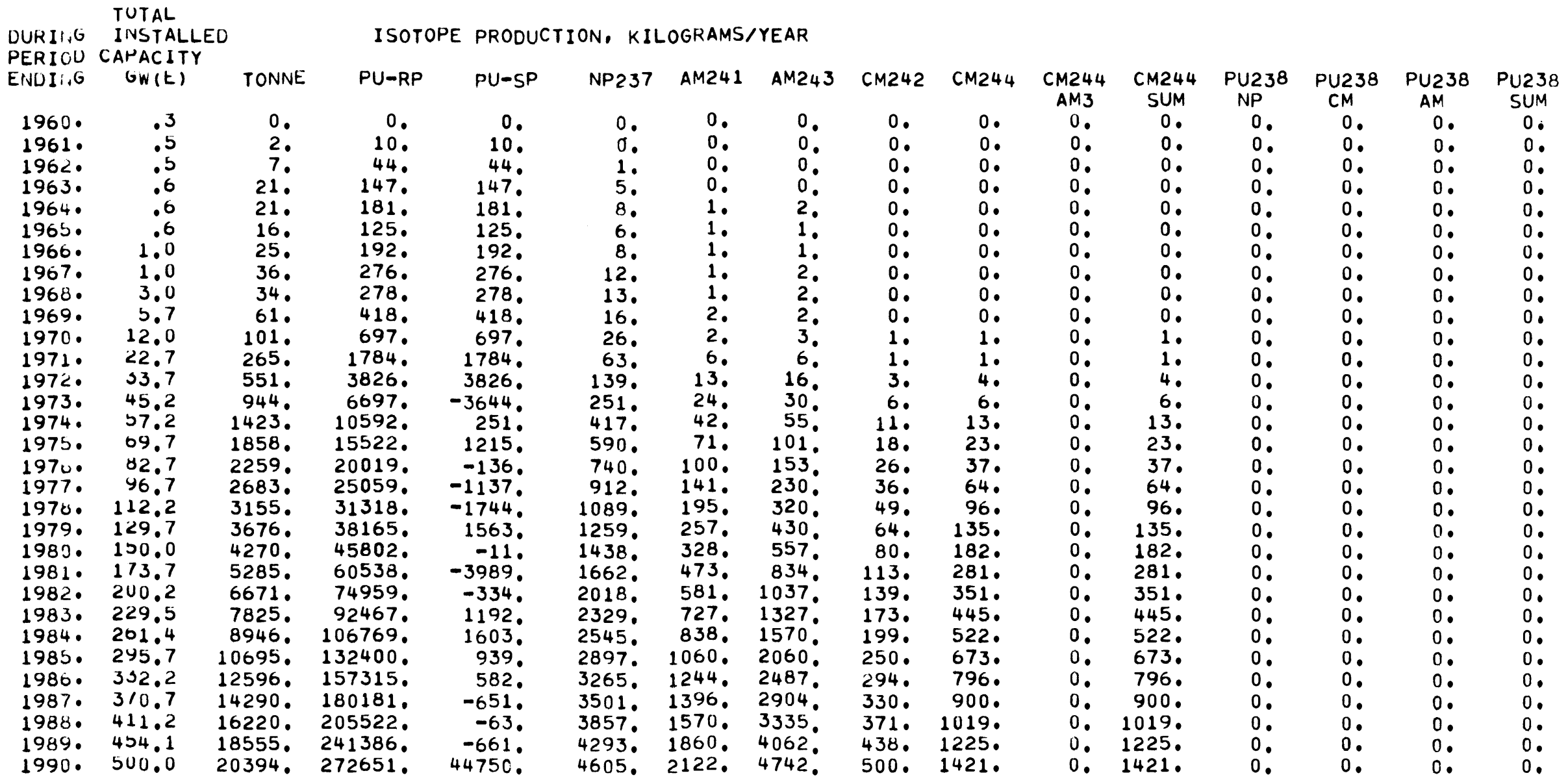


(NO LOSS BASIS)

URIING PERIOU ENDIING

1960.

1961.

1962.

1963.

$1964^{\circ}$

1965.

1966.

1967.

1960.

1969.

$1970^{\circ}$

1971.

1972.

1973.

1974 .

1975.

$19760^{\circ}$

1977.

1978.

$197 \% \circ$

1981.147 .6

1983.144 .9

$19840^{\circ} 221.9$

1985.250 .8

1986. 281.3

1987.

1980.

$1989^{\circ}$

313.2

$346.6 \quad 1267$

$301.7 \quad 143900$.

419.1156926 .
ISOTOPE PRODUCTION, KILOGRAMS/YEAR

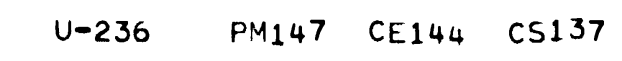

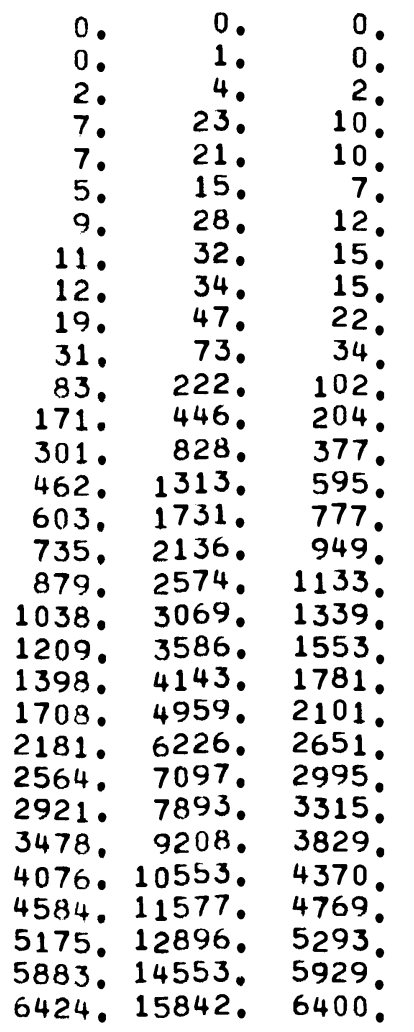

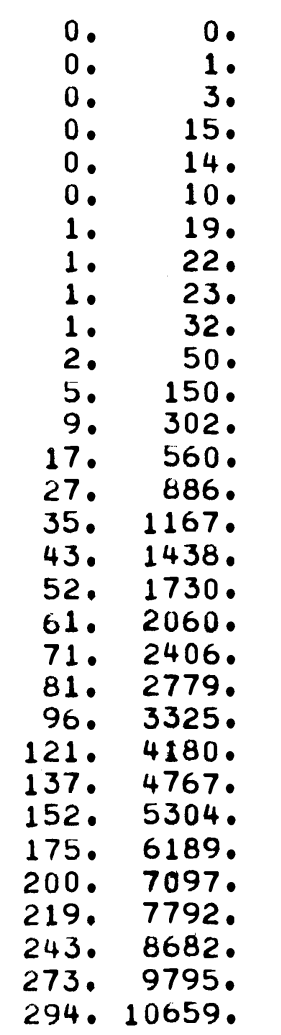


(NO LOSS BASIS

$$
\begin{aligned}
& \text { KR } \\
& \text { STABL }
\end{aligned}
$$

1960.

1961.

1962.

1963.

1964.

1965.

1960

1967.

1968.

1969.

1970 .

1971 .

1973.254 .6

$19740^{\circ} 310.5$

1975.483 .5

$1975^{\circ} 483.5$

$\begin{array}{ll}1976 . & 590.5 \\ 1977 . & 703.8\end{array}$

1978.831 .4

1979. 963.

$1980 \cdot 1104.2$

1981.1300 .5

1982.1640 .0

1983.1847 .5

1984. 2041.3

1985.2353 .4

$1986 \cdot 2682.2$

$1987 \cdot 2923.1$

1988.3242 .2

$1989 \cdot 3628.6$

1990.3913 .2

STABL
.0
.3

1.2
6.4
5.9

5.9
4.3

7.8

.1

120

369.

739.

1378 .

2191 .

2896.

3589.

4343.

5195 .

6090 .

7057 .

8496.

10638 .

12154.

13533 .

$H-3$
GRAMS

227.

18166.302 .

19957. 331.

22259. 369 .

25191 . 416 .

0 .

0.

1.

0 .

1.

1.

2.

13.

24.

50

62.

89.

04.

120.

27511 . 452 . 
CUMULATIVE ISOTOFE PRODUCTION AT D I S CHARGE

(NO LOSSES EXCEPT FOR RADIOACTIVE DECAY)

$$
L W R \cdot S \text { - PU RECYCLE }
$$

TOTAL

INS I ALLED IEAR CAPACITY EINDII,G GW IE)

ISOTOPE PRODUCTION, KGS

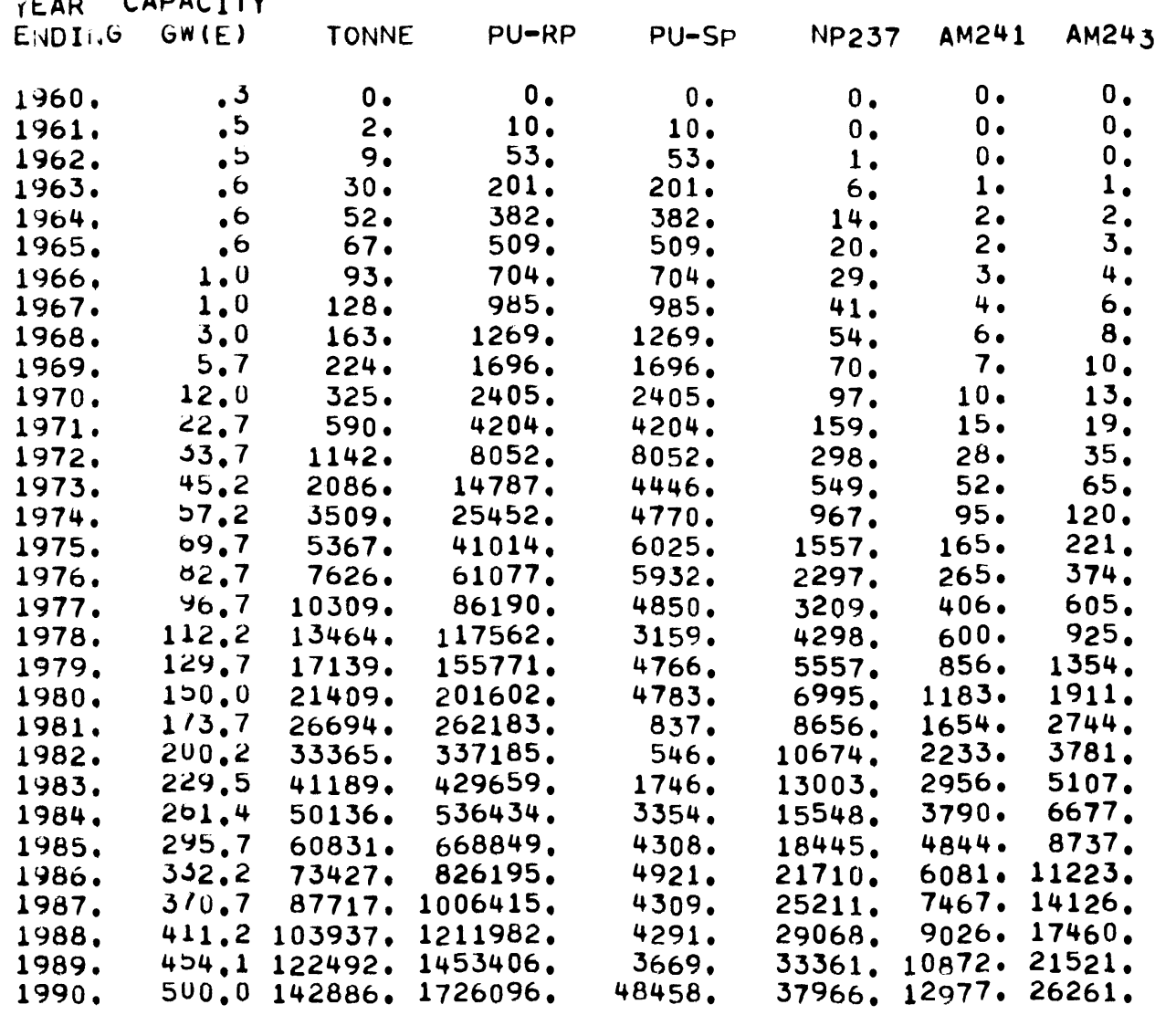

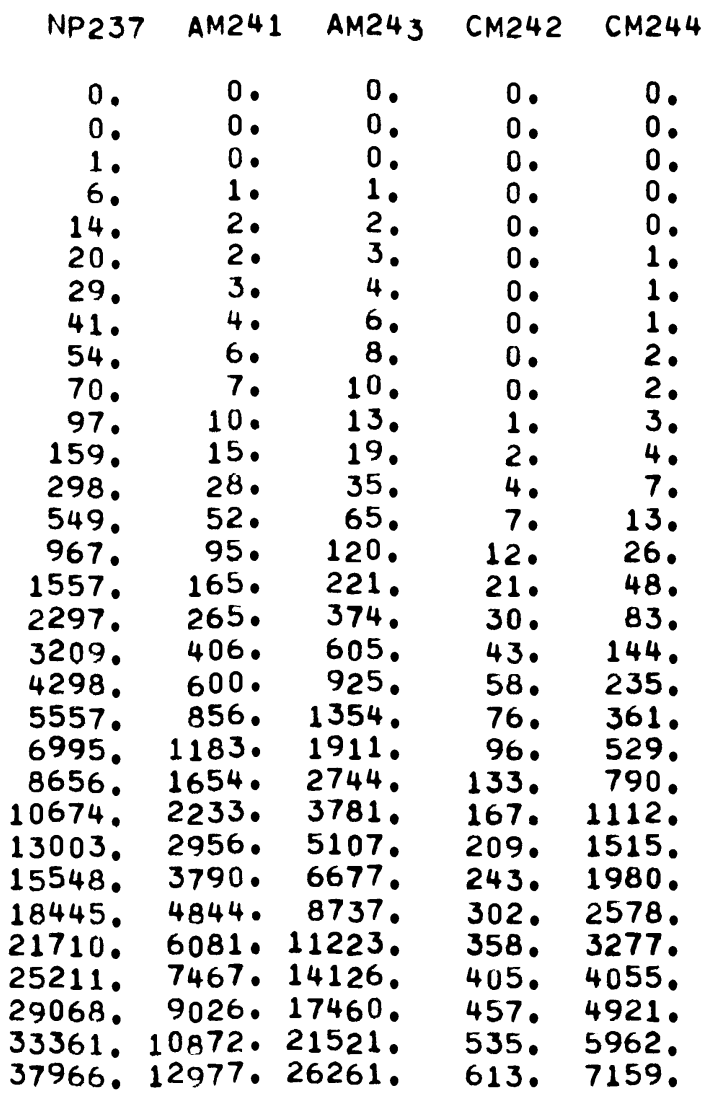

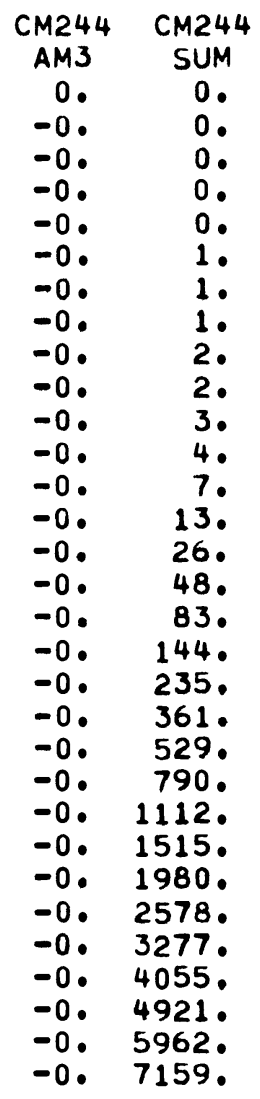

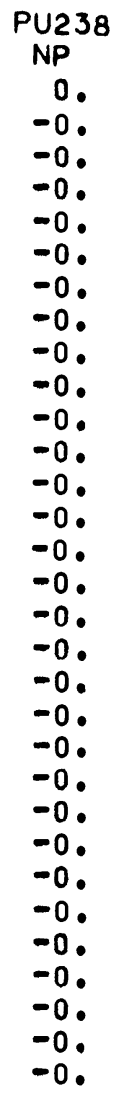

$P U 238$
$C M$
0
-0
-0
-0
-0
-0
-0
-0
-0
-0
-0
-0
-0
-0
-0
-0
-0
-0
-0
-0
-0
-0
-0
-0
-0
-0
-0
-0
-0
-0
-0
0

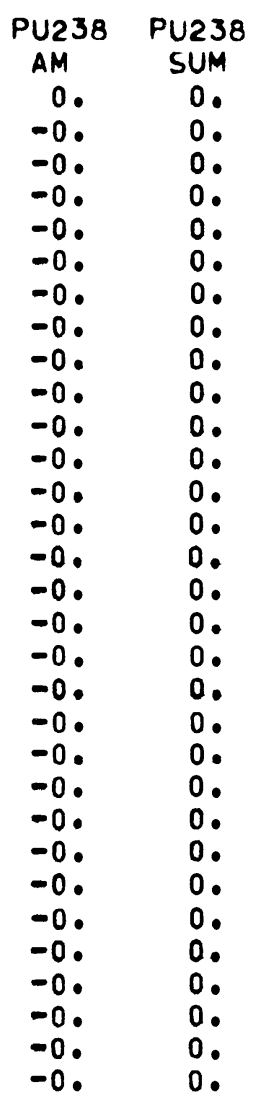


YEAR

1960.

1960.

$1961^{\circ}$

1963.

$1964^{\circ}$.

1966.

1967.

1968.

1970 .

$1971^{\circ}$

1972.

1973.

$1974^{\circ}$

1975.

1976.

$1978^{\circ}$

$1978^{\circ}$

1979.
1980.

1981 .

1982.

1214.2336857.

U-236

N, KGS

$$
\text { GW-YR U-235 }
$$$$
\text { U-2 }
$$

.2
.6
1.0
1.5

$2.1 \quad 440$.

3.4805 .

4.31103 .

$\begin{array}{rr}6.8 & 1405 . \\ 11.7 & 2000 .\end{array}$

21.93008

41.25658 .

108.910919.

136.932419.

216.248100 .

206.566971.

464.0114976 .

574.2145013 .

701.7179639 .

1019.3274942 .

1984.

1985.

1436.1407992.

1686.9492357.

1968.1591721.
2281.3703802 .

1988 .

2627.9830546 .

1989. 3009.6 974445 .

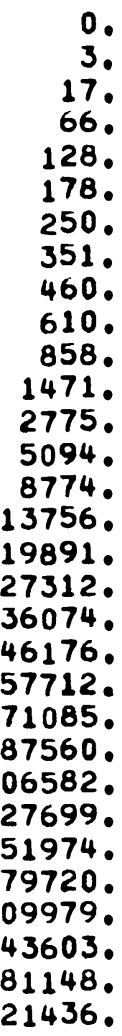

0.

CE144

CS137 SR-9

$\begin{array}{rr}0 . & 0 . \\ 0 . & 1 \circ \\ 2 . & 5 \circ \\ 8 . & 27 \circ \\ 10 . & 48 . \\ 9 . & 62 . \\ 12 . & 89 . \\ 17 . & 119 .\end{array}$

$19^{\circ} 1190$.

26. 193 .

42. 262.119.

100.479 .218$.

$\begin{array}{rrr}213^{\circ} & 914^{\circ} & 417 . \\ 389^{\circ} & 1721^{\circ} & 784 .\end{array}$

622. 2995. 1359.

859. 4657. 2103 .

1089. 6687. 3001.

1584. 11969. 5300。

$1860^{\circ} 15281.6723^{\circ}$

2163. 19075. 8340 .

2598. 23599. 10237 .

3249 . 29285. 12638.

4525. 42791. 18264 .

5339.51021 .21647$.

6272. 60409. 25488.

7164. 70606. 29634.

8122 . 81890. 34203.

10217.108254 • 44734. 1722.81453.48178.

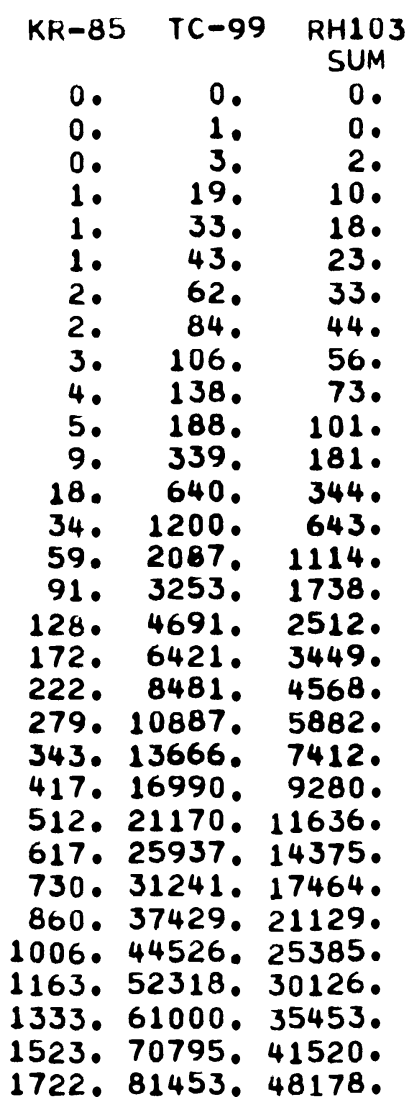

RU106 PT

XE CM242 AM241

1990.

3428.71131371 .321436 .

9478.

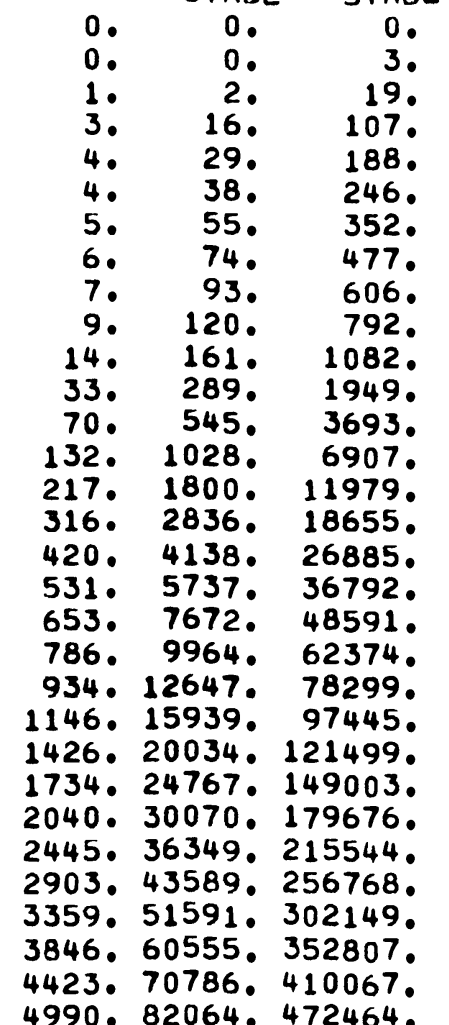

AM1 0.0.

$-0 . \quad-0$.

$\begin{array}{ll}-0 . & 0 . \\ -0 . & 1 .\end{array}$

$-0.26$.

$-0.10$.

$-0.17$.

-0. 26 .

$\begin{array}{ll}-0 . & 37 . \\ -0 . & 52 .\end{array}$

$-0.74$.

-0.112 .

$-0.185$.

$-0 . \quad 225 \circ$

$-0.267$.

-0. 321.

-0.375 .

-0. 418 .

-0.446 .

$\begin{array}{ll}-0 . & 488 . \\ -0 . & 531 .\end{array}$

-0.538 .

-0.542 .

-0.557 .

-0 . 586 .

-0. 624 .

-0. 668.

0.706.

4990. 82064. 472464. $\quad-0 . \quad 776$. 

CUMULATIVE I SOTOPE PROODUCTIIN I A T D I SCHARG (NO LOSSES EXCEPT FOR RADIOACTIVE DECAY)

PAGE 71

LWR $S$ - - PU RECYCLE

\section{ISOTOPE PRODUCTION. KGS}

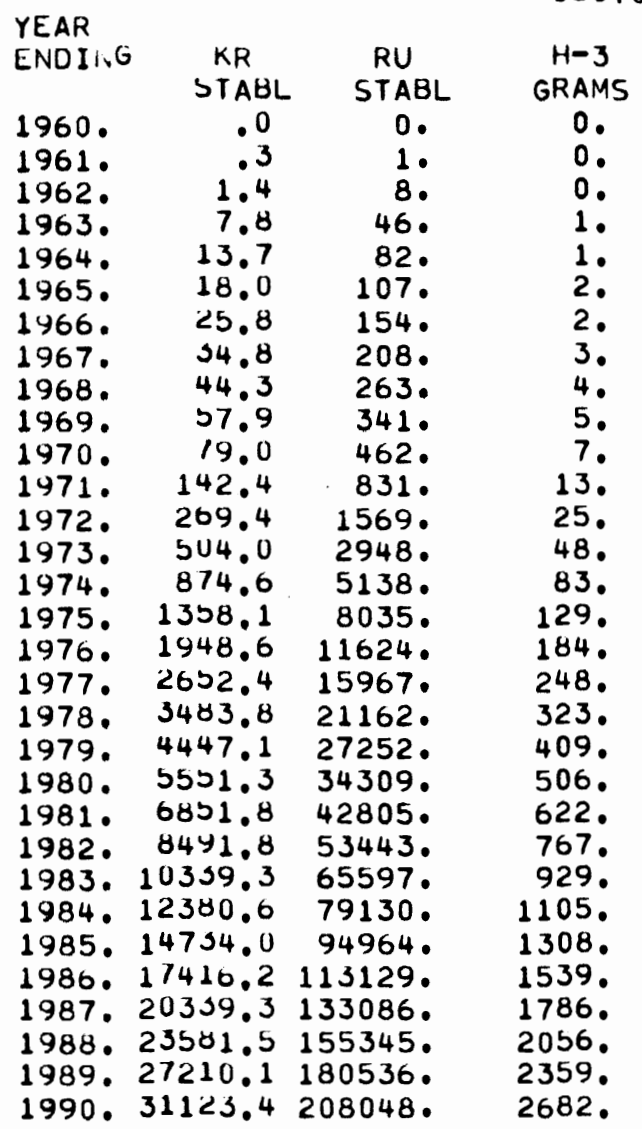


NET ANNUAL I SOTOPE A $V A$ I L A B I L I T Y

(WITH TARGET IRRADIATION, PROCESS LOSSES AND DECAY LOSSES)

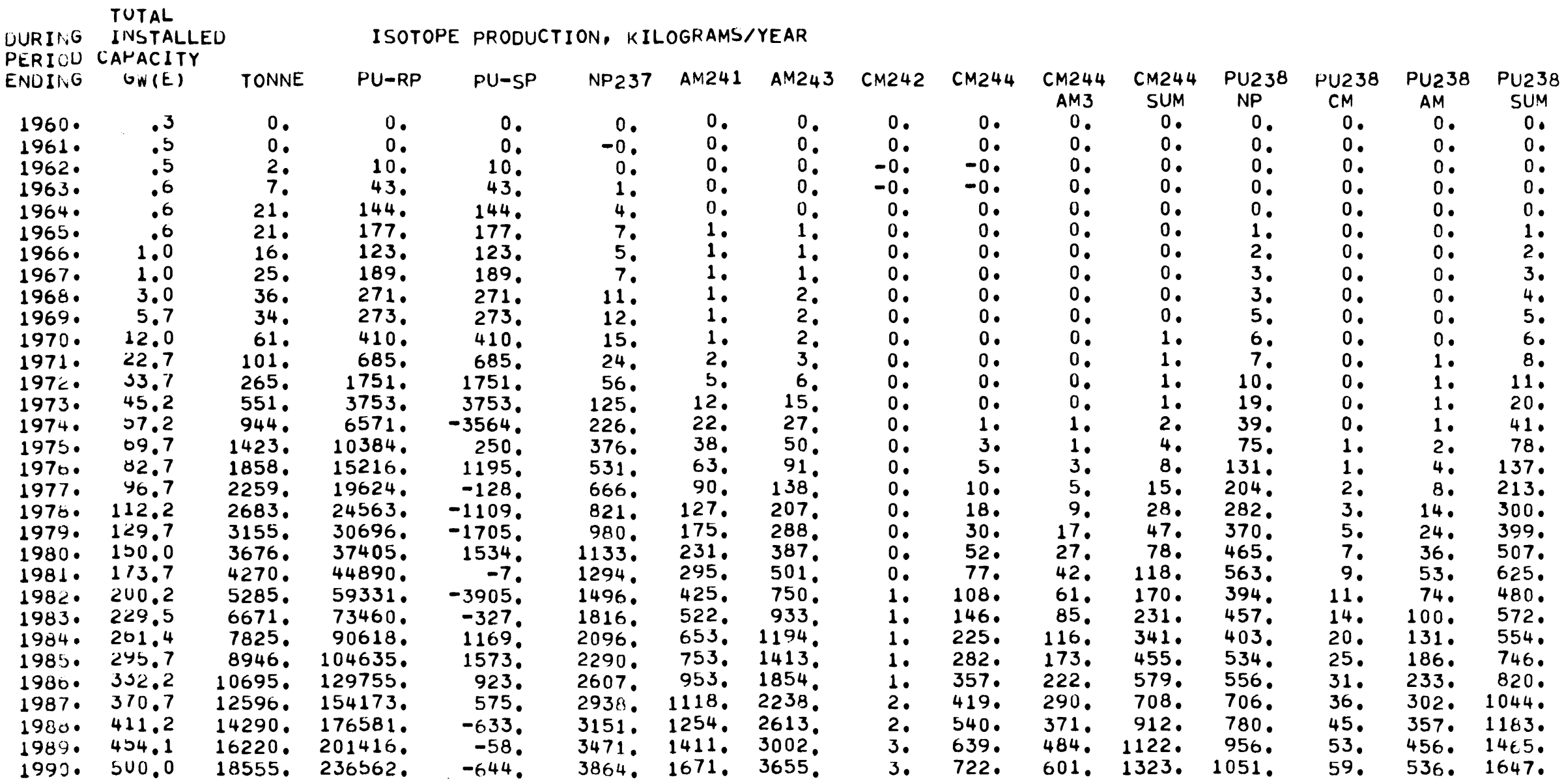


NET A N NUAL I SOT TOPE AVA I L A B I L I T Y

(WITH TARGET IRRADIATION. HROCESS LOSSES AND DECAY LOSSES)

$$
L W R \cdot S-\text { PU RECYCLE }
$$

,URIIVG PERIUU ENDIING

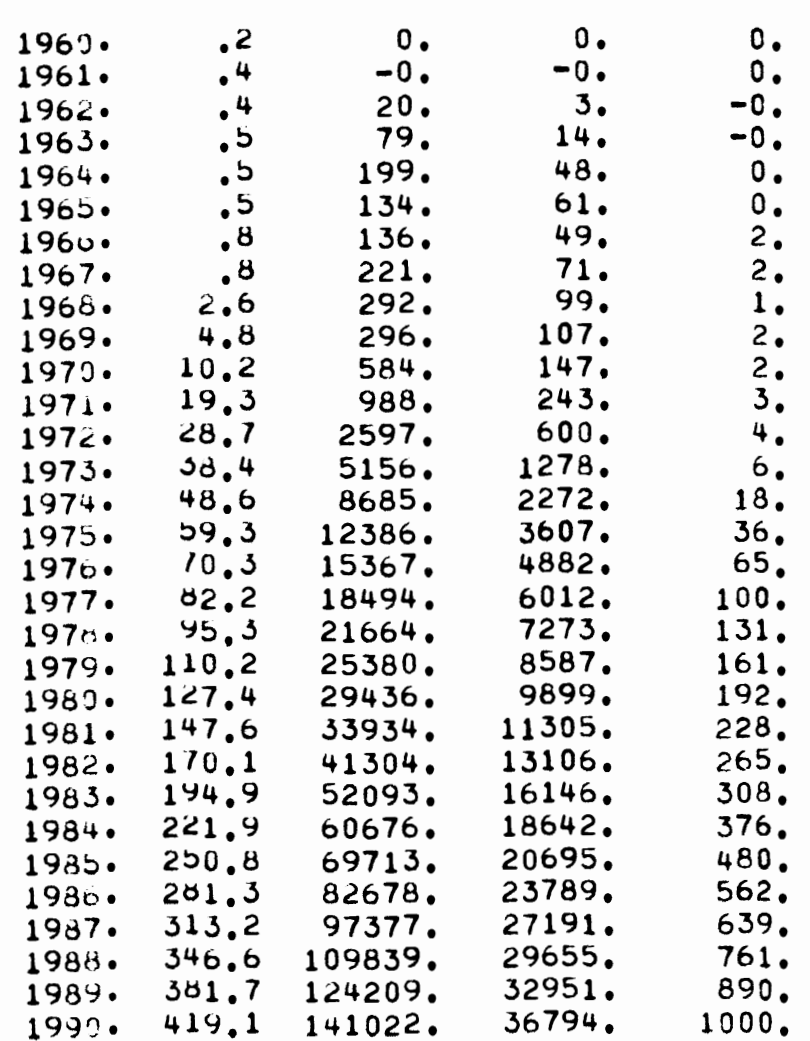

ISOTOPE PRODUCTION, KILOGRAMS/YEAR

PM147 CE144 CS137 SR-90

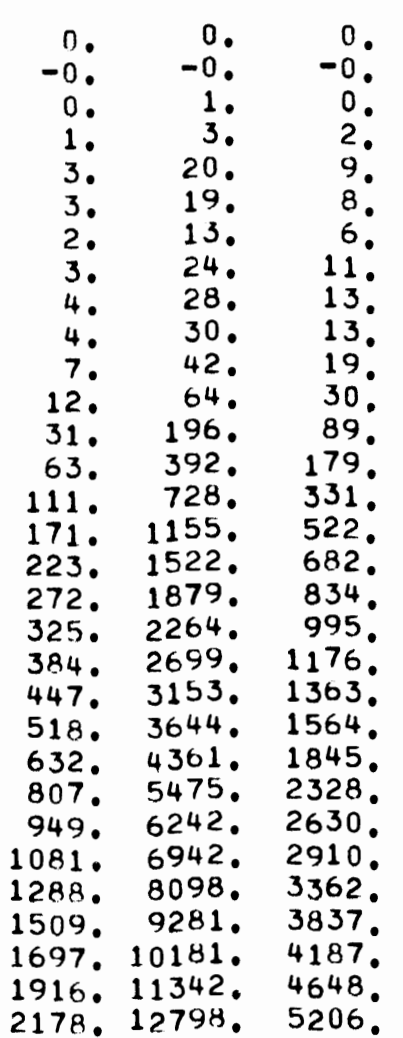

KK-8S TC-99 RH103 RU106

$$
\text { SUM }
$$

$\begin{array}{rr}0 . & 0 . \\ -0 . & -0 . \\ 0 . & 1 . \\ 0 . & 2 . \\ 0 . & 14 . \\ 0 . & 13 . \\ 0 . & 90^{\circ} \\ 0 . & 17 . \\ 1 . & 20 . \\ 1 . & 20 . \\ 1 . & 29 . \\ 1 . & 45 \circ \\ 4 . & 135 \circ \\ 8 . & 272 . \\ 15 . & 504 . \\ 23 . & 798 . \\ 30 . & 1050 . \\ 36 . & 1294 .\end{array}$

36.1294.

44.1557.

$51.1854 \circ 843$.

$60.2165^{\circ}$. 1183.

68. 2501. 1377.

81. 2992. 1681.

$102.3762^{\circ} 2120$.

115. 4290. 2465 .

128. 4774. 2780 .

i48. 5570. 3299.

169. 6388. 3830 .

185. 7013. 4267 .

205. 7814. 4794.

$2300^{\circ} 8816.5460$.

1
1
2
2
3
3
4
465
5
641
7
858
975
1125

STABL
CM242 AM241

AM1 PU1

$0 . \quad 0$.

0.0 .

$0 . \quad 0$.

0.00

0.2 .

$0.2 \quad 2$.

0.4.

0.6 .

0.8.

0.10 .

0.14.

$\begin{array}{llll}115^{\circ} & 781^{\circ} & 0 . & 19{ }^{\circ} \\ 230^{\circ} & 1569^{\circ} & 0 . & 34{ }^{\circ} \\ 435^{\circ} & 2893^{\circ} & 0 . & 64{ }^{\circ}\end{array}$

$\begin{array}{llll}230^{\circ} & 1569^{\circ} & 0 . & 34^{\circ} \\ 435^{\circ} & 2893^{\circ} & 0 . & 64{ }^{\circ}\end{array}$

695. 4564. 0.35 .

932. 6009. 0.38 .

18. 1172. 7407. 0.48.

$\begin{array}{llll}145 . & 1439.8917 . & 0 . & 47 .\end{array}$

07. 2063. 12404. 1.25.

43. 2414.14333. 1. 38.

06. 2963. 17232. 2.38 .

384. 3685.21648. 2० 7 .

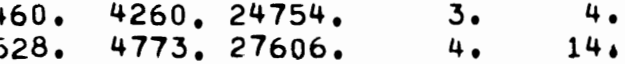

41. 5651.32281. 5. 27.

756. 6516.37102. 7.34.

858. 7202.40843. $\quad 8.39$.

975. $8067.45593^{\circ} \quad 10.34$. 
NET ANNUAL I SOT TOPEAVA I LABIL I T Y

(WITH TARGET IRRADIATION. PROCESS LOSSES AND DECAY LOSSES)

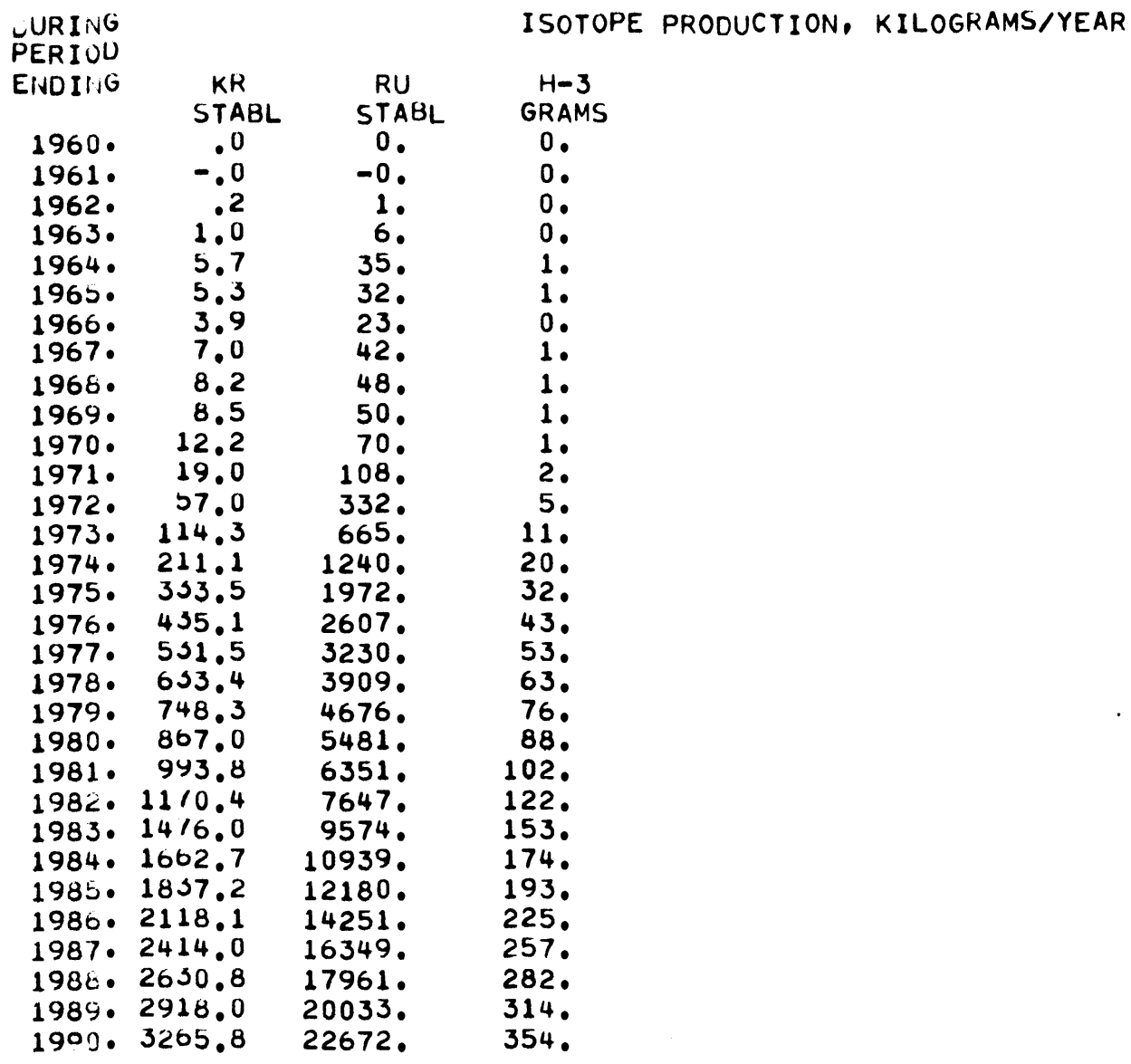


CUMULATIVE ISOTOPE AVA I LABILI T Y

PAGE 75

(WITH TARGET IRRAOIATION, PROCESS LOSSES AND DECAY LOSSES)

LWR $S$ - - PU RECYCLE

CASE 3

TOTAL

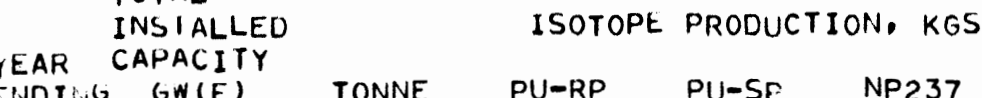

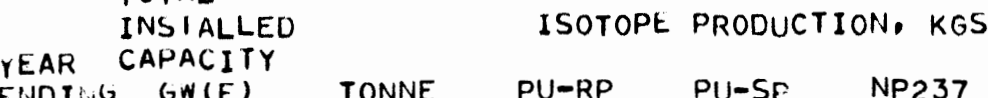

\begin{tabular}{|c|c|c|c|c|c|c|c|}
\hline 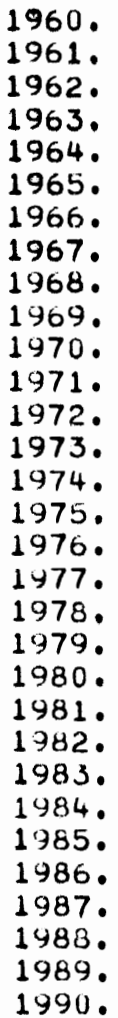 & $\begin{array}{r}.3 \\
.5 \\
.5 \\
.6 \\
.6 \\
.6 \\
1.0 \\
1.0 \\
3.0 \\
5.7 \\
12.0 \\
22.7 \\
33.7 \\
45.2 \\
57.2 \\
09.7 \\
02.7 \\
90.7 \\
112.2 \\
129.7 \\
150.0 \\
113.7 \\
200.2 \\
229.5 \\
201.4 \\
295.7 \\
352.2 \\
310.7 \\
411.2 \\
404.1 \\
500.0\end{array}$ & 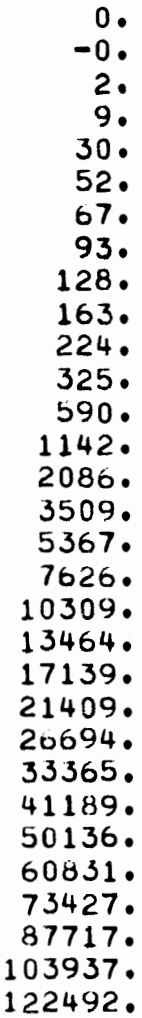 & 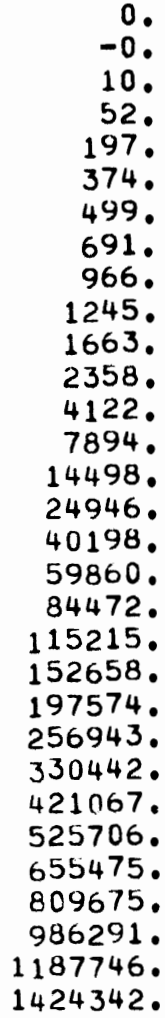 & 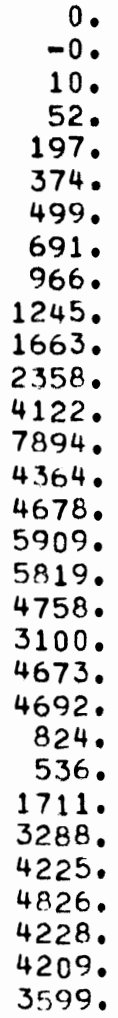 & 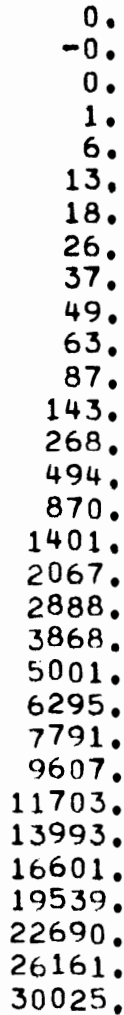 & $\begin{array}{r}0 . \\
-0 . \\
0 . \\
0 . \\
1 . \\
1 . \\
2 . \\
3 . \\
4 . \\
5 . \\
7 . \\
9 . \\
14 . \\
25 . \\
47 . \\
85 . \\
148 . \\
238 . \\
365 . \\
539 \\
770 \\
1063 . \\
1486 . \\
2006 . \\
2657 . \\
3406 . \\
4353 . \\
5464 . \\
6710 . \\
8111 . \\
9770 .\end{array}$ & 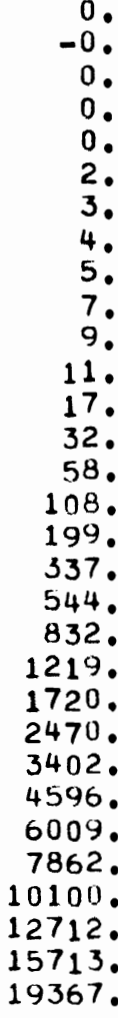 \\
\hline
\end{tabular}

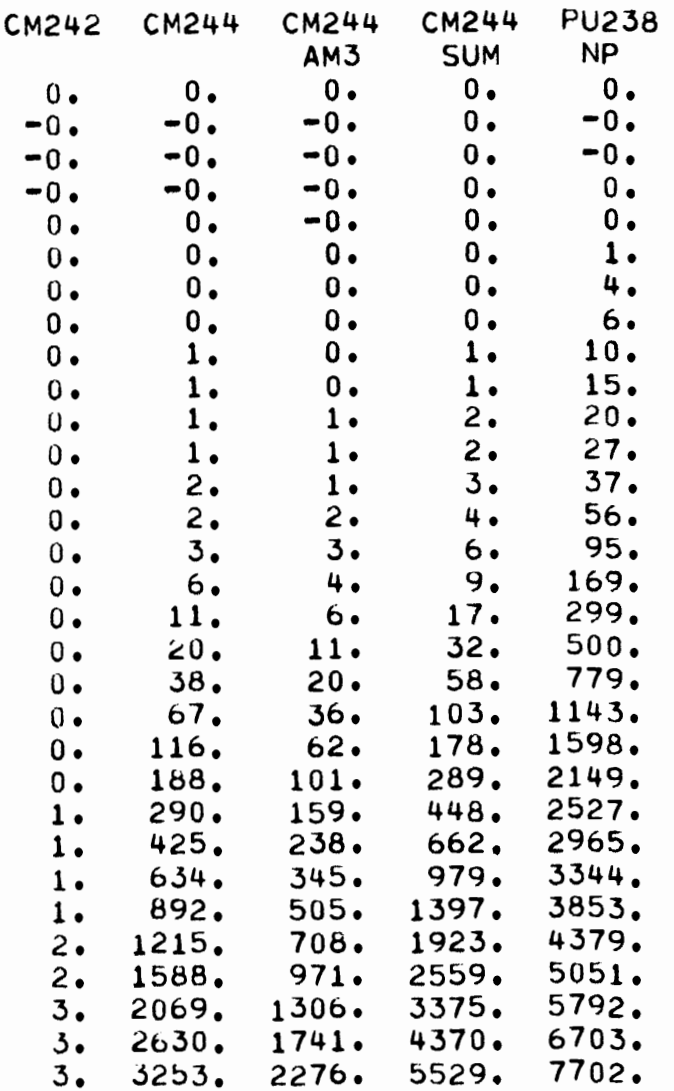

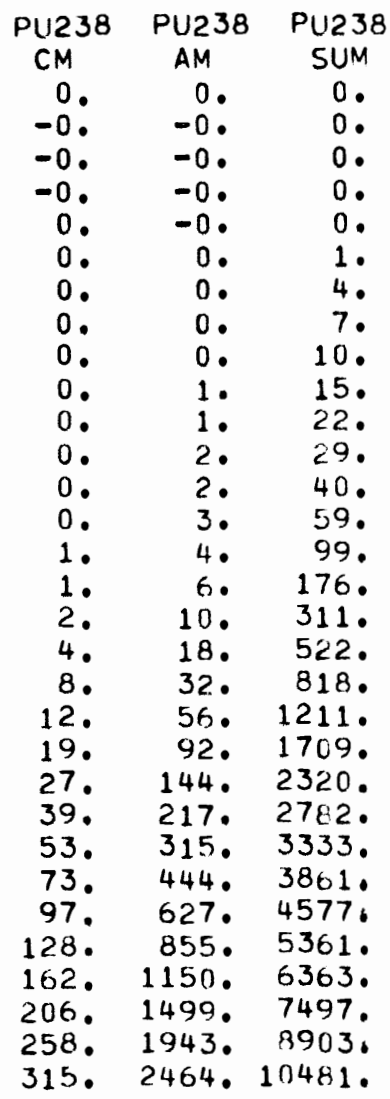


(WITH TARGET IRRADIATION, PROCESS LOSSES AND DECAY LOSSES)

ISOTOPE PRODUCTION, KGS

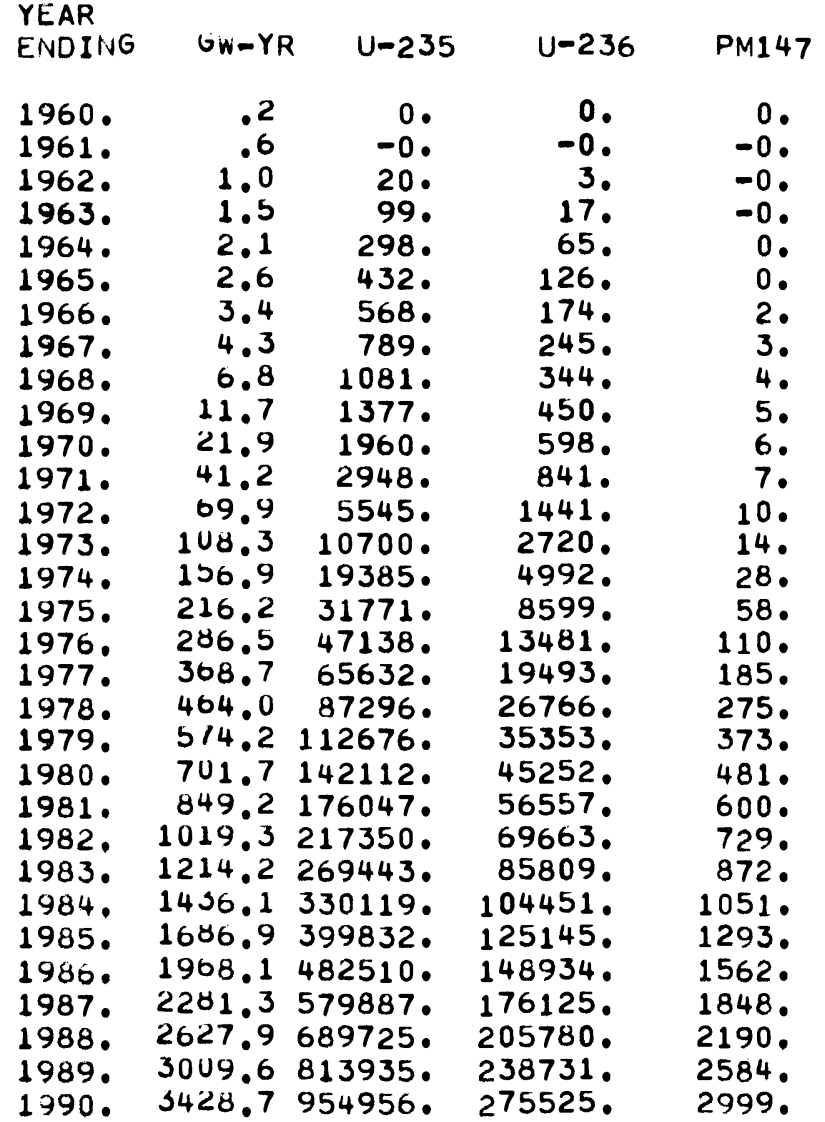

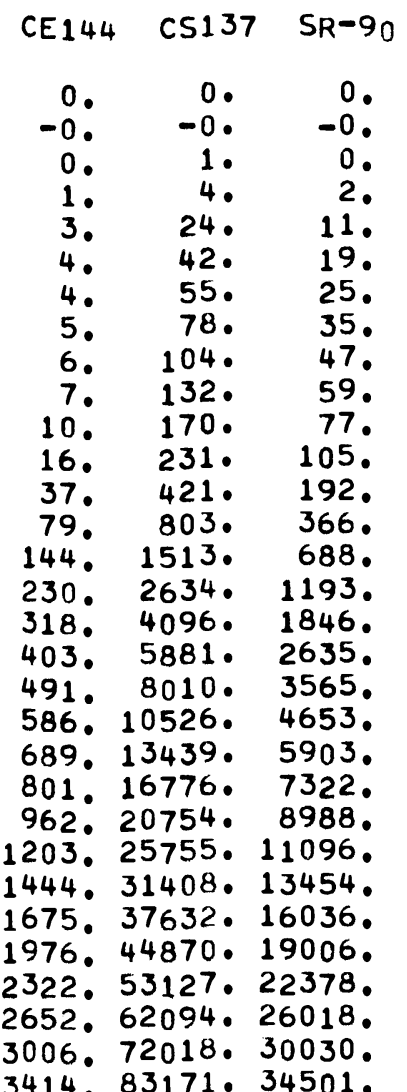

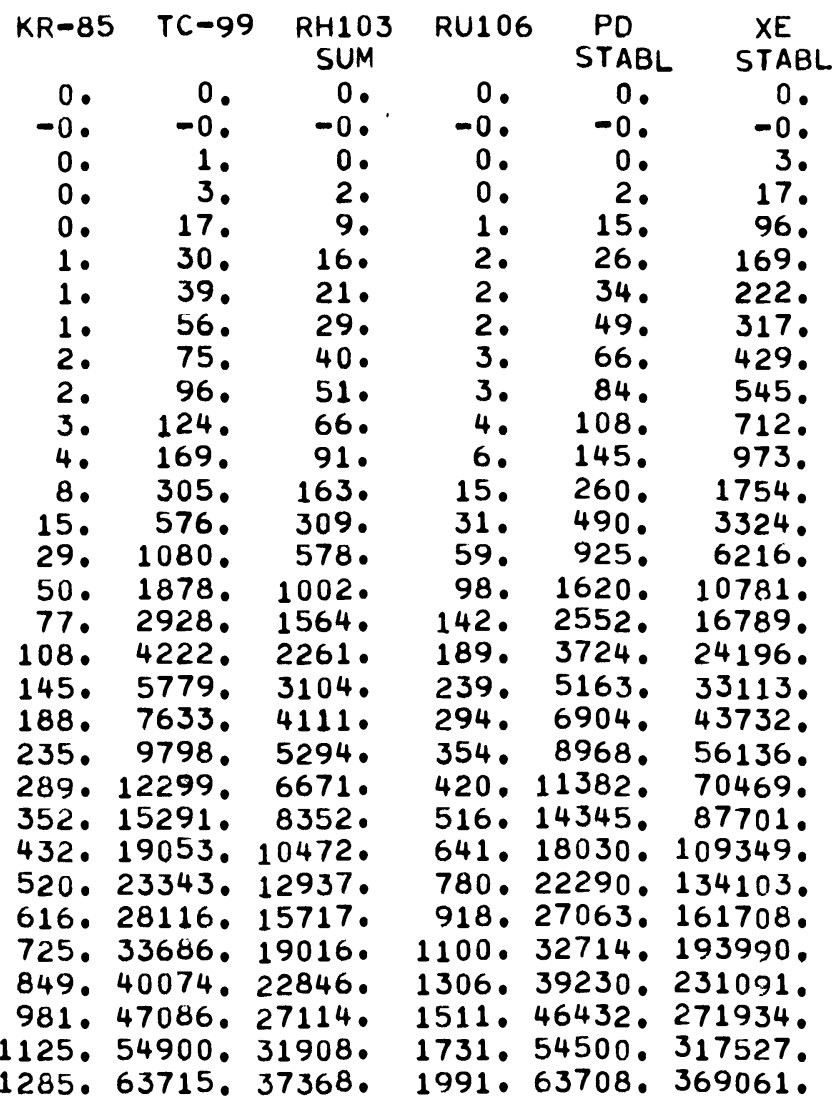

\begin{tabular}{|c|c|}
\hline $\begin{array}{c}\text { CM242 } \\
\text { AM1 }\end{array}$ & $\begin{array}{r}\text { AM24 } \\
\text { PU1 }\end{array}$ \\
\hline 0 & 0 \\
\hline-0 & -0 . \\
\hline-0 & -0 . \\
\hline $\begin{array}{l}-0 . \\
-0 .\end{array}$ & $\begin{array}{l}0 . \\
1 .\end{array}$ \\
\hline 0. & 2 . \\
\hline 0. & 5. \\
\hline 0 & 9. \\
\hline 0. & 15. \\
\hline 0. & 23. \\
\hline 0. & 33. \\
\hline 0. & 46. \\
\hline 0. & 65. \\
\hline $0 \bullet$ & 99. \\
\hline 0. & 163. \\
\hline $0 \bullet$ & 198. \\
\hline $\begin{array}{l}0 . \\
0 .\end{array}$ & $\begin{array}{l}236 \\
283\end{array}$ \\
\hline 0. & 330 \\
\hline 1. & 368. \\
\hline 1. & 393. \\
\hline 1. & 430 \\
\hline 3 & $\begin{array}{l}400 \\
474\end{array}$ \\
\hline 3. & 477. \\
\hline 5. & 491. \\
\hline 6. & 517. \\
\hline 8. & 550. \\
\hline 12. & 622 \\
\hline & \\
\hline
\end{tabular}


(WITH TARGET IRRADIATION, PROCESS LOSSES AND DECAY LOSSES)

ISOTOPE PRODUCTION, KGS

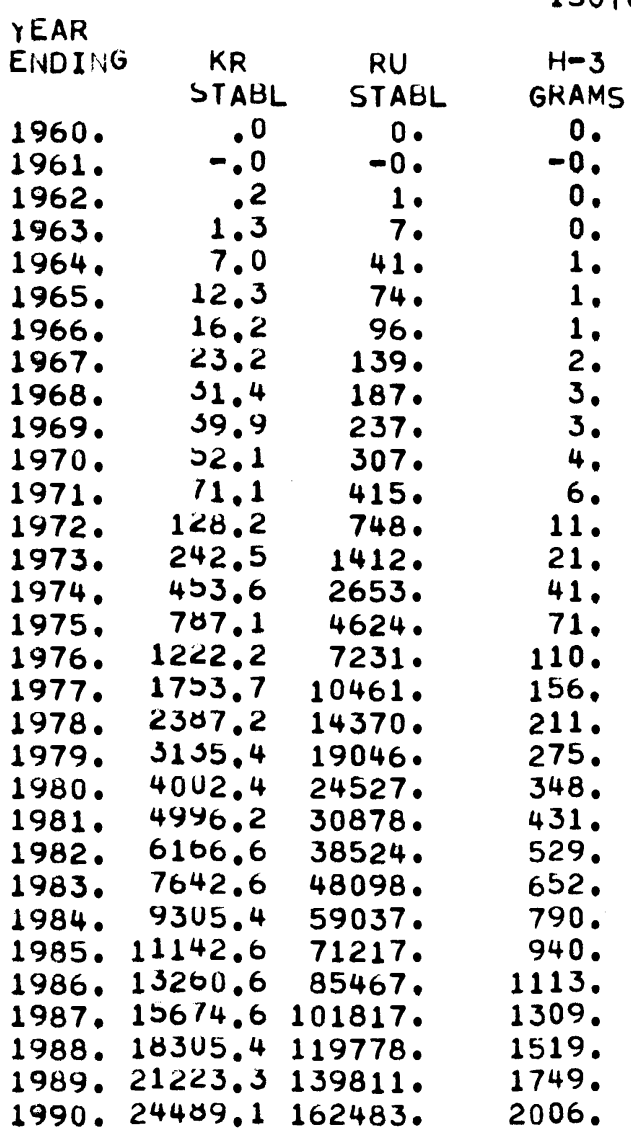


ISOTOPE PRODUCTION

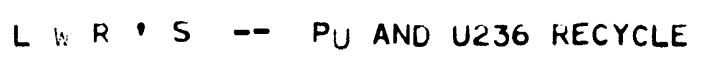




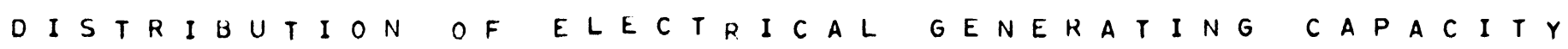

PAGE 78

LWR ' S - PU AND U236 RECYCLE

CASE $\quad 4$

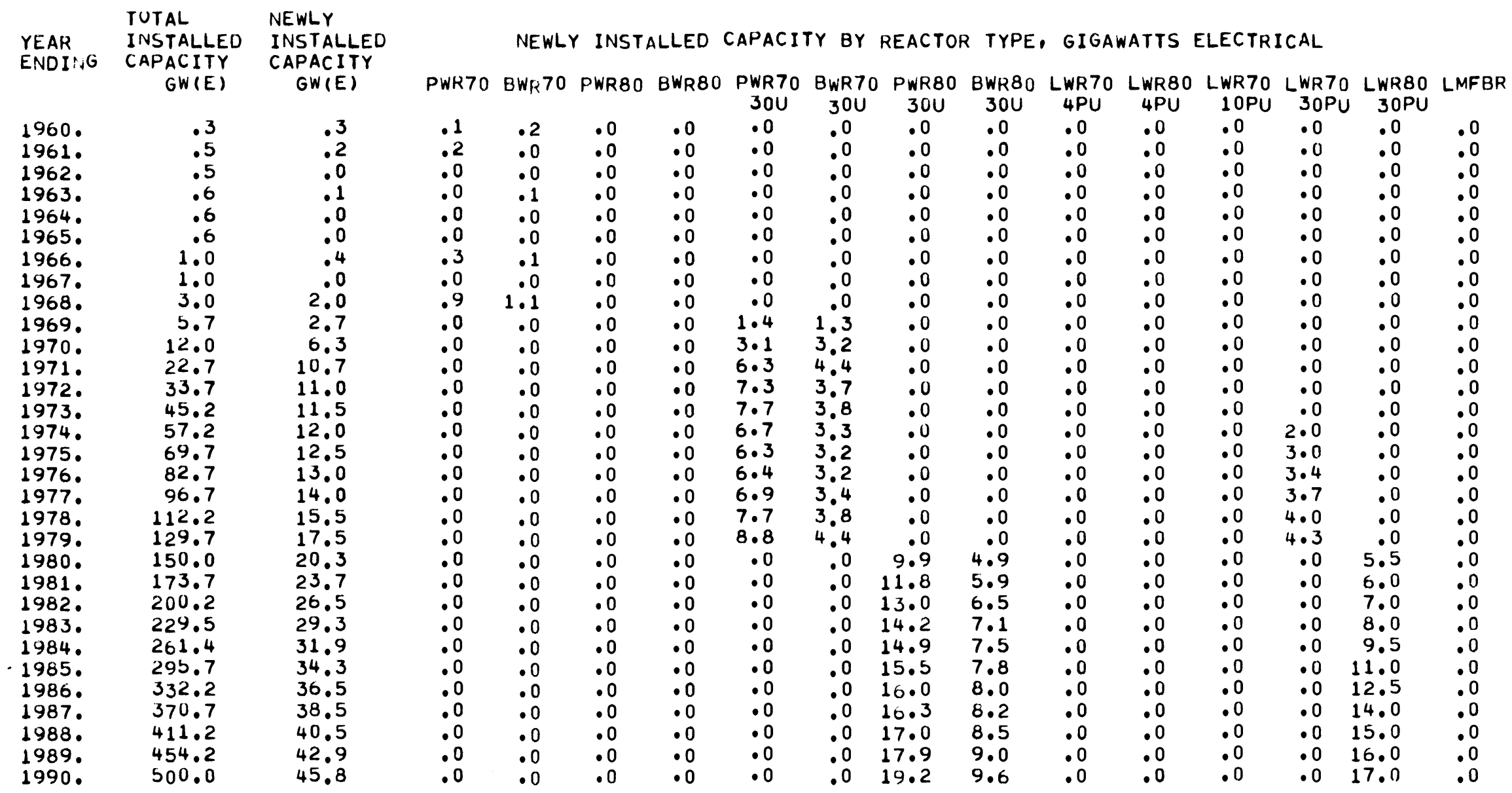


DISTRIBUTION OF ELECTRICAL GENERATINGACA PACITY

PAGE 79

$L R \cdot S-P$ PU AND U236 RECYCLE

CASE $\quad 4$

TUTAL NEWLY

YEAR INSTALLED INSTALLED

ENDIIV CAPACITY CAPACITY

GW(E) GW(E)

CUMULATIVE INSTALLED CAPACITY BY REACTOR TYPE, GIGAWATTS ELECTRICAL

PWR70 BWR70 PWR80 BWR80 PWR70 BWR70 PWR80 GWR80 LWR70 LWR80 LWR70 LWR70 LWR80 LMFBR

$\begin{array}{lrrrrr}1960 . & .3 & .3 & .1 & .2 & .0 \\ 1961 . & .5 & .2 & .2 & .2 & .0 \\ 1962 . & .5 & .0 & .2 & .2 & .0 \\ 1963 . & .6 & .1 & .2 & .4 & .0 \\ 1964 . & .6 & .0 & .3 & .4 & .0 \\ 1965 . & .6 & .0 & .3 & .4 & .0 \\ 1966 . & 1.0 & .4 & .6 & .4 & .0 \\ 1967 . & 1.0 & .0 & .6 & .4 & .0 \\ 1968 . & 3.0 & 2.0 & 1.5 & 1.5 & .0 \\ 1969 . & 5.7 & 2.7 & 1.5 & 1.5 & .0 \\ 1970 . & 12.0 & 6.3 & 1.5 & 1.5 & .0 \\ 1971 . & 22.7 & 10.7 & 1.5 & 1.5 & .0 \\ 1972 . & 33.7 & 11.0 & 1.5 & 1.5 & .0 \\ 1973 . & 45.2 & 11.5 & 1.5 & 1.5 & .0 \\ 1974 . & 57.2 & 12.0 & 1.5 & 1.5 & .0 \\ 1975 . & 69.7 & 12.5 & 1.5 & 1.5 & .0 \\ 1976 . & 82.7 & 13.0 & 1.5 & 1.5 & .0 \\ 1977 . & 96.7 & 14.0 & 1.5 & 1.5 & .0 \\ 1978 . & 112.2 & 15.5 & 1.5 & 1.5 & .0 \\ 1979 . & 129.7 & 17.5 & 1.5 & 1.5 & .0 \\ 1980 . & 150.0 & 20.3 & 1.5 & 1.5 & .0 \\ 1981 . & 173.7 & 23.7 & 1.5 & 1.5 & .0 \\ 1982 . & 200.2 & 26.5 & 1.5 & 1.5 & .0 \\ 1983 . & 229.5 & 29.3 & 1.5 & 1.5 & .0 \\ 1984 . & 261.4 & 31.9 & 1.5 & 1.5 & .0 \\ 1985 . & 295.7 & 34.3 & 1.5 & 1.5 & .0 \\ 1986 . & 332.2 & 36.5 & 1.5 & 1.5 & .0 \\ 1987 . & 370.7 & 38.5 & 1.5 & 1.5 & .0 \\ 1980^{\circ} & 411.2 & 40.5 & 1.5 & 1.5 & .0 \\ 1989 . & 454.2 & 42.9 & 1.5 & 1.5 & .0 \\ 1990 . & 500.0 & 45.8 & 1.5 & 1.5 & .0\end{array}$

$\begin{array}{rrrrr} & 30 U & 30 U & 30 U & 30 \\ .0 & .0 & .0 & .0 & .0 \\ .0 & .0 & .0 & .0 & .0 \\ .0 & .0 & .0 & .0 & .0 \\ .0 & .0 & .0 & .0 & .0 \\ .0 & .0 & .0 & .0 & .0 \\ .0 & .0 & .0 & .0 & .0 \\ .0 & .0 & .0 & .0 & .0 \\ .0 & .0 & .0 & .0 & .0 \\ .0 & .0 & .0 & .0 & .0 \\ .0 & 1.4 & 1.3 & .0 & .0 \\ .0 & 4.4 & 4.5 & .0 & .0 \\ .0 & 10.8 & 8.9 & .0 & .0 \\ .0 & 18.1 & 12.6 & .0 & .0 \\ .0 & 25.8 & 16.4 & .0 & .0 \\ .0 & 32.5 & 19.7 & .0 & .0 \\ .0 & 38.8 & 22.9 & .0 & .0 \\ .0 & 45.2 & 26.1 & .0 & .0 \\ .0 & 52.1 & 29.5 & .0 & .0 \\ .0 & 59.8 & 33.3 & .0 & .0 \\ .0 & 68.6 & 37.7 & .0 & .0 \\ .0 & 68.6 & 37.7 & 9.9 & 4.9 \\ .0 & 68.6 & 37.7 & 21.7 & 10.8 \\ .0 & 68.6 & 37.7 & 34.7 & 17.3 \\ .0 & 68.6 & 37.7 & 48.9 & 24.4 \\ .0 & 68.6 & 37.7 & 63.8 & 31.9 \\ .0 & 68.6 & 37.7 & 79.3 & 39.7 \\ .0 & 68.6 & 37.7 & 95.3 & 47.7 \\ .0 & 68.6 & 37.7 & 111.6 & 55.9 \\ .0 & 68.6 & 37.7 & 128.6 & 64.4 \\ .0 & 68.6 & 37.7 & 146.5 & 73.4 \\ .0 & 68.6 & 37.7 & 165.7 & 83.0\end{array}$

$4 P$
.0
.0
.0
.0
.0
.0
.0
.0
.0
.0
.0
.0
.0
.0
.0
.0
.0
.0
.0
.0
.0
.0
.0
.0
.0
.0
.0
.0
.0
.0
.0

$4 P$
.0
.0
.0
.0
.0
.0
.0
.0
.0
.0
.0
.0
.0
.0
.0
.0
.0
.0
.0
.0
.0
.0
.0
.0
.0
.0
.0
.0
.0
.0
.0

$\begin{array}{rrrr}.0 & .0 & .0 & .0 \\ .0 & .0 & .0 & .0 \\ .0 & .0 & .0 & .0 \\ .0 & .0 & .0 & .0 \\ .0 & .0 & .0 & .0 \\ .0 & .0 & .0 & .0 \\ .0 & .0 & .0 & .0 \\ .0 & .0 & .0 & .0 \\ .0 & .0 & .0 & .0 \\ .0 & .0 & .0 & .0 \\ .0 & .0 & .0 & .0 \\ .0 & .0 & .0 & .0 \\ .0 & .0 & .0 & .0 \\ .0 & .0 & .0 & .0 \\ .0 & 2.0 & .0 & .0 \\ .0 & 5.0 & .0 & .0 \\ .0 & 8.4 & .0 & .0 \\ .0 & 12.1 & .0 & .0 \\ .0 & 16.1 & .0 & .0 \\ .0 & 20.4 & .0 & .0 \\ .0 & 20.4 & 5.5 & .0 \\ .0 & 20.4 & 11.5 & .0 \\ .0 & 20.4 & 18.5 & .0 \\ .0 & 20.4 & 26.5 & .0 \\ .0 & 20.4 & 36.0 & .0 \\ .0 & 20.4 & 47.0 & .0 \\ .0 & 20.4 & 59.5 & .0 \\ .0 & 20.4 & 73.5 & .0 \\ .0 & 20.4 & 88.5 & .0 \\ .0 & 20.4 & 104.5 & .0 \\ .0 & 20.4 & 121.5 & .0\end{array}$


(NO LOSS BASIS)

TUTAL

DURII:G INSTALLED

PERIOU CAHACITY

ENDING GW(E)

ISOTOPE PRODUCTION, KILOGRAMS/YEAR

$\begin{array}{ll}1960^{\circ} & .3 \\ 1961 . & .5 \\ 1960^{\circ} & .5 \\ 1963^{\circ} & .6 \\ 1964^{\circ} & .6 \\ 1965^{\circ} & .6 \\ 1966^{\circ} & 1.0 \\ 1967 . & 1.0 \\ 1960^{\circ} & 3.0 \\ 1969 \circ & 5.7 \\ 1970^{\circ} & 12.0\end{array}$

TONNE

PU-RP

PU-SP

NP237 AM241 AM243 CM242 CM244

$\mathrm{CM} 244$
$\mathrm{AM} 3$

CM2 244
SUM

PU238
NP

PU238 PU238 PU238

$\begin{array}{rr}0 . & 0 \\ 20^{\circ} & 10^{\circ} \\ 71^{\circ} & 44^{\circ} \\ 21^{\circ} & 147{ }^{\circ} \\ 21^{\circ} & 181^{\circ} \\ 16^{\circ} & 125^{\circ} \\ 25^{\circ} & 190^{\circ} \\ 36^{\circ} & 276^{\circ} \\ 34^{\circ} & 278^{\circ} \\ 61^{\circ} & 4180^{\circ} \\ 101^{\circ} & 703{ }^{\circ} \\ 265^{\circ} & 1801^{\circ}\end{array}$

\section{10
44}

147.

181 .

125 .

192.

276.

278 .

$1970 . \quad 12.0$

$\begin{array}{ll}19711^{\circ} & 22.7 \\ 1972 . & 33.7\end{array}$

1973. 45.2

1974. 57.2

1975. 09.7

1970 ० 82.7

1977. $\quad 96.7$

$\begin{array}{ll}1978 . & 112.2 \\ 1979 . & 1<9.7\end{array}$

1980.150 .0

1981. 113.7

1982. 200.2

1983. 229.5

1984. 261.4

$\begin{array}{ll}1985 . & 295.7 \\ 1986 . & 352.2\end{array}$

1987.370 .7

1988. 411.2

$\begin{array}{ll}1989 . & 454.2 \\ 1990 . & 500.0\end{array}$

551.

3863 .

418.

1801 .

1801.

944.

6751.

$-143$.

1423. 10624. $283^{\circ}$.

$2204.19198^{\circ}$

$19198^{\circ}$

24038 .

3034 .

$36634 . \quad-631$.

-271 .

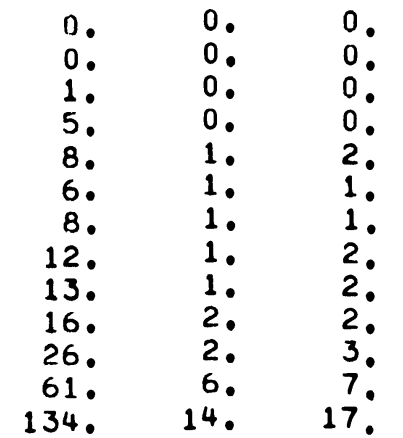

44915 .

59417.

73969.

6592.

7646. 105

105132.

10522 . 129349.

12561 . 156242 .

14176. 178861.

16130 . 204697.

17958.235819.

270296 .

-17
-2372

239.

398.

571 .

69. 100 .

95. 146

$\begin{array}{rrr}901 . & 133 . & 218 . \\ 1096 . & 182 . & 305\end{array}$

1321. 245. 414.

1596. 319. 545 .

1898. 451. 799.

2290 . 574. 1031。

2632. 725. 1334.

$-16$.

213.

3489. 1029. 2006

385 .

4064. 1231. 2469.

4480 . 1385. 2886.

$1182^{\circ}$

5024. 1569. 3334.

1182.
-2436.

$\begin{array}{lll}5024^{\circ} & 15699^{\circ} & 3334^{\circ} \\ 5423^{\circ} & 1834 . & 4017\end{array}$

43829 .

5985. 2116. $4741^{\circ}$.

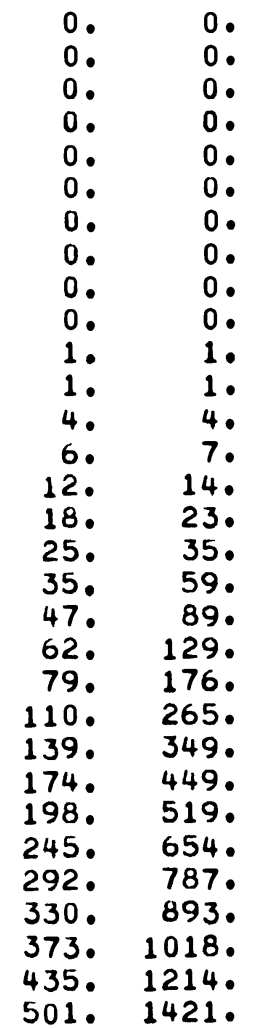

$0 . \quad 0.00$

0 .

0.

0 .

$0 . \quad 0$.

0 .

0 .

0 .

1

0.4

0.214.

35.

59.
89.

129.

176.

265.

349 .

449.

519.

654.

893

. 893

0 . 1018 .

0 . 1214

:.

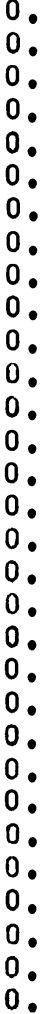

0.
0.
0.
0.
0.
0.
0.
0.
0.
0.
0.
0.
0.
0.
0.
0.
0.
0.
0.
0.
0.
0.
0.
0.
0.
0.
0.
0.
0.
0.
0.
0.

作

0 .

0 .

. 0 


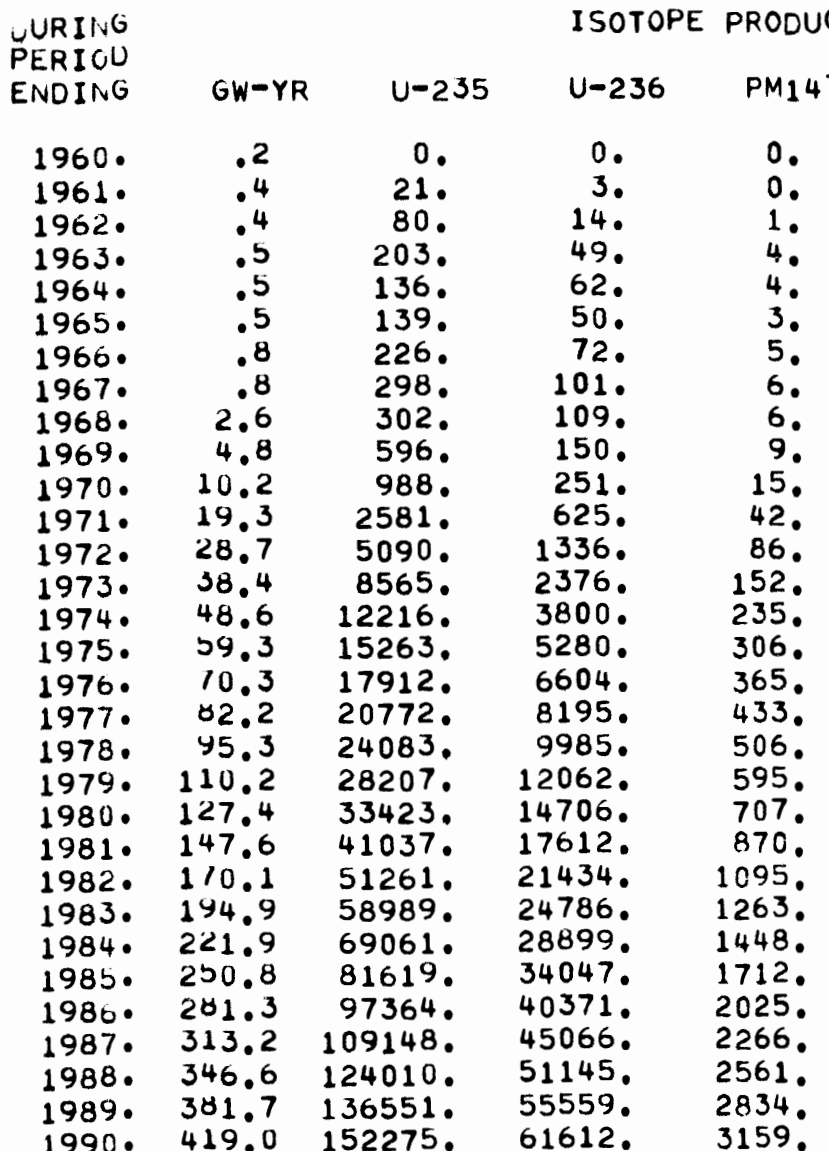

\begin{tabular}{|c|c|c|}
\hline CE144 & $\operatorname{cs} 137$ & $S R-90$ \\
\hline 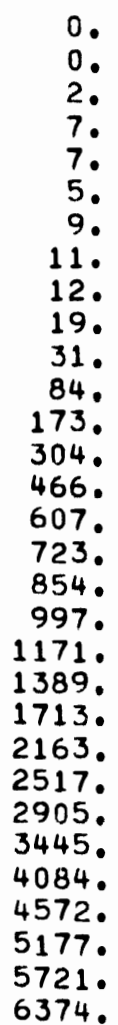 & 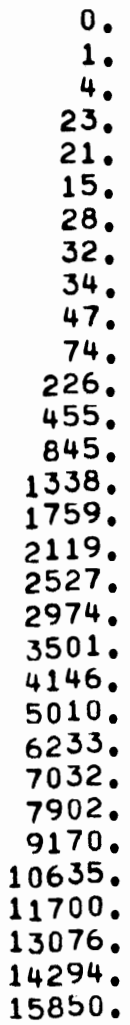 & 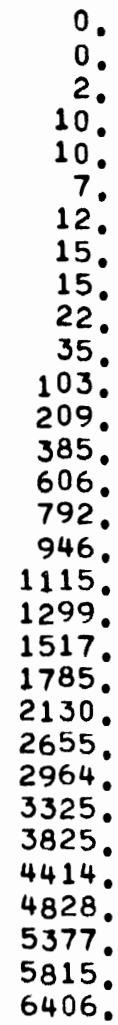 \\
\hline
\end{tabular}

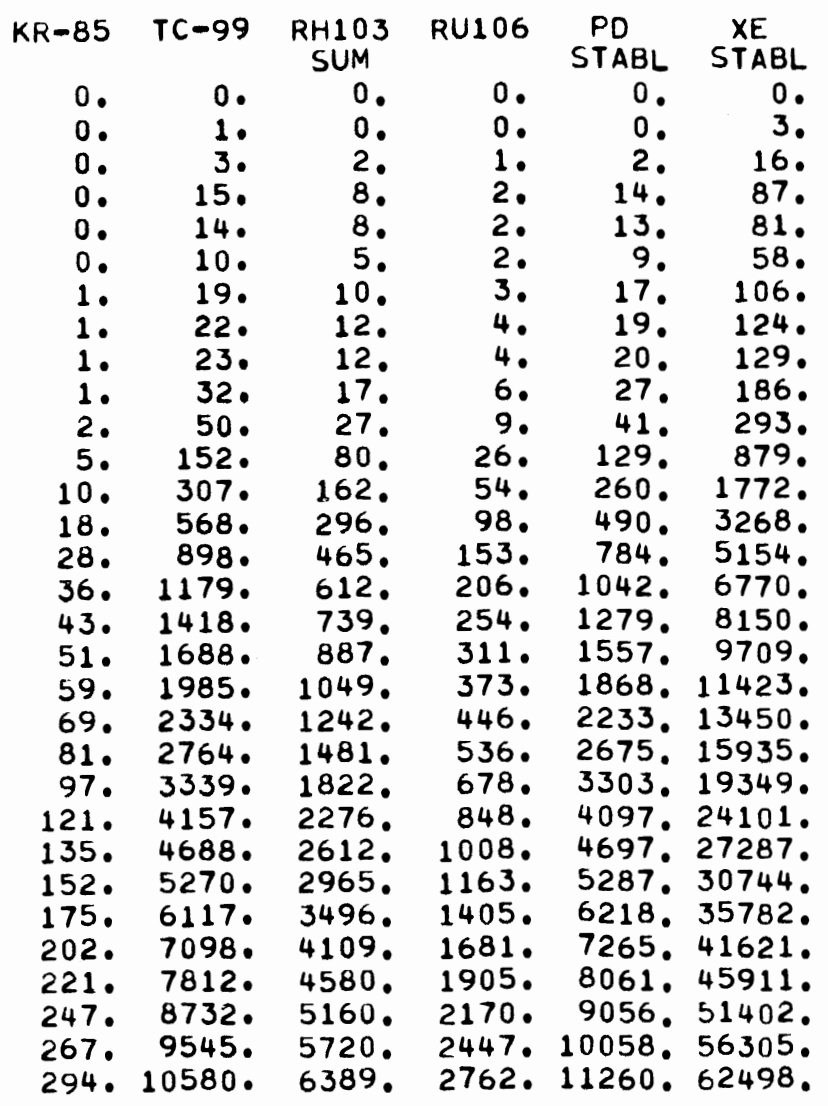

$\begin{array}{cc}\text { CM242 } & \text { AM241 } \\ \text { AM1 } & \text { PU1 } \\ 0 . & 0 . \\ 0 . & 0 . \\ 0 . & 0 . \\ 0 . & 0 . \\ 0 . & 2 . \\ 0 . & 3 . \\ 0 . & 5 . \\ 0 . & 6 . \\ 0 . & 9 . \\ 0 . & 11 . \\ 0 . & 15 . \\ 0 . & 22 . \\ 0 . & 38 . \\ 0 . & 73 . \\ 0 . & 72 . \\ 0 . & 76 . \\ 0 . & 83 . \\ 0 . & 81 . \\ 0 . & 71 . \\ 0 . & 54 . \\ 0 . & 49 . \\ 0 . & 49 . \\ 0 . & 28 . \\ 0 . & 39 . \\ 0 . & 39 . \\ 0 . & 41 . \\ 0 . & 36 . \\ 0 . & 40 . \\ 0 . & 40 . \\ 0 . & 51 . \\ 0 . & 30 . \\ & \end{array}$


ANNUAL ISOTOPE PRODUCTION A T D IS CHARG (NO LOSS BASIS)

UURIING

ENDING

1960.

1961.

1960 .

1963.

1964.

1965.

1966.

1967.

1969.

13.6

1971.21 .3

1971.04 .2

$\begin{array}{ll}1972 . & 129.1 \\ 1973 . & 238.5\end{array}$

1974.376 .6

1975. 4Y2.0

1976. 586.9

1977. 6Y1.5

1970. 805.1

1979. 939.9

1980.1106 .0

$1981 \cdot 1318.4$

8565

1841.810646

$1983 \cdot 1827.7 \quad 12045$.

$1984 \cdot 2046.6 \quad 13526$.

1985.2350 .215740 .

ISOTOPE PRODUCTION, KILOGRAMS/YEAR

. 2708.1

18278 .

1987.2908 .620145$.

1988.3290 .922533$.

1989.3503 .924726$.

1990.3911 .1227480 .

$H-3$

GRAMS

STABL 0.

0.0 .

0 .

1.

1.

1.

1.

2.

7

24.

1400.24.

531.51.

61.

73 .

86.

101.

120

145.

180

202 .

227.

263.

305.

335.

374.

408 .

453. 


\section{CUMULATIVE ISOTOPE PRODUCTION AT D I SCHARGE}

(NO LOSSES EXCEPT FOR RADIOACTIVE DECAY)

LWR ' S - PU AND U236 RECYCLE

TOTAL

INSTALLED ISOTOPE PRODUCTION, KGS

EAR CAPACITY

ENDIIG GW (E) TONNE PU-RP

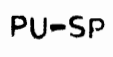

1960.

$1961^{\circ}$

1963.

1964.

1965.

1966.

1967.

1968.

1969.

1970.

1971.

1972.

1973.

1974.

1975.

1977.

1978.

1979.

1980 .

1981.

$1982^{\circ}$

1984.

1985 .

1986.

1987.

1988.

1989.

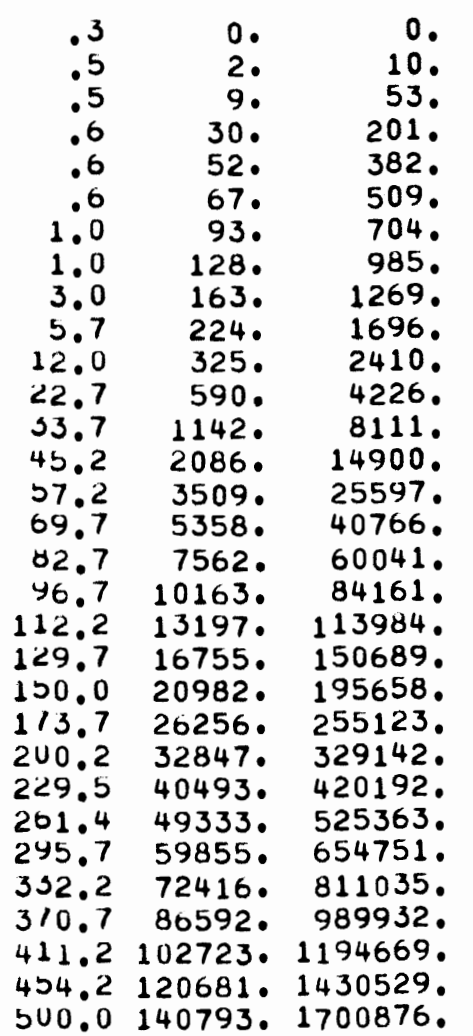

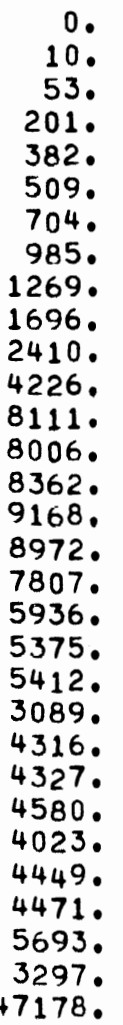

\begin{tabular}{|c|c|c|}
\hline & & \\
\hline 0. & 0. & 0. \\
\hline 0 . & $0 \bullet$ & 0. \\
\hline 1. & 0. & 0. \\
\hline 6. & 1. & 1. \\
\hline 14. & 2. & 2. \\
\hline 20. & 2 & 3. \\
\hline 29. & 3. & 4. \\
\hline $\begin{array}{l}41 . \\
54 .\end{array}$ & $\begin{array}{l}4 . \\
6 .\end{array}$ & $\begin{array}{l}0 . \\
8 .\end{array}$ \\
\hline 70. & 7. & 10. \\
\hline 97. & 10. & 13. \\
\hline 158. & 15. & 19. \\
\hline $\begin{array}{l}291^{\circ} \\
531 .\end{array}$ & $\begin{array}{l}29{ }^{\circ} \\
54 .\end{array}$ & $\begin{array}{l}37 . \\
68 .\end{array}$ \\
\hline 928. & $\begin{array}{r}97 . \\
166\end{array}$ & $\begin{array}{l}127 . \\
227 \circ^{\circ}\end{array}$ \\
\hline $2217^{\circ}$ & 261 . & 374 . \\
\hline 3118. & 393. & 591. \\
\hline $\begin{array}{l}4214^{\circ} \\
5535\end{array}$ & 574. & $\begin{array}{l}896 . \\
1310\end{array}$ \\
\hline $\begin{array}{l}5535 . \\
7130^{\circ}\end{array}$ & $1136^{\circ}$ & $\begin{array}{l}1310 . \\
1855^{\circ}\end{array}$ \\
\hline 9029 & 1586 & $2654^{\circ}$ \\
\hline 11318. & 2157. & $3684^{\circ}$ \\
\hline $\begin{array}{l}13950 . \\
16952 .\end{array}$ & $\begin{array}{l}2879^{\circ} \\
3703^{\circ}\end{array}$ & $\begin{array}{l}5018 . \\
6576 .\end{array}$ \\
\hline 204 & 4726 & \\
\hline $24505^{\circ}$ & 5950. & $\begin{array}{l}11049^{\circ} \\
13934^{\circ}\end{array}$ \\
\hline & $8884^{\circ}$ & $17266^{\circ}$ \\
\hline & $\begin{array}{l}10705 . \\
12805 .\end{array}$ & $\begin{array}{l}21282 . \\
26021 .\end{array}$ \\
\hline
\end{tabular}

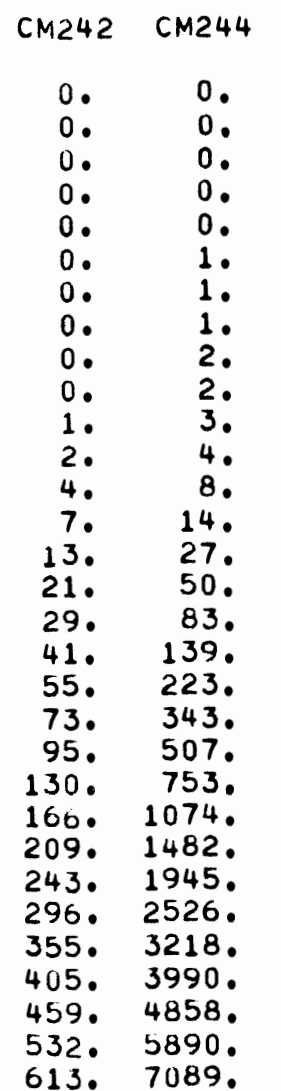

$\begin{array}{cc}\text { CM244 } & \text { CM244 } \\ A M 3 & \text { SUM } \\ 0 . & 0 . \\ -0 . & 0 . \\ -0 . & 0 . \\ -0 . & 0 . \\ -0 . & 0 . \\ -0 . & 1 . \\ -0 . & 1 . \\ -0 . & 1 . \\ -0 . & 2 . \\ -0 . & 2 . \\ -0 . & 3 . \\ -0 . & 4 . \\ -0 . & 8 . \\ -0 . & 14 . \\ -0 . & 27 . \\ -0 . & 50 . \\ -0 . & 83 . \\ -0 . & 139 . \\ -0 . & 223 . \\ -0 . & 343 . \\ -0 . & 507 . \\ -0 . & 753 . \\ -0 . & 1074 . \\ -0 . & 1482 . \\ -0 . & 1945 . \\ -0 . & 2526 . \\ -0 . & 3218 . \\ -0 . & 3990 . \\ -0 . & 4858 . \\ -0 . & 5890 \\ -0 . & 7089 .\end{array}$

$P U 23$
NP
0.
-0.
-0.
-0.
-0.
-0.
-0.
-0.
-0.
-0.
-0.
-0.
-0.
-0.
-0.
-0.
-0.
-0.
-0.
-0.
-0.
-0.
-0.
-0.
-0.
-0.
-0.
-0.
-0.
-0.
-0.

PU238 PU238 PU238

$\begin{array}{ccc}C M & A M & \text { SUM } \\ 0 . & 0 . & 0\end{array}$

$\begin{array}{rrr}0 . & 0 . & 0 . \\ -0 . & -0 . & 0 . \\ -0 & -0.0 & 0 .\end{array}$

$\begin{array}{lll}-0.0 & -0.0 & 0.0\end{array}$

$\begin{array}{lll}-0 . & -0 . & 0 . \\ -0 . & -0.0 & 0 .\end{array}$

$\begin{array}{lll}-0 . & -0 . & 0 . \\ -0 & -0.0 & 0\end{array}$

$\begin{array}{ll}-0 & -0 \\ -0 & -0\end{array}$

$-0 . \quad-0$.

$-0 . \quad-0$.

$-0 . \quad-0$.

-0 .

-0.
-0
-0

-0 .

-0 .

-0 .

$-0$

-0
-0

-0 .

-0 .

-0 .

-0
-0
-0

-0.
-0.

-0 .

-0 .

-0 .

-0 .

-0.
-0.

-0.
-0.
-0
-0

-0.
-0.

-0 .

-0 .

-0 .

-0.
-0.

-0 .

$-0$.

-0 .

-0 .

-0.
-0.

-0
-0

$-0$.

-0 .

$-0$.

-0.
-0.
-0 
CUMULATIVE I S OTOPE PRO DUCTION A T D I S C HAR GE (NO LOSSES EXCEPT FOR RADIOACTIVE DECAY)

LWR P - - PU AND U236 RECYCLE

CASE 4

YEAR

1960.

1961.

1962.

1963.

$1964^{\circ}$

1965.

1966.

1967.

1968.

1969.

1970 .

1971.

1972 .

1973.

1974 .

1975.

1976.

1977.

1978.

1979.

1980 .

1981.

1982 .

1983.

1984.

1985.

1986.

1987.

1988 .

1989.

1990 .
ISOTOPE PRODUCTION, KGS

$$
\text { GW-YR U-235 }
$$

$$
u-236
$$$$
\text { PM1 } 47
$$$$
\text { CE144 }
$$

CS137

$\begin{array}{rr}.2 & 0 . \\ .6 & 21 . \\ 1.0 & 101 . \\ 1.5 & 304 . \\ 2.1 & 440 . \\ 2.6 & 580 . \\ 3.4 & 8050 \\ 4.3 & 1103 . \\ 6.8 & 1405 . \\ 11.7 & 2000 . \\ 21.9 & 2988 . \\ 41.2 & 5569 \\ 09.9 & 10659 .\end{array}$

0.
37.
66.

128 .

128.
178.

250 .

351 .

460.

610.

862.

1486.

2823.

5198 .

8999.

14278 .

20882 .

29077 .

39062 .

51124.

65830 .

83442 .

849.2212137 .

104876.

129662 .

158562 .

192608.

232979 .

278045 .

329190 .

384749 .

2627.9803589 .

3009.6940140

446361 .
0.

0.

0.
0.
2.

7.

8.

11.

17.

23.

33.

68.

139.

260.

436.

643.

863.

1100 .

1358.

1646.

2402 .

2954.

3548 .

4192.

4955.

5858.

6797.

7819.

10030 。$$
10 .
$$

9.

17.

19. $150^{\circ}$

393. 1751. 798.

$627^{\circ} 3049.1385$.

866. 4738. 2143.

$10790^{\circ} 6749.3037$.

$\begin{array}{rrr}1298^{\circ} 9122^{\circ} & 4077^{\circ} \\ 1531^{\circ} & 11887^{\circ} \quad 5277^{\circ}\end{array}$

$1801^{\circ} 15116.6664^{\circ}$.

$2129 \cdot 18918.8287$.

3228 . 29192. 12619 .

3845,35557 . $15275^{\circ}$

4486 . 42646. 18226 .

5290 . 50842. 21606 .

6260 . 60316. 25492.

7147 . 70639. 29697.

8117.82102 . 34348.

$10100.108212 .44767^{\circ}$.

$K R-85$

$\begin{array}{cccc}\text { KR-85 } & \text { TC- } & \text { SUM } & \\ 0 . & 0 . & 0 . & 0 \\ 0 . & 1 . & 0 . & 0 \\ 0 . & 3 . & 2 . & 1 . \\ 1 . & 19 . & 10 . & 3 . \\ 1 . & 33 . & 18 . & 4 . \\ 1 . & 43 . & 23 . & 4 . \\ 2 . & 62 . & 33 . & 5 . \\ 2 . & 84 . & 44 . & 6 .\end{array}$

3. 106. 56. 
C UMULATIVE I SOTOPE PRODUCTION AT D I S CHARGE

PAGE 85 (NO LOSSES EXCEPT FOR RADIOACTIVE DECAY)

ISOTOPE PRODUCTION, KGS

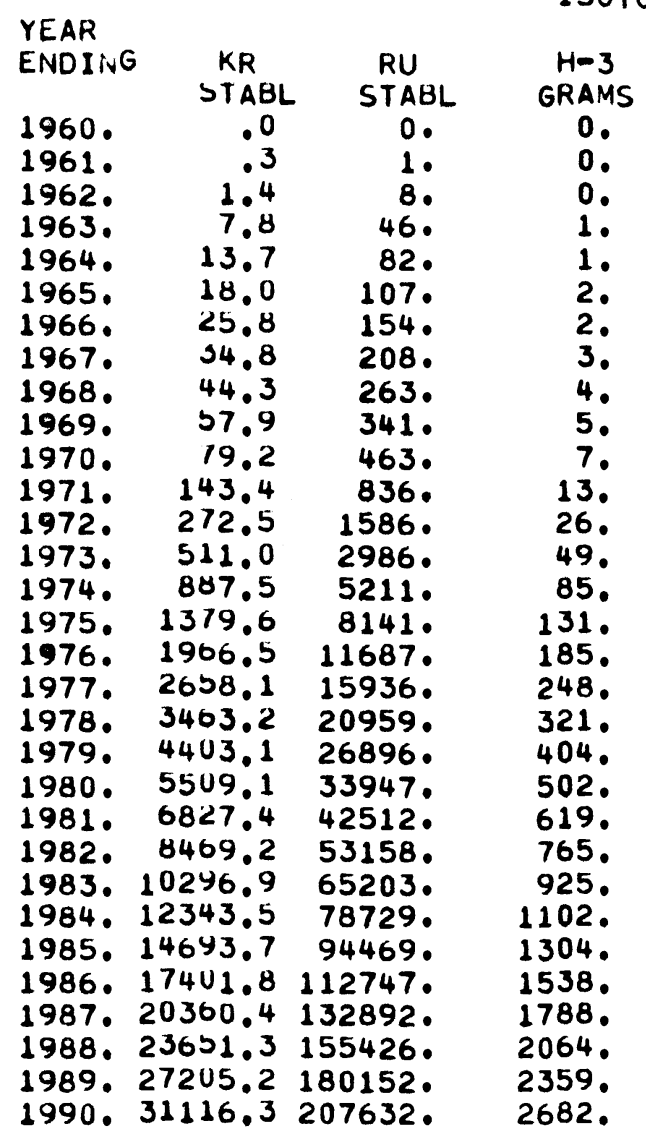


NET ANNUAL I SOTOPE AVAILABILI T Y

(WITH TARGET IRRADIATION, PROCESS LOSSES AND DECAY LOSSES)

TUTAL

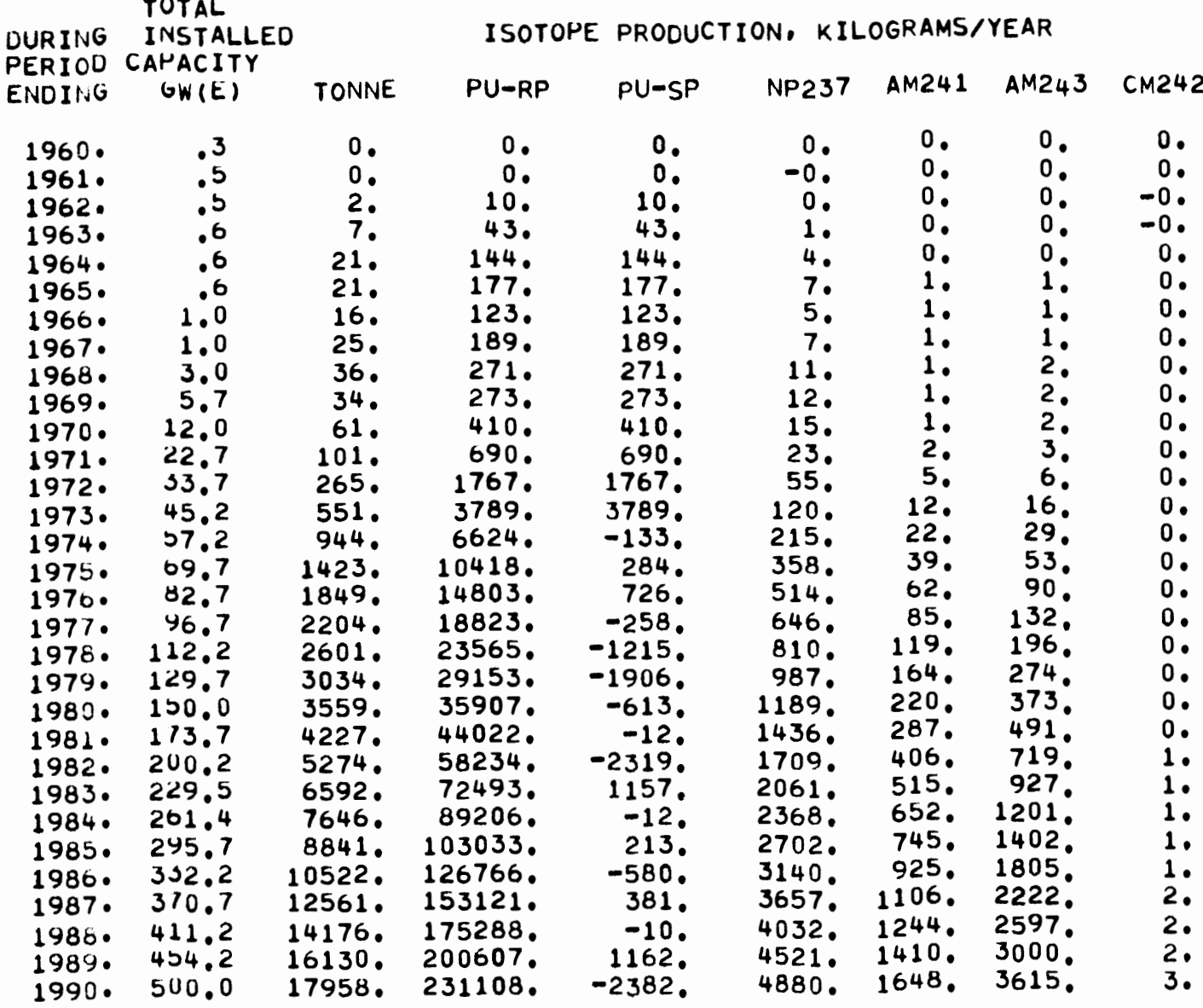

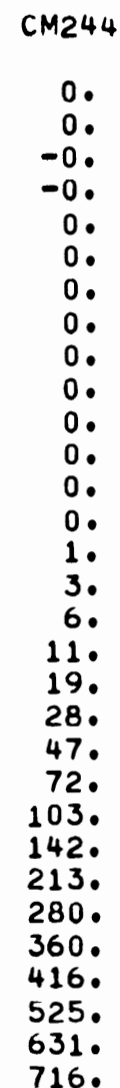

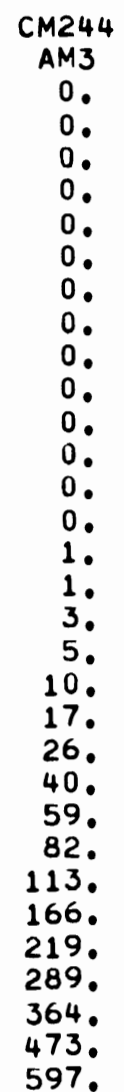
SUM

0.

0.

o.

0.

0.

0.

0 .

1.

1.

1.

4.

9. 126

16. 196.

46.361.

73. 460

112.

162.

224.

326.

446.

579.

705.

889.

1104.

1314.
NP 238

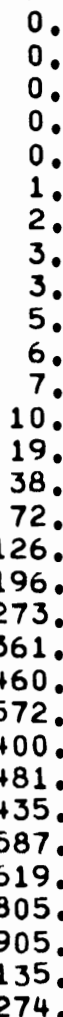

\begin{tabular}{|c|c|c|}
\hline 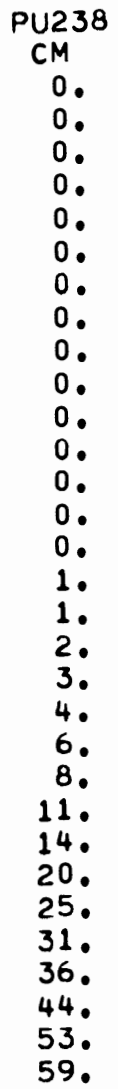 & 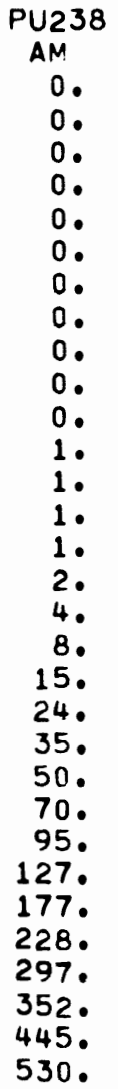 & 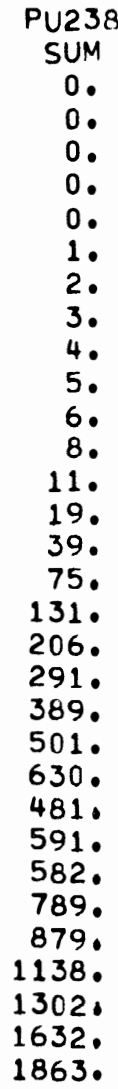 \\
\hline
\end{tabular}


NET ANNUAL ISOTOPE AVAILABILI T Y

(WITH TARGET IRRADIATION. PROCESS LOSSES AND DECAY LOSSES)

$$
L W R \cdot S \text {-- PU AND U236 RECYCLE }
$$

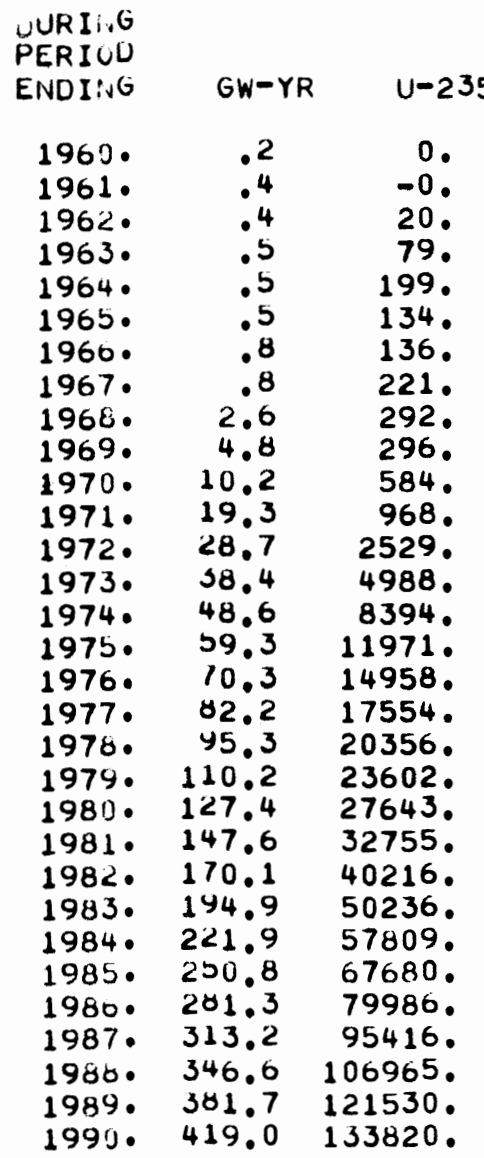

ISOTOPE PRODUCTION, KILOGRAMS/YEAR

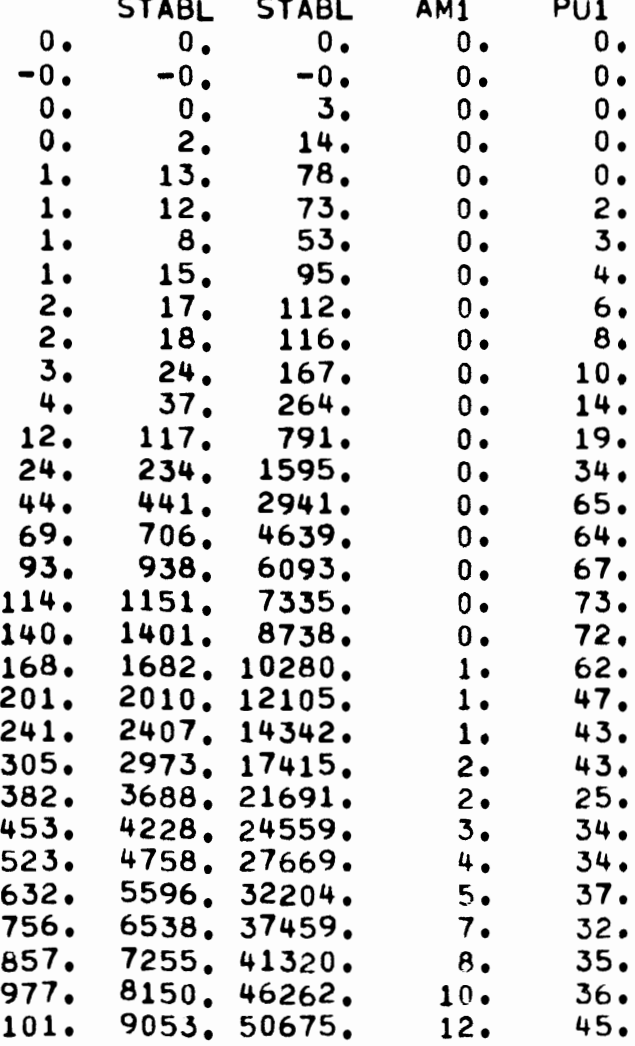


NET ANNUAL ISOTOOPE AVAILABILI T Y (WITH TARGET IRRADIATION. PROCESS LOSSES AND DECAY LOSSES)

$L W R \cdot S$ PU AND U236 RECYCLE
PAGE 88

CASE 4

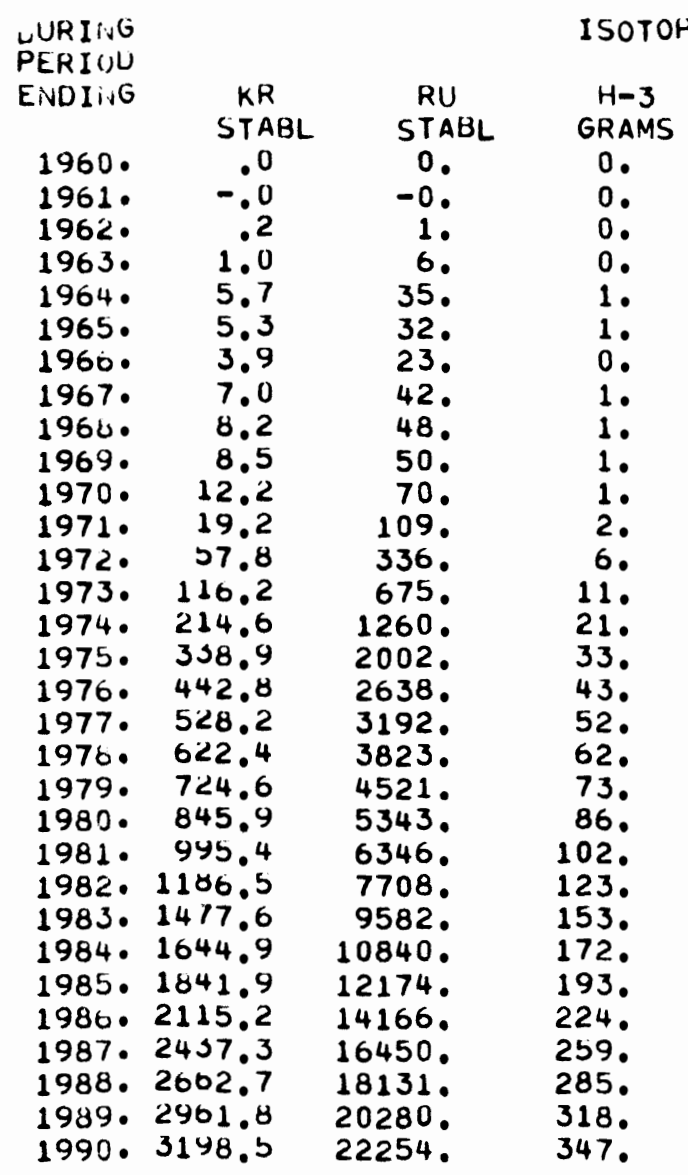


CUMULATIVE I SOTOPEA A T A I LA B I L I T Y

(WITH TARGET IRRAIIATION, PROCESS LOSSES AND DECAY LOSSES)

TUTAL

INS I ALLED

YEAR CAPACITY

ISOTOPE PRODUCTION, KGS

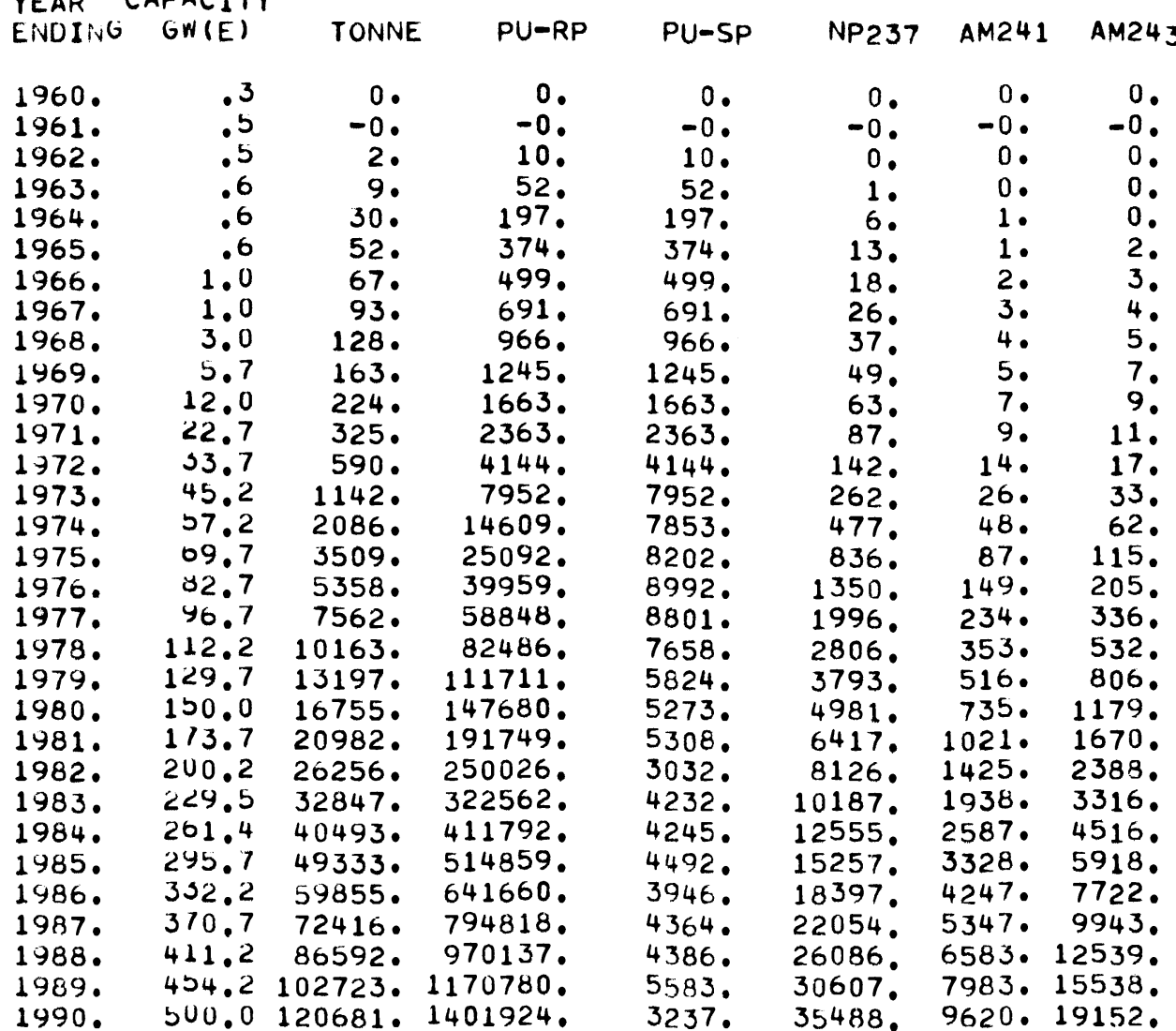

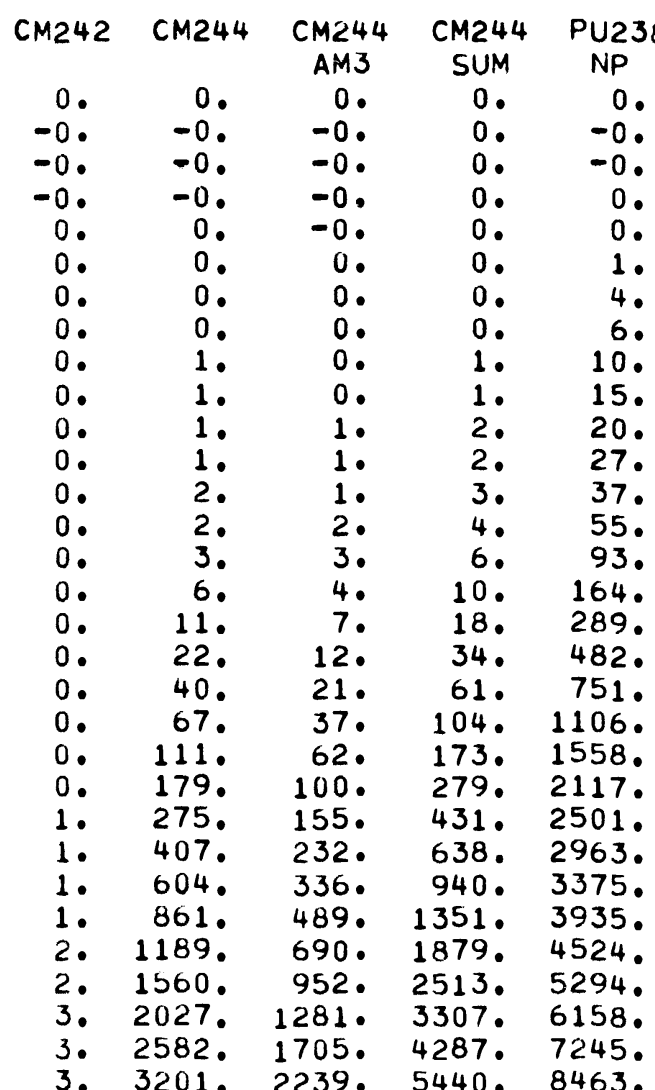

\begin{tabular}{|c|c|c|}
\hline PU238 & $\begin{array}{l}\text { PU238 } \\
\text { AM }\end{array}$ & PU23 \\
\hline 0. & 0. & 0. \\
\hline-0 . & -0 . & 0 . \\
\hline-0. & -0. & 0. \\
\hline-0 . & -0. & 0 . \\
\hline 0 . & -0 . & 0. \\
\hline 0. & 0. & 1. \\
\hline 0. & 0 . & 4. \\
\hline 0 . & 0. & 7. \\
\hline 0. & 0 . & 10. \\
\hline 0. & 1. & 15. \\
\hline 0 . & 1. & 22. \\
\hline 0 . & 2. & 29. \\
\hline 0 . & 2 . & 40. \\
\hline 0. & 3. & 59. \\
\hline 1. & 4. & 98. \\
\hline 1. & 6. & 171. \\
\hline $\begin{array}{l}2 . \\
5\end{array}$ & 10. & 301. \\
\hline $\begin{array}{l}5 . \\
8 .\end{array}$ & $\begin{array}{l}18 . \\
33 .\end{array}$ & $\begin{array}{l}505 . \\
792 .\end{array}$ \\
\hline 12. & 56. & 1175 \\
\hline 18. & 91. & 1667. \\
\hline $\begin{array}{l}27 . \\
38 .\end{array}$ & $\begin{array}{l}140 . \\
209 .\end{array}$ & $\begin{array}{l}2284^{\circ} \\
2747^{\circ}\end{array}$ \\
\hline 51. & 302 . & 3317 . \\
\hline 71. & 427. & 3873. \\
\hline 95. & 601. & 4631. \\
\hline 126. & 824. & 5474 . \\
\hline 161. & 1115. & 6570 . \\
\hline 203. & 1459. & 7820 . \\
\hline $\begin{array}{l}254 \\
312\end{array}$ & $\begin{array}{l}1892 . \\
2408\end{array}$ & $\begin{array}{r}9392 . \\
11182 .\end{array}$ \\
\hline
\end{tabular}


(WITH TARGET IRRACIATION, PROCESS LOSSES AND DECAY LOSSES)

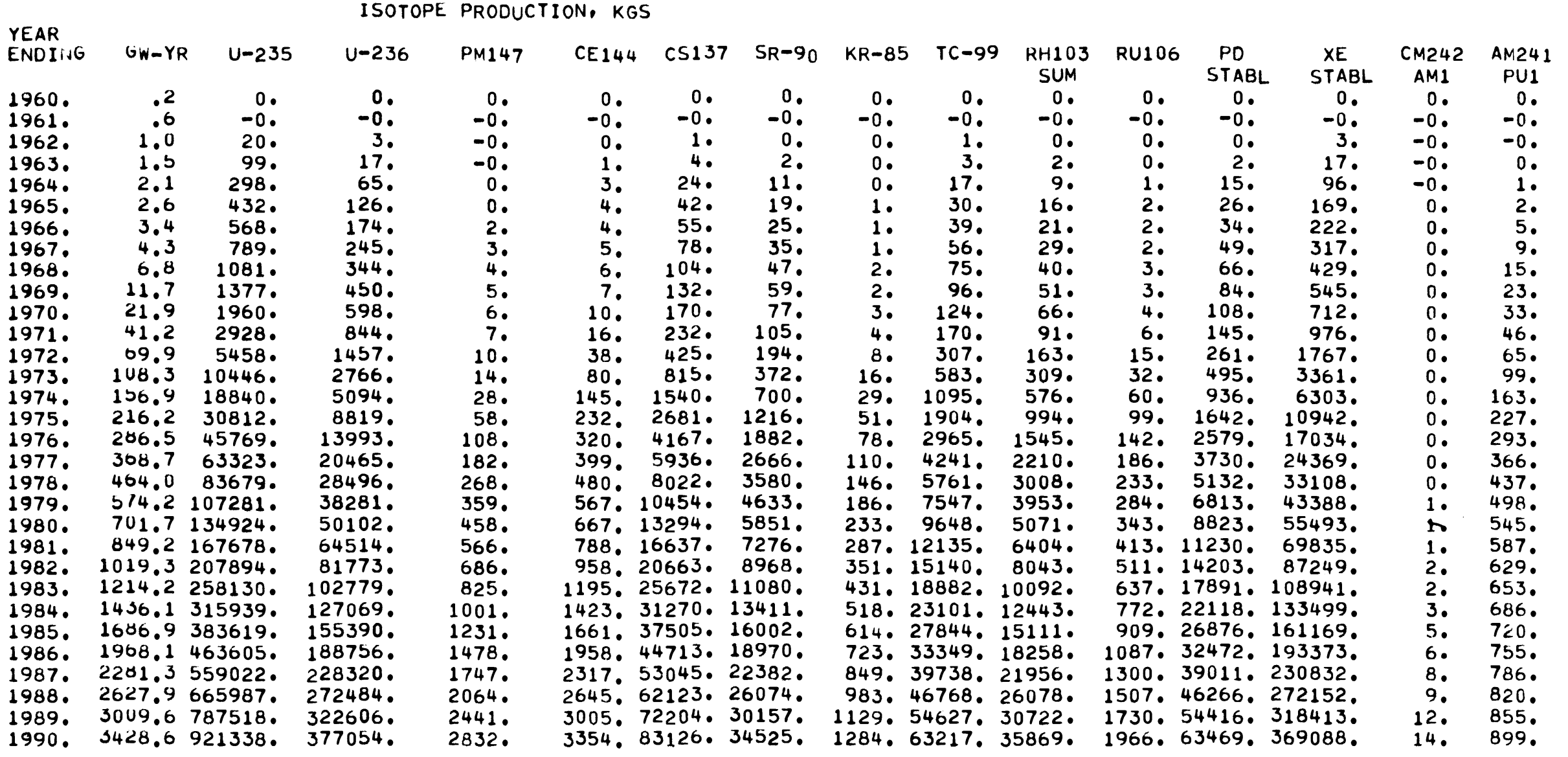


(WITH TARGET IRRADIATION. PROCESS LOSSES AND DECAY LOSSES)

ISOTOPE PRODUCTION, KGS

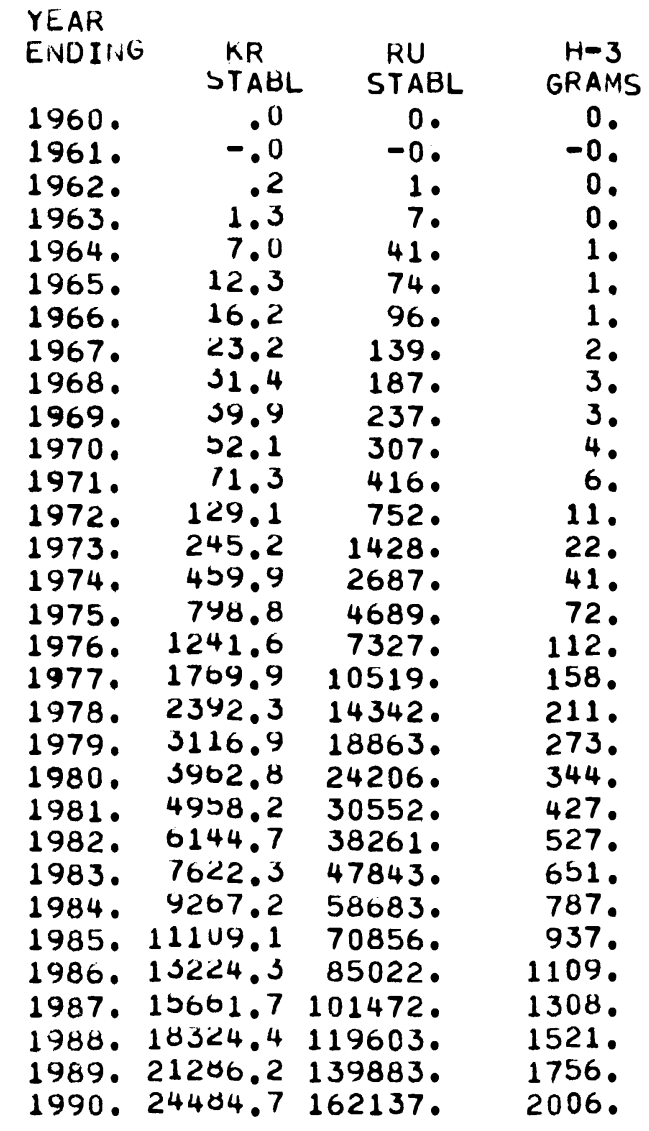


I S O T O P E PROAUCA I O N

LWR ' S - U236 RECYCLE WITH FAST REACTORS 
DISTRIBUTION OF ELECTRICAL GENERATINGACA PACITY

PAGE 92

LWR - S - U236 RECYCLE WITH FAST REACTORS

CASE 5

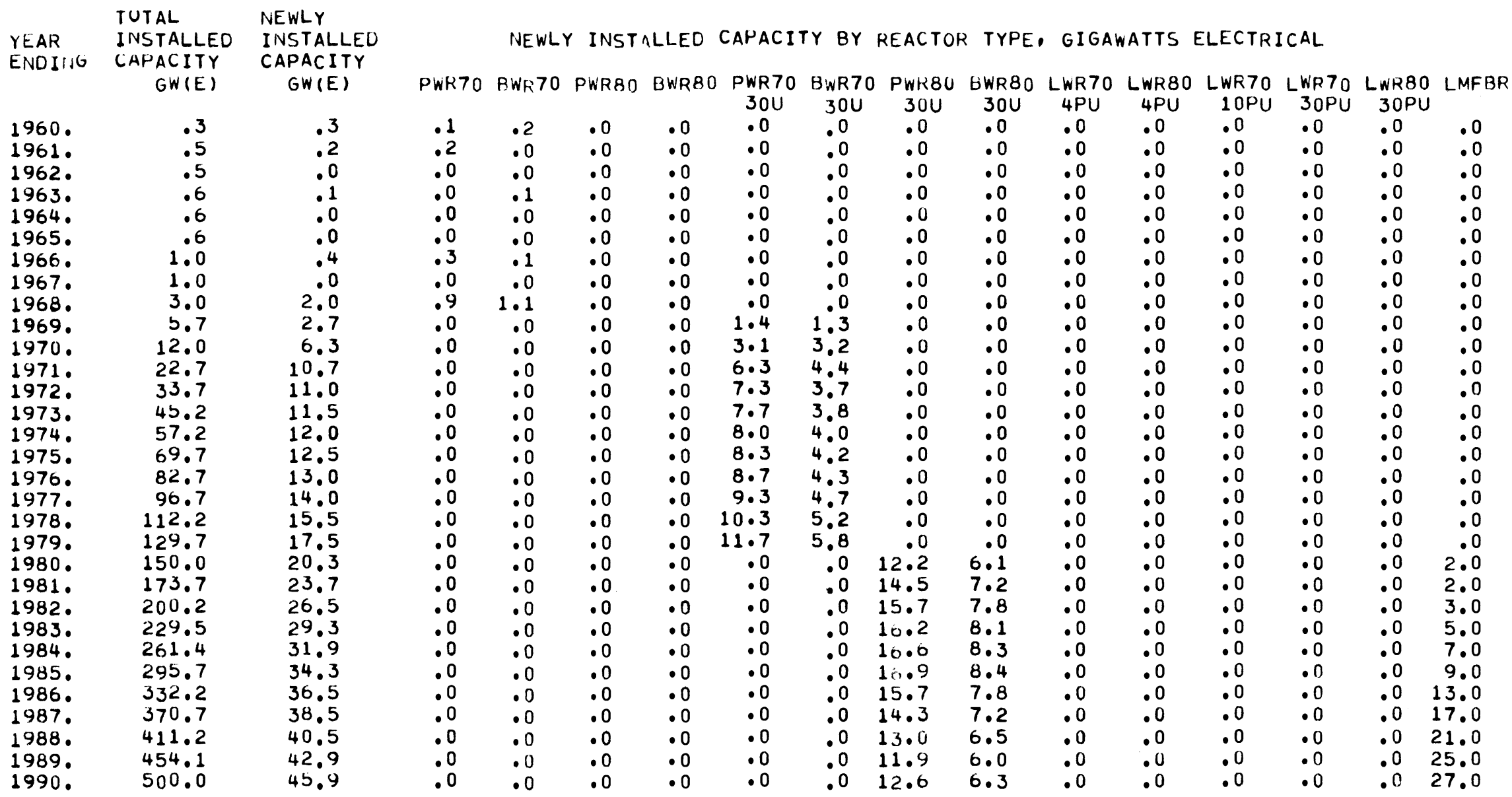


DISTR I BUTION OF ELECTRICAL GENERATINGACAPACITY

PAGE 93

LWR ' S - U236 RECYCLE WITH FAST REACTORS

CASE

TUTAL NEWLY

YEAR INSTALLED INSTALLED

EINDING CAPACITY CAPACITY

CUMULATIVE INSTALLED CAPACITY BY REACTOR TYPE, GIGAWATTS ELECTRICAL

GW(E) GW(E)

PWR70 BWR70 PWR80 BWR80 PWR70 BWR70 PWR80 BWR80 LWR70 LWR80 LWR70 LWR70 LWR80 LMFBR

\begin{tabular}{|c|c|}
\hline 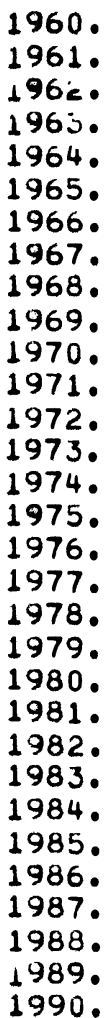 & $\begin{array}{r}.6 \\
.6 \\
1.0 \\
1.0 \\
3.0 \\
5.7 \\
12.0 \\
22.7 \\
33.7 \\
45.2 \\
57.2 \\
69.7 \\
82.7 \\
96.7 \\
112.2 \\
129.7 \\
150.0 \\
173.7 \\
200.2 \\
229.5 \\
261.4 \\
295.7 \\
332.2 \\
370.7 \\
411.2 \\
454.1 \\
500.0\end{array}$ \\
\hline
\end{tabular}

.3
.2
.0
.1
.0
.0
.4
.0
2.0
2.7
6.3
10.7
11.0
11.5
12.0
12.5
13.0
14.0
15.5
17.5
20.3
23.7
26.5
29.3
31.9
34.3
36.5
38.5
40.5
42.9
45.9

$\begin{array}{ll}.1 & .2 \\ .2 & .2 \\ .2 & .2 \\ .2 & .4 \\ .3 & .4 \\ .3 & .4 \\ .6 & .4 \\ .6 & .4 \\ 1.5 & 1.5 \\ 1.5 & 1.5 \\ 1.5 & 1.5 \\ 1.5 & 1.5 \\ 1.5 & 1.5 \\ 1.5 & 1.5 \\ 1.5 & 1.5 \\ 1.5 & 1.5 \\ 1.5 & 1.5 \\ 1.5 & 1.5 \\ 1.5 & 1.5 \\ 1.5 & 1.5 \\ 1.5 & 1.5 \\ 1.5 & 1.5 \\ 1.5 & 1.5 \\ 1.5 & 1.5 \\ 1.5 & 1.5 \\ 1.5 & 1.5 \\ 1.5 & 1.5 \\ 1.5 & 1.5 \\ 1.5 & 1.5 \\ 1.5 & 1.5 \\ 1.5 & 1.5 \\ & \end{array}$

.0
.0
.0
.0
.0
.0
.0
.0
.0
.0
.0
.0
.0
.0
.0
.0
.0
.0
.0
.0
.0
.0
.0
.0
.0
.0
.0
.0
.0
.0
.0

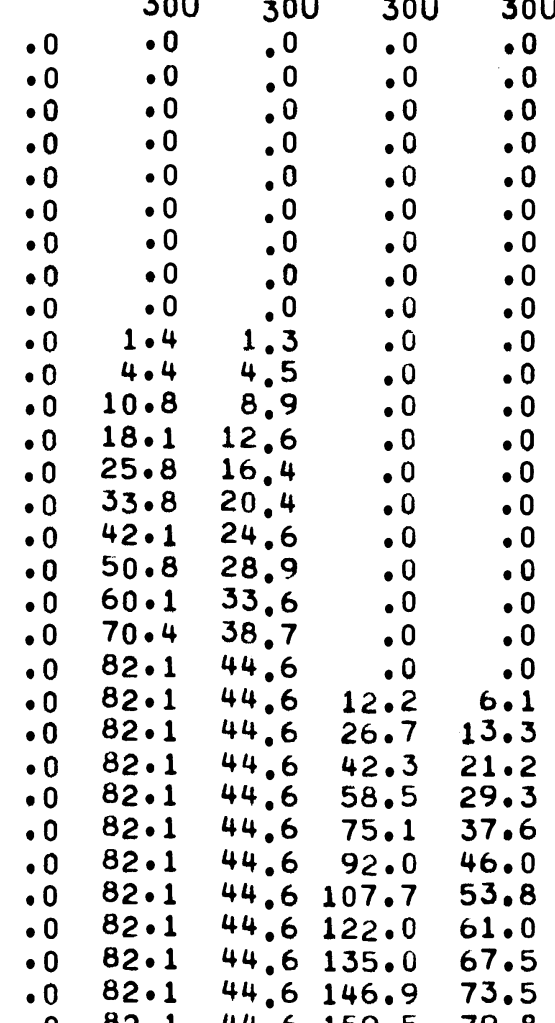

.0
.0
.0
.0
.0
.0
.0
.0
.0
.0
.0
.0
.0
.0
.0
.0
.0
.0
.0
.0
.0
.0
.0
.0
.0
.0
.0
.0
.0
.0
.0
.0

$\begin{array}{lll}.0 & .0 & .0 \\ .0 & .0 & .0 \\ .0 & .0 & .0 \\ .0 & .0 & .0 \\ .0 & .0 & .0 \\ .0 & .0 & .0 \\ .0 & .0 & .0 \\ .0 & .0 & .0 \\ .0 & .0 & .0 \\ .0 & .0 & .0 \\ .0 & .0 & .0 \\ .0 & .0 & .0 \\ .0 & .0 & .0 \\ .0 & .0 & .0 \\ .0 & .0 & .0 \\ .0 & .0 & .0 \\ .0 & .0 & .0 \\ .0 & .0 & .0 \\ .0 & .0 & .0 \\ .0 & .0 & .0 \\ .0 & .0 & .0 \\ .0 & .0 & .0 \\ .0 & .0 & .0 \\ .0 & .0 & .0 \\ .0 & .0 & .0 \\ .0 & .0 & .0 \\ .0 & .0 & .0 \\ .0 & .0 & .0 \\ .0 & .0 & .0 \\ .0 & .0 & .0 \\ .0 & .0 & .0\end{array}$

$\begin{array}{rr}.0 & .0 \\ .0 & .0 \\ .0 & .0 \\ .0 & .0 \\ .0 & .0 \\ .0 & .0 \\ .0 & .0 \\ .0 & .0 \\ .0 & .0 \\ .0 & .0 \\ .0 & .0 \\ .0 & .0 \\ .0 & .0 \\ .0 & .0 \\ .0 & .0 \\ .0 & .0 \\ .0 & .0 \\ .0 & .0 \\ .0 & .0 \\ .0 & .0 \\ .0 & 2.0 \\ .0 & 4.0 \\ .0 & 7.0 \\ .0 & 12.0 \\ .0 & 19.0 \\ .0 & 28.0 \\ .0 & 41.0 \\ .0 & 58.0 \\ .0 & 79.0 \\ .0 & 104.0 \\ .0 & 131.0\end{array}$



(NO LOSS BASIS)

TUTAL

DURIIYG INSTALLED PERIUU CAHACITY

ISOTOPE PRODUCTION, KILOGRAMS/YEAR

ENDIIVG GW(E)

TONNE

PU-RP

PUI-SP

NP237

AM241 AMQ

M243

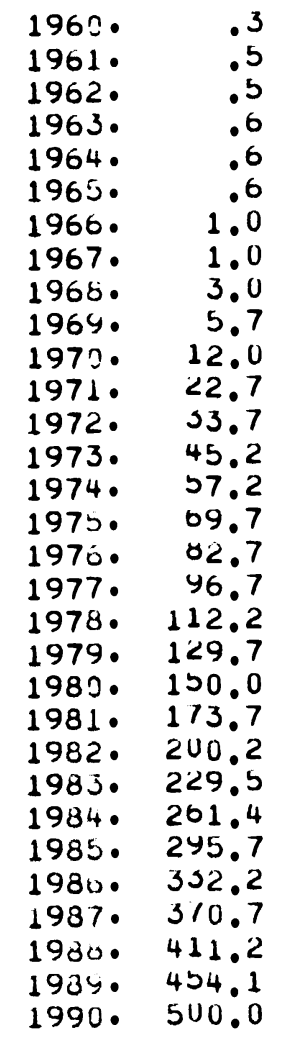

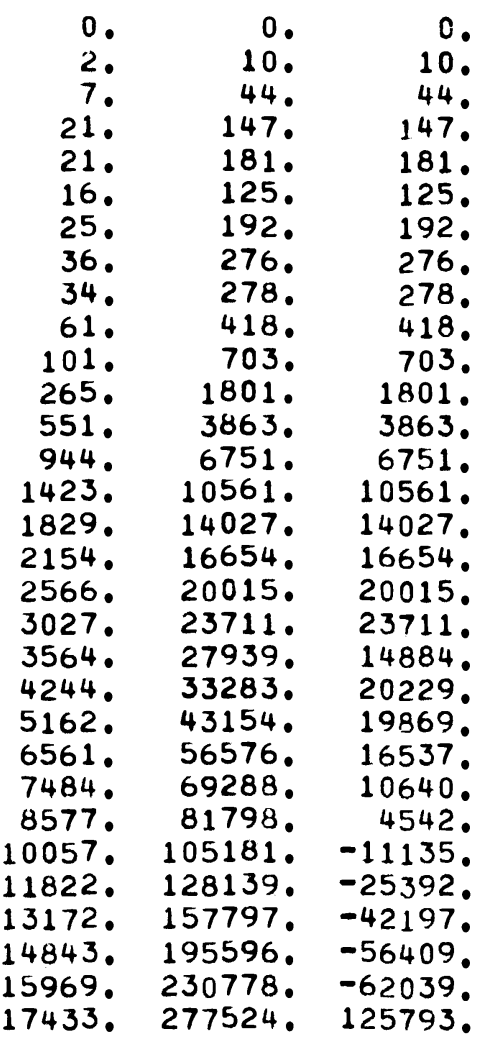

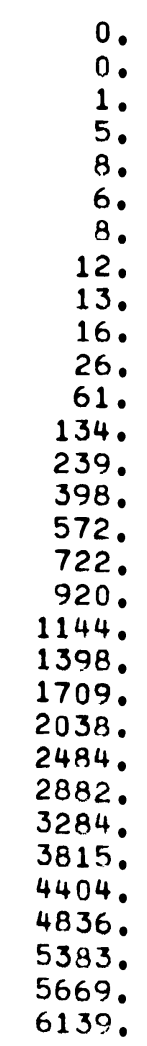

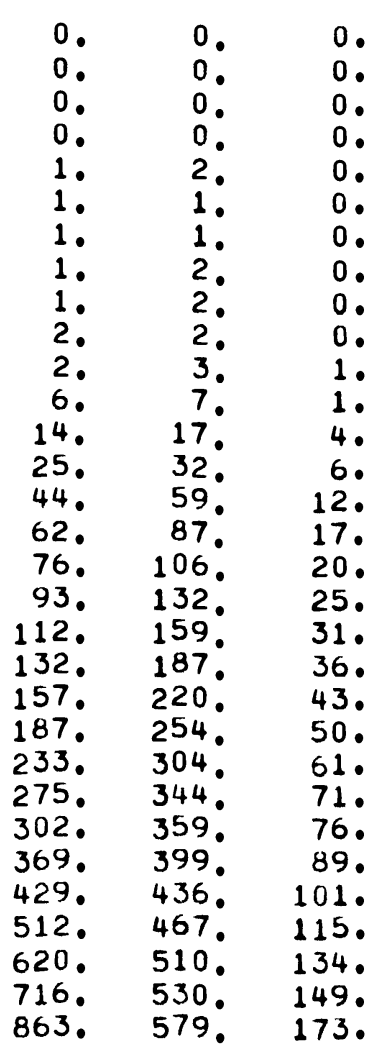

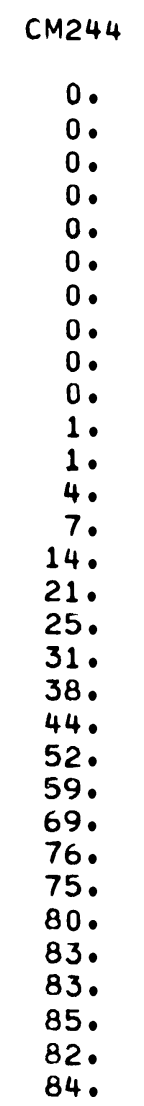

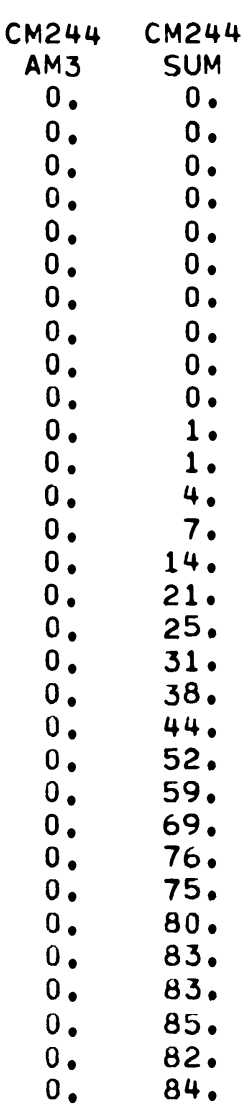

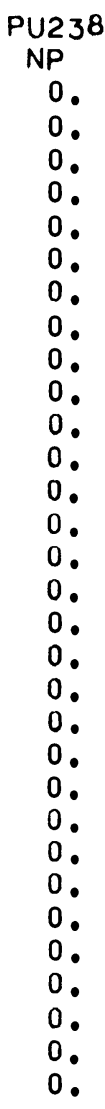

\begin{tabular}{|c|c|c|}
\hline $\begin{array}{l}\text { PU238 } \\
\text { CM }\end{array}$ & PU238 & PU238 \\
\hline 0 & 0 & 0 \\
\hline 0. & 0. & 0. \\
\hline 0. & 0 . & 0 . \\
\hline 0. & 0 . & 0. \\
\hline 0 . & 0 . & 0 . \\
\hline 0. & 0. & 0. \\
\hline 0. & 0. & 0. \\
\hline 0 . & 0 . & 0. \\
\hline 0 . & 0 . & 0 . \\
\hline 0. & 0. & 0. \\
\hline 0. & 0 . & 0 . \\
\hline 0. & 0 . & 0 . \\
\hline 0. & 0 . & 0 . \\
\hline 0. & 0 . & 0 . \\
\hline 0. & 0. & 0 . \\
\hline 0 . & 0. & 0 . \\
\hline 0. & 0 . & 0. \\
\hline 0. & 0 . & 0 . \\
\hline 0. & 0 . & 0 . \\
\hline 0 . & 0 . & 0 . \\
\hline 0. & 0 . & 0 . \\
\hline 0 . & 0 . & 0 . \\
\hline 0. & 0. & 0. \\
\hline 0 . & 0 . & 0 . \\
\hline 0. & 0. & 0 . \\
\hline 0 . & 0 . & 0. \\
\hline 0. & 0. & 0. \\
\hline 0. & 0 . & 0. \\
\hline 0. & 0. & 0. \\
\hline 0 . & 0. & 0. \\
\hline 0 . & 0 . & 0. \\
\hline
\end{tabular}


ANNUAL ISOTOPE PKODUCTION AT DISCHARGE PAGE 95

95

(NO LOSS BASIS)

LWR' 5 - U236 RECYCLE WITH FAST REACTORS CASE S

LUR IIVG

PERIOU

ENDING

1960.

1961.

1962.

1963.

1964 .

1965.

1960.

1967.

$1960^{\circ}$

1969.

1970 .

$1971^{\circ}$

1972.

1973.

1974.

$1975^{\circ}$

$1970 \circ$

$1977^{\circ}$

1978.

1980.

1981.

1982.

1983.

$1984^{\circ}$
$1985^{\circ}$

$1980^{\circ}$

1987.

$1980^{\circ}$

$1990^{\circ}$.
ISOTOPE PRODUCTION, KILOGRAMS/YEAR

$$
\text { GW-YR U-235 U-236 }
$$

.22

.4

21.

203 .

136.

139.

226.

298 .

302 .

596.

988.

2581 .

5090 .

8565 .

12216 .

18270 .

21765 .

25747 .

30527 .

36457 .

44223 .

63721 .

73900 .

86155 .

101229.

111481.

124374

$142686^{\circ}$

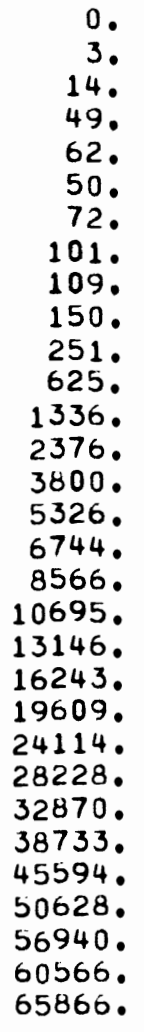

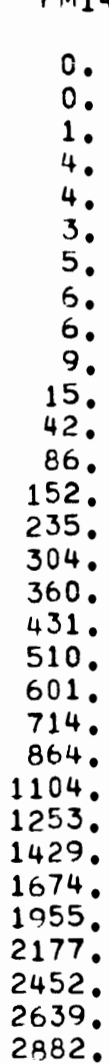

\begin{tabular}{|c|c|c|}
\hline$R R-85$ & $T C-99$ & $\begin{array}{c}\text { RH1 } 03 \\
\text { SUM }\end{array}$ \\
\hline 0 . & 0. & 0. \\
\hline 0. & 1. & 0 . \\
\hline 0 . & 3. & 2 \\
\hline 0 . & 15. & 8. \\
\hline 0 . & 14. & 8. \\
\hline 0. & 10. & 5. \\
\hline 1. & 19. & 10. \\
\hline 1. & 22. & 12. \\
\hline 1 . & 23. & 12. \\
\hline 1 . & 32. & 17. \\
\hline 2. & 50. & 27. \\
\hline 10 & 307 . & 162. \\
\hline 18. & 568. & 296. \\
\hline 28. & 898 & 465. \\
\hline $\begin{array}{l}36 . \\
43 .\end{array}$ & $\begin{array}{l}11744^{\circ} \\
1405^{\circ}\end{array}$ & $\begin{array}{l}604 . \\
718 .\end{array}$ \\
\hline 52. & $1697^{\circ}$ & 864. \\
\hline 02. & 2017 & 1023. \\
\hline 73. & 2377 & $1204^{\circ}$ \\
\hline 87. & $\begin{array}{l}2815 \\
3323\end{array}$ & 1428. \\
\hline $\begin{array}{l}103 . \\
130 .\end{array}$ & 4186 . & 2130 \\
\hline 145. & 4645 . & 2369. \\
\hline $1622^{\circ}$ & 5173. & 2654 \\
\hline $\begin{array}{l}186^{\circ} \\
212 .\end{array}$ & $\begin{array}{l}5949 . \\
6798 .\end{array}$ & $\begin{array}{l}3062 \\
3524\end{array}$ \\
\hline 232. & 7461 & 3872 \\
\hline 257. & $\begin{array}{l}8302 . \\
8795\end{array}$ & 4307 \\
\hline 295. & 9601 . & 4955 \\
\hline
\end{tabular}

.

RU106

PD

XE
STABL

CM242 AM241

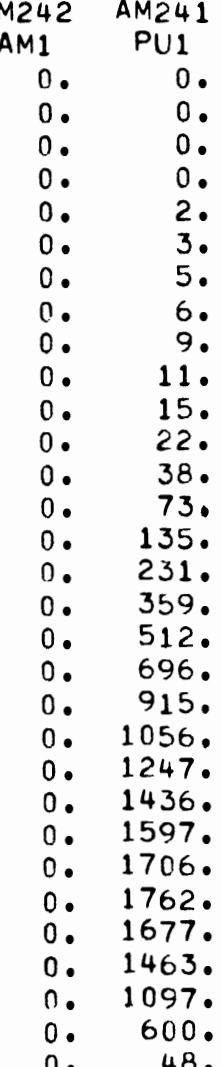



ANNUAL ISOTOPE PRODUCTION AT DISCHARGE (NO LOSS BASIS)

LWR - S - U236 RECYCLE WITH FAST REACTORS

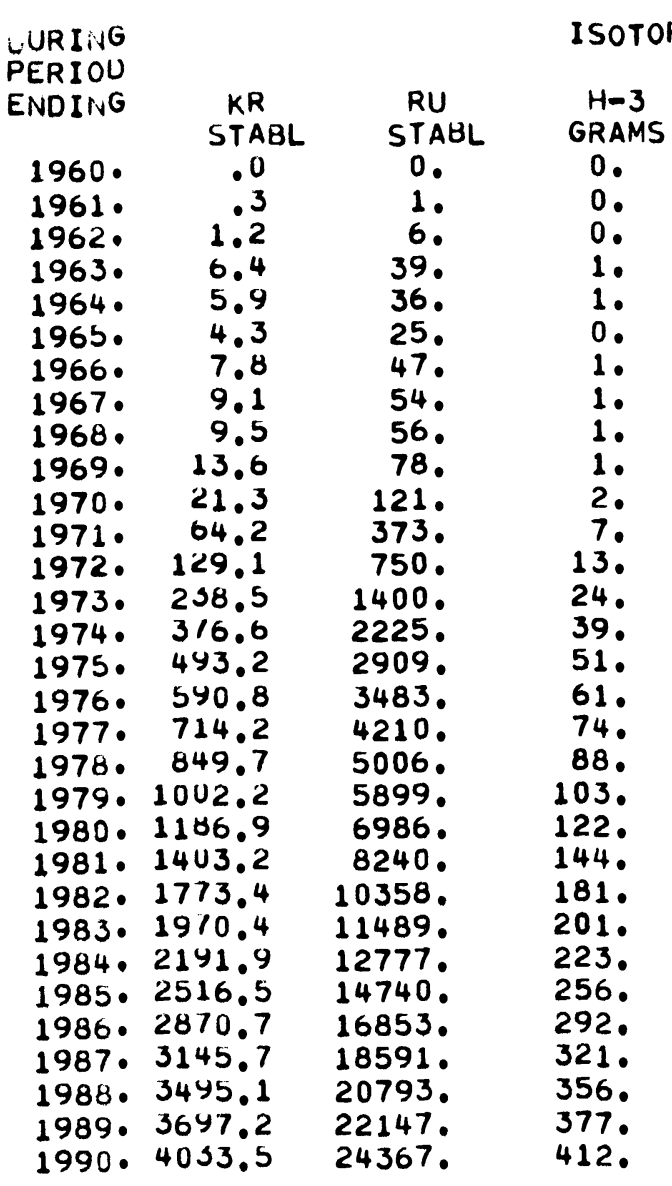


CUMULATIVE ISOTOPE PROODUCTION AT D I SCHARGE

PAGE 97

(NO LOSSES EXCEPT FOR RADIOACTIVE UECAY)

$L W R \cdot S$ - U236 RECYCLE WITH FAST REACTORS

CASE 5

TOTAL

INSIALLED ISOTOPE PRODUCTION, KGS

YEAR CAPACITY TONNE

PU-RP

PU-SP

NP237

AM241

1960.
1961.

1961.

1963.

$1964^{\circ}$

$1965^{\circ}$

1966.

1967.

1968.

1969.

1970 .

1971.

$1971^{\circ}$

1973.

1974.

1975.

1976.

1977.

1978.

1979.

1981.

1982.

1983.

1984.

1986.

1987.

1988.

1989.

1990.

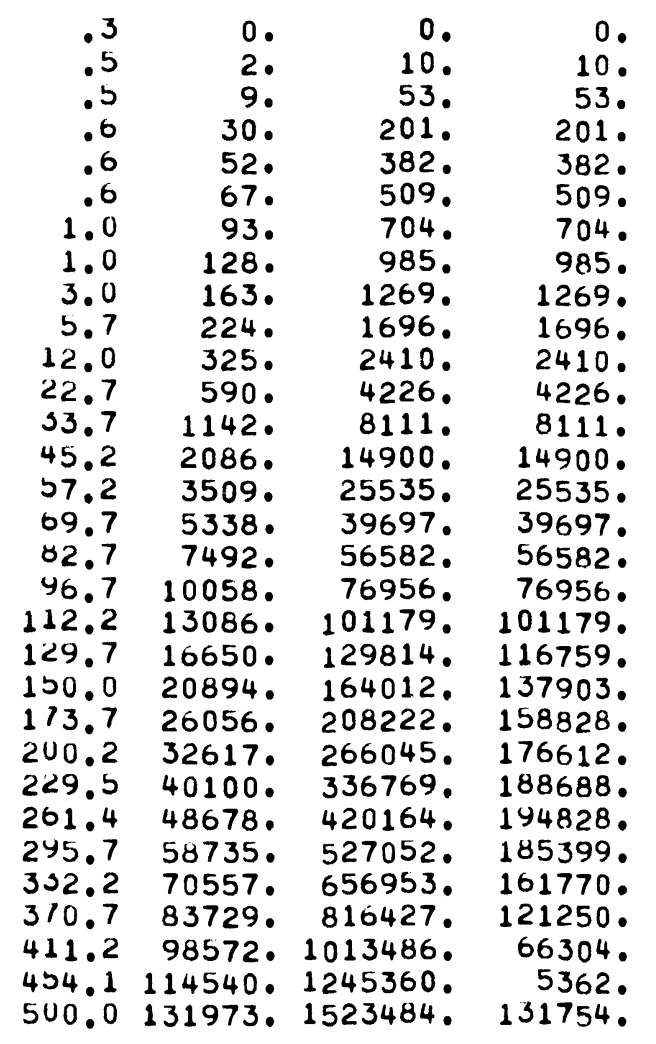

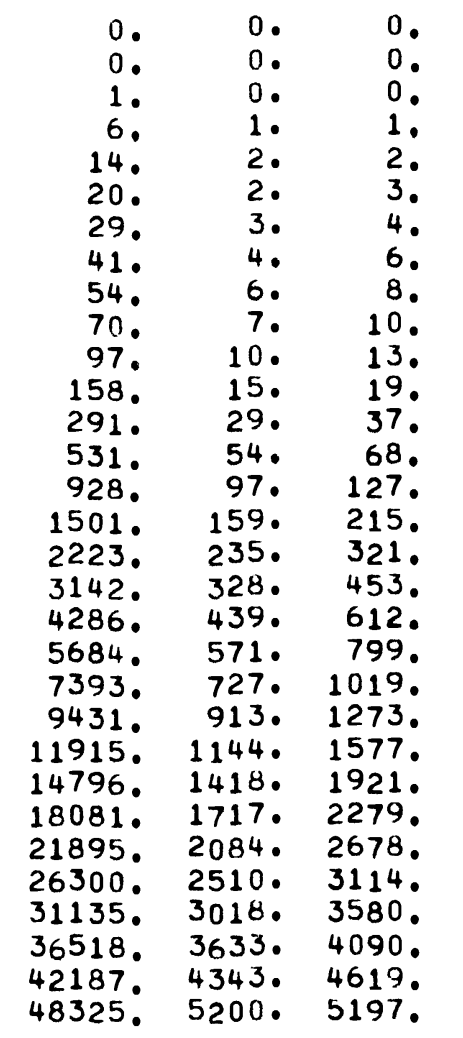

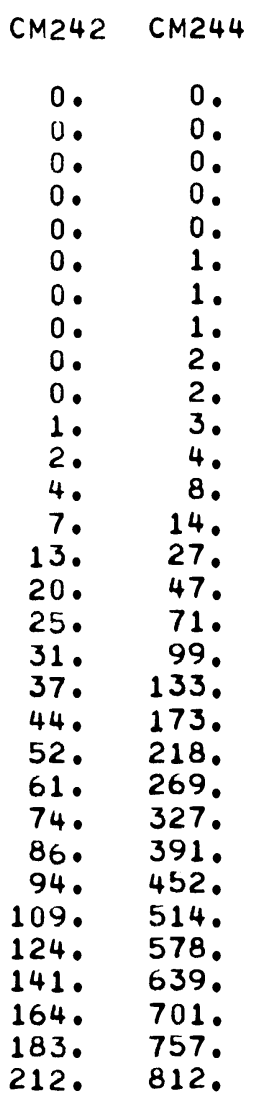

$C M 244$
$A M 3$
00
-00
-00
-00
-00
-00
-00
-00
-00
-00
-0
-0
-00
-00
-00
-00
-00
-00
-00
-00
-00
-00
-00
-00
-00
-00
-00
-00
-00
-00
-00

\begin{tabular}{|c|c|}
\hline \multirow{2}{*}{$\begin{array}{c}\text { CM244 } \\
\text { SUM }\end{array}$} & PU238 \\
\hline & NP \\
\hline 0 . & 0 . \\
\hline 0. & -0 \\
\hline 0. & -0 \\
\hline 0. & -0 \\
\hline 0. & -0 \\
\hline 1. & -0 \\
\hline 1. & -0. \\
\hline 1. & $\begin{array}{l}-0 . \\
-0 .\end{array}$ \\
\hline 2. & -0. \\
\hline 3. & -0. \\
\hline 4. & -0. \\
\hline 8. & -0 . \\
\hline 14. & -0. \\
\hline 27. & \\
\hline 47. & -0 . \\
\hline 71. & -0. \\
\hline $133^{\circ}$ & -0. \\
\hline 173. & -0 . \\
\hline $210^{\circ}$ & \\
\hline 327 . & -0 \\
\hline 391. & -0. \\
\hline 452 . & \\
\hline 514. & -0 . \\
\hline $\begin{array}{l}578 . \\
639\end{array}$ & -0. \\
\hline $\begin{array}{l}059 . \\
701 .\end{array}$ & \\
\hline 757. & -0 \\
\hline ? & \\
\hline
\end{tabular}

PU238
$C M$
0
-0
-0
-0
-0
-0
-0
-0
-0
-0
-0
-0
-0
-0
-0
-0
-0
-0
-0
-0
-0
-0
-0
-0
-0
-0
-0
-0
-0
-0
-0

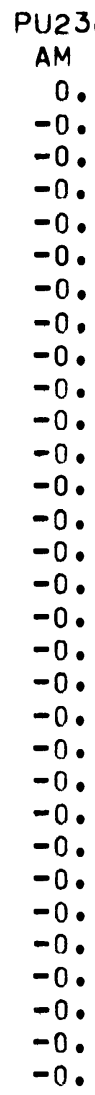

PU238

SUM 
ISOTOPE PRODUCTION, KGS

YEAR

\section{ENDING}

1960.

1961.

1962.

1963.

1964.

1965.

1966.

1967.

1968

1969.

1970.

1971.

1972.

1973.

1974.

1975.

1976.

1977

1978.

1979.

1980.

1981.

982

1983.

$1984^{\circ}$

1986.

1987 .

1988 .

1989.
1990.
GW-YR U-235

$u-236$

.2
.6
1.0
1.5
2.1
2.6
3.4
4.3
6.8

21.

21.

304.

440 .

580 .

805 .

1103.

1405 .

2192988 .

41.25569 .

09.910659 .

108.319225.

106.931440 .

216.246852 .

286.565123.

$464.0112634^{\circ}$.

574.2143161.

701.6179618 .

849.2223841 .

1019.2279666.

1214.1343387.

1436.0417287.

1686.8503442 .

1908.0604671 .

261.2716152.

3009.5972623 .

3428.51115309 .

0
37
17$$
128 .
$$

178

250 .

351 .

460 .

610 .

862

1486 .

2823.

5198 .

8999 .

14324 .

21068

29634.

40329

53475 .

69718 .

89327.

113441.

141669

213272 .

258866 .

309494 .

366434 .

427000 .

492866 .

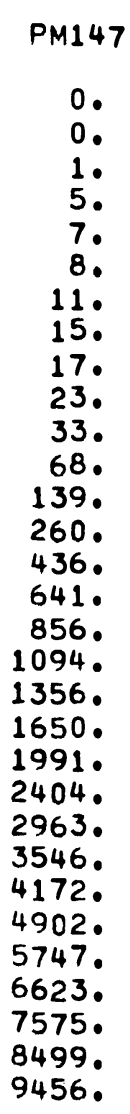

CE144 CS137 SR-9 0

1306. 9102. 4120 .

1560. 11909. 5388.

1851. 15190. 6870.

2199. 19051. 8613.

2660 . 23581 • 10660 .

3347 . 29289. 13247 .

3978 . 35550 . 16080.

4626. 42451 . 19200 .

5425. 50345. 22743 .

6360 . 59314. 26754.

7219.69057 . 31068.

8952.91059 .40656$.

9798.103234 . 45861 .

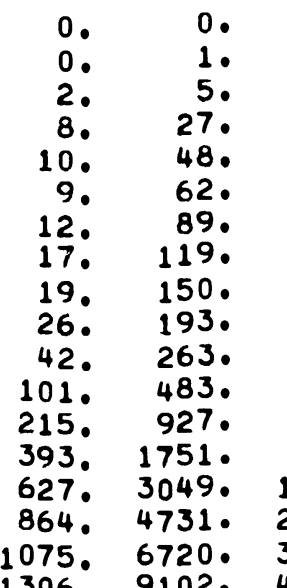

0.

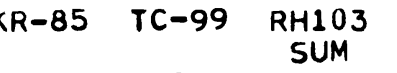

RU106

PT

XE CM242 AM241

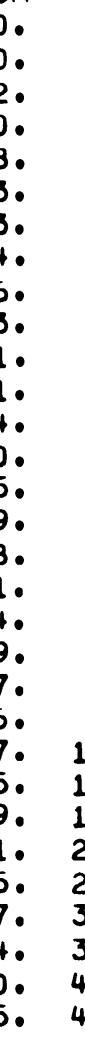

0.
0.
1.
3.
4.
4.
5.
6.
7.
9.

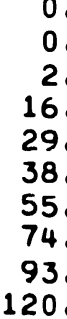

0 . 0 .

3.

19.

188 .

246 .

352 .

$477^{\circ}$

606.

792 .

14. 161. 1084 .

33. 291. 1963.

70. 550. 3735 .

133. 1040. 7003 .

220. 1824. 12157 .

310. 2848. 18896.

393. 4073. 26956 .

482 5554, 36691。

580.7314 .48262 .

690. 9385. 61905.

821.11838 . 78069 .

987. 14736. 97227.

1239. 18367. 121382。

1500 . 22422. 148310 。

1763. 26941. 178364 .

2139. 32254. 213045 .

2569. 38375. 252779.

3058. 45296. 296516.

1410.59435. 30554. 3652, 53223. 345320.

1788 . 77831. 40075 .

4969. 71677. 453975.

AM1 PUI

$\begin{array}{rr}0 . & 0 . \\ -0 . & -0 .\end{array}$

$-0.00$

$-0.12$

$-0.6$

$-0.10$.

-0 .

$-0.37$.

$-0.52$.

$-0.74$

$-0.112$

-0.185 .

$\begin{array}{ll}-0 . & 320 \\ -0 . & 550 .\end{array}$

$-0.909$.

-0. 1419.

$-0.2113$.

-0. 3025 .

-0. 4076.

- 0.5317.

-0.8332 .

-0.10026 .

$-0.11773$.

$-0.13432$.

-0 . 14875.

$-0.16525$.

-0.16548 . 
(NO LOSSES EXCEPT FOR RADIOACTIVE DECAY)

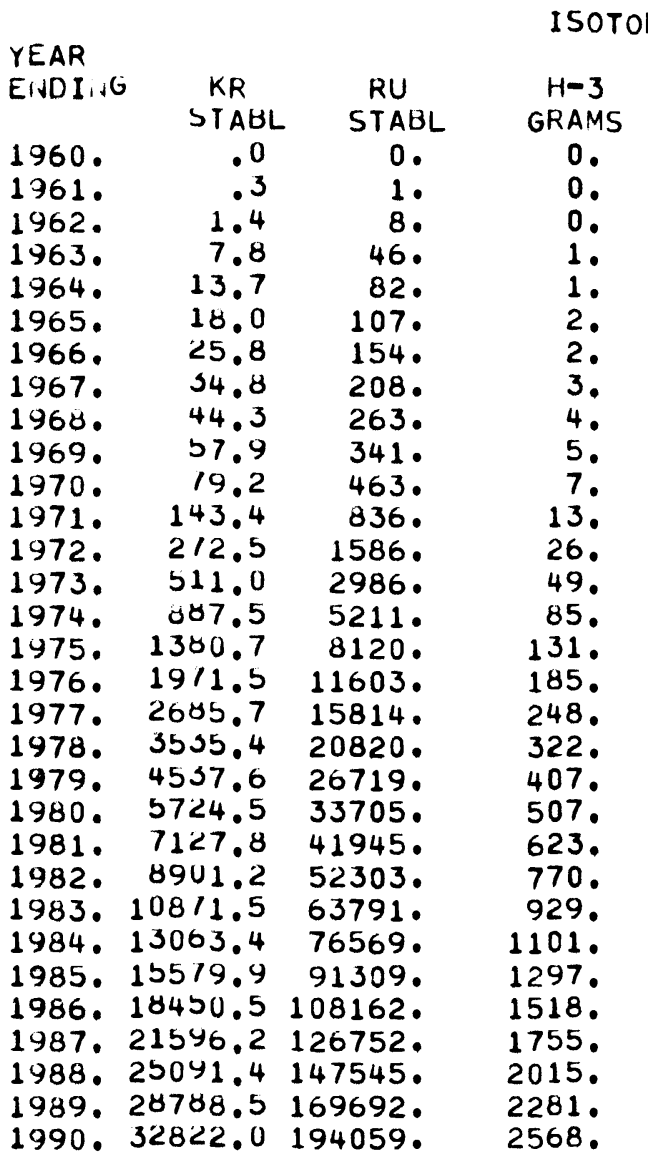


NET ANNUAL I SOTOPE AVA I LABILI T Y

PAGE 100

(WITH TARGET IRRADIATION. PROCESS LOSSES AND DECAY LOSSES)

LWR ' S - U236 RECYCLE WITH FAST REACTORS

CASE 5

TUTAL

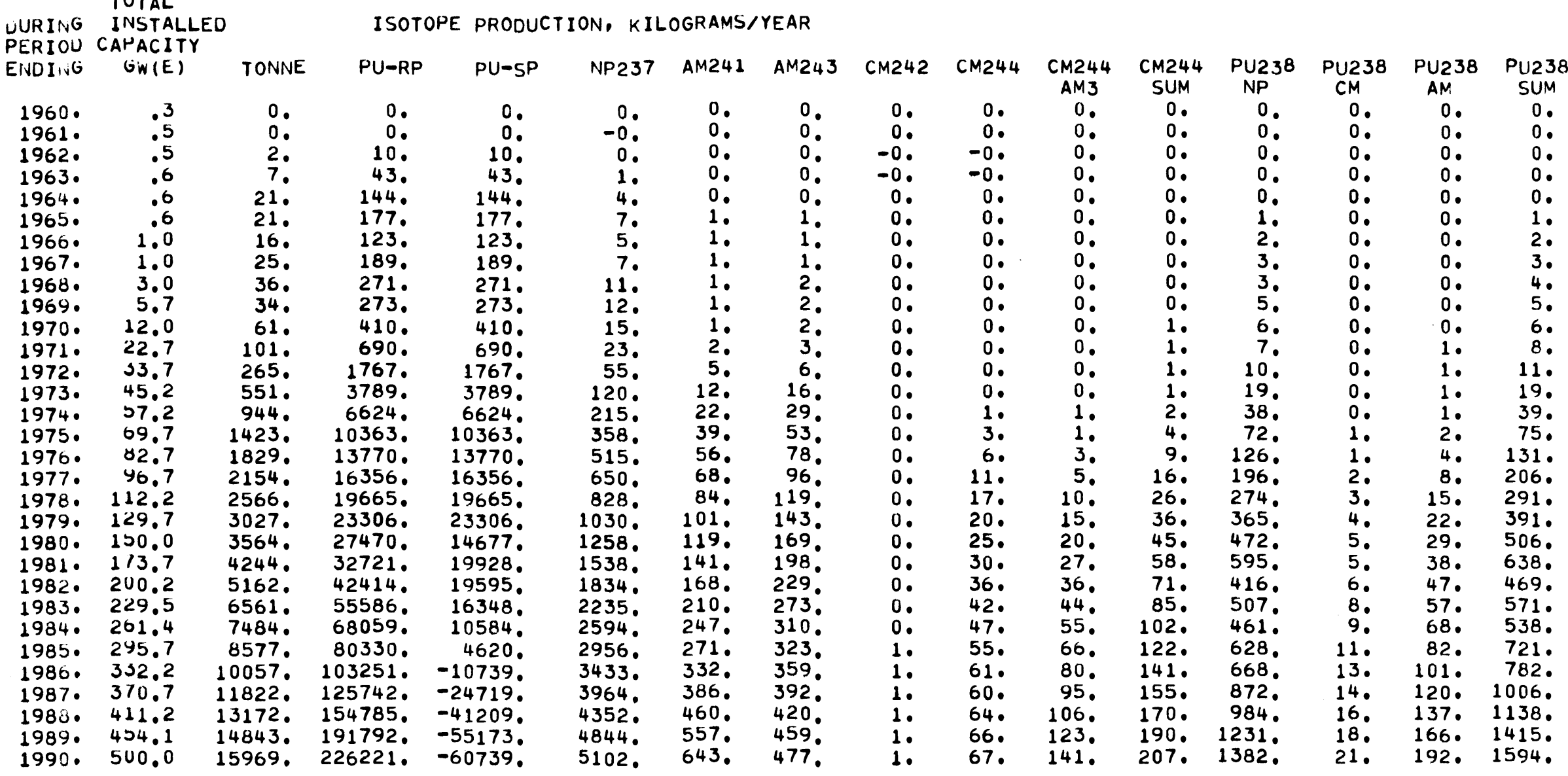


NET ANNUAL I SOTOP PE AVA I L A B I L I T Y

PAGE 101

(WITH TARGET IRRADIATION. PROCESS LOSSES AND DECAY LOSSES)

$L W R \cdot S$ - U236 RECYCLE WITH FAST REACTORS

CASE

5

UUR IYiG

EERIIIA

1960

$1960^{\circ}$

1961.

1963.

1964.

1965.

1960.

1907.

1968.

1970 .

1971.

1972 .

1973.

1974 .

1975 .

1976.

1977.

1970. Y5.

1980.127 .4

$1981 . \quad 147.6$

$1982 . \quad 110.1$

1984. 221.9

1985.250 .8

1986.281 .2

1987. 313.2

$1989.381 .7 \quad 109251$.

$1990^{\circ}$

199.

134.

136.

221 .

292.

296.

584.

968

2529

4988 .

8394.

15104.

17905 .

21330 .

25232 .

29916.

35728 .

43338 .

62447

62447

72422

99205 .

$121886^{\circ}$
GW-YR U-235

ISOTO

$0-236$

$$
\begin{array}{r}
0 \\
-0
\end{array}
$$

20.

129455.

0.
-0.
$30^{\circ}$
$14^{\circ}$
$48^{\circ}$
$61^{\circ}$
$43^{\circ}$
$71^{\circ}$
$99^{\circ}$
$107^{\circ}$
$147^{\circ}$
$246^{\circ}$
$610^{\circ}$
$1309^{\circ}$
$2328^{\circ}$
$3724^{\circ}$
$5219^{\circ}$
$6609^{\circ}$
$8394^{\circ}$
$10482^{\circ}$
$12883^{\circ}$
$15918^{\circ}$
$192170^{\circ}$
$23632^{\circ}$
$27664^{\circ}$
$32213^{\circ}$
$37958^{\circ}$
$44682^{\circ}$
$49616^{\circ}$
$55801^{\circ}$
$59355^{\circ}$

PM147
0.
0.
-0.
-0.
0.
0.
2.
2.
1.
2.
2.
3.
4.
6.
18.
36.
63.
98.
127.
150.
180.
213.
250.
298.
360.
460.
522.
596.
698.
815.
907.

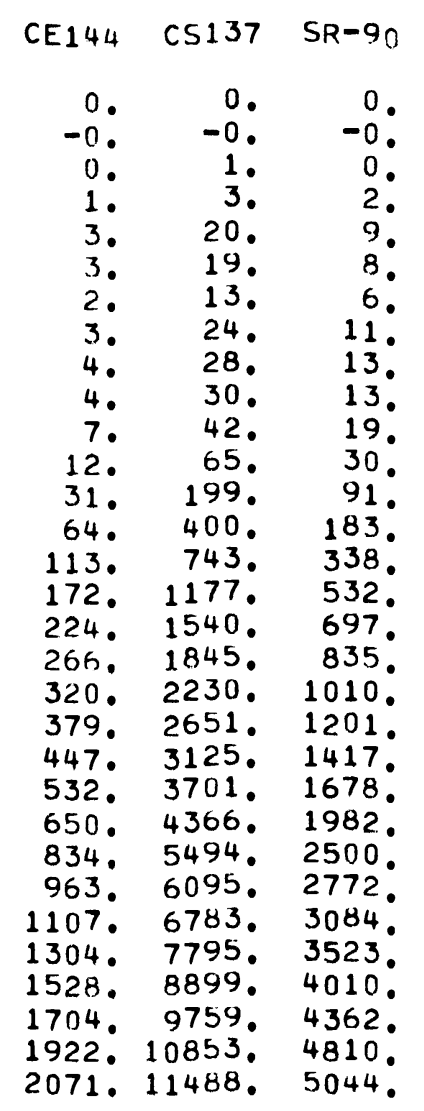

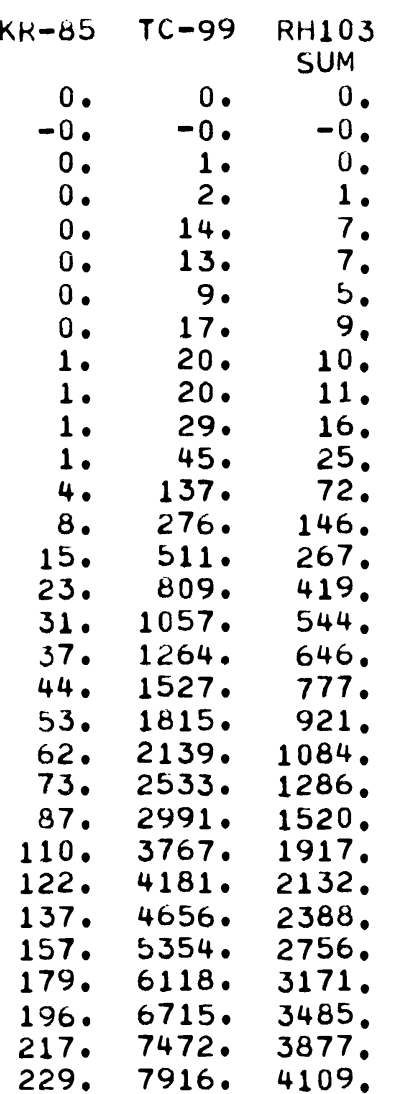

RU106

PD

XE
STABL

CM242 AM241

0.
-0.

-0.
0.

0.

1.

1.

1.

2.

3.

4.
$120^{\circ}$
24.

24.

44.
69.

90.

107.

129.

180. 1864. 12278 .

214. 2208. 14548 .

259. 2608, 17242 .

335. 3268. 21740.

396. 3649. 24235 .

456. 4068. 27049 .

566. 4782. 31213 .

675. 5509. 35761.

798. 6228. 39363.

955. 7134. 43924 .

1088. 7782. 46679.

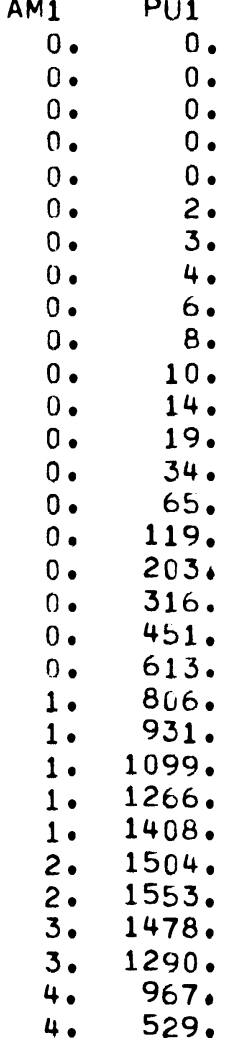


NET ANNUAL I SOTOPE AVAILABILI T Y

(WITH TARGET IRRADIATION. PROCESS LOSSES AND DECAY LOSSES)

UURIIVG

PERIOU

ENDING

1960

$1961^{\circ}$

1962.

1963.

1964.

$1965 \circ$

1965.

$1966^{\circ}$

1968.

1969.

1970 .

1971 .

1972.

1973. 110.2

1974.214 .6

1975. 358.9

1976. 443.9

1977. 531.7

1977. 642.8

$\begin{array}{ll}1978 . & 642.8 \\ 1979 . & 764.7\end{array}$

1979. 764.7

1980.942 .0

1981.1008 .2

$1982 \cdot 1262.9$

1983. 1546.1

1984.1773 .3

$1985.19 / 2.7$

$1986 \cdot 2264.8$

1987.2583 .6

1988.2831 .1

1989.3145 .6

1990.3327 .5
ISOTOPE PRODUCTION, KILOGRAMS/YEAR

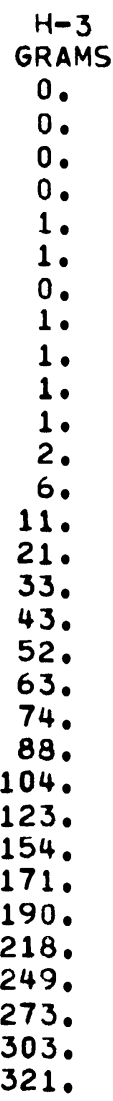


CUMULATIVE I SOTOPE A $V A$ A I L A B I L I T Y

PAGE 103

(WITH TARGET IRRADIATION, PROCESS LOSSES AND DECAY LOSSES)

TOTAL

INS I ALLED

YEAR CAPACITY

EIVDING GW(E)

TONNE PU-RP PU-SP

NP237 AM241 AM243 CM242

CM244

CM244

1960.

1961.

1963.

1904.

1965.

1900.

1368 .

1709 .

1970 .

1971 .

1972 .

1473 .

1974.

1975.

$1970^{\circ}$

1977.

1978.

1980 .

.3
.5
.5
.6
.6
.6

0 .

0
-0

2.

9.

1.0

1.0
3.0

5.7

12.0

22.7
53.7

33.7
45.2

57. 1142 .

69.22086.

82.75338.

96.77492

112.210058.

129.713086 .

$\begin{array}{rr}-0 . & -0 . \\ 10 . & 10 .\end{array}$

0.
-0.
0.

52.52.

374.

499.

374.

691.

499.

$966.966^{\circ}$

1245. 1245.

$1663^{\circ}$

2363. 2363 .

$4144^{\circ} 4144^{\circ}$

7952.7952.

14609 .

25037 .

38926.

55485 .

38926

75467 .

99224.

113.7 16650. 127308. 114514.

$\begin{array}{lll}20894^{\circ} & 160836 \circ & 135249^{\circ} \\ 26056 . & 204180^{\circ} & 155774^{\circ}\end{array}$

1982.

1983.

229.532617.

201.440100 . 330191. 185072.

295.748678 . 411929. 191100 .

$332.258735 \circ 516684.181865$.

310.7 70557. 643979. 158700.

411.283729.

1988.

404.198572 .

65086 .

1.

13.

18.

37.

49.

83.

87.
142.

262 .

477.

836.

1351 .

2000 .

2828.

5116.513 .719$.

$8488^{\circ} 820^{\circ} 1146$.

$10723^{\circ} 1028.1419$.

13317 . 1274.1729.

16273. 1543. 2051.

19706. 1873. 2410.

2256.2802.

28022. 2712. 3222.

$\begin{array}{lll}328666^{\circ} & 3265^{\circ} & 3680^{\circ} \\ 379680^{\circ} & 3903 . & 4157 .\end{array}$

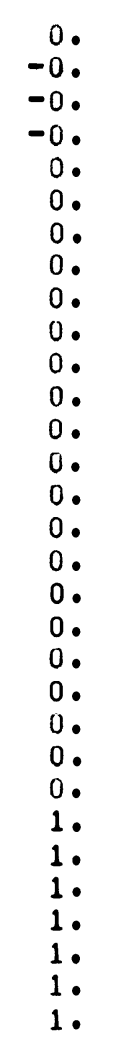

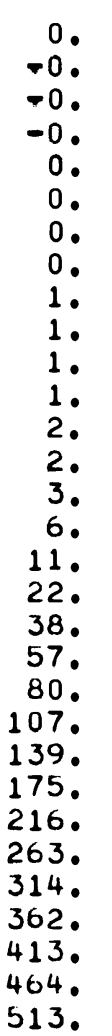

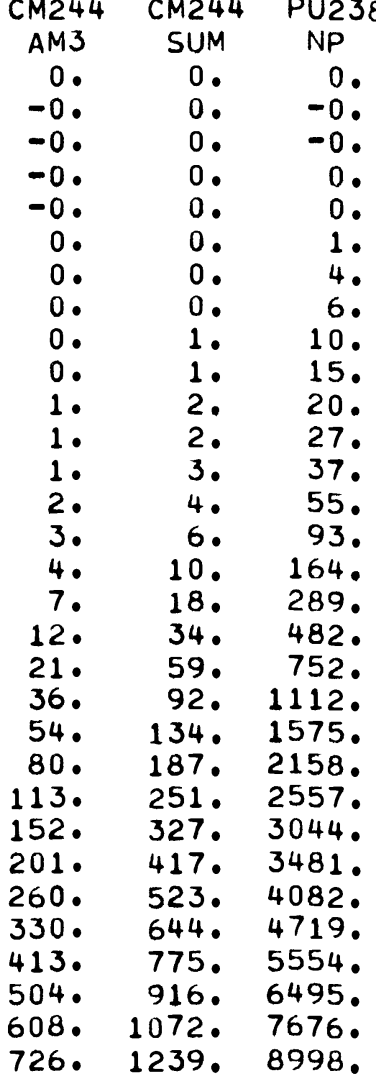

PU238 PU238 PU238

\begin{tabular}{|c|c|c|}
\hline$C M$ & $A M$ & SUM \\
\hline 0. & 0. & 0. \\
\hline-0. & -0. & 0. \\
\hline-0 . & -0. & 0 . \\
\hline-0 . & -0 . & 0. \\
\hline 0. & -0. & 0. \\
\hline 0. & 0. & 1. \\
\hline 0. & 0. & 4. \\
\hline 0 . & 0 . & 7. \\
\hline 0 . & 0 . & 10. \\
\hline 0 . & 1. & 15. \\
\hline 0 . & 1. & 22. \\
\hline 0. & 2. & 29. \\
\hline 0 . & 2. & 40. \\
\hline 0 . & 3. & 59. \\
\hline 1. & 4. & 98. \\
\hline 1. & 6. & 171. \\
\hline 2. & 10. & 301. \\
\hline 5. & 18. & 505. \\
\hline $\begin{array}{r}8 . \\
11 .\end{array}$ & $\begin{array}{l}33 . \\
54 .\end{array}$ & $\begin{array}{r}793 . \\
1178 .\end{array}$ \\
\hline 16. & 83. & 1674 . \\
\hline 21. & 120. & 2299. \\
\hline 27 . & 166. & 2751 . \\
\hline 35. & 222 . & 3301. \\
\hline 43. & 289. & 3813. \\
\hline 54. & 369. & $\begin{array}{l}4505 . \\
5250^{\circ}\end{array}$ \\
\hline 80. & $\begin{array}{l}467 . \\
584^{\circ}\end{array}$ & 6217. \\
\hline 95. & 717. & 7307 . \\
\hline 113. & $877^{\circ}$ & 8666 \\
\hline & • & 101 \\
\hline
\end{tabular}


C UMULATIVE I SOT OP E AVA I LAB I L I T Y

PAGE 104

(WITH TARGET IRRADIATION, PROCESS LOSSES AND DECAY LOSSES)

$L W R \cdot S$ - U236 RECYCLE WITH FAST REACTORS

CASE 5

ISOTOPE PRODUCTION, KGS

YEA

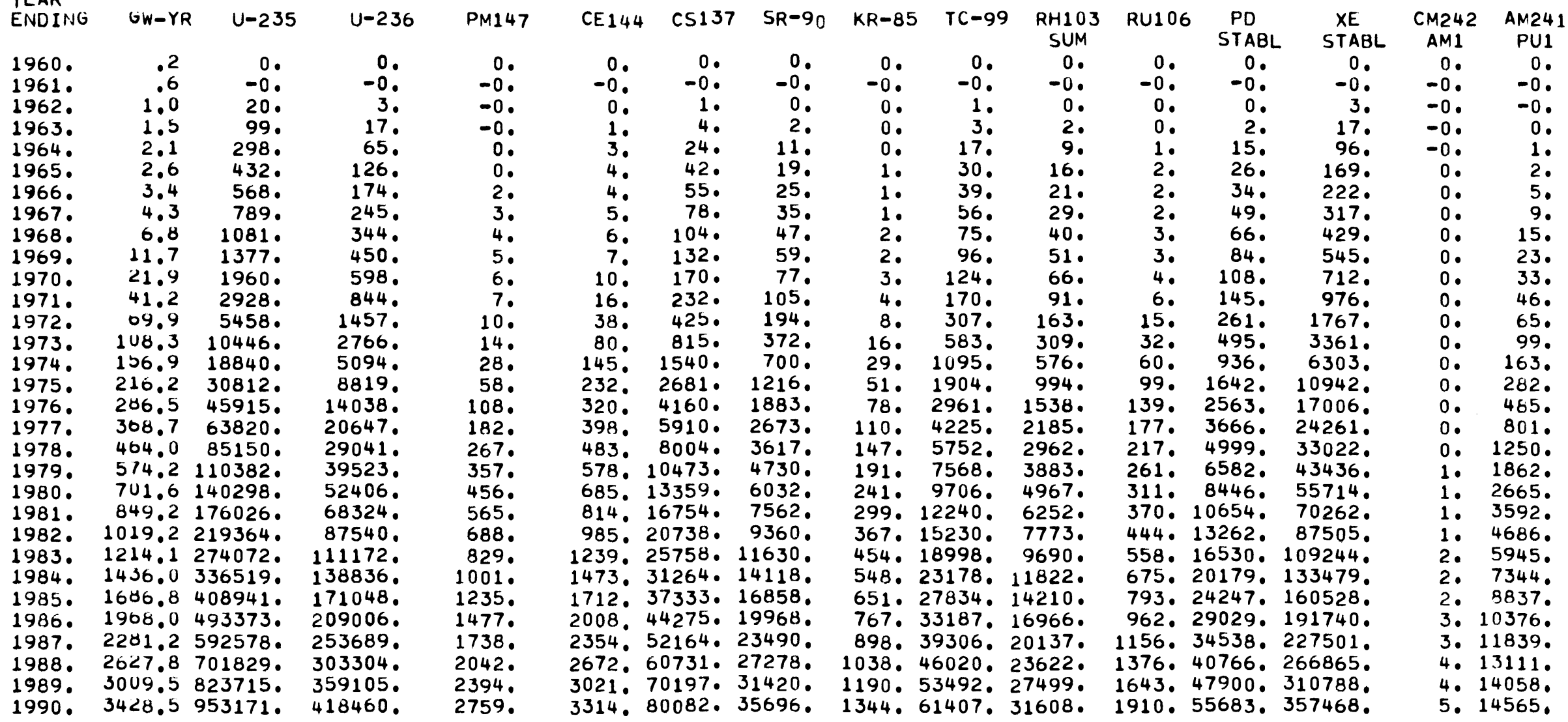


(WITH TARGET IRRADIATION, PROCESS LOSSES AND DECAY LOSSES)

ISOTOPE PRODUCTION, KGS

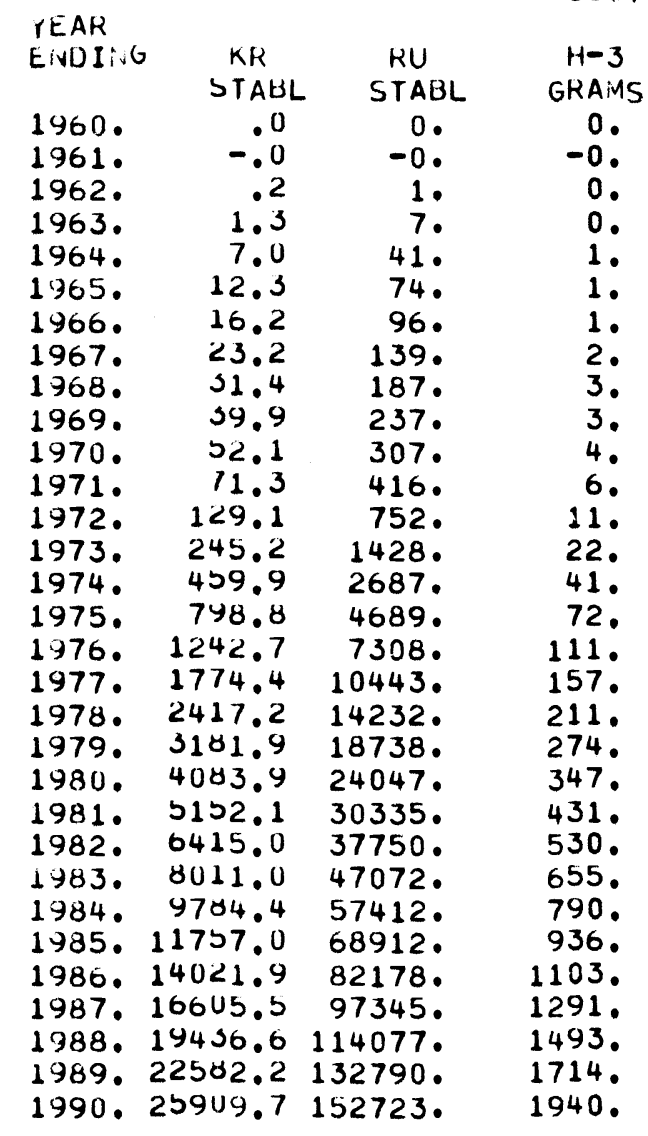





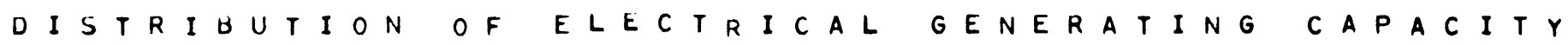

PAGE 106

LWR'S - PU \& U236 RECYCLE WITH FAST REACTORS

CASE 6

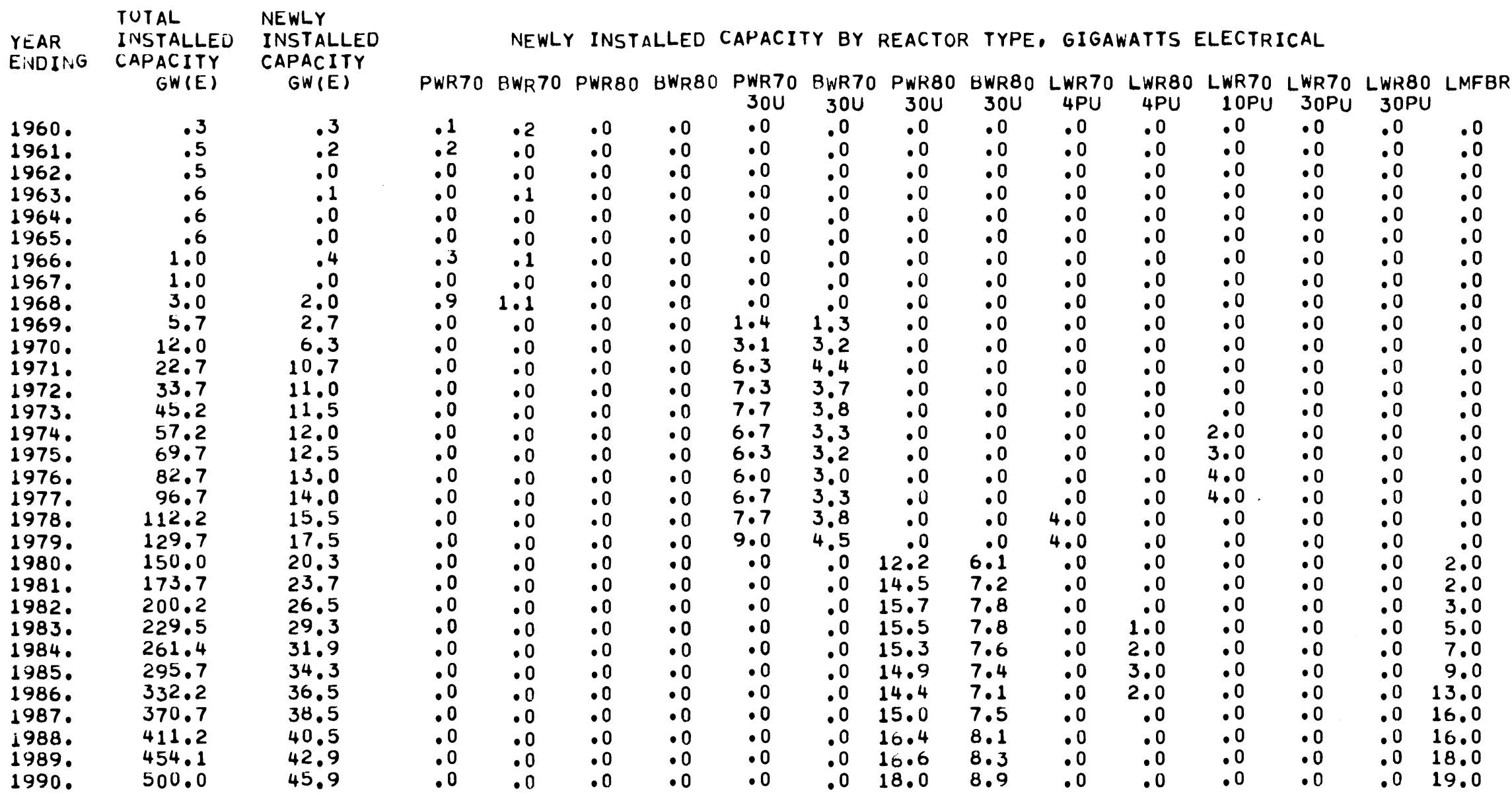


U I STRIBUTION OF ELECTRICAL GENERATIA G CA PACA T Y

PAGE 107

LWR'S - PU \& U236 RECYCLE WITH FAST REACTORS

CASE 6

$\begin{array}{lll} & \text { TUTAL } & \text { NEWLY } \\ \text { YEAR } & \text { INSTALLED } & \text { INSTALLED }\end{array}$

EINDIIG CAPACITY CAPACITY

GW(E) GW(E)

CUMULATIVE INSTALLED CAPACITY BY REACTOK TYPE, GIGAWATTS ELECTRICAL

$\begin{array}{lrrrrr}1960 . & .3 & .3 & .1 & .2 & .0 \\ 1961 . & .5 & .2 & .2 & .2 & .0 \\ 1962 . & .5 & .0 & .2 & .2 & .0 \\ 1963 . & .6 & .1 & .2 & .4 & .0 \\ 1964 . & .6 & .0 & .3 & .4 & .0 \\ 1965 . & .6 & .0 & .3 & .4 & .0 \\ 1960 . & 1.0 & .4 & .6 & .4 & .0 \\ 1967 . & 1.0 & .0 & .6 & .4 & .0 \\ 1968 . & 3.0 & 2.0 & 1.5 & 1.5 & .0 \\ 1969 . & 5.7 & 2.7 & 1.5 & 1.5 & .0 \\ 1970 . & 12.0 & 6.3 & 1.5 & 1.5 & .0 \\ 1971 . & 22.7 & 10.7 & 1.5 & 1.5 & .0 \\ 1972 . & 33.7 & 11.0 & 1.5 & 1.5 & .0 \\ 1973 . & 45.2 & 11.5 & 1.5 & 1.5 & .0 \\ 1974 . & 57.2 & 12.0 & 1.5 & 1.5 & .0 \\ 1975 . & 69.7 & 12.5 & 1.5 & 1.5 & .0 \\ 1976 . & 82.7 & 13.0 & 1.5 & 1.5 & .0 \\ 1977 . & 96.7 & 14.0 & 1.5 & 1.5 & .0 \\ 1978 . & 112.2 & 15.5 & 1.5 & 1.5 & .0 \\ 1979 . & 129.7 & 17.5 & 1.5 & 1.5 & .0 \\ 1980 . & 150.0 & 20.3 & 1.5 & 1.5 & .0 \\ 1981 . & 173.7 & 23.7 & 1.5 & 1.5 & .0 \\ 1982 . & 200.2 & 26.5 & 1.5 & 1.5 & .0 \\ 1983 . & 229.5 & 29.3 & 1.5 & 1.5 & .0 \\ 1984 . & 261.4 & 31.9 & 1.5 & 1.5 & .0 \\ 1985 . & 295.7 & 34.3 & 1.5 & 1.5 & .0 \\ 1986 . & 332.2 & 36.5 & 1.5 & 1.5 & .0 \\ 1987 . & 370.7 & 38.5 & 1.5 & 1.5 & .0 \\ 1988 . & 411.2 & 40.5 & 1.5 & 1.5 & .0 \\ 1989 . & 454.1 & 42.9 & 1.5 & 1.5 & .0 \\ 1990 . & 500.0 & 45.9 & 1.5 & 1.5 & .0\end{array}$

\begin{tabular}{|c|c|c|c|c|c|c|c|c|c|c|}
\hline sho & 30u & $\begin{array}{r}\text { SWR } \\
30 U\end{array}$ & $30 \mathrm{U}$ & $30 U$ & 4PU & $\begin{array}{l}\text { LWROL } \\
\text { 4PU }\end{array}$ & IOPU & $\begin{array}{l}\text { LWR } \\
\text { 3OPU }\end{array}$ & $\begin{array}{r}\text { LWR80 } \\
\text { 30PU }\end{array}$ & \\
\hline .0 & .0 & .0 & .0 & .0 & .0 & .0 & .0 & .0 & .0 & .0 \\
\hline$\cdot 0$ & $\cdot 0$ & .0 & .0 & .0 & .0 & .0 & .0 & .0 & .0 & .0 \\
\hline$\cdot 0$ & $\cdot 0$ & .0 & .0 & .0 & .0 & .0 & .0 & .0 & .0 & .0 \\
\hline .0 & .0 & .0 & .0 & .0 & .0 & .0 & .0 & .0 & .0 & .0 \\
\hline .0 & $\cdot 0$ & .0 & .0 & .0 & .0 & .0 & .0 & $\cdot 0$ & .0 & .0 \\
\hline .0 & .0 & .0 & .0 & .0 & .0 & .0 & .0 & .0 & .0 & .0 \\
\hline .0 & $\cdot 0$ & .0 & .0 & .0 & .0 & .0 & .0 & .0 & .0 & .0 \\
\hline$\cdot 0$ & $\cdot 0$ & .0 & .0 & $\cdot 0$ & .0 & .0 & .0 & .0 & .0 & .0 \\
\hline$\cdot 0$ & $\cdot 0$ & .0 & .0 & .0 & .0 & .0 & .0 & .0 & .0 & .0 \\
\hline .0 & $1 \cdot 4$ & 1.3 & .0 & .0 & .0 & .0 & .0 & .0 & .0 & .0 \\
\hline$\cdot 0$ & $4 \cdot 4$ & 4.5 & .0 & .0 & .0 & .0 & .0 & $\cdot 0$ & .0 & .0 \\
\hline .0 & $10 \cdot 8$ & 8.9 & .0 & .0 & .0 & .0 & .0 & .0 & .0 & .0 \\
\hline .0 & $18 \cdot 1$ & 12.6 & .0 & .0 & .0 & .0 & .0 & .0 & .0 & .0 \\
\hline$\cdot 0$ & $25 \cdot 8$ & 16.4 & .0 & .0 & .0 & .0 & .0 & $\cdot 0$ & .0 & .0 \\
\hline$\cdot 0$ & $32 \cdot 5$ & 19.7 & .0 & $\cdot 0$ & .0 & .0 & $2 \cdot 0$ & $\cdot 0$ & .0 & 0 \\
\hline .0 & 38.8 & 22.9 & .0 & .0 & .0 & .0 & 5.0 & .0 & .0 & .0 \\
\hline$\cdot 0$ & 44.8 & 25.9 & .0 & .0 & .0 & .0 & 9.0 & $\cdot 0$ & .0 & .0 \\
\hline$\cdot 0$ & 51.5 & 29.2 & .0 & $\cdot 0$ & $\cdot 0$ & .0 & $13 \cdot 0$ & $\cdot 0$ & .0 & .0 \\
\hline .0 & $59 \cdot 2$ & 33.0 & .0 & .0 & 4.0 & .0 & 13.0 & .0 & .0 & .0 \\
\hline$\cdot 0$ & $68 \cdot 2$ & 37.5 & .0 & .0 & 8.0 & .0 & 13.0 & $\cdot 0$ & .0 & .0 \\
\hline$\cdot 0$ & $68 \cdot 2$ & 37.5 & 12.2 & 6.1 & 8.0 & .0 & $13 \cdot 0$ & $\cdot 0$ & .0 & 2.0 \\
\hline$\cdot 0$ & $68 \cdot 2$ & 37.5 & $26 \cdot 7$ & $13 \cdot 3$ & $8 \cdot 0$ & .0 & $13 \cdot 0$ & $\cdot 0$ & $\cdot 0$ & 4.0 \\
\hline $\begin{array}{l}.0 \\
.0\end{array}$ & $\begin{array}{l}68.2 \\
68.2\end{array}$ & 37.5 & $\begin{array}{l}42.4 \\
57.9\end{array}$ & $\begin{array}{l}21.1 \\
28.9\end{array}$ & 8.0 & $\bullet 0$ & 13.0 & $\cdot 0$ & .0 & 7.0 \\
\hline .0 & 68.2 & 37.5 & 73.2 & 36.5 & 8.0 & 3.0 & 13.0 & .0 & .0 & 12.0 \\
\hline$\cdot 0$ & 68.2 & 37.5 & 88.1 & 43.9 & 8.0 & 6.0 & 13.0 & .0 & .0 & 28.0 \\
\hline$\cdot 0$ & $68 \cdot 2$ & 37.5 & 102.5 & 51.0 & 8.0 & 8.0 & 13.0 & .0 & .0 & 41.0 \\
\hline .0 & 68.2 & 37.5 & 117.5 & 58.5 & $8 \cdot 0$ & 8.0 & 13.0 & .0 & .0 & 57.0 \\
\hline$\cdot 0$ & $68 \cdot 2$ & 37.5 & 133.9 & 66.6 & 8.0 & 8.0 & 13.0 & $\cdot 0$ & .0 & 0.0 \\
\hline$\cdot 0$ & $68 \cdot 2$ & 37.5 & 150.5 & 74.9 & 8.0 & 8.0 & 13.0 & .0 & .0 & 91.0 \\
\hline$\cdot 0$ & $68 \cdot 2$ & 37.5 & 168.5 & 83.8 & 8.0 & 8.0 & 13.0 & .0 & .0 & 10.0 \\
\hline
\end{tabular}


ANNUAL ISOTOPE PRODUCT IO IN A T D I S C HARGE PAGE 108

(NO LOSS BASIS)

LWR'S - PU \& U236 RECYCLE WITH FAST REACTORS

CASE

TUTAL

UURIIVG INSTALLED
PERIUU CATACITY

PERIUO CAPACITY

ISOTOPE PRODUCTION. KILOGRAMS/YEAR

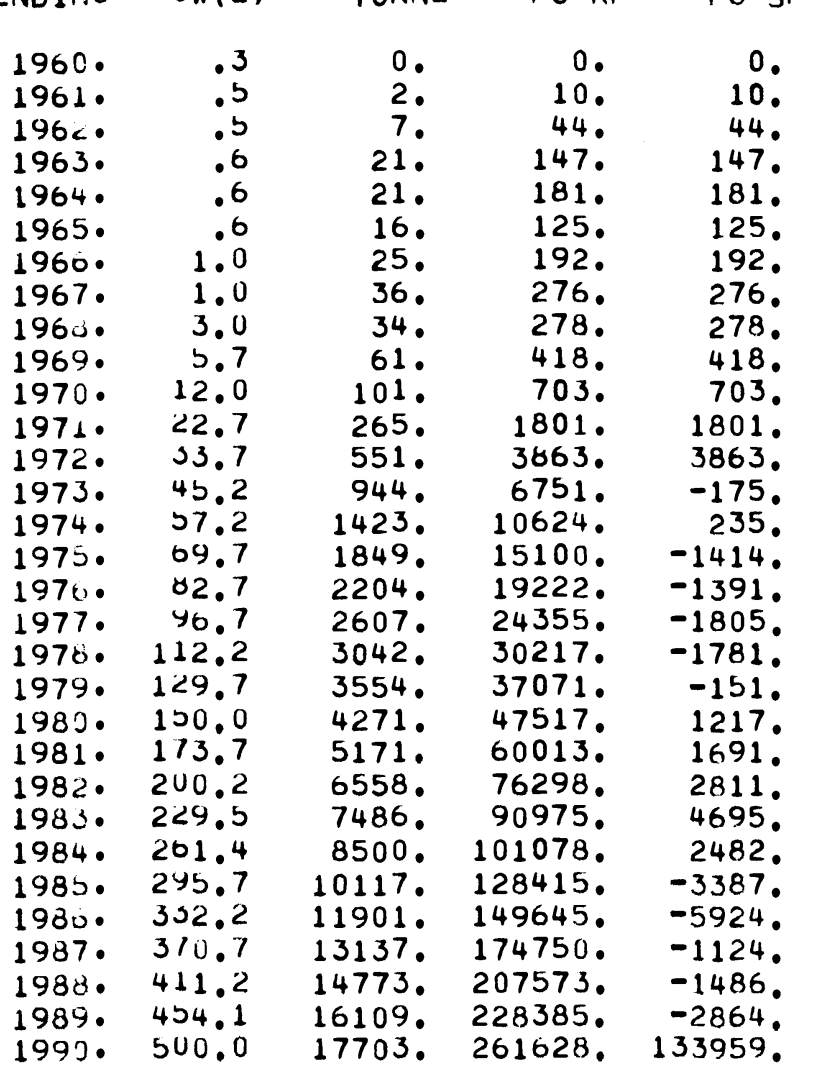

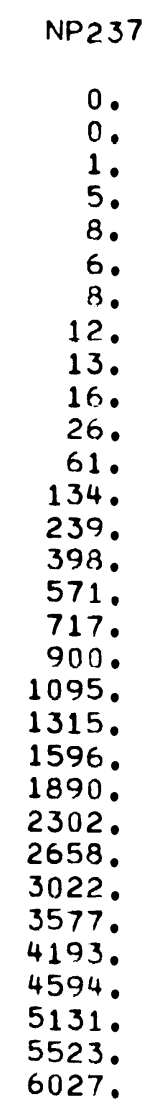

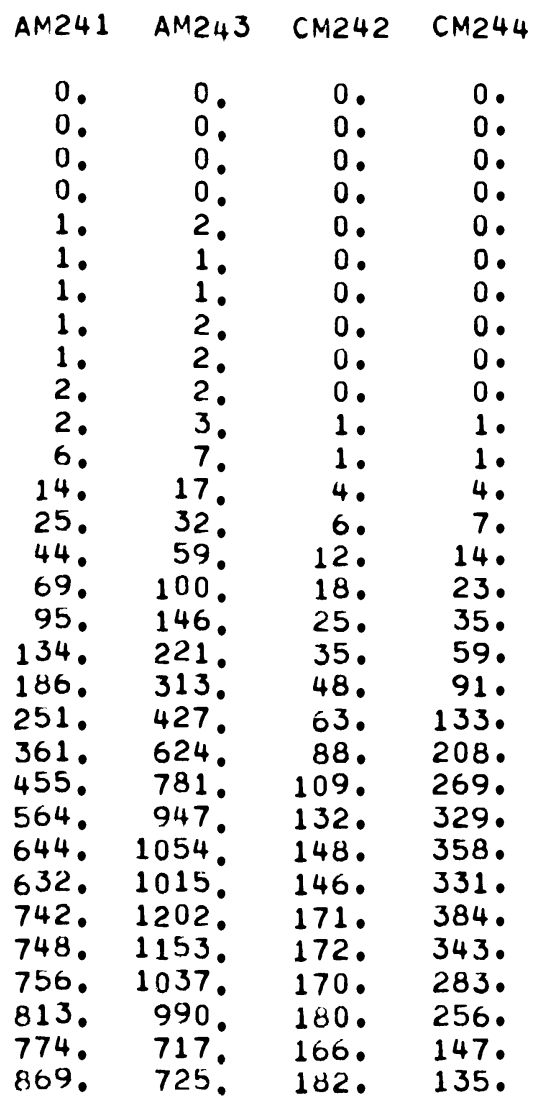

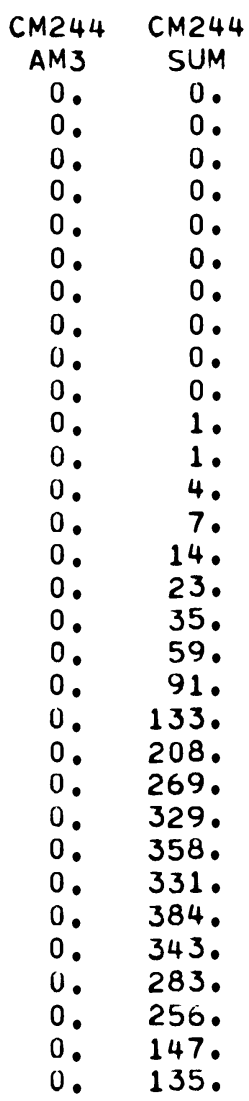

$P U 230$
$N P$.
0.
0.
0.
0.
0.
0.
0.
0.
0.
0.
0.
0.
0.
0.
0.
0.
0.
0.
0.
0.
0.
0.
0.
0.
0.
0.
0.
0.
0.

PU238
CM
0.
0.
0.
0.
0.
0.
0.
0.
0.
0.
0.
0.
0.
0.
0.
0.
0.
0.
0.
0.
0.
0.
0.
0.
00
0.
0.
00
0.
0.
0.
0

\begin{tabular}{|c|c|}
\hline \multirow{2}{*}{$\begin{array}{c}\text { PU238 } \\
\text { AM }\end{array}$} & Pu238 \\
\hline & SUM \\
\hline 0. & 0. \\
\hline 0. & 0 . \\
\hline 0. & 0. \\
\hline 0. & 0 . \\
\hline 0 . & 0 . \\
\hline 0. & 0. \\
\hline 0 . & 0 . \\
\hline 0. & 0. \\
\hline 0. & 0 . \\
\hline 0 • & $0 \cdot$ \\
\hline 0. & 0 . \\
\hline 0 . & 0 . \\
\hline 0. & 0 . \\
\hline 0. & 0. \\
\hline 0. & 0. \\
\hline $0 \bullet$ & 0 . \\
\hline 0 . & 0 . \\
\hline 0 . & 0 . \\
\hline 0. & 0 . \\
\hline 0 . & 0 . \\
\hline 0 . & 0. \\
\hline 0 . & 0 . \\
\hline 0 . & 0 . \\
\hline 0 • & 0 , \\
\hline 0 . & 0 . \\
\hline 0. & 0. \\
\hline 0 . & 0. \\
\hline 0 . & 0 . \\
\hline 0 . & 0 . \\
\hline 0. & 0 . \\
\hline 0 • & 0 . \\
\hline
\end{tabular}


(NO LOSS BASIS)

LURING PERION EivUIivg

1960

1961.

1962.

1963.

1964.

1963.

1966.

1967.

1968.

1969.

1970.

1971 .

1972.

1973.

1974.

1975

$1970^{\circ}$

1970 .

$1979^{\circ}$

1980.

1981.

1982.

1983.

$1984^{\circ}$

$1980^{\circ}$

1987.

1988.

$1989^{\circ}$
$1990^{\circ}$

\section{ISOTOPE PRODUCTION, KILOGRAMS/YEAR}

$G W-Y R \quad U-235$

$$
\text { PM1 } 147
$$

CE144

CS137 SR 90

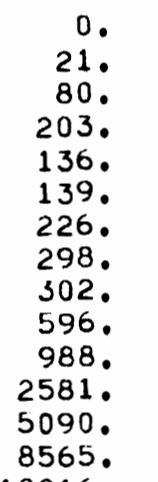

8565.

12216.

$17898^{\circ}$.

20701.

23990 .

27997 .

33417.

40980 .

52347 .

60295 .

69900 .

82808 .

98346.

$121934^{\circ}$.

$346.6 \quad 121934$.

419.1147309.

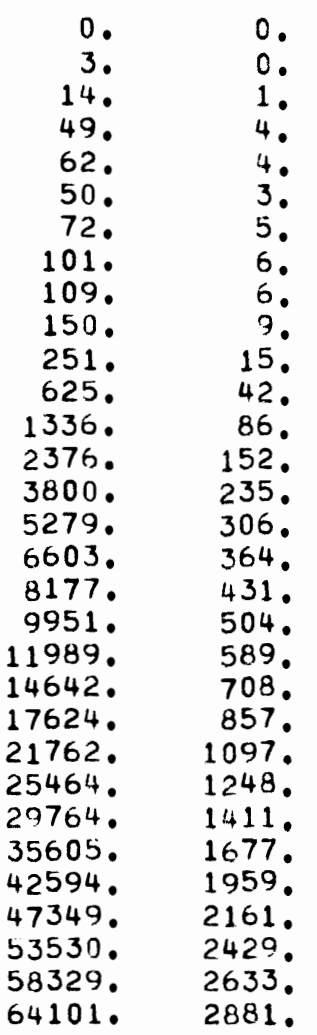

2881 .
31. 74. 147309. 64101 .

0.
0.
2.
7.
7.
5.

11.

11.

19.47.

84. 226.

173.455.

$466^{\circ} 1338$.

607. 1758 .

723.2119.

854 . 2528.

$198^{\circ} 2977^{\circ} 1297$.

$1400.4190^{\circ} 1793$.

$1714^{\circ} 4973.2127$.

2209. 6264. 2706.

2558: 6970. 3025 .

$\begin{array}{lll}2919 & 7647 . & 3352 . \\ 3482 & 8947 . & 3875 .\end{array}$

4083.10211 .4413

4514.11065 . 4759.

5088.12306 . 5261.

5529.13211.

$5611^{\circ}$

$6066.14500^{\circ}, 6137$.

\section{$K R-85$}

TC-99 RH103 RU106

PD

$X E$

CM242 AM241 SUM

0

STABL STABL

AM

P. PU1

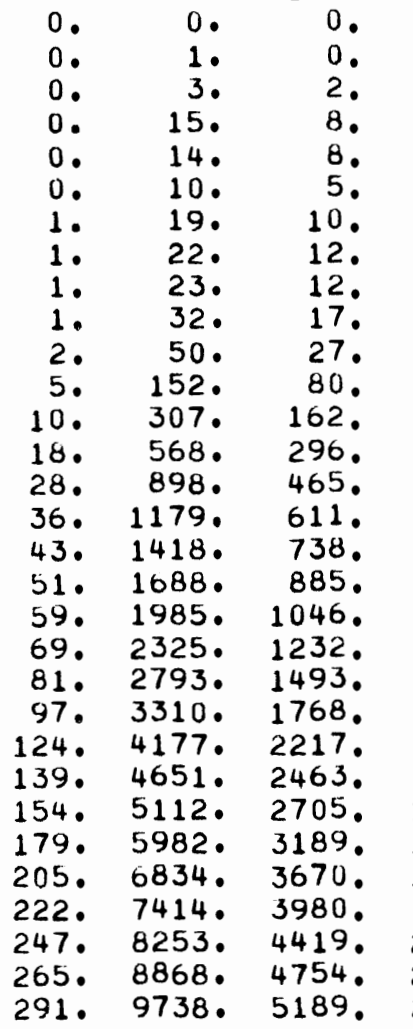

1.

2.

14.

2.

3. 17.

4.

6

26.

54.

98.

5154.

206. 1042. 6771 .

254. 1279. 8154

313. 1562. 9722 .

376. 1878. 11444.

448. 2237. 13419 .

551 . 2747. 16140。

660 . 3256. 19192.

835. 4031 . 24216 .

972. 4472.27064

1084. 4824. 29789

1373. 5820. 34989 .

1635. 6687. 40079 .

1899. 7413. 43610 .

2248. 8429. 48661 .

2548. 9217, 52437 .

2929. 10334. $57713^{\circ}$.

O. $\quad 0$.

0.0.

0.20

0.3.

0 . 5 .

0.9. 

(NO LOSS BASIS)

LWR'S - PU \& U236 RECYCLE WITH FAST REACTORS

LURIING

PERIUD

ISOTOPE PRODUCTION, KILOGRAMS/YEAR

ENDIIVG

1960.

1961.

1962.

1963.

$1964^{\circ}$

1965.

1960.

1967 .

1968.

1969.

1970

1972. 129.1

1973. 238.5

1974. $3 / 6.6$

1975. 442.1

1976. 587.0

1977. 6Y1.

1970.804 .2

1979. 934.2

$1980 \cdot 1110.0$

1981.1317 .9

1982. 1681.3

1983. 1883.3

$1984 \cdot 2087.8$

$1985 \cdot 2426.3$

$1986 \cdot 2769.4$

21075 .

22711.

$1990.3965 .7 \quad 25027$.

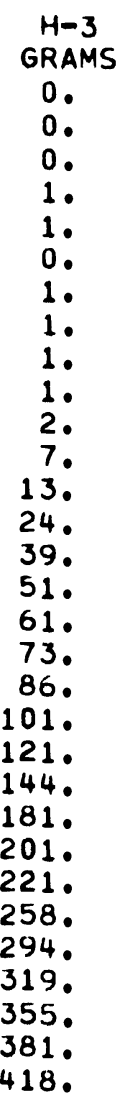


(NO LOSSES EXCEPT FOR RADIOACTIVE DECAY)

TUTAL

INS I ALLEU

YEAR CAPACITY

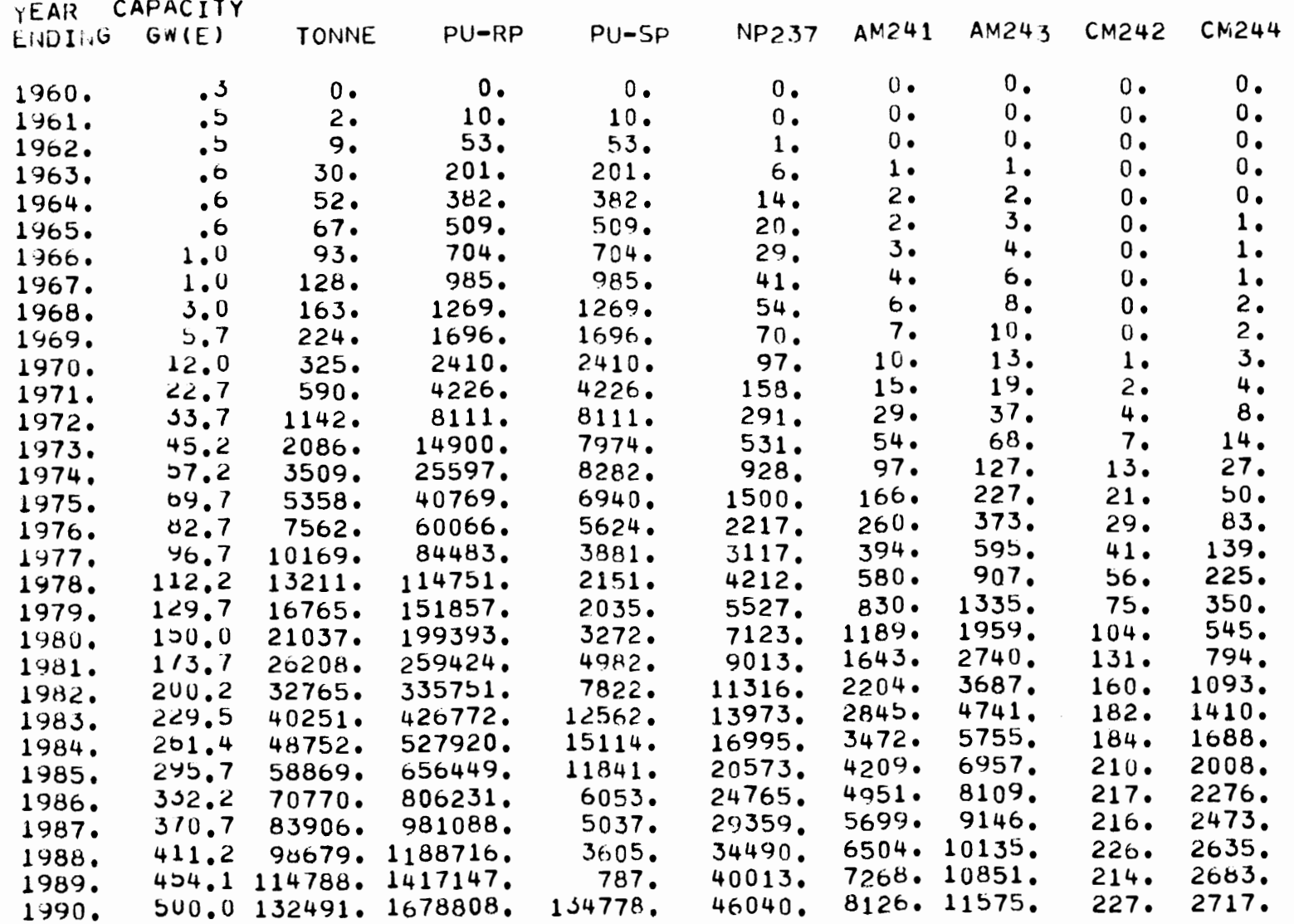

ISOTOPE PRODUCTION, KGS 
(NO LOSSES EXCEPT FOR RADIOACTIVE UECAY)

IEAR

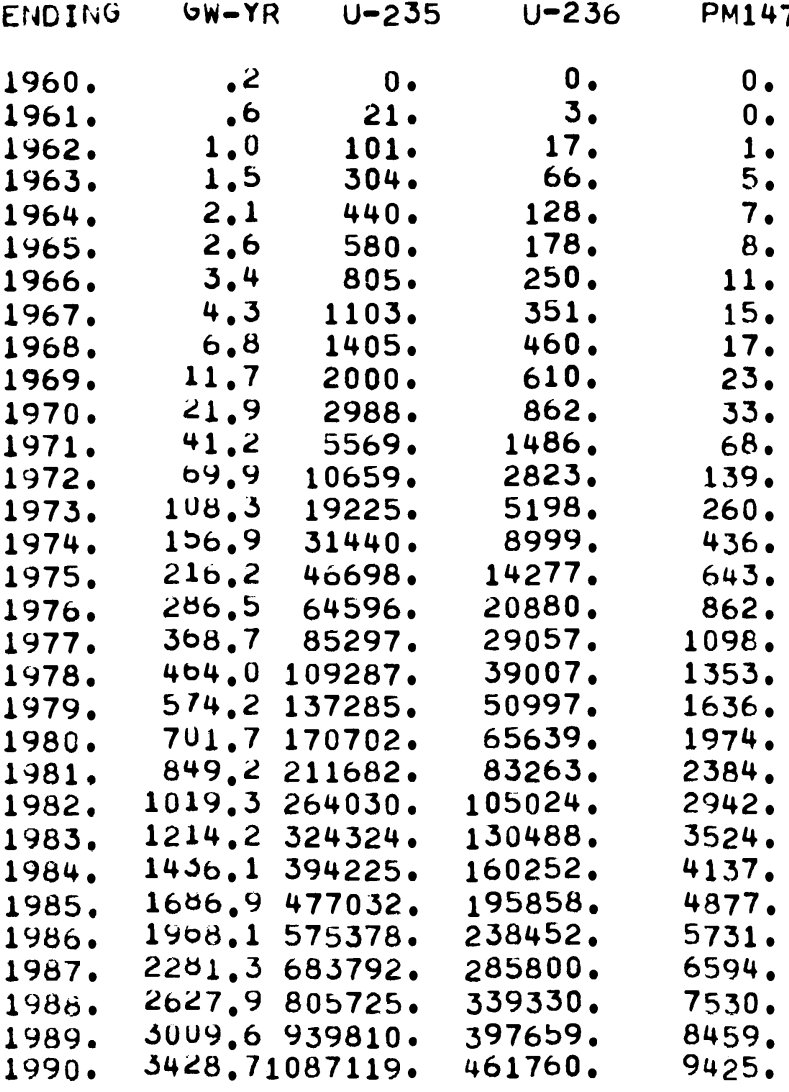

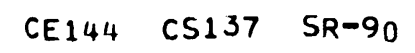


(NO LOSSES EXCEPT FOR RADIOACTIVE DECAY)

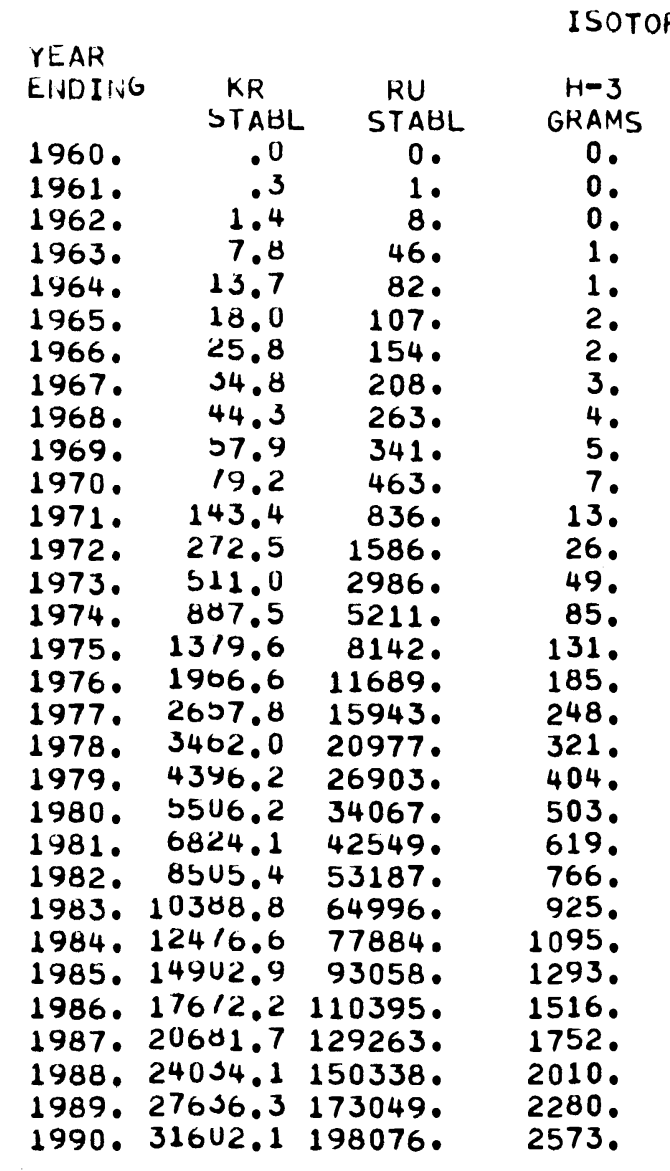


NET ANNUAL I SOOTOPE AVA ILA B I L I T Y

PAGE 114

(WITH TARGET IRRADIATION. PROCESS LOSSES AND DECAY LOSSES)

LWR'S - PU \& U236 RECYCLE WITH FAST REACTORS

CASE 6

TUTAL

UURING IIVSTALLED

PERIOU CARACITY

ENUIT:G UW(E)

ISOTOPE PRODUCTION, KILOGRAMS/YEAR

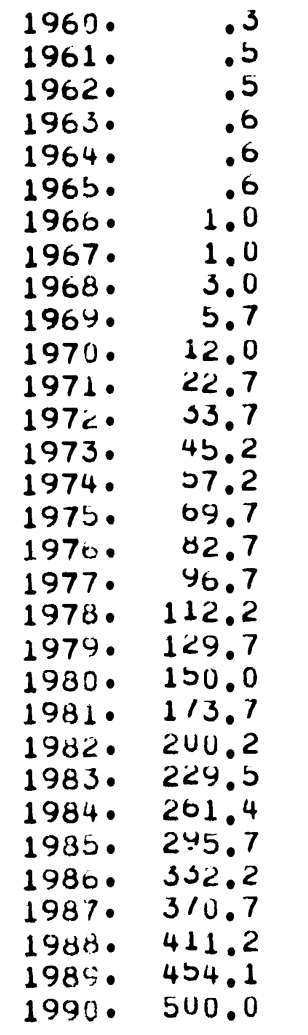

TONNE

PU-RP

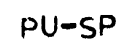

NP237

AM241 AM243 CM242 CM244

CM2 244
AM3

CM244

PU238

SUM NP

0.
0
2
7

10

21.

21.

43.
144.

0.
0.
10

144.

43.

$16.170^{\circ} 170^{\circ}$

25. $189^{\circ} 189^{\circ}$

36.

271.

273.

61.

101.

265.

551.

410.

690.

1767.

3789.

6624.

1423.

10418 .

14805 .

18843 .

2607

23872 .

29616.

36331 .

3554 .

46568 .

58815 .

74776 .

89163.

99068 .

8500 .

10117 .

125860 .

$11901^{\circ}$

146663 .

171260 .

14773.

203426.

271.

0
-0
0
1

0

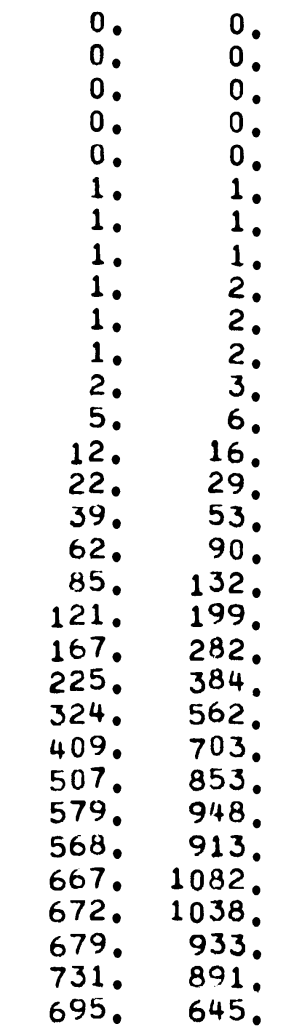

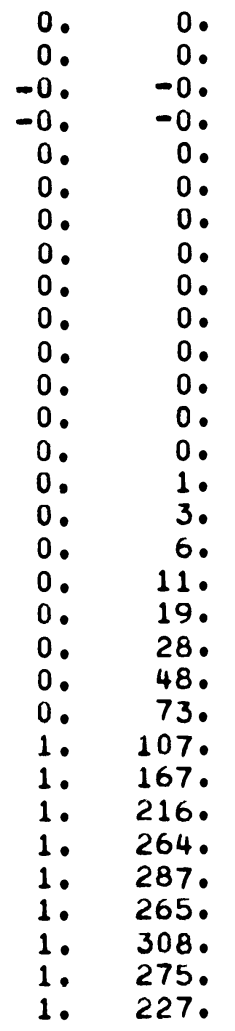

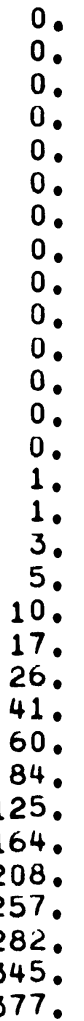

0.

0 .

0 .

0 .

0 .

0 .

410.

690.

3789 .

$-164$.

$237^{\circ}$

$-1379$

-1764 .

$-1742$.

$-146$.

1195

2759 .

4608 .

2443.

-5795 .

$-1096$.

-1452 .

4970 .

227.

377 .

0.

1.

1

.

2.

4

9. 126.

16. 196

28. 273 .

46. 361 .

74.460

114.570

$251^{\circ} \quad 481$.

341.434

428. 588 .

496. 621.

522.809

590. 918

603.1299.

\begin{tabular}{|c|c|c|}
\hline 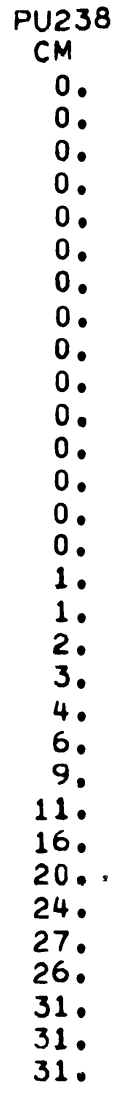 & 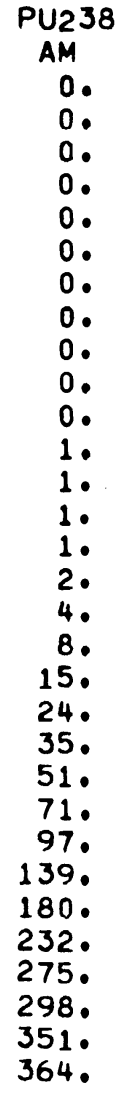 & 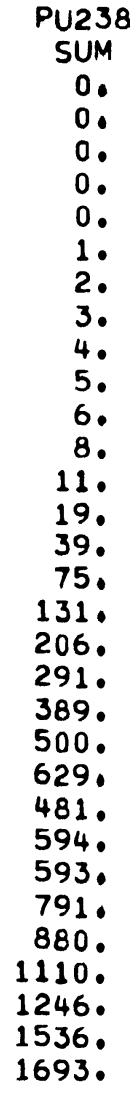 \\
\hline
\end{tabular}


NET ANNUAL I SOT TOPE AVAILABILITY

PAGE 115

(WITH TARGET IRRADIATION, PROCESS LOSSES AND DECAY LOSSES)

LWR'S - PU \& U236 RECYCLE. WITH FAST REACTORS

CASE

6

WUR IIVG

PERICU

ENDING

1960.
1961.

1961.

1963.

$1964^{\circ}$.

1965.

1966.

1967.

1968.

1969.

1970 .

1971.

1972.

1973.

1974.

$1970^{\circ}$

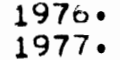

1978.

$1974^{\circ}$

$1980^{\circ}$

$1981^{\circ}$

1982.

$1985^{\circ}$

1984.

$1980^{\circ}$

1987.

$1980^{\circ}$.

$1989^{\circ}$
$1990^{\circ}$
ISOTOPE PRODUCTION, KILOGRAMS/YEAR

$G W-Y R \quad U-235 \quad U-236$

PM147
0.
0.
-0.
-0.
0.
0.
2.
2.
1.
2.
2.
3.
4.
$6 \circ$
18.
36.
63.
98.
127.
152.
180.
210.
246.
295.
357.
457.
520.
588.
699.
816.
900.

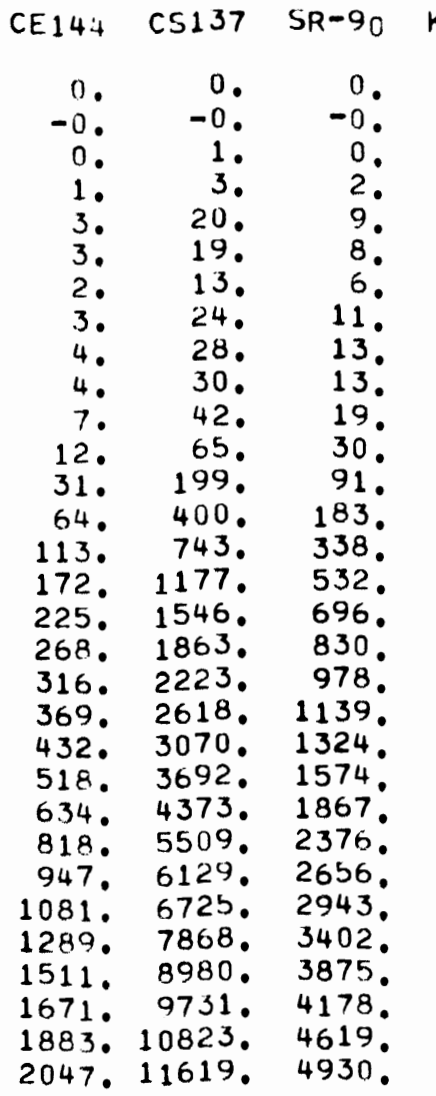

41742

52460 .

57162 .

900 .

\begin{tabular}{|c|c|c|}
\hline$k k-85$ & TC-99 & $\begin{array}{c}\text { RH103 } \\
\text { SUM }\end{array}$ \\
\hline 0 . & 0 . & 0 \\
\hline-0. & -0 . & -0. \\
\hline 0. & 1. & 0. \\
\hline 0 . & 2. & 1. \\
\hline 0 . & 14. & 7. \\
\hline 0 . & 13. & 7. \\
\hline 0 . & 9. & 5. \\
\hline 0 . & 17. & 9. \\
\hline 1. & 20. & 10. \\
\hline 1. & 20 . & 11. \\
\hline 1. & 29. & 16. \\
\hline 1. & 45. & 25. \\
\hline 4. & 137. & $\begin{array}{r}72 . \\
146 .\end{array}$ \\
\hline $15^{\circ}$ & 511. & 267 . \\
\hline 23. & 809. & 419. \\
\hline 30. & $\begin{array}{l}1061 . \\
1276\end{array}$ & $\begin{array}{l}550 . \\
664 .\end{array}$ \\
\hline 43. & $1519^{\circ}$ & $790^{\circ}$ \\
\hline 50. & 1786. & 942. \\
\hline $\begin{array}{l}58 . \\
69 .\end{array}$ & $\begin{array}{l}2092{ }^{\circ} \\
2514^{\circ}\end{array}$ & $\begin{array}{l}1109^{\circ} \\
1344^{\circ}\end{array}$ \\
\hline 82. & 2979. & 1591. \\
\hline 104. & 3759 • & $1995^{\circ}$ \\
\hline 117. & 4186 . & $2217^{\circ}$ \\
\hline $130^{\circ}$ & $\begin{array}{l}4600 . \\
5383 .\end{array}$ & $\begin{array}{l}2435^{\circ} \\
2870^{\circ}\end{array}$ \\
\hline $\begin{array}{l}151 . \\
173 .\end{array}$ & 6151. & 3303 . \\
\hline 187. & 6673. & 3582 . \\
\hline 208. & $\begin{array}{l}7428 . \\
7981 .\end{array}$ & $3977^{\circ}$ \\
\hline & & \\
\hline
\end{tabular}

RU106

STAB

XTE

CM242 AM241

$\begin{array}{rrr}0 . & 0 . & 0 . \\ -0 . & -0.0 & -0 . \\ 0.0 & 0.0 & 3 .\end{array}$

0.

1.

1.

1

1.

2.

4. 24.167.

$12.117^{\circ} 264^{\circ}$

12. $1170^{\circ} 791^{\circ}$

44. 441. 2941 .

69. 706. 4639.

93. 938.6094.

114. 1151. 7338 .

141. 1406. 8750 .

169. 1690. $10299^{\circ}$

202. 2014. 12077.

248. 2472. 14526.

297. 2930. 17273.

376. 3628. 21795.

437. 4025. 24357 .

488 . 4342. 26810 .

618. 5238. 31490.

736. 6018. 36071.

855. 6671. 39249.

1011. 7586. 43795.

8296. 47194 .

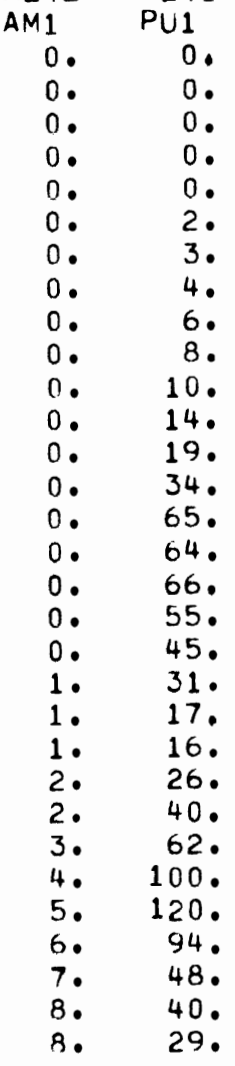


(WITH TARGET IRRADIATION. HROCESS LOSSES AND DECAY LOSSES)

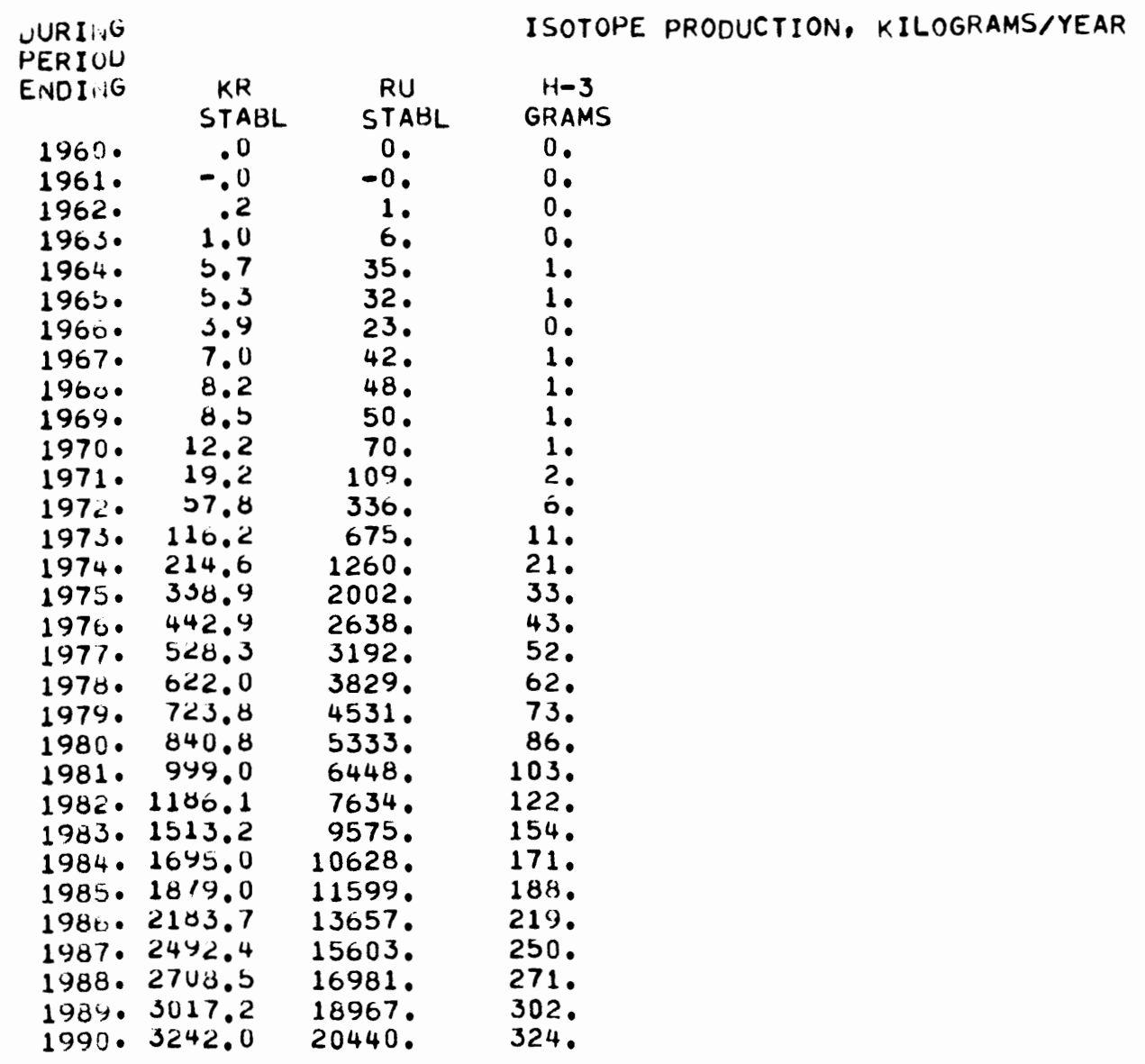


CUMULATIVE ISOTOPE AVA I LAB I L I T Y

PAGE 117

(WITH TARGET IRRADIATION. PROCESS LOSSES AND DECAY LOSSES)

TOTAL

INSI ALLED

YEAR CAPACITY

YEAR CAPACITY

TONNE PUTR

\begin{tabular}{|c|c|c|c|c|c|c|c|}
\hline 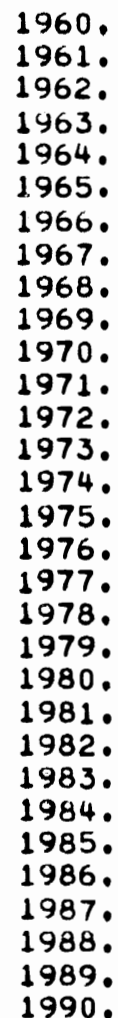 & $\begin{array}{r}.3 \\
.5 \\
.5 \\
.6 \\
.6 \\
.6 \\
1.0 \\
1.0 \\
3.0 \\
5.7 \\
12.0 \\
22.7 \\
33.7 \\
45.2 \\
37.2 \\
69.7 \\
82.7 \\
46.7 \\
112.2 \\
129.7 \\
130.0 \\
173.7 \\
200.2 \\
229.5 \\
261.4 \\
245.7 \\
352.2 \\
370.7 \\
411.2 \\
454.1 \\
500.0\end{array}$ & 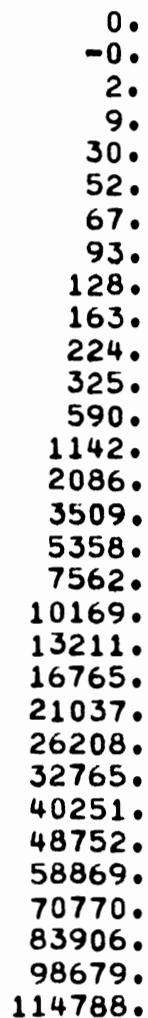 & 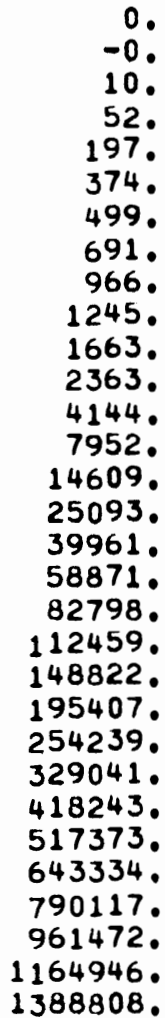 & 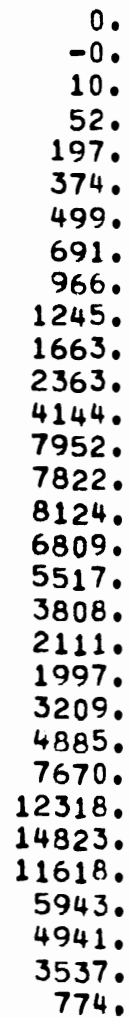 & 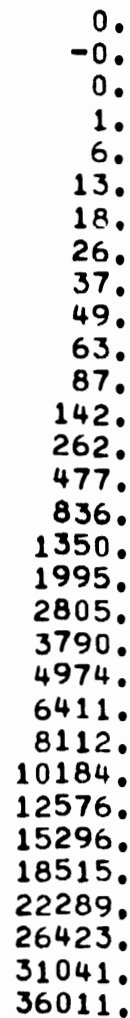 & 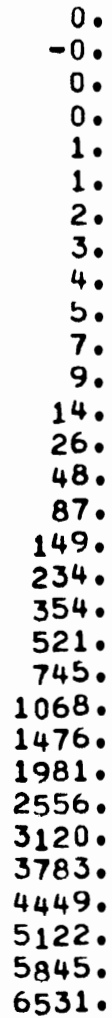 & 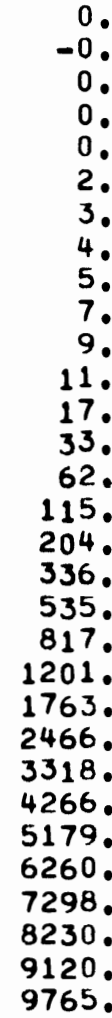 \\
\hline
\end{tabular}

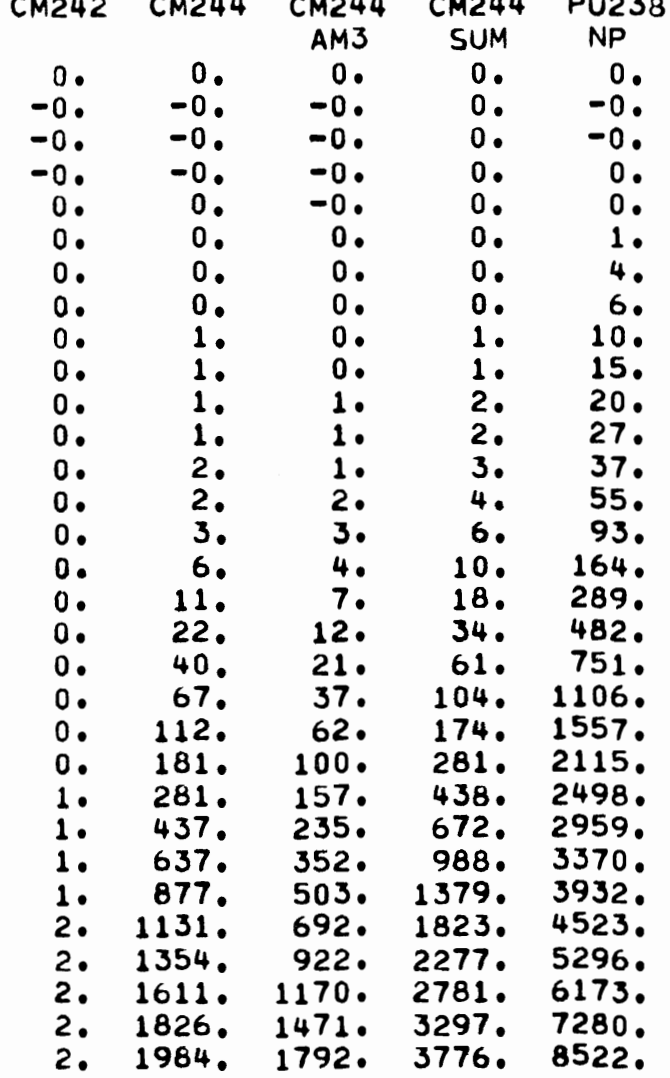

\begin{tabular}{|c|c|c|}
\hline $\begin{array}{c}\text { PU238 } \\
\text { CM }\end{array}$ & $\begin{array}{l}\text { PU238 } \\
\text { AM }\end{array}$ & $\begin{array}{l}\text { PU238 } \\
\text { SUM }\end{array}$ \\
\hline 0. & 0. & 0. \\
\hline-0 & -0. & 0. \\
\hline-0. & -0. & 0. \\
\hline-0 & -0. & 0. \\
\hline 0. & -0. & 0. \\
\hline 0. & 0. & 1 \\
\hline 0. & 0. & 4. \\
\hline 0. & 0. & 7. \\
\hline 0. & 0 . & 10. \\
\hline 0. & 1. & 15. \\
\hline 0. & 1. & 22. \\
\hline 0. & 2. & 29. \\
\hline 0. & 2 . & 40. \\
\hline 0. & 3. & 59. \\
\hline 1. & 4. & 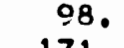 \\
\hline 1. & 6. & 171. \\
\hline & $\begin{array}{l}10 . \\
18 .\end{array}$ & $505^{\circ}$ \\
\hline & 33. & 792. \\
\hline 12. & 56. & $1175^{\circ}$ \\
\hline 18. & 91. & $1666^{\circ}$ \\
\hline 38. & 210 & 2746 . \\
\hline 53. & 306 & $3319^{\circ}$ \\
\hline 96 & 443. & 4647 . \\
\hline $122^{\circ}$ & 846. & 5490 . \\
\hline 147. & $1114^{\circ}$ & 65 \\
\hline $\begin{array}{l}177^{\circ} \\
206\end{array}$ & $\begin{array}{l}14030^{\circ} \\
1743\end{array}$ & 9. \\
\hline & D & \\
\hline
\end{tabular}


C UMULATIVE I SOT TOPE AVA I L A B I L I T Y

PAGE 118

(WITH TARGET IRRAIIATION, PROCESS LOSSES AND DECAY LOSSES)

LWR'S - PU \& U236 RECYCLE WITH FAST REACTORS

CASE

6

ISOTOPE PRODUCTION, KGS

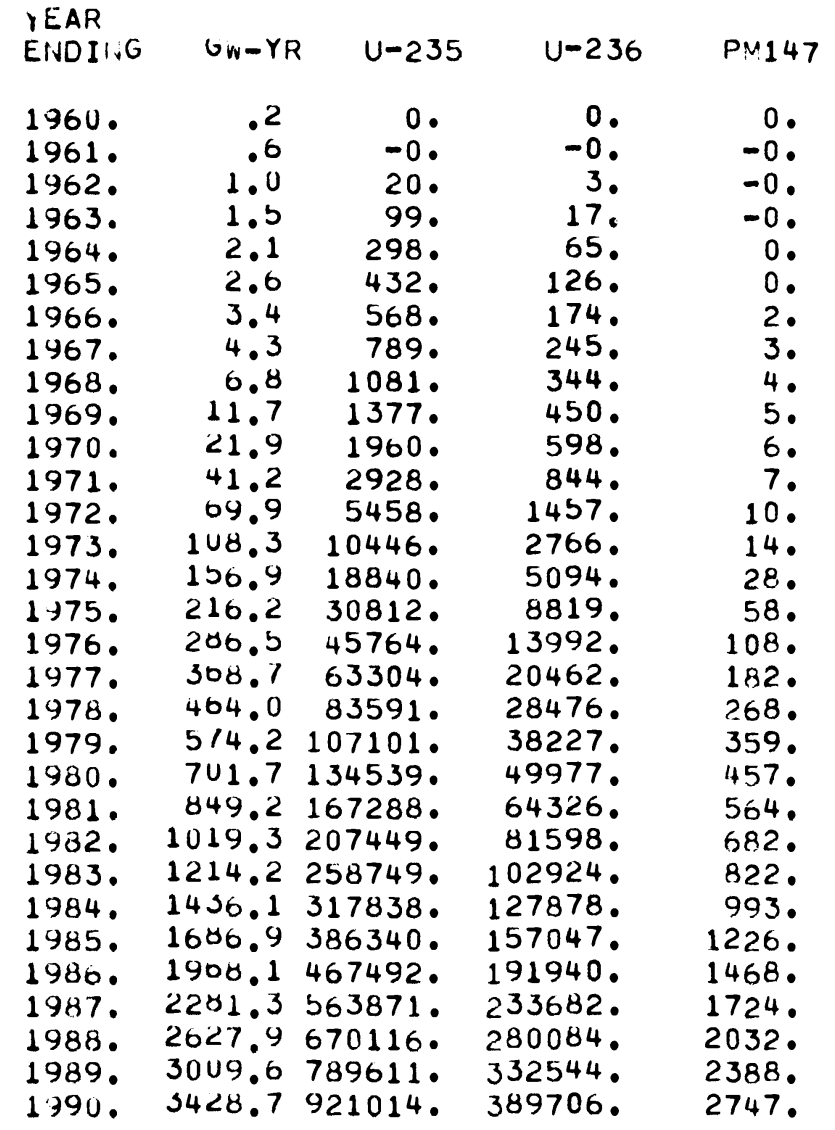

\begin{tabular}{|c|c|c|}
\hline CE 144 & $\operatorname{cs} 137$ & $S R-90$ \\
\hline $\begin{array}{r}0 . \\
-0 \\
0 \\
1 \bullet \\
3 \\
4 \\
4 \\
5 \\
6 \\
7 \\
10 \\
16 \\
38 \\
80 \\
145 \\
232 \\
320 \\
399 \\
480 \\
567 \\
665 \\
792 \\
960 \\
1212 \\
1446 \\
1675 \\
1978 \\
2325 \\
2627 \\
2964 \\
3266 \\
\end{array}$ & 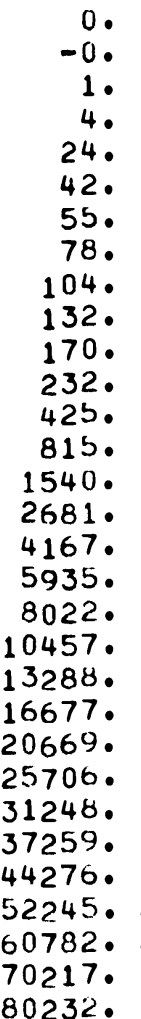 & $\begin{array}{r}0 . \\
-0 \\
0 \\
2 . \\
11 . \\
19 \\
25 . \\
35 . \\
47 . \\
59 \\
77 . \\
105 . \\
194 . \\
372 . \\
700 \\
1216 . \\
1882 . \\
2666 . \\
3579 \\
4631 \\
5841 \\
7272 \\
8962\end{array}$ \\
\hline
\end{tabular}

\begin{tabular}{|c|c|c|}
\hline \multicolumn{3}{|r|}{ RH1 } \\
\hline \multirow{2}{*}{0} & $U_{0}$ & 0 . \\
\hline & -0 . & -0 . \\
\hline $\begin{array}{r}-0 \\
0 .\end{array}$ & 1. & 0 . \\
\hline \multirow{2}{*}{$\begin{array}{l}0 . \\
0 .\end{array}$} & 3. & 2. \\
\hline & 17. & 9. \\
\hline 1. & 30 . & 16. \\
\hline 1. & 39. & 21. \\
\hline & 36. & 29. \\
\hline & 75 . & 40. \\
\hline \multirow{2}{*}{2.} & 96 & 51. \\
\hline & 124. & \\
\hline & $\begin{array}{l}170 . \\
307\end{array}$ & $\begin{array}{r}91^{\circ} \\
163^{\circ}\end{array}$ \\
\hline \multirow{3}{*}{$\begin{array}{l}16 . \\
29 \\
51 \\
78\end{array}$} & 583 & 309. \\
\hline & & $\begin{array}{l}576 . \\
994^{\circ}\end{array}$ \\
\hline & 2964 . & 1544. \\
\hline \multirow{5}{*}{$\begin{array}{l}110^{\circ} \\
145^{\circ} \\
186^{\circ} \\
232^{\circ} \\
287{ }^{\circ} \\
350^{\circ}\end{array}$} & 4240. & 2208 . \\
\hline & 5760 . & 3005 . \\
\hline & 7546 . & 3946. \\
\hline & & $\begin{array}{l}5055^{\circ} \\
6399 .\end{array}$ \\
\hline & 15131 . & 7990. \\
\hline 433. & 18890. & 9985. \\
\hline \multirow{2}{*}{$\begin{array}{l}523 . \\
620 . \\
733^{\circ}\end{array}$} & 23076 & 12201. \\
\hline & 27676 & 14636 . \\
\hline \multirow{2}{*}{$\begin{array}{l}860 . \\
994 .\end{array}$} & 39210. & $\begin{array}{l}17506 \\
20809 .\end{array}$ \\
\hline & 83. & 391. \\
\hline $\begin{array}{l}140 . \\
292 .\end{array}$ & 311. & 8368 . \\
\hline
\end{tabular}

RU106

PD

$X E$

CM242 AM241

$\begin{array}{ccccc}0 . & 0 . & 0 . & 0 . & 0 . \\ 0 & 0.0 & -0.0 & 0 . & 0.0\end{array}$

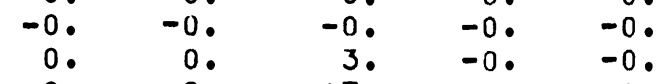

0. 2.017 .000$.

1. 15. 96. -0 .

$\begin{array}{lll}2 . & 26 . & 169 . \\ 2 . & 34 . & 222 .\end{array}$

2. 49. 317 .

3. 66.429.

3. 84. 545 .

4. 108.712

$\begin{array}{rrr}6^{\circ} & 145^{\circ} & 976 . \\ 15 . & 261 . & 1767 .\end{array}$

32. 495. 3361.

60. 936. 6303.

99. 1642. 10942

142. 2580.17036 .

185. 3731. 24374 .

233. 5137. 33124 .

286. 6828. 43423.

345. 8841. 55500。

420. 11313. 70026 .

507.14244 .87299

630.17872 .109094

752. 21897 . 133451 .

864. 26238. 160261.

1050 . 31476. 191751.

1261 . 37494. 227822.

1485. 44166. 267070 .

1754 . 51752. 310866 。

0.

0.

0.

0.

0 .

0.

$0 . \quad 09$

0.163.

- 227.

0.347.

0 . 392 .

1. 422.

1. 454

2. 479 .

3. 518 .

5. 580.

6. 799.

7. 892.

8. 939.

3266.80232. 34331. 1292.61292.32646. 2024.60048.358059. 10. 1005. 
(WITH TARGET IRRADIATION, PROCESS LOSSES AND DECAY LOSSES)

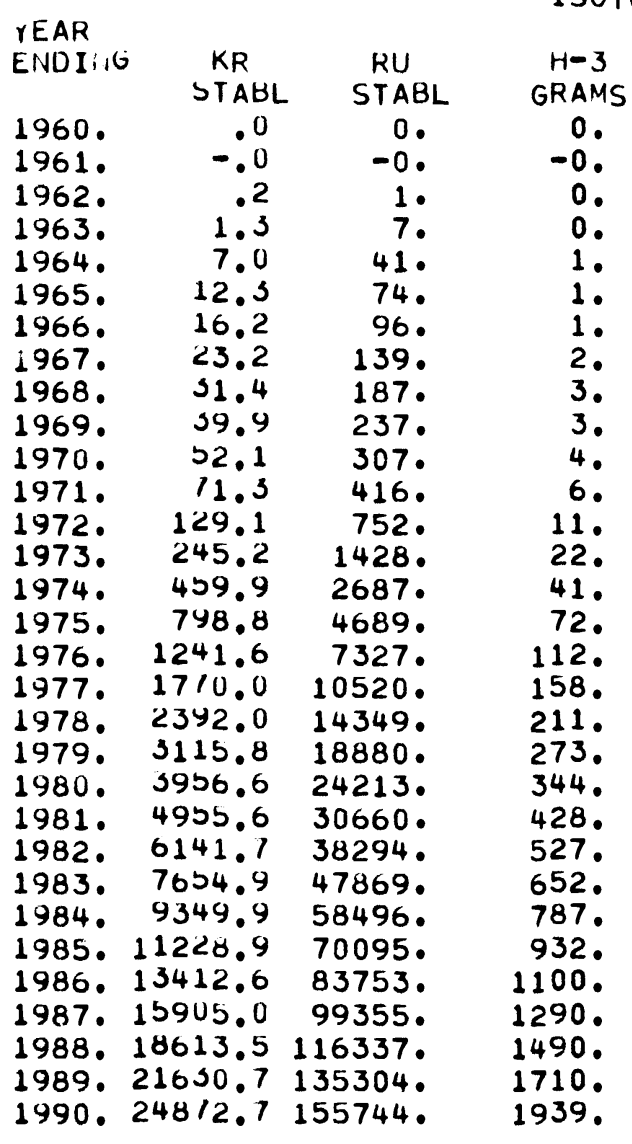


ISOTOPE PRODUCTION CASE 6 FOR 180 GW(E) AT 1980 
D I STR I BUTION OF ELECTRICAL GENERATIA G CA PACI T Y

PAGE 120

CASE 6 FOR 180 GW(E) AT 1980

CASE 7

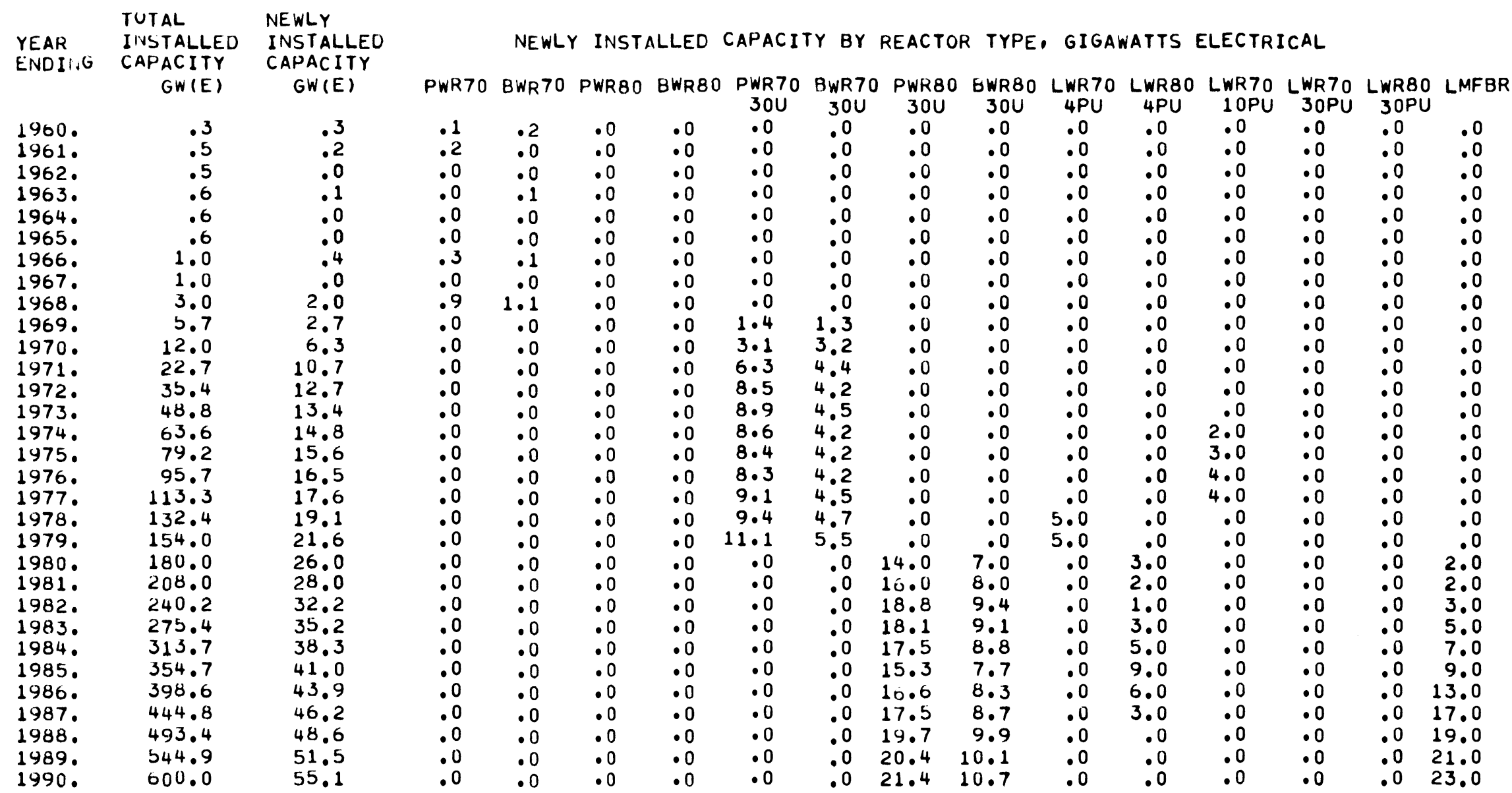




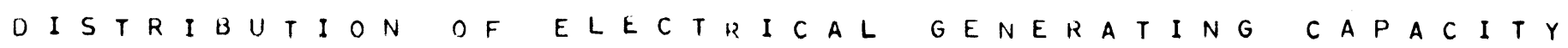

PAGE 121

CASE 6 FOR 180 GW(E) AT 1980

CASE 7

TUTAL NEWLY

\begin{tabular}{|c|c|c|c|c|c|c|c|c|c|c|c|c|c|c|c|c|}
\hline \multirow[t]{2}{*}{$\begin{array}{l}\text { YEAR } \\
\text { EINDING }\end{array}$} & $\begin{array}{l}\text { TUTAL } \\
\text { INSTALLEU } \\
\text { CANACITY }\end{array}$ & $\begin{array}{l}\text { NEWLY } \\
\text { INSTALLED } \\
\text { CAPACITY }\end{array}$ & CUMI & ULATIVE & E INST & ALLED & CAPACI & ITY BY & REACTOF & R TYPE & - GIGA & WATTS & ELECTRI & ICAL & & \\
\hline & $G W(E)$ & $G W(E)$ & PWR70 & BWR 70 & PWR80 & BWR8 0 & $\begin{array}{c}0 \text { PWR } 70 \\
30 U\end{array}$ & $\begin{array}{c}B \cdot N R 70 \\
30 U\end{array}$ & $\begin{array}{c}\text { PWK80 } \\
30 U\end{array}$ & $\begin{array}{c}\text { BWR80 } \\
30 U\end{array}$ & $\begin{array}{c}\text { LWR70 } \\
4 P U\end{array}$ & $\begin{array}{c}\text { LWR80 } \\
4 P U\end{array}$ & $\begin{array}{r}\text { LWR70 } \\
10 P U\end{array}$ & $\begin{array}{r}\text { LWR } 70 \\
30 P U\end{array}$ & $\begin{array}{r}\text { LWR8C } \\
30 P L\end{array}$ & $\begin{array}{l}0 \text { LMFBR } \\
U\end{array}$ \\
\hline 1960. & .3 & .3 & .1 & .2 & .0 & .0 & .0 & .0 & .0 & .0 & .0 & .0 & .0 & .0 & .0 & .0 \\
\hline 1961. & .5 & .2 & .2 & .2 & .0 & .0 & .0 & .0 & .0 & .0 & .0 & .0 & .0 & .0 & .0 & .0 \\
\hline 1962. & .5 & .0 & .2 & .2 & .0 & .0 & .0 & .0 & .0 & .0 & .0 & .0 & .0 & .0 & .0 & .0 \\
\hline 1963. & .6 & .1 & .2 & .4 & .0 & .0 & .0 & .0 & .0 & .0 & .0 & .0 & .0 & .0 & .0 & .0 \\
\hline 1964. & .6 & .0 & .3 & .4 & .0 & .0 & .0 & .0 & .0 & .0 & .0 & .0 & .0 & .0 & .0 & .0 \\
\hline 1965. & .6 & .0 & .3 & .4 & .0 & .0 & .0 & .0 & .0 & .0 & .0 & .0 & .0 & .0 & .0 & .0 \\
\hline 1966. & 1.0 & .4 & .6 & .4 & .0 . & .0 & .0 & .0 & .0 & .0 & .0 & .0 & .0 & .0 & .0 & .0 \\
\hline 1967. & 1.0 & .0 & .6 & .4 & .0 & .0 & .0 & .0 & .0 & .0 & .0 & .0 & .0 & .0 & .0 & .0 \\
\hline 1968. & 3.0 & 2.0 & 1.5 & 1.5 & .0 & .0 & .0 & .0 & .0 & .0 & .0 & .0 & .0 & .0 & .0 & .0 \\
\hline 1969. & 5.7 & 2.7 & 1.5 & 1.5 & .0 & .0 & 1.4 & 1.3 & .0 & .0 & .0 & .0 & .0 & .0 & .0 & .0 \\
\hline 1970. & 12.0 & 6.3 & 1.5 & 1.5 & .0 & .0 & 4.4 & 4.5 & .0 & .0 & .0 & .0 & .0 & .0 & .0 & .0 \\
\hline 1971. & 22.7 & 10.7 & 1.5 & 1.5 & .0 & .0 & 10.8 & 8.9 & .0 & .0 & .0 & .0 & .0 & .0 & .0 & .0 \\
\hline 1972. & 35.4 & 12.7 & 1.5 & 1.5 & .0 & .0 & 19.3 & 13.1 & .0 & .0 & .0 & .0 & .0 & .0 & .0 & .0 \\
\hline 1973. & 48.8 & 13.4 & 1.5 & 1.5 & .0 & .0 & 28.2 & 17.6 & .0 & .0 & .0 & .0 & .0 & .0 & .0 & .0 \\
\hline 1974. & 63.6 & 14.8 & 1.5 & 1.5 & .0 & .0 & 36.8 & 21.8 & .0 & .0 & .0 & .0 & 2.0 & .0 & .0 & .0 \\
\hline 19 & 79.2 & 15.6 & 1.5 & 1.5 & .0 & .0 & 45.2 & 26.0 & .0 & .0 & .0 & .0 & 5.0 & .0 & .0 & .0 \\
\hline 1976. & 95.7 & 16.5 & 1.5 & 1.5 & .0 & .0 & 53.5 & 30.2 & .0 & .0 & .0 & .0 & 9.0 & .0 & .0 & .0 \\
\hline $1977^{\circ}$ & 113.3 & 17.6 & 1.5 & 1.5 & .0 & .0 & $62 \cdot 6$ & & .0 & .0 & .0 & .0 & 13.0 & .0 & .0 & .0 \\
\hline 1978. & 132.4 & 19.1 & 1.5 & 1.5 & .0 & .0 & $72 \cdot 0$ & 39.4 & .0 & .0 & 5.0 & .0 & 13.0 & .0 & .0 & .0 \\
\hline 1979. & 154.0 & 21.6 & 1.5 & 1.5 & .0 & .0 & $83 \cdot 1$ & 44.9 & .0 & .0 & 10.0 & .0 & 13.0 & .0 & .0 & .0 \\
\hline $1980^{\circ}$ & 180.0 & 26.0 & 1.5 & 1.5 & .0 & .0 & $83 \cdot 1$ & 44.9 & 14.0 & 7.0 & 10.0 & 3.0 & 13.0 & .0 & .0 & 2.0 \\
\hline 1981. & 208.0 & 28.0 & 1.5 & 1. & .0 & .0 & $83 \cdot 1$ & 44.9 & 30.0 & 15.0 & 10.0 & 5.0 & 13.0 & .0 & .0 & 4.0 \\
\hline 1982. & 240.2 & 32.2 & 1.5 & 1 . & .0 & .0 & $83 \cdot 1$ & 44.9 & 48.8 & 24.4 & 10.0 & 6.0 & 13.0 & .0 & .0 & 7.0 \\
\hline 1983. & 275.4 & 35.2 & .5 & 1. & .0 & .0 & $83 \cdot 1$ & 44.9 & 66.9 & 33.5 & 10.0 & 9.0 & 13.0 & .0 & .0 & 12.0 \\
\hline 1984. & 313.7 & 38.3 & .5 & 1 . & .0 & .0 & $83 \cdot 1$ & 44.9 & 84.4 & 42.3 & 10.0 & 14.0 & 13.0 & .0 & .0 & 19.0 \\
\hline 1985. & 354.7 & 41.0 & 1.5 & 1. & .0 & .0 & 83.1 & 44.9 & 99.7 & 50.0 & 10.0 & 23.0 & 13.0 & .0 & 0 & 28.0 \\
\hline 1986. & 398.6 & 43.9 & 1.5 & 1. & .0 & .0 & $83 \cdot 1$ & 44.91 & 116.3 & 58.3 & 10.0 & 29.0 & 13.0 & .0 & .0 & 41.0 \\
\hline 1987. & 444.8 & 46.2 & 1.5 & 1. & .0 & .0 & $83 \cdot 1$ & 44.91 & 133.8 & 67.0 & 10.0 & 32.0 & 13.0 & .0 & .0 & 58.0 \\
\hline 1988. & 493.4 & 48.6 & .5 & 1 . & .0 & .0 & $83 \cdot 1$ & 44.91 & 153.5 & 76.9 & 10.0 & 32.0 & 13.0 & .0 & .0 & .0 \\
\hline & 544.9 & 51.5 & 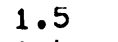 & & .0 & $\cdot 0$ & 83.1 & 44.91 & 173.9 & 87.0 & 10.0 & 32.0 & 13.0 & .0 & .0 & \\
\hline & 600.0 & $5 ל .1$ & 5 & & .0 & .0 & $83 \cdot 1$ & 91 & 195.3 & 97.7 & 10.0 & 32.0 & 13.0 & .0 & .0 & 121.0 \\
\hline
\end{tabular}


(NO LOSS BASIS)

CASE 6 FOR 180 GW(E) AT 1980

CASE 7

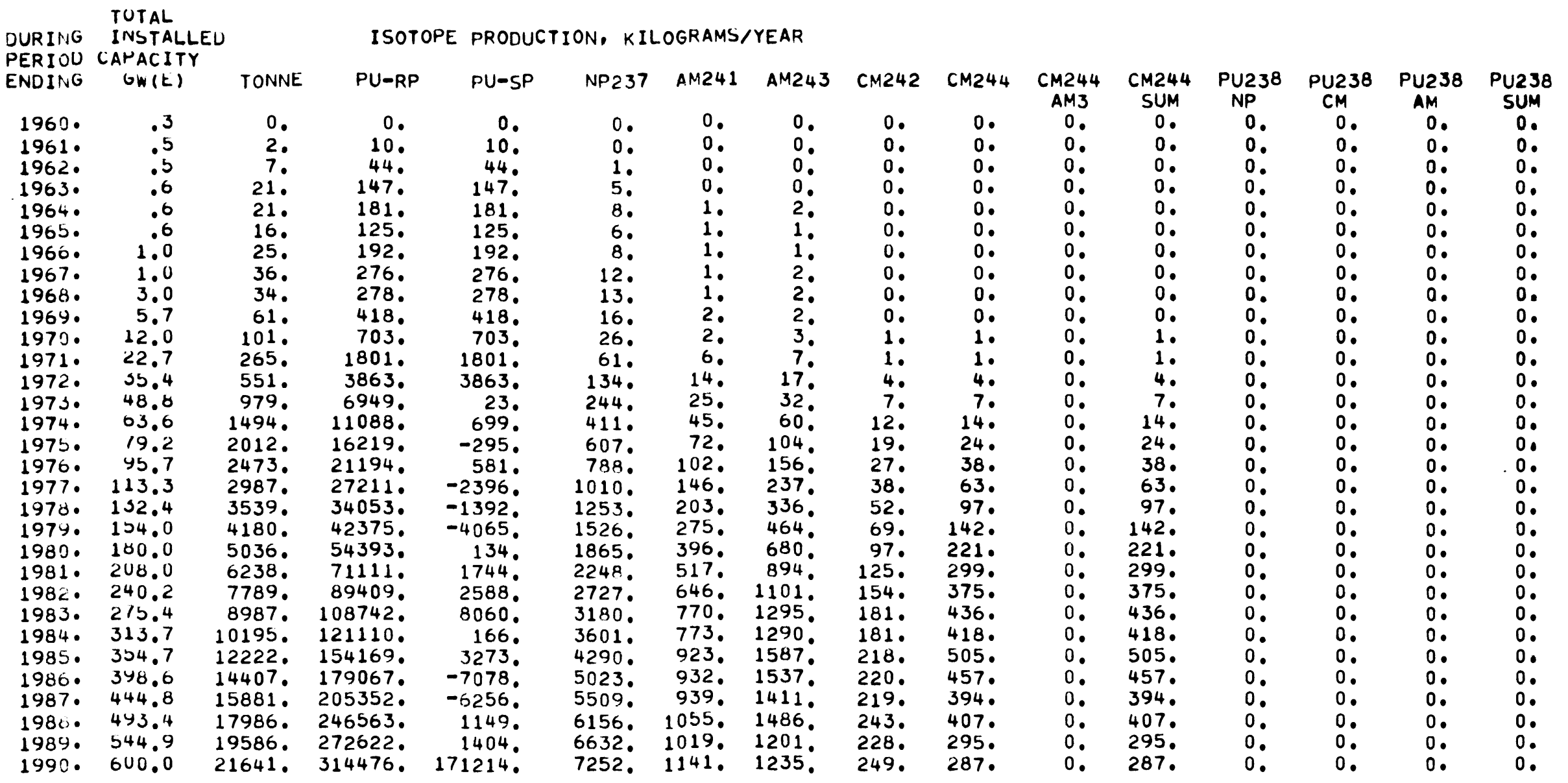


(NO LOSS BASIS)

DURING

PERIOU

ENDIIIG

\section{0.}

1961.

1963.

$1964^{\circ}$

1965.

$1960^{\circ}$

1967.

1968.

1969.

1970 .

1971.

1972.

1973.

$1974^{\circ}$

1975.

1976.

$1977^{\circ}$

1970.

1979 .

$1980^{\circ}$

1982.

1983.

$1984^{\circ}$

1985.

$1987^{\circ}$

$1980^{\circ}$

1989.

1990 .
ISOTOPE PRODUCTION, KILOGRAMS/YEAR

$$
G W-Y R \quad U-235
$$
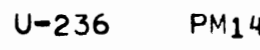

.2
.4
.4
.5
.5
.5
.8
.8
2.6
4.8
10.2

21.

80.

203.

136.

139.

226.

302 .

596 .

988 .

2581.

5090 .

8952 .

12925.

$20296^{\circ}$

20296.

$24019^{\circ}$

$33204^{\circ}$

39655 .

49176 .

61719.

71792 .

82979.

$\begin{array}{rr}340.9 & 98832 . \\ 337.7 & 117538 .\end{array}$

376.1130111 .

416.3147035.

$\begin{array}{ll}458.6 & 161993 .\end{array}$

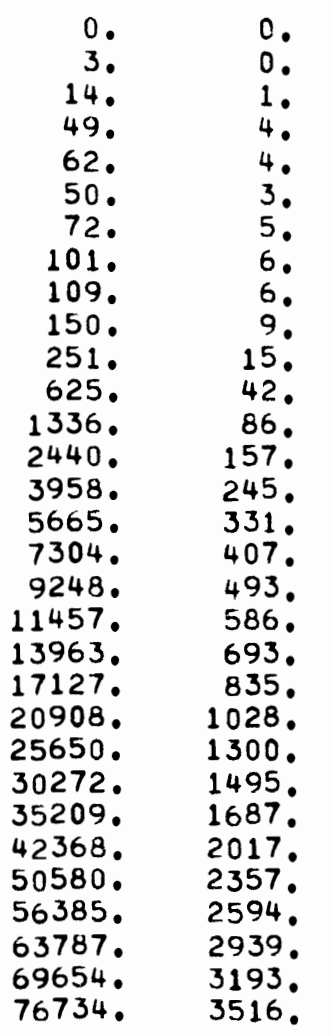

CE144 CS137

$S R=90$

$K R-85$

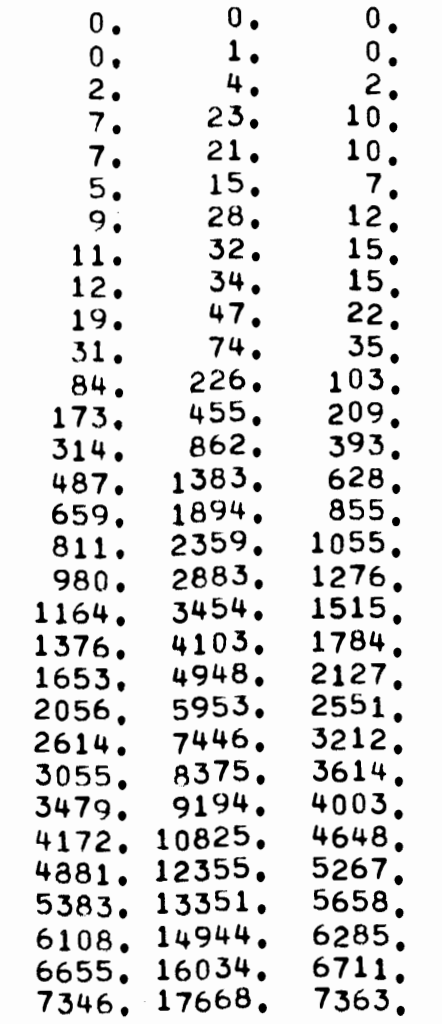

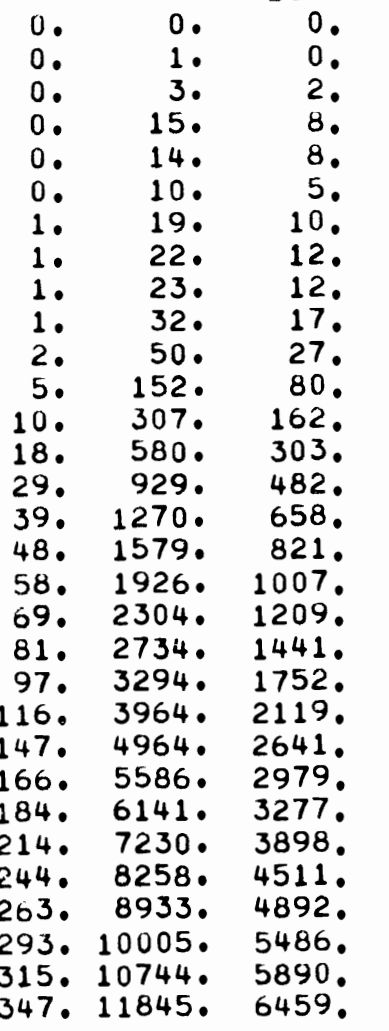

RU106 PD

0.
0.
1.
2.
2.
2.
3.
4.
4.
6.
9.
26.
54.
100.
159.

PD

XE
STABL

CM242 AM241

0.0

$\begin{array}{ll}2 . & 16 \\ 14 . & 87\end{array}$

13.

13. 51.

17. 106.

19. 124.

20.129.

41.293

129. 879 .

260.1772 .

$498^{\circ} 3338^{\circ}$

808 . 5334.

222. 1118. 7300 .

282. 1417.9084.

353. 1768. 11093 .

430. 2155. 13280 .

520. 2600. 15773 .

641. 3200. 19024

794. 3884. 22998

997. 4803. 28791.

1177. 5419. 32523 .

1312. 5861.35816 .

1668. 7121. 42320

2003. 8245. 48481 .

2306. 9102. 52598 .

$2733.10420,59044$.

3082. 11358. 63560 。

3561. 12762. 70229 . 

(NO LOSS BASIS)

\begin{tabular}{|c|c|c|c|}
\hline $\begin{array}{l}\text { UURIING } \\
\text { PERIOU }\end{array}$ & & & ISOTOF \\
\hline ENDING & $\begin{array}{c}K K \\
\text { STABL }\end{array}$ & $\begin{array}{c}R U \\
\text { STABL }\end{array}$ & $\begin{array}{c}H-3 \\
\text { GRAMS }\end{array}$ \\
\hline $1960^{\circ}$ & .0 & 0. & 0. \\
\hline $1961^{\circ}$ & .3 & 1. & 0. \\
\hline 1962. & 1.2 & 6. & 0. \\
\hline 1963. & 6.4 & 39. & 1. \\
\hline $1964^{\circ}$ & 5.9 & 36. & 1. \\
\hline $\begin{array}{l}1965^{\circ} \\
1960^{\circ}\end{array}$ & $\begin{array}{l}4.3 \\
7.8\end{array}$ & $\begin{array}{l}25 . \\
47 .\end{array}$ & $\begin{array}{l}0 . \\
1 .\end{array}$ \\
\hline $1967^{\circ}$ & 9.1 & 54. & 1. \\
\hline 1968. & 9.5 & 56. & 1. \\
\hline $1969^{\circ}$ & 13.6 & 78 . & 1. \\
\hline $\begin{array}{l}1970 . \\
1971 .\end{array}$ & $\begin{array}{l}21.3 \\
04.2\end{array}$ & $\begin{array}{l}121 . \\
373 .\end{array}$ & $\begin{array}{l}2 . \\
7 .\end{array}$ \\
\hline 1972. & 129.1 & 750 . & 13. \\
\hline 1973. & $\begin{array}{l}243.6 \\
389.6\end{array}$ & $\begin{array}{l}1426 . \\
2296\end{array}$ & $\begin{array}{l}25 . \\
40\end{array}$ \\
\hline $\begin{array}{l}1974{ }^{\circ} \\
1975^{\circ}\end{array}$ & $\begin{array}{l}389.6 \\
530.7\end{array}$ & $\begin{array}{l}2290^{\circ} \\
3153^{\circ}\end{array}$ & 55. \\
\hline $\begin{array}{l}1970^{\circ} \\
1977^{\circ}\end{array}$ & $\begin{array}{l}605.0 \\
741.6\end{array}$ & $\begin{array}{l}3944 . \\
4842 .\end{array}$ & $\begin{array}{l}68 . \\
84 .\end{array}$ \\
\hline $1970^{\circ}$ & 939.0 & 5825. & 100 \\
\hline $1979^{\circ}$ & $11 \cup 5.3$ & 6946. & 119. \\
\hline $\begin{array}{l}1980^{\circ} \\
1981 .\end{array}$ & $\begin{array}{l}1317.5 \\
1500.4\end{array}$ & $\begin{array}{r}8420 \\
10146\end{array}$ & $\begin{array}{l}143 . \\
172 .\end{array}$ \\
\hline 1982. & 1994.1 & 12654 & 215 \\
\hline $\begin{array}{l}1983 . \\
1984 .\end{array}$ & $\begin{array}{l}2246.3 \\
2488.5\end{array}$ & $\begin{array}{l}14222 \\
15540\end{array}$ & $\begin{array}{l}242 . \\
265\end{array}$ \\
\hline $1985^{\circ}$ & 2901.0 & $18417^{\circ}$ & 312 . \\
\hline $\begin{array}{l}1986^{\circ} \\
1987^{\circ}\end{array}$ & $\begin{array}{l}3292.3 \\
3508.9\end{array}$ & $\begin{array}{l}21083^{\circ} \\
22879^{\circ}\end{array}$ & $\begin{array}{l}356 . \\
384\end{array}$ \\
\hline $\begin{array}{l}1987^{\circ} \\
1980^{\circ}\end{array}$ & 3977.3 & $25726^{\circ}$ & 430 . \\
\hline $19899^{\circ}$ & 4274.6 & 27690 . & $\begin{array}{l}461 . \\
508 .\end{array}$ \\
\hline & 472 & & \\
\hline
\end{tabular}


(NO LOSSES EXCEPT FOR RADIOACTIVE LECAY)

TOTAL

INS I ALLED

YEAR CAPACITY

YEAR CAPACITY

ISOTOPE PRODUCTION, KGS

\begin{tabular}{|c|c|c|c|c|}
\hline 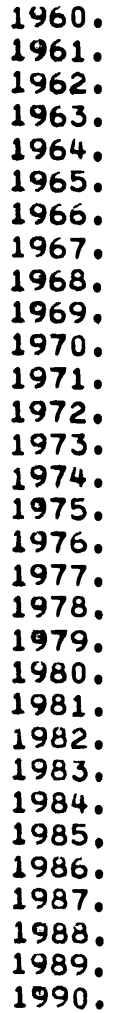 & $\begin{array}{r}.3 \\
.5 \\
.5 \\
.6 \\
.6 \\
.6 \\
1.0 \\
1.0 \\
3.0 \\
5.7 \\
12.0 \\
22.7 \\
35.4 \\
48.8 \\
63.6 \\
19.2 \\
95.7 \\
113.3 \\
132.4 \\
154.0 \\
180.0 \\
208.0 \\
240.2 \\
275.4 \\
313.7 \\
354.7 \\
348.6 \\
444.8 \\
493.4 \\
544.9 \\
600.0\end{array}$ & 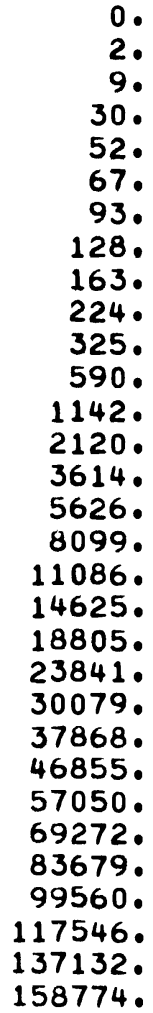 & $\begin{array}{r}0 . \\
10 . \\
53 . \\
201 . \\
382 . \\
509 . \\
704^{\circ} \\
985 . \\
1269 . \\
1696 . \\
2410 . \\
4226 . \\
8111 . \\
15098 . \\
26260 \\
42553 \\
63828 \\
91118 . \\
125256 . \\
167695 . \\
222140 . \\
293266 . \\
382693 . \\
491469 . \\
612636 . \\
766936 . \\
946135 . \\
1151651 . \\
1398314 . \\
1670982 . \\
1985514 .\end{array}$ & 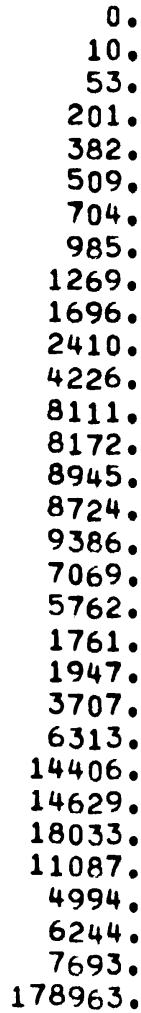 \\
\hline
\end{tabular}

\begin{tabular}{|c|c|c|}
\hline NP237 & AM241 & $A M 243$ \\
\hline $\begin{array}{c}0 \\
0 \\
1 \\
6 \\
14 \\
20 \\
29 \\
41 \\
54 \\
70 \\
97 \\
158 \\
291 \\
535 \\
946 \\
1553 \\
2341 \\
3351 \\
4604 \\
6130 \\
7995 \\
0243 \\
2969 \\
6149 \\
9750 \\
4040 \\
9063 \\
4572 \\
0728 \\
7360 \\
4613 \\
\end{array}$ & 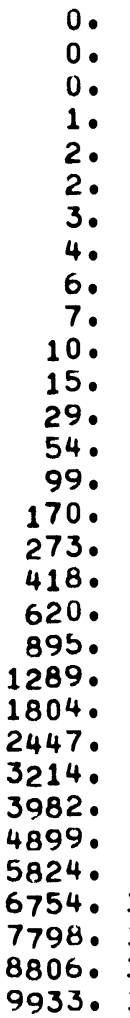 & 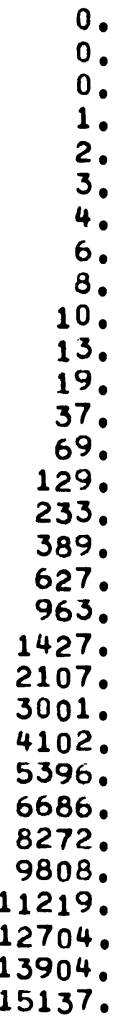 \\
\hline
\end{tabular}

\begin{tabular}{|c|c|}
\hline CM242 & CM244 \\
\hline 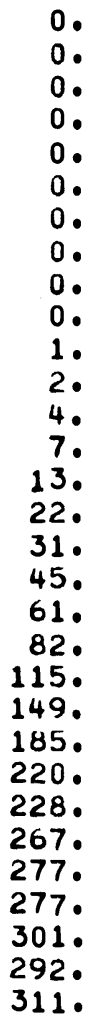 & 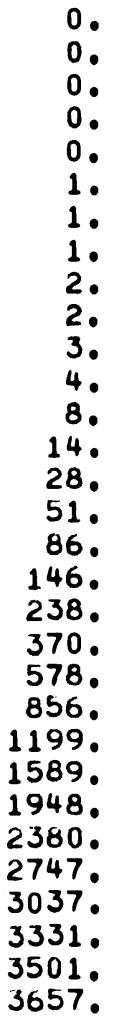 \\
\hline
\end{tabular}

$\begin{array}{cc}\text { CM244 } & \text { CM244 } \\ A M 3 & \text { SUM } \\ 0 . & 0 . \\ -0 . & 0 . \\ -0 . & 0 . \\ -0 . & 0 . \\ -0 . & 0 . \\ -0 . & 1 . \\ -0 . & 1 . \\ -0 . & 1 . \\ -0 . & 2 . \\ -0 . & 2 . \\ -0 . & 3 . \\ -0 . & 4 . \\ -0 . & 8 . \\ -0 . & 14 . \\ -0 . & 28 . \\ -0 . & 51 . \\ -0 . & 86 . \\ -0 . & 146 . \\ -0 . & 238 . \\ -0 . & 370 \\ -0 . & 578 . \\ -0 . & 856 . \\ -0 . & 1199 . \\ -0 . & 1589 . \\ -0 . & 1948 . \\ -0 . & 2380 . \\ -0 . & 2747 . \\ -0 . & 3037 . \\ -0 . & 3331 . \\ -0 . & 3501 . \\ -0 . & 3657 .\end{array}$

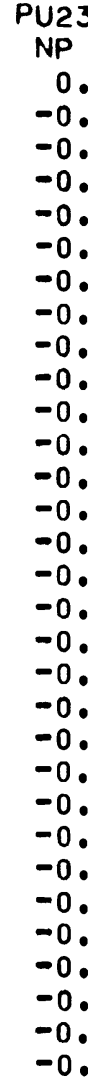

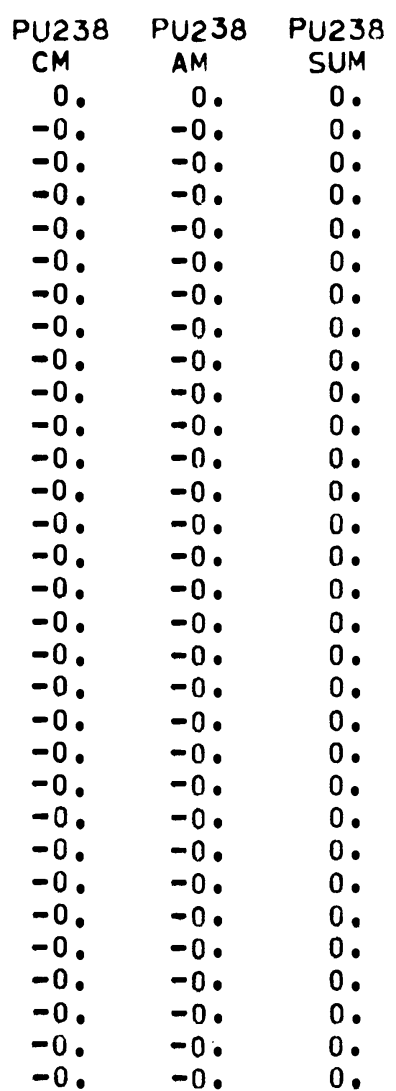




\section{(NO LOSSES EXCEPT FOR RADIOACTIVE UECAY)}

ISOTOPE PRODUCTION, KGS

YEAR

GW-YR U-235

$u-236$

PM1 147

CE 144

C5137

1960.

1961.

1962 .

1963.

1964.

1965.

1966.

1967 .

1968 .

1969.

1970 .

1971.

1972.

1973.

1974.

1976.

$1976^{\circ}$

1978 .

1979.

1980.

1981.

1982.

1983.

.20

.0
1.0

21.

1.5304 .

2.1440 .

2.6580 .

3.4805 .

4.31103.

6.81405 .

11.72000 .

21.92988 .

$\begin{array}{rr}41.2 & 5569 . \\ 11.3 & 10659 .\end{array}$

112.819612 .

106.932537.

204.249325.

315.669621 .

411.993640 .

524.4121903.

605.2155107 .

808.2194763.

0.

$189^{\circ} 243938$.

1422.9377449 .

1984. 1689.2460429.

1985. 1940.2559260 .

1986. 2327.9676798.

1987.

2744.0806909.

1988.3120 .3953945$.

$19900^{\circ} \quad 4082.51295197$.

0
3
17
66
128
178

128.

178

351.

351.

610 .

862.

1486

1486.

2823.

5262.

14885 .

22190 .

31438 .

42895 .

56858 .

73985 .

94893 .

120543.

150815 .

186024 .

228392 .

278971 .

335356

468798 .

545531 .

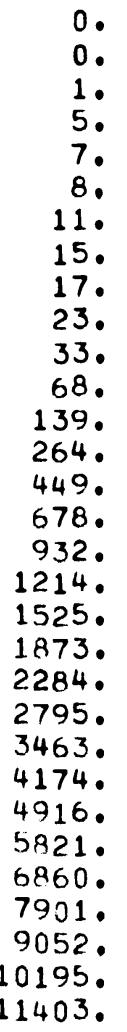

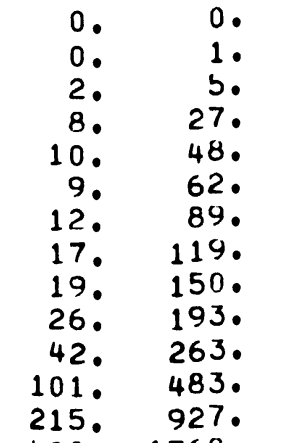

$S R-90$

KR-85 TC-99 RH103 RU106

PD XE CM242 AM241

402.1768 . 424.

653. 3110. 1414.

928. 4933. 2234.

1192. 7180. 3235.

1470 . 9899. 4432 .

1768.13126 . 5838 .

2103.16929 .7479$.

2519. 21491 . 9423 .

3092. 26953. 11744.

3886 . 33784 . $14669^{\circ}$

4653. 41388. 17924.

5393 . 49637. 21489 .

6391.59328 .25611$.
7509.70328 .30252$.

8472 . 82073 . 35171.

9593.95142 . 40596.

- 0.0000.

. 0

11706.124181 . 52545.

$\begin{array}{rrr}0 . & 1 . & 0 . \\ 0 . & 3 . & 2 . \\ 1 . & 19 . & 10 .\end{array}$

$1.233^{\circ} \quad 18$.

1. 43. 23 .

2. 62. 33.

$\begin{array}{rrr}2 . & 84 . & 44 . \\ 3 . & 106 . & 56 .\end{array}$

4. 138.73.

5. 189. 101 .

9. 341.6181.

$\begin{array}{rrr}18 . & 648 . & 348^{\circ} \\ 35 . & 1228^{\circ} & 646 .\end{array}$

62. 2157.1128 .

97. 3427 . 1786.

139. 5006. 2607.

245. 9236. $4822^{\circ}$.

311.11970 . 6263.

388 • 15264. 8015.

$4800^{\circ} 192280^{\circ} 101344^{\circ}$

725. 29777. 15754 .

864. 35918. 19032 .

1024 . 43148. 22930.

1204.51405 . 27441.

$1590^{\circ} 70342.37310^{\circ}$

1814.81087 .43708 .

0.
0.
3.

4.

$\begin{array}{ll}4 . & 38 \\ 5 . & 55\end{array}$

6. 74.

9. 120.0796

14. 161. 1084

33. 291. 1963

70. 550 . 3735 .

135. 1048. 7073 .

227. 1856. 12407.

335. 2974. 19707.

450. 4391. 28791.

578. 6159. 39885.

719. 8314. 53165 .

879. 10914. 68938.

1081. 14114. 87961.

1334. 17998. 110959。

1664 . 22801. 139750 。

2008. 28220. 172272.

2316. 34082. 208089

2826. 41202. 250408.

3416.49448 . 298889 .

4013 . 58550. 351487 .

4740 . 68969. 410531.

5452 . 80328 . 474091 .

2048.92932.50168.6287.93089.544320.

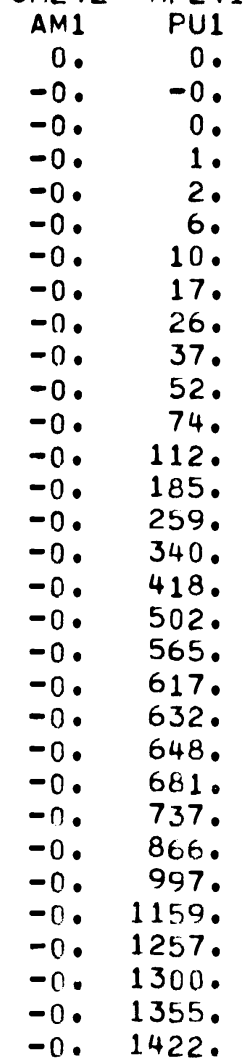




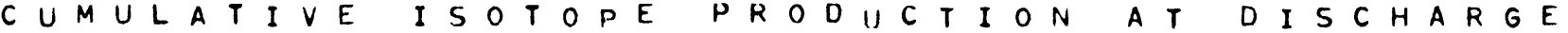 \\ (NO LOSSES EXCEPT FOR RADIOACTIVE LECAY)}

PAGE 127

ISOTOPE PRODUCTION, KGS

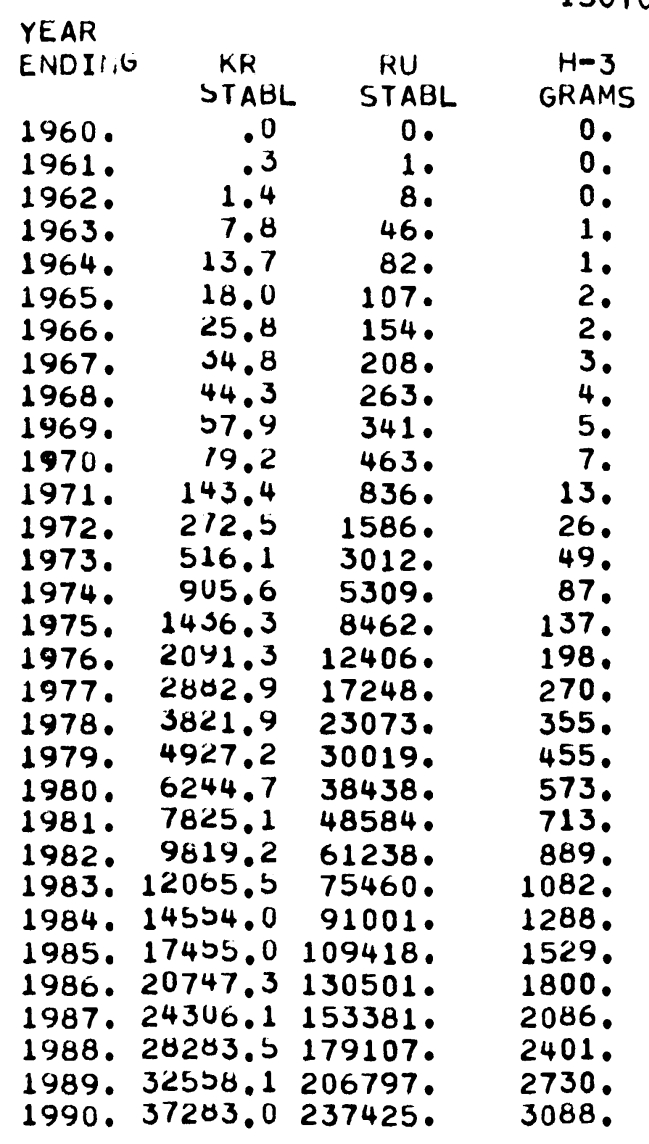


NET ANNUAL I SOT TOPE A $T$ A I L A B I L I T Y

PAGF 128

(WITH TARGET IRRADIATION. PROCESS LOSSES ANC DECAY LUSSES)

CASE O FOR 180 GW(E) AT 1980

CASE

7

TUTAL
LURIIVG INSTALLED

PERICUN CAHACITY

LINDIIG OW(E)

ISOTOPE PROCUCTION, KILOGRAMS/YEAR

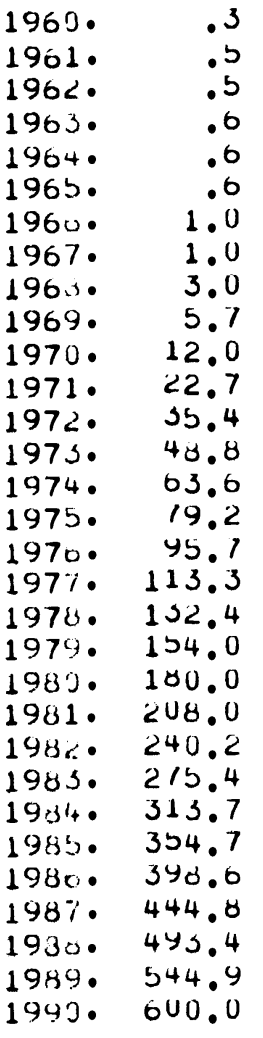

TONNE

PU-RP PU-SP

NP237

AM241 AM243

$\mathrm{CM} 242 \quad \mathrm{CM} 244$
$\mathrm{CM} 244$
$\mathrm{AM} 3$

CM244 PU238 PU238 PU238 PU238

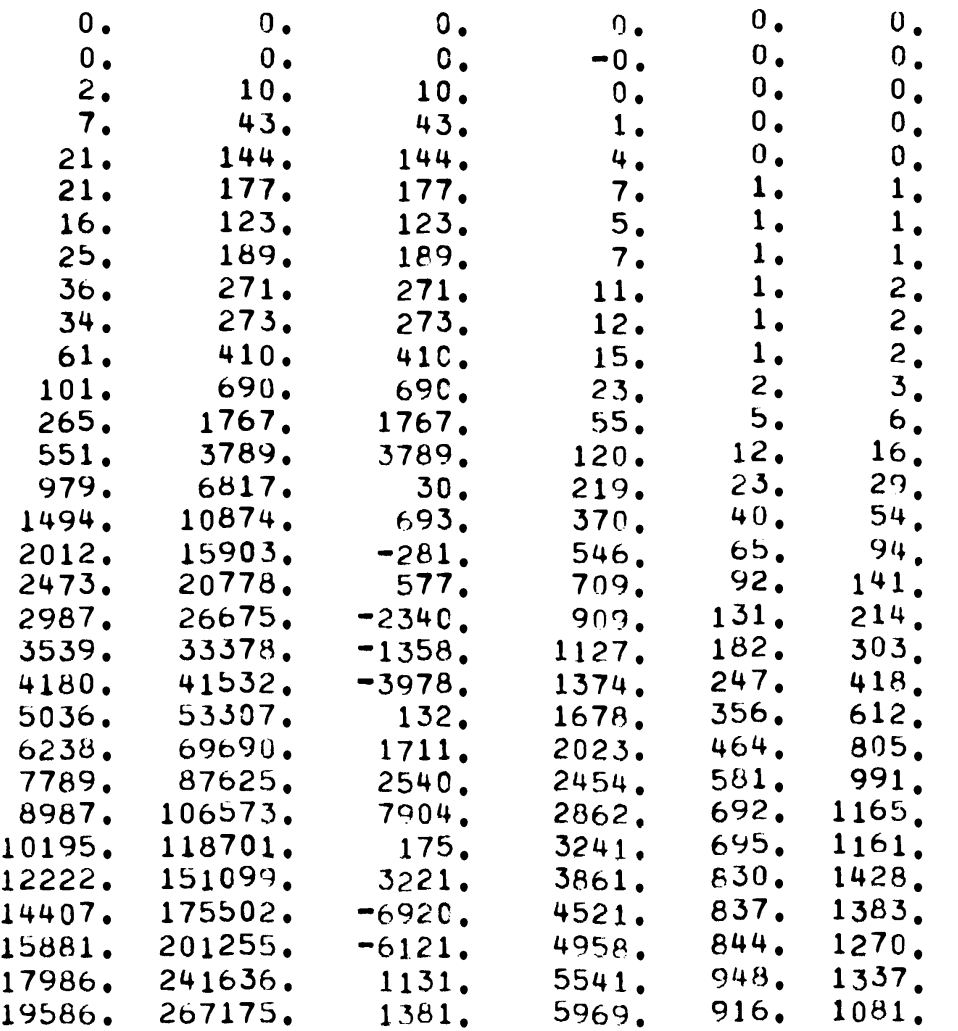

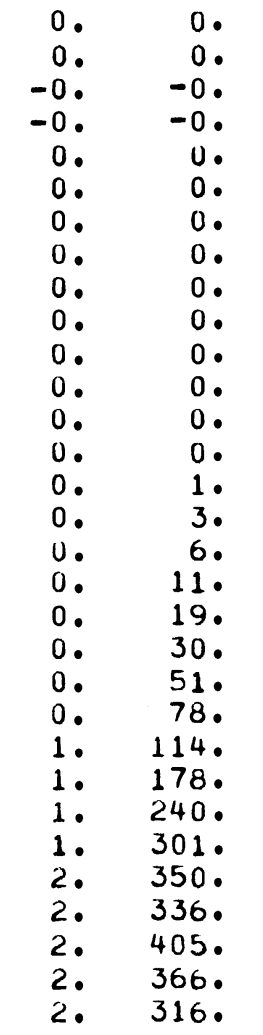

AM.
0.
0.
0.
0.
0.
0.
0.
0.
0.
0.
0.
0.
0.
1.
1.
3.
5.
10.
18.
28.
43.
64.
91.
136.
184.
236.
301.
339.
425.
469.

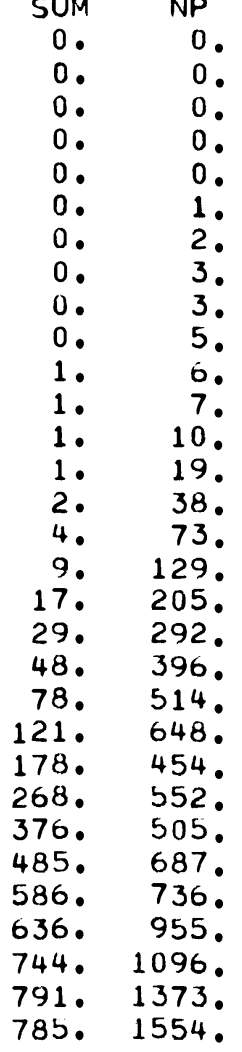

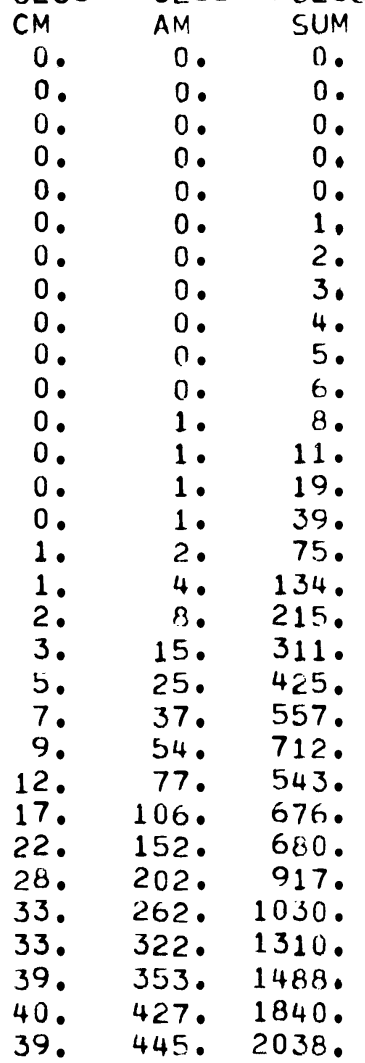


NET ANNUAL I SOT TOPE AVAILABILITY

PAGE 129

(WITH TARGET IRRADIATION, PROCESS LOSSES AND DECAY LOSSES)

CASE 6 FOR 180 GW(E) AT 1980

CASE 7

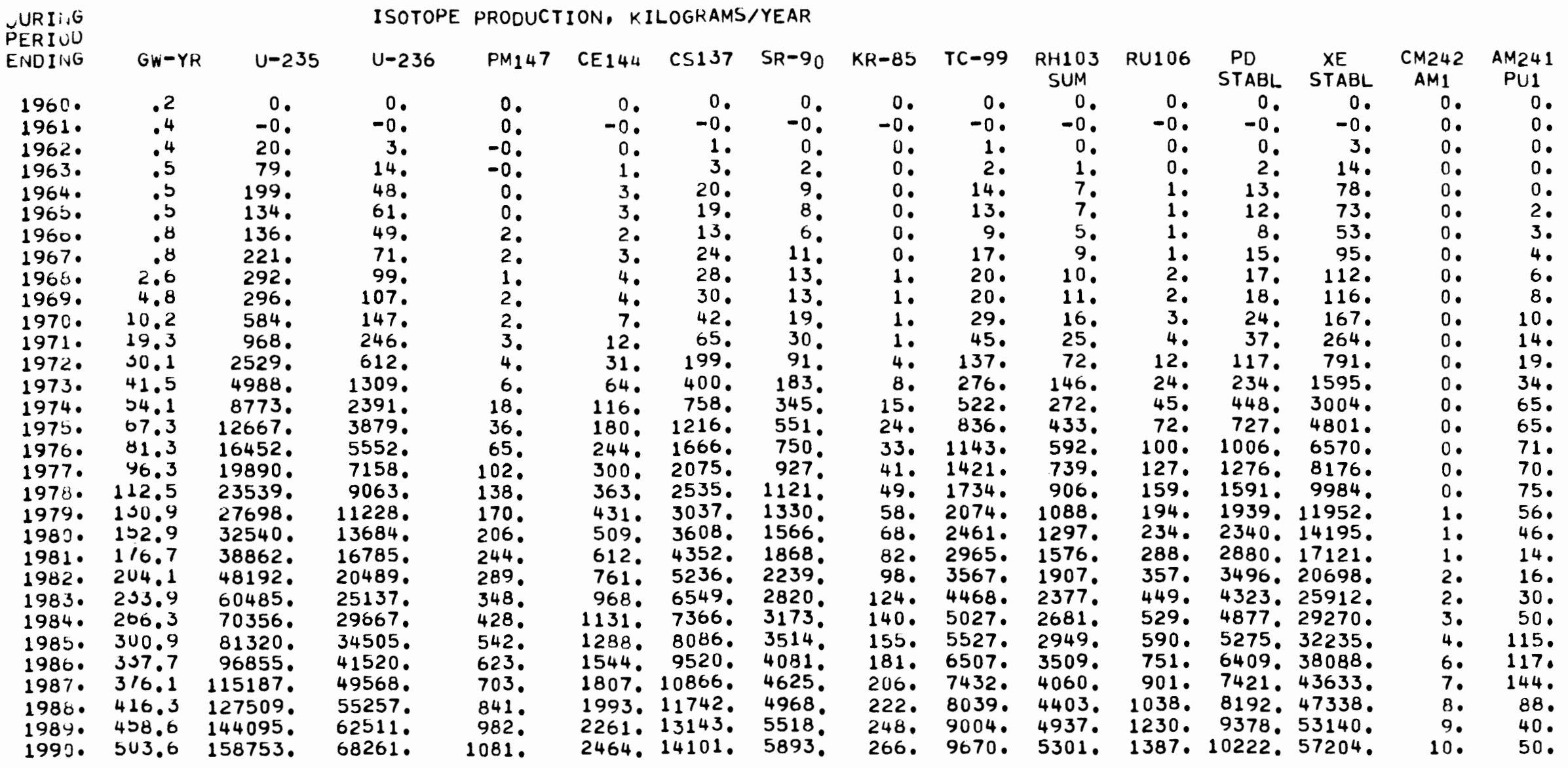


NET ANNUAL I SOTOPE AVAILABILITY PAGE 130 (WITH TARGET IRRADIATION, PROCESS LOSSES AND DECAY LOSSES)

1960.

1961.

STABL

1962.

.0
-.0

1963.

$1964 . \quad 5.7$

1965.503

1966.3 .9

1967. 7.0

1968.8 .2

1969.8 .5

1970.12 .2

1971. 19.2

1972. 07.8

1973.116 .2

$\begin{array}{ll}1973 . & 116.2 \\ 1974 . & 219.2\end{array}$

$\begin{array}{ll}1974 . & 219.2 \\ 1975 . & 350.6\end{array}$

1970. 417.6

1977. 589.5

1976. 712.4

1979. 845.1

1980.944 .8

$1981 \cdot 1185.7$

1982.1422 .3

1983. 1744.7

$1984 \cdot 2021.6$

1985.2239 .7

$1986 \cdot 2610.9$

1987.2903 .0

1988.3203 .0

1984.3519 .6

1990.3847 .2

$H-3$

GRAMS

0.0

-0.

1.

35 .

32 .

23.

23

42.

48.

70

109.

109

336.

675.

$1284^{\circ}$

2067.

2838 .

3549 .

4358 .

5242 .

6251.

7578 .

9131

0.
0.
0.

0 .

1.

1.

1.

1.

1.

2.

6.

21.

34.

47.

58.

85.

101.

122.

146.

183.

13986.

$16576.265^{\circ}$.

18975.302 .

20591.327.

23153.366.

392. 
CUMULATIVE I SOTOP

PAGE 131

(WITH TARGET IRRADIATION. PROCESS LOSSES AND DECAY LOSSES)

CASE 6 FOR 180 GW(E) AT 1980

CASE 7

TUTAL

INSIALLED ISOTOPE PRODUCTION, KGS

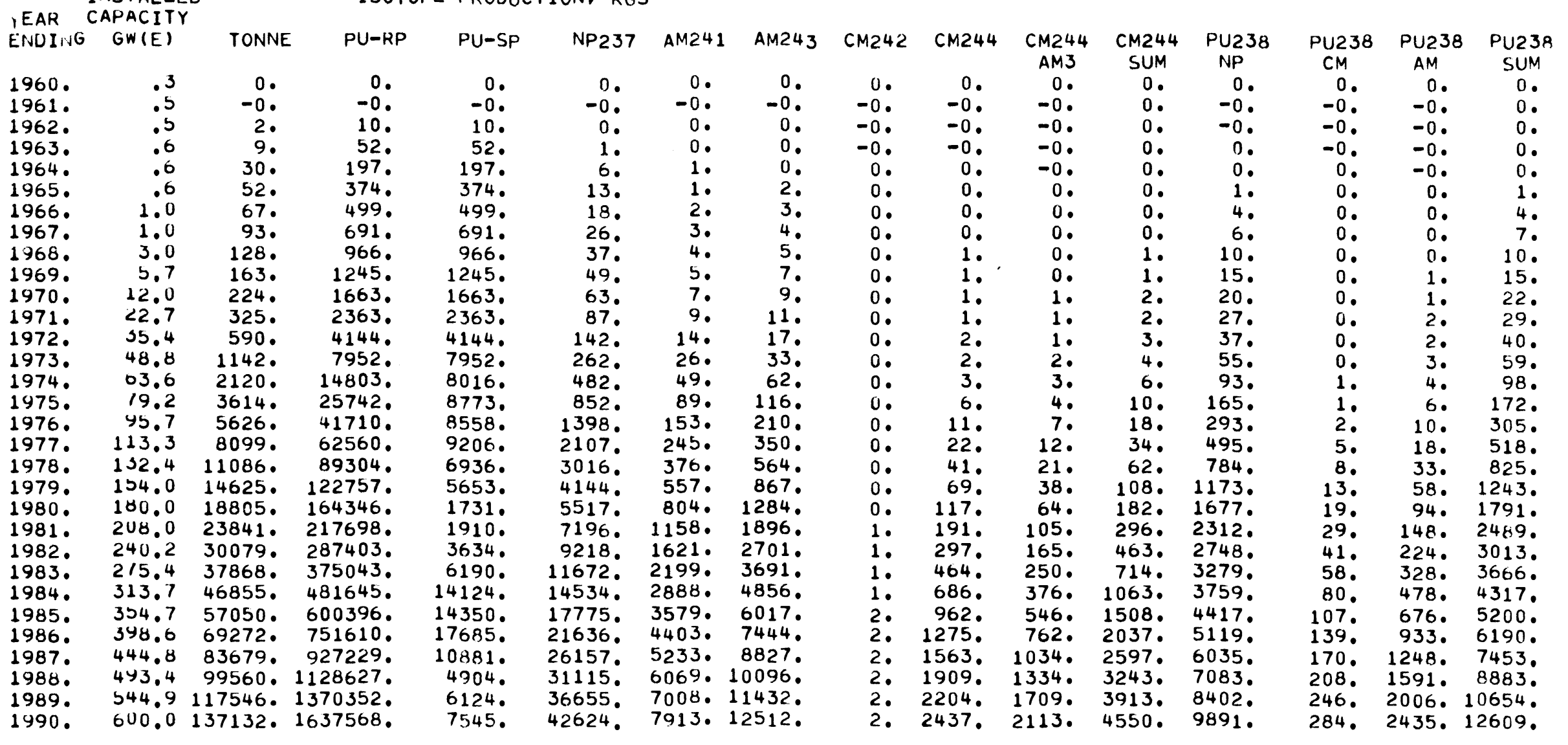


C UMULATIVE ISOTOPE AVAILABILIT Y

PAGE 132

(WITH TARGET IRRADIATION. PROCESS LOSSES AND DECAY LOSSES)

CASE 6 FOR 180 GW(E) AT 1980

CASE 7

YEAR

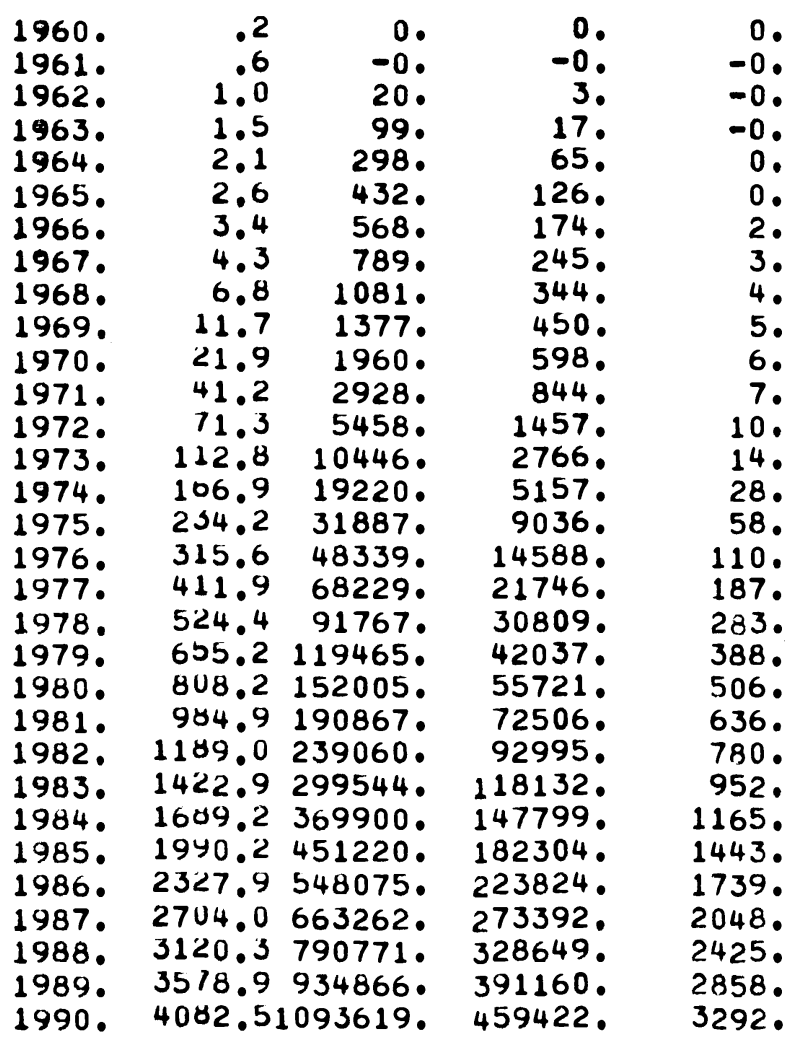

CE144 CS137 SR-9

KR-85 TC-99 RH103 RU106

PD

XE

CM242 AM241

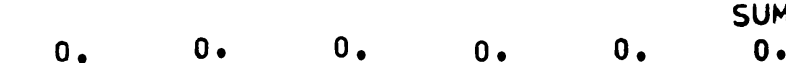

$\begin{array}{rr}0 . & 0 \\ -0 . & -0 \\ 0 . & \end{array}$

1. 24.

4. 42.

4. 55.

5. 78.

7. 132 .

10. 170 .

16. 232. 105 .

38. 425. 194.

$80 . \quad 815.372$.

242 . 2735. 1242 .

343. 4338. 1962.

441. 6314. 2840 。

544. 8705. $3891^{\circ}$.

655. 11544.5126.

932. 18900. 8274.

1144.23704 .10311$.

1438. 29711. 12879.

1723. 36398. 15737 .

1997. 43653. 18867 .

2366. 52175. 22487 .

2780 .61849.26561.

3136.72178 .30880

3551 . 83672. 35643 .

$\begin{array}{rr}0 . & 0 \\ -0 . & - \\ 0 . & \end{array}$

SUM

$-0$

0 .

STABL

STABL

AM1 PU1

0.1 .20

0.17.

1. 30

9.

$\begin{array}{lll}1 . & 39 . & 21 . \\ 1 . & 56 . & 29\end{array}$

2. 75.40.

2. 96.51.

124

4. 170.

16.583 .5309

30. 1105. 582 .

52. 1941. 1015.

82. 3084. 1607.

117. 4505. 2346 .

159. 6239. 3252.

262. 10773. 5637.

328. 13737. 7214.

405. 17305. 9121 .

504.21772 . 11498 .

612. 26799. 14179.

729. 32326. 17128 .

864 . 38833. 20637.

1016 • 46265. 24697 .

1349.63308 . 34037 .

1530 . 72978. 39337 .

1. 15

1.15

2.26.

2. 34.

3.

3. 84

4. 108.

6. 145.

15. 261

32.495

102.1670 .11167$.

151. 2677. 17736 .

202. 3952. 25912 .

260. 5543. 35896 .

324. 7482. 47848 。

396. 9822. 62044 .

486.12703 .79165 .

600. 16198. 99863.

749. 20521. 125775.

904. 25398. 155045.

1042. 30674. 187280.

1272. 37082. 225368 .

1537. 44503. 269000。

1806, 52695, 316338 .

2133 . 62073. 369478.

2453. 72295. 426682 .

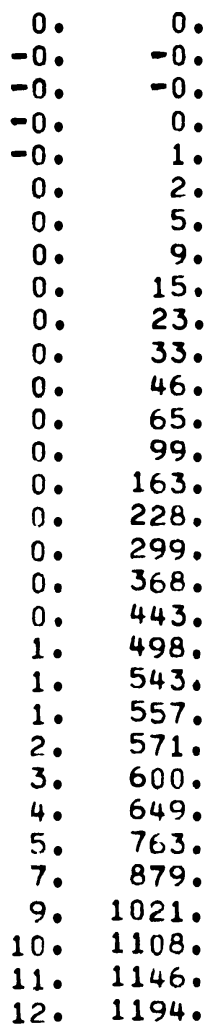


(WITH TARGET IRRADIATION, PROCESS LOSSES AND DECAY LOSSES)

ISOTOPE PRODUCTION, KGS

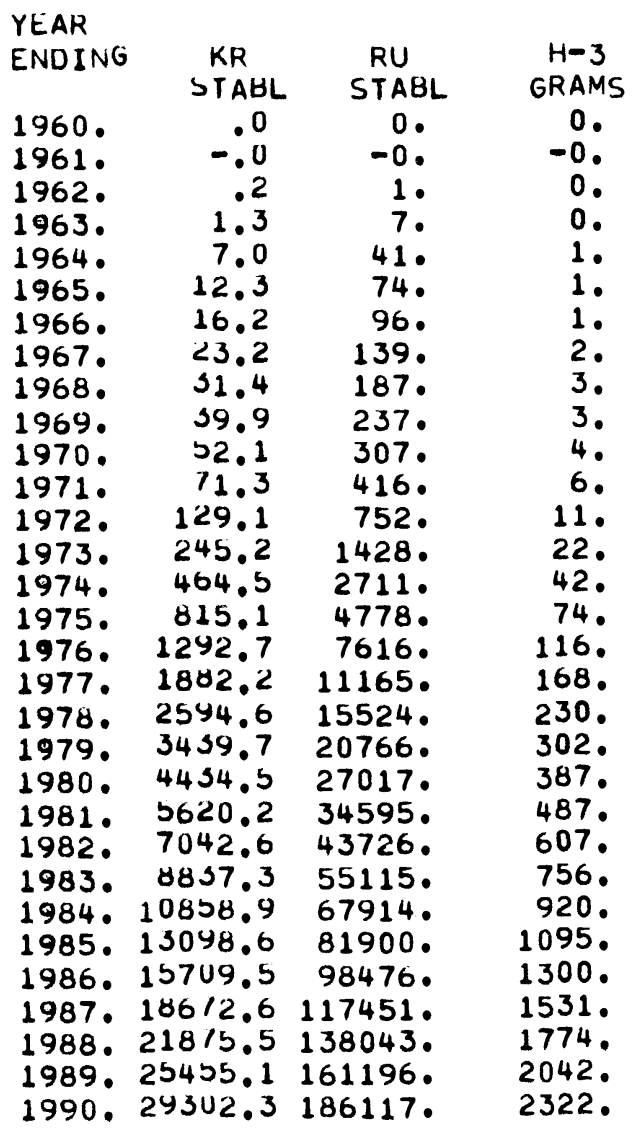




\section{ISOTOPE PROOUCTION}

CASE 6 FOR 120 GW(E) AT 1980 
DISTRIBUTION OF ELECTRICAL GENERATA NGACA PACITY

PAGE 134

CASE 6 FOR 120 GW(E) AT 1980

CASE 8

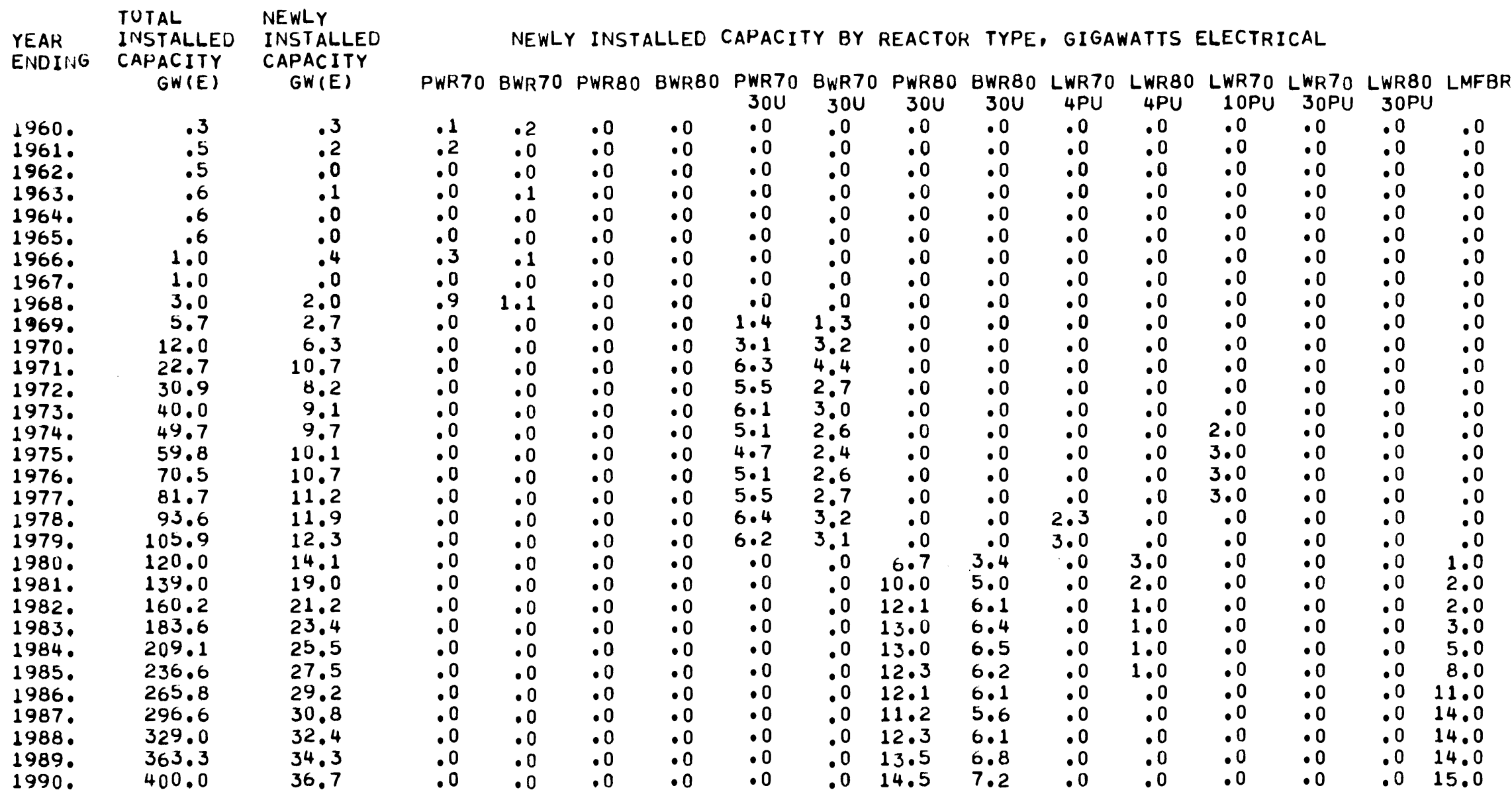


DISTRIBUTION OF ELECTRICAL GENERATINGACA PACITY

PAGE 135

CASE 6 FOR 120 GW(E) AT 1980

CASE 8

TUTAL NEWLY

YEAR INSTALLED INSTALLED

ENDING CAPACITY CAPACITY

CUMULATIVE INSTALLED CAPACITY BY REACTOR TYPE, GIGAWATTS ELECTRICAL

GW(E) GW(E)

PWR70 BWR70 PWR80 BWR80 PWR70 BWR70 PWR80 BWR80 LWR70 LWR80 LWR70 LWR70 LWR80 LMFBR

1960.

.3
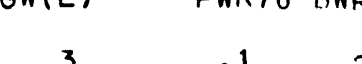

\begin{tabular}{|c|c|c|c|c|c|}
\hline WR 80 & BWR8 & $\begin{array}{c}\text { PWR70 } \\
30 U\end{array}$ & $\begin{array}{r}B W R 7 C \\
30 U\end{array}$ & $\begin{array}{c}\text { O PWR80 } \\
30 U\end{array}$ & $\begin{array}{c}\text { BWR } 80 \\
30 U\end{array}$ \\
\hline .0 & .0 & . 0 & .0 & .0 & .0 \\
\hline .0 & .0 & .0 & 0 & .0 & .0 \\
\hline .0 & .0 & . 0 & .0 & .0 & .0 \\
\hline . 0 & .0 & .0 & .0 & .0 & .0 \\
\hline . 0 & .0 & .0 & .0 & .0 & .0 \\
\hline . 0 & .0 & .0 & 0 & .0 & .0 \\
\hline .0 & .0 & .0 & .0 & .0 & .0 \\
\hline . 0 & .0 & .0 & .0 & .0 & .0 \\
\hline .0 & .0 & $\cdot 0$ & .0 & .0 & .0 \\
\hline .0 & .0 & 1.4 & 1.3 & .0 & .0 \\
\hline . 0 & .0 & 4.4 & 4.5 & .0 & .0 \\
\hline .0 & .0 & $10 \cdot 8$ & 8.9 & .0 & .0 \\
\hline .0 & .0 & $16 \cdot 3$ & 11.6 & .0 & .0 \\
\hline .0 & .0 & $22 \cdot 3$ & 14 & .0 & .0 \\
\hline . 0 & .0 & $27 \cdot 4$ & 17.3 & .0 & .0 \\
\hline . 0 & .0 & $32 \cdot 1$ & 19.7 & .0 & .0 \\
\hline . 0 & .0 & 37.2 & 22.3 & .0 & .0 \\
\hline - 0 & .0 & 42.7 & 25.0 & .0 & .0 \\
\hline .0 & $\cdot 0$ & 49.2 & 28.2 & .0 & .0 \\
\hline .0 & .0 & 55.4 & 31.3 & .0 & .0 \\
\hline - 0 & $\cdot 0$ & 55.4 & 31.3 & 6.7 & 3.4 \\
\hline $\begin{array}{r}.0 \\
.0\end{array}$ & $\because 0$ & $\begin{array}{l}55.4 \\
55.4\end{array}$ & $\begin{array}{l}31.3 \\
31 \cdot 3\end{array}$ & $\begin{array}{l}16.7 \\
28.8\end{array}$ & $\begin{array}{r}8.4 \\
14.5\end{array}$ \\
\hline .0 & .0 & 55.4 & 31.3 & 41.8 & 20.9 \\
\hline . 0 & .0 & 55.4 & 31.3 & 54.8 & 27.4 \\
\hline . 0 & .0 & 55.4 & 31.3 & 67.1 & 33.6 \\
\hline .0 & .0 & 55.4 & 31.3 & 79.2 & 39.7 \\
\hline .0 & .0 & 55.4 & 31.3 & 90.4 & 45.3 \\
\hline .0 & .0 & 55.4 & 31.3 & 102.7 & 51.4 \\
\hline . 0 & .0 & 4 & 31.3 & 116.2 & 58.2 \\
\hline . 0 & .0 & 5.4 & 31.3 & 130.7 & 65.4 \\
\hline
\end{tabular}

$\begin{array}{ccc}\text { LWR70 } & \text { LWR80 } & \text { LWR70 } \\ \text { 4PU } & 4 P U & 10 P U \\ .0 & .0 & .0 \\ .0 & .0 & .0 \\ .0 & .0 & .0 \\ .0 & .0 & .0 \\ .0 & .0 & .0 \\ .0 & .0 & .0 \\ .0 & .0 & .0 \\ .0 & .0 & .0 \\ .0 & .0 & .0 \\ .0 & .0 & .0 \\ .0 & .0 & .0 \\ .0 & .0 & .0 \\ .0 & .0 & .0 \\ .0 & .0 & .0 \\ .0 & .0 & 2.0 \\ .0 & .0 & 5.0 \\ .0 & .0 & 8.0 \\ .0 & .0 & 11.0 \\ 2.3 & .0 & 11.0 \\ 5.2 & .0 & 11.0 \\ 5.2 & 3.0 & 11.0 \\ 5.2 & 5.0 & 11.0 \\ 5.2 & 6.0 & 11.0 \\ 5.2 & 7.0 & 11.0 \\ 5.2 & 8.0 & 11.0 \\ 5.2 & 9.0 & 11.0 \\ 5.2 & 9.0 & 11.0 \\ 5.2 & 9.0 & 11.0 \\ 5.2 & 9.0 & 11.0 \\ 5.2 & 9.0 & 11.0 \\ 5.2 & 9.0 & 11.0 \\ & & \end{array}$

$\begin{array}{ccc}\text { LWR7O } & \text { LWR8 } \\ 30 P U & \text { LMFB } & \text { LMP } \\ .0 & .0 & .0 \\ .0 & .0 & .0 \\ .0 & .0 & .0 \\ .0 & .0 & .0 \\ .0 & .0 & .0 \\ .0 & .0 & .0 \\ .0 & .0 & .0 \\ .0 & .0 & .0 \\ .0 & .0 & .0 \\ .0 & .0 & .0 \\ .0 & .0 & .0 \\ .0 & .0 & .0 \\ .0 & .0 & .0 \\ .0 & .0 & .0 \\ .0 & .0 & .0 \\ .0 & .0 & .0 \\ .0 & .0 & .0 \\ .0 & .0 & .0 \\ .0 & .0 & .0 \\ .0 & .0 & .0 \\ .0 & .0 & 1.0 \\ .0 & .0 & 3.0 \\ .0 & .0 & 5.0 \\ .0 & .0 & 8.0 \\ .0 & .0 & 13.0 \\ .0 & .0 & 21.0 \\ .0 & .0 & 32.0 \\ .0 & .0 & 46.0 \\ .0 & .0 & 60.0 \\ .0 & .0 & 74.0 \\ .0 & .0 & 89.0\end{array}$




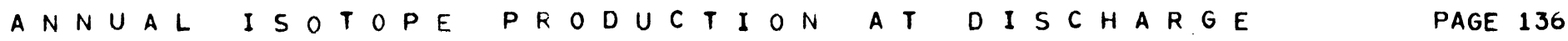

(NO LOSS BASIS)

CASE 6 FOR 120 GW(E) AT 1980

CASE 8

TUTAL

DURING INSTALLED

PERIOU CAHACITY

EINDIIVG

OW(E)

ISOTOPE PRODUCTION, KILOGRAMS/YEAR

\begin{tabular}{|c|c|c|c|c|}
\hline $\begin{array}{l}1960^{\circ} \\
1961^{\circ} \\
1962^{\circ} \\
1963^{\circ} \\
1964^{\circ} \\
1965^{\circ} \\
1966^{\circ} \\
1967^{\circ} \\
1968^{\circ} \\
1969^{\circ} \\
1970^{\circ} \\
1971^{\circ} \\
1972^{\circ} \\
1973^{\circ} \\
1974^{\circ} \\
1975^{\circ} \\
1976^{\circ} \\
1977^{\circ} \\
1978^{\circ} \\
19790^{\circ} \\
1980^{\circ} \\
1981^{\circ} \\
1982^{\circ} \\
19833^{\circ} \\
1984^{\circ} \\
1980^{\circ} \\
19866^{\circ} \\
1987^{\circ} \\
1988^{\circ} \\
1989^{\circ} \\
1990 \circ\end{array}$ & $\begin{array}{r}.3 \\
.5 \\
.5 \\
.6 \\
.6 \\
.6 \\
1.0 \\
1.0 \\
3.0 \\
5.7 \\
12.0 \\
22.7 \\
30.9 \\
40.0 \\
49.7 \\
59.8 \\
70.5 \\
81.7 \\
93.6 \\
105.9 \\
120.0 \\
139.0 \\
160.2 \\
183.6 \\
209.1 \\
236.6 \\
205.8 \\
296.6 \\
329.0 \\
303.3 \\
400.0\end{array}$ & 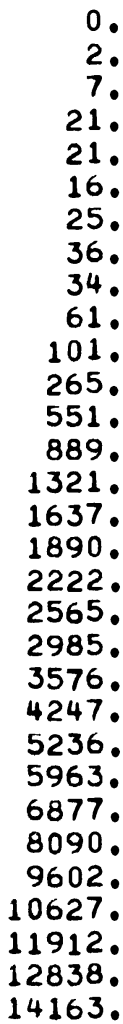 & 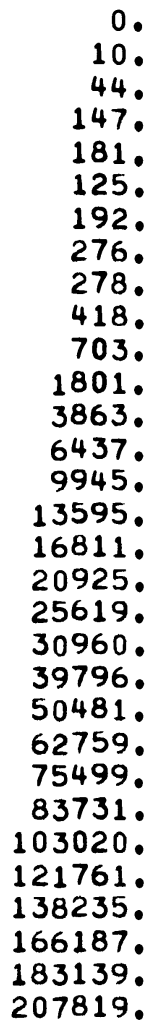 & 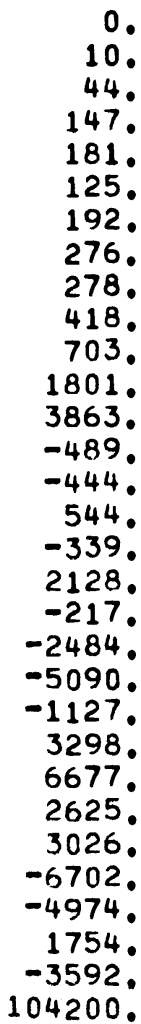 \\
\hline
\end{tabular}

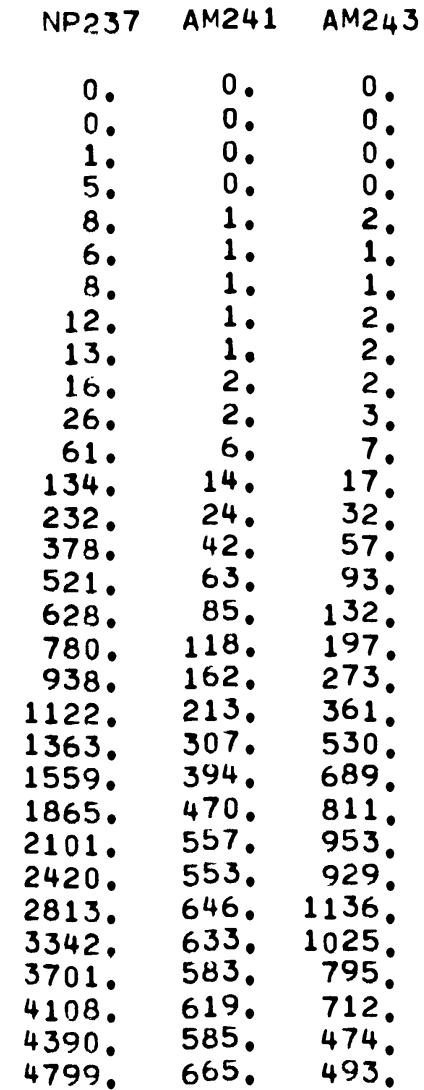

$\mathrm{CM} 242 \quad \mathrm{CM} 244$

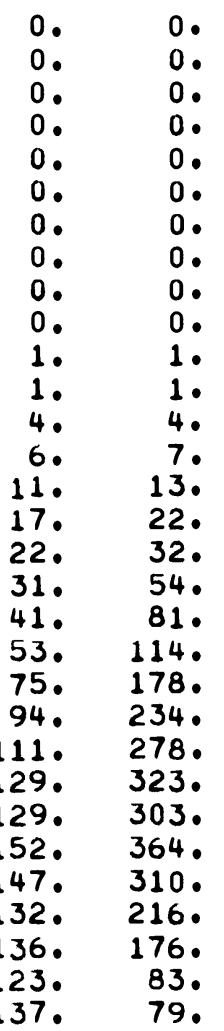

\begin{tabular}{|c|c|c|}
\hline $\begin{array}{c}C M 244 \\
\text { AM3 }\end{array}$ & $\begin{array}{c}\mathrm{CM} 244 \\
\text { SUM }\end{array}$ & $\begin{array}{l}\text { PU238 } \\
\text { NP }\end{array}$ \\
\hline 0 & 0. & 0. \\
\hline 0 . & 0. & 0 . \\
\hline 0. & 0. & 0. \\
\hline 0. & 0. & 0. \\
\hline 0. & 0. & 0. \\
\hline 0. & 0. & 0 . \\
\hline 0. & 0. & 0 . \\
\hline 0. & 0. & 0. \\
\hline 0. & 0. & 0. \\
\hline 0. & 0. & 0 . \\
\hline 0. & 1. & 0 . \\
\hline 0. & 1. & 0 . \\
\hline 0. & 4. & 0. \\
\hline 0. & 73 & $\begin{array}{l}0 . \\
0 .\end{array}$ \\
\hline 0 . & 22. & 0 \\
\hline 0. & 32 . & 0 . \\
\hline 0 . & 54. & 0 . \\
\hline 0. & 114. & 0. \\
\hline 0 . & 178. & 0 . \\
\hline 0. & 234. & 0. \\
\hline $\begin{array}{l}0 . \\
0 .\end{array}$ & $\begin{array}{l}278 \\
323\end{array}$ & $\begin{array}{l}0 . \\
0 .\end{array}$ \\
\hline 0. & 303. & 0 . \\
\hline 0. & 364. & 0 \\
\hline 0. & 310. & 0 \\
\hline 0. & $216 \circ$ & 0 . \\
\hline $\begin{array}{l}0 . \\
0 .\end{array}$ & $\begin{array}{r}176 \\
83\end{array}$ & 0 . \\
\hline 0. & 79. & 0. \\
\hline
\end{tabular}

$P U 23$
$C M$
0.
0.
0.
0.
0.
0.
0.
0.
0.
0.
0.
0.
0.
0.
0.
0.
0.
0.
0.
0.
0.
0.
0.
0.
0.
0.
0.
0.
0.
0.
0.

\begin{tabular}{|c|c|}
\hline $\begin{array}{c}\text { PU238 } \\
\text { AM }\end{array}$ & $\begin{array}{l}\text { PU238 } \\
\text { SUM }\end{array}$ \\
\hline 0. & 0. \\
\hline 0. & 0. \\
\hline 0. & 0. \\
\hline 0. & 0. \\
\hline 0. & 0. \\
\hline 0. & 0. \\
\hline 0. & 0. \\
\hline 0. & 0. \\
\hline 0. & 0. \\
\hline 0. & 0. \\
\hline 0. & 0. \\
\hline 0. & 0. \\
\hline 0 . & 0. \\
\hline 0. & 0. \\
\hline 0. & 0. \\
\hline 0. & 0. \\
\hline 0. & 0. \\
\hline 0. & 0. \\
\hline 0. & 0. \\
\hline 0. & 0. \\
\hline 0 • & 0. \\
\hline 0. & 0. \\
\hline 0. & 0. \\
\hline 0. & 0. \\
\hline 0. & 0. \\
\hline 0. & 0. \\
\hline 0. & 0. \\
\hline 0. & 0. \\
\hline 0. & 0. \\
\hline 0. & 0. \\
\hline 0. & 0. \\
\hline
\end{tabular}


(NO LOSS BASIS)

URIIVG

PERIUU

ENDING

1960.

1961.

1962.

1964.

1965.

1966.

1967.

$1960^{\circ}$

1969.

1970 .

1971.

1972.

1973.

1975.

1976.

1977 .

1978.

1979.

1980. 101.9

1981.118 .1

1982.156 .1

1983. 155.9

1984. 117.4

1985. 200.5

1986. 224.8

1987.250 .3

1988. 216.9

$1990^{\circ}$

\section{GW-YR U-235}

0 .

21.

203 .

136.

139.

226.

302 .

596.

988.

2581.

$5090^{\circ}$

7950 .

11205 .

13322 .

15240.

17589.
20202.

23672 .

27994 .

32850 .

40982.

$47019^{\circ}$.

05374.

79204.

88599.

99077.

107484.
118562 .
ISOTOPE PRODUCTION, KILOGRAMS/YEAR

$$
u-236
$$

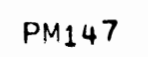

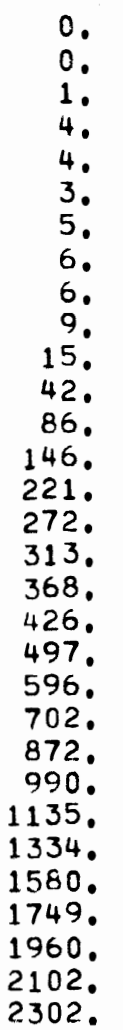

$K R-85$

$T C-99$

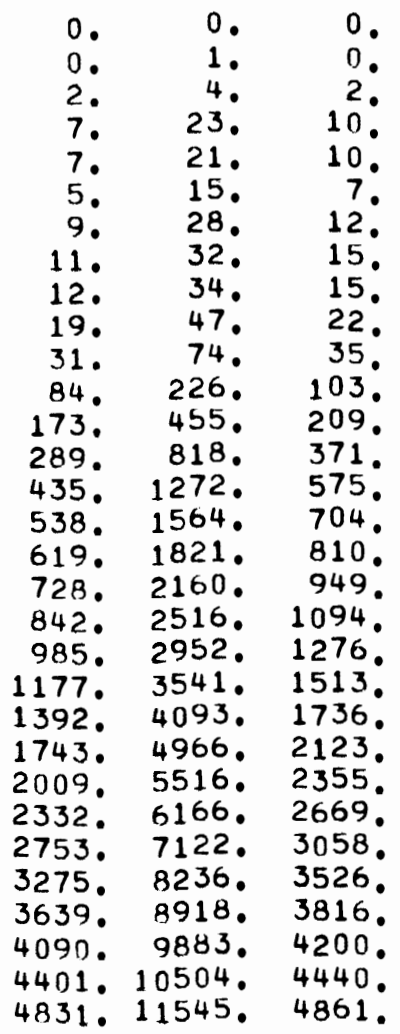

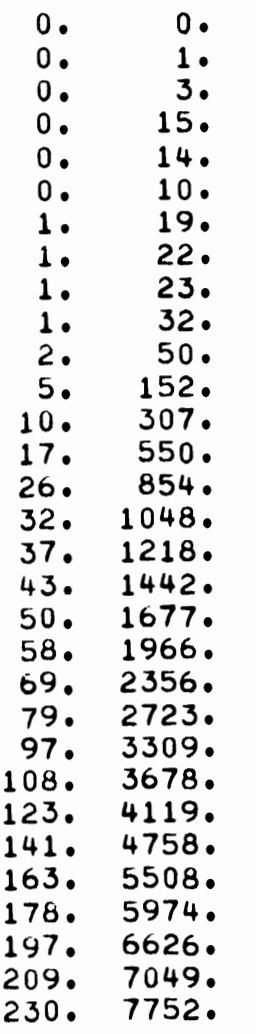

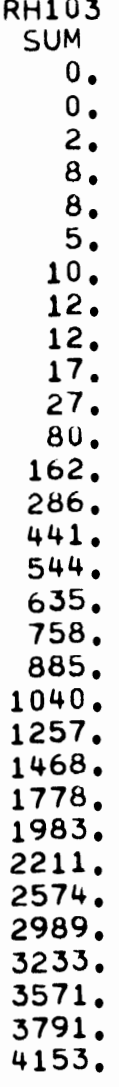

RU106

PTA

\begin{tabular}{|c|c|c|}
\hline $\begin{array}{l}0 . \\
0 . \\
1 . \\
2 . \\
2 . \\
20^{\circ} \\
3 . \\
40^{\circ} \\
40^{\circ} \\
60^{\circ} \\
90^{\circ} \\
26{ }^{\circ} \\
54^{\circ}\end{array}$ & 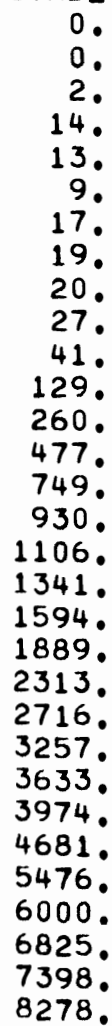 & 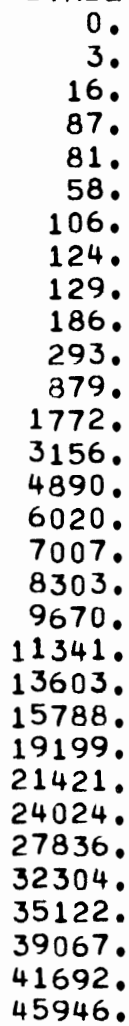 \\
\hline
\end{tabular}

CM242 AM241

AM1 PU1

$0 . \quad 0$.

0.0.

0.20 .

0.2 .

0.3.

0 . 5 .

D. $\quad 9$.

0.11.

0.22

0.38.

0.73

$\begin{array}{ll}0 . & 69 . \\ 0 . & 66 .\end{array}$

0.71 .

0.69.

0.89.

0 . 66 .

0.21.

0.11.

0.41.

$0.126^{\circ}$

0.154.

0.94.

0.51.

$\begin{array}{ll}0 . & 68 . \\ 0 . & 36 .\end{array}$ 

(NO LOSS BASIS)

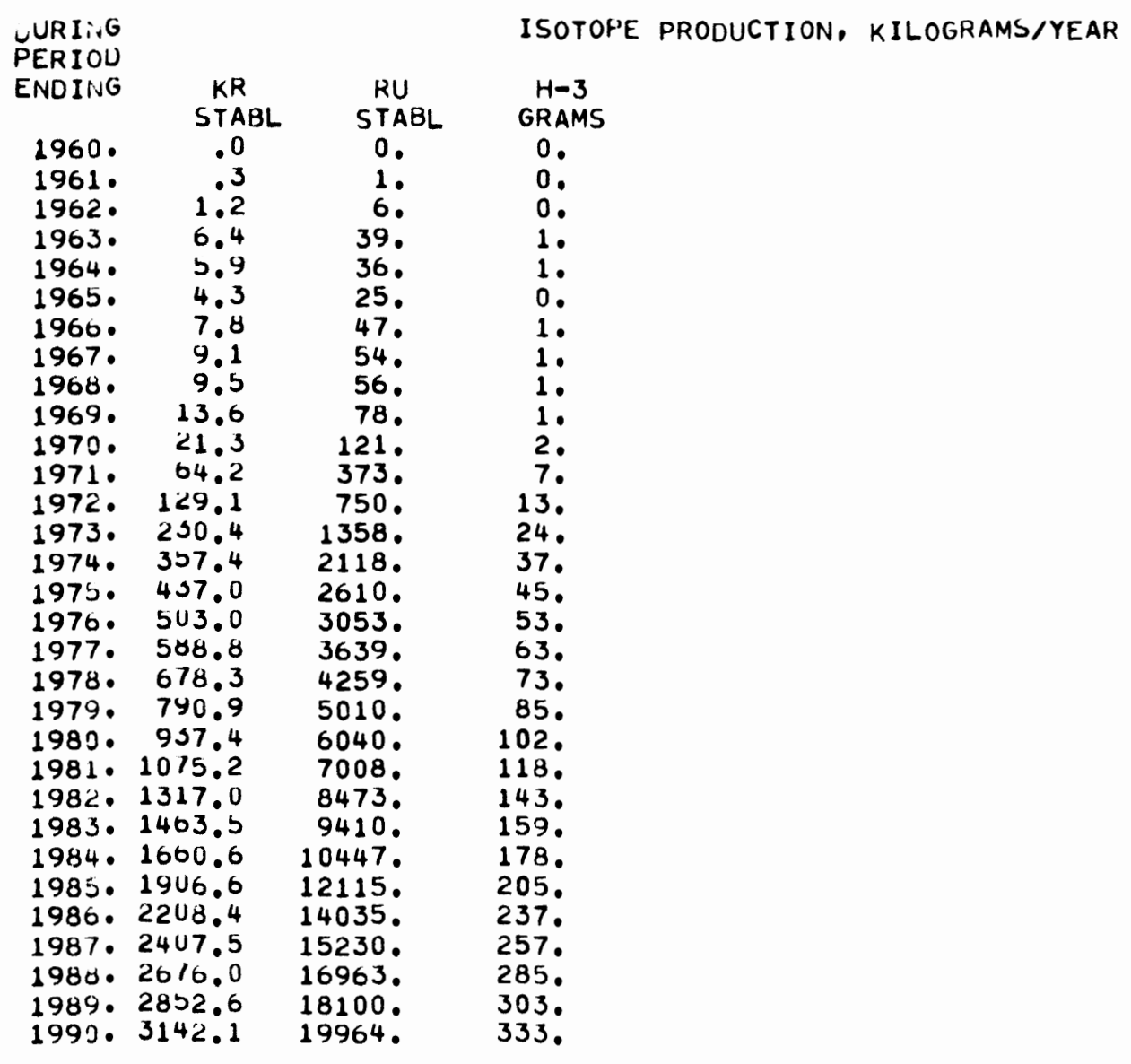


(NO LOSSES EXCEPT FOR RADIOACTIVE LECAY)

TOTAL

INS I ALLED

YEAR CAPACITY

ENDIIG GWIEI

ISOTOPE PRODUCTION, KGS

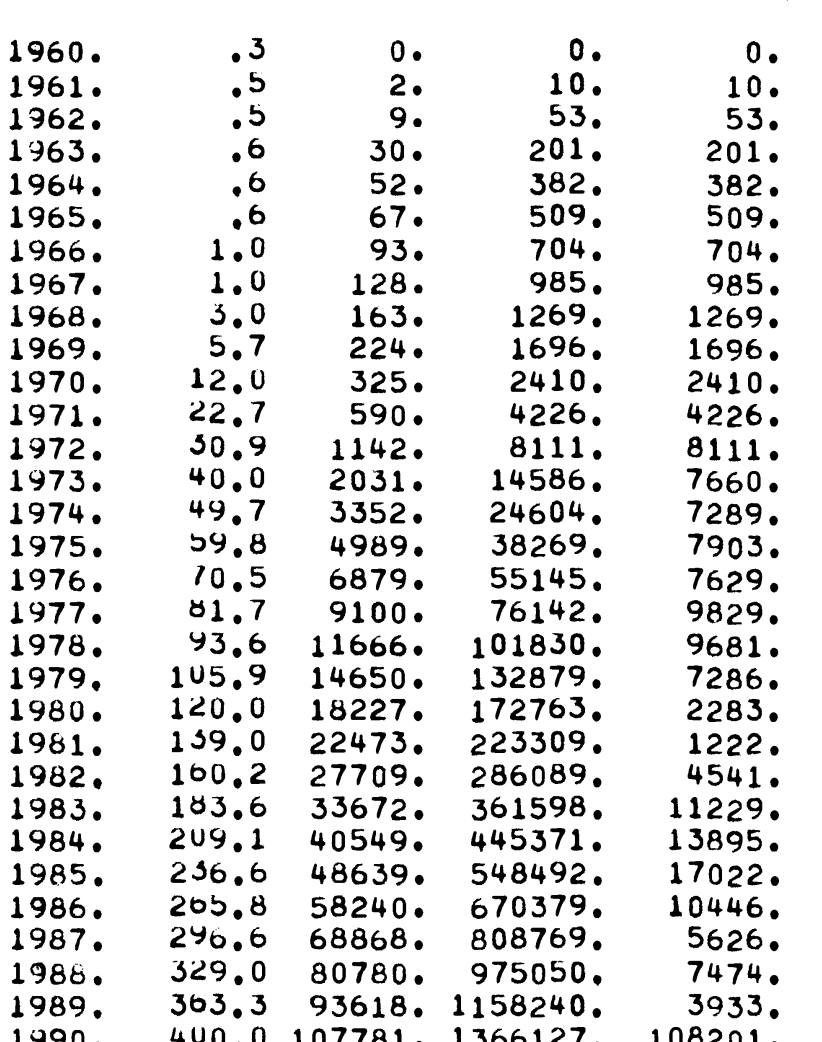

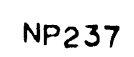

\begin{tabular}{|c|c|c|}
\hline 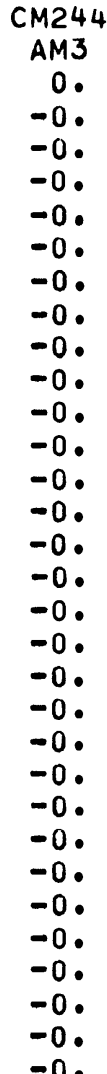 & 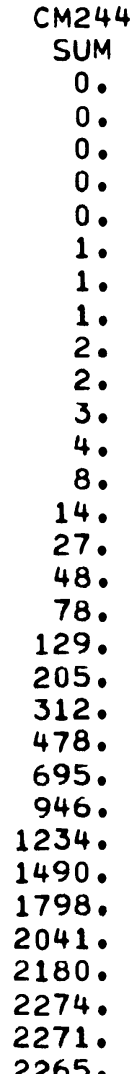 & 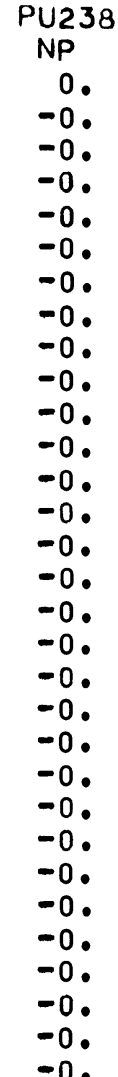 \\
\hline
\end{tabular}

$\begin{array}{ccc}\text { PU238 PU238 PU2.38 } & \\ \text { CM } & \end{array}$

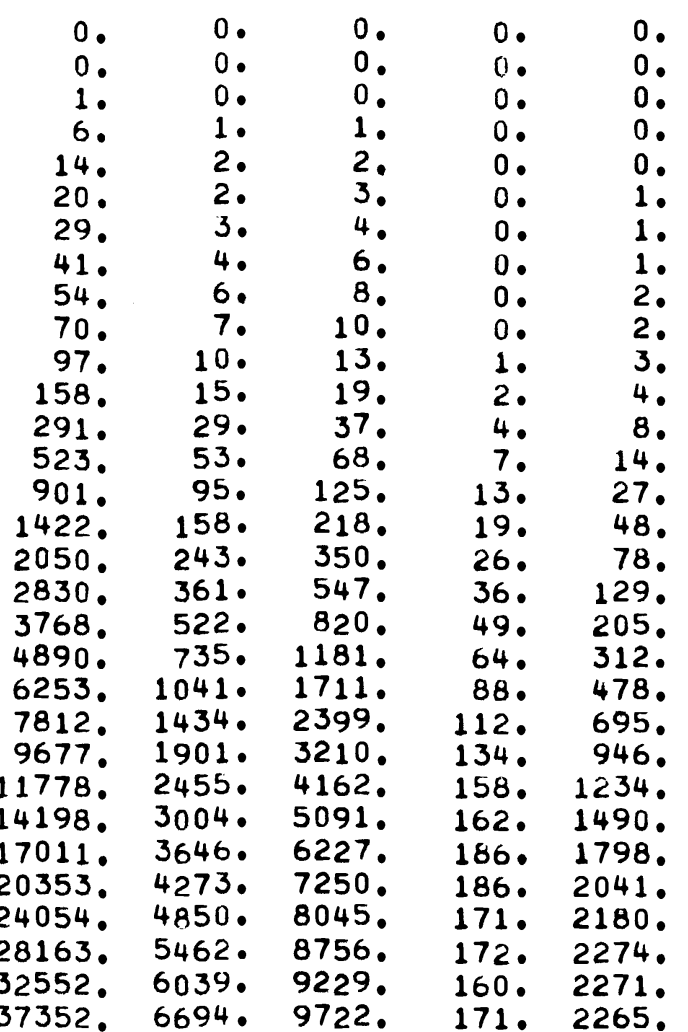

$-0.2265$.

-0 .

$\begin{array}{ll}C M & A M \\ 0 . & 0 . \\ -0 . & -0 . \\ -0 . & -0 . \\ -0 . & -0 . \\ -0 . & -0 . \\ -0 . & -0 . \\ -0 . & -0 . \\ -0 . & -0 . \\ -0 . & -0 . \\ -0 . & -0 . \\ -0 . & -0 . \\ -0 . & -0 . \\ -0 . & -0 . \\ -0 . & -0 . \\ -0 . & -0 . \\ -0 . & -0 . \\ -0 . & -0 . \\ -0 . & -0 . \\ -0 . & -0 . \\ -0 . & -0 . \\ -0 . & -0 . \\ -0 . & -0 . \\ -0 . & -0 . \\ -0 . & -0 . \\ -0 . & -0 . \\ -0 . & -0 . \\ -0 . & -0 . \\ -0 . & -0 . \\ -0 . & -0 . \\ -0 . & -0 . \\ -0 . & -0 . \\ & \end{array}$


YEAR ISOTOPE PRODUCTION, KGS

1960.

1961.

1962.

1963.

1964.

1965 .

1966.

1967 .

1968.

1969.

1970 .

1971 .

1972.

1973.

1974.

1975.

1976.

1977.

1978 .

1979.

1980.

1981.

1982.

1983.

1984.

1985 .

1986.

1988 .

1989.

1990 .
GW-YR U-235

$u-236$

.20.

$\begin{array}{rr}.6 & 21 . \\ 1.0 & 101 .\end{array}$

1.5304.

2.1440 .

2.6580 .

3.4805 .

4.31103 .

6.81405 .

11.72000 .

21.92988 .

41.25569.

07.510659 .

1U1.5 18609 .

143.829814.

194.643136 .

254.558376.

324.075965.

403.596167.

443.5119839.

79.4147833.

849.6221665 .

1005.5268684 .

1182.9324473.
1303.4389847.

1608.2469051 .

3.

17.

128.

178.

250.

351.

460

610 .

862.

1486 .

2823.

5097 .

8666.

13421.

19167.

26232.

34747 .

45000 .

57519 .

71945 .

89473.

109496 .

133264 .

161201 .

195089 .

233275 。

2135.4656727 . 276143.

2440.3764211 .322474 .

373524 .
PM1 47

CE144

$\operatorname{cS} 137$

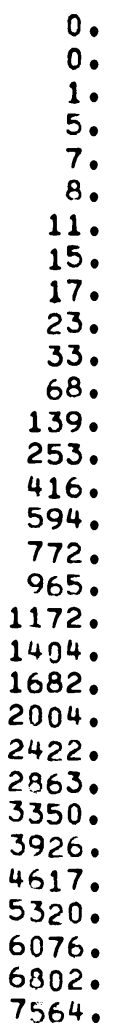

10.48.
0.
0.
2.
8.

10. 62 .

12. 89 .

17.119.

26. 193.

42. 263.

120.

101.483 .220$.

$\begin{array}{rrr}2150^{\circ} & 9277^{\circ} & 424^{\circ} \\ 378 . & 1724^{\circ} & 785 .\end{array}$

590 . 2957. 1340 .

781 . 4453. 2011 .

940. 6173. 2772.

1114. 8191. 3654 .

1300 . 10520. 4659.

1520 . 13231 . $5821^{\circ}$

1802 . 16470. 7193.

2133. 20187. 8752.

2620. 24692. 10661 .

3086. 29644. 12756.

3602 . 35133 . 15113 .

4235. 41453. 17802.

5017 . 48741. 20893.

5703 . 56547. 24197 .

6436. 65138. 27806.

7048 . 74154. 31566.

7730 . 84004 . 35655 .
$K R-85$

0.0 .00

$\begin{array}{lll}0 . & 1 . & 0 . \\ 0 . & 3 . & 2 .\end{array}$

1.19 .10$.

1. 33.418.

1. $43.230^{\circ}$

$\begin{array}{lll}2 . & 62 . & 33 . \\ 2 . & 84 . & 44^{\circ}\end{array}$

3. 106. 56.

4. 138. 73.

5. $189^{\circ} 101$.

18. 648. $344^{\circ}$.

34. 1198. 629.

58. 2052. 1070 .

87. 3100. 1615.

118. 4318. 2250.

$\begin{array}{lll}154^{\circ} & 5760^{\circ} \quad 30080^{\circ} \\ 194^{\circ} & 7437^{\circ} \quad 3893^{\circ}\end{array}$

240.9403 .4933$.

294. 11759. 6190.

354. 14483. 7658.

429. 17792. 9437 .

510 . 21469. 11420 .

601 . 25588. 13631 .

705. 30347. 16205.

824. 35855. 19193.

950 . 41828. 22426.

1088 . 48454. 25997 .

1229. 55503. 29788 .

1383.63254 . $33941^{\circ}$.
RU106

PD
STABL

XE CM242 AM241

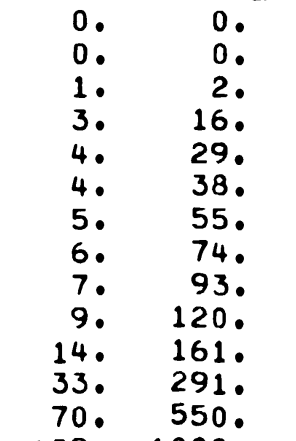

$120^{\circ} \quad 550^{\circ}$

209. 1777. 11781.

288. 2707. 17801.

364. 3813. 24807 .

451. 5154. 33111.

544. 6748. 42780 .

648. 8637. 54122 .

786. 10950. 67725 .

947. 13666. 83514 .

1152. 16923. 102712.

1371. 20556. 124133.

1583. 24531. 148157.

1884 . 29212. 175993 .

2276. 34688. 208297.

2668. 40688. 243419 。

3159. 47513. 282486.

3640 . 54911. 324178 .

4177 . 63189 . 370125 .
AM1 PUI

$0 . \quad 0$.

$-0 . \quad 0$

$-0.1$

$-0.2$

$-0.6$

$\begin{array}{ll}-0 . & 10 . \\ -0 . & 17\end{array}$

$-0 . \quad 37$.

$-0.52$.

-0.112 .

-0 . 185 .

$-0 . \quad 254$.

-0 . 391 .

$-0.459$.

-0.548 .

-0. 634.

$-0.719$.

-0.729 .

$-0.769$.

$-0.869$

$-0.993$

$-0.1239$.

-0. 1288.

-0.1353 .

$-0.1387$.
$-0.26$ 
(NO LOSSES EXCEPT FOR RADIOACTIVE UECAY)

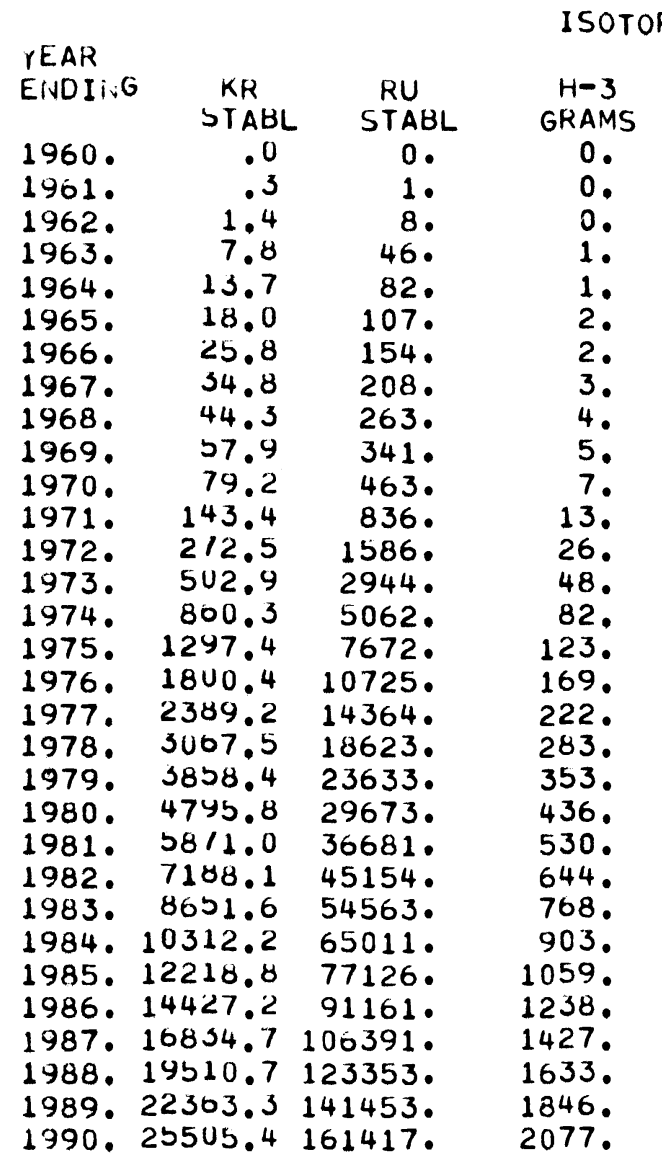


NET ANNUAL I SOTOPE AVAILABILITY

PAGE 142

(WITH TARGET IRRADIATION. PROCESS LOSSES AND DECAY LOSSES)

CASE 6 FOR 120 GW(E) AT 1980

CASE 8

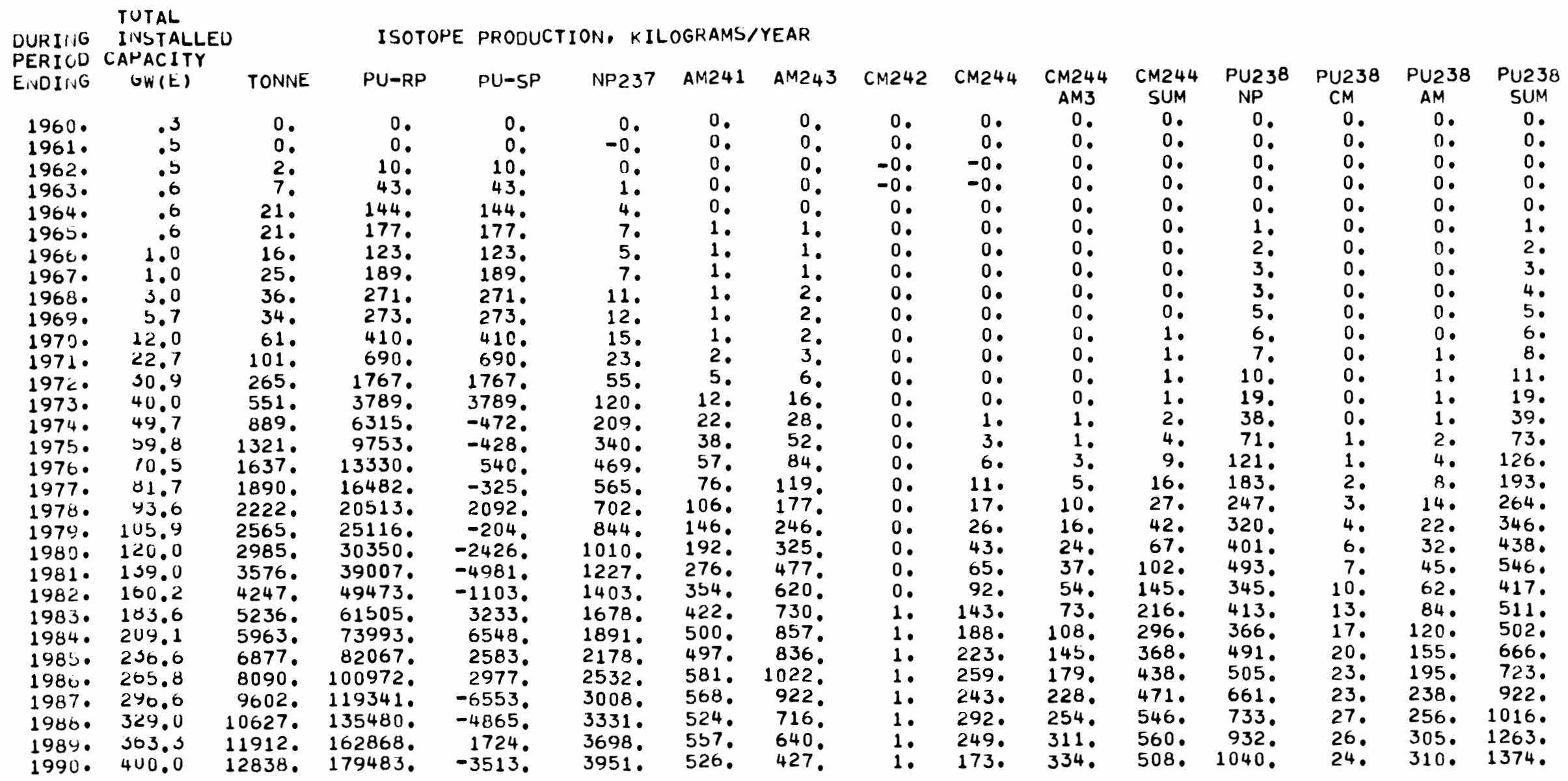


NET ANNUAL I SOTOPE AVAILAB I L I T Y

PAGE 143

(WITH TARGET IRRADIATION. PROCESS LOSSES AND DECAY LOSSES)

CASE 6 FOR 120 GW(E) AT 1980

CASE 8

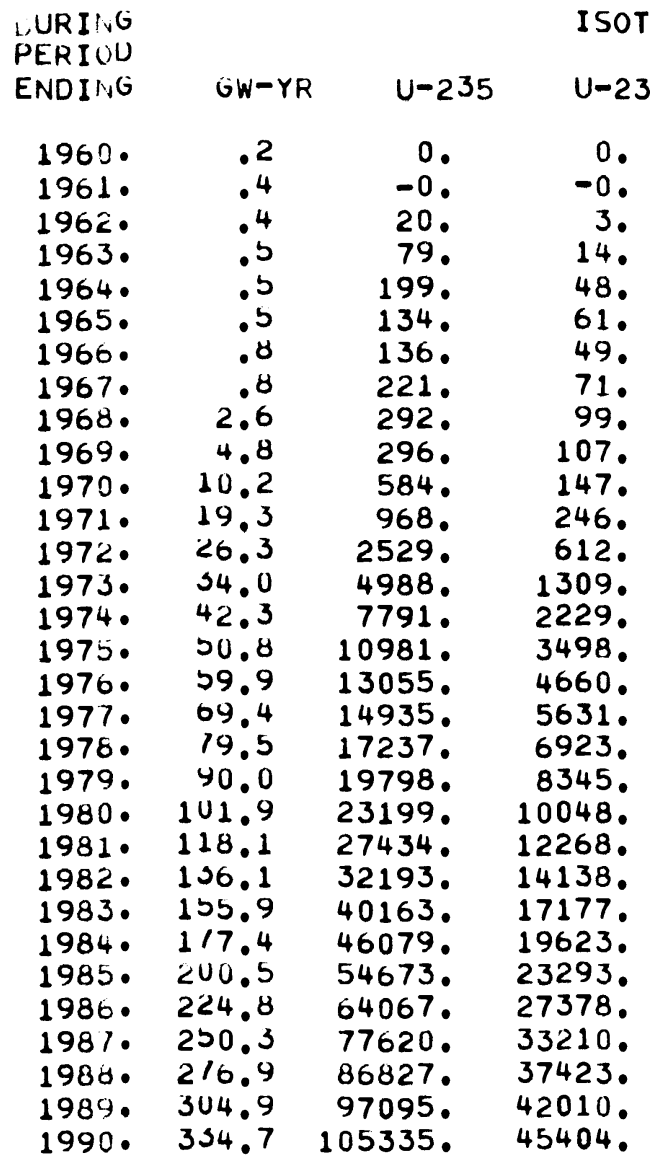

\section{SOTOPE PRODUCTION, KILOGRAMS/YEAR}

\begin{tabular}{|c|c|c|c|}
\hline$P M_{147}$ & CE144 & $\operatorname{cs} 137$ & $S R-90$ \\
\hline 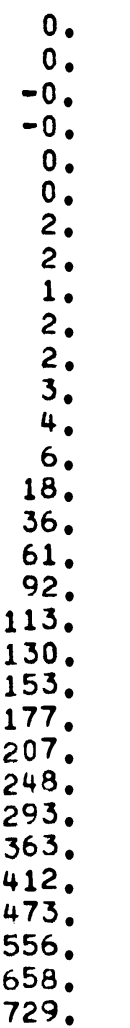 & 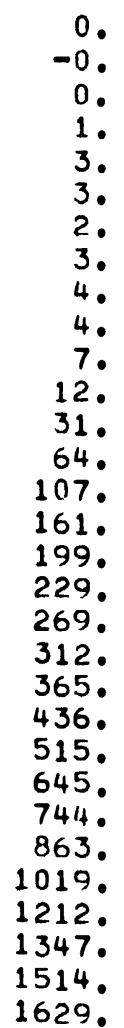 & $\begin{array}{r}0 . \\
-0 . \\
1 . \\
3 . \\
20 . \\
19 . \\
13 . \\
24 . \\
28 . \\
30 . \\
42 . \\
65 . \\
199 . \\
400 . \\
719 . \\
1119 . \\
1376 . \\
1602 . \\
1899 . \\
2213 . \\
2596 . \\
3114 . \\
3600 \\
4367 . \\
4851 . \\
5422 . \\
6264 . \\
7243 . \\
7843 . \\
8691 . \\
9237 .\end{array}$ & 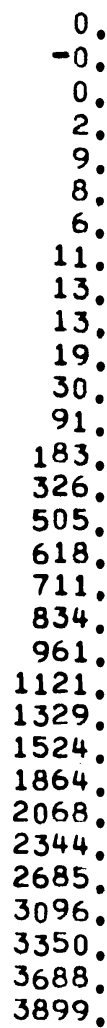 \\
\hline
\end{tabular}

\begin{tabular}{|c|c|c|}
\hline$K R-85$ & $T C-99$ & $\underset{\text { SUM }}{\text { RHIO3 }}$ \\
\hline 0 . & 0 . & 0. \\
\hline 0. & -0 . & -0 . \\
\hline 0. & 1. & 0. \\
\hline 0. & 2 . & 1. \\
\hline 0. & 14. & 7. \\
\hline 0 . & 13. & 7. \\
\hline 0 . & 9. & 5. \\
\hline 0. & 17. & 9. \\
\hline 1. & 20. & 10. \\
\hline 1. & 20. & 11. \\
\hline 1. & 29. & 16. \\
\hline 1. & $45^{\circ}$ & $\begin{array}{l}25 . \\
72 .\end{array}$ \\
\hline 8. & $276^{\circ}$ & 146. \\
\hline 14. & 495. & 257. \\
\hline 22. & 768. & 397. \\
\hline 31. & $\begin{array}{r}944^{\circ} \\
1096 .\end{array}$ & $\begin{array}{l}490^{\circ} \\
572 .\end{array}$ \\
\hline 36. & 1298. & 682. \\
\hline 42. & $1510^{\circ}$ & 797. \\
\hline $\begin{array}{l}49^{\circ} \\
58 .\end{array}$ & $\begin{array}{l}1769^{\circ} \\
2120^{\circ}\end{array}$ & $\begin{array}{r}930 . \\
1131 .\end{array}$ \\
\hline 67. & 2451 . & 1322. \\
\hline 82. & 2978. & 1601. \\
\hline 104. & 3707 . & 1990. \\
\hline 119. & 4282 . & 2317 . \\
\hline 138. & 4957 . & 2690. \\
\hline 166 & 5 & $3214^{\circ}$ \\
\hline 177. & J & 3412 . \\
\hline
\end{tabular}

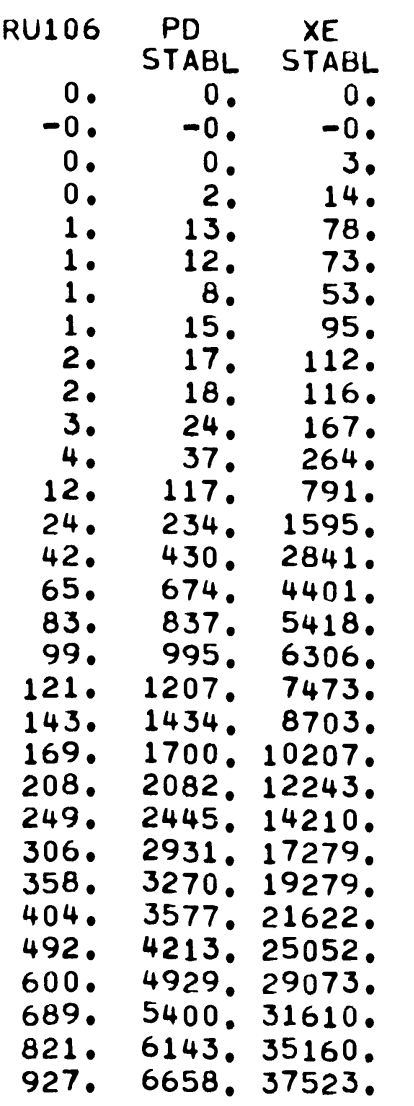

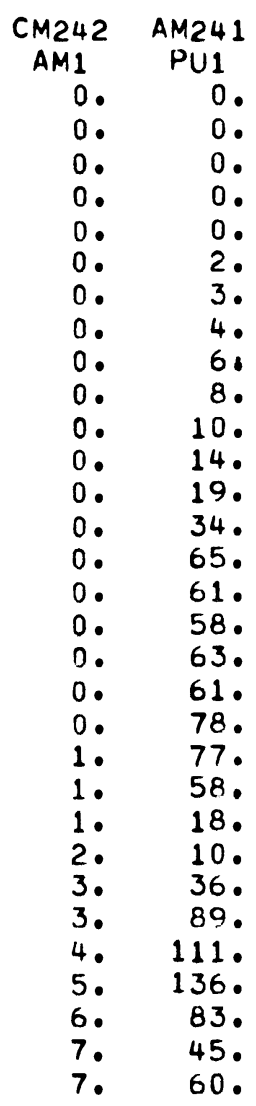


NET ANNUAL I SOTTOPE AVAILABILI T Y

(WITH TARGET IRRADIATION, NROCESS LOSSES ANU DECAY LOSSES)

CASE 6 FOR $120 \mathrm{GW}(\mathrm{E})$ AT 1980
PAGE 144

CASE

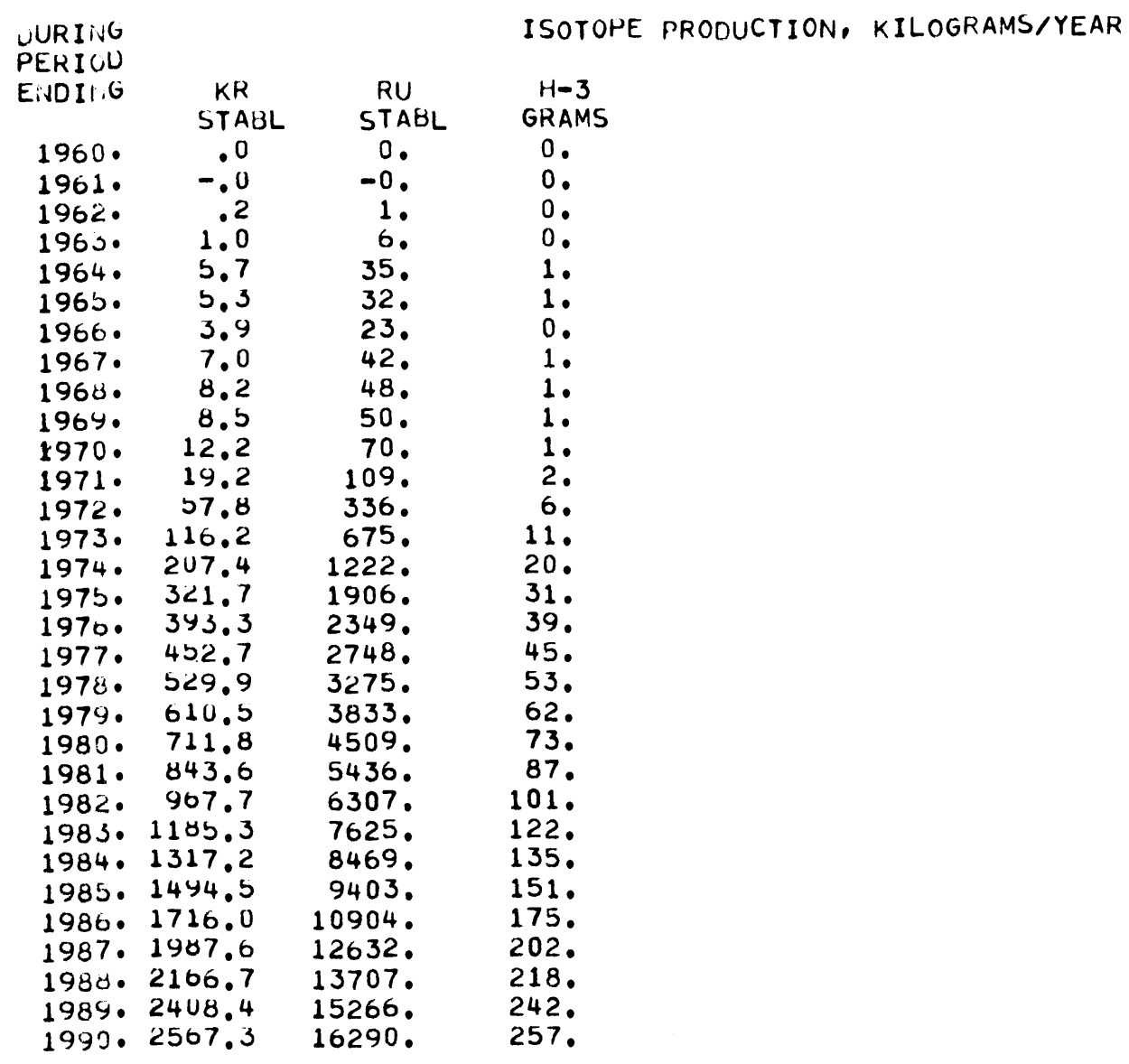


(WITH TARGET IRRACIATION. PROCESS LOSSES AND DECAY LOSSES)

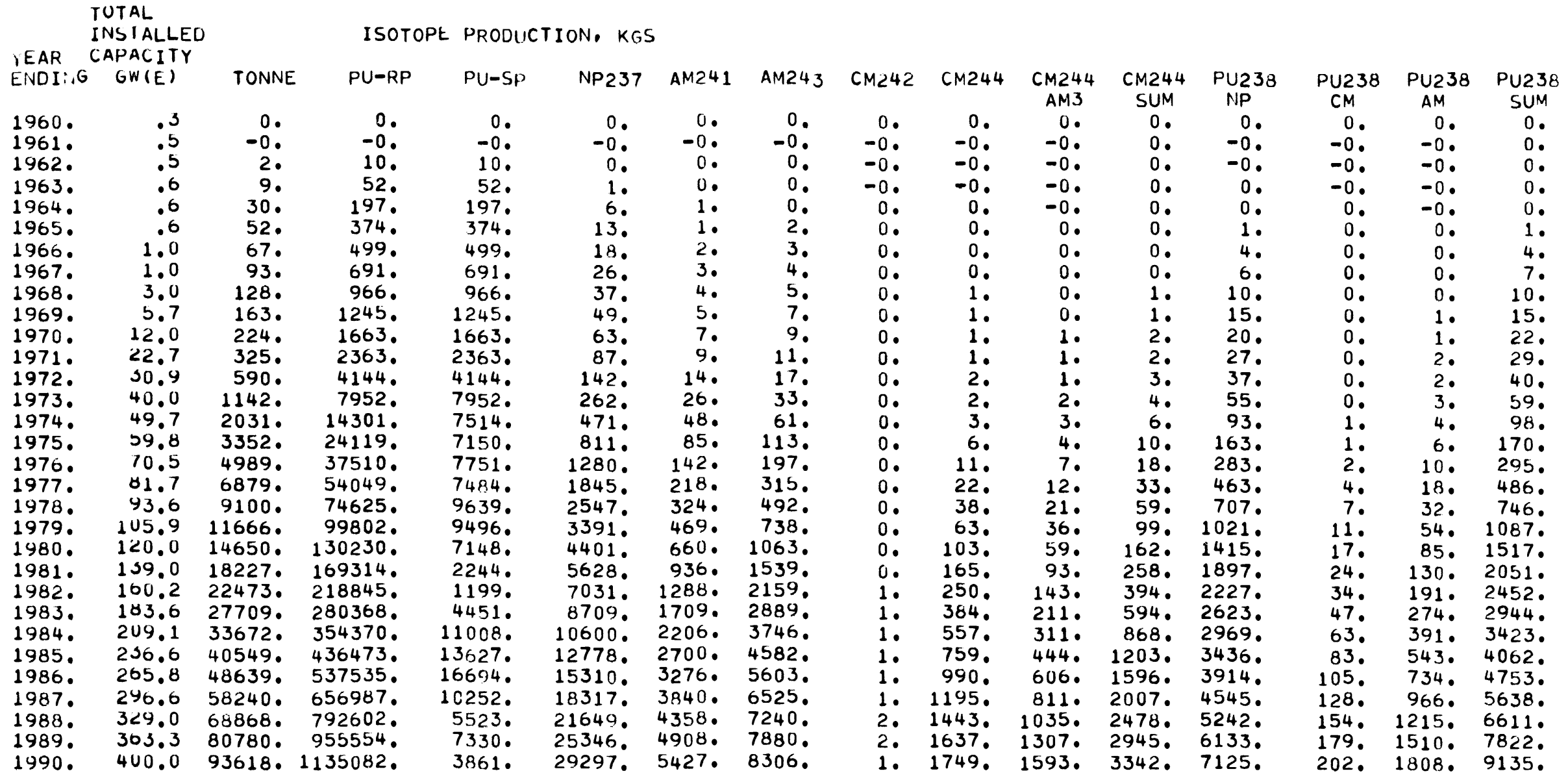


(WITH TARGET IRRADIATION, PROCESS LOSSES AND DECAY LOSSES)

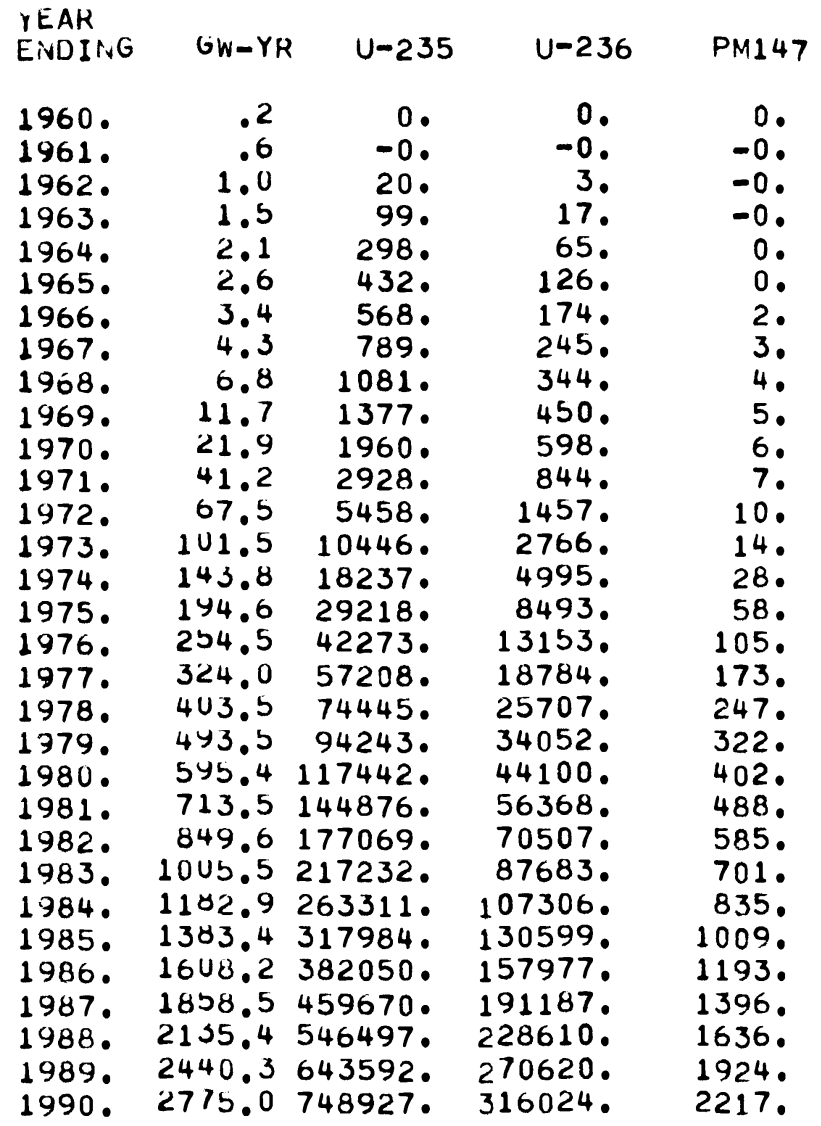

\begin{tabular}{|c|c|c|}
\hline CE 144 & CS137 & $S R-90$ \\
\hline 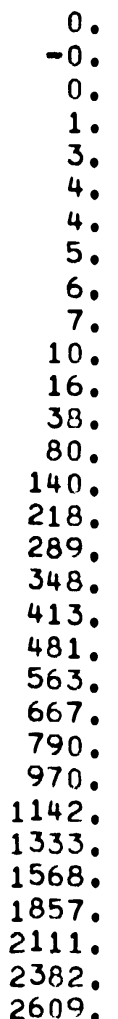 & 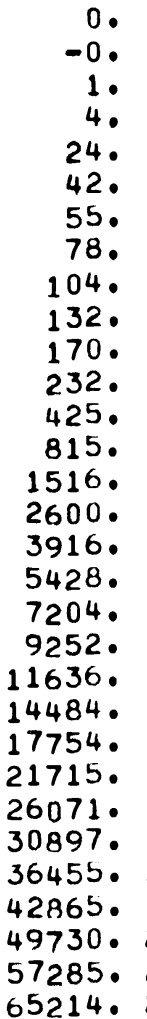 & 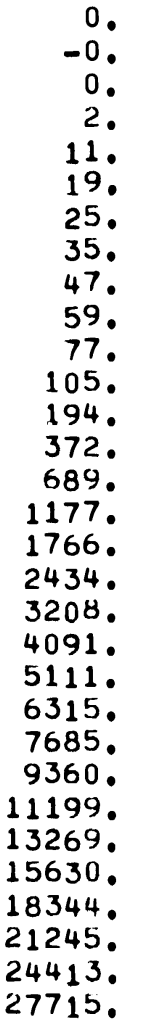 \\
\hline
\end{tabular}

\begin{tabular}{|c|c|c|}
\hline \multicolumn{3}{|r|}{$\begin{array}{c}\text { RH103 } \\
\text { SUM }\end{array}$} \\
\hline 0. & 0. & $\begin{array}{l}\text { SUM } \\
0 .\end{array}$ \\
\hline-0 & -0 & -0. \\
\hline 0 . & 1. & 0. \\
\hline 0 . & 3. & 2 \\
\hline 0. & 17. & 9. \\
\hline 1. & 30. & 16. \\
\hline 1. & 39. & 21. \\
\hline 1. & 56. & 29. \\
\hline 2 . & 75. & 40. \\
\hline 2. & 96. & 51. \\
\hline 3. & 124. & 66. \\
\hline $\begin{array}{l}4 . \\
8 .\end{array}$ & $\begin{array}{l}170 . \\
307 .\end{array}$ & $\begin{array}{r}91^{\circ} \\
163^{\circ}\end{array}$ \\
\hline 16. & 583. & 309 . \\
\hline 29. & 1078. & 566. \\
\hline $\begin{array}{l}49 . \\
73 .\end{array}$ & $1847^{\circ}$ & 963. \\
\hline 100 & 3886 . & 2025 . \\
\hline 130 . & 5184. & 2707 . \\
\hline 164. & 6694. & 3504 . \\
\hline $\begin{array}{l}202 . \\
248 .\end{array}$ & 8463 & 4440. \\
\hline $\begin{array}{l}248 . \\
299 .\end{array}$ & $\begin{array}{l}10583 . \\
13034\end{array}$ & $\begin{array}{l}5571 . \\
6893 .\end{array}$ \\
\hline 362. & 16013 . & 8493. \\
\hline 30 . & 19322. & 10278 \\
\hline & 23029 & 12268 . \\
\hline & 27312 . & 14584 \\
\hline 5 & 327 & $17274^{\circ}$ \\
\hline & 4 & 233 \\
\hline & 4 & 68 \\
\hline
\end{tabular}

\begin{tabular}{|c|c|c|}
\hline \multirow{2}{*}{\multicolumn{3}{|c|}{ RU106 }} \\
\hline & & \\
\hline \multirow{2}{*}{$\begin{array}{r}0 \\
-0\end{array}$} & 0 . & 0 . \\
\hline & -0 & -0 . \\
\hline \multirow{2}{*}{$\begin{array}{l}0 . \\
0 .\end{array}$} & 0 . & 3. \\
\hline & 2. & 17. \\
\hline \multirow{2}{*}{$\begin{array}{l}1 . \\
2 .\end{array}$} & 15. & 96. \\
\hline & 26. & 169. \\
\hline \multirow{2}{*}{$\begin{array}{l}2 . \\
2 .\end{array}$} & 34. & 222 . \\
\hline & 49. & 317. \\
\hline 3. & 66. & 429. \\
\hline \multirow{2}{*}{$\begin{array}{l}3 . \\
4 .\end{array}$} & 84. & 545. \\
\hline & 108. & 712. \\
\hline \multirow{4}{*}{$\begin{array}{l}6 . \\
15 . \\
32 \\
58 \\
94 .\end{array}$} & $\begin{array}{l}145 . \\
261 .\end{array}$ & $\begin{array}{r}976^{\circ} \\
1767 .\end{array}$ \\
\hline & 495 & 3361 . \\
\hline & 925. & 6202 \\
\hline & 1599. & 10603. \\
\hline \multirow{3}{*}{$\begin{array}{l}164^{\circ} \\
203 . \\
245 .\end{array}$} & 3431. & 22327 . \\
\hline & $4639^{\circ}$ & 29799. \\
\hline & 6073. & 38502 \\
\hline \multirow{6}{*}{$\begin{array}{l}292^{\circ} \\
354^{\circ} \\
426^{\circ} \\
519^{\circ} \\
617 . \\
712^{\circ} \\
848^{\circ}\end{array}$} & 7773. & 48710. \\
\hline & 9855. & 60953. \\
\hline & 15231 . & 92441 . \\
\hline & 18501. & $111720^{\circ}$ \\
\hline & 78. & 133342. \\
\hline & & \\
\hline \multirow{3}{*}{$\begin{array}{l}1024^{\circ} \\
1201 . \\
1422^{\circ} \\
1638^{\circ}\end{array}$} & 9. & 187467. \\
\hline & 0. & 9077. \\
\hline & 104? & $91760^{\circ}$ \\
\hline
\end{tabular}

CM242 AM241

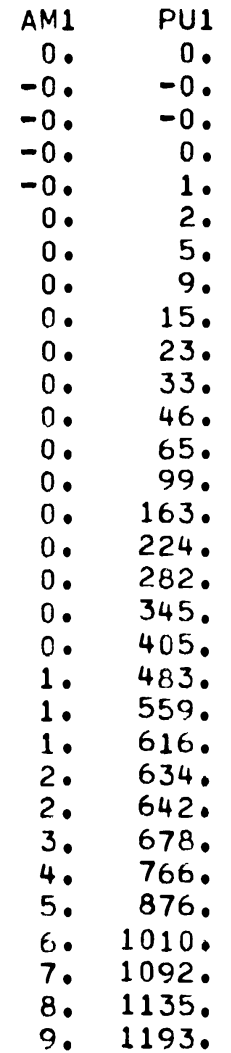


(WITH TARGET IRRADIATION, PROCESS LOSSES AND DECAY LOSSES)

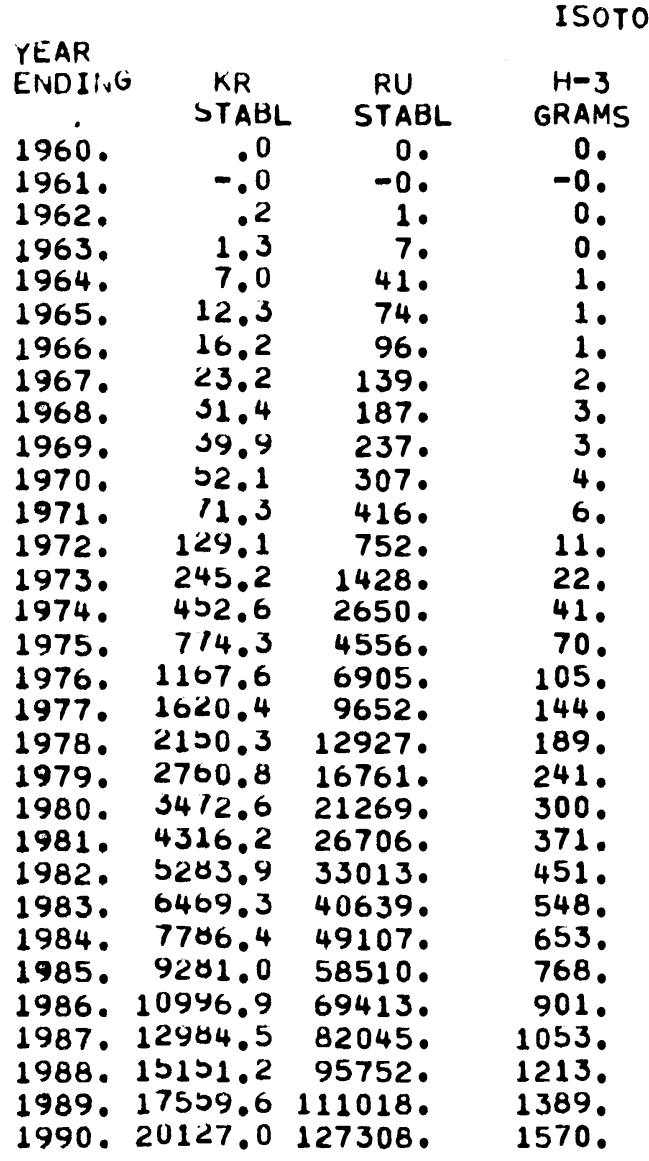




\section{DISTRIBUTION}

Number of Copies
Division of Technical Information Extension (UC-23)

Atomic Energy Commission, Washington, D.C.

Division of Production

F. P. Baranowski

Division of Reactor Development and Technology

J. Whitman

S. Strauch

D. Erb (3)

Division of Isotope Development

G. Y. Jordy (5)

J. Maddox

Division of Isotope Development, Space Nuclear Systems

J. Powers

IM. Gatanis

Atomic Energy Commission - Richland, Washington

C. L. Robinson (5)

M. R. Schneller (8)

Technical Information Library

AECOP, Oak Ridge, Tennessee

J. Schacter

Atomic Energy Commission, Chicago Patent Group

G. H. Lee

R. K. Sharp

Atomic Energy Commission, RDT Site Representative

P. G. Holsted (3)

NASA Lewis Research Center

NA.SA Lewis Research Center, Sandusky

Rand Corporation

Battelle Memorial Institute 
Douglas United Nuclear, Inc.

T. W. Ambrose

L. E. Kusler

L. W. Lang

R. Nilson

G. F. Owsley

W. K. Woods

Files (6)

Atlantic Richfield Hanford Company

S. J. Beard

J. S. Cochran (4)

O. F. Hill

W. W. Kinney

H. C. Rathvon

H. P. Shaw

A. E。 Smith

M。 J。Szulinski

R. E. Tomlinson

Document Classification and Custody (5)

Computer Science Corporation - Richland

R. J. Shields

Battelle-Northwest

F. W. Albaugh

J. M. Atwood

J. M. Batch

C.A. Bennett

T. K. Bierlein

A. G. Blasewitz

C. H. Bloomster

D. W. Bolme

J. G. Bradley

J. B. Burnham

R. E。 Burns

G. J. Busselman

J. L. Carter

C. R. Cooley

E. A. Coppinger

G. M. Dalen

F. G. Dawson, Jr。

D. E. Deonigi

D. R. deHalas

K. Drumheller

R. L. Engel

E. A. Eschbach (5)

E. A. Evans 
J. F. Fletcher

J. C. Fox

J. R. Fishbaugher

J. J. Fuquay

D. P. Granquist

D. R. Haffner

W. A. Haney

H. Harty

P. L. Hofmann

P. A. Horton

L. D. Jacobson

B. M. Johnson

R. A. R. Kent

D. P. Konichek

D. D. Lanning

B. B. Lane

R. C. Liikala

C. W. Lindenmeier

R. W. McKee (75)

E. T. Merrill

R. L. Moore

J. H. Nail

S. E. Nunn

H. M. Parker

A. M. Platt

W. W. Porath

R. G. Rau

W. A. Reardon

R. L. Reynolds

C. A. Rohrmann (5)

L. C. Schmid

E. A. Schnaible

A. M. Schneider

K. J. Schneider

L. C. Schwendiman

R. E. Shaver

R. E. Shippert

R. I. Smith

C. R. Tipton, Jr.

C. J. Touhill

R. E. Turley

H. H. Van Tuyl

E. E. Voiland

G. L. Wilfert

J. R. Worden

K. E. Yandon

Technical Information Files (5) 


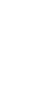

